\title{
Carboxylic Acids Under Vibrational Scrutiny: Experimental Reference Data to Benchmark Quantum Chemical Calculations
}

\author{
Dissertation \\ for the award of the degree \\ „Doctor rerum naturalium" \\ of the Georg-August-Universität Göttingen
}

within the doctoral program chemistry

of the Georg-August University School of Science (GAUSS)

submitted by

Katharina Meyer

from Stadthagen

Göttingen, 2019 


\section{Thesis Committee}

Prof. Dr. Martin A. Suhm, Institut für Physikalische Chemie

Prof. Dr. Dirk Schwarzer, Max-Planck-Institut für Biophysikalische Chemie

Prof. Dr. Ricardo A. Mata, Institut für Physikalische Chemie

\section{Members of the Examination Board}

\section{Reviewer:}

Prof. Dr. Martin A. Suhm, Institut für Physikalische Chemie

Second Reviewer:

Prof. Dr. Dirk Schwarzer, Max-Planck-Institut für Biophysikalische Chemie

\section{Further members of the Examination Board:}

Prof. Dr. Ricardo A. Mata, Institut für Physikalische Chemie

Prof. Dr. Jörg Behler, Institut für Physikalische Chemie

Dr. Oliver Bünermann, Institut für Physikalische Chemie

Dr. Sebastian Kruss, Institut für Physikalische Chemie

Date of the oral examination: 17.12.2019 


\section{Acknowledgements}

This work would not have been possible without the help and support of many people, for which I am very grateful.

First and foremost, I would like to thank my supervisor Martin Suhm for his continuous encouragement, guidance, inspiring and enthusiastic discussions, and all the opportunities he created for me ever since I joined his group.

I would also like to thank Dirk Schwarzer for undertaking the co-supervision of this work, Ricardo Mata for taking the time to be on my thesis committee as well as the other members of the examination board Jörg Behler, Oliver Bünermann, and Sebastian Kruss for taking the time to evaluate my work.

Furthermore, I would like to thank Andrew Ellis and Julia Davies for hosting me during a research stay at the University of Leicester, for introducing me to the helium nanodroplet experiment, and for the many helpful and thorough discussions about the results.

For the financial support, I would like to thank the German Research Foundation (Deutsche Forschungsgemeinschaft, DFG, projects 388861488/DFGSU121/7-1 and 389479699/GRK2455), without which this work would not have been possible. In addition, I would like to thank all members of the Research Training Group (RTG) "2455 Benchmark Experiments for Numerical Quantum Chemistry" for the welcoming and collaborative working atmosphere. I am also grateful for the financial support of the German Academic Exchange Service (Deutscher Akademischer Austauschdienst, DAAD), which funded my participation at the 22. Horizons in Hydrogen Bond Research (HBOND) Conference in Jyväskylä, Finland in 2017.

A lot of this work would not have been possible without the creativity and support from the institute workshops, especially the mechanical workshop team. I would like to thank Reinhard Hildebrandt, Volker Meyer, and Mike Zippert for their inventiveness with the various nozzle designs, several broken set-up parts, as well as the design of the new detector table.

During my time in the Suhm group, I had the great opportunity to work with many talented and motivated students who greatly contributed to this work - Sophie Schweer and Lea Leibold as part of their Bachelor theses and Beppo Hartwig and Michael Schwarzer during research internships.

I would like to thank all members of the Suhm group for their tremendous support, their patience with my lack of talent in table football matches and my excitement for semigood football teams, and for the fun times during conferences, group trips, and the many pizza/board games nights. I would like to thank Sönke Oswald, Hannes Gottschalk, Anja Poblotzki, and Thomas Forsting for their help with all kinds of experimental and nonexperimental troubles I encountered during my work. I want to thank Arman Nejad for 
our joint ventures with deuterated formic acid and his excitement in all discussions, scientific as well as non-scientific. I am also very grateful to Sönke Oswald, Arman Nejad, Anja Poblotzki, Hannes Gottschalk, and Nils Lüttschwager for proof-reading parts of this thesis and their helpful comments. Moreover, I would like to thank Petra Lawecki, not only for her help with administrative tasks, but also for her general support and encouragement throughout my whole time in the Suhm group.

Finally, I would like to thank my family and friends for their patience and continuous support. 


\section{Contents}

1 Introduction 1

2 Background 7

2.1 Vibrational Anharmonicity . . . . . . . . . . . . . . . . . . . 7

2.2 Supersonic Expansions . . . . . . . . . . . . . . . . . . . . . 8

3 Experimental and Theoretical Methods $\quad 15$

3.1 Experimental Set-ups . . . . . . . . . . . . . . . . 15

3.1 .1 The Chilli Jet . . . . . . . . . . . . . . . . 15

3.1 .2 The Filet Jet . . . . . . . . . . . . . . . . . . . . . 26

3.1 .3 The Curry Jet. . . . . . . . . . . . . . . . . . 27

3.1.4 Summary of the Differences of the Three Jet Set-ups . . . . . . . 30

3.1.5 Concentration Determination . . . . . . . . . . . . 31

3.1.6 The Helium Nanodroplet Set-up . . . . . . . . . . . . . . . . . . . 32

3.2 Theoretical Methods . . . . . . . . . . . . . . . . . . . 33

3.2.1 Band Position Assignments . . . . . . . . . . . . . . . . 33

3.2.2 Performance Tests of Different Methods . . . . . . . . . . . . . 35

4 Monomers $\quad 37$

4.1 Introduction . . . . . . . . . . . . . . . . . . . 37

4.2 trans-Formic Acid Fundamentals . . . . . . . . . . . . . . . 41

4.2 .1 Literature values . . . . . . . . . . . . . . . . . . . 44 41

4.2 .2 Fermi resonance $\nu_{5}$ and $2 \nu_{9} \ldots \ldots \ldots \ldots$. . . . . . . . . . . . . . . . . . . . . . . 44

4.3 cis-Formic Acid Fundamentals . . . . . . . . . . . . . . . . . . . . . . . . 49

4.3.1 Vibrational Detection of cis-Formic Acid . . . . . . . . . . . . . . 49

4.3.2 Hot Band Assignments of trans-Formic Acid . . . . . . . . . . . . . . 55

4.3.3 Summary of cis-Formic Acid Fundamentals . . . . . . . . . . . . 58

4.4 Vibrational Benchmark . . . . . . . . . . . . . . . . . . 59

4.4.1 Instabilities of DFT Functionals . . . . . . . . . . . . . . 66

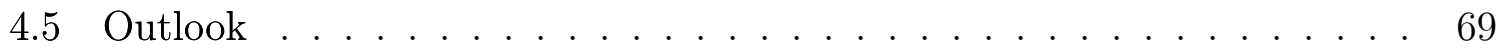

4.5.1 Introduction . . . . . . . . . . . . . . . . 69

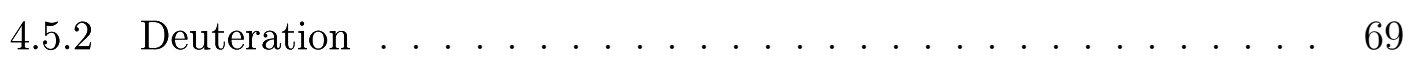

4.5.3 cis-trans-Isomerism of Larger Carboxylic Acids . . . . . . . . . . 77

4.5 .4 Summary . . . . . . . . . . . . . . . . . . . 79

5 Globally and Locally Stable Dimers $\quad 81$

5.1 Introduction . . . . . . . . . . . . . . . . . . 81 
5.2 Helium Nanodroplet Spectra . . . . . . . . . . . . . . . . . . . . 85

5.2.1 Experimental Spectra. . . . . . . . . . . . . . 85

5.2.2 Comparison to Quantum Chemical Calculations . . . . . . . . . . 87

5.2.3 Differences in Formic and Acetic Acid Local Minima . . . . . . . 93

5.3 Exciton Coupling in Homo and Hetero Dimers of Carboxylic Acids . . . . 95

5.3.1 Structures and Dissociation Energies of Homo and Hetero Dimers 98

5.3.2 FTIR and Raman Jet Spectra of Hetero Dimers . . . . . . . . . . . . 99

5.3.3 Exciton Shifts and Couplings . . . . . . . . . . . . . 105

5.3.4 Vibrational Benchmark . . . . . . . . . . . . . . . . 107

5.3 .5 Outlook ............................ 114

5.3 .6 Summary . . . . . . . . . . . . . . . . . 117

6 Aggregation Dynamics: Trimers and Metastable Dimers 121

6.1 Introduction . . . . . . . . . . . . . . . . . . . . . . 121

6.2 Formic Acid Trimers . . . . . . . . . . . . . . . . . . . . 123

6.2.1 Trimer structures . . . . . . . . . . . . . . . . 123

6.2.2 FTIR and Raman Spectra . . . . . . . . . . . . . . . . 125

6.2.3 Aggregation Dynamics in Supersonic Expansions . . . . . . . . . 128

6.3 Acetic and Pivalic Acid Trimers . . . . . . . . . . . . . . . 135

6.3.1 Comparison to the Formic Acid Spectra . . . . . . . . . . . . . 135

6.3.2 Acetic Acid Trimers . . . . . . . . . . . . . . . . . . . . . . 137

6.3 .3 Pivalic Acid Trimers . . . . . . . . . . . . . . . . . . . 141

$6.4 \mathrm{Z}$ bands . . . . . . . . . . . . . . . . . . . . . . 144

6.5 Summary and Outlook . . . . . . . . . . . . . . . . 151

7 Dimer Assemblies $\quad 155$

7.1 Introduction . . . . . . . . . . . . . . . . . . . . 155

7.2 High Concentration Measurements . . . . . . . . . . . . . . 158

7.3 Influence of the Carrier Gas . . . . . . . . . . . . . . . . . . . 161

7.3.1 Comparison to Quantum Chemical Calculations . . . . . . . . . 163

7.3.2 Formation of Dimer Assemblies . . . . . . . . . . . . . . . 166

7.4 Outlook . . . . . . . . . . . . . . . . . . . . . 169

7.5 Summary . . . . . . . . . . . . . . . . . . . 172

8 Summary and Outlook $\quad 175$

A Appendix 213

A.1 Experimental and Theoretical Methods . . . . . . . . . . . . . . . . 213

A.1.1 Chilli Jet . . . . . . . . . . . . . . . . . . . . . . 213

A.1.2 FTIR Components and Settings . . . . . . . . . . . . . . . 215

A.2 Carboxylic Acid Monomers . . . . . . . . . . . . . . . . . . . . . 219

A.2.1 Scaled Harmonic Frequency Calculations . . . . . . . . . . . . . 219

A.2.2 Vibrational Benchmark: C-H Stretching Vibration . . . . . . . . 219

A.2.3 Instabilities of DFT Functionals . . . . . . . . . . . . . . . 219 
A.2.4 C-H stretching vibration of HCOOD . . . . . . . . . . . . . 221

A.2.5 Fully Deuterated Formic Acid . . . . . . . . . . . . . . . . . 222

A.2.6 C-O stretching vibration of HCOOD . . . . . . . . . . . . . . . . . . . 222

A.2.7 Cis-Acetic and -Pivalic Acid Measurements . . . . . . . . . . . . . 224

A.3 Carboxylic Acid Dimers . . . . . . . . . . . . . . . . . . . 226

A.3.1 Setting Sensitivity of VPT2 Calculations . . . . . . . . . . 226

A.3.2 Combination Vibrations of the $\mathrm{F}_{\mathrm{C}} \mathrm{F}$ dimer . . . . . . . . . . . . 230

A.3.3 Coupling Diagrams of the Homo and Hetero Dimers . . . . . . . . 231

A.3.4 Anharmonic Frequency Calculations of the Formic Acid Dimer . . 231

A.4 Carboxylic Acid Trimers . . . . . . . . . . . . . . . . . . 236

A.4.1 Formic Acid Trimers . . . . . . . . . . . . . . . . . . . 236

A.4.2 Acetic Acid Higher-Energy Dimers and Trimers . . . . . . . . . . 237

A.4.3 Pivalic Acid Higher-Energy Dimers . . . . . . . . . . . . . . . . 239

A.4.4 Mixed Trimers . . . . . . . . . . . . . . . . . . . . . 239

A.4.5 Performance of Quantum Chemical Methods . . . . . . . . . . . 241

A.5 Dimer Assemblies . . . . . . . . . . . . . . . . . . . . . . . . . 242

A.6 Summary of Key Experimental Observables for Benchmarking Quantum

Chemical Methods . . . . . . . . . . . . . . . . . . 243

A.6.1 The Formic Acid Monomer . . . . . . . . . . . . . . . . . . 243

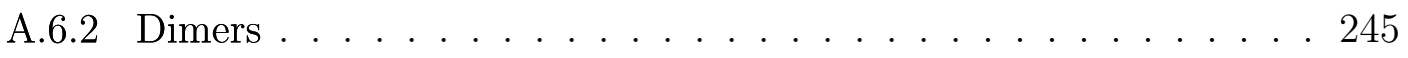





\section{Introduction}

Vibrational spectroscopy is an effective and powerful tool for gaining insight into the dynamical behaviour and structural properties of molecules and their clusters. The interpretation of vibrational spectra and assignment of bands, however, can be very challenging and difficulties increase significantly with the system size due to spectral congestion. One way of dealing with spectral ambiguity is to use multi-experimental approaches such as joint conventional FTIR and Raman spectroscopy studies $[1,2]$ or their combination with isomer-selective techniques such as IR/UV spectroscopy $[1,3-8]$. The latter comes at the cost of system limitations, in this case, the requirement for an UV chromophore. For precise structural information, combined vibrational and rotational spectroscopy studies are a prominent choice [7-10]. In addition to these experimental means, one of the most important resources for spectral analysis are quantum chemical calculations. These are typically performed within the double harmonic approximation, meaning that the potential energy function of the system is described by uncoupled quadratic potentials and that the property surfaces (e.g. dipole moment and polarisability) are assumed to depend linearly on atomic displacements. With this approach, good estimates of relative quantities such as band position shifts can be obtained. However, the harmonic frequencies of (intramolecular) vibrations are typically higher than the (anharmonic) experimental values [11]. One pragmatic way of dealing with the neglect of anharmonicity as well as errors resulting from shortcomings of the underlying electronic structure treatment is to introduce scaling factors [12, 13]. Two strategies are established - uniform scaling of harmonic frequencies [11, 12, 14-19], either globally or for different spectral regions, and empirical scaling of harmonic force constants [20-29] where each type of vibration (stretching, bending, torsion) is treated separately [29].

While for band position assignments of the fundamentals of a molecule scaled harmonic frequencies might suffice, little to no information about Fermi resonances, overtones, combination and hot bands are attainable without an explicit treatment of anharmonicity. That, however, is cost-intensive and limited to relatively small molecules [2931]. With increasing computer power and developments of more effective computational methods, larger systems come into reach [32]. Among the most popular methods for anharmonic description of these is second-order vibrational perturbation theory (VPT2), which treats anharmonicity as a small correction to the harmonic oscillator ensemble [33-35]. VPT2 is implemented in common quantum chemical program packages such as Gaussian [36], ORCA [37, 38], Molpro [39], or CFOUR [40] and has been shown to perform particularly well for relatively rigid molecules [32], but reaches its limits for highly anharmonic, large amplitude motions that diverge from the equilibrium geometry [32]. A way to systematically account for anharmonicity is to start with a mean-field description, i.e., the vibrational self-consistent field (VSCF) approach [41-46]. In analogy to 
electronic structure methods, refined extensions to VSCF are vibrational Møller-Plesset perturbation theory (VMP2) [47-49], vibrational configuration interaction (VCI), and vibrational coupled cluster theory (VCC) [50-52]. Another ansatz is based on solving the time-dependent Schrödinger equation, as done in the multi-configuration time-dependent Hartree (MCTDH) method [53].

To enable further progress of anharmonic vibrational frequency calculations, rigorous benchmarking of the results is crucial. A suitable reference system for such a benchmark must fulfil several requirements: Desirably, the selected molecules are small and rigid, so that these are amenable for various theoretical treatments from relatively simple approximations up to high levels of theory. While the global minimum structures are vital in this endeavour, spectroscopic data on higher-energy conformers is equally as important, because otherwise a thorough validation of the globality of a potential energy surface (PES) prediction is not feasible. Furthermore, to provide enough flexibility, the size and therefore the complexity of the system should be easily increasable, as the agreement of a theoretical method with only one experimental data point has no predictive significance - it can merely be a result of fortuitous error compensation. From an experimental perspective, particularly those molecules or molecular clusters that have been critically examined by a multitude of different techniques are an excellent fit for such a study, as ambiguous assignments are typically well-known, so that these can be tackled by the interplay of theoretical predictions and specifically tailored experiments. Hence, theory can be as much of service to experiment than the other way around.

Another crucial aspect for benchmarking, in addition to an appropriate choice of system, is the type of experiment. For a meaningful comparison, experimental conditions such as environment or temperature must match those assumed in the theoretical model as closely as possible. For example, a spectrum of molecules embedded in solvent at room temperature does not suffice to benchmark quantum chemical calculations of isolated molecules at $0 \mathrm{~K}$ in the gas phase. For the anharmonic description of small reference systems, however, such calculations are the standard. A suitable technique for benchmarking these is a supersonic expansion, where a gas is rapidly cooled below its condensation point by expanding it from a high pressure source through an orifice into a low pressure background. If the conditions are chosen accordingly, the expansion leads to substantial translational, rotational, and to some extent also vibrational cooling, while the molecules and molecular clusters remain isolated in the gas phase [54-56]. The advantage compared to gas phase spectra is that thermal shifts of bands are largely avoided, which is why supersonic jet techniques are used throughout this work.

One example of an accomplished reference system for benchmarking are carboxylic acid monomers and dimers, with formic acid as the simplest and experimentally best characterised one (see Chapters 4 and 5 for references). The monomers exhibit rotational isomerism around the $\mathrm{C}-\mathrm{O}$ bond resulting in two $C_{\mathrm{s}}$-symmetric cis- and transconformers, as shown in Figure 1.1. The higher-energy cis-rotamer of formic acid cF is experimentally not nearly as well characterised as the global minimum trans-form $\mathrm{F}$ - only one out of its nine fundamentals had been assigned in the gas phase before 2018 [57]. The reason for this lack of data is the low gas phase abundance of merely $0.1 \%$ at room temperature [58]. In stark contrast to the gas phase, cis-formic acid has 

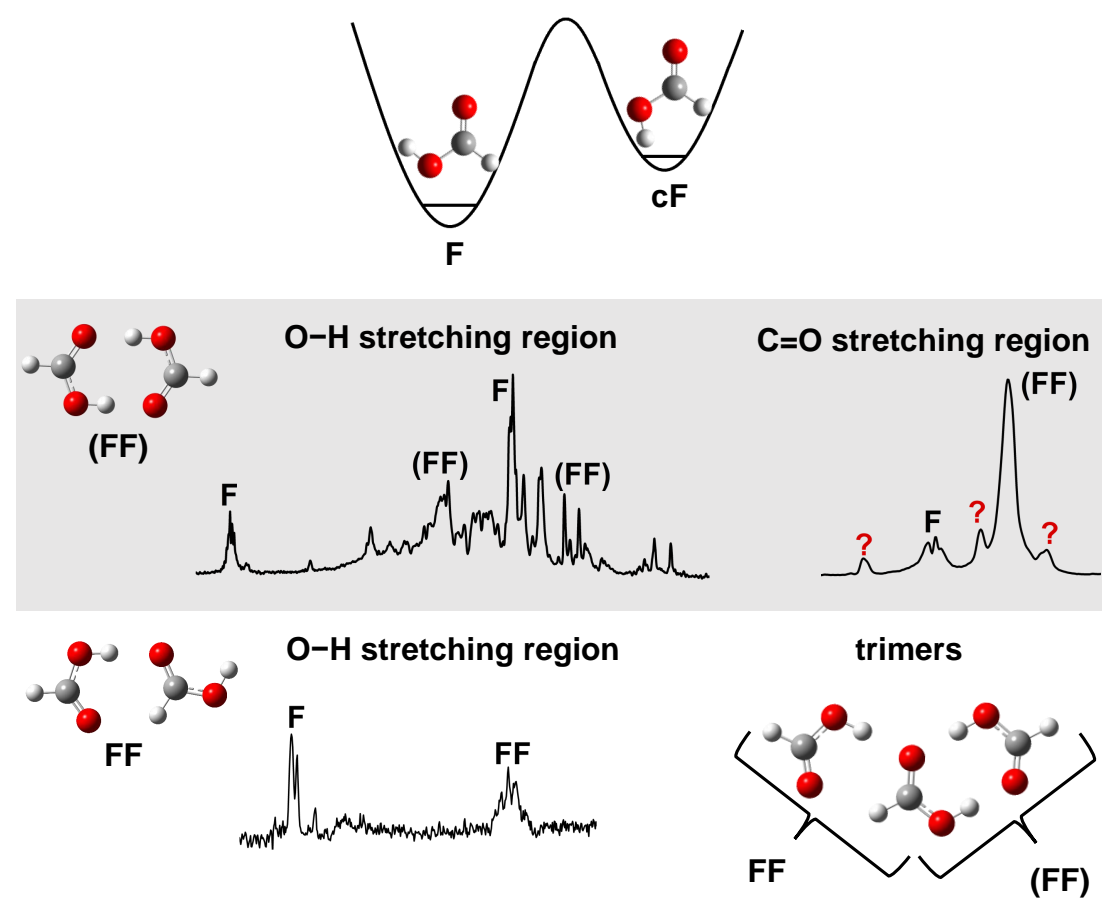

Figure 1.1: Schematic representation of topics of interest regarding the vibrational characterisation of formic acid and its hydrogen bonded clusters - the cis-trans-isomerism of the monomers, the $\mathrm{O}-\mathrm{H}$ and $\mathrm{C}=\mathrm{O}$ stretching region of the global minimum dimer $(\mathrm{FF})$, the significantly simpler $\mathrm{O}-\mathrm{H}$ stretching region of the higher-energy dimer $\mathrm{FF}$, as well as the structure and vibrational characterisation of the trimer.

been thoroughly studied in solid rare gas matrices via $\mathrm{O}-\mathrm{H}$ overtone excitation from the global minimum trans-form [59, 60]. The downside of cryogenic matrices is the host-guest interaction that shifts the vibrational bands of the trapped species relative to the isolated molecules in the gas phase. The sign and magnitude of matrix shifts can vary for different vibrations, as will be shown in several examples throughout this thesis. Consequently, matrix isolation spectroscopy is ultimately not suitable for benchmarking gas phase calculations.

The dimers of carboxylic acids form a doubly hydrogen bonded ring $\left(C_{2 \mathrm{~h}}\right.$ point group symmetry, see Figure 1.1). For a full vibrational characterisation, both infrared and Raman spectroscopy are needed, as the rule of mutual exclusion applies due to the inversion symmetry, meaning that the vibrations are split into pairs of exclusively Raman and IR active modes. However, beyond the pure necessity given by the molecular symmetry, Raman studies on carboxylic acid monomers and their clusters are surprisingly sparse [61-68] in comparison to the multitude of infrared studies (see Chapters 4 and 5 for references), even though Raman spectroscopy can provide complementary information, when assignments are in doubt, as will be showcased in several examples throughout this work.

One of the most interesting features of the global minimum dimers is the resonancebroadened $\mathrm{OH}$ stretching region, which is exemplarily shown for the formic acid dimer 
$(\mathrm{FF})$ in Figure 1.1. Despite many experimental and theoretical efforts [69-81], the interpretation of its resonance pattern still remains somewhat elusive. The local minimum structure closest in energy to the cyclic dimers is bound via just one strong $\mathrm{O}-\mathrm{H} \cdots \mathrm{O}=\mathrm{C}$ hydrogen bond in addition to a weaker $\mathrm{C}-\mathrm{H} \cdots \mathrm{O}=\mathrm{C}$ contact ( $\mathrm{FF}$ in Figure 1.1). These locally stable dimers were shown to be an intermediate in the formation of the cyclic dimers [82] and exhibit a distinctly narrower $\mathrm{OH}$ stretching region [83, 84], as illustrated for FF in Figure 1.1. Scrutinising the spectral differences between both dimer structures might therefore facilitate a better understanding of the ongoing dynamics. However, as the global minimum dimers dominate the spectra, weaker features of higher-energy dimers are difficult to identify in supersonic expansions [79]. An environment that is more suitable for their characterisation are helium nanodroplets, where the doubly hydrogen bonded cyclic dimers do not form as a result of the unique dipole-dipole driven cluster growth conditions within the droplets [83]. Helium nanodroplets reach a temperature as low as $0.37 \mathrm{~K}$ via evaporative cooling [85, 86], so that thermal effects on the spectra are negligible. Band position shifts caused by the host-guest interactions with the surrounding helium are typically smaller than for cryogenic matrices and on the order of just a few $\mathrm{cm}^{-1}$ [85]. However, larger shifts are found for hydrogen bonded clusters [87]. This is a result of a stiffening of the hydrogen bonds by the surrounding helium [88]. The resulting shift scales approximately linearly with the hydrogen bond induced downshift, as schematically shown for several examples in Figure 1.2. Thus, for a benchmark of the results, this helium solvent shift needs to be accounted for, which is done in this work by compressing the spectra accordingly, as will be detailed in Chapter 5.

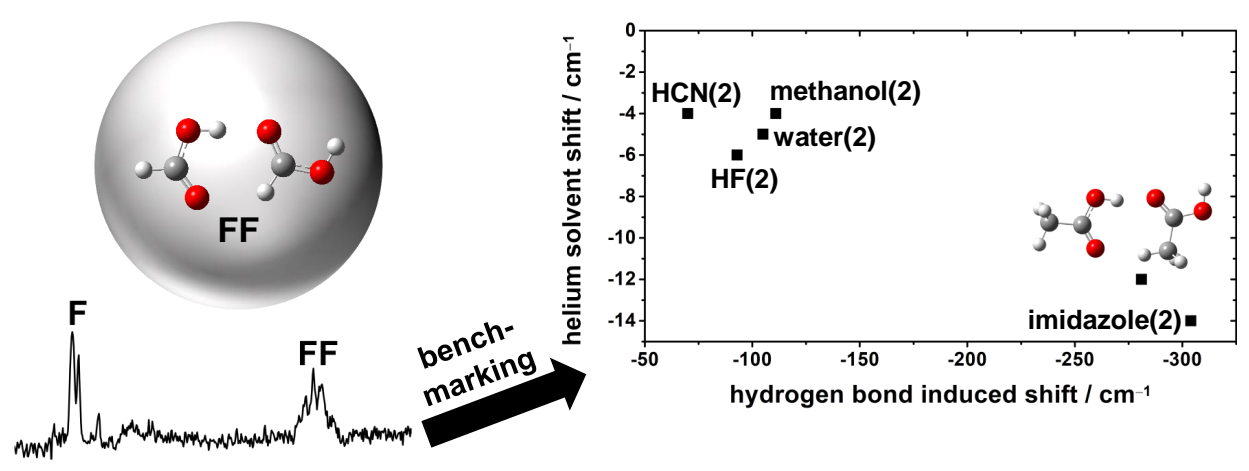

Figure 1.2: Solvent shift caused by the stiffening of hydrogen bonds in helium nanodroplets for dimers (2) of HCN [89-91], methanol [92, 93], water [94-96], HF [97-99], imidazole [2, 100], and acetic acid $[79,84]$. Its magnitude depends approximately linearly on the hydrogen bond induced downshift of the dimer bands with respect to the monomer. For benchmarks with quantum chemical gas phase calculations, this helium solvent shift needs to be accounted for.

So far, key features of carboxylic acid monomers and homo dimers have been pointed out. Another important class of clusters are those composed of different monomers. Hetero dimers of carboxylic acids are a popular subject for microwave spectroscopy studies due to their permanent electric dipole moment [101-111], but have been barely characterised vibrationally [112-115]. These mixed clusters, however, provide a valuable 
performance test for quantum chemical calculations, as these do not necessarily describe all systems equally well, i.e., the sign of errors can vary for different components (and vibrations). For vibrational benchmarks, the available reference data base must be significantly enhanced, which is attempted in this work by measuring their $\mathrm{C}=\mathrm{O}$ stretching spectra, as detailed in Chapter 5.

Even though the $\mathrm{C}=\mathrm{O}$ stretching region of carboxylic acids appears to be much simpler than the $\mathrm{OH}$ stretching region and has been characterised for several acids [68, 116-118], some bands are still unassigned, as exemplarily shown for formic acid in Figure 1.1. Possible origins of these bands could be Fermi resonances, the aforementioned higher-energy dimers, or even larger clusters such as trimers [82, 119]. Particularly the distinction between the latter two is difficult, as carboxylic acid trimers are composed of a cyclic dimer to which another monomer is docking via the formation of a strong $\mathrm{O}-\mathrm{H} \cdots \mathrm{O}$ in addition to a weaker $\mathrm{C}-\mathrm{H} \cdots \mathrm{O}$ hydrogen bond, which is the same binding motif found for the higher-energy dimers (cf. Figure 1.1) [79]. The experimental interpretation of the spectra therefore relies heavily on the quality of affordable theoretical descriptions. The employed methods need to be well understood for the monomer and global minimum dimer, so that these can be a valuable guide in more difficult assignments. Obviously, the best experimental aid are multi-spectroscopic approaches such as combined FTIR and Raman studies. Throughout the course of this work, a third technique is used that is called FTIR imaging, which enables the space-resolved, synchronous recording of 4096 FTIR spectra in a $(10 \times 10) \mathrm{mm}^{2}$ large area of a supersonic expansion. Here, it is applied to scrutinise dynamical phenomena such as cluster formation and decomposition in detail, thus, facilitating a snapshot of the processes in different parts of

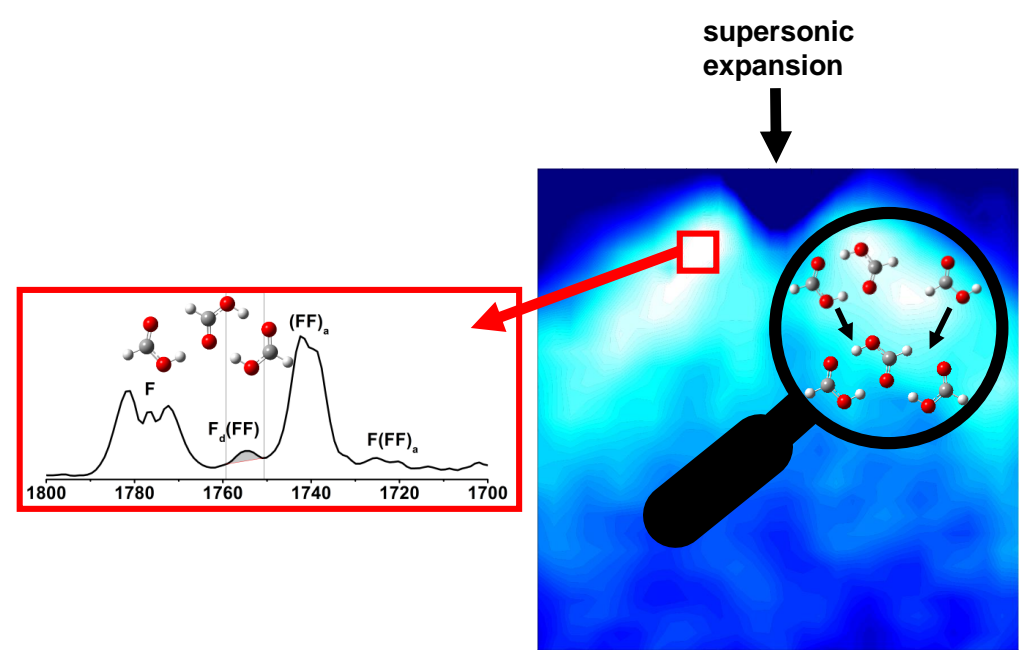

Figure 1.3: Density map of formic acid trimers in a supersonic expansion of formic acid in helium $(c<0.2 \%)$ at a reservoir pressure of 500 mbar obtained by integrating over a formic acid trimer band in 4096 space-resolved FTIR spectra in an expansion area of $(10 \times 10) \mathrm{mm}^{2}$. The propagation direction of the flow from the nozzle is indicated by an arrow. Such density maps allow for allocation of areas of largest trimer formation, as illustrated by the magnifying glass. 
the expansion, as schematically visualised in Figure 1.3. Its first and until 2017 only gas phase application was performed for nitrous oxide, where general cluster growth, temperature, and density evolution were examined in supersonic expansions [120]. In this work, its versatility is extended to obtain more detailed information on specific cluster sizes, in this case, carboxylic acid dimers, trimers, and dimer assemblies.

In summary, the aim of this work is to provide accurate experimental reference data on carboxylic acids and their small hydrogen bonded clusters in a multi-experimental study using conventional FTIR and Raman spectroscopy in supersonic expansions, FTIR imaging, and IR depletion spectroscopy in helium nanodroplets to challenge and test the performance of modern quantum chemical calculations. By examining three carboxylic acids, namely formic, acetic, and pivalic acid, as well as their mixed clusters, not only differences in their aggregation behaviour can be addressed, but equally as important, the challenge for theory is gradually increasable, i.e., the performance of a method can be tested for more than one system and cluster size. However, even on the experimental side, further progress is vitally needed, which will be detailed in the respective chapters of this thesis. These are sorted by size beginning from the monomers in Chapter 4, extending further from globally and locally stable dimers in Chapter 5, to general aggregation dynamics of metastable dimers and trimers in Chapter 6, and ending with dimer assemblies such as tetramers in Chapter 7. 


\section{Background}

Within this chapter, a brief introduction into vibrational anharmonicity as well as supersonic expansion is given, on which the discussion of the experimental spectra recorded throughout this work is based on.

\subsection{Vibrational Anharmonicity}

A simple model to describe molecular vibrations is the harmonic oscillator. In the harmonic approximation, the $n$ vibrations of a polyatomic molecule are described by uncoupled, quadratic potentials $V$ following Hooke's law. The energy levels are equally spaced and depend linearly on the vibrational quantum number $v$. If the dipole and dipole polarisability depend linearly on displacement, only transitions between neighbouring $v$ are allowed. For an anharmonic treatment, coupling between the $n$ modes, higher-order curvature of the potential $V$ (mechanical anharmonicity), and non-linearity of the dipole moment and the polarisability with displacement (electric anharmonicity) need to be included [121]. One of the simplest anharmonic approaches is based on second-order perturbation theory (PT2). It introduces binary couplings between the modes and cubic and quartic terms in the potentials [122]. The anharmonic term energies that follow from this model are shown in Equation 2.1. $x_{i j}$ represent the diagonal $(i=j)$ and off-diagonal $(i \neq j)$ anharmonicity constants, which describe the deviations from the quadratic potential of the mode $i$ and its binary couplings to other modes $j$.

$$
G\left(v_{1}, v_{2}, \ldots v_{n}\right)=\sum_{i}^{n} \omega_{i}\left(v_{i}+\frac{1}{2}\right)+\sum_{i \leq j}^{n, n} x_{i j}\left(v_{i}+\frac{1}{2}\right)\left(v_{j}+\frac{1}{2}\right)
$$

The diagonal anharmonicity $x_{i i}$ of mode $i$ can be deduced from its fundamental $\tilde{\nu}_{i}^{(1 \leftarrow 0)}$ (Equation 2.2) and overtone transitions $\tilde{\nu}_{i}^{(2 \leftarrow 0)}$ (Equation 2.3), as shown in Equation 2.4.

$$
\begin{aligned}
& \tilde{\nu}_{i}^{(1 \leftarrow 0)}=\omega_{i}+2 x_{i i}+\frac{1}{2} \sum_{j \neq i} x_{i j} \\
& \tilde{\nu}_{i}^{(2 \leftarrow 0)}=2 \omega_{i}+6 x_{i i}+\sum_{j \neq i} x_{i j} \\
& x_{i i}=\frac{1}{2}\left(\tilde{\nu}_{i}^{(2 \leftarrow 0)}-2 \cdot \tilde{\nu}_{i}^{(1 \leftarrow 0)}\right)
\end{aligned}
$$


The off-diagonal contributions $x_{i j}$ are typically determined from combination bands $\tilde{\nu}_{i+j}^{(1 \leftarrow 0)+(1 \leftarrow 0)}$ by subtracting the respective fundamentals $\tilde{\nu}_{i}^{(1 \leftarrow 0)}$ and $\tilde{\nu}_{j}^{(1 \leftarrow 0)}$ (Equation 2.5).

$$
\tilde{\nu}_{i+j}^{(1 \leftarrow 0)+(1 \leftarrow 0)}=\tilde{\nu}_{i}^{(1 \leftarrow 0)}+\tilde{\nu}_{j}^{(1 \leftarrow 0)}+x_{i j}
$$

Alternatively, these can be ascertained from hot bands $\tilde{\nu}_{i+j}^{(1 \leftarrow 0)+(1 \leftarrow 1)}$ (Equation 2.6), i.e., vibrational transitions originating from thermally populated (low-lying) energy levels.

$$
\tilde{\nu}_{i+j}^{(1 \leftarrow 0)+(1 \leftarrow 1)}=\tilde{\nu}_{i}^{(1 \leftarrow 0)}+x_{i j}
$$

For the formic acid monomer, a comparison of the results retrieved with both approaches can be found in Chapter 4 .

\subsection{Supersonic Expansions}

The overall aim of this work is to scrutinise the vibrational spectra of carboxylic acids and their small hydrogen-bonded clusters to challenge the predictive power of modern quantum chemical calculations. Evidently, experimental reference data for such a benchmark needs to be as accurate as possible. One of the most important tools in this endeavour is ro-vibrational cooling, which greatly reduces the spectral complexity and therefore, the ambiguity. However, cooling of the sample of interest is ultimately limited by condensation, which in turn shifts and broadens bands. An ideal spectroscopic reference system would therefore exhibit isolated molecules or molecular clusters free of perturbations in the gas phase at temperatures close to $0 \mathrm{~K}$. A state of matter that approaches these desired conditions closely is a supersonic expansion.

To prepare a supersonic expansion, a gas is expanded from a high pressure reservoir $p_{0}$ through an orifice into a low pressure background $p_{\mathrm{BG}}$, typically an evacuated chamber. If the mean free path of the particles is considerably smaller than the dimensions of the orifice, numerous collisions take place when the gas enters the lower pressure chamber. These collisions reduce the population of excited rotational and vibrational energy levels in favour of translation in direction of the expansion. Hence, the random motion in the reservoir is converted into directed mass flow at the expense of internal energy of the gas.

Initially, the flow can be described as isentropic, as equilibrium between the internal degrees of freedom is maintained by the high collision frequency and gas viscosity and heat transfer effects can be neglected due to the high flow rate $[55,123]$. The density of the flow decreases rapidly with distance, as exemplarily shown for an expansion of nitrous oxide in helium in Figure 2.1. The same applies to the temperature and collision frequency. Its mean velocity $u$, on the other hand, rises steeply and reaches $98 \%$ of its terminal value within a few orifice diameters [123]. The ratio of $u$ and the speed of sound $a$ is called the Mach number $M$. The substantial cooling in the supersonic expansion reduces the local speed of sound $a$ which is the dominating factor for terminal 

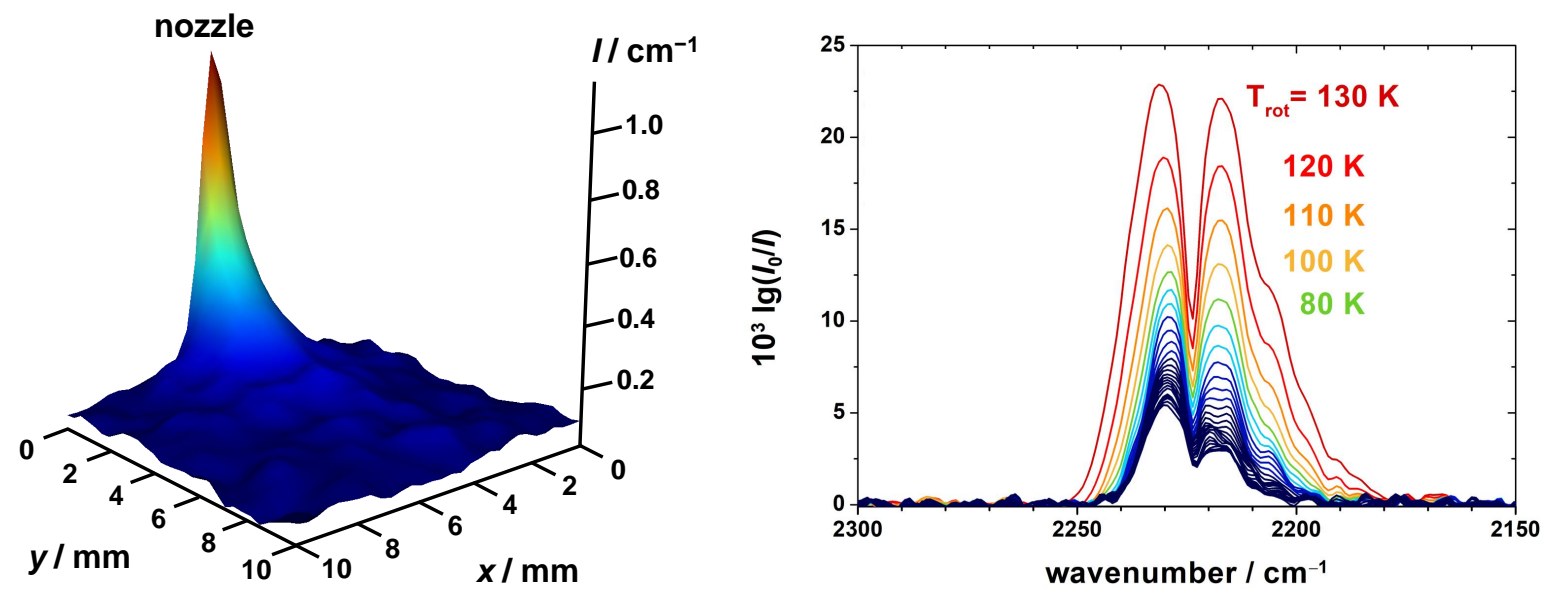

Figure 2.1: Left: density decrease in a supersonic expansion of nitrous oxide $\left(\mathrm{N}_{2} \mathrm{O}, 23 \%\right.$ in helium) recorded at a reservoir pressure of 300 mbar and with 155 scans. The density of $\mathrm{N}_{2} \mathrm{O}$ is visualised via integration of the $\nu_{3}$ band (see right side) in 4096 space-resolved FTIR spectra recorded in an expansion area of $(10 \times 10) \mathrm{mm}^{2}$. The band integral increases from blue, over green and yellow up to red. The FTIR spectra shown on the right-hand side correspond to those measured along the propagation axis of the flow at $x=5 \mathrm{~mm}$. The nozzle distance ( $y$ axis) at which the spectra have been recorded can be inferred from the integral height, which is colour coded to match the space-resolved density plot on the left side. Further details on the technique are given in Section 3.1.1. The rotational temperatures $T_{\text {rot }}$ listed have been calculated from the distance of the maxima of the $\mathrm{P}$ and $\mathrm{R}$ rotational branches according to Equation 2.8, with the gas phase reference of $\Delta \tilde{\nu}_{\mathrm{PR}, \mathrm{RT}}^{\max }=21 \mathrm{~cm}^{-1}$ from Ref. [124]. Further details can be found in the text.

$M$ values $\gg 1$ [54], whereas the flow velocity $u$ is almost constant throughout the expansion [123].

When the collision frequency approaches zero, the degrees of freedom fall out of equilibrium, which marks the transition from a continuum to a free molecular flow [55]. As the vibrational relaxation in a supersonic expansion is least efficient, vibrational degrees of freedom freeze out first, followed by rotational, and at last, translational degrees of freedom. Hence, the terminal vibrational $T_{\mathrm{vib}}$, rotational $T_{\text {rot }}$, and translational temperatures $T_{\text {trans }}$ differ significantly, whereby $T_{\text {vib }}>T_{\text {rot }}>T_{\text {trans }}$. The rotational temperatures are crucial for the spectral analysis throughout this work, as $T_{\text {rot }}$ dominates the band widths.

There are various ways of determining rotational temperatures, e.g., via empirical fitting [125] or from the intensities of rovibrational transitions under the assumption of a Boltzmann population of the energy levels [126]. If the rotational lines are not well separated due to limited spectral resolution, as in this work $\left(2 \mathrm{~cm}^{-1}\right.$ for the FTIR and $1 \mathrm{~cm}^{-1}$ for Raman measurements, see Section 3), $T_{\text {rot }}$ can also be estimated from the distance of the maxima of the $\mathrm{P}$ and $\mathrm{R}$ rotational branches $\Delta \tilde{\nu}_{\mathrm{PR}}^{\max }$, as shown for diatomic molecules in Equation 2.7, where $B$ is the rotational constant [127]. 


$$
\Delta \tilde{\nu}_{\mathrm{PR}}^{\max }=\sqrt{\frac{8 k T_{\text {rot }} B}{h c}}
$$

The rotational temperature can be calculated with respect to a reference spectrum at a known temperature and $\Delta \tilde{\nu}_{\mathrm{PR}}^{\max }$ value without information on $B$ and without strict limitation to linear tops via its general proportionality to the square root of the temperature (Equation 2.8).

$$
T_{\text {rot,jet }}=\left(\frac{\Delta \tilde{\nu}_{\mathrm{PR}, \text { jet }}^{\max }}{\Delta \tilde{\nu}_{\mathrm{PR}, \mathrm{RT}}^{\max }}\right)^{2} \cdot T_{\mathrm{rot}, \mathrm{RT}}
$$

The density plot shown in Figure 2.1 has been obtained from the integrated intensity of the $\nu_{3}$ band of $\mathrm{N}_{2} \mathrm{O}$ in 4096 space-resolved FTIR spectra. This asymmetric stretching vibration of $\mathrm{N}_{2} \mathrm{O}$ exhibits well separated $\mathrm{P}$ and $\mathrm{R}$ branches (see Figure 2.1). With the gas phase separation $\Delta \tilde{\nu}_{\mathrm{PR}, \mathrm{RT}}^{\max }$ of $21 \mathrm{~cm}^{-1}$ at room temperature $(\approx 300 \mathrm{~K})$ [128], the rotational temperature at the nozzle throat is estimated to $130 \mathrm{~K}$ and decreases with distance, as expected (see Figure 2.1). The large disparity to the $300 \mathrm{~K}$ in the reservoir can be explained with substantial cooling taking place inside the nozzle. For $\mathrm{N}_{2} \mathrm{O}$, this analysis is limited by cluster formation, as the cluster bands overlap with the monomer band. Consequently, the rotational temperature has solely been determined for small nozzle distances where cluster formation is negligible.

The free molecular flow region of a supersonic expansion is excellent for spectral interrogation, as the molecules and clusters have reached a highly non-equilibrium state - they are cooled below their condensation point, yet present in the gas phase [54]. This state continues into a region where the density of the expansion falls below that of the background gas and the flow overexpands. As information is conveyed at the speed of sound, the supersonic flow is blind towards the boundaries imposed by the low pressure background it is expanded into, yet it must ultimately conform to these [123]. This occurs in form of shock waves, where the supersonic flow is re-compressed via collisions with the background gas. Shock waves are characterised by a steep gradient in various properties such as the temperature, density, and entropy [56]. The molecular flow velocities are randomised [55]. The dimensions of a shock wave are determined by the local mean free path [123] and their form and location varies with the reservoir and background pressure ratio and the shape of the orifice $[56,125]$. In principle, one can distinguish between two classes of orifices - circular (pinhole nozzles with diameter $D$ ) or rectangular orifices (slit nozzles of length $L$ and width $D$ ). ${ }^{1}$

The flow from a pinhole nozzle is axisymmetric and that of a slit nozzle planar. Thus, the effective absorption path achievable with a slit nozzle is longer, which significantly enhances the sensitivity for absorption measurements [132]. It is one of the main reasons why slit nozzles are used throughout this work. In addition, the density decrease with nozzle distance $y$ (see Figure 2.1) is less steep, as it depends linearly on $1 / y$, whereas it

\footnotetext{
${ }^{1}$ There are more complex, flow confining nozzle forms such as conical or lavar nozzles, which will not be addressed here. Details can be found in Refs. [56, 129-131] (and Refs. therein).
} 


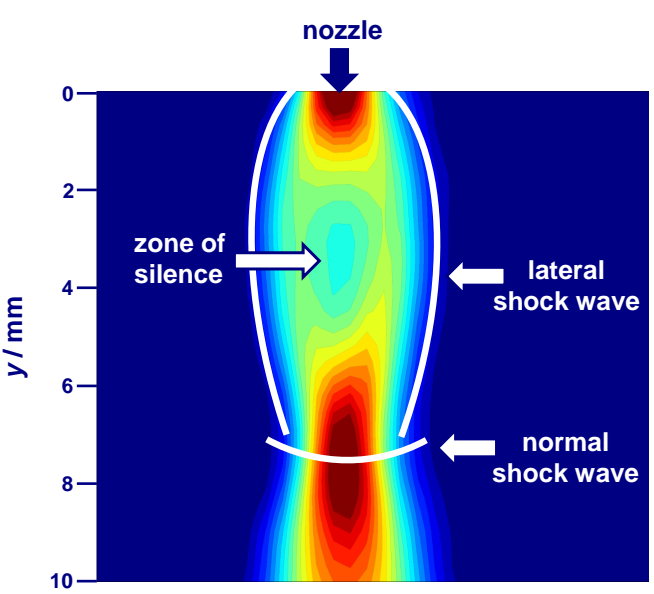

Figure 2.2: Density map of a supersonic expansion of formic acid in helium (concentration $c<0.2 \%$ ) from a slit nozzle (length $L=20 \mathrm{~mm}$ and width $D=0.5 \mathrm{~mm}$ ) at a reservoir pressure $p_{0}$ of $300 \mathrm{mbar}$ and a background pressure $p_{\mathrm{BG}}$ of $10 \mathrm{mbar}$. The integrated intensity over the $\mathrm{C}=\mathrm{O}$ stretching region retrieved from $64 \times 64$ FTIR spectra recorded in an expansion area of $(10 \times 10) \mathrm{mm}^{2}$ serves as a measure of density. The band integral increases from blue, over green and yellow up to red. White lines indicate the location of shock waves.

decreases with $1 / y^{2}$ for axisymmetric flows [132, 133]. Consequently, the number of twoand three-body collisions is larger for slit nozzles [56]. As a result, the internal degrees of freedom have a greater chance of equilibrating with the cold translational bath, which reduces the differences between the individual temperatures [134]. The larger number of three-body collisions enhances cluster formation [56, 135, 136]. Another advantage of slit nozzle expansions is a reduced Doppler line width $[56,135]$. Due to the aforementioned limited spectral resolution, the latter is not relevant for this work.

The shock wave pattern of a slit nozzle expansion is shown in Figure 2.2. The area of minimal density corresponds to the free molecular flow region. It is also referred to as zone of silence, as acoustic waves cannot travel through it due to the near-zero speed of sound [56]. Sideways, the flow is encompassed by lateral shock waves and in propagation direction by the normal shock wave. While the latter marks the transition between a super- $(M>1)$ and a subsonic flow $(M<1)$, lateral shock waves reduce the flow speed significantly, yet the Mach number $M$ remains above one [56]. Dependent on the experimental conditions chosen (pressures and nozzle width), the lateral shock waves can cross each other before or after the normal shock wave. An empirical correlation of their crossing point with respect to the nozzle is given in Ref. [56]. The distance of the normal shock wave to the nozzle $y_{\mathrm{M}}$ depends on the nozzle dimensions as well as reservoir and background pressures. For slit lengths between $1 \ll L / D<50$ and large nozzle distances ${ }^{2}$ (requiring high pressure ratios), Equation 2.9 was reported to be a good approximation [125].

\footnotetext{
${ }^{2}$ In Ref. [125], large nozzle distances are defined as $y_{\mathrm{M}}>(L / D)^{0.94} \cdot D$.
} 


$$
y_{\mathrm{M}}=0.67 D \cdot \sqrt{\frac{p_{0}}{p_{\mathrm{BG}}}}\left(\frac{L}{D}\right)^{0.47}
$$

An example is the expansion shown in Figure 2.1, which has been carried out with a $L / D=40$ slit nozzle. According to Ref. [125], Equation 2.9 can be applied for $y_{\mathrm{M}}>16 \mathrm{~mm}$. With the ratio of reservoir to background pressure of (300 mbar/0.6 mbar), a shock wave distance of $42 \mathrm{~mm}$ is obtained. However, one should keep in mind that shock waves do not remain at a steady position during the expansion, but move closer to the nozzle as the background pressure rises during a pulse. At its onset, $p_{\mathrm{BG}}$ typically amounts to $<0.2 \mathrm{mbar}$, which corresponds to a shock wave distance of $y_{\mathrm{M}}>73 \mathrm{~mm}$. To obtain uniform conditions, spectroscopic measurements are therefore started after a short stabilisation time.

For $y_{\mathrm{M}}$ values below $16 \mathrm{~mm}$, i.e., at low pressure ratios such as those applied for the measurements shown in Figure 2.2, Beylich predicted a linear dependence of the shock wave distance on the ratio of reservoir and background pressure [125]. An experimental study by Zischang and Suhm confirmed this linear dependence [120]. Their empirically determined proportionality factor is shown in Equation 2.10 and amounts to 0.37. It is reasonably close to the predicted value by Beylich (0.30) [125]. If Equation 2.10 is applied to the expansion shown in Figure 2.2, the shock wave location is predicted to $5.5 \mathrm{~mm}$, which roughly matches the onset of the density increase at the normal shock wave.

$$
y_{\mathrm{M}}=0.37 D \cdot \frac{p_{0}}{p_{\mathrm{BG}}}
$$

After the normal shock wave, the gas re-expands which is followed by an additional recompression. These density fluctuations are shown in Figure 2.3. The density maximum decreases with each re-expansion [125]. A thorough characterisation of these shock oscillations can be found in Refs. [120, 137-141].

After the outline of various flow parameters such as temperature, density, type of nozzle, and shape of the expansion, the composition of the flow shall be briefly addressed. For spectroscopic applications, the substance of interest is typically seeded to a small percentage (typically below 1\%) in a carrier gas. A popular choice are monoatomic rare gases such as helium, as these are inert and exhibit no rotational or vibrational degrees of freedom that require cooling [55]. The properties of the expansion are dominated by the carrier gas and the seeded species is accelerated to its speed via binary collisions. At large size and mass differences between the (light) carrier gas and the (heavy) analyte, the seed molecule tends to lag behind, which is known as the "velocity slip effect" [136, 142, 143]. It can be minimised by using heavier carrier gases, higher reservoir pressures, and a larger slit width $D$ [143]. A related advantage of heavier carrier gases is their enhanced cooling efficiency $[55,143,144]$. Most of the initial collisions behind the nozzle serve to align the velocity of seed and carrier, whereas the low-energy collisions occurring subsequently result in internal cooling [145]. A heavier carrier gas exhibits a lower terminal velocity, so that fewer collisions are required to equilibrate the speed of the seed and carrier gas and thus, enhances the possibility of internal cooling [145]. This 


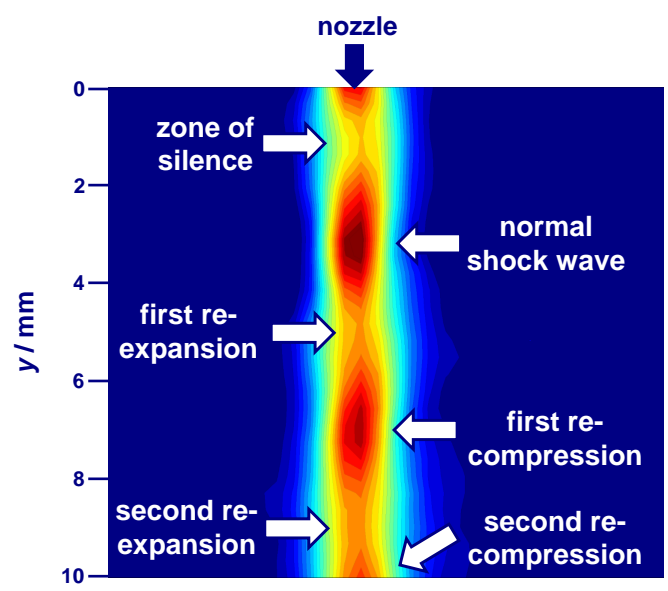

Figure 2.3: Density map of a supersonic expansion of formic acid in helium (concentration $c<0.2 \%$ ) from a slit nozzle (length $L=20 \mathrm{~mm}$ and width $D=0.5 \mathrm{~mm}$ ) at a reservoir pressure $p_{0}$ of 300 mbar and a background pressure $p_{\mathrm{BG}}$ of $40 \mathrm{mbar}$. The integrated intensity over the $\mathrm{C}=\mathrm{O}$ stretching region retrieved from $64 \times 64$ FTIR spectra recorded in an expansion area of $(10 \times 10) \mathrm{mm}^{2}$ serves as a measure of density. The band integral increases from blue, over green and yellow up to red. The location of the oscillating shock waves is marked with arrows.

also leads to stronger cluster formation. On the downside, the heavier carrier gases tend to form van-der-Waals complexes with the analyte [55], which results in nanocoating of the substances [146-149]. A strong nanosolvation can reproduce the band position shift in cryogenic matrices [149]. This will be illustrated for argon expansions in Chapter 7. For benchmarking, such environmental effects are undesirable, which is why helium is used for all other supersonic expansions performed in this work. As will be seen in Chapter 6 , the rotational temperatures reduce down to about $20 \mathrm{~K}$, which is sufficient for spectral analysis.

Lastly, the expansion type shall be briefly addressed. In principle, supersonic expansions can be conducted in a pulse sequence or continuously. Pulsed expansions yield higher densities, facilitate a more efficient internal cooling, and reduce the gas consumption [56, 132], which is why they are used when possible. The exceptions are Raman experiments, which benefit from longer accumulation times of the scattered light and thus, a continuous operation. The FTIR imaging experiments visualising shock waves in Figures 2.2 and 2.3 were also carried out by expanding the gas continuously. Further details can be found in Chapter 6 . 



\section{Experimental and Theoretical Methods}

\subsection{Experimental Set-ups}

Within this thesis, three supersonic jet set-ups were used, which will be introduced in this section - a chemical imaging, a conventional FTIR, and a Raman jet apparatus. In laboratory jargon, these are called the chilli, the filet, and the curry jet. All are acronyms, which explain their function. As the main experimental focus was on the chilli jet, it will be explained in more detail, whereas the other two set-ups will only be briefly addressed. Furthermore, in a collaboration with the group of Prof. Andrew Ellis at the University of Leicester, a helium nanodroplet experiment was conducted, which is detailed at the end of this section.

\subsubsection{The Chilli Jet}

Chilli stands for chemical imaging by spatially resolved infrared spectroscopy. This technique is also called FTIR imaging and enables the space-resolved synchronous recording of FTIR spectra with a focal plane array (FPA) detector. In this work, a $64 \times 64$ pixel FPA is used to image a $(10 \times 10) \mathrm{mm}^{2}$ large area of a supersonic expansion.

While there are barely any gas phase applications of FTIR imaging [120], it is a well-established technique for condensed phase studies. Examples include biomedical applications [150-163], polymer studies [164-169], forensic science [170-172], and the analysis of historical objects such as paintings [173-176] or books [177]. The first application of FTIR imaging to supersonic expansions was realised by Zischang and Suhm in 2013, who imaged the cluster formation of nitrous oxide $\left(\mathrm{N}_{2} \mathrm{O}\right)$ in a slit jet expansion, visualised shock wave patterns, and characterised rotational temperatures [120]. Within the framework of this thesis, the applicability of FTIR imaging is extended to carboxylic acid clusters, which exhibit well-separated, bright vibrational bands in the $\mathrm{C}=\mathrm{O}$ stretching region. This facilitates a cluster-specific scrutiny of their aggregation behaviour, which could only be generally characterised for $\mathrm{N}_{2} \mathrm{O}$, as all cluster bands overlap [120]. The jet apparatus was initially built by J. Zischang and is thoroughly described in her dissertation [124]. Here, the main focus is on the instrumental changes implemented during this work. Initially, the sample preparation and expansion are described followed by a characterisation of the nozzle designs, an outline of the optical components, and a summary of the data pre-processing and noise randomisation. 


\section{Experimental and Theoretical Methods}

\section{Preparation of the Supersonic Expansion}

The supersonic gas mixtures are prepared by seeding the sample substance in a carrier gas, typically helium. This is done in a temperature-controlled saturator, where the substance of interest is filled in as a liquid or solid. In case of solids, their surface area is enhanced by grinding the substance in a mortar, when possible. Concentrations of the analyte are determined by the vapour pressure at the set saturator temperature and the applied carrier gas pressure (typically between $1.5-1.8$ bar). As the chilli jet reservoirs and feed lines are non-heatable, the upper analyte concentration is limited by its vapour pressures just below room temperature to avoid substance condensation at the walls of the apparatus. Two saturators are available per set-up and can be cooled down to $-(30-40){ }^{\circ} \mathrm{C}$ with a cryostat. The substance concentrations used throughout this work are typically well below $1 \%$ in helium. Further details on concentration determination can be found in Section 3.1.5.

The chilli jet has two reservoirs - one is made of glass and one of metal. Both are of similar size (65 L and $69 \mathrm{~L}$, respectively). Each is equipped with three solenoid valves (ASCO Numantics), which connect it with the two saturators and a third gas cylinder for further dilution of the analyte-carrier-gas mixtures. Hence, two substances kept in separate saturators can be mixed into one reservoir or filled into separate reservoirs. The mixing or helium dilution ratio can be controlled via the opening and closing times of the solenoid valves.

From the reservoir, the supersonic expansion takes place through a slit nozzle into the vacuum chamber, which is pumped down by up to two pump racks with a combined pumping speed of $2500 \mathrm{~m}^{3} \mathrm{~h}^{-1}$. Each is equipped with a dry rotary piston and two roots pumps. To avoid large background pressure rises during pulses that shift shock waves into the field of view (cf. Chapter 2), a large buffering volume of up to $23 \mathrm{~m}^{3}$ connects the jet chamber and the pumps. Dependent on the reservoir pressures chosen (typically between $300-700 \mathrm{mbar}$ ), as well as the nozzle dimensions and pulse length described below, the background pressure rises up to $2-3$ mbar during a pulse. Before the next pulse, $p_{\mathrm{BG}}$ is pumped down to $<0.2 \mathrm{mbar}$.

The gas flow through the nozzle is controlled by a solenoid valve (Parker Lucifer), which is located at the nozzle feed line, as visualised in Figure 3.1. Its opening time is set with a pulse generator (IOTA ONE), which is synchronised with the spectrometer scan via a TTL communication box. Throughout this work, predominantly pulsed expansions are performed. The minimum pulse duration is limited by the spectrometer scan time. For stabilisation, the pulse starts shortly before a scan and should ideally end immediately afterwards. Unfortunately, it is not possible to send a TTL signal to the pulse generator, once the spectrometer is ready for measurement. Instead, the signal is sent when the mirrors of the interferometer are moving into starting position. Thus, if these are at maximum displacement, the scan will start at a later time. This introduces a substantial jitter of $1-2 \mathrm{~s}$ that has to be accounted for in the length of the gas pulses. This, in combination with the comparatively low mirror velocity of $5 \mathrm{kHz}^{1}$ limited by the

\footnotetext{
${ }^{1}$ For conventional FTIR set-ups like the filet [178] (described in Section 3.1.2) or muesli jet [124], the mirror velocity amounts to $80 \mathrm{kHz}$.
} 


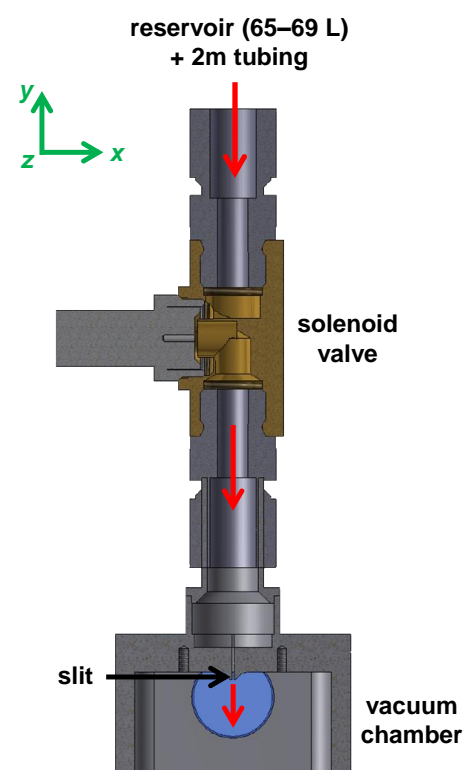

Figure 3.1: Technical drawing of the solenoid valve and the single slit nozzle, provided by R. Hildebrandt from the mechanical workshop. The red arrows mark the gas flow through the nozzle.

slow read-out time of the focal plane array (FPA) detector, results in an overall pulse duration of $4.5 \mathrm{~s}$. In-between pulses, an evacuation time is set until which the pressure in the vacuum chamber $p_{\mathrm{BG}}$ has recovered $(<0.2 \mathrm{mbar})$. This typically takes between $5-45 \mathrm{~s}$, depending on pumping capacity and stagnation pressure.

In addition to the sample spectrum, 20 background (substance-free) spectra are recorded prior to the gas pulse and are subsequently co-added. This measurement sequence of background and sample scans is automated with a macro. The measurement macro can be found in Ref. [124].

To study the aggregation behaviour in shock waves, these need to be shifted into the $(10 \times 10) \mathrm{mm}^{2}$ wide imaged area. According to Equation 2.10, this requires a low reservoir to background pressure ratio, which is achieved by manually restricting the pumping speed with a butterfly valve, which connects the jet chamber with the buffer volume and pumps. The solenoid valve at the nozzle is opened permanently. Once a constant background pressure is reached, typically $10-40$ mbar, the spectrometer scan is started manually. The two examples recorded throughout the course of this work were shown in Figures 2.2 and 2.3 and will be more thoroughly analysed in Chapter 6 (Figure $6.5)$.

\section{Nozzle Types}

Two nozzle designs are employed, a single and a double slit nozzle. Both are depicted in Figure 3.2. The slit length $L$ and width $D$ of both are identical and amount to $20 \mathrm{~mm}$ and $0.5 \mathrm{~mm}$, respectively. The slit length corresponds to the absorption path, as the IR beam is located perpendicular to the expanding flow in the $z$ plane of Figure 3.1. The 


\section{Experimental and Theoretical Methods}
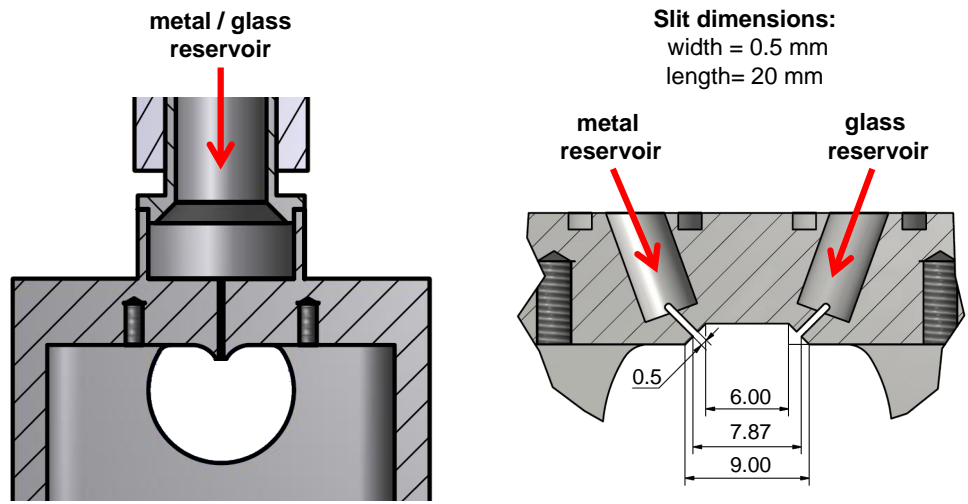

Figure 3.2: Technical drawing of the single and the double slit nozzle, provided by R. Hildebrandt from the mechanical workshop. All dimensions are given in $\mathrm{mm}$. Red arrows indicate the gas flow.

single slit nozzle deviates from the original design by J. Zischang in two ways. First of all, the absorption path has been enhanced from $10 \mathrm{~mm}$ to $20 \mathrm{~mm}$ based on preliminary studies of Zischang [124], who recorded the light intensity of a U-shaped metal piece with varying length on the detector and found that the spatial resolution is only significantly compromised for those over $20 \mathrm{~mm}$. Additionally, the nozzle shape was altered. This re-design is based on a more efficient noise-reduction, which will be detailed below.

The two slits of the double slit nozzle are converging in a $90^{\circ}$ angle. Each feed line is connected to one of the reservoirs. Hence, two solenoid valves are required. The middistance between both slits amounts to $7.5 \mathrm{~mm}$. In an initial design (see Figure A.1), there was no distance between the slits to mimic a slit nozzle containing 10 of such converging slits, which was constructed to overlap supersonic expansions in a conventional FTIR jet set-up called the muesli jet ${ }^{2}[124]$. However, no clear evidence of mixing of these could be observed [124]. With FTIR imaging, this phenomenon was scrutinised in more detail. Performance tests within the framework of the bachelor thesis of S. Schweer showed that the density of the interacting expansions is too high so that they repel each other [179]. In addition, indications for turbulences were found. An FTIR image of the interfering expansions is shown in Figure A.2. The increased distance between the slits of the new design reduces the density if both collide and allows for cluster stability measurements in shock waves, as will be detailed in Chapters 6 and 7 .

\section{Gas Flow through the Double Slit Nozzle}

Similar to the density decrease in a supersonic expansion shown in Figure 2.1, which was recorded with the single slit nozzle (Figure 3.2), the gas flow through the double slit nozzle shall be briefly characterised within this section. Two test gases are used for this analysis, namely nitrous oxide $\left(\mathrm{N}_{2} \mathrm{O}\right)$ and hexafluoroethane $\left(\mathrm{C}_{2} \mathrm{~F}_{6}\right)$. Both are strong IR absorbers, which enable high signal-to-noise ratios in the spectra, in spite of relatively

\footnotetext{
${ }^{2}$ The acronym muesli refers to the nozzle shape and its planned applicability for mixing supersonic jets and stands for multiple equidistant slits.
} 


\section{Experimental and Theoretical Methods}

few scans averaged (75). As the $\mathrm{C}_{2} \mathrm{~F}_{6}$ signal is much stronger than the $\mathrm{N}_{2} \mathrm{O}$ band in the measured spectral region $\left(1360-1200 \mathrm{~cm}^{-1}\right), \mathrm{C}_{2} \mathrm{~F}_{6}$ has been seeded in helium at a mixing ratio of $1: 12$. The double expansion of $\mathrm{N}_{2} \mathrm{O}$ and $\mathrm{C}_{2} \mathrm{~F}_{6}$ in helium is shown in Figure 3.3. To visualise the density evolution, the $\nu_{1}$ band of $\mathrm{N}_{2} \mathrm{O}$ at $1285 \mathrm{~cm}^{-1}$ and the $\nu_{7}$ band of $\mathrm{C}_{2} \mathrm{~F}_{6}$ at $1252 \mathrm{~cm}^{-1}$ are integrated. The integrated intensity of $\mathrm{N}_{2} \mathrm{O}$ is displayed as a blue and that of $\mathrm{C}_{2} \mathrm{~F}_{6}$ as a red colour map (Figure 3.3). To illustrate how both expansions interact, their spatially resolved integrated intensities are additionally overlayed by using coloured lines. Light colours represent high and dark colours low signal intensities. The slit positions are indicated by arrows.

As seen in Figure 3.3, both supersonic flows collide within the first third of the $(10 \times$ 10) $\mathrm{mm}^{2}$ wide imaged area. The flow pattern directly behind the nozzle is governed by the Bernoulli effect ${ }^{3}$, which states that the pressure that a fluid (gas or liquid) exerts is determined by its speed [181]. With increasing flow rate, the pressure decreases, which pulls the two expansions inwards, so that these do not collide in the $90^{\circ}$ angle given by the nozzle geometry (cf. Figure 3.2). Upon collision, both flows repel each other and continue side by side. A spectrum in the collision zone is shown in Figure 3.3. The bands of both substances can be seen in the same spectrum, and are thus located at the same point in space in the $2 \mathrm{D}$ image. This could suggest that the penetration depth of both flows is sufficient for studying reactive processes such as isotopic exchange reactions or the formation of hetero clusters. However, tests where $\mathrm{C}_{2} \mathrm{~F}_{6}$ in helium is replaced with a formic acid in helium expansion and nitrous oxide with an acetic, pivalic, or $N, N$ dimethylformamide in helium expansion showed that no mixed clusters are formed when both collide, although these mixed clusters remain stable in the collision zone when the substances are premixed and expanded against a pure helium expansion and cluster formation is favoured due to the high collision frequency. Hence, the fact that the $\mathrm{N}_{2} \mathrm{O}$ and the $\mathrm{C}_{2} \mathrm{~F}_{6}$ bands are seen in the same spectrum and their corresponding signals in the half of the other flow is most likely a result of turbulences along the absorption path caused by the high density upon collision. In order to study reactive processes, such viscous flow needs to be avoided. One way of achieving this is to reduce the reservoir pressure. Tests with $p_{0}$ values of $20-50$ mbar still indicate some extent of repulsion of the expansions (see Figure A.5 in the Appendix). For future tests, one could exploit pure substance expansions of reactive gases (without carrier gas). To reduce the reservoir pressure further, flow meters have to be utilised, as 20 mbar is the currently lowest feasible pressure, limited by the back filling sensitivity of the reservoir.

In addition to the high density, this region of interfering lateral shock waves of both expansions is also characterised by a temperature increase. The rotational temperature can be estimated from the distance of the maxima of the $\mathrm{P}$ and $\mathrm{R}$ rotational branches $\Delta \tilde{\nu}_{\mathrm{PR}}^{\max }$ of the bands (Equation 2.8). As mentioned in Section 2, nitrous oxide is an ideal test gas for temperature determination, if cluster formation is avoided. This applies to the shock wave spectrum shown in Figure 3.3. A gas phase spectrum of $\mathrm{N}_{2} \mathrm{O}$ recorded at $\approx 300 \mathrm{~K}$ serves as a reference for this temperature estimate. For $\mathrm{N}_{2} \mathrm{O}$, it is shown in red

${ }^{3}$ Named after Daniel Bernoulli and described in his book 'Hydrodynamica sive de viribus et motibus fluidorum commentarii'[180] published in 1738. 
in Figure 3.3. The room temperature $\Delta \tilde{\nu}_{\mathrm{PR}, \mathrm{RT}}^{\max }$ value amounts to $25 \mathrm{~cm}^{-1}$ and reduces to $(22 \pm 2) \mathrm{cm}^{-1}$ in the area of interacting barrel shocks (black square in Figure 3.3). This yields a rotational temperature of $(230 \pm 30) \mathrm{K}$. As will be detailed in Chapter 6 , the temperature in an unperturbed (single slit) expansion is an order of magnitude lower. This relatively high temperature when both expansions collide is particularly interesting as it counteracts cluster stability, whereas the associated density increase at the shock wave promotes it. Such cluster stability studies will be detailed in Chapters 6 and 7 .

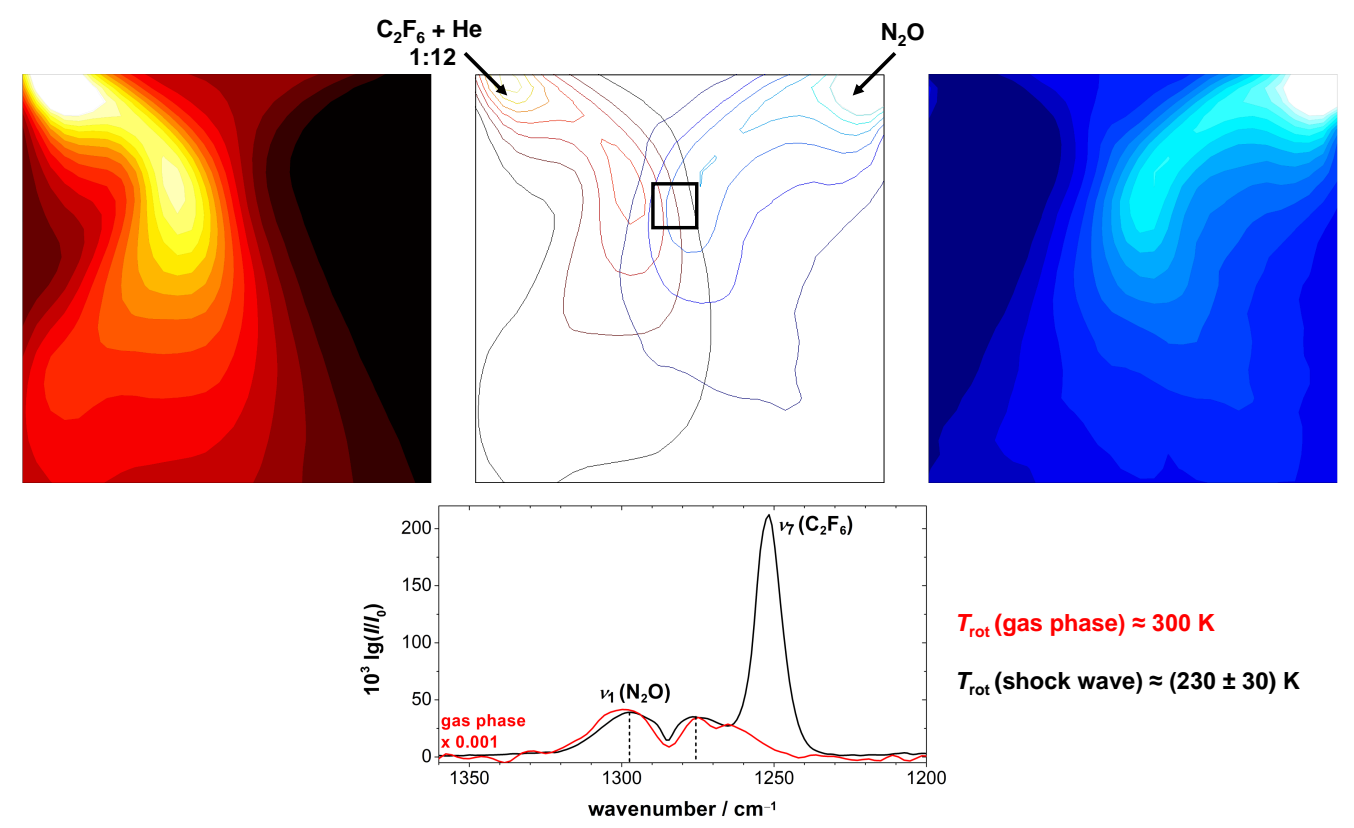

Figure 3.3: Gas flow through the double slit nozzle (Figure 3.2) in a double expansion of hexafluoroethane (red) in helium (mixing ratio 1:12, left slit) and nitrous oxide (blue, right slit) at a reservoir pressure of $500 \mathrm{mbar}$. The number of scans amounts to 75 . The two flows are visualised by integrating over the $\nu_{1}$ band of $\mathrm{N}_{2} \mathrm{O}$ centred at $1285 \mathrm{~cm}^{-1}$ and the $\nu_{7}$ band of $\mathrm{C}_{2} \mathrm{~F}_{6}$ at $1252 \mathrm{~cm}^{-1}$ in 4096 space-resolved FTIR spectra of a $(10 \times 10) \mathrm{mm}^{2}$ wide area of the expansion behind the nozzle exit. The bands are labelled according to the Herzberg nomenclature [182]. The integrated intensity is shown with colours - either in form of a colour map (integration over one band) or with coloured lines to illustrate the evolution of both band integrals in one plot. Light colours represent high and dark colours low band integrals. The location of the nozzle slits is indicated by arrows. Below, an FTIR spectrum is plotted in black, which has been spatially averaged over $6 \times 6$ pixels, depicted as a black square in the $2 \mathrm{D}$ evolution of the band integrals. In red, a gas phase spectrum of $\mathrm{N}_{2} \mathrm{O}$ is shown, which has been provided by J. Zischang. The rotational temperature of the black spectrum has been determined from the distance of the maxima of the $\mathrm{P}$ and $\mathrm{R}$ rotational branches (Equation 2.8) to (230 \pm 30$) \mathrm{K}$.

\section{Spectrometer and Optics}

The FTIR spectrometer used is the Vertex 70v from Bruker, equipped with a RockSolid $^{\mathrm{TM}}$ interferometer [183], which contains cube corner mirrors. The advantage of these 


\section{Experimental and Theoretical Methods}

over flat mirrors is their robustness towards (mirror) tilts [184]. The employed beam splitter is composed of potassium bromide. A filter wheel in-between light source and interferometer allows for optical filters to be placed inside the spectrometer. A list of typically used IR band pass filters can be found in Table A.3 in the appendix. Most commonly, a mid-infrared filter is employed that is transparent between $1900-1600 \mathrm{~cm}^{-1}$, with an exceptionally high transmittance of approximately 0.9 (see Figure A.3). According to the order of purchase, it is abbreviated to F18 (for details, see Table A.3). As the imaging detector used is sensitive to overexposure, light attenuators have to be placed inside the beam path in addition to the band pass filters. Two types are available - a perforated metal piece and several layers of fly screen, typically five are used. The transmittance curves of both can be found in Figure 3.4 in the appendix. Obviously, the required attenuation level depends on the spectral width of the band pass filter.

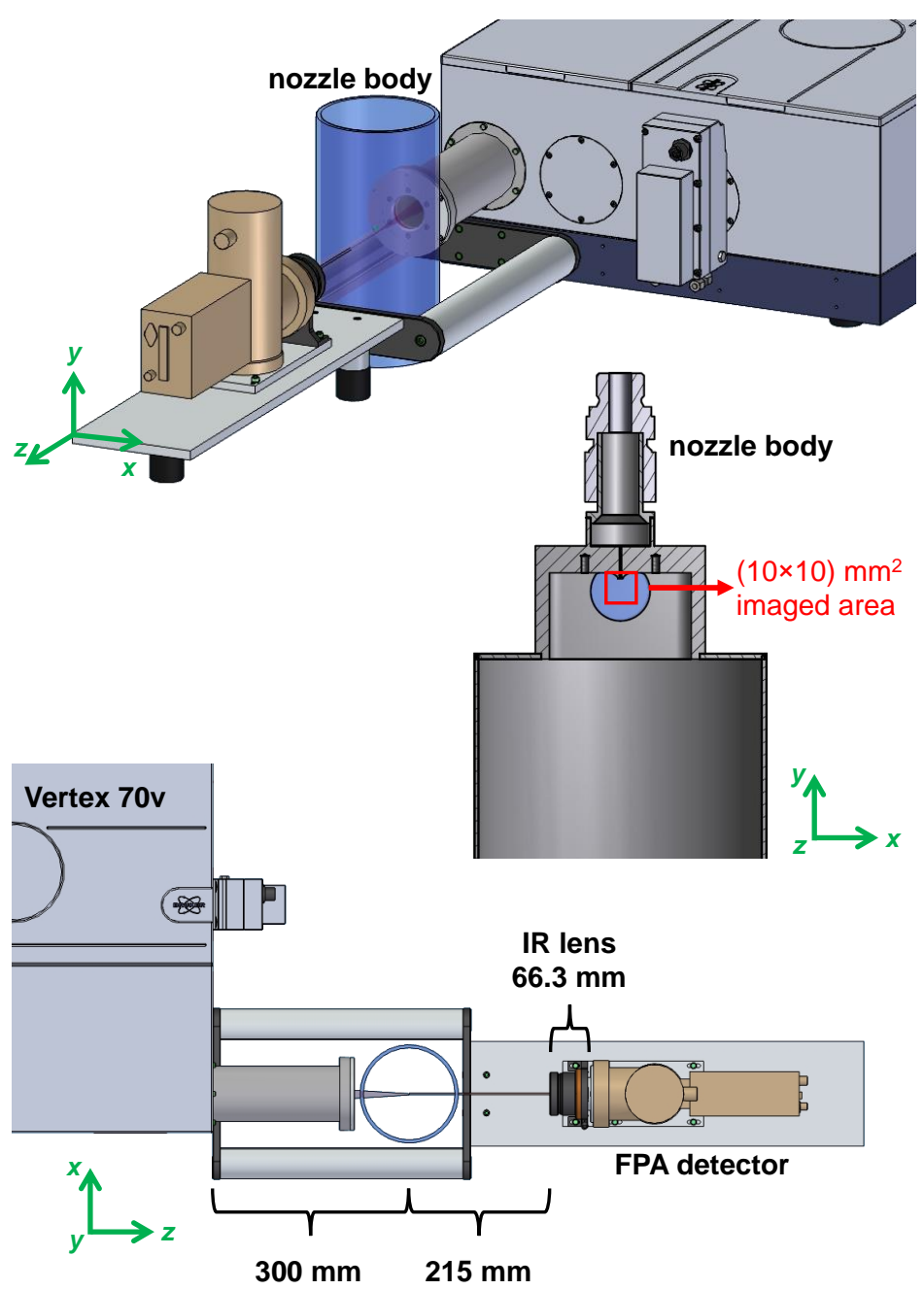

Figure 3.4: Technical drawings of the optical path, provided by the Bruker Corporation. The technical drawing of the nozzle body has been supplied by R. Hildebrandt (mechanical workshop). 


\section{Experimental and Theoretical Methods}

For F18, five layers of fly screen are sufficient. Additionally, the light attenuators also somewhat compensate for the non-uniformity of the detector illumination.

A globar (silicon carbide rod, heated to $1500 \mathrm{~K}$ [121]) serves as a mid-infrared light source. The modulated IR beam is loosely focussed onto the slit nozzle exit with a parabolic mirror, where its diameter amounts to roughly $10 \mathrm{~mm}$, as illustrated in Figure 3.4. Behind the nozzle, the IR beam is imaged onto the focal plane array (FPA) detector with a mid-infrared $(3-5 \mu \mathrm{m})$ IR lens $(f=50 \mathrm{~mm}$, ASIO Series, Janos Technology). Its transmittance curve is depicted in Figure 3.5. As expected, barely any light reaches the detector without the IR lens ( $I_{0}$ measurement), which results in $T$ values above unity and causes the spike-like artefacts. Its maximum transmittance is reached between $2600-$ $1700 \mathrm{~cm}^{-1}$, where all spectra have been measured. At higher wavenumbers, it decreases significantly, which limits its applicability in the $\mathrm{OH}$ stretching region. The magnification ratio $A$ with the currently placed IR lens amounts to 0.25 , which is determined by the distance of the detector to the IR lens and that of the IR lens with respect to the nozzle, which are kept fixed for all measurements throughout this work (see Figure 3.4). A list of all measurement parameters can be found in Table A.1 in the appendix.

The FPA detector is composed of $64 \times 64$ mercury cadmium telluride detector elements (pixels). Each pixel has a size of $(40 \times 40) \mu \mathrm{m}^{2}$. Hence, the overall detector size amounts to $(2.56 \times 2.56) \mathrm{mm}^{2}$. In accordance with the magnification ratio $A$, the imaged area of the expansion amounts to $(10 \times 10) \mathrm{mm}^{2}$ (see Figure 3.4). The 4096 spectra are thus recorded with a spatial resolution of $0.16 \mathrm{~mm}$. However, the effective spatial resolution depends on the data pre-processing described below.

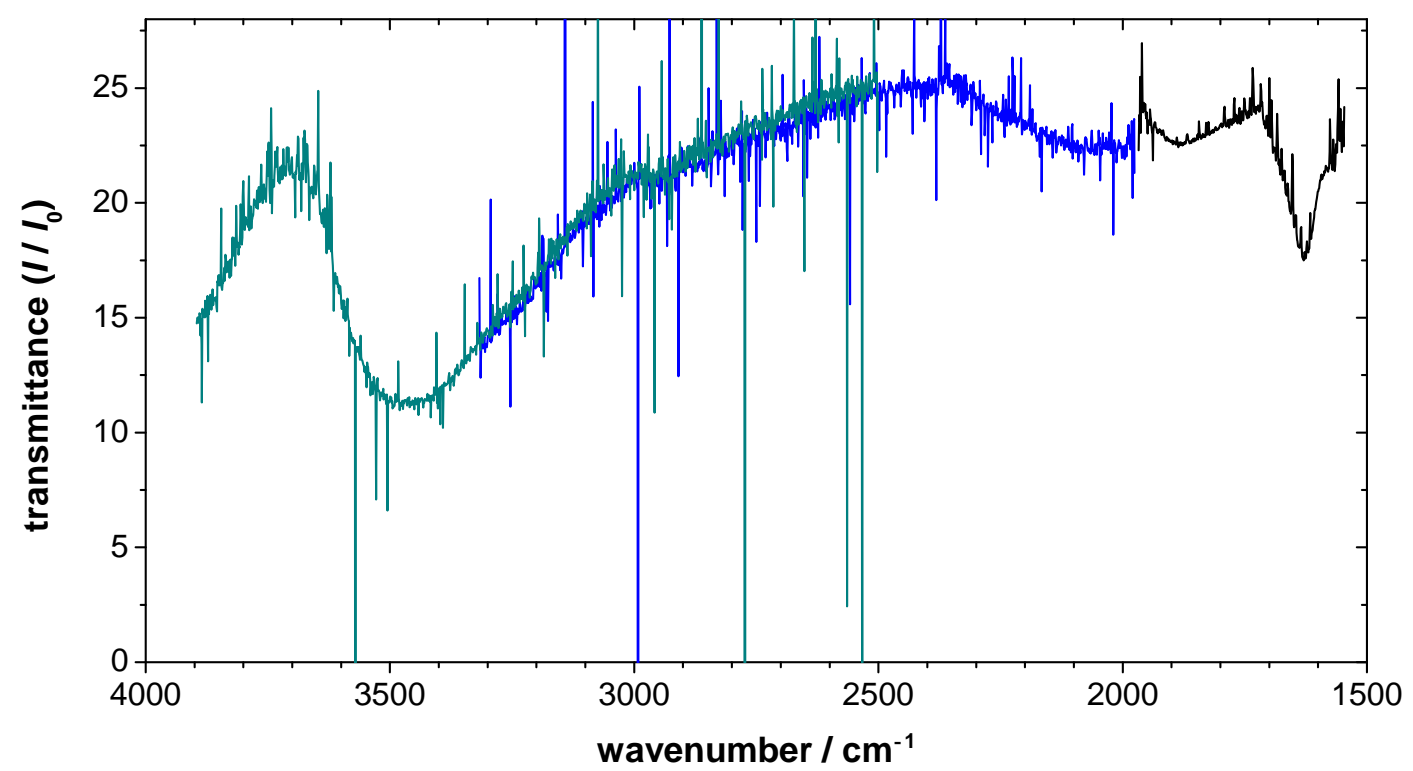

Figure 3.5: Transmittance of the IR lens measured with three filters (transparent spectral window). Black: F18 $\left(1900-1600 \mathrm{~cm}^{-1}\right)$, blue: F2 $\left(3300-2000 \mathrm{~cm}^{-1}\right)$, and cyan: F13 $(4100-$ $\left.2500 \mathrm{~cm}^{-1}\right)$. A full list of filters can be found in Table A.3 in the appendix. $I_{0}$ has been recorded without the IR lens and correspondingly, $I$ with it. 


\section{Data Pre-Processing}

During each gas pulse, 4096 space-resolved FTIR spectra are recorded simultaneously. One way of visualising spectral changes with nozzle distance is to determine spectral quantities such as band intensities, integrals, or signal-to-noise ratios for each spectrum and assign this value to each pixel. Within this work, this is typically done for integrated intensities as a measure of the density of the species of interest. An example is shown in Figure 3.6, where the integrated intensity of the asymmetric stretching band of nitrous oxide $\left(\mathrm{N}_{2} \mathrm{O}\right)$ is plotted in an expansion of $20 \% \mathrm{~N}_{2} \mathrm{O}$ in helium. The two dimensional evolution of the band integral is fairly noisy. To smoothen the data, three steps of data pre-processing are performed, namely replacement of faulty pixels, binning, and a Gauss-type weighting scheme.

Damaged pixels can be identified by plotting the root mean square deviation (RMSD) of the noise in substance-free spectra recorded in the evacuated jet-chamber for all 4096 pixels. A three dimensional plot can be found in Figure 3.7. 19 spectra exhibit an exceptionally high noise level and correspond to such faulty pixels. Their number has increased by three compared to Ref. [124]. A full list of the respective pixels can be found in Table A.4 in the appendix. Their spectra are replaced by those of adjacent pixels.

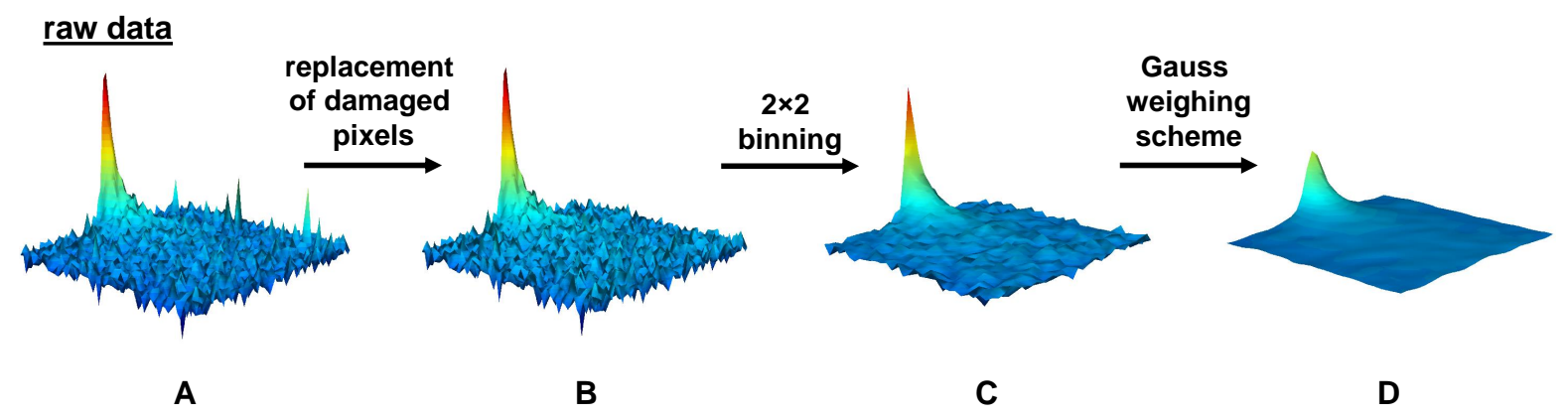

Figure 3.6: Data pre-processing to reduce the image noise visualised for the band integral over the asymmetric stretching vibration $\nu_{3}$ of nitrous oxide $\left(\mathrm{N}_{2} \mathrm{O}\right)$ measured in supersonic jet expansions of $20 \% \mathrm{~N}_{2} \mathrm{O}$ in helium at a reservoir pressure of 300 mbar. The number of scans amounts to 155 scans.

As can be seen in Figure 3.7, the noise in the spectra of the functioning pixels is not perfectly uniform. This slight heterogeneity of the illumination of the pixels causes an image noise, which is compensated by spatially averaging $2 \times 2$ pixel blocks $(2 \times 2$ binning). The effect on the spectra is illustrated in panel $\mathrm{C}$ of Figure 3.6. This binning reduces the overall number of spectra to $32 \times 32$. An additional smoothing is achieved by weighing the spectrum of a pixel with those of its eight neighbours in a Gauss-type manner. The spectrum itself contributes to $100 \%$, the spectra of the four adjacent pixels by $70 \%$, and those of the diagonal neighbours by $50 \%$. Obviously, this weighing scheme cannot be applied to the outermost pixel ring, as one set of neighbouring pixels is missing, which reduces the overall number of spectra to $30 \times 30$, yet its effect on the twoor three-dimensional images of the expansion outweighs this loss (cf. Figure 3.6). As a 


\section{Experimental and Theoretical Methods}

consequence of the data pre-processing, the spatial resolution is reduced to $<0.4 \mathrm{~mm}$ $(0.31 \mathrm{~mm})$, which is sufficient for an analysis of the aggregation behaviour of carboxylic acids in supersonic expansions, as will be seen in Chapters 6 and 7 .

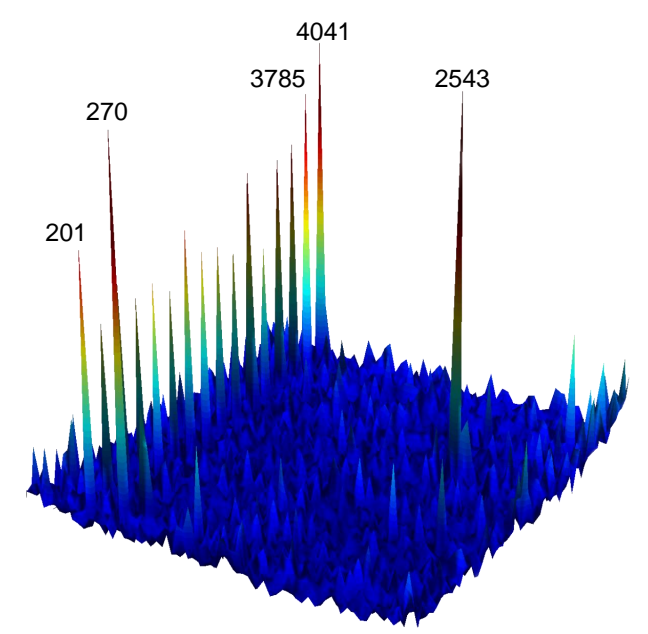

Figure 3.7: Root mean square deviation (RMSD) of the noise between $1900-1600 \mathrm{~cm}^{-1}$ in substance-free FTIR spectra of the $64 \times 64$ pixel focal plane array (FPA) detector. The RMSD value is encoded in the signal height ( $z$ dimension) as well as the colour, whereby the RMSD increases from blue over green and yellow up to red. Pixel numbers are given for spectra with particularly high RMSD values. A full list of these faulty pixels can be found in Table A.4 in the appendix.

\section{Noise randomisation}

One of the main challenges of FTIR imaging is the low signal-to-noise ratio compared to conventional FTIR spectroscopy. The reasons are two-fold: The amount of photons per pixel is drastically smaller for an FPA compared to a standard (single element) FTIR detector of the same size [186]. Secondly, the electronics of area detectors are more compact, which also raises the overall noise level [186]. Moreover, focal plane array detectors exhibit an additional, pixel-spanning noise component. Each detector element has its own readout circuit, to avoid signal transfer. However, due to the complexity of the electronics and the spatial constraints, this so called cross-talk between pixels cannot be entirely inhibited [187]. The effect on the spectra is visualised in Figure 3.8. 16 absorbance spectra are shown, which were averaged over blocks of $8 \times 8$ pixels along the two image diagonals measured in FTIR spectra of the evacuated vacuum chamber (without the substance expansion). The pixels-spanning noise is clearly visible in the common noise features. If adjacent pixel blocks $\left(\mathrm{A}^{\prime}\right)$ are added or subtracted, these uniform features are intensified $(\mathrm{B})$ or reduced $(\mathrm{C})$. In case of pure random noise, there should be no difference. However, the randomisation of the noise by subtracting spectra with the same common noise features offers an efficient way of reducing it. In case of substance measurements, spectra of sample-free or at least low-absorption regions can be exploited to reduce it. These have to be from the same measurements, as the noise 


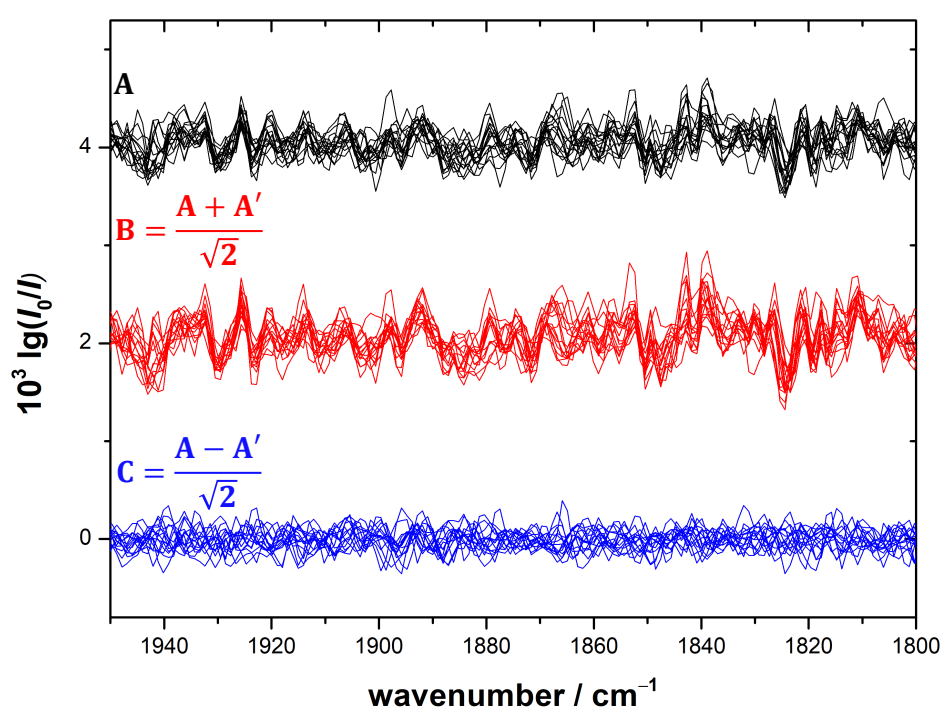

Figure 3.8: Substance-free absorbance spectra visualising the pixel-spanning noise of FPA detectors. A shows 16 such spectra averaged over blocks of $8 \times 8$ pixels along the two image diagonals. B shows the result of their summation with adjacent pixel blocks (A') and $\mathrm{C}$ the corresponding subtraction. Adapted from J. Chem. Phys. 147, 144305 (2017) (Ref. [185]), with the permission of AIP Publishing.

features (maxima and minima) vary. Additionally, the amount of subtracted spectra has to be high enough so that the random noise component is not enhanced.

Expansion zones with barely any sample signal can be found on the far left and right from the nozzle exit (cf. Figure 2.1). To enhance these areas, the nozzle shape has been adjusted to a protruding design compared to Ref. [124], as visualised in Figure 3.9. With this re-design, typically two pixel blocks of $14 \times 24$ low-absorbance spectra can be used for the noise-reduction. These are visualised as white boxes in the two-dimensional integrated intensity of the $\mathrm{C}=\mathrm{O}$ stretching vibration of the formic acid dimer in Figure 3.9. Their averaged spectrum is shown in panel A of that figure. Raw and pixelspanning noise corrected substance-spectra are shown in panels B and C. These have been averaged over $6 \times 6$ pixels along the expansion centre. The cross-talk induced noise component can be clearly seen in spectra with low overall signal intensities, e.g., at greater nozzle distances, and in spectral windows without vibrational features, e.g., between $1720-1700 \mathrm{~cm}^{-1}$. Altogether, this noise randomisation is particularly crucial when band intensities are near noise level and is performed when possible throughout this work. 


\section{Experimental and Theoretical Methods}
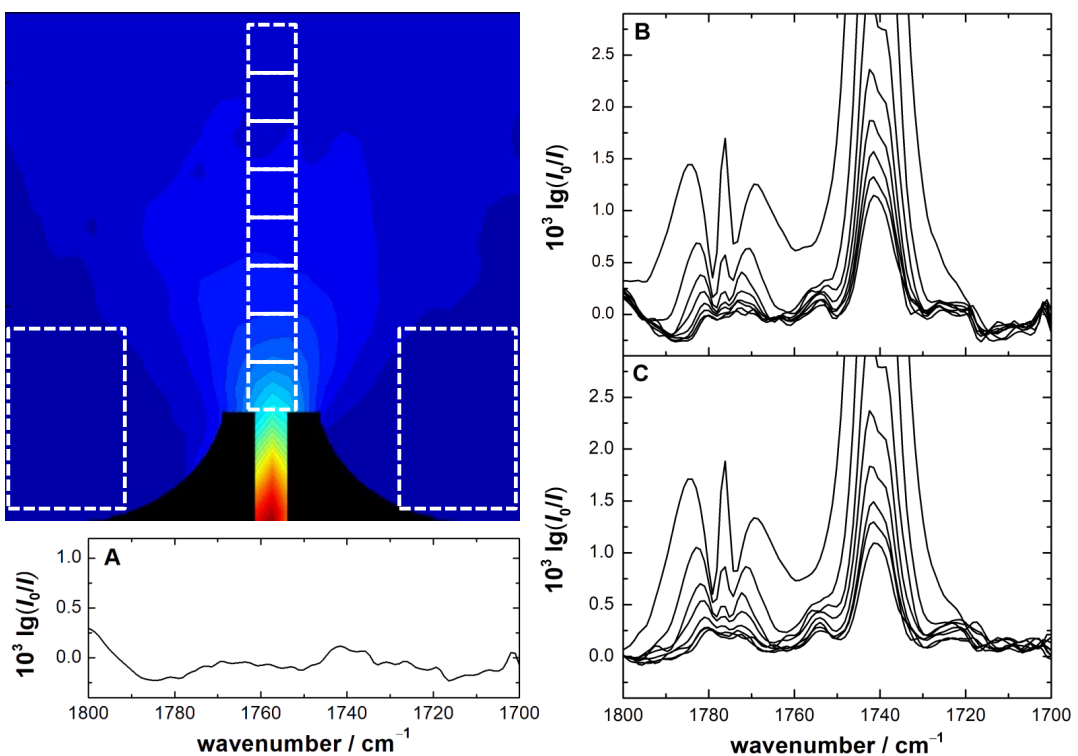

Figure 3.9: Two-dimensional integrated intensity of the antisymmetric $\mathrm{C}=\mathrm{O}$ stretching vibration of the formic acid dimer $\left(1741 \mathrm{~cm}^{-1}\right)$ in FTIR jet spectra of formic acid in helium $(c<0.1 \%)$ at a reservoir pressure of 500 mbar averaged over 100 scans. The band integral is increasing from blue, over green and yellow up to red. The nozzle is overlayed in black. In panel A, a low-absorbance spectrum is shown, which has been averaged over two $14 \times 24$ pixel blocks left and right from the nozzle, visualised as white boxes. This spectrum has been subtracted from eight substance spectra along the expansion centre (panel B) to obtain the pixel-spanning noise-corrected spectra in panel C. The eight substance spectra were averaged over $6 \times 6$ pixels. Adapted from J. Chem. Phys. 147, 144305 (2017) (Ref. [185]), with the permission of AIP Publishing.

\subsubsection{The Filet Jet}

The distinct advantage of the chilli jet is the dynamical spectral information attainable, i.e., the kinetic perspective on cluster formation and decomposition, yet the comparably low signal-to-noise ratio is one of its main challenges. Particularly fine details of spectra such as low intensity bands or band shapes, are difficult to characterise. An apparatus optimised for the measurement of rotationally cold FTIR jet spectra with a high-signalto-noise ratio is the filet jet. One of its main characteristics is the fine but lengthy slit nozzle, which is $0.2 \mathrm{~mm}$ wide and $600 \mathrm{~mm}$ long. For comparison, the absorption path of the chilli jet is 30 times shorter. The apparatus has been built by Nicole Borho and is thoroughly described in her PhD thesis [188]. Further advances such as an extension of the spectral range are described in Refs. [118, 178, 189-192], whereby the most thorough and detailed description of the operation principle can be found in Ref [193]. Here, only the relevant common features and differences to the chilli jet are pointed out.

The sample preparation and gas feed lines are similar and the buffer volume $\left(23 \mathrm{~m}^{3}\right)$ and pump racks $\left(2500 \mathrm{~m}^{3} / \mathrm{h}\right)$ are shared amongst both apparatuses. The gas pulses of the filet jet are considerably shorter $(147 \mathrm{~ms})$. The main reason is the 16 times faster 


\section{Experimental and Theoretical Methods}

interferometer sampling $(80 \mathrm{kHz})$. Additionally, the synchronisation between spectrometer scan and pulse generator is superior for the former and does not result in a jitter. Optical components such as filters, light sources, and beam splitter are the same, yet the spectrometer of the filet-jet is an older model (IFS 66v/S by Bruker). A list of these and the measurement parameters can be found in Table A.2 in the appendix.

In addition to the nozzle, the most important differences of the two FTIR set-ups are the detector and IR beam path. For the filet jet, a single element detector is utilised, which is of similar size compared to the the area detector (MCT: $(2 \times 2) \mathrm{mm}^{2}$, MCT FPA: $\left.(2.56 \times 2.56) \mathrm{mm}^{2}\right)$. The distance of IR beam and nozzle of that apparatus amounts to $10 \mathrm{~mm}$ in the focal point. With the $10 \mathrm{~mm}$ width of the IR beam (in its focus), a large expansion area is covered with one filet jet spectrum, whereas the space-resolved FTIR spectra of the chilli jet give a detailed view of the cluster formation in a $(10 \times 10) \mathrm{mm}^{2}$ wide window directly behind the nozzle. In conclusion, both set-ups have their distinct advantages over the other, which will be exploited accordingly throughout this thesis.

\subsubsection{The Curry Jet}

The interplay of different experimental techniques and theoretical methods for the vibrational scrutiny of carboxylic acid monomers and clusters is a crucial aspect of this work. The indispensability of Raman spectroscopy in this endeavour lies not only in the fact that half of the vibrational modes of the inversion symmetric carboxylic acid dimers are exclusively Raman active (rule of mutual exclusion), but it also provides complementary information for the characterisation of monomers, as some of the fundamentals are barely IR active. A plethora of further examples will be given throughout this thesis. In line with this complementary quest, the description of the curry jet set-up will focus on the similarities and differences to the FTIR jet set-ups and highlight its unique features.

The curry acronym stands for classical unrestricted Raman spectroscopy. The apparatus has been initially built by Philipp Zielke [194], and further improved in the context of various PhD theses including Tobias Wassermann [195], Zhifeng Xue [68], Nils Lüttschwager [196], Katharina Otto [197], Sebastian Bocklitz [198], and Thomas Forsting [199], where more detailed descriptions can be found.

Compared to the FTIR set-ups, the curry jet is more versatile in terms of substance concentration as well as expansion conditions, which is illustrated in the schematic depiction of the set-up shown in Figure 3.10. In addition to the two (only one is depicted in Figure 3.10) cryostatically cooled saturators present for all jet set-ups, it is also equipped with a heatable saturator. In this work, it is only used for the pivalic acid Raman spectra depicted in Figure 6.12, which have been measured at saturator temperatures of $40-50{ }^{\circ} \mathrm{C}$. The overall most important feature for this work is the heatable nozzle $\theta_{\mathrm{n}}$ and feed line of a total length of $2 \mathrm{~m}$ [199]. The highest temperature $\theta_{\mathrm{n}}$ feasible amounts to $190{ }^{\circ} \mathrm{C}$. This aspect of the set-up can be used to thermally enhance the population of higher-energy structures, e.g., cis-amides [197, 199], and is employed for the carboxylic acid monomer measurements discussed in Chapter 4. All other spectra have been measured with both set to room temperature.

As opposed to the IR set-ups, the substance-in-carrier gas mixture is expanded con- 


\section{Experimental and Theoretical Methods}

tinuously from a $4.7 \mathrm{~L}$ reservoir through a $0.15 \mathrm{~mm}$ wide and $4 \mathrm{~mm}$ long slit nozzle into the jet chamber, which is evacuated with a pump rack containing two roots and a rotary vane pump with a pumping speed of $500 \mathrm{~m}^{3} / \mathrm{h}$. The chosen reservoir pressures amount to $0.5-1.5$ bar, which results in background pressures of up to $2-3$ mbar during measurements.

The expansion is probed by a $532 \mathrm{~nm}$ continuous-wave frequency-doubled Nd:YVO 4 diode pumped solid state (DPSS) laser (Millennia eV, Spectra-Physics) with a laser power of $25 \mathrm{~W}$ from down below (see Figure 3.10). The distance between the laser beam and the nozzle is typically varied between $1-3 \mathrm{~mm}$. To maximise the signal, it is set to $1 \mathrm{~mm}$ throughout this work. A half-wave plate can be placed inside the optical path of the laser to change its polarisation with respect to the scattering plane from perpendicular to parallell ( $x z$ plane in Figure 3.10). This is used for the depolarisation measurements shown in Figure 4.8 (Chapter 4). Details on those can be found below. The scattered radiation is collected perpendicular to both the expanding flow and the laser with a camera objective (Nikkor, $f=50 \mathrm{~mm}$ ) and focussed onto the entrance slit $(75 \mu \mathrm{m})$ of a $1 \mathrm{~m}$ monochromator (McPherson, Model 2051, f /8.7, grating: 1200/mm) with a convex lens. An edge filter is placed before the monochromator to quench the Rayleigh light. A liquid nitrogen cooled charge-coupled device (CCD) camera (Princeton Instruments, PyLoN 400B) serves as a detector, which exhibits 1340 pixel columns for spectral dispersion and 400 pixel rows for vertical binning of the photon signal. The spectral resolution varies slightly over the investigated spectral range and amounts on average to about $1 \mathrm{~cm}^{-1}$ [199]. Typical data acquisition times vary between $120-300 \mathrm{~s}$. Cosmic ray artefacts are removed by comparison of independent scans within a sequence. The wavenumber calibration is achieved via the emission spectrum of neon and krypton lamps and their known vacuum lines taken from the NIST data base [200]. The overall wavenumber uncertainty of the Raman spectra amounts to $1-2 \mathrm{~cm}^{-1}$. Further details on the automated data preprocessing sequence can be found in the $\mathrm{PhD}$ thesis of $\mathrm{T}$. Forsting [199].

\section{Depolarisation measurements}

As detailed above, the scattered radiation is detected orthogonal to the incident radiation (laser) and the expanding flow. This $90^{\circ}$ geometry is transferred into a Cartesian coordinate system in the inset of Figure 3.10 for illustration. The laser beam is located on the $z$ axis and the detector on the $x$ axis, and hence, the $x z$ plane is the scattering plane. The polarisation of incident and scattered light is given with respect to the scattering plane. Laser (incident) light with a polarisation parallel to the scattering plane is labelled $\|^{\mathrm{i}}$ and incident light with perpendicular polarisation accordingly $\perp^{\mathrm{i}}$. As mentioned above, the default is $\perp^{\mathrm{i}}$. Scattered radiation of both polarisations $\left(\perp^{\mathrm{s}}\right.$ and $\left.\|^{\mathrm{s}}\right)$ is detected simultaneously with the set-up.

Whether the polarisation of the incident light is retained or changed after scattering depends on the symmetry of the molecular vibration. Complete preservation is limited to totally symmetric vibrations of optically isotropic molecules [202]. Non totally symmetric vibrations reduce the polarisation. If the vibration is totally symmetric, but 


\section{Experimental and Theoretical Methods}

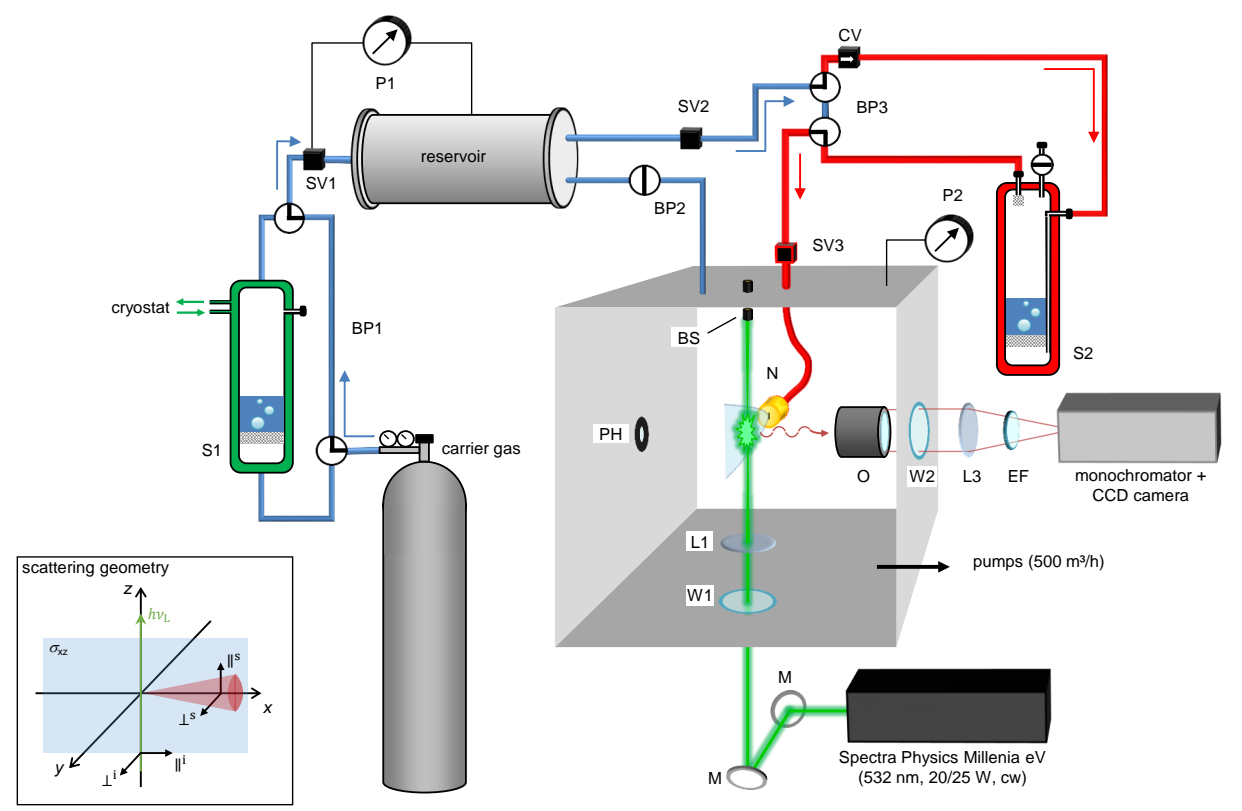

Figure 3.10: Schematic depiction of the curry jet setup by T. Forsting, reprinted with adaptations from Ref. [201] licensed under CC BY-NC-ND 4.0 with permission from T. Forsting. The following abbreviations are used (in alphabetical order): BP: bypass, BS: beam stop, CV: check valve, EF: edge filter, L: focus lens, N: nozzle, M: laser mirror, O: objective, P: pressure gauge, $\mathrm{PH}$ : pinhole, S: saturator, SV: solenoid valve, W: window. Red lines represent heatable parts of the apparatus.

the molecule anisotropic, the polarisation is largely retained [202]. The degree to which depolarisation occurs is called $\delta$ and can be determined via the comparison of intensity measurements of scattered radiation with parallelly $I\left(90^{\circ} ;\left\|^{\mathrm{i}}, \perp^{\mathrm{s}}+\right\|^{\mathrm{s}}\right)$ and perpendicularly polarised incident light $I\left(90^{\circ} ; \perp^{\mathrm{i}}, \perp^{\mathrm{s}}+\|^{\mathrm{s}}\right)$, as shown in Equation 3.1, with $\alpha$ and $\gamma$ the invariants of the polarisability tensor [68]. $\gamma$ contains both diagonal and off-diagonal elements and $\alpha$ only depends on the former. For non totally symmetric vibrations, a depolarisation ratio $\delta$ of $6 / 7$ is obtained, as $\alpha=0$ [202] (cf. Equation 3.1). It is lower for totally symmetric vibrations $(\alpha \neq 0)$ and varies between $0 \leq \delta<6 / 7$.

$$
\delta=\frac{I\left(90^{\circ} ;\left\|^{\mathrm{i}}, \perp^{\mathrm{s}}+\right\|^{\mathrm{s}}\right)}{I\left(90^{\circ} ; \perp^{\mathrm{i}}, \perp^{\mathrm{s}}+\|^{\mathrm{s}}\right)}=\frac{6 \gamma^{2}}{45 \alpha^{2}+7 \gamma^{2}} \leq \frac{6}{7}
$$

Hence, the comparison of spectra measured with parallely and perpendicularly polarised laser light can help to distinguish the symmetry of vibrations. Within this work, this comparison is done by subtracting spectra measured with parallelly polarised laser light $\|^{\mathrm{i}}$ scaled by $7 / 6$ from those of the standard measurement $\left(\perp^{\mathrm{i}}-7 / 6 \cdot \|^{\mathrm{i}}\right)$, which cancels out bands of non totally symmetric modes. Note that Equation 3.1 is only valid under the assumption that the detection efficiency of $\perp^{\mathrm{s}}$ and $\|^{\mathrm{s}}$ is the same as well as the experimental conditions. However, the grating of the monochromator disperses scattered radiation with parallel polarisation $\|^{\text {s }}$ less efficiently, which is not accounted 
Table 3.1: Comparison of the slit width $D$ and length $L$ employed for the three jet set-ups.

\begin{tabular}{ccc}
\hline \hline set-up & $D / \mathrm{mm}$ & $L / \mathrm{mm}$ \\
\hline curry jet & 0.15 & 4 \\
filet jet & 0.2 & 600 \\
chilli jet & 0.5 & 20 \\
\hline \hline
\end{tabular}

for in this work. Further details can be found in Ref. [196].

\subsubsection{Summary of the Differences of the Three Jet Set-ups}

The differences in expansion conditions of the three jet set-ups previously introduced will be briefly summarised within this section. As the sample preparation is identical for all set-ups, it is not addressed in detail. The concentration determination is described hereafter.

The four dominating factors that cause differences of the supersonic flows probed with each set-up are the type of expansion (continuous or pulsed), the reservoir pressure, the dimensions of the slit nozzle, as well as the nozzle distance chosen for measurements. To facilitate long acquisition times of the scattered radiation, the supersonic expansions of the Raman set-up are continuous, whereas the expansions of the FTIR experiments are pulsed with the aforementioned exception (Figure 6.5).

A direct comparison of reservoir pressures $p_{0}$ of different experiments is misleading, as these are measured in the reservoir instead of at the nozzle throat, where the stagnation pressure is lower. The extent of the pressure drop depends on the dimensions (length and diameter) of the corresponding feed line, which is overall largest for the curry jet. Hence, at a given reservoir pressure, its effective pressure will be smallest.

The width $D$ of the nozzle utilised for the imaging experiments is $2-3$ times larger than those of the conventional FTIR and Raman set-ups (see Table 3.1). Thus, at a given reservoir pressure and nozzle distance, the cluster formation is most pronounced for the chilli jet (cf. Chapter 2). The nozzle length $L$ has no critical influence on the cluster formation and is longer for the FTIR set-ups as it defines the absorption path length (Table 3.1). The largest impact on the expansion conditions is given by the area of spectral interrogation and its position relative to the nozzle. The filet jet spectra cover a wide range of the expansion $(10 \mathrm{~mm}$ nozzle distance, $10 \mathrm{~mm}$ IR beam diameter in the focus point). The Raman jet spectra, on the other hand, are recorded directly behind the nozzle at the centre line of the expansion ( $1 \mathrm{~mm}$ nozzle distance). Therefore, the extent of cluster formation is lower for the latter. The imaging experiments probe a $(10 \times 10) \mathrm{mm}^{2}$ large expansion area behind the nozzle. Considering the difference in nozzle widths (Table 3.1), the experimental conditions of the Raman jet experiments are best compared to those at a nozzle distance of $3 \mathrm{~mm}$ of the chilli jet. 


\section{Experimental and Theoretical Methods}

\subsubsection{Concentration Determination}

The acid concentrations in this work are estimated from the temperature dependence of their vapour pressure and are given in percent with respect to the helium partial pressure, which was varied between 1.5-1.8 bar. Further dilution of the substance in helium mixtures is possible via an additional helium feed line, as detailed above. Moreover, for the hetero dimer spectra shown in Chapter 5, different acids were mixed. These substance or helium admixing ratios were typically varied between 1:1 and 1:10.

Formic acid has the highest vapour pressure of all substances used in this work with $56.7 \mathrm{mbar}$ at room temperature $(298 \mathrm{~K})$ [203]. For comparison, those of acetic and pivalic acid only amount to 20.8 mbar [204] and 1.2 mbar [205] at $298 \mathrm{~K}$, respectively. As such, formic acid was typically measured at temperatures below its melting point of $8.2{ }^{\circ} \mathrm{C}[206]$. As a solid, the vapour pressure was determined with respect to the triple point temperature $(281.4 \mathrm{~K})[206]$ and pressure $(17.72 \mathrm{mmHg})[207]$ and the sublimation enthalpy $\left(60.1 \mathrm{~kJ} \mathrm{~mol}^{-1}\right)[208,209]$. If measured as a liquid, it was estimated from the Antoine parameters $\left(\mathrm{A}=2.00121, \mathrm{~B}=515.0 \mathrm{~K} \mathrm{bar}^{-1}\right.$, and $\left.\mathrm{C}=-139.408 \mathrm{~K}\right)$ listed in Reference [208], which were extrapolated from Reference [203] by the authors (National Institute of Standards and Technology). The vapour pressures of the deuterated isotopologues of formic acid (HCOOD and DCOOD) are assumed to be identical to that of $\mathrm{HCOOH}$, as the general uncertainty of the concentration determination introduces a larger error.

Acetic acid melts at $16.6{ }^{\circ} \mathrm{C}$ [210] and was measured as a solid, apart from the highconcentration spectra shown in Chapter 7. The vapour pressures were taken from Refs. [211] (solids) and [212] (liquid). The melting point of pivalic acid lies above room temperature $\left(36^{\circ} \mathrm{C}[213]\right)$, which is why it has always been measured as a solid apart from the Raman measurements with the heatable saturator shown in Figure 6.12. The vapour pressures were taken from Reference [205]. The supplier and purities of the acids, noble (helium and argon), and test gases (nitrous oxide and hexafluoroethane) are summarised in Table 3.2.

Generally, one should keep in mind that the estimated vapour pressures and the resulting concentrations are solely upper limits as equilibrium between the solids or liquids and the gas phase is not fully reached in the saturator due to the short waiting times in between different measurements and the re-filling of the reservoirs during a pulse. The concentration uncertainty is enhanced for solids due to large changes in surface area during measurements. The temperature-dependent equilibrium constant of monomers and dimers in the gas phase adds additional uncertainty. Hence, the concentrations listed throughout this work serve as a measure of number density in the expansions and should only be compared relative to each other. For carboxylic acid dimers, the IR intensity and Raman scattering cross-sections of the $\mathrm{C}=\mathrm{O}$ stretching vibrations are comparatively constant so that for the mixed spectra in Chapter 5 , the relative concentrations can be compared via the band intensities of the homo dimers (in cases where these do not overlap). 
Table 3.2: CAS numbers, suppliers, and purities of the carboxylic acids and gases used for the jet measurements throughout this work. Formic acid- $d$ corresponds to HCOOD and formic- $d$ acid- $d$ to DCOOD.

\begin{tabular}{cccc}
\hline \hline acid & CAS number & supplier & purity \\
\hline \multirow{2}{*}{ formic acid } & $64-18-6$ & $\begin{array}{c}\text { Acros Organics } \\
\text { Emsure }\end{array}$ & $\begin{array}{c}\geq 9 \%, 98+\% \\
98-100 \%\end{array}$ \\
formic acid- $d$ & $925-94-0$ & abcr & $95 \%$ in $\mathrm{D}_{2} \mathrm{O}, 98 \%$ deuterium \\
formic- $d$ acid- $d$ & $920-42-3$ & Eurisotop & $95 \%$ in $\mathrm{D}_{2} \mathrm{O}, 98 \%$ deuterium \\
acetic acid & $64-19-7$ & Acros Organics & $95 \%$ in $\mathrm{D}_{2} \mathrm{O}, 98 \%$ deuterium \\
& & Fluka Chemika & $99.8 \%$ \\
pivalic acid & $75-98-9$ & Fluka & $\geq 99 \%$ \\
helium & $7440-59-7$ & Alfa Aesar & $99 \%$ \\
argon & $7440-37-1$ & Linde & $99 \%$ \\
nitrous oxide & $10024-97-2$ & Linde & $99.996 \%$ \\
hexafluoroethane & $76-16-4$ & Linde & $99.999 \%$ \\
\hline \hline
\end{tabular}

\subsubsection{The Helium Nanodroplet Set-up}

IR depletion spectra of formic acid in helium nanodroplets were recorded in collaboration with the group of Prof. Andrew Ellis at the University of Leicester. A detailed description of the set-up can be found in Refs. [84, 93, 214, 215]. Here, a brief discussion of the general approach is presented.

Helium nanodroplets of a size of about 5000 helium atoms were obtained by expanding helium (99.9999\% purity) at 32 bar through a pinhole nozzle ( 5 um diameter) cooled down to $16 \mathrm{~K}$. The droplet size can be adjusted by variation of the helium backing pressure and the nozzle temperature [216]. The dopant is added in a pick-up cell using a needle valve to control its partial pressure. For formic acid (99\%, SERVA), a partial pressure of $4 \cdot 10^{-6}$ mbar was used. As detailed in Section 5.2, this partial pressure corresponds to an average pick-up of roughly one formic acid molecule per droplet.

To record IR spectra, the doped droplets are overlapped with a tunable IR laser beam from an optical parametric amplifier (OPA, LaserVision) after the pick-up chamber. The OPA laser has a band width of $4 \mathrm{~cm}^{-1}$ and operates at a repetition rate of $10 \mathrm{~Hz}$ [84]. Following IR excitation, the doped droplets are ionized via electron impact at $90 \mathrm{eV}$ in a quadrupole mass spectrometer. IR spectra are obtained by measuring the change in ion signal as a function of laser wavelength. If the IR frequency of the laser is resonant with a vibration of the dopant, heat is dispersed into the droplet as a result of the absorption. This causes an evaporation of helium and therefore, a reduction of the geometrical cross-section of the droplet, which can be detected as a depletion of the ion signal in the respective mass-channel. These IR depletion spectra can be recorded for 
different mass-channels. Apart from measuring the ion signal at a fixed mass-to-charge ratio, it is also possible to record mass spectra over a large range of masses.

The geometry of the helium nanodroplet set-up is such that the tunable IR beam passes the ionisation region of the quadropole mass spectrometer. Consequently, background signal of cations may arise when the ionisation occurred prior to IR excitation. To ensure that the vibrational features in the mass-channels are not due to IR absorption of cations but neutrals, additional spectra with enhanced background signal were measured and subtracted from the raw data. This was necessary for mass channel $m / z=19$.

\section{Gas phase simulation}

In helium nanodroplets, a solvent shift has been observed for hydrogen bonded complexes, which increases linearly with associated downshift of the vibrational band [87]. To account for this, gas phase spectra have been simulated by compressing the raw helium nanodroplet spectra by an estimated upper bound of the solvent shift of about $5 \%$ of the hydrogen bond induced downshift. This approximate value has been obtained by an extrapolation of the reported shifts from Ref. [87] with an intercept at zero. The conversion formula applied is shown below, where $\tilde{\nu}_{\mathrm{He}}$ represents the helium nanodroplet band positions, $\tilde{\nu}_{\mathrm{M}}$ the monomer band position $\left(3570 \mathrm{~cm}^{-1}\right)$ [79], and $\tilde{\nu}$ the approximated gas phase value.

$$
\tilde{\nu}=\frac{20 \tilde{\nu}_{\mathrm{He}}+\tilde{\nu}_{\mathrm{M}}}{21}
$$

This transformation is used to get a rough estimate of the gas phase band position and is by no means an attempt of an exact conversion.

\subsection{Theoretical Methods}

Quantum chemical calculations within this work were exploited for two purposes - to support spectral assignments and to test the applicability of commonly used methods for the vibrational descriptions of the systems studied within this work in form of benchmarks. Obviously, the requirements for the two approaches are different and will be detailed below. The quantum chemical program packages used are Gaussian 09 (revision E.01) [36] and ORCA (version 4.0.1) [37, 38].

\subsubsection{Band Position Assignments}

For spectral assignments, the conformational space was searched. The structure motifs of the carboxylic acid monomers and their hydrogen bonded clusters have been thoroughly characterised in the literature both experimentally $[217,218]$ as well as theoretically $[83$, 219,220 ], which this work is mostly based on. For geometry optimisations and harmonic frequency calculations, the B3LYP functional [221, 222] is used in combination with Grimme's D3 dispersion correction [223] and Becke-Johnson damping [224], as it has shown to be a good compromise between accuracy of absolute and relative band 


\section{Experimental and Theoretical Methods}

Table 3.3: DFT integration grid, optimisation criteria, and density fitting settings used for B3LYP-D3(BJ) calculations in the program packages ORCA (version 4.0.1) [37, 38] and Gaussian 09 [36].

\begin{tabular}{cccc}
\hline \hline program & integration grid & optimisation & density fitting \\
\hline Gaussian & ultrafine & tight & yes \\
ORCA & grid5 & tight & no \\
\hline \hline
\end{tabular}

positions and cost efficiency [225]. All band position predictions were performed with Gaussian 09 [36], apart from the calculations shown in Chapter 6, which were carried out with the ORCA program package $[37,38]$. The DFT integration grid and optimisation criteria used for both program packages are summarised in Table 3.3. All harmonic frequency calculations were performed without the use of symmetry $\left(C_{1}\right)$, unless otherwise noted. The differences in harmonic $\mathrm{C}=\mathrm{O}$ stretching frequencies obtained with both quantum chemical program packages amount to $1-2 \mathrm{~cm}^{-1}$, but can be larger for other fundamentals. These differences are mainly caused by the different implementation of B3LYP [36, 37].

Basis sets are adjusted to the system size. For carboxylic acid monomers and dimers, an augmented correlation consistent basis set by Dunning [226-228] was utilised, namely aug-cc-pVTZ, which is abbreviated to aVTZ throughout this work. For trimer and tetramer calculations, the smaller def2-TZVP basis set of the Ahlrichs group has been employed [229]. Relative energy differences as well as dissociation energies are corrected for vibrational zero-point energy. Band position assignments are based on scaled, harmonic frequency calculations. In case of the two rotamers of the formic acid monomer studied in Chapter 4, the (harmonic) band positions are scaled to the respective fundamental of the global minimum trans-structure. The band positions of the higher-energy dimer studied in helium nanodroplets in Chapter 5 are scaled to the $\mathrm{OH}$ stretching vibration of the formic acid monomer at $3570 \mathrm{~cm}^{-1}$. All other harmonic band positions throughout this work are scaled to the antisymmetric $\mathrm{C}=\mathrm{O}$ stretching vibration of the formic acid dimer at $1741 \mathrm{~cm}^{-1}$.

The Raman scattering cross-sections $\sigma$ in the $90^{\circ}$ scattering geometry can be determined from the Raman activity $A$ provided by Gaussian 09 [36] and ORCA [37, 38], the vibrational temperature $T$, here assumed to be $100 \mathrm{~K}$, the predicted wavenumber $\tilde{\nu}_{i}$ of the mode $i$, its degeneracy $g_{i}$, and the laser wavelength $\tilde{\nu}_{L}$, as shown in Equation 3.3. A detailed derivation can be found in the $\mathrm{PhD}$ thesis of T. Wassermann [195]. The grating of the monochromator disperses the perpendicularly polarised laser light $\perp^{\mathrm{s}}$ more effectively than that with parallel polarisation $\|^{\mathrm{s}}[196]$. This effect has not been accounted for in this work, as the Raman scattering cross-sections $\sigma$ are only compared relative to one another.

$$
\sigma=g_{i} \cdot \frac{2 \pi^{2} h}{45 c \tilde{\nu}_{i}} \cdot \frac{\left(\tilde{\nu}_{\mathrm{L}}-\tilde{\nu}_{i}\right)^{3} \cdot \tilde{\nu}_{\mathrm{L}}}{1-\exp \left(-\frac{h c \tilde{\nu}_{i}}{k T}\right)} \cdot A
$$




\subsubsection{Performance Tests of Different Methods}

For harmonic (carboxylic acid dimers) as well as anharmonic vibrational benchmarks (formic acid monomer as well as global minimum and higher-energy dimer), the performance of different DFT functionals, semi-empirical, as well as ab initio methods was examined. The DFT methods tested include PBE0-D3, M06-2X, and $\omega$ B97-XD in addition to B3LYP-D3(BJ) and B2PLYP-D3(BJ). Two semi-empirical methods were chosen, namely PBEh-3c developed by the Grimme group and PM3. In addition to MP2, spincomponent-scaled (SCS)-MP2 using the resolution of identity (RI) approximation has been picked as a second ab initio method. The SCS-MP2 and PBEh-3c calculations have been exclusively performed with the ORCA program package (version 4.0.1) [37, 38], whereas all other calculations have been carried out in Gaussian 09 [36]. A summary of all methods, their parameters, and references can be found in Table 3.4.

Anharmonic vibrational frequency calculations were performed with vibrational perturbation theory (VPT2) as implemented in Gaussian 09 [36], as it has shown to perform reasonably well for small, relatively rigid molecules [32]. The default version has been used that removes resonances in a pre-screening and treats these variationally. This default version is called generalised VPT2 (GVPT2). As will be detailed in Chapter 4.4, VPT2 calculations using DFT methods are, among other things, integration grid size sensitive. For M06-2X, this is well-known [230, 231]. Therefore, two grid sizes were tested, namely the pruned ultrafine grid utilised for all harmonic vibrational frequency calculations (Table 3.3) and the pruned superfine grid. Pruned hereby refers to a minimisation of the number of grid points while remaining a chosen level of accuracy [36]. The ultrafine grid exhibits 99 radial shells and 590 points per shell $(99,590)$. It is larger than the default grid (fine grid, $(75,302)$ ) of Gaussian 09 [36]. The superfine is the next-larger grid and has 150 shells with 974 points per shell $(150,974)$ for the elements used in this work [36].

Additionally, the sensitivity of the VPT2 calculations towards the implementation of symmetry and the optimisation settings was explored. The rotamers of the formic acid monomer exhibit $C_{\mathrm{s}}$ point group symmetry. The same applies for the higher-energy dimer of formic acid studied in Chapter 5. The global minimum dimer of formic acid exhibits the highest point symmetry $\left(C_{2 \mathrm{~h}}\right)$. The convergence criteria of the geometry optimisation were varied from opt $=$ tight (standard, see Table 3.3) to opt = very tight. The resulting eight anharmonic vibrational frequency calculations for DFT methods (two grids, two optimisation criteria, and two symmetries) were averaged. For ab initio methods such as MP2, these reduce to four (no integration grid). The standard deviation of the band positions and intensities is given as measure of sensitivity. A detailed description of these effects will be given in Section 4.4.

For the monomer benchmark, the augmented quadruple zeta basis set aug-cc-pVQZ (aVQZ) by Dunning [226, 227] is used. For the basis set sensitive methods, additional double- (aVDZ) and triple-zeta (aVTZ) calculations were performed. All VPT2 calculations of dimers have been carried out with the aVTZ basis set. Further details can be found in the respective chapters. 
3 Experimental and Theoretical Methods

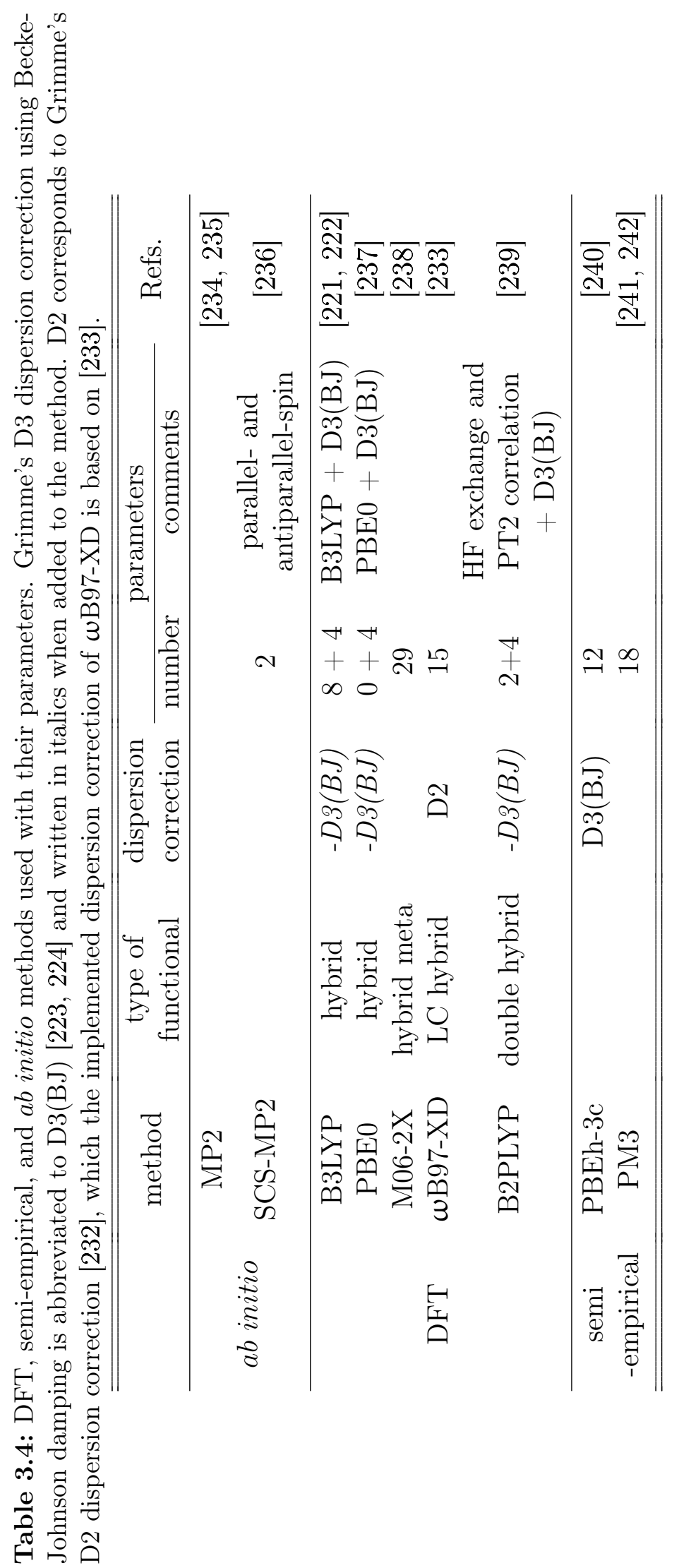




\section{Monomers}

\subsection{Introduction}

The dynamics of molecules or molecular systems are governed by their multidimensional potential energy hypersurface (PES), an important conceptual tool which originates from the Born-Oppenheimer approximation [243]. The first numerical PES has been developed by Eyring and Polyani in 1931 for $\mathrm{H}_{3}$ [244, 245], which was employed to determine the reaction rate of $\mathrm{H}_{2}+\mathrm{H}$ one year later [246]. In addition to reaction kinetics, another large field of application of potential energy surfaces is high-resolution molecular spectroscopy. Particularly for small molecules and their clusters, a myriad of spectroscopically accurate potential energy surfaces is available. Typical examples are carbon dioxide $\mathrm{CO}_{2}[247,248]$, water $\mathrm{H}_{2} \mathrm{O}$ [249-254] and small water clusters $\left(\mathrm{H}_{2} \mathrm{O}\right)_{2-3}$ [255-257], ammonia $\mathrm{NH}_{3}$ [258-262], hydrogen peroxide $\mathrm{H}_{2} \mathrm{O}_{2}$ [263-265], formaldehyde $\mathrm{H}_{2} \mathrm{CO}[266,267]$, the hydrogen fluoride dimer $(\mathrm{HF})_{2}$ [268-271], methane $\mathrm{CH}_{4}$ [272-275], and methanol $\mathrm{CH}_{3} \mathrm{OH}$ [276-278]. In order to critically examine their quality, accurate experimental benchmark data is needed. In the gas phase, vibrational spectroscopy of such reference systems is normally focussed on the global minimum structures, as these are most abundant at room temperature. Consequently, the potential energy surface near that region can be well tested, yet critical evaluation of higher energy regimes, i.e., local minima or saddle points is obviously not feasible to the same extent. Consequently, for an assessment of the globality of a potential energy surface prediction, the vibrational scrutiny of local minima needs to be pushed forward, which is the main motivation behind this chapter.

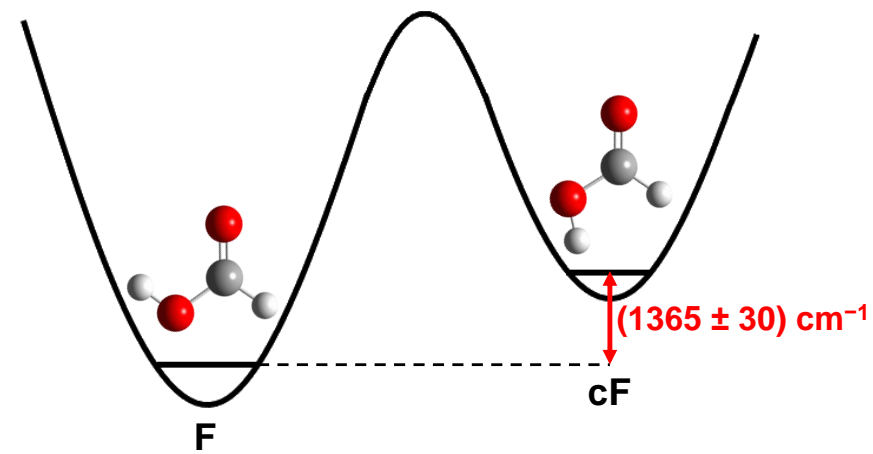

Figure 4.1: Most stable structures of trans- $(\mathrm{F})$ and cis-formic acid (cF), calculated at the B3LYP-D3(BJ)/aVTZ level with the experimentally determined energy difference by Hocking [58]. 
Examples of small systems with characteristic higher-energy structures are the tautomers hydrogen cyanide and hydrogen isocyanide HCN/HNC [279] and formaldehyde $\mathrm{H}_{2} \mathrm{CO}$, whose photodissociation products form a near-isoenergetic van der Waals complex $\left(\mathrm{H}_{2}-\mathrm{CO}\right)$ [280, 281], which is challenging to describe by theory. A next-larger system of interest is the pentatomic, $C_{\mathrm{s}}$-symmetric formic acid molecule, which will be examined throughout this work. It exhibits rotational isomerism around the $\mathrm{C}-\mathrm{O}$ bond, as shown in Figure 4.1. The global minimum form is historically labelled trans-formic acid, yet the $\mathrm{C}=\mathrm{O}$ and $\mathrm{O}-\mathrm{H}$ groups are in cis-configuration (cf. Figure 4.1). Here, the historic labels will be used.

The spectroscopic interest in formic acid is widespread with a manifold of rotational $[58,217,282-292]$ and vibrational studies on the trans-monomer $[60,62,65,68,293-$ 315]. The first ones date back to the late nineteen-thirties [316, 317]. Formic acid is also a popular reference system for quantum chemical calculations [32, 296, 298, 318-328]. The most recent, full set of high-resolution experimental [305] as well as anharmonic $a b$ initio band positions [328] of all fundamentals of trans-formic acid can be found in Table 4.1. A schematic visualisation of the nine normal modes of formic acid is shown in Figure 4.2. Four of these are stretching vibrations $\nu(\mathrm{O}-\mathrm{H}, \mathrm{C}-\mathrm{H}, \mathrm{C}=\mathrm{O}$, and $\mathrm{C}-\mathrm{O})$, three in-plane $\delta(\mathrm{H}-\mathrm{C}-\mathrm{O}, \mathrm{H}-\mathrm{O}-\mathrm{C}, \mathrm{O}-\mathrm{C}=\mathrm{O})$, and two out-of-plane bending vibrations $\gamma(\mathrm{C}-\mathrm{H}, \mathrm{O}-\mathrm{H})$. The stretching and in-plane bending vibrations are of $A^{\prime}$ symmetry and the two out-of-plane bending vibrations of $A^{\prime \prime}$ symmetry. All fundamentals are labelled according to the Herzberg nomenclature [182], where the vibrations are grouped by symmetry (in descending order) and sorted by energy within a group.

A trans-formic acid topic of interest in recent years concerns a Fermi resonance of the $\mathrm{O}-\mathrm{H}$ in-plane bending vibration $\nu_{5}$ and the overtone of the $\mathrm{O}-\mathrm{H}$ torsion $2 \nu_{9}[315$, 327, 328]. Both band positions are listed in Table 4.2. Theoretical [327, 328] as well as experimental studies [315] suggest a label switch compared to previous experimental assignments such as that of Freytes and co-workers [305]. Here, additional evidence will be given to support it by a combined FTIR and Raman jet study. An important aspect throughout this work is the contrasting juxtaposition of FTIR and Raman data, when necessary. As will be seen in this case and in many more examples in the following chapters, for a full comprehension of the vibrational dynamics, the combination of both techniques is beneficial. Besides, the general amount of Raman gas phase data on the trans-formic acid monomer $\mathrm{F}$ is surprisingly scarce $[62,68]$. The first relevant Raman spectroscopic measurement of formic acid has been performed by Bertie and Michaelian in 1982 [62]. It was focussed on the detection of both the monomer and the dimer [62], so that fine details of the monomer signals are not as easily extractable, as these are in some cases overlapping with dimer bands. The same is valid for the Raman jet study by Xue from 2011 [68]. In the present work, pure monomer spectra are recorded by thermal dissociation of clusters, which nicely complement the available IR data, particularly for combination band assignments and the Fermi resonance of $\nu_{5}$ and $2 \nu_{9}$.

The cis-rotamer of formic acid $\mathrm{cF}$ is not nearly as well characterised in the gas phase. The reason for this is its low gas phase abundance of just $0.1 \%$ at room temperature governed by the large energy difference between both forms, which was determined to $(1365 \pm 30) \mathrm{cm}^{-1}$ in microwave spectroscopy measurements by Hocking in 1976 [58]. As 
Table 4.1: Gas phase band positions (exp., in $\mathrm{cm}^{-1}$ ) of the fundamentals and the overtone of the $\mathrm{O}-\mathrm{H}$ out-of-plane bending vibration of trans- and cis-formic acid measured with highresolution infrared spectroscopy by Freytes and co-workers [305] (trans-formic acid) and by Baskakov et al. [57] (cis-formic acid). For comparison, calculated (theo.) values by Richter and Carbonnière are listed, who constructed a potential energy surface at the CCSD(T)-F12a/aVTZ level and predicted the vibrational energy levels with the multi-configuration time-dependent Hartree (MCTDH) method [328]. The symmetry $\left(\mathrm{A}^{\prime}\right.$ and $\left.\mathrm{A}^{\prime \prime}\right)$ and respective assignments of the ten modes are given, whereby $\nu$ indicates stretching, $\delta$ in-plane, and $\gamma$ out-of-plane bending vibrations. A label switch of the Fermi resonance of $\nu_{5}$ and $2 \nu_{9}$ of trans-formic acid has been suggested by Refs. [315, 327, 328], which is why the band positions are displayed in italics. Newly determined cis-formic acid band positions obtained within the framework of this work are marked with bold letters. These are published in Refs. [329, 330].

\begin{tabular}{|c|c|c|c|c|c|c|}
\hline \multirow{2}{*}{ symmetry } & \multirow{2}{*}{ mode } & \multirow{2}{*}{ assignment } & \multicolumn{2}{|c|}{ trans } & \multicolumn{2}{|l|}{ cis } \\
\hline & & & exp. ${ }^{[305]}$ & theo. ${ }^{[328]}$ & exp. & theo. ${ }^{[328]}$ \\
\hline \multirow{8}{*}{$\mathrm{A}^{\prime}$} & $\nu_{1}$ & $\nu(\mathrm{OH})$ & 3570.5 & 3567 & $3637^{\mathrm{a}}$ & 3631 \\
\hline & $\nu_{2}$ & $\nu(\mathrm{C}-\mathrm{H})$ & 2942.06 & 2937 & $2873^{\mathrm{a}}$ & 2871 \\
\hline & $\nu_{3}$ & $\nu(\mathrm{C}=\mathrm{O})$ & 1776.86 & 1774 & $1818^{\mathrm{a}}$ & 1810 \\
\hline & $\nu_{4}$ & $\delta(\mathrm{H}-\mathrm{C}-\mathrm{O})$ & 1380 & 1375 & & 1383 \\
\hline & $\nu_{5}$ & $\delta(\mathrm{H}-\mathrm{O}-\mathrm{C})$ & 1223 & 1301 & & 1246 \\
\hline & $2 \nu_{9}$ & $2 \gamma(\mathrm{O}-\mathrm{H})$ & 1306.2 & 1216 & & 957 \\
\hline & $\nu_{6}$ & $\nu(\mathrm{C}-\mathrm{O})$ & 1104.85 & 1106 & $1093^{\mathrm{a}}$ & 1097 \\
\hline & $\nu_{7}$ & $\delta(\mathrm{O}-\mathrm{C}=\mathrm{O})$ & 626.16 & 623 & & 652 \\
\hline \multirow{2}{*}{$\mathrm{A}^{\prime \prime}$} & $\nu_{8}$ & $\gamma(\mathrm{C}-\mathrm{H})$ & 1033.47 & 1032 & & 1011 \\
\hline & $\nu_{9}$ & $\gamma(\mathrm{O}-\mathrm{H})$ & 640.72 & 637 & $493.420509(8)^{\mathrm{b}}$ & 491 \\
\hline $\begin{array}{l}\mathrm{a} \text { this work } \\
\mathrm{b} \text { Ref. [57] }\end{array}$ & & & & & & \\
\hline
\end{tabular}

indicated in Figure 4.1, the torsional barrier between both rotamers of formic acid is also high. It was estimated to $4842 \mathrm{~cm}^{-1}$ with respect to the bottom of the trans-potential well [331]. The rotational spectrum of cis-formic acid recorded by Hocking corresponds to its first spectroscopy detection in the gas phase, which was followed up by two further microwave studies $[217,331]$. The distinct detection advantage of this technique is the large dipole moment of cis-formic acid compared to its trans-form (cF: 3.79(1) D [58], F: $1.42 \mathrm{D}$ [217]) that somewhat compensates for its low abundance. The first, and until 2018, only vibrational gas phase band position of cis-formic acid has been determined by Baskakov and co-workers thirty years later in 2006. In a high resolution FTIR study, they have characterised the $\mathrm{O}-\mathrm{H}$ out-of-plane bending vibration $\nu_{9}$ (torsion) [57]. The key advantage of that cis-formic acid fundamental is the large spectral separation of nearly $150 \mathrm{~cm}^{-1}$ to the corresponding trans-vibration (cf. Table 4.1). Within the framework of this thesis, four new band positions of cis-formic acid were recorded with Raman jet spectroscopy by using thermal excitation to increase the population of cis-formic acid 


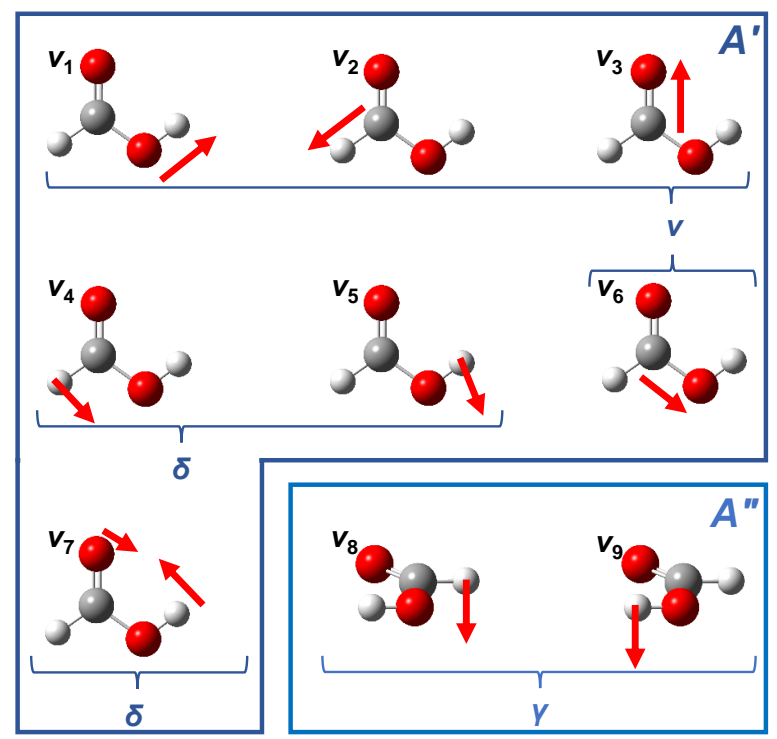

Figure 4.2: Schematic representation of the nine fundamentals of trans-formic acid. These can be divided into stretching vibrations $\nu$, in-plane $\delta$, and out-of-plane bending vibrations $\gamma$. The in-plane $\nu$ and $\delta$ vibrations are of $A^{\prime}$ and the out-of-plane $\gamma$ vibrations of $A^{\prime \prime}$ symmetry.

by an order of magnitude to $1-2 \%$. The results have been published in two Refs. in 2018 [329] and 2019 [330], which this chapter is partially based on.

In stark contrast to the gas phase, the cis-rotamer $\mathrm{cF}$ of formic acid has been thoroughly characterised in solid rare gas matrices [59, 60, 311, 314, 332-337]. The first vibrational study on cis-formic acid was performed by Pettersson, Lundell, Khriachtchev, and Räsänen in a solid argon matrix in 1997 [59]. A significant population of $\mathrm{cF}$ was obtained via $\mathrm{OH}$ overtone excitation of the trans-form [59]. This pioneering work was followed up by a vast number of cryomatrix studies on such higher-energy rotamers of carboxylic acids [60,314, 334, 337-342]. Matrix isolation spectroscopy, however, is not suitable for benchmarking gas phase calculations, as the vibrational bands are shifted compared to the gas phase, due to the perturbation that the matrix environment imposes on vibrations of the trapped species. As will be shown in Section 4.3, these shifts can be substantial and of opposite sign for different vibrations. Another recent condensed phase study on the cis-trans-isomerism of carboxylic acids was performed by Giubertoni, Sofronov, and Bakker in 2019 who measured the deuterated formic acid isotopologue HCOOD in deuterated water and acetonitrile solutions at room temperature and found a total of $20-30 \%$ of the cis-rotamer [343]. These pronounced differences to the gas phase are unexpected and an interesting starting point for future studies of the dynamics in solution.

Since formic acid is a reasonably small model system, it also enables an anharmonic vibrational frequency calculations using vibrational perturbation theory (VPT2), which has proven to be sufficient for the description of small, relatively rigid molecules [344]. In a study of formic and acetic acid and their nitrogen clusters as benchmarking model systems, VPT2 calculations have indeed proven to be adequate for formic acid, yet first 
insufficiencies were identified for acetic acid due to the large amplitude methyl torsions [79]. Due to the fourfold increase of cis-formic acid benchmark data achieved in this work, a more balanced benchmark is feasible as both the global and local minimum description of quantum chemical methods can be analysed. Additionally, the performance of two recent sets of high-level anharmonic ab initio band positions of all fundamentals of cis- and trans-formic acid by Tew and Mizukami [327] as well as Richter and Carbonnière [328] can be tested. Their trans-formic acid band position predictions are fairly similar, yet larger discrepancies are seen for the cis-rotamer. As both compared their cis-formic acid band position predictions to argon matrix values [60], a critical evaluation of the performance of these methods was not feasible. The new reference data on cis-formic acid enable an unbiased analysis, as both theoretical studies [327, $328]$ were published prior to it [329, 330]. This benchmark can be found in Section 4.4, yet initially, the focus is on trans-formic acid.

\section{2 trans-Formic Acid Fundamentals}

\subsubsection{Literature values}

As mentioned in the previous section, there have been numerous vibrational studies of trans-formic acid. The band positions of the most relevant ones are summarised in Table 4.2. Within this work, Raman spectra of all fundamentals of F (see Figure 4.3) have been recorded with the Raman jet apparatus described in Section 3.1.3. It exhibits a heatable nozzle and feed line, which cannot only be exploited to enhance the population of higher-energy structures such as cis-formic acid [329, 330] or cis-amides [197, 199], but also for thermal dissociation of clusters, enabling the measurement of pure monomer spectra. The thus obtained band positions are listed alongside the literature values in Table 4.2. The agreement with previous Raman jet data [68] and gas phase data from Bertie and Michaelian [62] from 1982 is satisfactory. The deviations amount to just $1 \mathrm{~cm}^{-1}$, which falls within the band position uncertainty of the Raman jet set-up used (cf. Section 3.1.3). The only exception is the aforementioned Fermi resonance, that will be discussed separately below. The exact band centres of $\nu_{8}$ and $\nu_{9}$ cannot be clearly ascertained from the spectrum and are therefore not listed in Table 4.2, but their approximate position is shown in Figure 4.3.

The band positions determined with infrared spectroscopy fluctuate more over time, but the agreement of the Raman jet band positions with the most recent, complete set of FTIR data from 2002 by Freytes and co-workers [305] as well as the high-resolution data of Baskakov and et al. [303, 306] is within $1 \mathrm{~cm}^{-1}$. The FTIR and Raman data recorded in solid rare gas matrices deviate considerably from the gas phase or jet values - not only in band position but also in sign of the matrix shift. While polar modes such as $\mathrm{C}=\mathrm{O}$ and $\mathrm{O}-\mathrm{H}$ stretching vibrations are downshifted in an argon matrix, the $\mathrm{C}-\mathrm{H}$ stretching vibration as well as the $\mathrm{C}-\mathrm{H}$ out-of-plane bending vibration are upshifted (cf. Table 4.2). Furthermore, different trapping sites inside the matrix cause additional splittings, as can be seen for the data of Maçôas and co-workers [60]. In addition to 


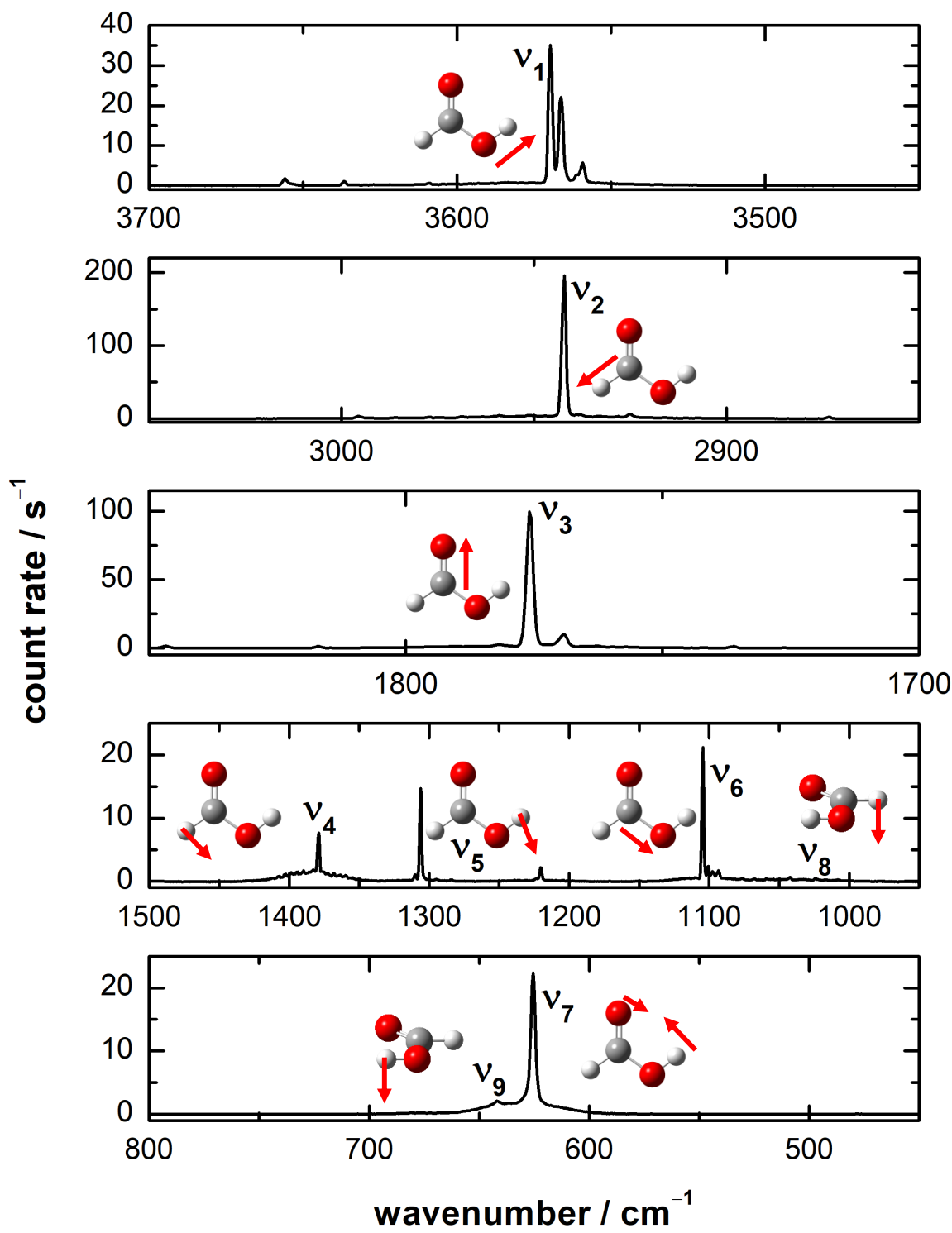

Figure 4.3: Raman jet spectra of all fundamentals of trans-formic acid ( $c<0.2 \%$ in helium), measured at a reservoir pressure of 1 bar and a nozzle temperature of $\theta_{\mathrm{n}}=190{ }^{\circ} \mathrm{C}$.

argon, matrix isolation spectra of trans-formic acid have also been measured in other matrix hosts such as neon [298, 335], nitrogen [345], or molecular hydrogen [309, 336]. 


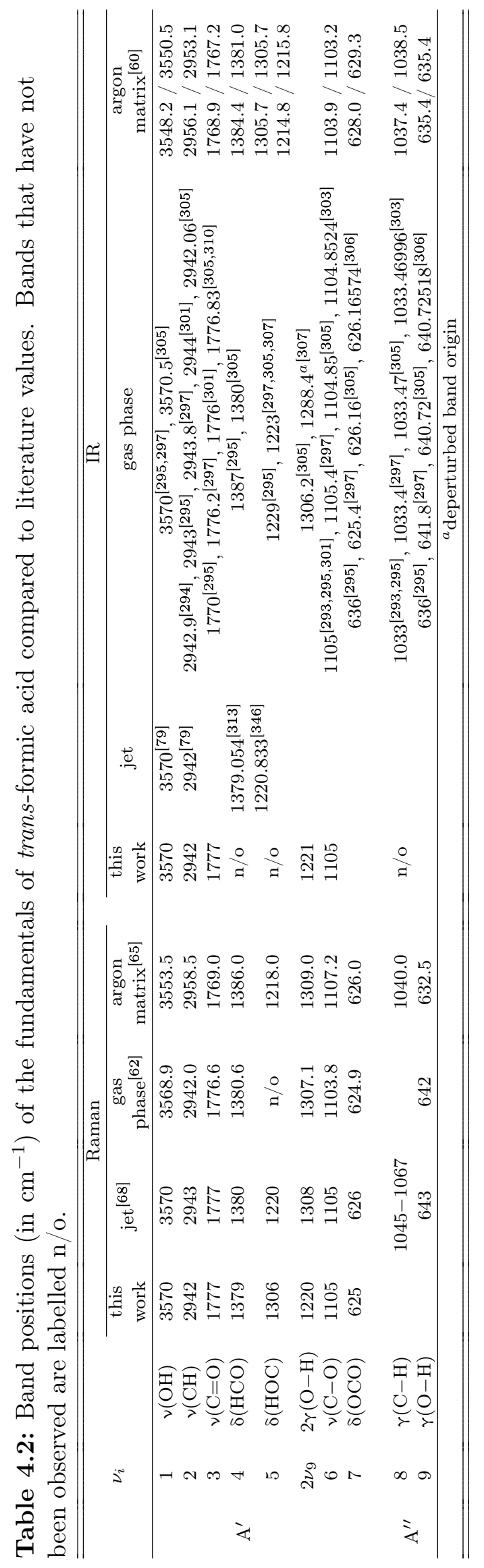

4 Monomers 


\subsubsection{Fermi resonance $\nu_{5}$ and $2 \nu_{9}$}

For a closer analysis of the assignment of $\nu_{5}$ and $2 \nu_{9}$, FTIR spectra have been recorded at three concentrations to contrast these with the previously shown Raman spectra (Figure 4.3). The results are depicted in Figure 4.4A. To ensure a good signal-tonoise ratio, the FTIR spectra have been averaged over around 2000 scans each. For reference, typically less than 200 scans are recorded for all other FTIR measurements shown in this work. The FTIR spectra exhibit one broad feature that extends from $1240-1210 \mathrm{~cm}^{-1}$. This broad band profile has been analysed by Goroya and co-workers in a high resolution IR spectroscopy study [346]. The formic acid monomer band was determined to $1220.83329(10) \mathrm{cm}^{-1}$ and three dimer bands were identified at $1219.71 \mathrm{~cm}^{-1}$, $1225.35 \mathrm{~cm}^{-1}$, and $1233.95 \mathrm{~cm}^{-1}$. The latter two were assigned to the $\left(\nu_{10}+\nu_{15}\right) \mathrm{com}$ bination band and the antisymmetric $\mathrm{C}-\mathrm{O}$ stretching fundamental $\left(\nu_{22}\right)$, respectively. The band at $1217.71 \mathrm{~cm}^{-1}$ was also attributed to a combination vibration, with several options discussed [346].

As seen in the overview of the mid-infrared region in Figure $4.4 \mathrm{~B}$, the IR bands of the $\mathrm{C}=\mathrm{O}\left(\nu_{3}\right)$ and $\mathrm{C}-\mathrm{O}$ stretching vibrations $\left(\nu_{6}\right)$ of the formic acid monomer are similarly broad and asymmetric. For a closer analysis of the broad feature between $1240-1210 \mathrm{~cm}^{-1}$, these two bands are plotted below the highest concentrated spectrum of Figure 4.4A and the dimer band position assignments of Ref. [346] in Figure 4.5. By assuming a similar band shape, one obtains $1221 \mathrm{~cm}^{-1}$ as a the monomer band position, which matches the value by Goroya and co-workers [346]. A suitable spectral region to estimate the abundance of monomers and dimers in the spectra is the $\mathrm{C}=\mathrm{O}$ stretching region, as the bands of hydrogen bonded clusters such as the formic acid dimer (FF) are clearly separated from the monomer band $\left(1777 \mathrm{~cm}^{-1}\right)$, as shown in the overview over the mid-infrared region depicted in Figure 4.4B. A small fraction of (FF) can be seen at $1741 \mathrm{~cm}^{-1}$ (antisymmetric $\mathrm{C}=\mathrm{O}$ stretching vibration) in the higher concentrated spectra, which is barely visible in the spectra at the lowest concentration. From the calculated, harmonic IR intensities at the B3LYP-D3(BJ)/aVTZ level (monomer $I_{\mathrm{F}}=373 \mathrm{~km} \mathrm{~mol}^{-1}$, dimer $I_{(\mathrm{FF})}=822 \mathrm{~km} \mathrm{~mol}^{-1}$ ), the monomer-to-dimer ratios in the spectra are estimated to 20:1,33:1, and 130:1. This confirms that the spectrum recorded at the lowest formic acid concentration is nearly cluster-free. Absolute line intensities are available for formic acid monomer fundamentals [301, 308, 310] (and Refs. therein) and vary from the B3LYP-D3(BJ) prediction by as much as $30 \%\left(284 \pm 43 \mathrm{~km} \mathrm{~mol}^{-1}\right)$ for $\nu_{3}$ [310]. Since the monomer band intensities are compared relative to those of the dimer, the overall errors are likely considerably smaller. Hence, the results can serve as a rough estimate.

In a previous study of Freytes and co-workers, the maximum $\left(1223 \mathrm{~cm}^{-1}\right)$ of the broad band in Figure 4.4 was assigned to the fundamental $\nu_{5}$ [305]. The overtone band $2 \nu_{9}$ in that same work has been observed at $1306.2 \mathrm{~cm}^{-1}$. Here, this band is only vaguely seen in the highest concentrated spectrum, if at all. This is likely due to the low concentration in the jet expansions $(0.01-0.05 \%)$ and the lower sensitivity compared to the high resolution FTIR spectra reported by Freytes and co-workers, who used a varying absorption path length of $0.2-4.8 \mathrm{~m}$ with a resolution of $0.002 \mathrm{~cm}^{-1}$ [305]. Their assignment of fundamental and overtone is based on the higher intensity of the lower wavenumber band 
Table 4.3: Harmonic band positions $\omega_{e}$ (in $\mathrm{cm}^{-1}$ ), IR intensities $I$ (in $\mathrm{km} \mathrm{mol}^{-1}$ ), and Raman scattering cross-sections $\sigma\left(10^{-36} \mathrm{~m}^{2} \mathrm{sr}^{-1}\right)$ of the fundamentals trans-formic acid, calculated at the B3LYP-D3(BJ)/aVTZ level.

\begin{tabular}{cccc}
\hline \hline$\nu_{i}$ & $\omega_{e}$ & $I$ & $\sigma$ \\
\hline 1 & 3716 & 60 & 76 \\
2 & 3049 & 40 & 172 \\
3 & 1811 & 373 & 40 \\
4 & 1402 & 2 & 19 \\
5 & 1299 & 9 & 10 \\
6 & 1122 & 262 & 12 \\
7 & 629 & 42 & 35 \\
8 & 1052 & 2 & 6 \\
9 & 675 & 137 & 4 \\
\hline \hline
\end{tabular}

compared to the signal at $1306.2 \mathrm{~cm}^{-1}$ and was confirmed by Baskakov and co-workers in 2006, who analysed the hot band $\left(2 \nu_{9}-\nu_{9}\right)$ [307]. In 2019, the topic was revisited by Hull and co-workers, who measured high-resolution torsional spectra of trans-formic acid [315]. This work was triggered by two ab initio VCI [327] and MCDTH studies [328] of (cis-and) trans-formic acid, both of which strongly suggested a re-assignment of these bands. By an intensity analysis of various transitions of the hot bands $\left(2 \nu_{9}-\nu_{9}\right)$ and $\left(\nu_{5}-\nu_{9}\right)$, Hull and co-workers were able to show that $2 \nu_{9}$ is in fact lower in wavenumber than $\nu_{5}$ [315], meaning that the band at $1306.2 \mathrm{~cm}^{-1}$ is the fundamental and accordingly, the band at $1221 \mathrm{~cm}^{-1}$ [346] the overtone.

Within this work, additional Raman spectra in the $\nu_{5}$ and $2 \nu_{9}$ region are provided (Figure 4.4). One of the many advantages of exploiting the interplay of Raman and FTIR jet spectroscopy can be seen when comparing the harmonically calculated IR intensities and Raman scattering cross-sections of the fundamentals of trans-formic acid listed in Table 4.3. $\nu_{4}$ and $\nu_{8}$ correspond to the weakest IR active formic acid monomer fundamentals with an IR intensity of just $2 \mathrm{~km} \mathrm{~mol}^{-1}$ and were therefore not observed in the FTIR spectra in Figure 4.4B. Both fundamentals could be detected in the corresponding Raman spectra, tough $\nu_{8}$ just very weakly (Figure 4.3), as its Raman scattering crosssection is also among the smallest of all fundamentals and amounts to about a third of that of $\nu_{4}$. Furthermore, the Raman set-up used in this work is very versatile - both the nozzle and feed line are heatable, which facilitates the use of higher concentrations $(0.2 \%$ compared to $0.01-0.05 \%$ for the IR spectra), as low cluster abundance is always ensured by the thermal dissociation of preformed clusters. At the same time, low-lying energy levels are thermally populated, which leads to an increase in the number of hot bands. Both can be distinguished from fundamentals, overtones, and combination bands, as the cluster bands decrease with increasing nozzle temperature if scaled to these, whereas hot bands increase. In Figure 4.4A, the spectra have been intensity-scaled to the band at $1306 \mathrm{~cm}^{-1}$. Hot bands are labelled $\mathrm{H}$ and the cluster bands $\mathrm{C}$. As expected, the band at $1220 \mathrm{~cm}^{-1}$ shows the same scaling behaviour as the band at $1306 \mathrm{~cm}^{-1}$. The small 
band position difference of $1 \mathrm{~cm}^{-1}$ in the IR $\left(1221 \mathrm{~cm}^{-1}\right)$ and Raman spectra $\left(1220 \mathrm{~cm}^{-1}\right)$ can be attributed Raman frequency calibration (see Section 3.1.3), which introduces an uncertainty of about $1-2 \mathrm{~cm}^{-1}$. The band at $1306 \mathrm{~cm}^{-1}$ is around 7 times more intense than the band at $1220 \mathrm{~cm}^{-1}$ (Figure 4.4B), meaning that the Fermi resonance between both is overall fairly weak. In the Raman spectra of Bertie and Michaelian, the latter was not observed, likely due to its lower intensity, whereby the overtone $2 \nu_{9}$ was assigned to $1307.1 \mathrm{~cm}^{-1}$ [62]. The Raman matrix isolation spectra measured by Olbert-Majkut and co-workers [65] show two monomer bands at $1309 \mathrm{~cm}^{-1}$ and at $1218.0 \mathrm{~cm}^{-1}$. Despite its higher intensity, the former was attributed to the overtone $2 \nu_{9}$ and the latter to the fundamental [65].

Fermi resonances between fundamentals and overtones are expected to exhibit the same intensity ratio in IR and Raman spectra, as the overtones usually have a near zero intensity (dark states) and only gain intensity via the wavefunction mixing with the fundamental (bright state). Therefore, this intensity mismatch is unexpected. One possible explanation could be that the overtone coincidentally has a higher intensity (IR intensity or Raman scattering cross-section) than the fundamental, which results in the unexpected case of a fundamental stealing intensity from an overtone in one of the spectra (IR or Raman).

The Raman scattering-cross sections of overtones and combination bands tend to be particularly small and are typically about two orders of magnitude weaker than fundamentals $[347,348]$. As this intensity difference is less pronounced in the infrared, IR spectra tend to be more complicated than Raman spectra [202]. Hence, it is likelier that the overtone has a higher IR intensity relative to the fundamental than a larger Raman scattering-cross section. Moreover, the overall Raman intensity of the band at $1306 \mathrm{~cm}^{-1}$ is similar with respect to the fundamentals in that region, namely $\nu_{4}$ and $\nu_{6}$ (see Figure 4.4B). From the harmonically predicted scattering cross-sections of all three fundamentals $\nu_{4}, \nu_{5}$, and $\nu_{6}$ (Table 4.3), this is expected, if the band at $1306 \mathrm{~cm}^{-1}$ was $\nu_{5}$. Due to the intensity redistribution caused by the wavefunction mixing, the crosssection prediction for $\nu_{5}$ will obviously slightly change if anharmonicity is introduced. Nonetheless, considering the small intensity of the second band at $1220 \mathrm{~cm}^{-1}$, the order of magnitude should still roughly match experiment. In the infrared, the most intense trans-formic acid fundamentals are the two $\mathrm{CO}$ stretching vibrations $(\mathrm{C}=\mathrm{O}$ and $\mathrm{C}-\mathrm{O}$, cf. Figure 4.4B and Table 4.3), whereas $\nu_{5}, \nu_{4}$, and $\nu_{8}$ exhibit by far the lowest IR intensities (Table 4.3). With that in mind, the likeliest explanation is that the IR intensity of the overtone $2 \nu_{9}$ exceeds that of $\nu_{5}$, resulting in the rare case of a fundamental stealing intensity from an overtone, whereas in case of the Raman spectra, it is the other way around, resulting in the differing IR and Raman intensity patterns.

In summary, in line with previous theoretical [327, 328] and experimental evidence [315], the Raman jet spectra in this work encourage a reassignment of the $\mathrm{O}-\mathrm{H}$ in-plane bending vibration $\nu_{5}$ to $1306 \mathrm{~cm}^{-1}$ and the overtone of the $\mathrm{O}-\mathrm{H}$ out-of-plane bending vibration $2 \nu_{9}$ to $1221 \mathrm{~cm}^{-1}$. Any resonance between the two states appears to be weak, with less than $10 \%$ intensity redistribution. This example is just one of a few debates surrounding trans-formic acid assignments, which can clearly benefit from additional Raman data. Several more examples are given in the next section. 


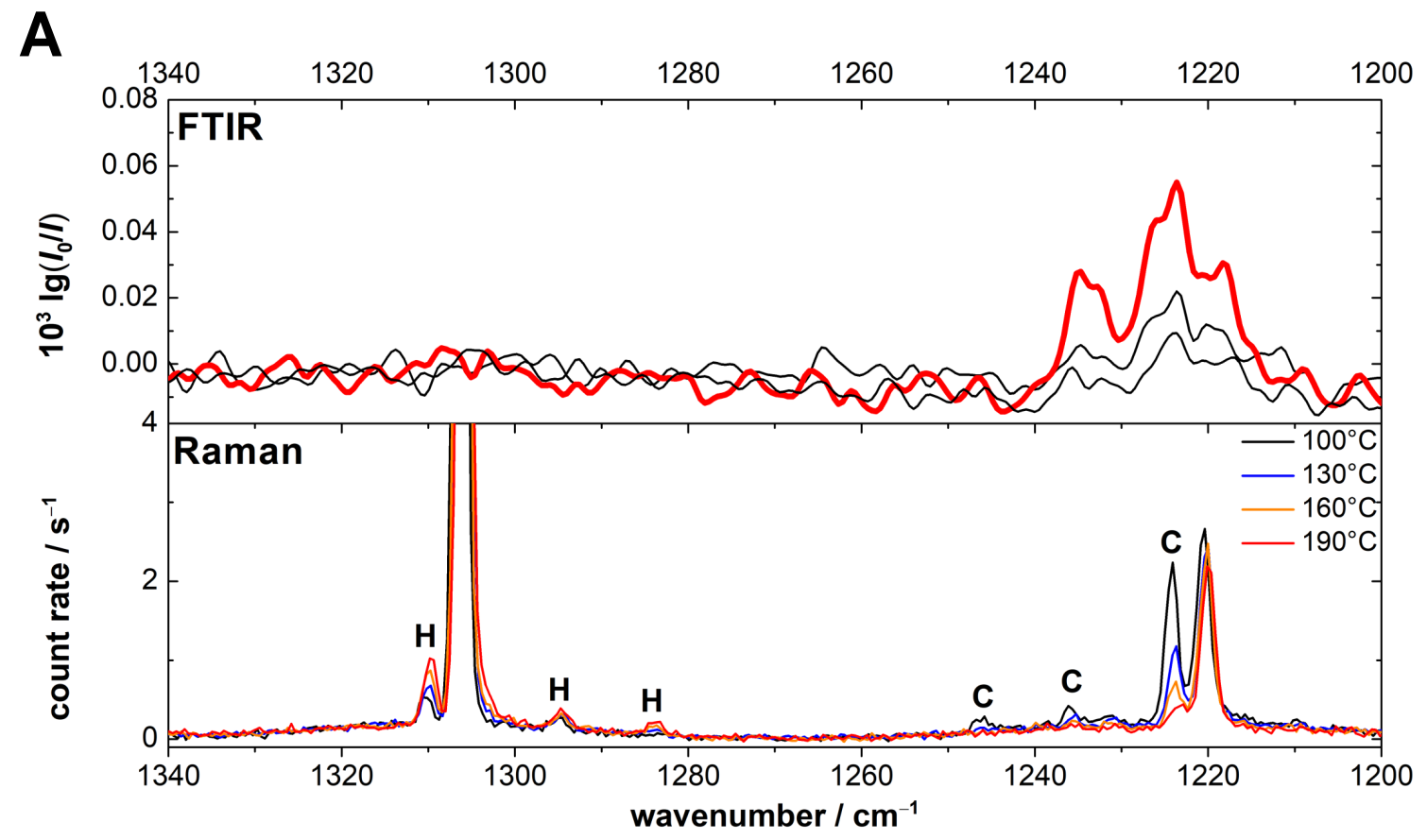

B

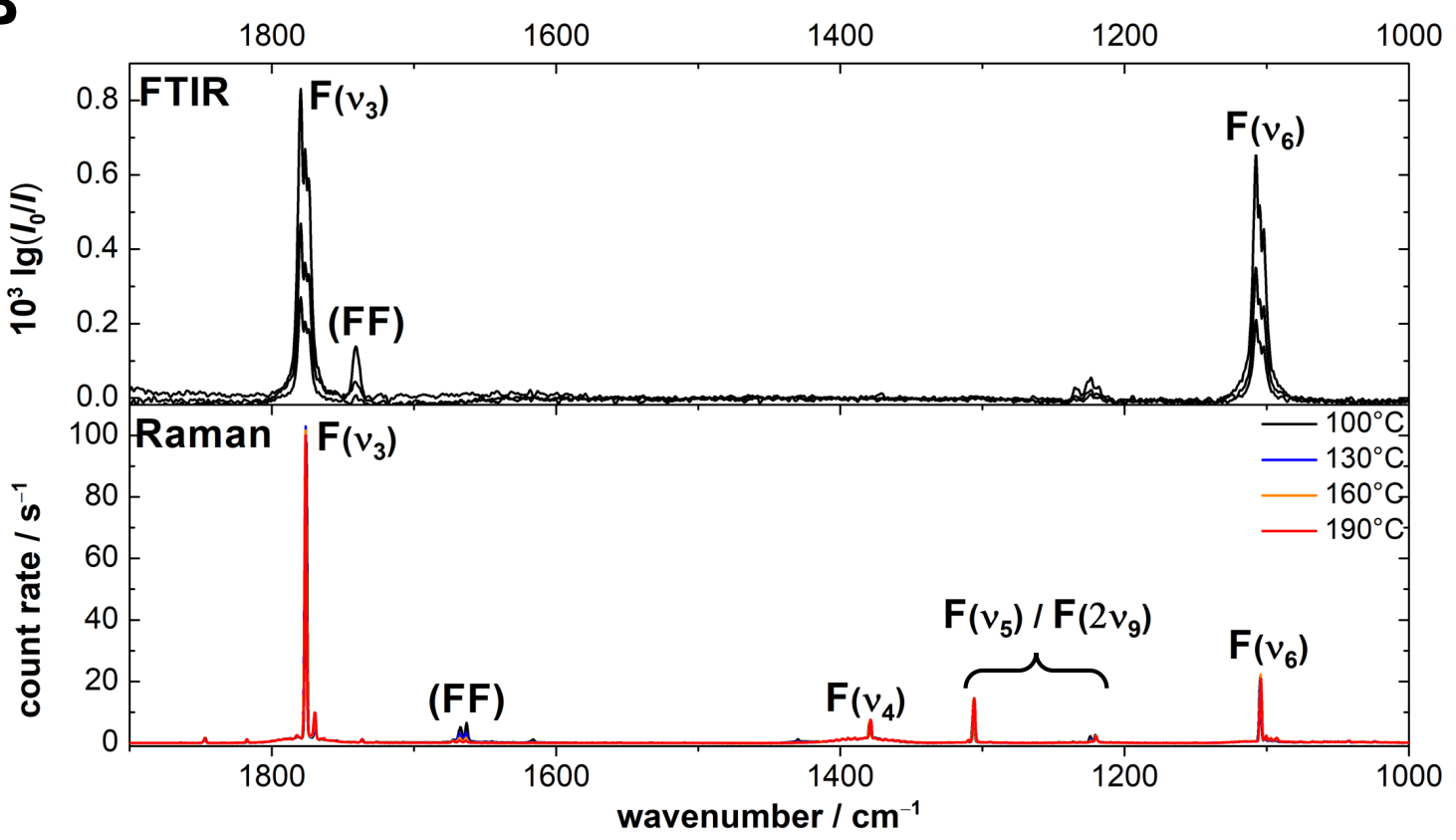

Figure 4.4: A: FTIR jet spectra (top) of formic acid at a reservoir pressure of $560 \mathrm{mbar}$ and increasing concentrations of $0.01 \%$ in helium (2000 scans), $0.02 \%$ in helium (1750 scans), and $0.05 \%$ in helium (2130 scans). The Raman jet spectra of formic acid $(<0.2 \%$ in helium) are shown below and have been measured at a reservoir pressure of 1 bar, increasing nozzle temperatures of $\theta_{\mathrm{n}}=100-190{ }^{\circ} \mathrm{C}$, and acquisition times of 30-35 min. B: FTIR and Raman jet spectra of formic acid between $1900-1000 \mathrm{~cm}^{-1}$ measured under the same conditions as those in panel A. 


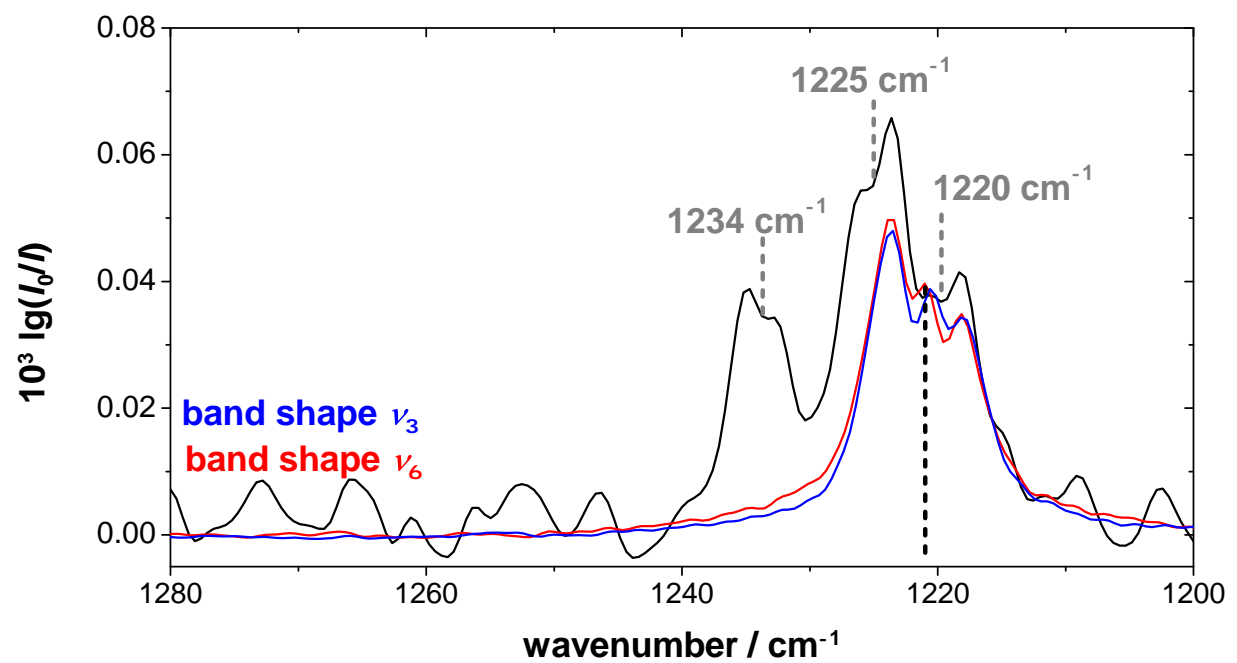

Figure 4.5: FTIR jet spectrum of formic acid in helium $(<0.05 \%)$ recorded at a reservoir pressure of $560 \mathrm{mbar}$ averaged over 2130 scans. In blue and red, intensity-scaled (red: 0.08, blue: 0.06 ) and wavenumber-shifted $\nu_{3}$ (blue, band centre: $1777 \mathrm{~cm}^{-1}$, shifted by $-556 \mathrm{~cm}^{-1}$ ) and $\nu_{6}$ (red, band centre: $1105 \mathrm{~cm}^{-1}$, shifted by $+116 \mathrm{~cm}^{-1}$ ) bands of formic acid are shown for identification of the band centre of the formic acid monomer band $\left(1221 \mathrm{~cm}^{-1}\right.$, black dotted line). Band positions of the formic acid dimer at $1234 \mathrm{~cm}^{-1}\left(\nu_{22}\right)$, at $1225 \mathrm{~cm}^{-1}\left(\nu_{10}+\nu_{15}\right)$, and $1220 \mathrm{~cm}^{-1}$ (combination band with unknown origin) are marked in grey and were taken from Ref. [346]. 


\section{3 cis-Formic Acid Fundamentals}

\subsubsection{Vibrational Detection of cis-Formic Acid}

As previously mentioned, the nozzle and feed line of the Raman jet apparatus are heatable. The upper temperature feasible amounts to $190{ }^{\circ} \mathrm{C}$, which translates into a $c i s$-formic acid population of $1-2 \%$, if differences in the partition function are disregarded. For this estimate, the harmonically calculated energy difference at the B3LYPD3(BJ)/aVTZ level has been used $\left(15.9 \mathrm{~kJ} \mathrm{~mol}^{-1}\right)$, which is just outside the error bar of the experimental value by Hocking $\left(16.33(36) \mathrm{kJ} \mathrm{mol}^{-1}\right)$ [58]. For successful detection, cis-formic acid fundamentals with a particularly high Raman scattering cross-section have to be chosen to somewhat compensate for its low abundance. The harmonically calculated Raman scattering cross-sections of all fundamentals of both rotamers are depicted in Figure 4.6. The scattering cross-sections $\sigma$ are largest for the stretching vibrations of cis-formic acid, namely $\nu_{1}(\mathrm{O}-\mathrm{H}), \nu_{2}(\mathrm{C}-\mathrm{H}), \nu_{3}(\mathrm{C}=\mathrm{O})$, and $\nu_{6}(\mathrm{C}-\mathrm{O})$. In addition, the $\sigma$ values are mostly similar for both rotamers with three exceptions the $\mathrm{C}-\mathrm{O}$ stretching vibration $\left(\nu_{6}\right)$, the $\mathrm{O}=\mathrm{C}-\mathrm{O}$ in-plane-bending vibration $\left(\nu_{7}\right)$, and the $\mathrm{O}-\mathrm{H}$ out-of-plane bending vibration $\left(\nu_{9}\right)$. For $\nu_{6}$ and $\nu_{9}$, the scattering cross-section of cis-formic acid is larger, whereas it is the other way around for $\nu_{7}$. As $\sigma$ of $\nu_{9}$ is overall weak, the most promising cis-formic acid vibrations for Raman detection are the stretching vibrations.

The spectra of $\nu_{1}, \nu_{2}, \nu_{3}$, and $\nu_{6}$ can be found in Figure 4.7 for four nozzle temperatures $\left(100-190{ }^{\circ} \mathrm{C}\right)$. To assist spectral assignments, harmonically calculated, $\mathrm{F}\left(\nu_{i}\right)$-scaled band positions of cis- and trans-formic acid have been plotted below the spectra. As throughout this (and the next) chapter, these harmonic vibrational frequency calculations have been performed at the B3LYP-D3(BJ)/aVTZ level to leave enough extension potential for larger clusters and anharmonic corrections. The spectra of each temperature series have been scaled to the respective trans-formic acid fundamental of lowest intensity. Since the nozzle orifice narrows with nozzle temperature due to thermal expansion, one would expect the overall lowest signal intensity for the $190{ }^{\circ} \mathrm{C}$ spectrum. However, concentration fluctuations can change this expected order. As aforementioned, by increasing the nozzle temperature, cluster bands can be distinguished from fundamentals and hot bands of the monomer, as the cluster bands decrease due to thermal dissociation, whereas the intensity of hot bands is enhanced due to thermal population. Due to the intensity scaling, monomer fundamentals, combination and overtone bands have the same intensity throughout the temperature series. There are two types of formic acid hot bands - isomeric hot bands, i.e., cis-formic acid bands, and hot bands originating from thermally populated low-lying energy levels of trans-formic acid. The former can be distinguished from the latter by a comparison of the band position and the intensity ratio with respect to the trans-formic acid fundamental. The isomeric hot bands are based on a different part of the potential energy surface (PES) connected by the $\mathrm{O}-\mathrm{H}$ torsion, while the hot bands originating from low-lying energy levels of trans-formic acid are based on the same structure. These $\left(\nu_{i}+\nu_{j}-\nu_{j}\right)$ hot bands are shifted from the respective fundamental by the coupling matrix element $x_{i j}$ of the two levels involved, 


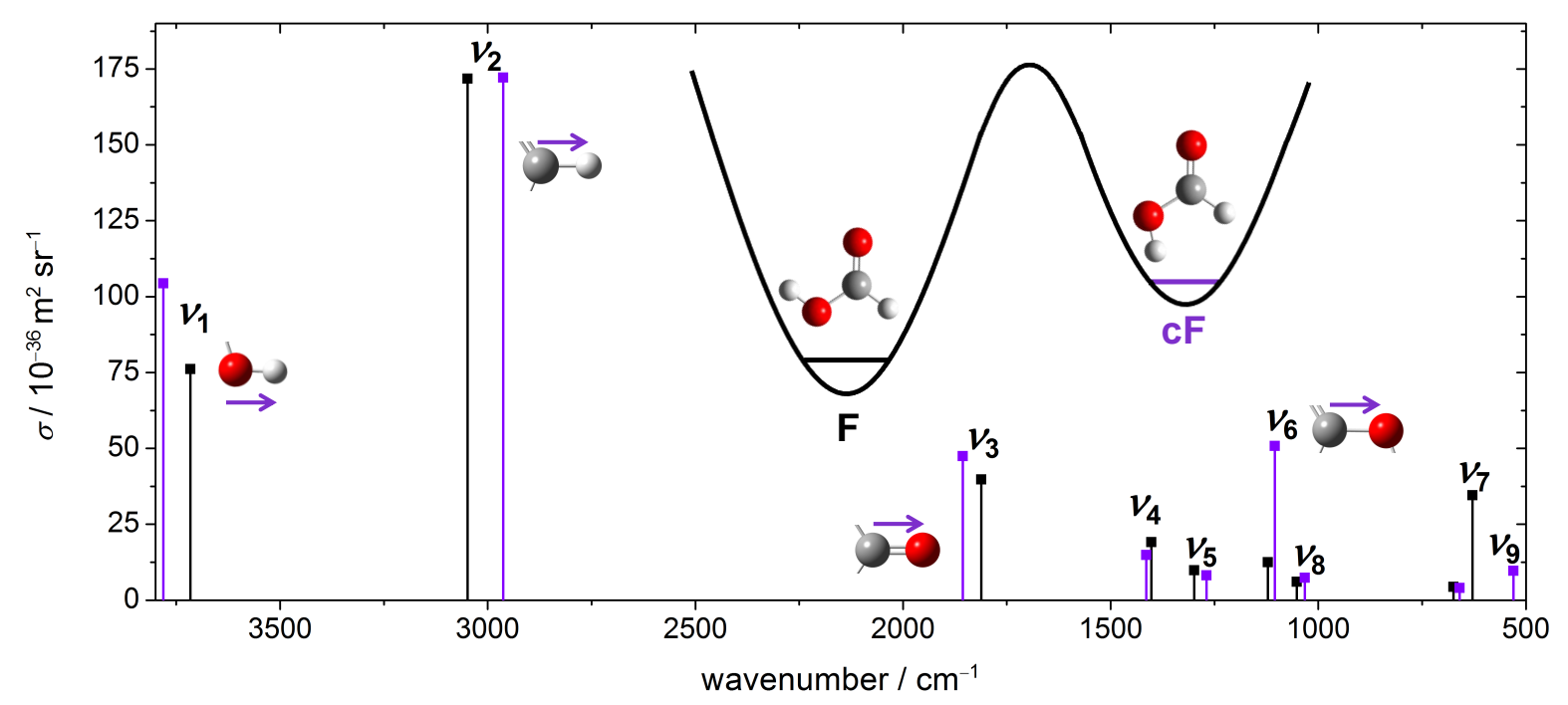

Figure 4.6: Predicted band positions and Raman scattering cross-sections $\sigma$ of all nine fundamentals of cis- (violet) and trans-formic acid (black), calculated at B3LYP-D3(BJ)/aVTZ level. Reprinted from Ref. [330] - Published by The Royal Society of Chemistry, licensed under CC BY 3.0.

where $\nu_{i}$ is the fundamental and $\nu_{j}$ the thermally populated energy level (Equation 2.6, Section 2.1). For a $\left(2 \nu_{i}-\nu_{i}\right)$ hot band, this difference amounts to $2 x_{i i}$. Moreover, the intensity of a hot band with respect to the trans-fundamental largely reflects the respective excitation energy, i.e., the energy of the thermally populated low-lying energy level or, in case of an isomeric hot band, the energy difference between both rotamers.

The $\mathrm{O}-\mathrm{H}$ stretching region of formic acid features one distinct hot band at $3637 \mathrm{~cm}^{-1}$, which deviates from the $\mathrm{F}\left(\nu_{1}\right)$-scaled harmonic prediction of the $\mathrm{cF}$ band position by $5 \mathrm{~cm}^{-1}$. The intensity compared to the fundamental of trans-formic acid $\left(3570 \mathrm{~cm}^{-1}\right)$ amounts to $2 \%$ at $190{ }^{\circ} \mathrm{C}$, if the different scattering cross-sections of both rotamers are taken into account $\left(\sigma_{\mathrm{F}} / \sigma_{\mathrm{cF}}=0.729\right)$, which matches the expected $\mathrm{cF}$ population $(1-2 \%)$. As such, the band at $3637 \mathrm{~cm}^{-1}$ can be assigned to $\nu_{1}$ of cis-formic acid. The two additional bands downshifted compared to $\mathrm{F}$ at $3566 \mathrm{~cm}^{-1}$ and $3560 \mathrm{~cm}^{-1}$ are most likely trans-formic acid combination bands involving the $\mathrm{C}-\mathrm{H}$ stretching vibration $\nu_{2}\left(2942 \mathrm{~cm}^{-1}\right)$ and the lowest-lying fundamentals $\nu_{9}\left(641 \mathrm{~cm}^{-1}\right)$ and $\nu_{7}\left(626 \mathrm{~cm}^{-1}\right)$, which profit from the large scattering cross-section of $\nu_{2}$. Both are in reasonably good agreement with available theory predictions of Tew and Mizukami $\left(\nu_{2}+\nu_{7}=3558 \mathrm{~cm}^{-1}\right.$; $\nu_{2}+\nu_{9}=3571 \mathrm{~cm}^{-1}$, in Fermi resonance with $\left.\nu_{3}+3 \nu_{9}\right)$ [327] and Richter and Carbonnière $\left(\nu_{2}+\nu_{7}=3566 \mathrm{~cm}^{-1}\right)$ [328]. A similar triad of the $\mathrm{OH}$ stretching band was observed in helium nanodroplets by Madeja and co-workers, who ascribed it to Fermi and Coriolis resonances [349].

The $\mathrm{C}-\mathrm{H}$ stretching region of formic acid is ideal for hot band assignments, due to the large scattering cross-section of $\nu_{2}$ (cf. Figure 4.6), yet spectrally more congested as cluster bands are barely separated from those of the monomer. The broad fine structured socket of $\nu_{2}$ of trans-formic acid at $2942 \mathrm{~cm}^{-1}$ is due to the rovibrational $\mathrm{O}$ and $\mathrm{S}$ 
Table 4.4: Calculated anharmonic (VPT2) band positions $\left(\right.$ in $\mathrm{cm}^{-1}$ ) of trans-formic acid alongside calculated diagonal (italics) and off-diagonal anharmonicity constants $x_{2 i}, x_{3 i}$, and $x_{6 i}\left(\right.$ in $\left.\mathrm{cm}^{-1}\right)$ of $\nu_{2}, \nu_{3}$, and $\nu_{6}$ with all nine fundamentals. Reprinted from Ref. [330] - Published by The Royal Society of Chemistry, licensed under CC BY 3.0.

\begin{tabular}{ccccc}
\hline \hline \multirow{2}{*}{$\nu_{i}$} & band position & \multicolumn{3}{c}{ B3LYP-D3(BJ)/aVTZ VPT2 } \\
& & $\boldsymbol{x}_{\boldsymbol{2} \boldsymbol{i}}$ & $\boldsymbol{x}_{\boldsymbol{3} \boldsymbol{i}}$ & $\boldsymbol{x}_{\boldsymbol{6} \boldsymbol{i}}$ \\
\hline 1 & 3533 & -3.5 & -1.1 & -0.9 \\
2 & 2892 & -61.9 & -13.4 & -2.3 \\
3 & 1779 & -13.4 & -9.3 & -4.1 \\
4 & 1374 & -20.4 & -0.3 & -6.2 \\
5 & 1219 & -6.1 & +2.8 & -14.3 \\
6 & 1089 & -2.3 & -4.1 & -6.2 \\
7 & 622 & +0.4 & -6.2 & -3.7 \\
8 & 1031 & -17.1 & -5.4 & -3.7 \\
9 & 643 & -2.8 & -0.4 & -5.6 \\
\hline \hline
\end{tabular}

branches. Between $2970-2945 \mathrm{~cm}^{-1}$ and $2935-2925 \mathrm{~cm}^{-1}$, there are several bands that decrease in intensity with temperature, namely cluster bands. The pronounced band at $2996 \mathrm{~cm}^{-1}$ remains approximately constant with temperature, meaning that it can most likely be attributed to a combination band or overtone of trans-formic acid. Additionally, there are two distinct bands that increase in intensity with temperature at $2925 \mathrm{~cm}^{-1}$ and at $2873 \mathrm{~cm}^{-1}$, i.e., hot bands. Next to the fundamental at $2938 \mathrm{~cm}^{-1}$ is a band whose intensity is slightly decreasing with increasing nozzle temperature up to $160^{\circ} \mathrm{C}$. In the $190^{\circ} \mathrm{C}$ spectrum, the band has the same intensity as in the lowest nozzle temperature spectrum $\left(100^{\circ} \mathrm{C}\right)$. This could be a result of an overlap of a hot band with a cluster band - the former increasing and the latter decreasing in intensity with temperature.

The calculated and $\nu_{2}(F)$-scaled band position of the $\mathrm{C}-\mathrm{H}$ stretching vibration of cis-formic acid $\left(2859 \mathrm{~cm}^{-1}\right)$ is downshifted by $14 \mathrm{~cm}^{-1}$ from the hot band at $2873 \mathrm{~cm}^{-1}$. The band position mismatch was smaller for the cis-O-H stretching vibration with a deviation of solely $5 \mathrm{~cm}^{-1}$. However, a Fermi resonance of the $\mathrm{C}-\mathrm{H}$ stretching fundamental and the overtone of the $\mathrm{C}-\mathrm{H}$ bending vibration $2 \nu_{4}$ is predicted for cis-formic acid [327, 328], which is also found by the B3LYP-D3(BJ)/aVTZ VPT2 calculations. Due to such stretch-bend Fermi resonances, the $\mathrm{C}-\mathrm{H}$ stretching vibration is notoriously harder to predict by theory, which is why such an inferior agreement is not surprising. If the intensity ratio of this potential cis-formic acid band is compared to the transfundamental and insignificantly corrected by the cross-section ratio $\left(\sigma_{\mathrm{F}} / \sigma_{\mathrm{cF}}=0.998\right)$, this results in $1 \%$ of cis-formic acid. As described above, this matches the expected $\mathrm{cF}$ population at $190{ }^{\circ} \mathrm{C}$, so that the band at $2873 \mathrm{~cm}^{-1}$ is assigned to the $\mathrm{C}-\mathrm{H}$ stretching vibration of $\mathrm{cF}$.

To identify the origin of the second hot band at $2925 \mathrm{~cm}^{-1}$, the diagonal and offdiagonal anharmonicity matrix elements $x_{2 i}$ of $\nu_{2}$ with all other vibrational energy levels can be compared to the experimental shift of that band with respect to the fundamental 

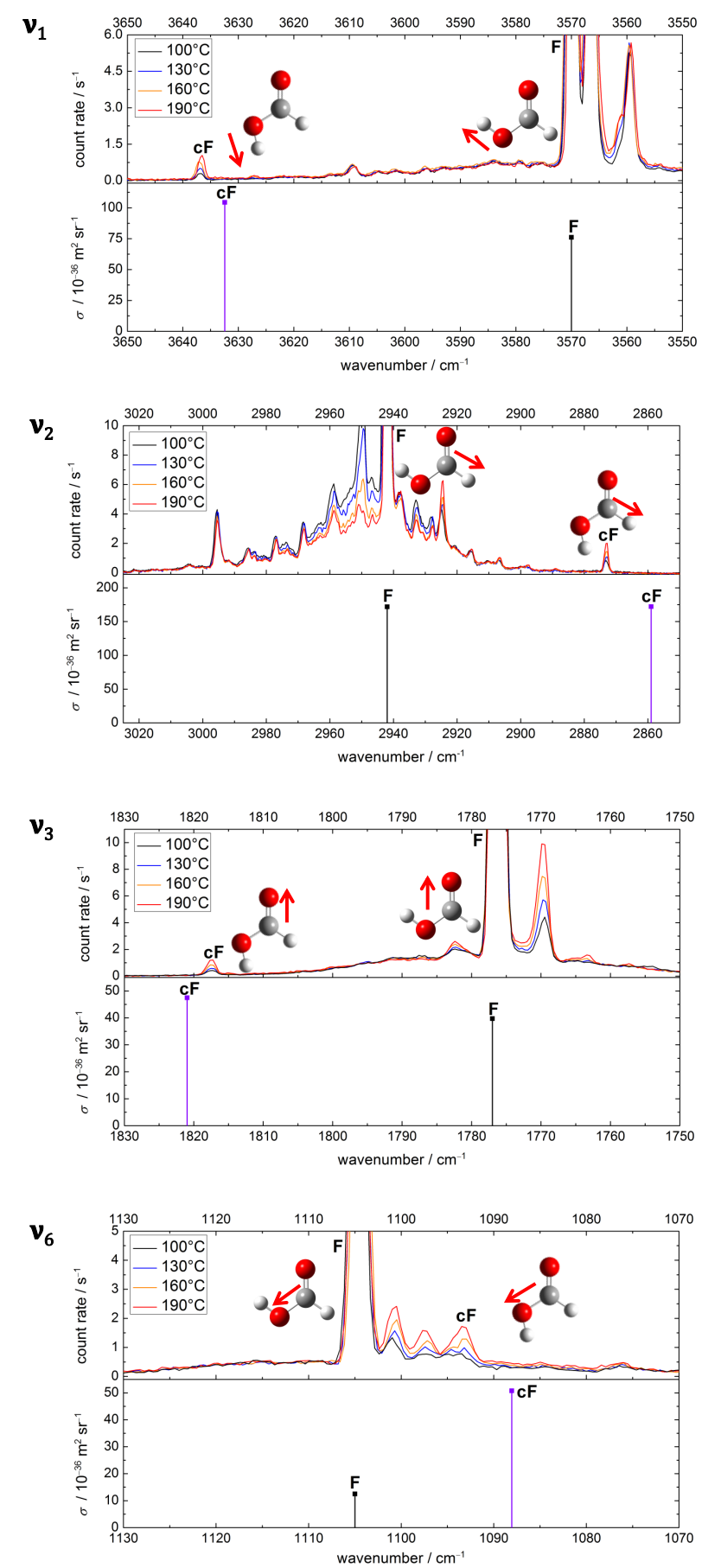

Figure 4.7: Raman jet spectra of formic acid $(<0.2 \%)$ in helium recorded at a reservoir pressure of 1.0 bar with increasing nozzle temperatures $\theta_{\mathrm{n}}$ of $100-190^{\circ} \mathrm{C}$. Four spectral regions are covered at four temperatures with data acquisition times between 28 and 63 minutes. Within a spectral region, the spectra have been intensity-scaled to the trans-formic acid monomer band $\mathrm{F}$ with the lowest concentration amongst the four temperatures. Below each temperature series, calculated, $\nu_{i}(\mathrm{~F})$-scaled harmonic band positions (B3LYP-D3(BJ)/aVTZ level) of cF and F are shown, which are additionally listed in Table A.5 in the appendix. Reprinted from Ref. [330] Published by The Royal Society of Chemistry, licensed under CC BY 3.0. 
$\left(-17 \pm 1 \mathrm{~cm}^{-1}\right)$. The calculated $x_{2 i}$ values are listed in Table 4.4. Hot bands originating from vibrational modes above $\nu_{4}$ are discarded, as their thermal population at $190{ }^{\circ} \mathrm{C}$ is fairly small $(\leq 0.4 \%)$. The expected thermal population of $\nu_{4}\left(1374 \mathrm{~cm}^{-1}\right)$ is similar to the cis-trans energy difference (experimental: $(1365 \pm 30) \mathrm{cm}^{-1}$, harmonically calculated: $1329 \mathrm{~cm}^{-1}$, B3LYP-D3(BJ)/aVTZ) and amounts to $1 \%$ at the highest nozzle temperature $\left(190^{\circ} \mathrm{C}\right)$. The hot band from the lowest-lying energy level $\nu_{7}$ is predicted to overlap with the fundamental and hot bands originating from $\nu_{9}$ and $\nu_{6}$ are predicted to be downshifted by $-2.3 \mathrm{~cm}^{-1}\left(\nu_{2}+\nu_{6}-\nu_{6}\right)$ and $-2.8 \mathrm{~cm}^{-1}\left(\nu_{2}+\nu_{9}-\nu_{9}\right)$. Consequently, the band at $2938 \mathrm{~cm}^{-1}$ could be a result of a cluster band overlapping with (two) hot bands. By measuring Raman spectra at lower concentration, it will be shown that this is indeed the case (see below). Initially, however, the focus is on the clearly separated hot band at $2925 \mathrm{~cm}^{-1}$. As can be seen in Table 4.4, the off-diagonal anharmonicity matrix element $x_{28}$ amounts to $-17.1 \mathrm{~cm}^{-1}$, which exactly matches the shift of that hot band with respect to the fundamental $\left(2942-2925 \mathrm{~cm}^{-1}\right)$. To verify this assignment, the observed intensity with respect to the fundamental is compared to the expected population of $\nu_{8}$ at $190{ }^{\circ} \mathrm{C}(4 \%)$. For this analysis, it is assumed that the scattering cross-section of that hot band $\left(\nu_{2}+\nu_{8}-\nu_{8}\right)$ is identical to the fundamental $\nu_{2}$, which is reasonable, as the structure will not change significantly. The observed population of $3 \%$ matches within the accuracy the expected $4 \%$, so that the peak at $2925 \mathrm{~cm}^{-1}$ can be assigned to $\left(\nu_{2}+\nu_{8}-\nu_{8}\right)$.

Spectra with a lower formic acid concentration can be found in Figure 4.8. Additionally, the polarisation of the laser with respect to the scattering plane (Figure 3.10) has been varied. These depolarisation measurements can help to reduce the ro-vibrational structure and separate it from overlapping totally symmetric vibrations of formic acid ( $\mathrm{A}^{\prime}$ symmetry). A brief introduction into the theoretical background is given in Section 3.1.3. Compared to the spectra at higher concentrations in Figure 4.7, cluster bands are barely present. Solely the cluster band centred at around $2951 \mathrm{~cm}^{-1}$ can be seen in the spectrum of lowest nozzle temperature. Under these conditions, it can be seen that the band at $2938 \mathrm{~cm}^{-1}$ indeed shows hot band behaviour. Due to the partial overlap with the fundamental and the potential overlap of two hot bands, the intensity analysis is error-prone, so that the assignment to $\left(\nu_{2}+\nu_{6}-\nu_{6}\right)$ and $\left(\nu_{2}+\nu_{9}-\nu_{9}\right)$ is just speculative. By subtracting a scaled spectrum $(7 / 6)$ of a measurement with parallelly polarised laser light from one with perpendicularly polarised light (the standard measurement setting), the rotational $\mathrm{O}$ and $\mathrm{S}$ branches completely diminish. The $\mathrm{C}-\mathrm{H}$ stretching fundamental ( $\mathrm{A}^{\prime}$ symmetry), the hot bands, as well as the $\mathrm{C}-\mathrm{H}$ stretching fundamental of cis-formic acid remain. Additional bands can be seen at $2960 \mathrm{~cm}^{-1}$ and $2928 \mathrm{~cm}^{-1}$, which were previously hidden under the $\mathrm{O}$ and $\mathrm{S}$ branches. These show the same temperature behaviour as the fundamental, meaning that these are overtones or combination bands of trans-formic acid. Exact assignments are not possible, as the MCDTH approach by Richter and Carbonnière predicts several combination bands in that spectral region [328], whereas the VCI calculations of Tew and Mizukami feature no suitable candidates [327]. The same applies to the B3LYP-D3(BJ)/aVQZ VPT2 calculations shown in this work. Another band with the same temperature behaviour is seen at $2996 \mathrm{~cm}^{-1}$, which is close to the $\left(\nu_{3}+2 \nu_{9}\right)$ combination band prediction of the VCI $\left(3003 \mathrm{~cm}^{-1}\right)$ [327] and 


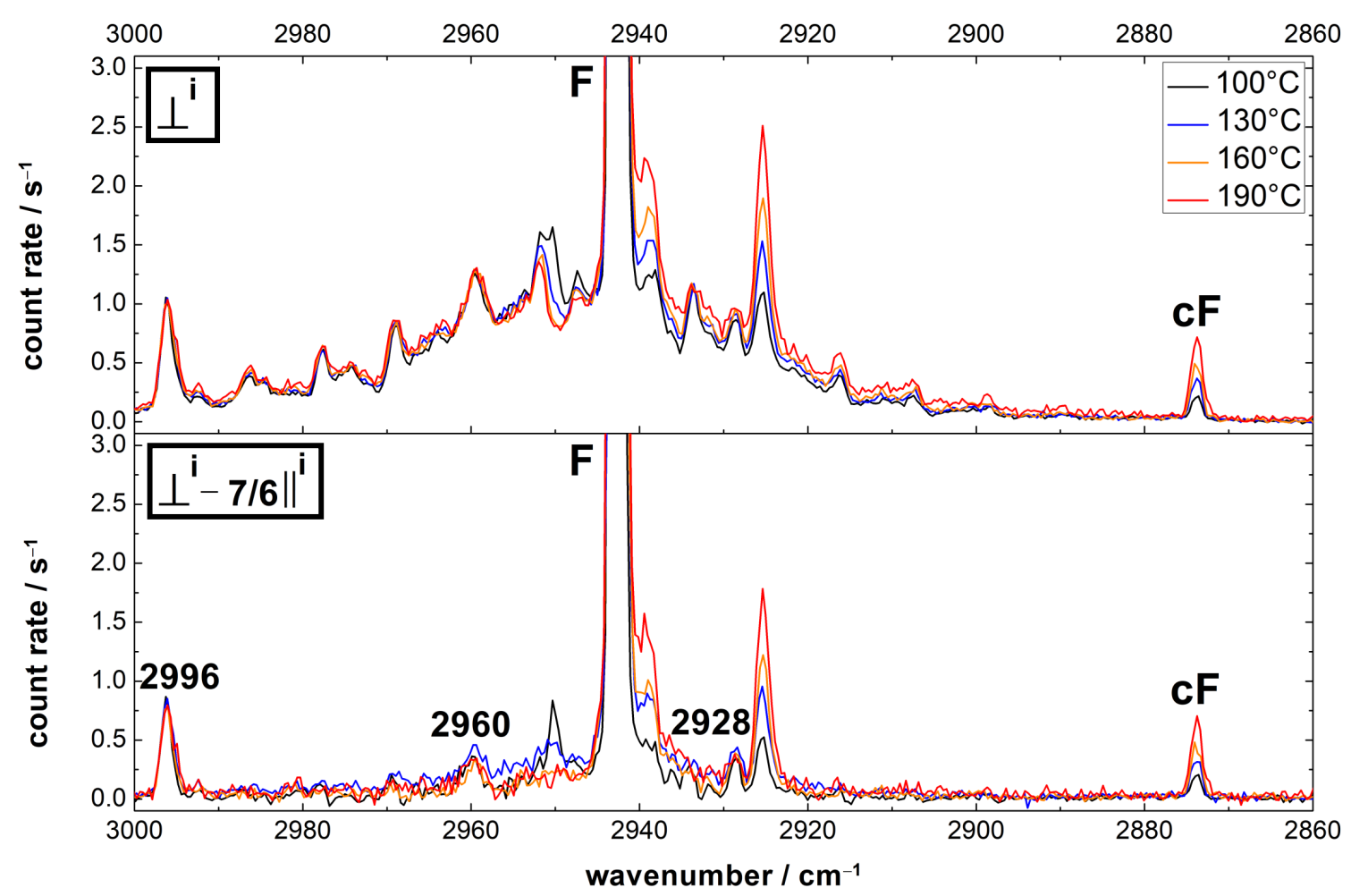

Figure 4.8: Top: Raman jet spectra of formic acid $(<0.1 \%)$ in helium in the $\mathrm{C}-\mathrm{H}$ stretching region recorded at a reservoir pressure of 0.5 bar with increasing nozzle temperatures $\theta_{\mathrm{n}}$ of $100-190^{\circ} \mathrm{C}$ and data acquisition times of $30-35$ minutes. The polarisation of the incident laser light was perpendicular with respect to the scattering plane. For details see inset of Figure 3.10. Bottom: Subtraction of scaled $(7 / 6)$ Raman jet spectra with parallel polarised laser light $\left(\|^{\mathrm{i}}\right)$ from those with perpendicular polarised light $\left(\perp^{\mathrm{i}}\right)$. The experimental conditions are identical.

MCTDH calculations $\left(2989 \mathrm{~cm}^{-1}\right)$ [328]. For both, $\left(\nu_{3}+2 \nu_{9}\right)$ is predicted in Fermi resonance with $\left(\nu_{3}+\nu_{5}\right)$. Another possibility would be the $\left(\nu_{3}+2 \nu_{7}\right)$ combination band that is predicted to be slightly higher in energy (VCI: $3027 \mathrm{~cm}^{-1}$ [327], MCTDH: $3008 \mathrm{~cm}^{-1}$ [328]).

The $\mathrm{C}=\mathrm{O}$ stretching region of formic acid is spectrally less congested. Four hot bands can be seen, whereby two are up- and two are downshifted with respect to the fundamental. The most upshifted hot band at $1818 \mathrm{~cm}^{-1}$ deviates from the harmonically calculated, $\nu_{3}(\mathrm{~F})$-scaled band position of cis-formic acid by solely $3 \mathrm{~cm}^{-1}$. The observed intensity of that band amounts to $1 \%$ of $\mathrm{F}\left(1777 \mathrm{~cm}^{-1}\right)$ and is in reasonably good agreement with the expected cis-formic acid population if corrected for the scattering cross-section ratio $\left(\sigma_{\mathrm{F}} / \sigma_{\mathrm{cF}}\right.$ of 0.837$)$, so that this band can be assigned to the $\mathrm{C}=\mathrm{O}$ stretching vibration of $c i s$-formic acid. To determine the thermally populated low-lying energy level of the other hot bands, the band position shifts of $+6 \mathrm{~cm}^{-1},-7 \mathrm{~cm}^{-1}$, and $-13 \mathrm{~cm}^{-1}$ from the fundamental are compared to the anharmonicity matrix elements (Table 4.4). A hot band originating from the lowest energy level $\nu_{7}$ is expected to be 
shifted by $-6.2 \mathrm{~cm}^{-1}$ from $\mathrm{F}$, which is in good agreement with the $-7 \mathrm{~cm}^{-1}$ observed. The intensity of $10 \%$ of $\nu_{3}$ agrees reasonably well with the expected $14 \%$, so that it can most likely be attributed to $\left(\nu_{3}+\nu_{7}-\nu_{7}\right)$. The hot band $\left(\nu_{3}+\nu_{9}-\nu_{9}\right)$ is expected to overlap with the fundamental and $\left(\nu_{3}+\nu_{8}-\nu_{8}\right)$ might overlap with the hot band originating from $\nu_{7}$. However, due to its lower thermal population, its contribution is distinctly smaller. The hot band originating from $\nu_{6}$ is expected to fall between $\left(\nu_{3}+\nu_{7}-\nu_{7}\right)$ and $\nu_{3}$. Since none of the $x_{3 j}$ values match the two bands at $1783 \mathrm{~cm}^{-1}$ and $1764 \mathrm{~cm}^{-1}$, no further assignments are possible. This clearly highlights the indispensability of the $\nu_{2}$ region for hot band assignments due to the large scattering cross-section of the $\mathrm{C}-\mathrm{H}$ stretching vibration.

The $\mathrm{C}-\mathrm{O}$ stretching region features three hot bands at $1101 \mathrm{~cm}^{-1}, 1097 \mathrm{~cm}^{-1}$, and $1093 \mathrm{~cm}^{-1}$ in addition to the fundamental of trans-formic acid $\left(1105 \mathrm{~cm}^{-1}\right)$. The anharmonicity matrix elements (Table 4.4) $x_{67}$ and $x_{68}$ are with $-3.7 \mathrm{~cm}^{-1}$ identical and $x_{69}$ and $x_{66}$ with about $-6 \mathrm{~cm}^{-1}$ very similar $\left(-5.6 \mathrm{~cm}^{-1}\right.$ and $\left.-6.2 \mathrm{~cm}^{-1}\right)$. A $\left(2 \nu_{i}-\nu_{i}\right)$ hot band such as $\left(2 \nu_{6}-\nu_{6}\right)$, however, is shifted by $2 x_{i i}$ from the fundamental and therefore, $\left(2 \nu_{6}-\nu_{6}\right)$ cannot contribute to the second hot band at $1097 \mathrm{~cm}^{-1}$. The intensities of the hot bands downshifted by $4 \mathrm{~cm}^{-1}$ and $7 \mathrm{~cm}^{-1}$ amount to $7 \%$ and $3 \%$ of $\nu_{6}(\mathrm{~F})$. Altogether, it seems likely that these are a result of overlapping hot bands originating from $\nu_{7}$ and $\nu_{8}$ $\left(1101 \mathrm{~cm}^{-1}\right)$ as well as $\nu_{9}\left(1097 \mathrm{~cm}^{-1}\right)$. The third hot band is shifted by $-11 \mathrm{~cm}^{-1}$ from the fundamental, which is close to the prediction of $2 x_{66}\left(-12.4 \mathrm{~cm}^{-1}\right)$. The intensity of the band, however, is with $7 \%$ of $\nu_{6}$ considerably larger than the $3 \%$ expected from thermal population of $\nu_{6}$. Since the intensities of the other hot bands were equal to or smaller than the expected values, such a pronounced outlier seems unlikely. Another contributor could be the $\left(\nu_{6}+\nu_{5}-\nu_{5}\right)$ hot band $\left(x_{56}=-14.3 \mathrm{~cm}^{-1}\right)$, though the thermal population of $\nu_{5}$ at $190{ }^{\circ} \mathrm{C}$ is even lower $(2 \%)$. The $\mathrm{C}-\mathrm{O}$ stretching vibration of cis-formic acid is predicted at $1088 \mathrm{~cm}^{-1}$ by the $\nu_{6}(\mathrm{~F})$-scaled, harmonic B3LYP-D3(BJ)/aVTZ calculations, $5 \mathrm{~cm}^{-1}$ downshifted from the hot band at $1093 \mathrm{~cm}^{-1}$. Considering the about four times larger scattering cross-section of $\mathrm{cF}\left(\sigma_{\mathrm{F}} / \sigma_{\mathrm{cF}}=0.246\right)$, the observed intensity ratio matches the value expected from thermal population. Therefore, the band can most likely be assigned to the $\mathrm{C}-\mathrm{O}$ stretching vibration of cis-formic acid.

Before the newly determined band positions of the cis-formic acid fundamentals are compared to literature data obtained in a solid argon matrix in Subsection 4.3.3, the hot band assignments of trans-formic acid will be summarised.

\subsubsection{Hot Band Assignments of trans-Formic Acid}

Five hot bands of trans-formic acid were tentatively assigned, namely $\left(\nu_{2}+\nu_{8}-\nu_{8}\right)$, $\left(\nu_{2}+\nu_{9}-\nu_{9}\right),\left(\nu_{3}+\nu_{7}-\nu_{7}\right),\left(\nu_{6}+\nu_{7}-\nu_{7}\right),\left(\nu_{6}+\nu_{9}-\nu_{9}\right)$. All but $\left(\nu_{2}+\nu_{8}-\nu_{8}\right)$ are predicted to overlap with hot bands from less populated states, which is the reason for the uncertainty of most of the assignments. The band positions of all hot band contributions are summarised in Table 4.5.

As detailed in Section 2.1, within the PT2 model, the anharmonicity matrix elements $x_{i j}$ can be determined via hot band measurements, as a $\left(\nu_{i}+\nu_{j}-\nu_{j}\right)$ hot band is shifted by $x_{i j}$ from the fundamental $\nu_{i}$ (Equation 2.6). In addition, $x_{i j}$ can be obtained from the 
combination band $\left(\nu_{i}+\nu_{j}\right)$ via subtraction of the corresponding fundamentals $\nu_{i}$ and $\nu_{j}$ (Equation 2.5). In both cases, this is under the assumption that no Fermi resonances occur and only binary combinations exist. Combination bands and overtones of the formic acid monomer were measured by Freytes and co-workers with high-resolution FTIR spectroscopy [305]. Additional combination bands involving $\nu_{5}$ and $\nu_{6}$ were reported by Perrin, Vander Auwera, and Zelinger [310]. The band positions of the hot bands can thus be estimated from the reported combination bands via $x_{i j}$ and vice versa. The results are summarised in Table 4.5 in comparison to the anharmonic ab initio values calculated by Tew and Mizukami [327] as well as Richter and Carbonnière [328].

As expected, not all values in the table coincide, which can indicate misassignments. Here, only the most relevant will be pointed out. For the hot band in the $\mathrm{C}=\mathrm{O}$ stretching region at $1770 \mathrm{~cm}^{-1}$, a perfect match between the possible $\left(\nu_{3}+\nu_{8}-\nu_{8}\right)$ contribution and its prediction from $\left(\nu_{3}+\nu_{8}\right)$ and the respective fundamentals can be seen, yet for the dominant (higher populated) $\left(\nu_{3}+\nu_{7}-\nu_{7}\right)$ contribution, a discrepancy of $20 \mathrm{~cm}^{-1}$ is observed. The hot band assignment of this work as well as the prediction of $\left(\nu_{3}+\nu_{7}\right)$ agree much better with the calculated values of Refs. [327] and [328]. The combination band assignment of Freytes et al. [305] yields an anharmonicity matrix element $x_{37}$ of $-27 \mathrm{~cm}^{-1}$. Such a strong coupling between the two modes is highly unexpected. Close to $\left(\nu_{3}+\nu_{7}\right)$, another band is reported in that Reference (at $2400.2 \mathrm{~cm}^{-1}$ ), which was assigned to the overtone of $\nu_{5}$. That band agrees much better with the hot band prediction and the calculated values of Refs. [327] and [328]. Moreover, it yields a more reasonable off-diagonal anharmonicity constant of $-3 \mathrm{~cm}^{-1}$.

Another large mismatch is observed for the hot bands in the $\mathrm{C}-\mathrm{O}$ stretching region. If the combination bands $\left(\nu_{6}+\nu_{7}\right)$ and $\left(\nu_{6}+\nu_{9}\right)$ are predicted from the dominating hot bands $\left(\nu_{6}+\nu_{7}-\nu_{7}\right)$ and $\left(\nu_{6}+\nu_{9}-\nu_{9}\right)$, the results are exactly vice versa $-\left(\nu_{6}+\nu_{7}\right)$ is observed at $1737.96 \mathrm{~cm}^{-1}$ in Ref. [310] and predicted to $1727 \mathrm{~cm}^{-1}$, whereas $\left(\nu_{6}+\right.$ $\left.\nu_{9}\right)$ is reported at $1726.40 \mathrm{~cm}^{-1}$ [310] and estimated to $1738 \mathrm{~cm}^{-1}$. The resulting offdiagonal anharmonicity matrix elements of the combination band assignments of Perrin and co-workers [310] amount to $+7 \mathrm{~cm}^{-1}\left(x_{67}\right)$ and $-19 \mathrm{~cm}^{-1}\left(x_{69}\right)$. Both values seem implausible. If these assignments were switched, the $x_{i j}$ values are more reasonable with $-4.6 \mathrm{~cm}^{-1}\left(x_{67}\right)$ and $-7.6 \mathrm{~cm}^{-1}\left(x_{69}\right)$. The anharmonic ab initio predictions of Refs. [327] and [328] support such a level switch. Besides, at the band positions of the respective hot band predictions (Table 4.5), no bands can be observed in the Raman spectra in Figure 4.7. Altogether, the most plausible explanation is a misassignment of the two bands reported in Ref. [310]. As aforementioned, this comparison of hot and combination band assignments has its limits, yet such distinct differences point at obvious flaws in assignments. For full verification, additional spectroscopic as well as quantum chemical evidence is necessary, which can hopefully be trigged by this Raman jet study.

\footnotetext{
${ }^{1}$ In their publication, all off-diagonal anharmonicity matrix elements $x_{i j}$ listed, are divided by a factor of 2 in disagreement with the definition given here (Equation 2.5). In this work, the $x_{i j}$ values have been determined from the reported combination bands and the corresponding fundamentals.
} 
4 Monomers

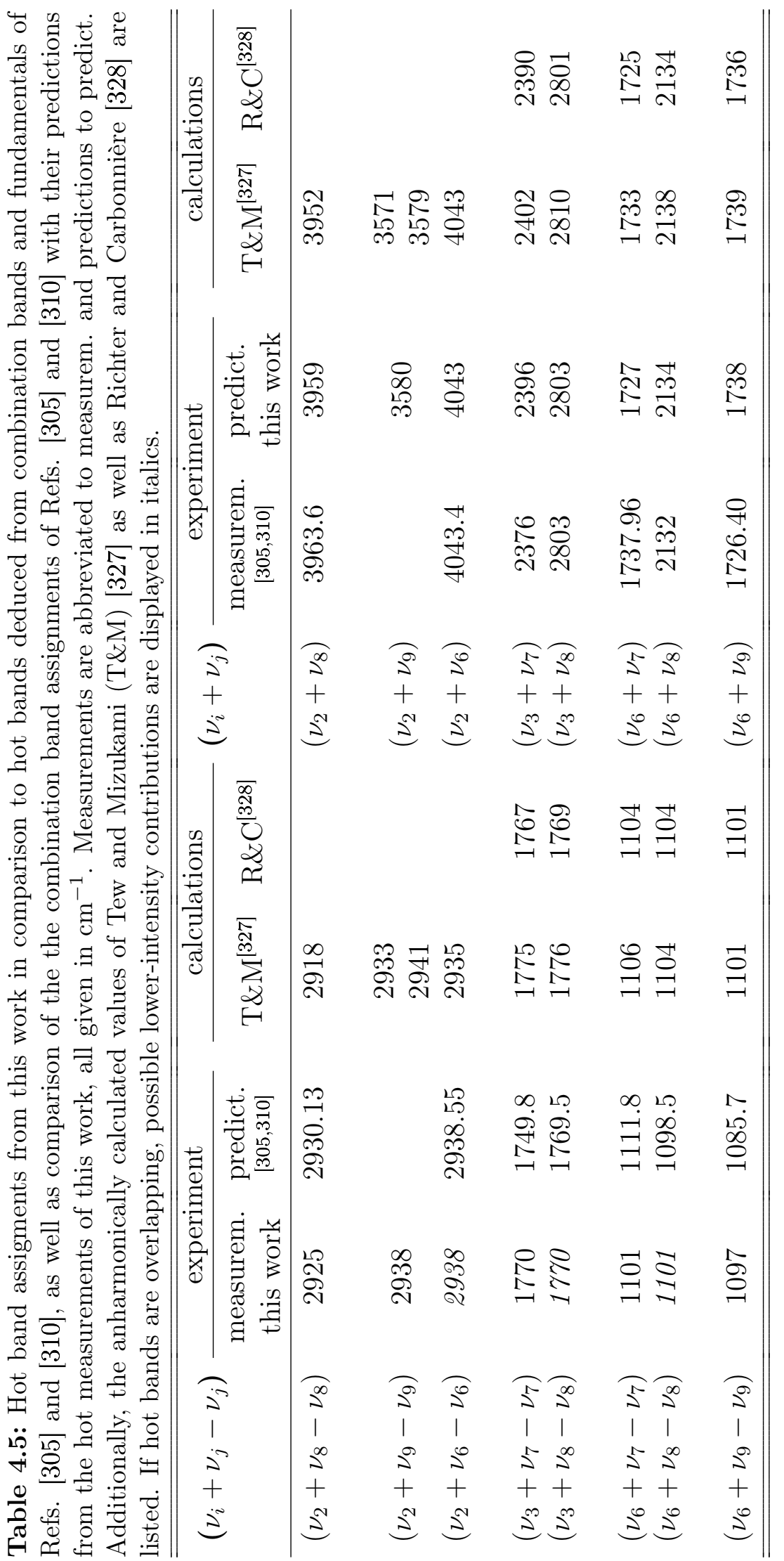


Table 4.6: Band positions of the fundamentals $\nu_{i}$ of $c i s$-formic acid (in $\mathrm{cm}^{-1}$ ) obtained in a supersonic jet expansion probed with Raman spectroscopy (this work) compared to literature values. Reprinted with adaptations from Ref. [330] - Published by The Royal Society of Chemistry, licensed under CC BY 3.0.

\begin{tabular}{|c|c|c|c|c|c|}
\hline$\nu_{i}$ & jet (this work) & gas phase [57] & $\operatorname{shift}(\mathrm{cF}-\mathrm{F})^{\mathrm{a}}$ & Ar matrix ${ }^{\mathrm{b}}[60]$ & matrix shift ${ }^{\mathrm{c}}$ \\
\hline 1 & 3637 & & +67 & $3617.2 / 3615.9$ & $-20 /-21$ \\
\hline 2 & 2873 & & -69 & $2899.5 / 2896.3$ & $+27 /+23$ \\
\hline 3 & 1818 & & +41 & $1808.0 / 1806.9$ & $-10 /-11$ \\
\hline 4 & & & & / 1391.8 & \\
\hline 5 & & & & $1243.4 / 1248.9$ & \\
\hline 6 & 1093 & & -11 & $1107.3 / 1104.6$ & $+14 /+12$ \\
\hline 7 & & & & 662.3 / 662.3 & \\
\hline 8 & & & & & \\
\hline 9 & & $493.420509(7)$ & $-147.30467(1)$ & $502.9 / 505.3$ & $+9.5 /+11.9$ \\
\hline \multicolumn{6}{|c|}{$\begin{array}{l}\text { a gas phase band position shifts from the trans- to the cis-rotamer } \\
\text { bite } 1 / \text { site } 2 \text { are two dominant trapping sites }\end{array}$} \\
\hline
\end{tabular}

\subsubsection{Summary of cis-Formic Acid Fundamentals}

All stretching vibrations of cis-formic acid and the respective shifts between the cis- and trans-rotamers are summarised in Table 4.6 alongside the band position of $\nu_{9}$ published in 2006 by Baskakov and co-workers [57] and values obtained in a solid argon matrix by Maçôas and et al. [60].

Eight of the nine fundamentals of cis-formic acid have been determined in an argon matrix [60]. The missing fundamental is $\nu_{8}$, which has a predicted (harmonic) IR intensity near-zero $\left(\approx 0.01 \mathrm{~km} \mathrm{~mol}^{-1}\right.$, at the B3LYP-D3(BJ)/aVTZ level). Due to its overall small Raman scattering cross-section (cf. Figure 4.6), it currently not accessible with the approach described here. Two dominant trapping sites of cis-formic acid were found in an argon matrix. The band positions shifts relative to the gas phase range from $-20 \mathrm{~cm}^{-1}$ (side 1 ) and $-21 \mathrm{~cm}^{-1}$ (side 2) to $+27 \mathrm{~cm}^{-1}$ and $+23 \mathrm{~cm}^{-1}$, respectively. These matrix shifts are not only on the same order of magnitude as the cis-trans band position differences, but they also change sign. Note that the sign of the gas phase trans-to-cis shift is, perhaps coincidentally, always opposite to the sign of the vacuumto-matrix shift. As seen for the trans-fundamentals, the bands of the $\mathrm{O}-\mathrm{H}$ and $\mathrm{C}=\mathrm{O}$ stretching vibrations are downshifted in the matrix, whereas the bands of the $\mathrm{C}-\mathrm{H}$ and $\mathrm{C}-\mathrm{O}$ stretching vibrations as well as the $\mathrm{O}-\mathrm{H}$ torsional mode are upshifted. In some cases, the sign and magnitude of the argon matrix shifts differs for both rotamers, as seen for the $\mathrm{C}-\mathrm{O}$ stretching vibration, which is slightly downshifted for trans-formic acid (cf. Table 4.2) and upshifted for $\mathrm{cF}$. Therefore, these values cannot simply be estimated by adding or subtracting a shift to all bands, which clearly highlights the need of additional perturbation-free cis-formic acid data. However, given the four new cis-formic acid band 
positions in addition to the value for the $\mathrm{OH}$ torsional mode from 2006 [57], a balanced vibrational benchmark including both the local and the global minimum is feasible for the first time.

\subsection{Vibrational Benchmark}

Vibrational benchmarks of electronic structure methods require reliable treatments of the nuclear quantum dynamics. Here, the VPT2 method is employed for this purpose, but two variational methods are added for the indirect assessment of the VPT2 approach. The electronic structure methods tested in this benchmark are popular DFT functionals for this system such as B3LYP-D3(BJ), PBE0-D3(BJ), M06-2X, $\omega$ B97-XD, and the double hybrid B2PLYP-D3(BJ). Additionally, the performance of an ab initio method is examined, in this case, MP2. For reference, PM3 is also included. As a basis set, if one is necessary, the augmented quadruple-zeta basis set by Dunning $[226,227]$ is chosen, which is abbreviated to aVQZ. For the basis set sensitive methods MP2 and B2PLYP-D3(BJ), additional double- (aVDZ) and triple-zeta (aVTZ) calculations were performed. The integration grid chosen for all calculations within this subsection is the pruned ultrafine grid. It is composed of 99 radial shells and 590 angular points per shell $(99,590)$ and is finer than the default grid $(75,302)$ of Gaussian 09 [36]. All calculations were carried out in $C_{1}$ symmetry with the tight optimisation of Gaussian 09 [36]. Band position deviations are found when the DFT integration grid size is increased, a more rigorous optimisation setting (very tight) is chosen, and the $C_{\mathrm{s}}$ symmetry is employed. The extent of these discrepancies depends highly on the method chosen. A closer examination of these effects can be found in the next section.

The experimental observables that will be benchmarked are the five cis-formic acid band positions and the band position shifts between both rotamers. For a fair comparison between experiment and theory, the experimental uncertainty of the band positions needs to be accounted for. As detailed in Section 3.1.3, the band position error of the Raman set-up amounts to about $1-2 \mathrm{~cm}^{-1}$. The half width at half maximum (HWHM) of the bands shown in Figure 4.7 is roughly $2 \mathrm{~cm}^{-1}$, which leads to a conservative error estimate of $\pm 2 \mathrm{~cm}^{-1}$ for the band positions. For the shifts, correspondingly twice the band position error is used $\left( \pm 4 \mathrm{~cm}^{-1}\right)$. These experimental errors are displayed in Figure 4.9 as green ellipses. Since the out-of-plane bending vibration $\nu_{9}$ has been determined in a high resolution FTIR measurement with a precision on the order of $10^{-5} \mathrm{~cm}^{-1}$ and a slightly lower accuracy [57], the band position and shift are displayed by the tip of an arrow. Consequently, an exact match with $\nu_{9}$ is virtually impossible, whereas the uncertainty of the Raman jet measurements imposes a realistic challenge on theory.

The results of this vibrational benchmark are summarised in Figure 4.9. Before the individual performance of the methods is examined, a few general trends will be discussed. Due to error cancellation, the prediction of a relative quantity such as the band position shift between both rotamers is obviously less-error prone compared to an absolute value. It is therefore not surprising that the $\mathrm{cF}$ band position is only predicted correctly once $\left(\nu_{6}\right.$ with MP2/aVQZ), whereas the shift is predicted correctly for more methods 


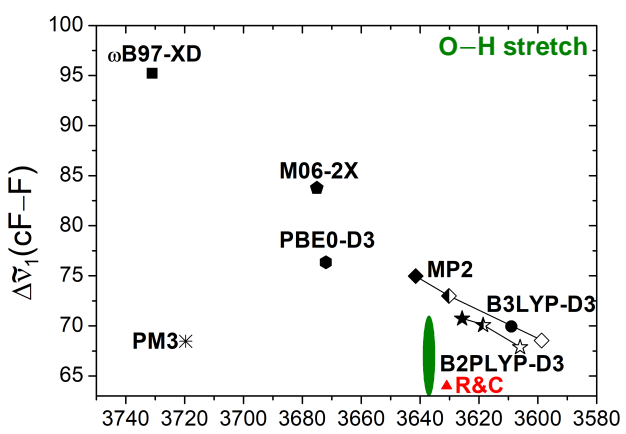

$\tilde{v}_{1}(\mathrm{cF})$
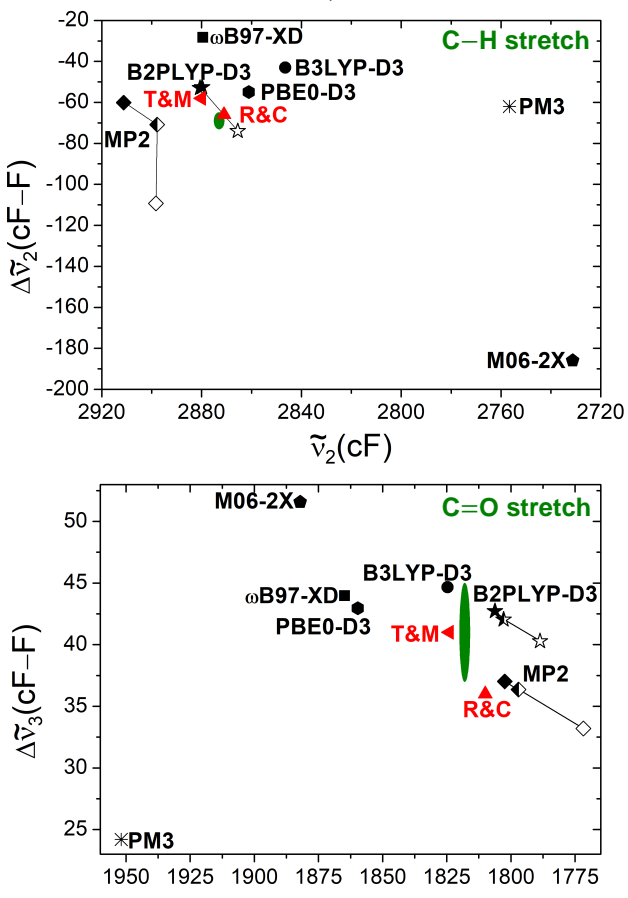

$\tilde{v}_{3}(\mathrm{cF})$
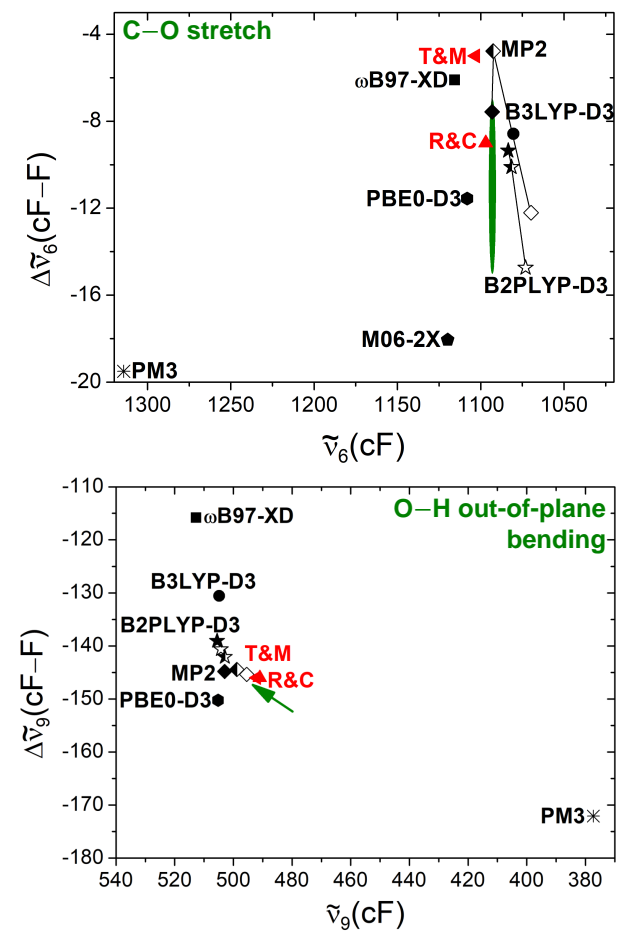

Figure 4.9: Anharmonic (VPT2), theoretical prediction of the shift between the band positions of the cis- and trans-rotamers of formic acid for five fundamentals $\left(\nu_{1}, \nu_{2}, \nu_{3}, \nu_{6}\right.$, and $\left.\nu_{9}\right)$ plotted against the absolute band positions of the cis-form. The basis set size is encoded in the filling of the symbols. All black, filled symbols have been calculated with an aVQZ basis set. For the half-empty and the empty symbols smaller basis sets have been used, namely aVTZ and aVDZ. The red triangles represent the band positions and shifts obtained from VCI calculations of Tew and Mizukami [327] (T\&M) and MCTDH calculations of Richter and Carbonnière [328] (R\&C). The green ellipsis shows agreement with experiment, whereby the size indicates the conservatively estimated experimental accuracy of $\pm 2 \mathrm{~cm}^{-1}$ for $\tilde{\nu}_{i}(\mathrm{cF})$ and $\pm 4 \mathrm{~cm}^{-1}$ for $\Delta \tilde{\nu_{i}}(\mathrm{cF}-\mathrm{F})$. The experimental band positions of the stretching vibrations $\left(\nu_{1}, \nu_{2}, \nu_{3}, \nu_{6}\right)$ have been obtained in this work and the values of the torsional modes $\nu_{9}$ of both rotamers have been taken from Refs. [306] and [57]. Since $\nu_{9}$ has been measured with high precision and accuracy for both isomers $[57,306]$, the exact band positions and shifts are shown by the tip of an arrow. The band position and shift of the $\mathrm{OH}$ out-of-plane bending vibration $\nu_{9}$ predicted at M06-2X/aVQZ VPT2 level are not shown due to their large, absolute deviation. Further details can be found in the text. Reprinted from Ref. [330] - Published by The Royal Society of Chemistry, licensed under CC BY 3.0. 
and vibrations. As indicated before, the $\mathrm{C}-\mathrm{H}$ stretching vibration imposes the largest challenge on theory due to stretch-bend Fermi resonances, even though these very pronounced resonances are included in the VPT2 code as implemented in Gaussian 09 [36]. As a result, the methods scatter the most for $\nu_{2}$, which can be seen by the small size of the green ellipsis in the plot. In contrast, the $\mathrm{C}=\mathrm{O}$ and $\mathrm{C}-\mathrm{O}$ stretching vibrations are overall best described by the methods. The $\mathrm{O}-\mathrm{H}$ stretching and the $\mathrm{O}-\mathrm{H}$ out-of-plane bending vibrations lie in-between. Having these varying levels of difficulty can hugely benefit the distinction of good from semi-good and overall bad methods. Generally, the failure of a method to describe the vibrations of both rotamers of formic acid correctly can either come from the electronic structure calculation or the vibrational treatment. As an attempt to separate the two, the overall structural agreement of the methods is assessed via the comparison with the experimental values of the rotational constants of cis- and trans-formic acid determined by Winnewisser and co-workers [217], followed by an examination of the vibrational benchmark. The results are summarised in Table 4.7.

The overall best agreement is seen for B3LYP-D3(BJ), with small overestimations of $A_{0}$ and small underestimations of $B_{0}$ and $C_{0}$, whereby the deviations are smaller for the cis-rotamer. If the rotational constants are averaged, an overall underestimation of solely $-0.1 \%(\mathrm{cF})$ and $-0.2 \%(\mathrm{~F})$ is found. The performance of B2PLYP-D3(BJ) and MP2 is only slightly inferior. The maximum of the deviations amounts to $-0.6 \%$ $\left(B_{0}, \mathrm{~B} 2 \mathrm{PLYP}-\mathrm{D} 3(\mathrm{BJ})\right)$ and $-0.7 \%\left(B_{0}, \mathrm{MP} 2\right)$. Both perform equally well for cis- and trans-formic acid. The mismatch of the averaged rotational constants with respect to experiment are as low as $-0.3 \%$ (B2PLYP-D3(BJ)) and $-0.3 \%$ (MP2). Slightly larger deviations of up to $1.4 \%$ are observed for M06-2X, $\omega$ B97-XD, and PBE0-D3, where all rotational constants are consistently overestimated. By far the largest deviations are obtained for PM3, with an underestimation of $A_{0}$ of nearly $20 \%$. However, since $A_{0}$ is under- and $B_{0}$ and $C_{0}$ are overestimated, the averaged deviation is with about $-1.2 \%$ (F) and $-1.3 \%(\mathrm{cF})$ only slightly larger compared to M06-2X, $\omega$ B97-XD, and PBE0D3 (cf. Table 4.7). Hence, this comparison already provides a first distinction of the methods.

As expected, the performance of PM3 for the determination of $\tilde{\nu}_{i}(\mathrm{cF})$ and $\Delta \tilde{\nu}_{i}(\mathrm{cF}-\mathrm{F})$ is also insufficient - it is the only method that fails to predict the energetic order of the vibrations correctly with $\nu_{6}$ and $\nu_{4}$ switched. Apart from two shifts, namely $\Delta \tilde{\nu}_{1}(\mathrm{cF}-\mathrm{F})$ and $\Delta \tilde{\nu}_{2}(\mathrm{cF}-\mathrm{F})$, no other value of the ten shifts and band positions is predicted within the error bars. Methods with a similarly bad performance are M06-2X and $\omega$ B97-XD. These exhibit particularly drastic deviations for the $\mathrm{O}-\mathrm{H}$ stretching vibration $\nu_{1}$ and the $\mathrm{O}-\mathrm{H}$ torsion $\nu_{9}$ (cf. Figure 4.9). Moreover, the M06-2X results show a pronounced deviation for the $\mathrm{C}-\mathrm{H}$ stretching vibration $\nu_{2}$ of $\mathrm{cF}$, which is caused by a level switch of the two resonance partners - the overtone of the $\mathrm{C}-\mathrm{H}$ bending vibration $2 \nu_{4}$ and $\nu_{2}$. If the labels are switched, the band position agreement with experiment increases significantly from an underestimation of $-142 \mathrm{~cm}^{-1}$ for $\nu_{2}(\mathrm{cF})$ to an overestimation of $+10 \mathrm{~cm}^{-1}$, yet the mismatch of the shift increases. This is illustrated in detail in Figure A.6 in Appendix A.2.2. It is not observed for the other methods, which predict the energy order correctly. Another deficiency of M06-2X is the incorrect sign of the total anharmonicity of the torsional mode $\nu_{9}$ of cis-formic acid. The anharmonic correction 
is positive $\left(+163 \mathrm{~cm}^{-1}\right)$, resulting in an overestimation of the band position. As the anharmonicity of the respective mode of trans-formic acid is severely underestimated, the band position shift is overestimated by $+308 \mathrm{~cm}^{-1}$. Due to these large mismatches, the data point is not shown in Figure 4.9.

B3LYP-D3(BJ) and PBE0-D3(BJ) exhibit a similar performance - B3LYP-D3(BJ) predicts the band position shifts correctly in three of the five cases and PBE0-D3(BJ) in two. The deviations of the band positions are similar. As the molecular structure prediction of B3LYP-D3(BJ) is better, it is preferred to the PBE0-D3(BJ) methodology.

The overall best methods for the determination of cis-formic acid fundamentals and cis-trans band position shifts with VPT2 are MP2 and B2PLYP-D3(BJ). All band position shifts apart from $\nu_{9}$ agree with experiment (with at least one of the three basis set sizes), whereby the shifts are mostly underestimated for B2PLYP-D3(BJ). MP2 overshoots in case of $\nu_{1}$ and $\nu_{2}$ with increasing basis set size and performs particularly well for the lower frequency vibrations $\nu_{3}, \nu_{6}$, and $\nu_{9}$. In case of $\nu_{6}$, the band position is predicted correctly. Desirably, the agreement with experiment increases with basis set size for both methodologies.

More elaborate ways of calculating anharmonic vibrational frequencies are shown by the groups of Tew and Mizukami (T\&M) [327] as well as Richter and Carbonnière (R\&C) [328]. Tew and Mizukami fitted a potential energy surface to $17076 \operatorname{CCSD}(\mathrm{T})\left(\mathrm{F} 12^{*}\right) /$ ccpVTZ-F12 energies and calculated the vibrational energy levels with vibrational configuration interaction (VCI). The isomerisation pathway leading to the higher-energy cis-form was described by an internal coordinate path Hamiltonian [327]. Richter and Carbonnière fitted their potential energy surface at the CCSD(T)-F12a/aug-cc-pVTZ level using valence coordinates. The vibrational energies were obtained by the multiconfiguration time-dependent Hartree (MCTDH) method [328]. More details on both methods can be found in the respective References [327, 328]. Their $\mathrm{cF}$ band position and $\mathrm{cF}-\mathrm{F}$ shift predictions are shown as red triangles in Figure 4.9. 
4 Monomers

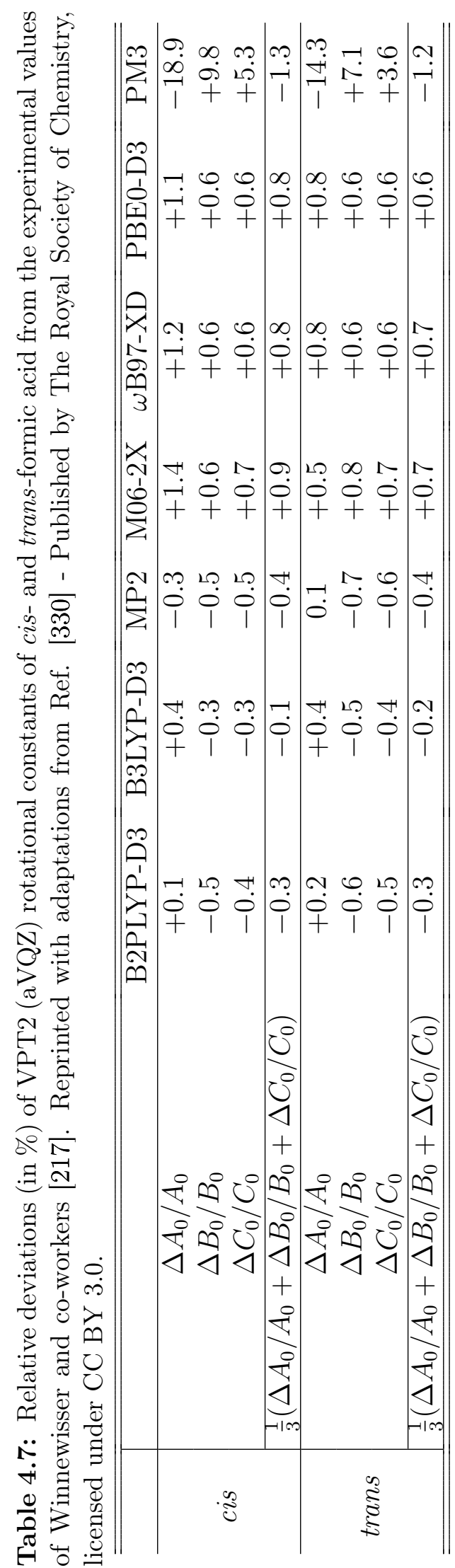


Since the $\mathrm{O}-\mathrm{H}$ stretching vibration of cis-formic acid is not included in Ref. [327], Tew and Mizukami's results can only be compared to four band positions and shifts. The four $\mathrm{cF}$ band positions $\left(\nu_{2}, \nu_{3}, \nu_{6}\right.$ and $\left.\nu_{9}\right)$ as well as two band position shifts are overestimated $\left(\Delta \nu_{2}\right.$ and $\left.\Delta \nu_{6}\right), \Delta \nu_{3}$ is predicted correctly, and $\Delta \nu_{9}$ deviates by $-1 \mathrm{~cm}^{-1}$. This overestimation of the cis-formic acid band positions and the missing value of $\nu_{1}(\mathrm{cF})$, could indicate a convergence issue of these calculations. In comparison to the B2PLYP-D3(BJ)/aVQZ VPT2 and MP2/aVQZ VPT2 results, the deviations of Tew and Mizukami are slightly smaller at the expense of higher computational costs. The results of Richter and Carbonnière, on the other hand, clearly exhibit the overall best agreement with experiment. The shift is predicted correctly in three cases $\left(\Delta \nu_{1}, \Delta \nu_{2}\right.$, and $\left.\Delta \nu_{6}\right)$, and is just outside the area of experimental agreement for $\Delta \nu_{3}\left(36 \mathrm{~cm}^{-1} \mathrm{com}-\right.$ pared to $\left.41 \pm 4 \mathrm{~cm}^{-1}\right)$. For $\Delta \nu_{9}$, its deviation amounts to $-1 \mathrm{~cm}^{-1}$ and the cis-formic acid band position deviates by $2 \mathrm{~cm}^{-1}$. The $\mathrm{C}-\mathrm{H}$ stretching vibration is predicted correctly.

Apart from these benchmarking plots, another way of visualising the predictive power of anharmonic vibrational frequency calculations is shown in Figure 4.10 in form of spiderweb diagrams. These are composed of octagons of various sizes. Each axis of the octagons represents one of the stretching vibrations. The division into cis-formic acid band position and cis-trans-shift, abbreviated to $c_{i}$ and $\Delta_{i}$, is retained by the two directions of each axis. The deviation from experiment is illustrated by the distance from the origin of the diagram: A point on a node on the smallest octagon translates into agreement within the experimental confidence interval (green ellipsis in Figure 4.9, $\pm 2 \mathrm{~cm}^{-1}$ for $c_{i}$, and $\pm 4 \mathrm{~cm}^{-1}$ for $\Delta_{i}$ ). Consequently, a point on a node on the next larger octagon translates into a mismatch of twice the size of the experimental confidence interval and accordingly, a deviation of $n$ times the experimental uncertainty is shown by a point on the $n$th node in these diagrams. Note that the origin itself cannot be met due to the band position uncertainty of the Raman jet experiments. If a value is met, over-, or underestimated, is shown by the colour shade. A dark colour represents overand a light colour underestimation. An indeterminate sign is shown by an intermediate shade. The symbols used are the same as in Figure 4.9 .

The spiderweb diagrams of the two most consistent VPT2 results (MP2/aVQZ VPT2 and B2PLYP-D3(BJ)/aVQZ VPT2) as well the approaches of Tew and Mizukami [327] and Richter and Carbonnière [328] are depicted in Figure 4.10. It is directly apparent that the MP2/aVQZ VPT2 methodology exhibits an inferior performance compared to the B2PLYP-D3(BJ) approach, as $\mathrm{c}_{2}$ is largely overestimated (19 experimental confidence intervals) and $c_{3}$ largely underestimated (8 confidence intervals). Nonetheless, for $\mathrm{c}_{1}, \mathrm{c}_{6}$, and $\Delta_{2}, \mathrm{MP} 2$ is slightly better than B2PLYP-D3(BJ). The observed tendency of the VCI calculations of Tew and Mizukami [327] to overestimate the band positions and shifts can be clearly seen by the dark coloured symbols, apart from $\Delta_{3}$, which matches experiment. The fact that all points fall closer to the centre of the spiderweb diagram and vary more in colour shade, undoubtedly visualise the superiority of the MCTDH calculations of Richter and Carbonnière over all other approaches. 
B2PLYP-D3(BJ)/aVQZ VPT2

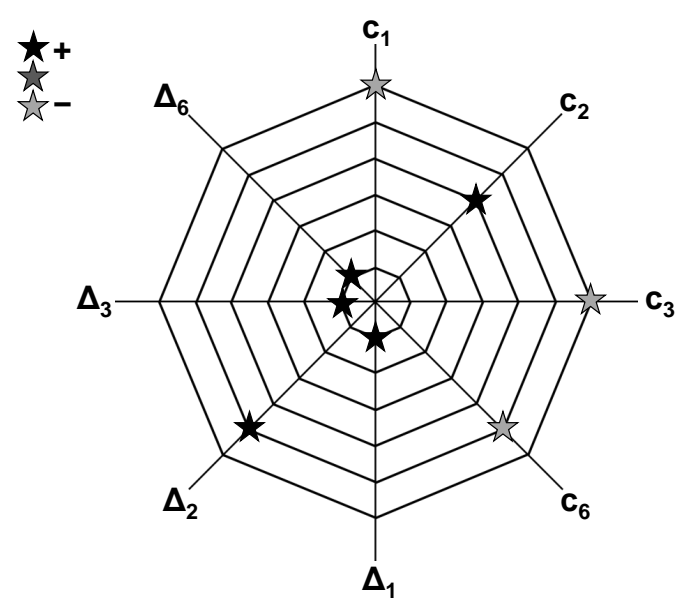

$\mathrm{T} \& \mathrm{M}$

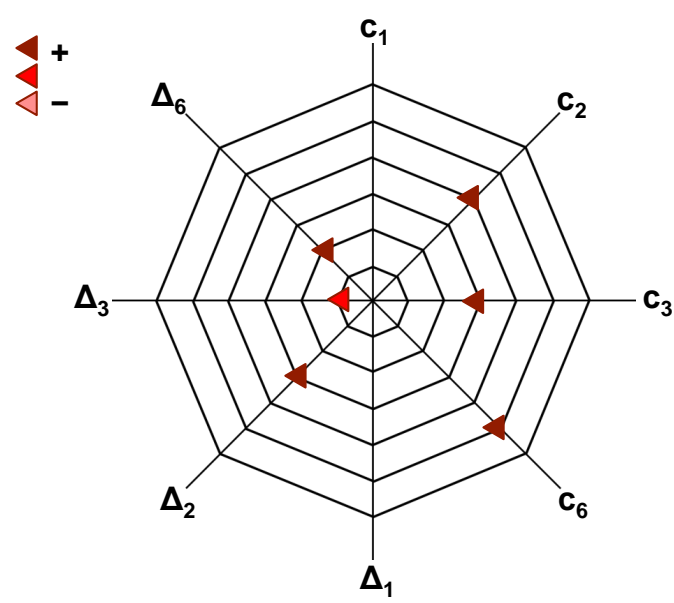

MP2/aVQZ VPT2

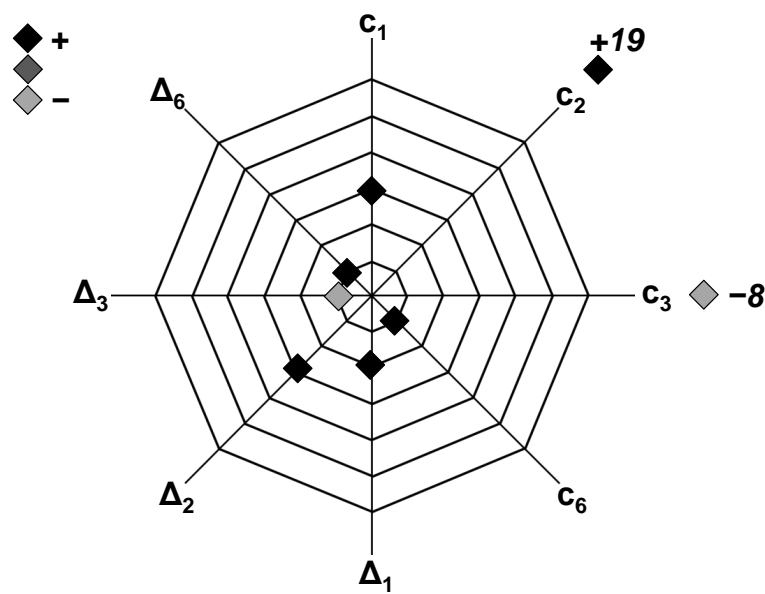

$R \& C$

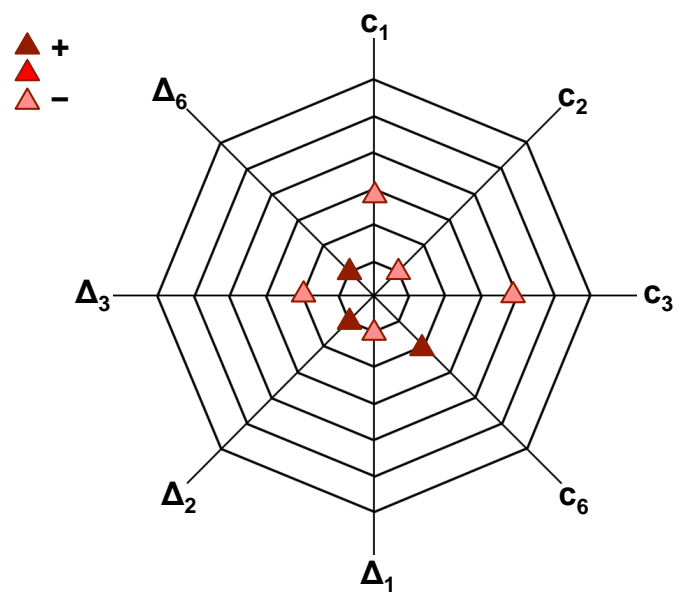

Figure 4.10: Agreement of the predicted anharmonic band positions of the stretching vibrations of cis-formic acid $\left(\mathrm{c}_{1,2,3,6}\right)$ as well as band position shifts between cis- and trans-formic acid $\left(\Delta_{1,2,3,6}\right)$ with experiment. The octagon size displays the deviation from experiment in units of experimental confidence interval, i.e., the smallest octagon represents agreement within the error bars of $\pm 2 \mathrm{~cm}^{-1}$ for $\mathrm{c}$ and $\pm 4 \mathrm{~cm}^{-1}$ for $\Delta$ (green ellipses in Figure 4.9) and the $n$th octagon agreement within $n$ experimental confidence intervals. The methods tested are the VCI calculations of Tew and Mizukami (T\&M) [327], the MCTDH calculations of Richter and Carbonnière (R\&C) [328], as well as VPT2 calculations at the B2PLYP-D3(BJ)/aVQZ and at the MP2/aVQZ level. The symbols used are the same as in Figure 4.9. The color shade shows whether the experimental observable is overestimated (+), underestimated (-), or met. Reprinted with adaptations from Ref. [330] - Published by The Royal Society of Chemistry, licensed under CC BY 3.0. 


\subsubsection{Instabilities of DFT Functionals}

Up until this point, all vibrational frequency calculations shown in this chapter have been performed in $C_{1}$ symmetry, with the tight optimisation, and the ultrafine integration grid of Gaussian 09 [36]. In order to explore the sensitivity of the anharmonically calculated band positions towards these settings, additional calculations have been carried out with the larger superfine grid $(150,974), C_{\mathrm{s}}$ symmetry, and the very tight optimisation. For this comparison, the four stretching vibrations as well as the $\mathrm{OH}$ torsion $\left(\nu_{9}\right)$ of cisand trans-formic acid are considered. To quantify the impact of settings, the mean absolute deviations (MAD) of the ten band positions are compared when the symmetry is enhanced from $C_{1}$ to $C_{\mathrm{s}}$ at a given DFT integration grid and optimisation setting. Additionally, the band position differences upon increasing the DFT integration grid size from ultrafine to superfine are compared. The resulting eight MAD values (four for MP2 and PM3) are plotted for each method in Figure 4.11 and are additionally listed in Table 4.8 for the DFT methods.

For PM3 and MP2, negligible band position variations $\left(\leq 0.1 \mathrm{~cm}^{-1}\right)$ are seen when

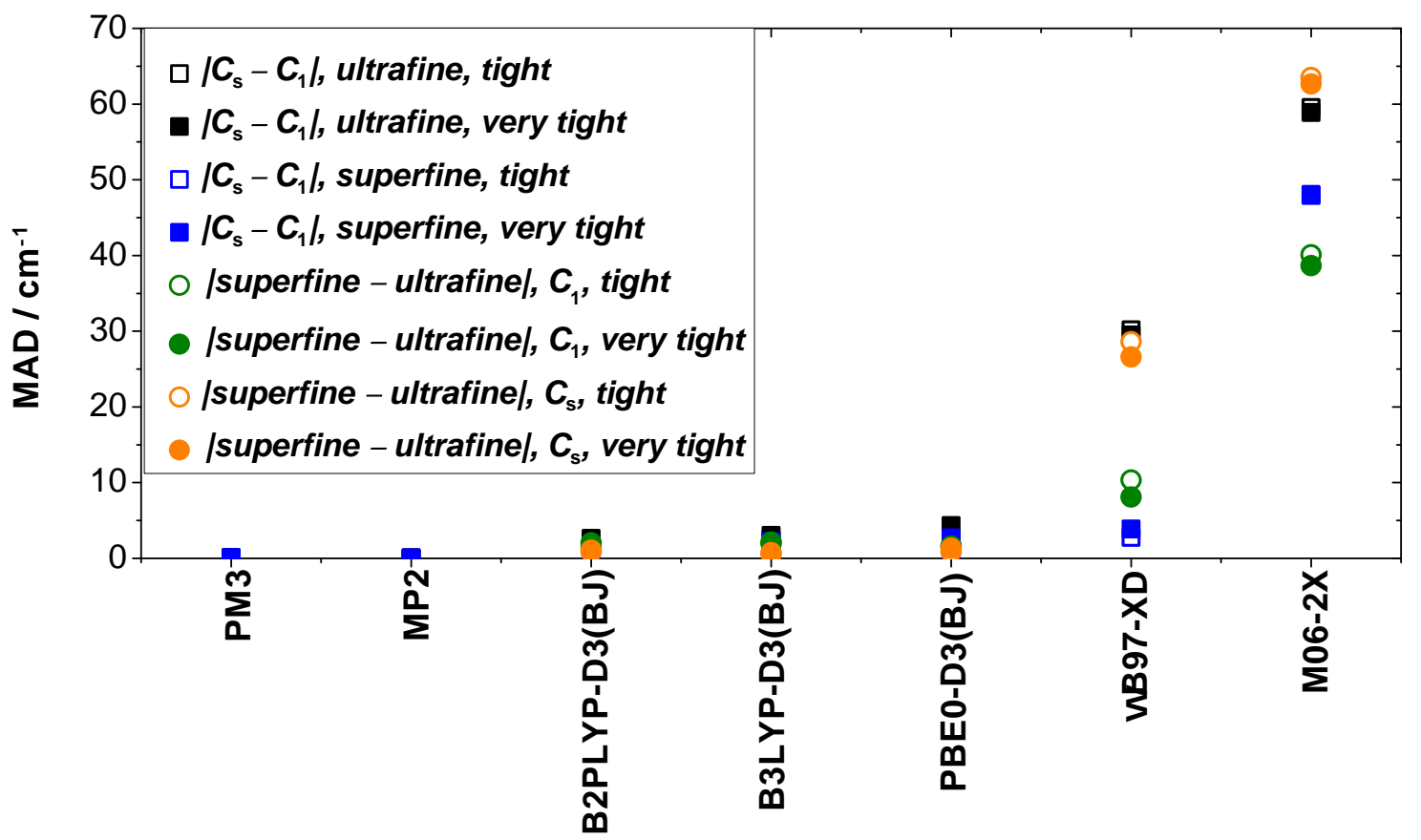

Figure 4.11: Mean absolute deviations (MAD, in $\mathrm{cm}^{-1}$ ) of anharmonically (VPT2) calculated band positions of the stretching vibrations $\left(\nu_{1}, \nu_{2}, \nu_{3}\right.$, and $\left.\nu_{6}\right)$ and the $\mathrm{O}-\mathrm{H}$ out-of-plane bending vibration $\left(\nu_{9}\right)$ of cis- and trans-formic acid resulting from the usage of symmetry $\left(C_{\mathrm{s}}\right)$ compared to no symmetry $\left(C_{1}\right)$ or the increase of the DFT integration grid size (superfine integration grid compared to the ultrafine integration grid) for tight and very tight optimisation, as implemented in Gaussian 09. Reprinted with adaptations from Ref. [330] - Published by The Royal Society of Chemistry, licensed under CC BY 3.0. 
Table 4.8: Mean absolute deviations (MAD) of anharmonically (VPT2) calculated band positions of cis- and trans-formic acid when the molecular symmetry is varied $\left(C_{\mathrm{s}}\right.$ compared to $C_{1}$ ) and the integration grid size (ultrafine or superfine) and optimisation settings (tight or very tight, abbreviated to $\mathrm{t}$ and $\mathrm{vt}$ ) are kept constant and mean absolute deviations when the integration grid size is increased from ultrafine to superfine at either $C_{1}$ or $C_{\mathrm{s}}$ symmetry and tight or very tight optimisation. The vibrations considered are the stretching vibrations of cisand trans-formic acid $\left(\nu_{1}, \nu_{2}, \nu_{3}\right.$, and $\left.\nu_{6}\right)$ as well as the $\mathrm{O}-\mathrm{H}$ out-of-plane bending vibration $\nu_{9}$.

\begin{tabular}{|c|c|c|c|c|c|c|c|c|}
\hline & \multicolumn{4}{|c|}{$\overline{\mathrm{C}_{\mathrm{s}}-C_{1}}$} & \multicolumn{4}{|c|}{ superfine - ultrafine } \\
\hline & \multicolumn{2}{|c|}{ ultrafine } & \multicolumn{2}{|c|}{ superfine } & \multicolumn{2}{|c|}{$C_{1}$} & \multicolumn{2}{|c|}{$C_{\mathrm{s}}$} \\
\hline & $\mathrm{t}$ & $\mathrm{vt}$ & $\mathrm{t}$ & $\mathrm{vt}$ & $\mathrm{t}$ & $\mathrm{vt}$ & $\mathrm{t}$ & $\mathrm{vt}$ \\
\hline B2PLYP-D3(BJ) & 1.7 & 2.6 & 0.8 & 0.6 & 1.5 & 2.0 & 0.8 & 1.1 \\
\hline B3LYP-D3(BJ) & 2.5 & 3.0 & 1.6 & 2.2 & 2.1 & 1.9 & 0.8 & 0.6 \\
\hline PBE0-D3(BJ) & 2.1 & 4.3 & 0.8 & 2.7 & 1.6 & 1.6 & 0.8 & 1.3 \\
\hline$\omega \mathrm{B} 97-\mathrm{XD}$ & 30.2 & 29.5 & 2.8 & 3.9 & 10.3 & 8.1 & 28.6 & 26.6 \\
\hline M06-2X & 59.5 & 58.9 & 48.0 & 47.9 & 40.1 & 38.7 & 63.5 & 62.6 \\
\hline
\end{tabular}

the symmetry is enhanced or the very tight optimisation is used. B3LYP-D3(BJ), B2PLYP-D3(BJ), and PBE0-D3(BJ) show small band position discrepancies on the order of $1-4 \mathrm{~cm}^{-1}$ with the coarser grid when $C_{\mathrm{s}}$ instead of $C_{1}$ symmetry is employed. Expectedly, these differences decrease with increasing grid size to $1-3 \mathrm{~cm}^{-1}$. The overall impact of the optimisation settings is on the same order of magnitude $\left(1-2 \mathrm{~cm}^{-1}\right)$.

A severe symmetry effect is seen for $\omega$ B97-XD and M06-2X, where the band positions vary on average by $30 \mathrm{~cm}^{-1}$ and $59-60 \mathrm{~cm}^{-1}$, respectively, when the symmetry is restricted to $C_{\mathrm{s}}$ instead of to $C_{1}$ and the ultrafine integration grid is used. As seen before, these symmetry effects decrease if a finer integration grid is employed. This is particularly pronounced for $\omega \mathrm{B} 97-\mathrm{XD}$, where band position variations reduce down to $3-4 \mathrm{~cm}^{-1}$. In contrast, the average mismatch between the band positions still amounts to $48 \mathrm{~cm}^{-1}$ for M06-2X. As for the other DFT methods, the influence of the optimisation settings is comparably small with differences of $1-2 \mathrm{~cm}^{-1}$.

Upon increasing the DFT integration grid size at a given symmetry and optimisation setting, similarly large mismatches are found. Small deviations of $1-2 \mathrm{~cm}^{-1}$ occur for the more reliable DFT methods (B2PLYP-D3(BJ), B3LYP-D3(BJ), and PBE0-D3(BJ)). These are considerably larger for $\omega$ B97-XD and M06-2X, with differences of up to $27-$ $29 \mathrm{~cm}^{-1}$ and $63-64 \mathrm{~cm}^{-1}$, respectively. For both, these deviations are more pronounced for the $C_{\mathrm{s}}$ symmetry, whereas for the other methods, it is the other way around. Also, one should keep in mind that values in Table 4.8 are averaged over five vibrations of two rotamers. The individual outliers are distinctly larger. Generally, the $\mathrm{O}-\mathrm{H}$ stretching as well as the $\mathrm{O}-\mathrm{H}$ out-of-plane bending vibration are the most sensitive vibrations to these symmetry, optimisation, and integration grid size effects, whereas the $\mathrm{C}=\mathrm{O}$ and $\mathrm{C}-\mathrm{O}$ stretching vibrations are affected the least.

To decide which combination of symmetry, grid size, and optimisation setting yields 


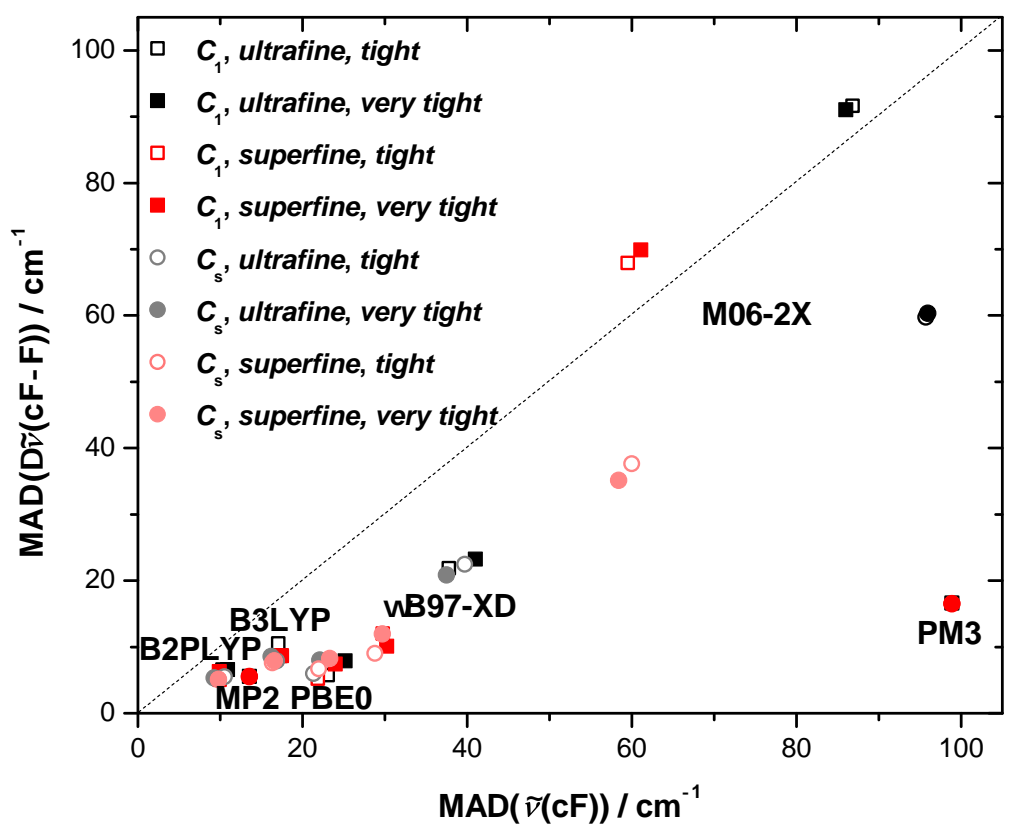

Figure 4.12: Mean absolute deviations (MAD, in $\mathrm{cm}^{-1}$ ) of anharmonically (VPT2) calculated band positions of the stretching vibrations $\left(\nu_{1}, \nu_{2}, \nu_{3}\right.$, and $\left.\nu_{6}\right)$ and the $\mathrm{O}-\mathrm{H}$ out-of-plane bending vibration $\left(\nu_{9}\right)$ of $c i s$-formic acid and the corresponding shifts between both rotamers $\Delta \nu(\mathrm{cF}-\mathrm{F})$ with respect to the experimental values listed in Table 4.1. The calculations have been performed with with varying settings (symmetry, optimisation, and DFT integration grid), as listed in the inlet.

the best agreement with experiment, the mean absolute deviations of the cis-formic acid band positions $\tilde{\nu}(\mathrm{cF})$ and cis-trans shifts for all five vibrations considered in this work (Table 4.1) are visualised in Figure 4.12 for all eight possible setting combinations (four for MP2 and PM3). The MAD values are additionally listed in Tables A.6 (DFT methods) and A.7 (MP2 and PM3) in the appendix. As seen before, the prediction of the band position shift between both rotamers is more accurate for almost all methods used. The exception is M06-2X, where the MAD values are larger for the shift when $C_{1}$ symmetry is employed. Of all variations, the $C_{\mathrm{s}}$ symmetry and the finer integration grid tend to give the best overall agreement with experiment - for some methods with tight (e.g. $\omega \mathrm{B} 97-\mathrm{XD})$ and for others with very tight optimisation (e.g. M06-2X).

Altogether, it can be concluded that for the more reliable DFT methods such as B3LYP-D3(BJ), PBE0-D3(BJ), and B2PLYP-D3(BJ), small variations in band position occur when the integration grid size and the symmetry are increased and the optimisation settings are narrowed (very tight). On average, these are smaller than the experimental uncertainty for the band positions and shifts. Therefore, Figure 4.9 would barely change. The extent of the deviations for M06-2X is in all cases unreasonable - particularly for the larger, computationally more demanding integration grid. These pronounced DFT integration grid effects are well known for M06-2X [230, 231]. It is therefore crucial to critically evaluate the impact of the integration grid, symmetry, and optimisation criteria when utilising DFT methods for vibrational perturbation theory calculations. 
Further examples are given throughout the next chapter. For an unbiased comparison, all VPT2 calculations hereinafter are averaged over these settings and displayed with their standard deviation as error bars.

\subsection{Outlook}

\subsubsection{Introduction}

As the available set of cis-formic acid fundamentals has been significantly enhanced with the Raman jet measurements shown in this work, their applicability to other systems shall be explored. An obvious choice are the deuterated isotopologues of formic acid, as these facilitate an extension of the available vibrational benchmark data base of this popular reference system. Unfortunately, no high level ab initio calculations are available for deuterated formic acid isotopologues, so that an additional motivation is to trigger these by providing new band positions. A brief discussion can be found in the following section. Additionally, the use of larger carboxylic acids for the scrutiny of the cis-trans isomerisation is examined. As the cis-formic acid abundance at the upper nozzle temperature $\left(190{ }^{\circ} \mathrm{C}\right)$ is with $1-2 \%$ rather low, the focus is on systems with smaller energy differences.

\subsubsection{Deuteration}

As for hydrogenated formic acid, all the fundamentals of the trans-rotamers of the three deuterated isotopologues (HCOOD, DCOOH, and DCOOD) have been assigned in the gas phase $[62,63,68,293,294,297,313,350-363]$. The only exception is the $\mathrm{C}-\mathrm{H}$ out-of-plane bending vibration of HCOOD, where solely solid rare gas matrix values are available [298, 364]. To ensure comparability, the fundamentals of the deuterated isotopologues of formic are labelled in analogy to $\mathrm{HCOOH}$. Consequently, the $\mathrm{C}-\mathrm{H}$ out-of-plane bending vibration of HCOOD corresponds to $\nu_{8}$. For the cis-rotamers of HCOOD, DCOOH, and DCOOD, no gas phase data are available. As for $\mathrm{HCOOH}$, their fundamentals have been measured in solid rare gas matrices via laser excitation of the trans-form for HCOOD [311,364] and DCOOH [60] by the Khriachtchev group. As detailed above, this lack of perturbation-free cis-formic acid data on the deuterated isotopologues can be closed by measuring Raman jet data exploiting thermal excitation. Firstly, suitable spectral regions need to be chosen. Therefore, the Raman scattering cross-sections of all the fundamentals of the two rotamers of HCOOD, DCOOH, and DCOOD have been calculated harmonically at the B3LYP-D3(BJ)/aVTZ level. The results are shown alongside the data on $\mathrm{HCOOH}$ in Figure 4.13. 


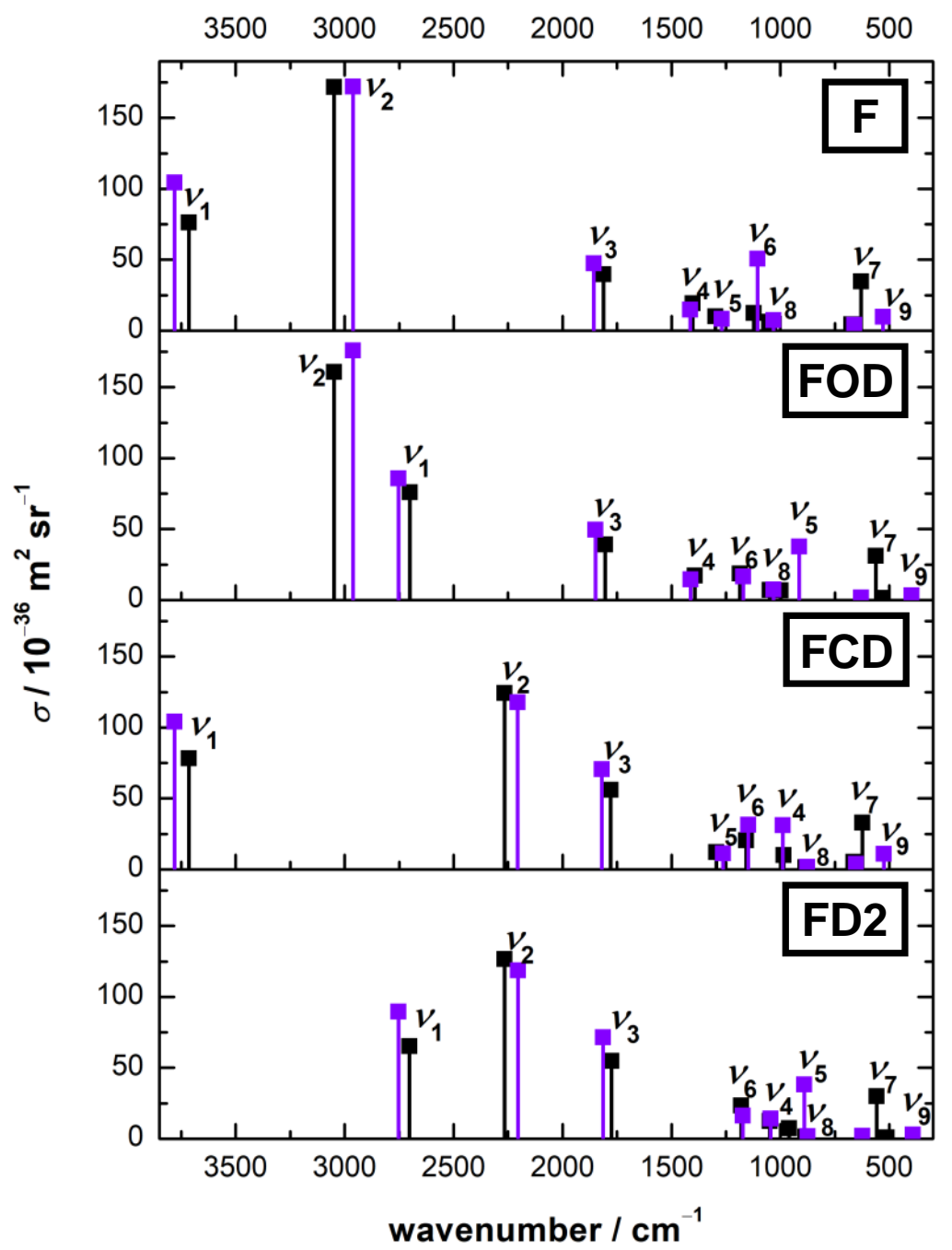

Figure 4.13: Harmonically calculated (B3LYP-D3(BJ)/aVTZ) Raman scattering crosssections of all the fundamentals of formic acid and its deuterated species FOD (HCOOD), FCD $(\mathrm{DCOOH})$, and FD2 (DCOOD). The results for $\mathrm{HCOOH}$ were shown previously in Figure 4.3.

As expected, the harmonic band positions and Raman scattering cross-sections of the $\mathrm{O}-\mathrm{H}, \mathrm{O}-\mathrm{D}, \mathrm{C}-\mathrm{H}$, and $\mathrm{C}-\mathrm{D}$ stretching vibrations are very similar for the isotopologues with the same groups. However, this might not necessarily be the case if anharmonicity is accounted for, as mode coupling can occur. Examples are the $\mathrm{C}-\mathrm{D}$ stretching vibration of DCOOD [62] and $\mathrm{C}-\mathrm{H}$ stretching vibration of HCOOD. The latter will be discussed in detail below. Unsurprisingly, the most unaffected vibrations by deuteration are the $\mathrm{C}=\mathrm{O}$ stretching vibration $\nu_{3}$ and the $\mathrm{O}-\mathrm{C}=\mathrm{O}$ bending vibration $\nu_{7}$. Again, Fermi resonances can cause distortions to the similarity of the spectra, as has been reported for $\nu_{3}$ of DCOOH [357] and DCOOD [62]. For the two out-of-plane bending vibrations $\nu_{8}$ and $\nu_{9}$, deuteration of the involved groups $(\mathrm{C}-\mathrm{D}$ or $\mathrm{O}-\mathrm{D})$ leads to near-zero Raman scattering cross-sections. Clearly the most interesting vibrations are the in-plane $\mathrm{H} / \mathrm{D}-\mathrm{O}-\mathrm{C}$ bending vibration $\nu_{5}$ and the $\mathrm{C}-\mathrm{O}$ stretching vibration $\nu_{6}$. 
As aforementioned, the Raman scattering cross-section of $\nu_{6}$ of cis- $\mathrm{HCOOH}$ is distinctly larger compared to trans-HCOOH (roughly four times), which enabled an assignment despite the low cis-abundance. This scattering cross-section advantage of the cis-rotamer shrinks for DCOOH to about one and a half and diminishes completely for the other oxygen deuterated isotopologues (DCOOD and HCOOD). Thus, it is likely too weak to be observed with the currently achievable nozzle temperatures of the set-up. However, for these two isotopologues, a different cis-rotamer vibration becomes accessible, namely $\nu_{5}$, the in-plane-bending vibration of the $\mathrm{O}-\mathrm{D}$ group. The Raman scattering cross-sections of the cis-rotamers are about three (DCOOD) and nearly six times (HCOOD) larger than those of the trans-forms. Consequently, deuteration does not only lead to more reference data points on the cis-trans-isomerism of formic acid, it also provides access to a different vibration and therefore, will facilitate an even more balanced vibrational benchmark.

As this section serves as an outlook, the advantages of deuteration will only be briefly illustrated for HCOOD, which is the most promising system for the band position assignment of $\nu_{5}$ of its cis-form. Firstly, Raman jet spectra of the $\mathrm{O}-\mathrm{D}\left(\nu_{1}\right), \mathrm{C}-\mathrm{H}\left(\nu_{2}\right)$, $\mathrm{C}=\mathrm{O}$ stretching vibrations $\left(\nu_{3}\right)$, as well as the $\mathrm{O}-\mathrm{D}$ in-plane-bending vibration $\left(\nu_{5}\right)$ will be shown with a focus on straightforward assignments of cis-HCOOD bands, followed by a VPT2 benchmark based on the findings of Section 4.4.1.

The vibrational spectra in the $\mathrm{O}-\mathrm{D}$ stretching region look similar compared to the $\mathrm{O}-\mathrm{H}$ stretching region of $\mathrm{HCOOH}$. Combination bands of $\nu_{2}$ with the lowest frequency vibrations cannot be seen due to the higher wavenumber of the $\mathrm{C}-\mathrm{H}$ compared to the $\mathrm{O}-\mathrm{D}$ stretching vibration. The cis-HCOOD band is predicted to be upshifted compared to the trans-band $\left(2631 \mathrm{~cm}^{-1}\right)$. And indeed, a band increasing in intensity with temperature can be seen at $2686 \mathrm{~cm}^{-1}$, which deviates from the harmonic, $\operatorname{FOD}\left(\nu_{1}\right)$-scaled B3LYP-D3(BJ)/aVTZ prediction by $6 \mathrm{~cm}^{-1}$. This underestimation of the band position is similar to the one observed for $\nu_{1}$ of $\mathrm{HCOOH}\left(5 \mathrm{~cm}^{-1}\right)$. The harmonically calculated, zero-point corrected energy difference (B3LYP-D3(BJ)/aVTZ) of cis- and trans$\mathrm{HCOOD}$ is nearly the same as for $\mathrm{HCOOH}\left(15.8 \mathrm{~kJ} \mathrm{~mol}^{-1}\right.$ compared to $\left.15.9 \mathrm{~kJ} \mathrm{~mol}^{-1}\right)$. Hence, the same cis-abundance of about $1-2 \%$ is expected at $190{ }^{\circ} \mathrm{C}$. From the experimental band integrals, $1 \%$ of cis-formic acid is obtained, if the slightly different scattering cross-sections $\left(\sigma_{\mathrm{F}} / \sigma_{\mathrm{cF}}=0.885\right)$ are taken into account. Hence, the band at $2686 \mathrm{~cm}^{-1}$ can be assigned to the $\mathrm{O}-\mathrm{D}$ stretching vibration of cis-HCOOD. In addition to HCOOD bands, a band of deuterated water $\left(\mathrm{D}_{2} \mathrm{O}\right)$ can be seen at $2671 \mathrm{~cm}^{-1}$. In the spectrum at $130{ }^{\circ} \mathrm{C}$, the $\mathrm{D}_{2} \mathrm{O}$ concentration was slightly higher compared to the other spectra, likely because that spectrum was, contrary to custom, measured last of the temperature series after waiting for the nozzle to cool down.

Similarly to $\mathrm{HCOOH}$, the $\mathrm{C}-\mathrm{H}$ stretching region of HCOOD is spectrally more congested. Two strong bands with near-identical intensities can be seen at $2955 \mathrm{~cm}^{-1}$ and $2939 \mathrm{~cm}^{-1}$ (cf. Figure A.7 in the appendix). In previous Raman gas phase spectra, a doublet was observed at $2943 / 2939 \mathrm{~cm}^{-1}$ by Xue [68] and at $2941.8 / 2938.2 \mathrm{~cm}^{-1}$ by Bertie and co-workers [63]. In the latter reference, the peaks were assigned to a Fermi resonance of $\nu_{2}$ and the $\left(\nu_{3}+\nu_{6}\right)$ combination band [63]. Additional Raman jet measurements of Xue, however, showed that the band at $2943 \mathrm{~cm}^{-1}$ is not present as strongly 
$\mathbf{v}_{1}$
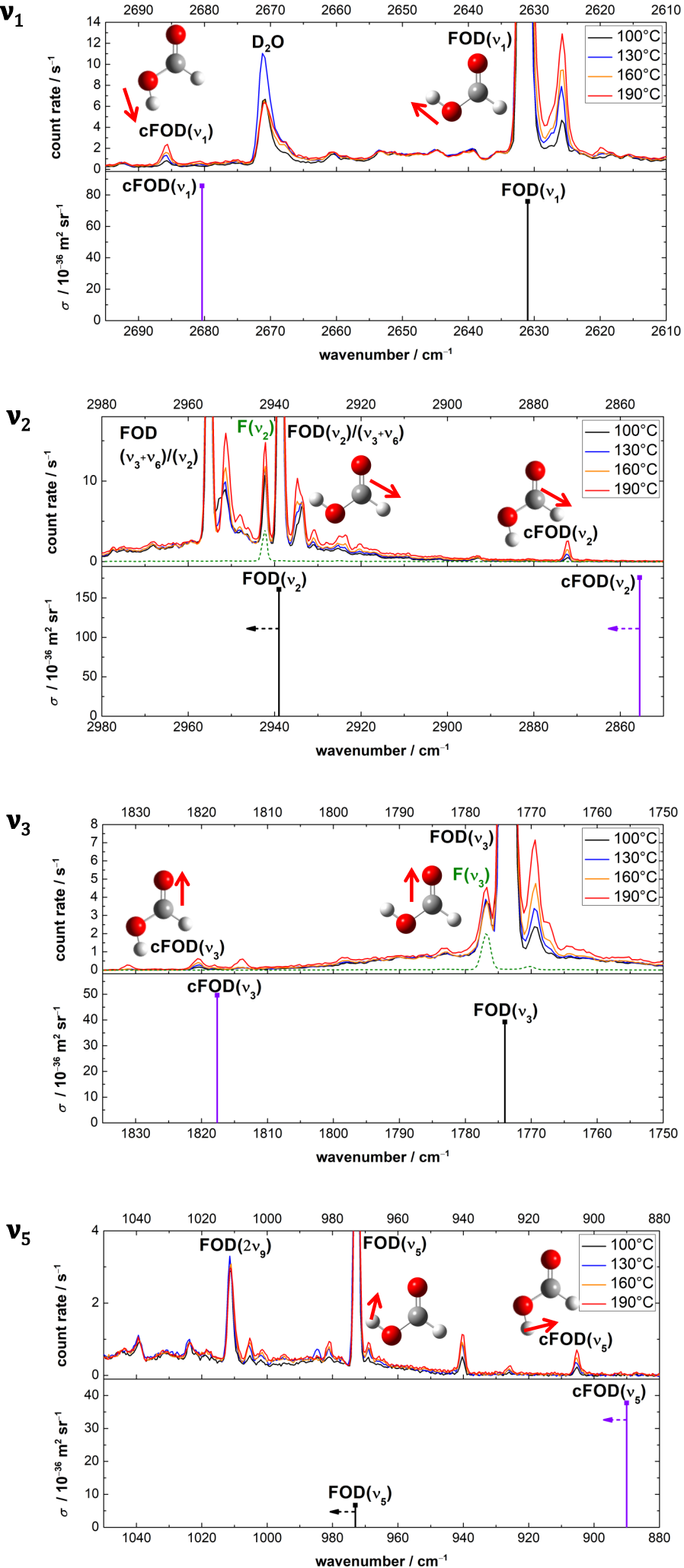

Figure 4.14: Raman jet spectra of HCOOD $(<0.2 \%)$ in helium, recorded at a reservoir pressure of $0.3 \mathrm{bar}$, increasing nozzle temperature of $100-190{ }^{\circ} \mathrm{C}$ and acquisition times of 25-50 minutes. The spectra have been intensity scaled to the trans-HCOOD (FOD) fundamental of lowest intensity in each spectral region. Bands arising from deuterated water, are labelled $\mathrm{D}_{2} \mathrm{O}$. Additionally, scaled $\mathrm{HCOOH}(\mathrm{F})$ spectra have been plotted in green, dashed lines in the $\mathrm{C}-\mathrm{H}$ and $\mathrm{C}=\mathrm{O}$ stretching region, as $\mathrm{HCOOH}$ impurities can be seen. The amount of $\mathrm{HCOOH}$ in the spectra amounts to about $5 \%$. Below the spectra, harmonic and $\operatorname{FOD}\left(\nu_{i}\right)$-scaled band positions are shown, calculated at the B3LYP-D3(BJ)/aVTZ level. Arrows at the band positions of $\nu_{2}$ and $\nu_{5}$ of cis- and trans-HCOOD indicate the shift upon scaling these to Fermi resonance corrected band centres according to Equation 4.1. Further details can be found in the text. 
in the supersonic expansion, which was attributed to the fact that $\mathrm{O}-\mathrm{H} / \mathrm{D}$ exchange is much faster in the gas phase, meaning that the band at $2943 \mathrm{~cm}^{-1}$ is in fact $\mathrm{HCOOH}$ [68]. This can be confirmed in this work, where a weak band of $\nu_{2}$ of $\mathrm{HCOOH}$ can also be seen at $2942 \mathrm{~cm}^{-1}$. According to the manufacturers (abcr and Eurisotop, cf. Table 3.2), the deuterated formic acid used in this work had a weight percentage of $95 \%$ of HCOOD in deuterium oxide and in total $98 \%$ deuterium. With additional Raman measurements in the $\mathrm{O}-\mathrm{H}$ stretching region as well as fitting of spectra of $\mathrm{HCOOH}$ in the $\mathrm{C}-\mathrm{H}$ stretching region and below $1900 \mathrm{~cm}^{-1}$, the $\mathrm{O}-\mathrm{H} / \mathrm{D}$ exchange ratio in the spectra in Figure 4.14 was determined to $5 \%$ throughout all measurements. Therefore, dominant contributions of hydrogenated formic acid can be ruled out. If the observed experimental band positions of $\nu_{3}\left(1774 \mathrm{~cm}^{-1}\right)$ and $\nu_{6}\left(1177 \mathrm{~cm}^{-1}\right)$ are added, $2951 \mathrm{~cm}^{-1}$ is obtained. Including the B2PLYP-D3(BJ)/aVQZ VPT2 prediction of $x_{36}\left(-2.6 \mathrm{~cm}^{-1}\right)$, yields $2948 \mathrm{~cm}^{-1}$ as an estimate for $\left(\nu_{3}+\nu_{6}\right)$. As the lower wavenumber band of the resonance doublet has a slightly higher intensity, it is tentatively assigned to the fundamental $\nu_{2}$ of HCOOD. The reason why the band at $2955 \mathrm{~cm}^{-1}$ was not observed in the previous Raman jet study of Xue [68] are the distinctly higher concentrations of $0.8-1.4 \%$ used, compared to the $<0.2 \%$ in this work. In the $\mathrm{O}-\mathrm{D}$ stretching region of the dimer in that same work $\left(2400-2000 \mathrm{~cm}^{-1}\right)$, several bands can be seen, whereas the spectra shown here, are cluster free, pure monomer spectra. Hence, the band in Reference [68] is likely an overlap of monomer and dimer bands (or even larger clusters), which are expected to be barely shifted in the $\mathrm{C}-\mathrm{H}$ stretching region, due to the low sensitivity of these vibrations towards hydrogen bonding.

In addition to a broad hot band structure between $2960-2910 \mathrm{~cm}^{-1}$, a clearly separated hot band can be seen at $2872 \mathrm{~cm}^{-1}$, which is very close to the band position of $\nu_{2}$ of cis-HCOOH $\left(2873 \mathrm{~cm}^{-1}\right)$. It deviates by $16 \mathrm{~cm}^{-1}$ from the harmonic, FODscaled B3LYP-D3(BJ)/aVTZ prediction for cis-HCOOD, which is again similar to the mismatch of $14 \mathrm{~cm}^{-1}$ observed for cis-HCOOH. Under the assumption that the combination band $\left(\nu_{3}+\nu_{6}\right)$ exhibits a negligible Raman scattering cross-section (dark state), the band position of the $\mathrm{C}-\mathrm{H}$ stretching vibration can be deconvoluted by setting the centre of mass of both as the band position. It can be calculated according to Equation 4.1 from the integral over the product of band position $\tilde{\nu}$ and observed Raman intensity $I(\tilde{\nu})$ divided by the integral over $I(\tilde{\nu})$. For the two bands, it amounts to $2946 \mathrm{~cm}^{-1}$. If scaled to that deconvoluted band position, the agreement of $\nu_{2}$ of $c i s-H C O O D$ with the hot band $2872 \mathrm{~cm}^{-1}$ is enhanced (mismatch of $9 \mathrm{~cm}^{-1}$ instead of $16 \mathrm{~cm}^{-1}$ ). This is illustrated by arrows in Figure 4.14. The observed intensity ratio of the band integrals scaled by the Raman scattering cross-sections $\left(\sigma_{\mathrm{F}} / \sigma_{\mathrm{cF}}=0.915\right)$ amounts to about $1-3 \%$ and depends strongly on how much hot band and rotational substructure is integrated over. Nonetheless, these values agree still fairly well with the $1-2 \%$ expected from thermal population. As such, the band at $2872 \mathrm{~cm}^{-1}$ can be assigned to cis-HCOOD.

$$
\tilde{\nu}=\frac{\int I(\tilde{\nu}) \cdot \tilde{\nu} \cdot d \tilde{\nu}}{\int I(\tilde{\nu}) \cdot d \tilde{\nu}}
$$

In the $\mathrm{C}=\mathrm{O}$ stretching region, two hot bands can be seen at $1820 \mathrm{~cm}^{-1}$ and $1769 \mathrm{~cm}^{-1}$ 
in addition to the trans-HCOOH and HCOOD fundamentals. The band position of the hot band at $1820 \mathrm{~cm}^{-1}$ agrees well with the prediction for the cis-rotamer. The same applies to its intensity, so that this band is assigned to $\nu_{3}$ of cis-HCOOD.

The band position of the $\mathrm{D}-\mathrm{O}-\mathrm{C}$ in-plane bending vibration $\nu_{5}$ of trans-HCOOD is located at $973 \mathrm{~cm}^{-1}$ and is downshifted with respect to the overtone of the $\mathrm{O}-\mathrm{D}$ torsion $2 \nu_{9}$ at $1011 \mathrm{~cm}^{-1}$. Both have been examined in a high-resolution FTIR study by Tan and co-workers, who identified significant wavefunction mixing between both [354]. Further indication that the high intensity of the overtone $2 \nu_{9}$ is a result of a Fermi resonance is obtained by comparing the spectra to those of DCOOD, whose $\mathrm{O}-\mathrm{D}$ torsion $\nu_{9}$ is most similar to HCOOD. These are shown in Figure A.8 in Appendix A.2.5. Around the anharmonically calculated band position of $2 \nu_{9}$, no band with pronounced intensity can be seen. Hence, its Raman scattering cross-section is presumably negligible, so that the high intensity of $2 \nu_{9}$ in case of HCOOD is indeed likely a result of wavefunction mixing with $\nu_{5}$. For the hydrogenated formic acid, a strong Fermi resonance between $2 \nu_{9}$ and $\nu_{5}$ is observed, but the energetic order of fundamental and overtone was shown to be the other way around (cf. Figure 4.4 in Section 4.2). As for $\nu_{2}$, the band position is deconvoluted with Equation 4.1. Scaling to the resulting band position $\left(981 \mathrm{~cm}^{-1}\right)$ is indicated by arrows in the stick spectrum.

There are four hot bands downshifted with respect to $\nu_{5}$ at $969 \mathrm{~cm}^{-1}, 940 \mathrm{~cm}^{-1}$, $926 \mathrm{~cm}^{-1}$, and $905 \mathrm{~cm}^{-1}$ in addition to those between $2 \nu_{9}$ and $\nu_{5}$. The predicted shift of cis-HCOOD is $-83 \mathrm{~cm}^{-1}$. The nearest hot band $\left(905 \mathrm{~cm}^{-1}\right)$ is shifted by $-68 \mathrm{~cm}^{-1}$ from the trans-fundamental. The intensity of that band amounts to $8 \%$ of $\nu_{5}$ of transHCOOD. If the different scattering cross-sections of both rotamers are accounted for $\left(\sigma_{\mathrm{F}} / \sigma_{\mathrm{cF}}=0.178\right)$, this translates into $1 \%$ of cis-HCOOD. The order of magnitude of the overestimation of the downshift of $15 \mathrm{~cm}^{-1}$ has also been seen for the $\mathrm{C}-\mathrm{H}$ stretching vibration with $16 \mathrm{~cm}^{-1}$ and reduces to $8 \mathrm{~cm}^{-1}$ for the deconvoluted band position. Moreover, the intensity of the nearest other hot band is too small in addition to the large band position error of $36 \mathrm{~cm}^{-1}$ it would result in. Consequently, the band at $905 \mathrm{~cm}^{-1}$ is assigned to $\nu_{5}$ of $c i s-\mathrm{HCOOD}$.

The detection of $\nu_{6}$ of cis-HCOOD was explored, but was not successful, due to its lower scattering cross-section with respect to cis-HCOOH (cf. Figure 4.13). The corresponding spectra can be found in Figure A.9 in the appendix. All band positions of the cis-HCOOD fundamentals as well as the shifts between the cis- and trans-rotamers are summarised in Table 4.9 in comparison to values obtained in a solid argon matrix by Domanskaya et al. [364]. As seen before, the matrix shifts of the fundamentals can have a positive or negative sign and exhibit slightly different values compared to cisand trans-HCOOH.

The vibrational benchmark of the four fundamentals and shifts of HCOOD determined with Raman jet spectroscopy is shown in Figure 4.15. Again, the experimental uncertainty $\left( \pm 2 \mathrm{~cm}^{-1}\right.$ for the band positions and $\pm 4 \mathrm{~cm}^{-1}$ for the shifts) is expressed in form of ellipses. As large grid size and symmetry instabilities were found for the VPT2 calculations with M06-2X and $\omega \mathrm{B} 97-\mathrm{XD}$ in the benchmark of cis- and trans-fundamentals of $\mathrm{HCOOH}$, these methods will not be employed here. PM3 is also discarded due to its overall bad performance. For the remaining three DFT methods (B3LYP-D3(BJ), 
Table 4.9: Band positions of the fundamentals $\nu_{i}$ of $c i s$-HCOOD (in $\mathrm{cm}^{-1}$ ) obtained in a supersonic jet expansion probed with Raman spectroscopy (this work) in comparison with values obtained in a solid argon matrix [364].

\begin{tabular}{|c|c|c|c|c|}
\hline$\nu_{i}$ & jet (this work) & $\operatorname{shift}(\mathrm{cFOD}-\mathrm{FOD})^{\mathrm{a}}$ & Ar matrix ${ }^{b}$ & matrix shift ${ }^{c}$ \\
\hline 1 & 2686 & +55 & 2668.7 & -17 \\
\hline 2 & 2872 & -67 & 2895.3 & +23 \\
\hline 3 & 1820 & +46 & 1821.0 / 1799.3 / 1788.9 & $+1 /-21 /-31$ \\
\hline 4 & & & $1395.4 / 1391.0$ & \\
\hline 5 & 905 & -68 & $911.2 / 910.5$ & $+6 /+6$ \\
\hline 6 & & & 1164.0 & \\
\hline 7 & & & $633.0 / 633.6$ & \\
\hline 8 & & & 1041 & \\
\hline 9 & & & & \\
\hline \multicolumn{5}{|c|}{$\begin{array}{l}\text { a } \text { gas phase band position shifts from the trans- to the cis-rotamer } \\
\text { b site } 1 / \text { site } 2 / \text { site } 3 \text { are three dominant trapping sites }\end{array}$} \\
\hline
\end{tabular}

PBE0-D3(BJ), and B2PLYP-D3(BJ)), eight calculations have been performed by varying the DFT integration grid size (ultrafine and superfine), the optimisation settings (tight or very tight), and the symmetry $\left(C_{1}\right.$ and $\left.C_{\mathrm{s}}\right)$. For MP2, these reduce to four. The results have each been averaged and are displayed alongside the standard deviation $\sigma$ as error bars in Figure 4.15. The largest $\sigma$ is observed for the cis-trans band position shift of the $\mathrm{O}-\mathrm{D}$ stretching vibration with B3LYP-D3(BJ), which amounts to $\pm 3 \mathrm{~cm}^{-1}$. As this value and all others are below the experimental uncertainty, these are uncritical. Unfortunately, there are no high level anharmonic vibrational frequency calculations like those of Refs. [327] and [328] available for the deuterated isotopologues, so that this benchmark is limited to vibrational perturbation theory.

Firstly, a few general trends will be discussed before the individual performance of the methods is ranked. Unsurprisingly, the agreement with experiment is significantly better for the band position shifts. The smallest deviations are seen for the $\mathrm{O}-\mathrm{D}$ and the $\mathrm{C}=\mathrm{O}$ stretching vibrations, where the predictions of nearly all methods lie within the experimental confidence interval of $\pm 4 \mathrm{~cm}^{-1}$. The exceptions are MP2/aVDZ and $\mathrm{MP} 2 / \mathrm{aVQZ}$ for the shift of the $\mathrm{C}=\mathrm{O}$ stretching vibration, which is underestimated in both cases. The reason for the rather large underestimation is a Fermi resonance of $\nu_{3}$ of cis-HCOOD with the overtone of $\nu_{5}$ predicted by the MP2 VPT2 calculations. The band position, on the other hand, is predicted correctly only in two cases, namely with PBE0-D3(BJ)/aVQZ $\left(\nu_{5}\right)$ and with B2PLYP-D3(BJ)/aVDZ $\left(\nu_{2}\right)$. As the band position deviations of the other modes are distinctly larger, this is just a coincidence. The overall smallest mismatches with respect to the experimental band position are seen for the $\mathrm{D}-\mathrm{O}-\mathrm{C}$ in-plane bending vibration $\nu_{5}$, which is underestimated by all methods. As seen in Figure 4.15, by using the deconvoluted band position of trans-HCOOD $\left(981 \mathrm{~cm}^{-1}\right.$ 

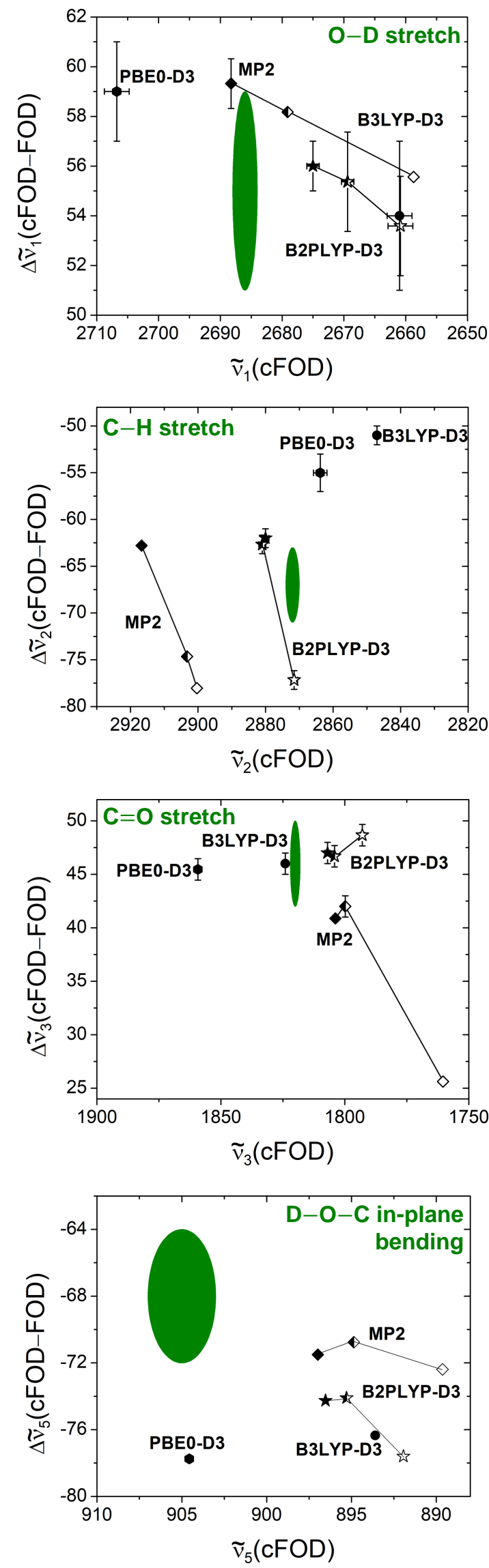

Figure 4.15: Anharmonic (VPT2) vibrational benchmark of the $\mathrm{O}-\mathrm{D}, \mathrm{C}-\mathrm{H}$, $\mathrm{C}=\mathrm{O}$ stretching vibrations as well as the $\mathrm{D}-\mathrm{O}-\mathrm{C}$ in-plane bending vibration of cis-HCOOD at various levels of theory. The basis set used for all filled symbols is an augmented quadruple-zeta basis set (aVQZ). For half-empty and empty symbols, augmented triple- (aVTZ) and double-zeta (aVDZ) basis sets have been employed. The VPT2 band positions have been calculated for eight settings (four for MP2) by varying the symmetry $\left(C_{1}\right.$ or $\left.C_{\mathrm{s}}\right)$, the DFT integration grid size (ultrafine or superfine), and the optimisation settings (tight or very tight). The results were subsequently averaged and are displayed with their standard deviation as error bars. The green ellipses in each plot represent the experimental uncertainty of $\pm 2 \mathrm{~cm}^{-1}$ for the band positions and $\pm 4 \mathrm{~cm}^{-1}$ for the shifts between the cis- and trans-HCOOD fundamentals. Further details can be found in the text. 
instead of $973 \mathrm{~cm}^{-1}$ ), the underestimation of the cis-trans band position shifts can be reduced. This Fermi resonance $\left(2 \nu_{9}\right.$ and $\left.\nu_{5}\right)$ of trans-HCOOD is not predicted by the VPT2 calculations.

The overall agreement of the predicted band positions and shifts for the methods is slightly better compared to $\mathrm{HCOOH}$ and as for $\mathrm{HCOOH}$, the best overall results can be achieved with the B2PLYP-D3(BJ)/aVQZ methodology. For the calculations in $C_{1}$ symmetry, with the ultrafine integration grid, and the tight optimisation, the mean absolute deviation (MAD) of the predicted band positions amounts to 5 experimental confidence intervals and agreement within $\pm 4 \mathrm{~cm}^{-1}$ is achieved for the shifts. For comparison, the MAD values amount to 6 experimental confidence intervals for the band positions and to two for the shifts for $\mathrm{HCOOH}$, if the same settings and number of vibrations are compared.

In conclusion, the deuterated isotopologues of formic acid are ideal for an extension of the data base on local minima of this popular reference system. The Raman jet measurements of these are currently ongoing. These new band positions will hopefully trigger further growth on both the theory and the experimental side.

\subsection{3 cis-trans-Isomerism of Larger Carboxylic Acids}

A recent spectroscopic and computational study of difluoro acetic acid by Bhattacharya and co-workers [365] has predicted a low cis-trans energy difference of $2.2 \mathrm{kcal} \mathrm{mol}^{-1}$ $\left(9.2 \mathrm{~kJ} \mathrm{~mol}^{-1}\right)$ at the $\omega \mathrm{B} 97-\mathrm{XD} / 6-311 \mathrm{G}++(\mathrm{d}, \mathrm{p})$ level. Vibrational bands of three rotamers were assigned in the matrix isolation spectra - two in trans- and one in cisconfiguration of the $\mathrm{OH}$ group [365] without conformational excitation via laser excitation. This indicates that fluorination might lower the cis-trans energy difference significantly and will therefore be examined alongside chlorination. An overview of the cis-trans-energy differences of the explored carboxylic acids is shown in Figure 4.16. The system size feasible for such a study is limited by the vapour pressure of the acids. As opposed to the infrared jet apparatuses used in this work, the Raman jet set-up allows for substances to be heated above room temperature in a heatable saturator and feed line. Nonetheless, thermal decomposition can occur. Pivalic acid, that is $\left(\mathrm{CH}_{3}\right)_{3} \mathrm{CCO}_{2} \mathrm{H}$, has been measured with the Raman set-up before to characterise its dimers [68]. Hence, its vapour pressure will certainly be high enough for the planned monomer measurements. The same is valid for propiolic acid $\left(\mathrm{HC}_{2} \mathrm{CO}_{2} \mathrm{H}\right)$ [68]. With regard to the halogenated species, 3,3,3-trifluoro-2-(trifluoromethyl) propionic acid (abbreviated to TFTFPA) is the largest carboxylic acid considered in this exploratory study. It has a lower boiling point than pivalic acid $\left(125{ }^{\circ} \mathrm{C}[366]\right.$ compared to $\left.164{ }^{\circ} \mathrm{C}[213]\right)$.

The predicted cis-trans-energy differences (B3LYP-D3(BJ)/aVTZ) of acetic and pivalic acid are larger than those of formic acid $\left(20.4 \mathrm{~kJ} \mathrm{~mol}^{-1}\right.$ and $20.2 \mathrm{~kJ} \mathrm{~mol}^{-1}$, respectively). Raman spectra of both acids were recorded with the heatable nozzle, yet no spectral signatures of the cis-rotamers were found, illustrating that the energy difference of $15.9 \mathrm{~kJ} \mathrm{~mol}^{-1}$ might be close to the detection limit with currently accessible nozzle temperatures. The spectra of both can be found in Figures A.10 and A.11 in the appendix. 
While additional methyl or tert-butyl groups as for acetic and pivalic acid lead to an increase of the cis-trans-energy difference, electron withdrawing groups reduce it (Figure 4.16), as anticipated from the study of Bhattacharya et al. [365]. The lowest energy difference is predicted for monofluoro acetic acid. The fluorine atom can be in cis- or trans-position with respect to the carboxyl group. Together with the cis- and trans-position of the $\mathrm{O}-\mathrm{H}$ group, four conformers exist. The global minimum is the trans-OH-cis-fluorine-rotamer. The energy difference to its cis-OH-form amounts to $+23.4 \mathrm{~kJ} \mathrm{~mol}^{-1}$. The all-trans monomer is $0.6 \mathrm{~cm}^{-1}$ higher in energy than the global minimum. For the cis-OH-rotamer of that structure, an additional $\mathrm{O}-\mathrm{H} \cdots \mathrm{F}$ contact exist, which reduces their energy difference to $+0.9 \mathrm{~kJ} \mathrm{~mol}^{-1}$. With respect to the global minimum structure (trans-OH-cis-fluorine-rotamer), it is just $+1.5 \mathrm{~kJ} \mathrm{~mol}^{-1}$ higher in energy.

Another very suitable system is propiolic acid, which is the simplest acetylenic carboxylic acid. While the predicted cis-trans-energy difference is larger than that of the mono- and difluoro acetic acid derivates (cf. Figure 4.16), it is more amenable for vibrational perturbation theory calculations due to its stiffness, whereas large amplitude motions such as the $\mathrm{CH}_{2} \mathrm{~F} / \mathrm{CHF}_{2}$ torsions of mono- and difluoro acetic acid are known to be inadequately described by VPT2. The predicted cis-trans-energy difference amounts to $10.9 \mathrm{~kJ} \mathrm{~mol}^{-1}$, which yields an expected amount $6 \%$ of the cis-rotamer at the highest feasible nozzle temperature of the Raman set-up $\left(190^{\circ} \mathrm{C}\right)$. A B3LYP-D3(BJ)/aVTZ VPT2 study from 2016 predicts a near-identical energy difference of $2.67 \mathrm{kcal} \mathrm{mol}^{-1}$ $\left(11.1 \mathrm{~kJ} \mathrm{~mol}^{-1}\right)$ [367]. The first spectroscopic study of its cis-trans-isomerism has been performed in 2006 by Isoniemi and co-workers in solid argon, krypton, and xenon ma-

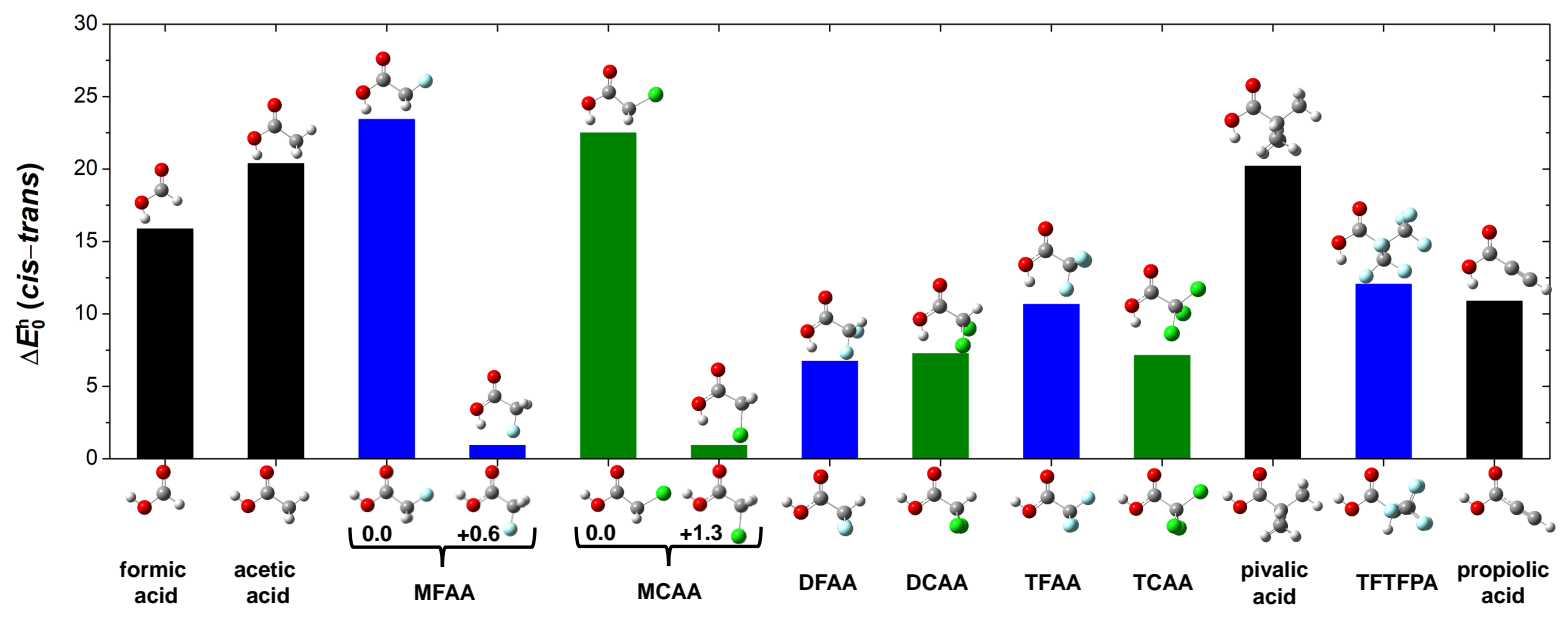

Figure 4.16: Zero-point corrected, harmonic cis-trans energy differences of carboxylic acids, calculated at the B3LYP-D3(BJ)/aVTZ level. MFAA stands for monofluoro acetic acid, MCAA for monochloro acetic acid, DFAA for difluoro acetic acid, DCAA for dichloro acetic acid, TFAA for trifluoro acetic acid, TCAA for trichloro acetic acid, and TFTFPA for 3,3,3-trifluoro-2(trifluoromethyl) propionic acid. 
tricies [340]. A follow up work has been published in 2019 by Lopes, Nikitin, and Fausto, who explored this isomerism as well as the back-tunneling in nitrogen matricies [342]. Its VPT2 applicability has been explored by Shiekh and Kaur who simulated an IR spectrum of propiolic acid using B3LYP-D3(BJ)/aVTZ VPT2 [367]. In conclusion, it is the most promising system for benchmarking anharmonic vibrational frequency calculations, yet the fluorinated and chlorinated acids are preferable when very high cis-rotamer concentrations are desired.

\subsubsection{Summary}

The content of this chapter is best summarised in Figure 4.17. The four stretching vibrations of the higher-energy cis-rotamer of formic acid have been determined in a supersonic expansion exploiting thermal excitation to enhance the population of that local minimum by an order of magnitude compared to room temperature $(1-2 \%$ compared to $0.1 \%$ ). The uniqueness of this approach lies in the rapid jet cooling after the excitation, which traps the conformational (and vibrational) excitation, but cools the rotational temperature down to a few tens of $\mathrm{K}$, which greatly simplifies the Raman spectra recorded.

In this way, four new reference data points on cis-formic acid were obtained in addition to the only value known before 2018 by Baskakov and co-workers [57]. These new data points did not only enable a first critical examination of modern, high-level ab initio quantum chemical calculations from 2016 [327] and 2018 [328], whose cis-formic acid results were previously compared to matrix shifted data, but they also revealed insufficiencies of vibrational perturbation theory calculations when combined with DFT methods. Here, B3LYP-D3(BJ), PBE0-D3(BJ), B2PLYP-D3(BJ), $\omega$ B97-XD, and M06$2 \mathrm{X}$ were tested. The VPT2 band positions of these methods depend on the integration

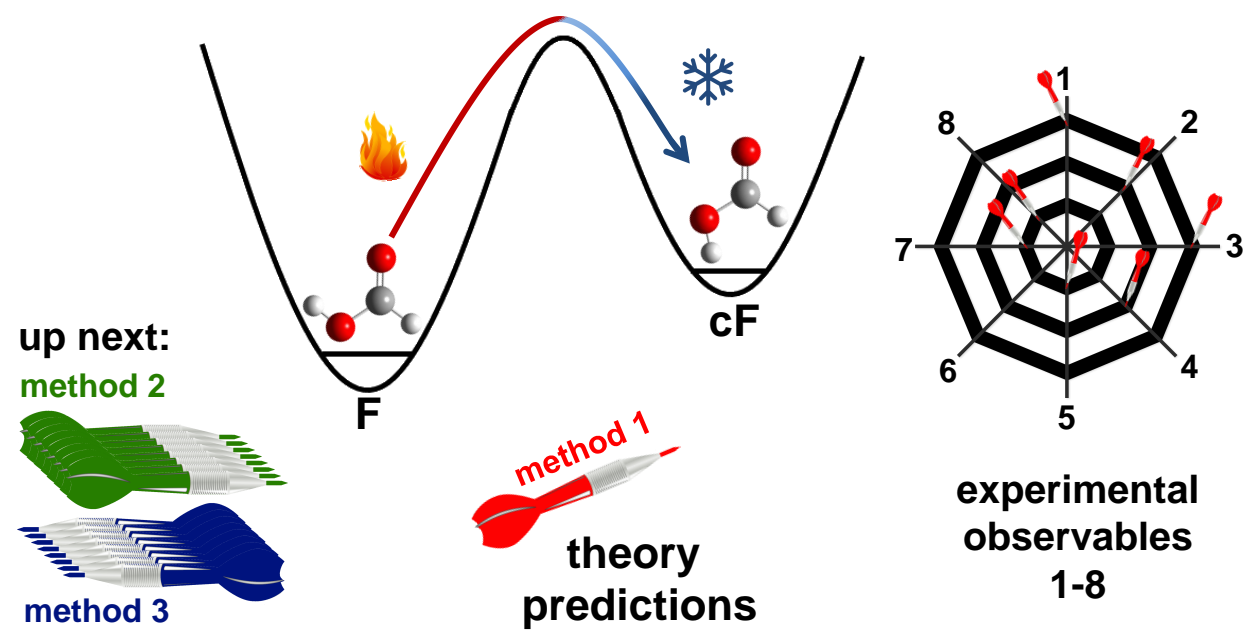

Figure 4.17: The vibrational scrutiny of $c i s$-formic acid fundamentals for benchmarking quantum chemistry. Reprinted from Ref. [330] - Published by The Royal Society of Chemistry, licensed under CC BY 3.0. 
grid size, optimisation setting, and symmetry chosen. Obviously, the effects are also visible in the harmonic band positions, yet an order of magnitude smaller. The severity differs for the methods, with extremely large deviations for M06-2X and $\omega$ B97-XD, where even the harmonic band positions vary by a few $\mathrm{cm}^{-1}$. Hence, vibrational perturbation theory calculations employing both must be treated very carefully.

Furthermore, starting points for future studies were explored. Most promising are the deuterated isotopologues of formic acid, where no gas phase band positions of the local minima are available. A first study of HCOOD highlighted the advantages of deuteration, namely that the vibrations are more harmonic and therefore easier to describe by theory and experimentally, a new vibration becomes accessible with the experimental approach shown here. The band position of the $\mathrm{O}-\mathrm{D}$ in-plane bending vibration $\nu_{5}$ was determined in addition to the $\mathrm{O}-\mathrm{D}, \mathrm{C}-\mathrm{H}$, and $\mathrm{C}=\mathrm{O}$ stretching vibrations. Additional studies on the deuterated species will also shed further light on the suggested re-assignment of the Fermi resonance of $\nu_{5}$ and $2 \nu_{9}$ of trans- $\mathrm{HCOOH}$ [315, 327, 328], which was confirmed by Raman jet data in this work. One central objective of this work is to use the interplay of different spectroscopic techniques, namely conventional FTIR and Raman jet spectroscopy, and as will be seen in Chapter 6, also FTIR imaging, to provide more spectroscopic reference data to trigger further theoretical studies on small reference systems such as formic acid. Along the way, deficiencies of conventionally used quantum chemical methods shall be pointed out to enhance the understanding of how and when to use these most efficiently and even more importantly, which to avoid. The next chapter will build on what has been learned so far about the formic acid monomer, to apply this to hydrogen bonded carboxylic acid dimers. 


\section{Globally and Locally Stable Dimers}

\subsection{Introduction}

Understanding fundamental intermolecular interactions such as hydrogen bonding is critically important, as these are ubiquitous in nature. Examples are the properties and the structure of bulk water [368], enzyme catalysis [369-372], protein folding [373], the triple helix of collagen [374], and DNA, where doubly and triply hydrogen bonded base pairs hold the two DNA strands together [375]. The vibrational characterisation of such biomolecules is very challenging due to the high congestion of the spectra, even when investigated isolated in the gas phase, as well as their demanding quantum chemical description [376, 377]. Thus, in order to characterise these complex systems, particularly their structure, stability, and reactivity, simple model systems are essential. For base pairing in the DNA, carboxylic acid dimers fulfil this purpose, as these are similarly bound via two strong linear hydrogen bonds (cf. Figure 5.1) These dimers, especially the formic acid dimer, have been widely studied experimentally [62, 64-67, 82-84, 119, 191, 335, 378-389] as well as theoretically [69, 73, 75, 78, 80, 81, 390-397].

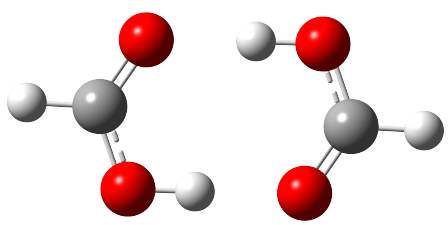

Figure 5.1: Global minimum equilibrium structure of the formic acid dimer (FF), calculated at the B3LYP-D3(BJ)/aVTZ level.

For a vibrational scrutiny, the $\mathrm{OH}$ stretching region is ideal, as it is most sensitive to hydrogen bonding. In case of carboxylic acid dimers, it exhibits a complex, broad resonance structure which extents over roughly $800 \mathrm{~cm}^{-1}\left(3300-2500 \mathrm{~cm}^{-1}\right)$, as shown for formic acid in Figure 5.2. For the formic acid monomer, on the other hand, this vibrational coupling is generally small $[305,349]$. The reason for these pronounced differences is the hydrogen bond that shifts the $\mathrm{OH}$ stretching fundamentals into close proximity to combination and overtone vibrations. These dark states then light up due to wavefunction mixing with the fundamental (bright state).

The $\mathrm{OH}$ stretching region of carboxylic acid dimers is not yet fully understood, even though extensive efforts have been made towards this goal [69-74, 76-78, 80, 81, 398]. 


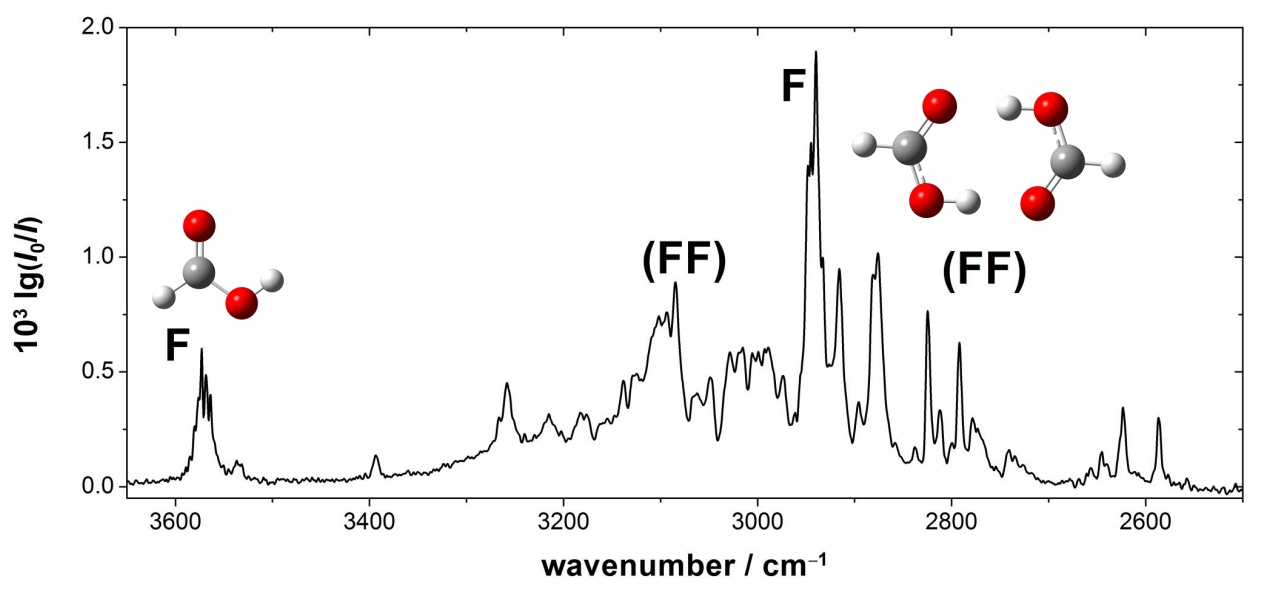

Figure 5.2: IR jet spectrum of formic acid in the $\mathrm{OH}$ stretching region. F marks monomer bands and $(\mathrm{FF})$ bands of the doubly hydrogen bonded cylic dimer.

One strategy to disentangle the resonance pattern is to find a (carboxylic acid) cluster that does not show these resonances at all, i.e., a cluster where the hydrogen bond is weaker and therefore, the distance of the $\mathrm{OH}$ fundamental to potential resonance partners larger. In order to understand the onset of such resonances, the hydrogen bond can then be strengthened via substitution, to see when a first lighting up of dark states occurs. The simplest systems for such a study are metastable carboxylic acid dimers, as these exhibit solely one strong, linear $\mathrm{O}-\mathrm{H} \cdots \mathrm{O}$ hydrogen bond if any [83]. Obviously, such a study is not feasible in supersonic expansions, where the global minimum dimer dominates the spectrum and higher-energy structures are not formed to a large extent. Helium nanodroplets, on the other hand, provide an ideal environment, as the global minimum does not form due to unfavourable dipole-dipole interactions during aggregation [83, 84].

In 2019, Davies and co-workers studied local mimima of acetic acid in helium nanodroplets [84]. Both dimer structures identified exhibit sharp $\mathrm{OH}$ stretching bands, i.e., no signs of resonances were found. Compared to rare gas matrices, where the global as well as local minima of carboxylic acid dimers were extensively studied [82, 380, 386, 389, 399-403], band position shifts due to environmental influences relative to the gas phase are distinctly lower and site splittings are not present, as the molecules can freely translate and rotate inside the droplet [85], although some hindrance of the motion may occur, as illustrated in Refs. [404-406]. This enhances the comparability with quantum chemical calculations without the need of a challenging description of the matrix environment on the theory side, though recent progress has been made by Ito in 2019, who has simulated the argon matrix spectrum of the formic acid dimer [397]. However, hydrogen bonds are still found to be somewhat strengthened by the surrounding helium [88]. An empirical analysis by the Miller group showed that this solvent shift scales approximately linearly with the hydrogen bond induced downshift [87]. An upper bound is estimated from Ref. [87] to about $5 \%$ of the downshift (see Section 3.1.6). If this estimate is applied to singly hydrogen bonded system like the hydrogen fluoride 
(HF), hydrogen cyanide (HCN), water, methanol, or imidazole dimer, the gas phase band position can be reproduced with an accuracy of $\pm 1 \mathrm{~cm}^{-1}$, as shown in Table 5.1, illustrating that this estimate works well. For larger hydrogen bonded clusters such as water trimers, tetramers, and pentamers, the deviations from the reported gas phase values of Ref. [94] increase significantly. This is not surprising, as the simple picture of a caged hydrogen bond does not hold true for the highly coupled hydrogen bonds in these clusters. Altogether, this model gives a valuable prediction of the solvent shift in helium nanodroplets, if the cooperativity of the hydrogen bonds is weak.

Table 5.1: Band positions (in $\mathrm{cm}^{-1}$ ) of hydrogen bonded clusters $\tilde{\nu}_{\mathrm{C}}$ measured in the gas phase (gp) and in helium nanodroplets (HeD) in comparison with gas phase band positions estimated from the helium nanodroplet solvent shift (HeD shift), empirically analysed in Ref. [87]. The clusters are abbreviated to molecule(cluster size). For the respective monomer vibrations $\nu_{\mathrm{M}}$, gas phase band positions are used. In case of water, the average over the symmetric $\left(\nu_{1}\right.$, $\left.3657 \mathrm{~cm}^{-1}\right)$ [94] and antisymmetric stretching vibration $\left(\nu_{3}, 3756 \mathrm{~cm}^{-1}\right)$ [95] of the monomer is taken as a reference. For water clusters, the dominant IR active vibration of the Davydov multiplet is chosen.

\begin{tabular}{ccccc}
\hline \hline system & $\tilde{\nu}_{\mathrm{M}}$ & $\tilde{\nu}_{\mathrm{C}}(\mathrm{gp})$ & $\tilde{\nu}_{\mathrm{C}}(\mathrm{HeD})$ & $\tilde{\nu}_{\mathrm{C}}$ (gp estimate) \\
\hline $\operatorname{HF}(2)$ & $3961^{[407]}$ & $3868^{[98]}$ & $3862^{[99]}$ & $\leq 3867$ \\
$\mathrm{HCN}(2)$ & $3312^{[89]}$ & $3242^{[90]}$ & $3238^{[91]}$ & $\leq 3242$ \\
water$(2)$ & $3707^{\mathrm{a}}$ & $3602^{[94]}$ & $3597^{[96]}$ & $\leq 3602$ \\
methanol$(2)$ & $3686^{[92]}$ & $3575^{[92]}$ & $3571^{[93]}$ & $\leq 3576$ \\
imidazole(2) & $3518^{[2]}$ & $3214^{[2]}$ & $3200^{[100]}$ & $\leq 3215$ \\
\hline water $(3)$ & $3707^{\mathrm{a}}$ & $3533^{[94]}$ & $3529^{[96]}$ & $\leq 3535$ \\
water $(4)$ & $3707^{\mathrm{a}}$ & $3401^{[94]}$ & $3394^{[96]}$ & $\leq 3407$ \\
water $(5)$ & $3707^{\mathrm{a}}$ & $3355^{[94]}$ & $3353^{[96]}$ & $\leq 3367$ \\
\hline a $\frac{1}{2}\left(\nu_{1}{ }^{[94]}+\nu_{3}{ }^{[95]}\right)$ & & & \\
\hline \hline
\end{tabular}

Oswald and co-workers measured FTIR jet spectra of acetic acid and were able to assign an $\mathrm{OH}$ stretching vibration of a local minimum structure of the acetic acid dimer [79]. This metastable dimer is composed of a strong $\mathrm{O}-\mathrm{H} \cdots \mathrm{O}$ and a weaker $\mathrm{CH}_{3} \cdots \mathrm{O}$ hydrogen bond (cf. Figure 5.3). An FTIR jet spectrum measured under similar conditions is shown in Figure 5.3 alongside an IR depletion spectrum of higher-energy acetic acid dimers in helium nanodroplets (black spectrum, bottom panel). Three bands are seen in the helium nanodroplet spectrum downshifted with respect to the acetic acid monomer band at $3585 \mathrm{~cm}^{-1}$. The band furthest downshifted from the monomer at $3292 \mathrm{~cm}^{-1}$ is assigned to the bound $\mathrm{OH}$ stretching vibration of a dimer with similar hydrogen bonds compared to the local minimum structure found in the jet, yet the hydrogen of the free $\mathrm{OH}$ group points upwards with respect to the plane of the $\mathrm{O}-\mathrm{H} \cdots \mathrm{O}$ hydrogen bond [84]. With calculations at the B3LYP-D3/aVTZ level, this structure is not a minimum and converges to the nearly planar structure shown in Figure 5.3. The MP2/6-311 $(2+, 2+) \mathrm{G}(2 \mathrm{~d}, 2 \mathrm{p})$ calculations of Ref. [84] found both. In a study of mixed 


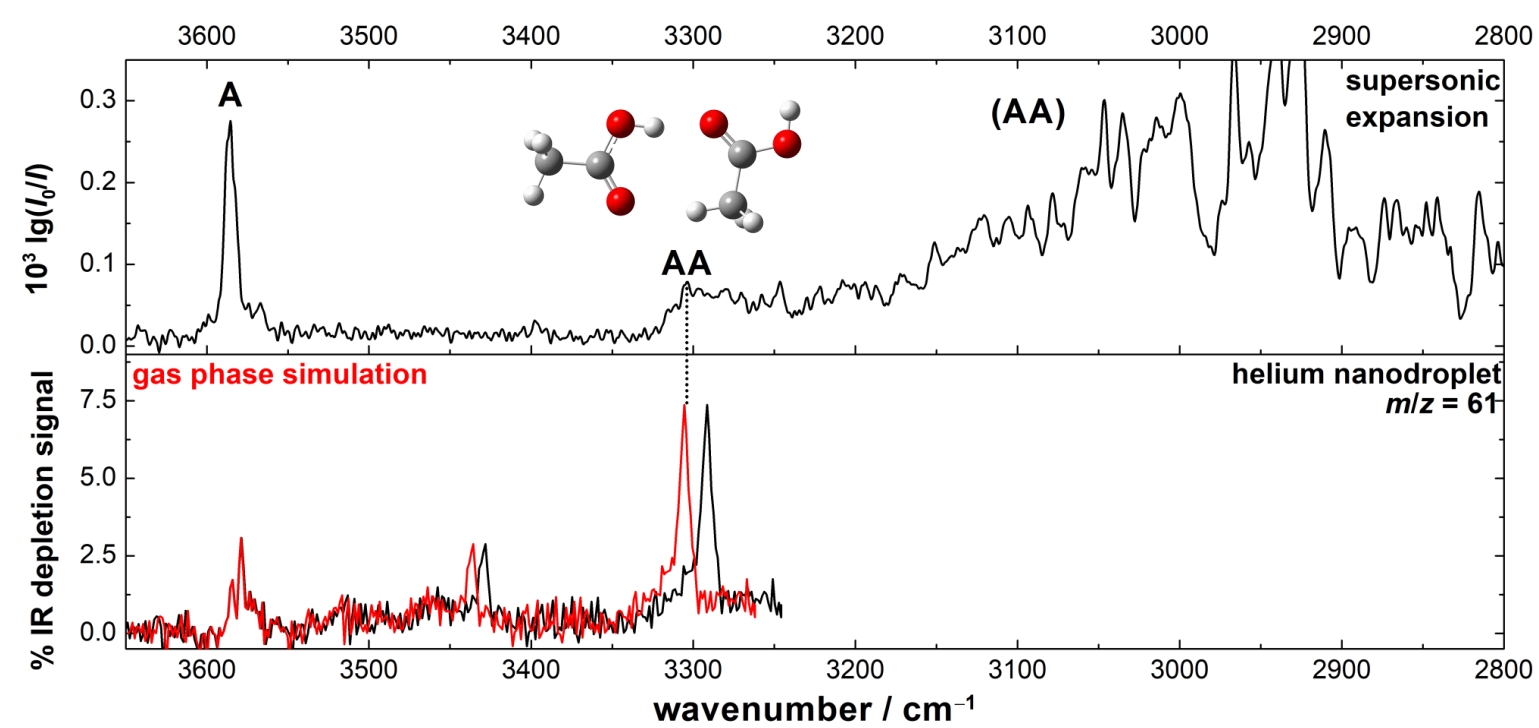

Figure 5.3: Top: FTIR jet spectrum of acetic acid in the $\mathrm{OH}$ stretching region. The assigned local minimum dimer structure is shown, which has been calculated at the B3LYPD3(BJ)/aVTZ level. The band assignments are taken from Ref. [79]. Bottom: IR depletion spectrum of acetic acid measured in helium nanodroplets at $m / z=61$ (black). A gas phase spectrum was simulated by compressing the helium nanodroplet spectrum by $5 \%$ (red).

acetic and methyl acetate dimers by Emmeluth and Suhm [71], calculations without dispersion correction at the B3LYP/6-31 $+\mathrm{G}^{*}$ and the MP2/6-311 $++\mathrm{G}^{*}$ level show different angles between the OCO planes of both monomer entities of the dimer shown in Figure 5.3. For B3LYP, the angle amounts to $0^{\circ}$ (planar) and for MP2, an angle of $25^{\circ}$ is obtained. Therefore, it is likely that these structural differences are solely dependent on the level of theory and that both experimental structures found are analogous. If the aforementioned maximum helium solvent shift (with Equation 3.2 estimated to $-14 \mathrm{~cm}^{-1}$ ) is accounted for (see Figure 5.3), the simulated gas phase band position of this locally stable dimer $\left(3306 \mathrm{~cm}^{-1}\right)$ basically coincides with the gas phase band position of the AA dimer $\left(3304 \mathrm{~cm}^{-1}\right)$ of Ref. [79], especially considering the broadness of the jet band and its underlying socket. This example clearly illustrates that the gas phase correction works well for hydrogen bonded systems with a low degree of cooperativity.

The formic acid monomer [349, 408] and a metastable dimer [83] have also been studied in helium nanodroplets. The bound $\mathrm{OH}$ stretching vibration of this higher-energy dimer was observed when a strong electric field was applied $\left(90 \mathrm{kV} \mathrm{cm}{ }^{-1}\right)$, which results in preferential alignment of dipole moments (pendular-state spectroscopy) [83]. The signal-to-noise ratio of that band was distinctly inferior compared to spectra in the free $\mathrm{CH}$ and $\mathrm{OH}$ stretching regions in that same work. The band extends from roughly $3270-3210 \mathrm{~cm}^{-1}$ and has several maxima. This could be a first hint of resonances, yet no exact conclusions were feasible. In the following section, newly measured helium nanodroplet spectra of the formic acid dimer are shown, which are the result of a collaboration with the group of Prof. Andrew Ellis at the University of Leicester. The results 
of this collaboration have been published in Reference [409], which Section 5.2 is based on.

\subsection{Helium Nanodroplet Spectra}

\subsubsection{Experimental Spectra}

A mass spectrum of formic acid doped helium nanodroplets ranging from $m / z 19$ to 96 is shown in Figure 5.4. The strongest signals include $m / z=19,29,45,47$, and 93 . These correspond to $\mathrm{H}_{3} \mathrm{O}^{+}, \mathrm{HCO}^{+}, \mathrm{COOH}^{+},(\mathrm{HCOOH}) \mathrm{H}^{+}$, and $(\mathrm{HCOOH})_{2} \mathrm{H}^{+}$ions, respectively. Formic acid has a molecular mass of $46 \mathrm{amu}$. Hence, all vibrational spectra of mass channels above $m / z=46$ cannot feature any monomer contribution. Likewise, the IR depletion spectrum of mass channel 93 can only contain vibrational bands of clusters larger than the dimer (molecular mass $92 \mathrm{amu}$ ). Here, the focus is on mass channels 19 and 29 .

The difference between the IR depletion spectra of these two mass channels (Figure 5.4) are two additional bands at $3570 \mathrm{~cm}^{-1}$ and at $3533 \mathrm{~cm}^{-1}$ at $\mathrm{m} / z=29$. These can be attributed to the formic acid monomer $\mathrm{OH}$ stretching vibration and the $\mathrm{C}=\mathrm{O}$ stretching overtone and match the respective gas phase values [79]. It makes sense that the formic acid monomer is not featured in the IR spectrum of $m / z=19$, as it has only two hydrogen atoms and thus, cannot fragment to $\mathrm{H}_{3} \mathrm{O}^{+}$. Apart from the monomer signals, two additional bands can be seen - one sharp signal barely downshifted from the monomer $\mathrm{OH}$ stretching vibration at $3561 \mathrm{~cm}^{-1}$ and one broad band extending from roughly $3266-3194 \mathrm{~cm}^{-1}$ with three pronounced maxima at $3247 \mathrm{~cm}^{-1}, 3236 \mathrm{~cm}^{-1}$, and $3225 \mathrm{~cm}^{-1}$. The highest intensity of that band is at the centre $\left(3236 \mathrm{~cm}^{-1}\right)$. From the positions of the two bands relative to the free $\mathrm{OH}$ stretching vibration of the monomer, it is likely that the former belongs to a free (dangling, non hydrogen bonded) OH group, and the latter to an $\mathrm{OH}$ group involved in hydrogen bonding. The spectral signatures observed here match the spectra reported by the groups of Havenith and Miller [83]. This first analysis of the band positions also confirms that the doubly hydrogen bonded global minimum structure of the formic acid dimer is not formed inside the helium nanodroplets, as its bands extent over a distinctly larger spectral range $\left(3300-2500 \mathrm{~cm}^{-1}\right)$.

Since there can be several possible neutral species contributing to each mass channel, i.e., several fragmentation pathways leading to the same ion, the dominant neutral species needs to be identified. For that, IR depletion spectra are recorded with increasing pick-up cell pressure (PUCP). The bands of interest are then integrated and their pressure dependence is plotted. As the doping of the droplets in the pick-up chamber is a statistical process, the expected pressure dependence for the uptake of $N=1,2,3, \ldots, i$ molecules can be described by a Poisson distribution. The PUCP curves of Ref. [409] show that the band integral of the free $\mathrm{OH}$ stretching vibration at $3561 \mathrm{~cm}^{-1}$ in mass channel $m / z=19$ follows the expected Poisson distribution for $N=2$ at partial pressures below $8 \times 10^{-6}$ mbar. Above that value, the band integrals start to deviate, likely due to contributions of clusters larger than the dimer, e.g. the trimer. Since a partial 

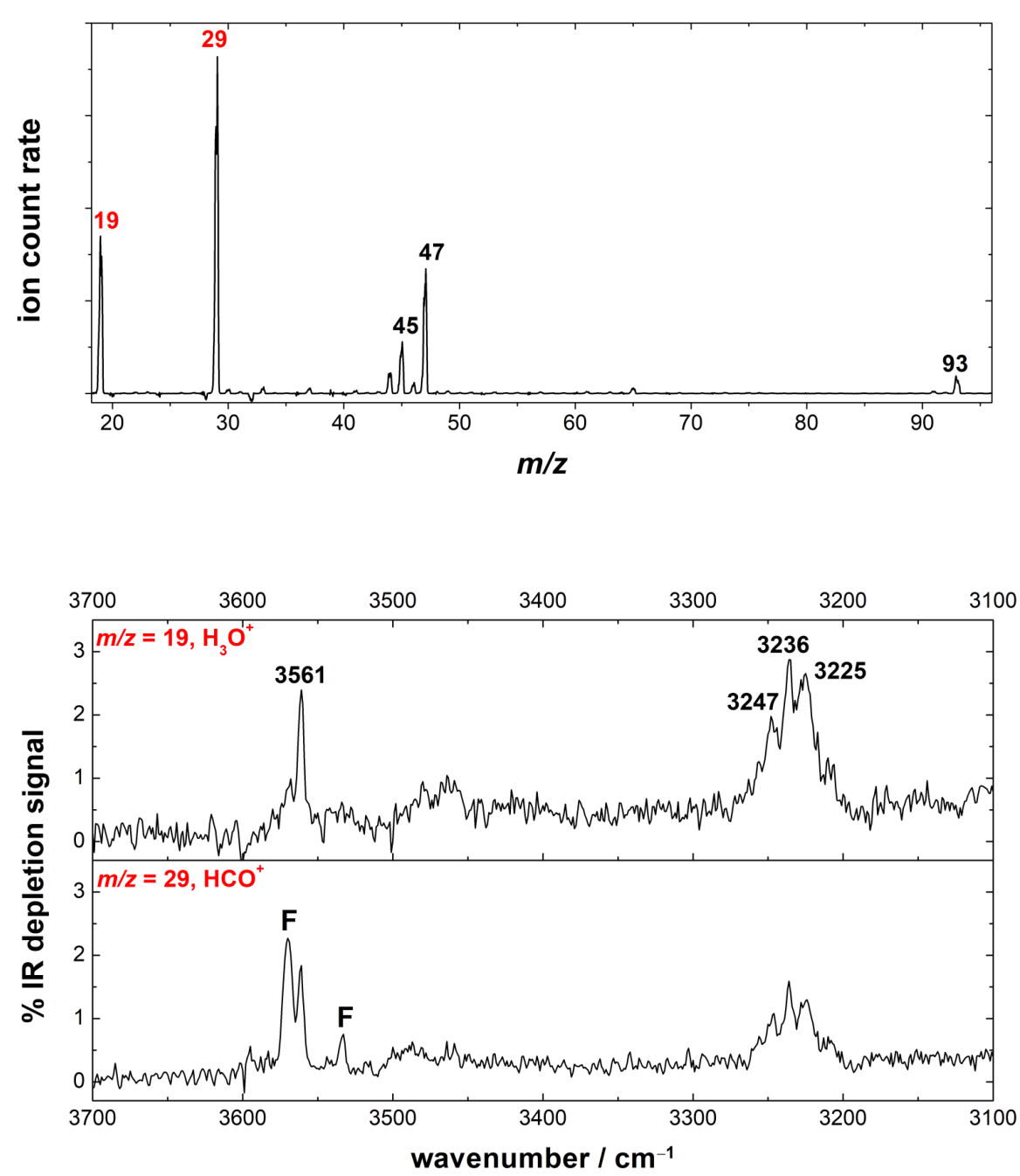

Figure 5.4: Mass spectrum obtained using electron ionization of helium nanodroplets doped with formic acid at a partial pressure of $4 \times 10^{-6}$ mbar. The background signal measured in the absence of dopant has been subtracted from the spectrum. Below the mass spectrum, infrared depletion spectra are shown, which are recorded by monitoring changes in the mass-selected ion yield at $m / z 19$ and $m / z 29$. For each spectrum the average number of dopant molecules per nanodroplet is close to one. Adapted from Ref. [409] with permission from the PCCP Owner Societies. 
pressure of $4 \times 10^{-6}$ mbar was used throughout this work, trimer contributions to that mass channel $(m / z=19)$ are negligible. As expected, the pressure dependence of the $\mathrm{OH}$ stretching vibration of the monomer matches the Poisson distribution of $N=1$. The pressure where on average one formic acid molecule is picked up per droplet is given by the maximum of the $N=1$ PUCP curve and amounts to $3.8 \times 10^{-6}$ mbar, which is just below the pressure chosen for all measurements $\left(4 \times 10^{-6} \mathrm{mbar}\right)$.

\subsubsection{Comparison to Quantum Chemical Calculations}

In order to compare the vibrational spectra measured in helium nanodroplets to quantum chemical predictions and assess the performance of different methods, it is important to account for the band position shift of hydrogen bonded clusters relative to the gas phase. Similar to Figure 5.3, gas phase spectra have been simulated by compressing those measured in helium nanodroplets by $5 \%$. The results can be found in Figure 5.5. The maximum of the broad band is shifted by $+16 \mathrm{~cm}^{-1}\left(3252 \mathrm{~cm}^{-1}\right)$ with respect to its helium nanodroplet band position. As seen in Table 5.1, this shift is likely the upper bound of the gas phase value. Consequently, the actual gas phase band position of the bound $\mathrm{OH}$ stretching vibration will lie in-between both spectra. As the width of this broad band is four times larger $\left(72 \mathrm{~cm}^{-1}\right)$, a sensible comparison with quantum chemical calculations is feasible despite the solvent shift.

In Reference [83], the conformational space of the formic acid dimer was searched and six local minimum structures were identified in addition to the global minimum. These are shown in Figure 5.6. The structures are labelled according to their hydrogen bond topology. A monomer with an $\mathrm{OH}$ group involved in hydrogen bonding to a $\mathrm{C}=\mathrm{O}$ group has the index $\mathrm{C}$ (carbonyl) and accordingly, an $\mathrm{O}-\mathrm{H} \cdots \mathrm{O}-\mathrm{H}$ hydrogen bond has the index A (alcohol). Free $\mathrm{OH}$ groups have no index. Further, dimers with both $\mathrm{OH}$ groups involved in hydrogen bonding, e.g., the global minimum, are enclosed by parentheses. This dimer is then abbreviated to $\left(\mathrm{F}_{\mathrm{C}} \mathrm{F}_{\mathrm{C}}\right)$. There are three dimers composed of at least one strong hydrogen bond, namely $\mathrm{F}_{\mathrm{C}} \mathrm{F}, \mathrm{F}_{\mathrm{A}} \mathrm{F}$, and $\left(\mathrm{F}_{\mathrm{A}} \mathrm{F}_{\mathrm{C}}\right) . \mathrm{F}_{\mathrm{C}} \mathrm{F}$ and $\mathrm{F}_{\mathrm{A}} \mathrm{F}$ both have a weaker $\mathrm{C}-\mathrm{H} \cdots \mathrm{C}=\mathrm{O}$ contact in addition to the strong hydrogen bond. The $\left(\mathrm{F}_{\mathrm{A}} \mathrm{F}_{\mathrm{C}}\right)$ dimer forms a cycle composed of bent $\mathrm{O}-\mathrm{H} \cdots \mathrm{O}-\mathrm{H}$ and $\mathrm{O}-\mathrm{H} \cdots \mathrm{O}=\mathrm{C}$ hydrogen bonds. The three remaining dimers are FF-type structures and feature no strong hydrogen bonds. These dimers are solely bound via weaker $\mathrm{C}-\mathrm{H} \cdots \mathrm{O}=\mathrm{C}$ and $\mathrm{C}-\mathrm{H} \cdots \mathrm{O}-\mathrm{H}$ contacts. Consequently, none of these structures can result in the vibrational features observed, as both $\mathrm{OH}$ stretching vibrations will be barely shifted from the monomer band position. These structures will therefore not be considered further.

The vibrational frequencies of the three local minima $\mathrm{F}_{\mathrm{C}} \mathrm{F}, \mathrm{F}_{\mathrm{A}} \mathrm{F}$, and $\left(\mathrm{F}_{\mathrm{A}} \mathrm{F}_{\mathrm{C}}\right)$ as well as the global minimum $\left(\mathrm{F}_{\mathrm{C}} \mathrm{F}_{\mathrm{C}}\right)$ have been calculated at various levels of theory including B3LYP-D3(BJ), PBEh-3c, M06-2X, B2PLYP-D3(BJ), MP2, and SCS-MP2. The basis set for all methods is aVTZ. All frequency calculations were performed within the double harmonic approximation. The band positions were subsequently scaled to the band position of the $\mathrm{OH}$ stretching vibration of the (trans-) formic acid monomer $\mathrm{F}$ at $3570 \mathrm{~cm}^{-1}$. The scaling factors for all methods are listed in Figure 5.5. Before the individual agreement of the methods with experiment is compared, general trends seen 


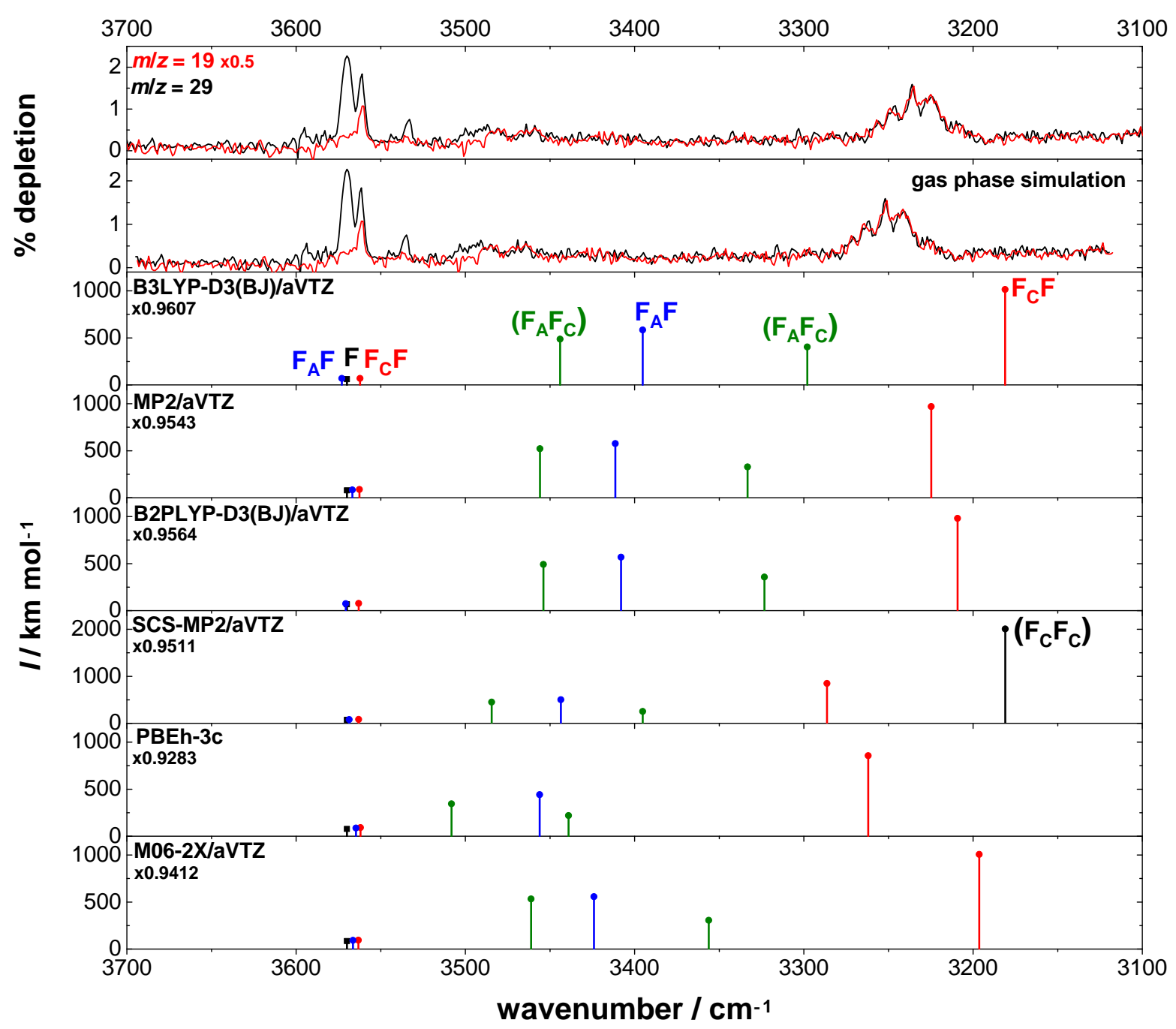

Figure 5.5: IR depletion spectra of formic acid doped helium nanodroplets of mass channels 19 and 29, recorded at a formic acid partial pressure of $4 \times 10^{-6}$ mbar. The intensity of the depletion signal of mass channel 19 was scaled by 0.5. Additionally, gas phase simulations of the helium nanodroplet spectra are shown, which were obtained by compressing these by $5 \%$. More details can be found in Section 3.1.6. Below the spectra, $\mathrm{F}\left(\nu_{1}\right)$-scaled harmonic frequency calculations are shown at various levels of theory. The scaling factors and methods can be found in the respective panels. Adapted from Ref. [409] with permission from the PCCP Owner Societies. 

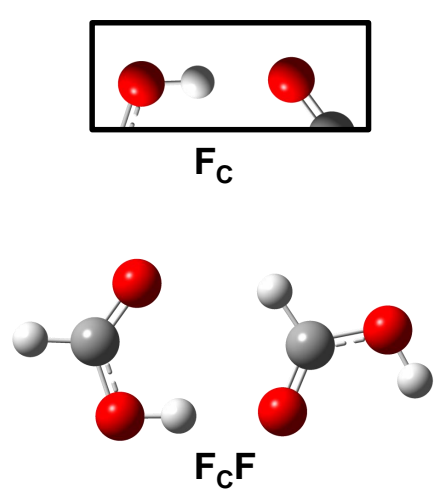

FF-structures
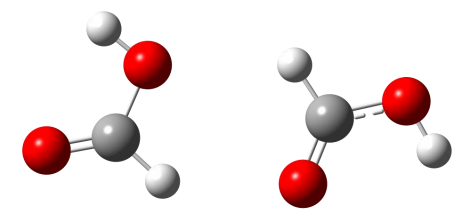

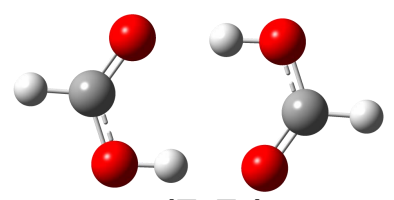

$\left(F_{\mathrm{c}} \mathrm{F}_{\mathrm{C}}\right)$
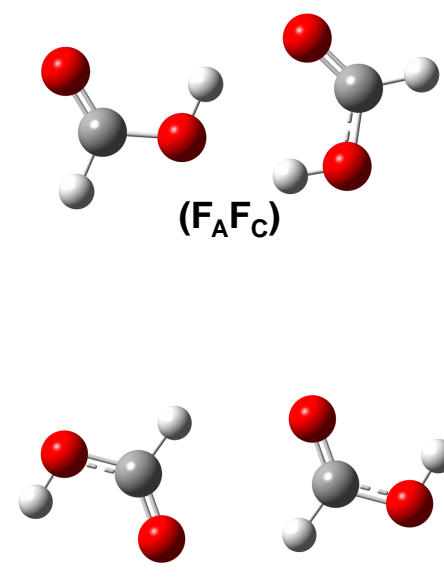

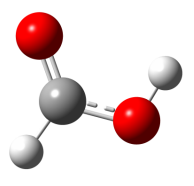

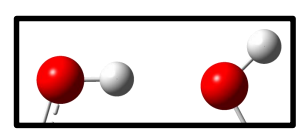

$\mathbf{F}_{\mathrm{A}}$
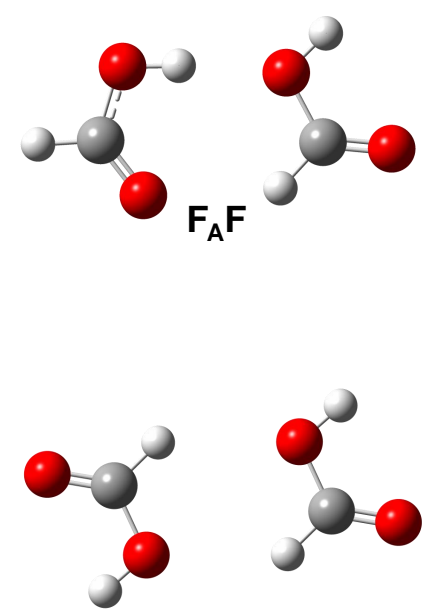

Figure 5.6: Global and local minimum structures of the formic acid dimer, calculated at the MP2/aVTZ level. Adapted from Ref. [409] with permission from the PCCP Owner Societies.

for all calculations will be discussed.

As both $\mathrm{OH}$ groups of $\left(\mathrm{F}_{\mathrm{A}} \mathrm{F}_{\mathrm{C}}\right)$ are involved in hydrogen bonding, the two $\mathrm{OH}$ stretching vibrations of that dimer are considerably downshifted with respect to the monomer. Since there are no additional bands observed between the unbound $\mathrm{OH}$ stretching vibration at $3561 \mathrm{~cm}^{-1}$ and the broad band at $3236 \mathrm{~cm}^{-1}$, it can be concluded that $\left(\mathrm{F}_{\mathrm{A}} \mathrm{F}_{\mathrm{C}}\right)$ is not formed in the helium nanodroplets, at least not to a significant extent. The $\mathrm{O}-\mathrm{H} \cdots \mathrm{O}-\mathrm{H}$ hydrogen bond of the $\mathrm{F}_{\mathrm{A}} \mathrm{F}$ dimer is weaker compared to the $\mathrm{O}-\mathrm{H} \cdots \mathrm{O}=\mathrm{C}$ bond of $\mathrm{F}_{\mathrm{C}} \mathrm{F}$. This translates into a smaller downshift of the bound $\mathrm{OH}$ stretching vibration of $\mathrm{F}_{\mathrm{A}} \mathrm{F}$ with respect to the monomer. Its downshift is also notably smaller than observed experimentally and can therefore also be ruled out. Besides, DFT methods such as B3LYP are known to overestimate the downshifts of $\mathrm{OH}$ stretching vibrations [192, 225, 410]. The overall likeliest structure to be featured in the helium nanodroplets is the $\mathrm{F}_{\mathrm{C}} \mathrm{F}$ dimer, whose band positions are closest to experiment for all methods.

When the individual performances are compared, the overall best agreement with the helium nanodroplet spectrum is achieved with the MP2/aVTZ methodology. For the acetic acid local minimum dimers observed in helium nanodroplets, harmonic MP2 calculations were also most compatible with experiment [84]. Compared to the gas phase simulation spectrum, both the PBEh-3c and MP2/aVTZ calculations agree well. SCSMP2 is the only other method apart from PBEh-3c that tends to overestimate the band position of the bound $\mathrm{OH}$ stretching vibration. All other methods underestimate it. Such good agreement of (scaled) harmonic frequency calculations with the anharmonic 
experiment for hydrogen bond downshifts of this magnitude is somewhat coincidental and likely based on cancellation of anharmonic contributions, as seen for the methanol dimer [411], where the net anharmonicity of bound $\mathrm{OH}$ stretching vibration amounts to just $10 \%$ of the downshift, because the diagonal and off-diagonal contributions largely compensate. Another example of this are imidazole clusters [2].

To examine whether the band of the bound $\mathrm{OH}$ stretching vibration of $\mathrm{F}_{\mathrm{C}} \mathrm{F}$ could be broadened due to coupling to combination and overtone vibrations, additional anharmonic vibrational frequency calculations have been performed using vibrational pertubation theory as implemented in Gaussian 09 [36]. $\mathrm{F}_{\mathrm{C}} \mathrm{F}$ is a relatively rigid molecule, so that VPT2 is expected to still show a reasonably good performance [32]. However, as seen in the previous chapter, DFT methods exhibit a sensitivity towards the integration grid, optimisation settings, and symmetry chosen, which was particularly severe for M06-2X. Other methods such as B3LYP-D3(BJ) and B2PLYP-D3(BJ) showed small deviations on the order of a few wavenumbers. Here, these three methods will be tested in addition to MP2. As for the harmonic vibrational frequency calculations, an aVTZ basis set is used. The resulting eight anharmonic vibrational frequency calculations (two DFT integration grids, two symmetries, and two optimisation settings) are averaged and displayed with their standard deviation as a measure of sensitivity in Figure 5.7. The individual results for all settings are displayed in Figures A.12-A.15 in the appendix. The anharmonically calculated band positions have been subsequently scaled to the free $\mathrm{OH}$ stretching vibration of the formic acid monomer $\left(3570 \mathrm{~cm}^{-1}\right)$, in order to remove imperfections in the description of the $\mathrm{OH}$ stretching potential. The individual scaling factors for each method are displayed in Figure 5.7 and are rewardingly close to 1 . The calculated IR intensities of all monomer bands have been enhanced by a factor of 2 , to roughly match the monomer to dimer abundance in the spectra. Besides, all six intermolecular vibrations of the $\mathrm{F}_{\mathrm{C}} \mathrm{F}$ dimer have been neglected for the following analysis, as these tend to be poorly described by VPT2. Further details on these can be found in Ref. [409]. Firstly, the influence of the optimisation settings for the VPT2 calculations will be examined, followed by a discussion of the overall agreement with experiment.

The error bars of the band intensities are negligible for all methods. Similarly small deviations are seen for the monomer band positions. The exception is M06-2X with a monomer $\mathrm{O}-\mathrm{H}$ band position error $\sigma(\tilde{\nu})$ of $\pm 35 \mathrm{~cm}^{-1}$. These standard deviations $\sigma(\tilde{\nu})$ are even larger for two dimer fundamentals with $\pm 62 \mathrm{~cm}^{-1}$ (free $\mathrm{O}-\mathrm{H}$ stretching vibration) and $\pm 51 \mathrm{~cm}^{-1}$ (bound $\mathrm{O}-\mathrm{H}$ stretching vibration). This confirms that M06-2X is not a suitable electronic structure method for these anharmonic vibrational frequency calculations. Therefore, it will not be considered further within this thesis. The band position errors decrease from B3LYP-D3(BJ) $\left( \pm 10 \mathrm{~cm}^{-1}\right.$ and $\left.\pm 9 \mathrm{~cm}^{-1}\right)$, over B2PLYP-D3(BJ) $\left( \pm 6 \mathrm{~cm}^{-1}\right.$ and $\left.\pm 9 \mathrm{~cm}^{-1}\right)$, to MP2 $\left( \pm 1 \mathrm{~cm}^{-1}\right.$ and $\left.\pm 1 \mathrm{~cm}^{-1}\right)$, which is unsurprisingly the overall the most reliable method in terms of setting sensitivity. In conclusion, this analysis clearly shows that integration grid size, symmetry, and optimisation criteria need to be critically evaluated when using DFT methods for vibrational perturbation theory calculations.

The B3LYP-D3(BJ)/aVTZ VPT2 calculations predict several combination bands with particularly high intensity around the hydrogen bonded $\mathrm{OH}$ stretching fundamental of 


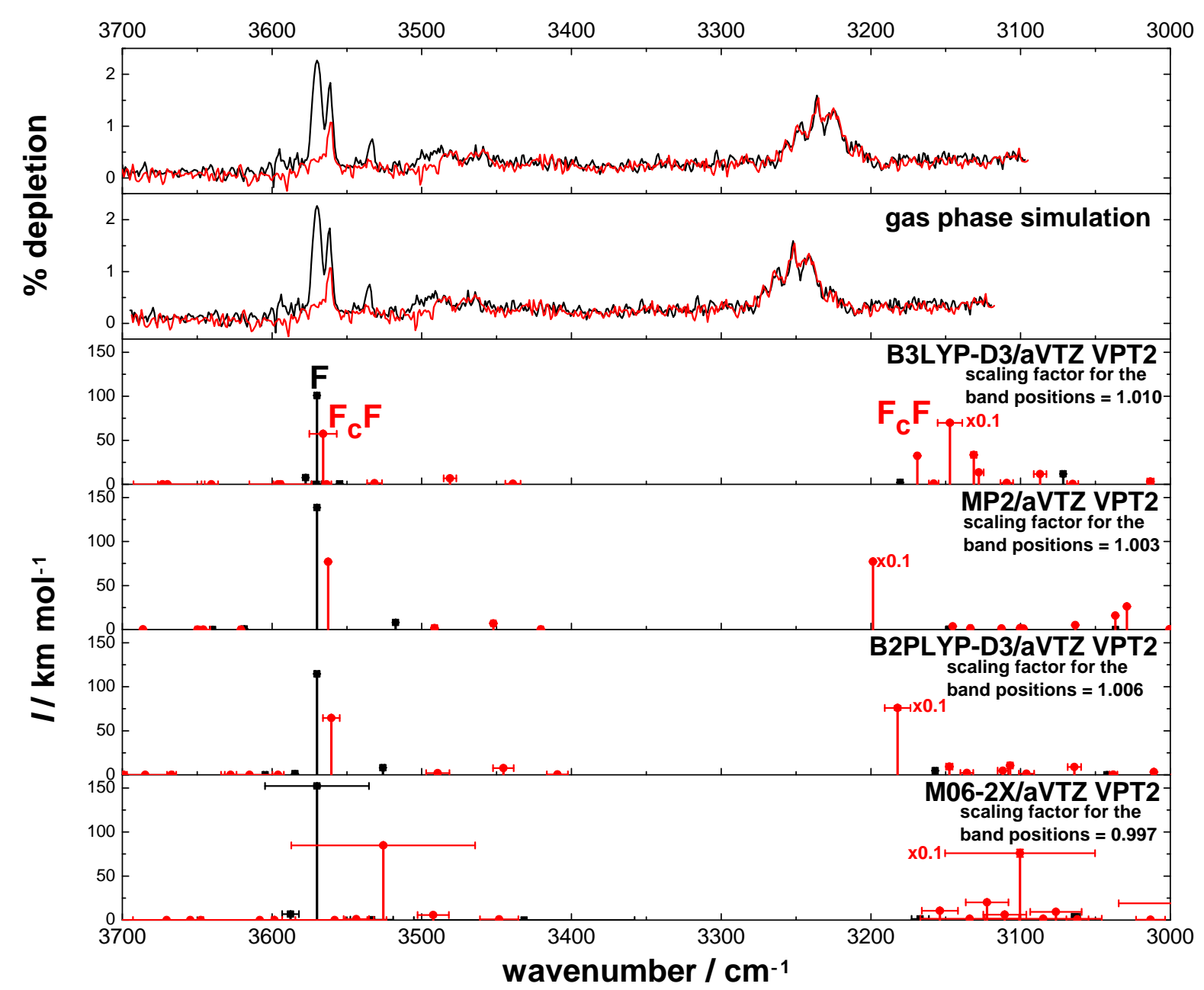

Figure 5.7: IR depletion spectrum of formic acid in helium nanodroplets of mass channels 19 and 29 at a partial pressure of $4 \times 10^{-6}$ mbar. The intensity of the depletion signal of mass channel 19 was scaled by 0.5. Additionally, gas phase simulations of the helium nanodroplet spectra are shown, which were obtained by compressing these by $5 \%$. More details can be found in Section 3.1.6. Below the spectra, anharmonic (VPT2), $\mathrm{F}\left(\nu_{1}\right)$-scaled frequency calculations are shown at various levels of theory. The scaling factors can be found in the respective panels. The calculations have been averaged over two optimisation settings (Gaussian 09 keywords tight and very tight [36]), two DFT integration grid sizes (ultrafine and superfine), and $C_{1}$ as well as $C_{\mathrm{s}}$ symmetry. The standard deviations of the methods are shown as error bars. To match the experimentally observed abundance of monomers and dimers, the monomer band intensities have been scaled by a factor of 2. Adapted from Ref. [409] with permission from the PCCP Owner Societies. 
the $\mathrm{F}_{\mathrm{C}} \mathrm{F}$ dimer. The involved modes are combinations of $\mathrm{C}=\mathrm{O}$ stretching and $\mathrm{C}-\mathrm{H}$ in-plane bending vibrations (see Figure A.16). Their band positions are summarised in Table A.8 in the appendix. The shape of this resonance pattern is not largely dependent on the symmetry, optimisation, and DFT integration grid settings chosen (cf. Figure A.13). It qualitatively matches the experimentally observed pattern, if the intensity of the fundamental is scaled by 0.1 . This huge scaling factor suggests that the wavefunction mixing is massively underestimated by VPT2 and that higher order couplings than the ones included in the VPT2 methodology might have a significant contribution. Additionally, some deviations could also be caused by the neglect of the intermolecular vibrations and deficiencies in the underlying potential energy hypersurface, which may introduce mismatches between the experimentally observed coupling partners.

Clearly, this coupling is very sensitive to the distance of the fundamental with respect to the dark states, and therefore, to the method used. As this distance is larger for MP2/aVTZ, wavefunction mixing is not observed to a significant extent. In case of B2PLYP-D3(BJ)/aVTZ, the bright state is shifted more towards these dark states, so that these have a slightly higher intensity. In conclusion, it seems plausible that the broad structure of the bound $\mathrm{OH}$ stretching vibration of the $\mathrm{F}_{\mathrm{C}} \mathrm{F}$ dimer is caused by coupling to dark states, though to a lower extent than for the global minimum dimer. Unsurprisingly, none of the VPT2 calculations shown here can predict it correctly for the right reasons. Insufficiencies are also seen for the monomer vibrations, where the overtone of the $\mathrm{C}=\mathrm{O}$ stretching vibration is predicted to be upshifted relative to the $\mathrm{OH}$ stretching band rather than downshifted for B3LYP-D3(BJ)/aVTZ and M06-2X/aVTZ. The other two methods underestimate its band position by $-16 \mathrm{~cm}^{-1}$ (MP2/aVTZ) and $-7(1) \mathrm{cm}^{-1}$ (B2PLYP-D3(BJ)/aVTZ). Overall, the VPT2 calculations shown here can at best provide a qualitative explanation of the spectral features observed. For a quantitative analysis, high level ab initio anharmonic vibrational frequency calculations are needed.

An experimental way to understand the resonance pattern better is to utilise deuteration. As the combination bands predicted to be in Fermi resonance with the bright $\mathrm{OH}$ fundamental involve $\mathrm{C}-\mathrm{H}$ in-plane bending vibrations (cf. Appendix A.3.2), helium nanodroplet spectra of DCOOH might be very insightful. The absence of these vibrations in case of acetic acid could potentially also be a reason for the different band shapes observed for both acids.

The broad structure of the bound $\mathrm{OH}$ stretching vibration of the $\mathrm{F}_{\mathrm{C}} \mathrm{F}$ dimer has also been observed in solid rare gas matrices [389, 399]. Whereas the spectral width can always be blamed on site splittings for the solid matrices, such an interpretation is not likely for helium nanodroplets. The reported band position in argon and nitrogen matrices are listed in Table 5.2 alongside the helium nanodroplet values of Madeja and co-workers [83, 349] and those obtained in this work. For the formic acid monomer, additionally the gas phase values of Ref. [79] are listed. The $\mathrm{F}_{\mathrm{C}} \mathrm{F}$ dimer has not been observed in the gas phase apart from a study of Balabin, who reported the band position of a $\mathrm{C}-\mathrm{O}-\mathrm{H}$ out-of-plane bending vibration at $864.1(2.1) \mathrm{cm}^{-1}$ [412]. However, until now, this finding has not been confirmed by other techniques in the gas phase. The monomer band positions measured in helium nanodroplets agree with the gas phase 
Table 5.2: Band positions of the formic acid monomer $\mathrm{F}$ and metastable dimer $\mathrm{F}_{\mathrm{C}} \mathrm{F}$ compared to literature values. Bands that have not been observed are marked n/o. OH stretching vibrations are labelled $\nu(\mathrm{OH})$, whereby the bound and free $\mathrm{OH}$ stretching vibration of the dimer are indicated by the superscripts b and f. $2 \nu(\mathrm{CO})$ represents the overtone of the $\mathrm{C}=\mathrm{O}$ stretching vibration of the formic acid monomer. The $\nu(\mathrm{OH})$ value of Ref. [349] has been estimated as the band centre. Adapted from Ref. [409] with permission from the PCCP Owner Societies.

\begin{tabular}{|c|c|c|c|c|c|c|}
\hline & \multirow{2}{*}{$\begin{array}{c}\text { gas phase } \\
\text { Oswald et al. }{ }^{[79]}\end{array}$} & \multicolumn{2}{|c|}{ helium nanodroplet } & \multicolumn{2}{|c|}{ matrix } \\
\hline & & & this work & Madeja et al. . $^{[83,349]}$ & $\operatorname{argon}^{[399]}$ & nitrogen ${ }^{[389]}$ \\
\hline \multirow{2}{*}{$\mathrm{F}$} & $\nu(\mathrm{OH})$ & 3570 & 3570 & $3569(1)$ & 3550 & 3528.2 \\
\hline & $2 \nu(\mathrm{CO})$ & $3538 / 3533$ & 3533 & 3532.7 & & \\
\hline \multirow[t]{8}{*}{$\mathrm{F}_{\mathrm{C}} \mathrm{F}$} & $\nu(\mathrm{OH})^{\mathrm{f}}$ & $\mathrm{n} / \mathrm{o}$ & 3561 & 3560 & 3540 & 3509.2 \\
\hline & $\nu(\mathrm{OH})^{\mathrm{b}}$ & $\mathrm{n} / \mathrm{o}$ & 3247 & around 3225 & 3184 & 3154.1 \\
\hline & & & 3236 & & 3168 & 3136.4 \\
\hline & & & 3225 & & 3154 & 3128.0 \\
\hline & & & & & 3142 & 3114.4 \\
\hline & & & & & 3101 & 3100.5 \\
\hline & & & & & & 3084.2 \\
\hline & & & & & & 3019.5 \\
\hline
\end{tabular}

values. As discussed for the cis-and trans-rotamers of the formic acid monomer, the vibrational bands measured in cryomatrices are distinctly shifted with respect to the gas phase, whereby this shift is more pronounced for nitrogen than argon. A closer examination of shifts in an argon matrix can be found in Chapter 7 .

\subsubsection{Differences in Formic and Acetic Acid Local Minima}

In an FTIR study of acetic acid local minima in helium nanodroplets by Davies et al. [84], two higher-energy dimer structures were found, whereby one is similar to the $\mathrm{F}_{\mathrm{C}} \mathrm{F}$ dimer and would translate into an $\mathrm{A}_{\mathrm{C}} \mathrm{A}$-type structure. As detailed above, the band position of the bound $\mathrm{OH}$ stretching vibration of that structure closely resembles the band position found in a supersonic expansion by Oswald et al. [79], even more so if the helium nanodroplet solvent shift is accounted for. The structural differences predicted by quantum chemical calculations in both works are minor, so that it is likely that both groups found the same structure. The second local minimum of acetic acid is an $\mathrm{A}_{\mathrm{A}} \mathrm{A}$-type structure, which is not observed for formic acid.

Considering the structural similarities of both local minima, it is somewhat odd that in case of formic acid, the $\mathrm{F}_{\mathrm{C}} \mathrm{F}$ dimer was not found in an FTIR jet study by Oswald and co-workers in the $\mathrm{OH}$ stretching region, even though the corresponding acetic acid local minimum was identified [79]. For a clearer understanding of these differences, FTIR jet spectra of both acids are plotted alongside the helium nanodroplet spectra and their gas phase simulations. The results are shown in Figure 5.8. 


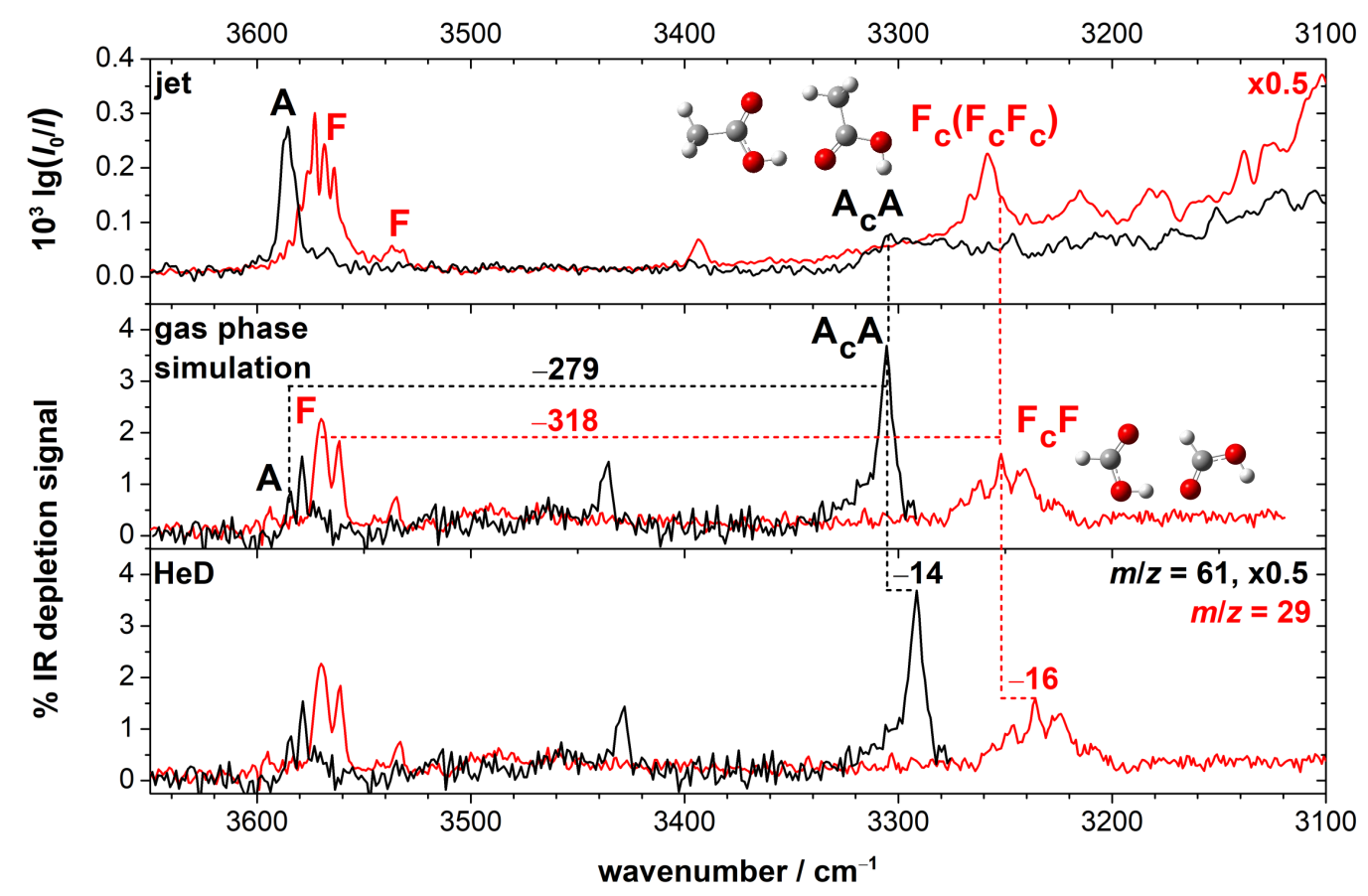

Figure 5.8: Top: FTIR jet spectra of formic (red) and acetic acid (black) in the $\mathrm{OH}$ stretching region. The band assignments are taken from Ref. [79]. The intensity of the formic acid spectrum has been scaled by 0.5. Middle: Gas phase simulation spectra of formic and acetic acid, obtained by compressing the helium nanodroplet spectra shown below by $5 \%$ according to Equation 3.2. The estimated hydrogen bond induced downshifts of the bound $\mathrm{OH}$ stretching vibration of $\mathrm{A}_{\mathrm{C}} \mathrm{A}\left(279 \mathrm{~cm}^{-1}\right)$ and $\mathrm{F}_{\mathrm{C}} \mathrm{F}\left(318 \mathrm{~cm}^{-1}\right)$ with respect to the monomer are displayed. Bottom: IR depletion spectra of formic $(m / z=29)$ and acetic acid $(m / z=61)$ measured in helium nanodroplets (HeD). The intensity of the acetic acid spectrum has been scaled by 0.5 . The estimated helium solvent shifts with respect to the gas phase simulation spectra are listed. The harmonically calculated (B3LYP-D3(BJ)/aVTZ) structures of $\mathrm{A}_{\mathrm{C}} \mathrm{A}$ and $\mathrm{F}_{\mathrm{C}} \mathrm{F}$ are shown as insets.

The band of the bound $\mathrm{OH}$ stretching vibration of $\mathrm{F}_{\mathrm{C}} \mathrm{F}$ is distinctly broader due to the wavefunction mixing with dark states caused by the closer proximity of the fundamental with respect to combination bands. As a result, the band is relatively shallow and therefore, more difficult to detect. Additionally, its downshift with respect to the free $\mathrm{OH}$ stretching vibration of the monomer $\left(3570 \mathrm{~cm}^{-1}\right)$ is larger. Using the simulated gas phase band positions, one obtains shifts of $-318 \mathrm{~cm}^{-1}$ for $\mathrm{F}_{\mathrm{C}} \mathrm{F}$ and $-279 \mathrm{~cm}^{-1}$ for $\mathrm{A}_{\mathrm{C}} \mathrm{A}$. As seen in Figure 5.8, the $\mathrm{F}_{\mathrm{C}} \mathrm{F}$ band would overlap with the broad resonance structure of the global minimum dimer $\left(\mathrm{F}_{\mathrm{C}} \mathrm{F}_{\mathrm{C}}\right)$ as a consequence of its larger downshift, if it was present in the expansions. Hence, the $\mathrm{F}_{\mathrm{C}} \mathrm{F}$ band could easily be hidden underneath the trimer band at $3258 \mathrm{~cm}^{-1}$ assigned by Oswald and co-workers [79], particularly considering the broadness of all bands. In contrast, the extent of overlap with the resonance structure of the global minimum dimer $\left(\mathrm{A}_{\mathrm{C}} \mathrm{A}_{\mathrm{C}}\right)$ is smaller for $\mathrm{A}_{\mathrm{C}} \mathrm{A}$, which is why it is easier detectable. Altogether, the spectral differences found by Oswald and co-workers [79] do 
not necessarily have to reflect upon changes in the aggregation behaviour of the two acids, but could merely be due to poor spectral separation of the formic acid bands.

Altogether, due to this high spectral congestion of the $\mathrm{OH}$ stretching region of carboxylic acids, it is clearly not the most suitable for cluster band assignments. As such, the remaining part of this chapter will focus on the $\mathrm{C}=\mathrm{O}$ stretching region, where hydrogen bond induced downshifts separate monomer from cluster bands whilst simultaneously, fewer resonance partners are present. The $\mathrm{C}=\mathrm{O}$ stretching region is much narrower, it extends only over roughly $200 \mathrm{~cm}^{-1}\left(1800-1600 \mathrm{~cm}^{-1}\right)$ - a small fraction of the about $1100 \mathrm{~cm}^{-1}\left(3600-2500 \mathrm{~cm}^{-1}\right)$ wide $\mathrm{OH}$ stretching region.

\subsection{Exciton Coupling in Homo and Hetero Dimers of Carboxylic Acids}

So far, the metastable, $C_{\mathrm{s}}$-symmetric $\mathrm{F}_{\mathrm{C}} \mathrm{F}$ dimer was scrutinised with vibrational spectroscopy. The doubly hydrogen bonded cyclic dimer, however, exhibits $C_{2 \mathrm{~h}}$ symmetry, resulting in interchangeable $\mathrm{C}=\mathrm{O}$ and $\mathrm{O}-\mathrm{H}$ oscillators. In an uncoupled picture, the local monomer vibrations would therefore be pairwise degenerate. Due to intermolecular coupling caused by hydrogen bonding, the degeneracy is revoked and the vibrations are split in pairs of symmetric (gerade) and antisymmetric (ungerade) vibrations, whereby the symmetric vibrations are Raman active and the antisymmetric vibrations IR active. This splitting of the dimer modes is called Davydov or (vibrational) exciton splitting and is abbreviated to $\Delta a s$ in this work. It amounts to twice the coupling matrix element between both oscillators [413]. The term is referring to electronic excitons and Davydov splittings in molecular crystals [414], for which the double hydrogen bonded carboxylic acid dimers serve as a model system [73, 415-418]. Within this work, both terms will be used interchangeably.

As two types of $\mathrm{C}=\mathrm{O}$ stretching vibrations will be discussed in this section, the nomenclature of the structural dimer labels is adjusted accordingly. This is done by using an additional index outside the parenthesis that symbolises the symmetry of the vibration - an a for antisymmetric and an $\mathrm{s}$ for a symmetric vibration. Hence, the two $\mathrm{C}=\mathrm{O}$ stretching vibrations of the global minimum dimer are abbreviated to $\left(\mathrm{F}_{\mathrm{C}} \mathrm{F}_{\mathrm{C}}\right)_{\mathrm{a}}$ and $\left(\mathrm{F}_{\mathrm{C}} \mathrm{F}_{\mathrm{C}}\right)_{\mathrm{s}}$. Since solely these dimers are considered in this section, the nomenclature will be shortened to $(\mathrm{FF})_{\mathrm{a} / \mathrm{s}}$ by omitting the indices that represent the type of hydrogen bond.

The coupling diagram of the formic acid dimer is shown in Figure 5.9. The dimer vibrations are shifted from the free $\mathrm{C}=\mathrm{O}$ stretching vibrations of the monomers by $\Delta a$ (antisymmetric $\mathrm{C}=\mathrm{O}$ stretching vibration) and $\Delta s$ (symmetric $\mathrm{C}=\mathrm{O}$ stretching vibration) and are separated by the Davydov splitting $\Delta a s$. For an analysis of this mode coupling pattern, the focus is on the $\Delta a$ and $\Delta a s$ values, as $\Delta s$ can be determined from the two. From FTIR and Raman jet studies it is known that the $\mathrm{C}=\mathrm{O}$ stretching vibrations of other carboxylic acid dimers, e.g., acetic acid, vary significantly from those of the formic acid dimer $[68,70,117]$. Thus, their coupling diagrams will also differ. The 


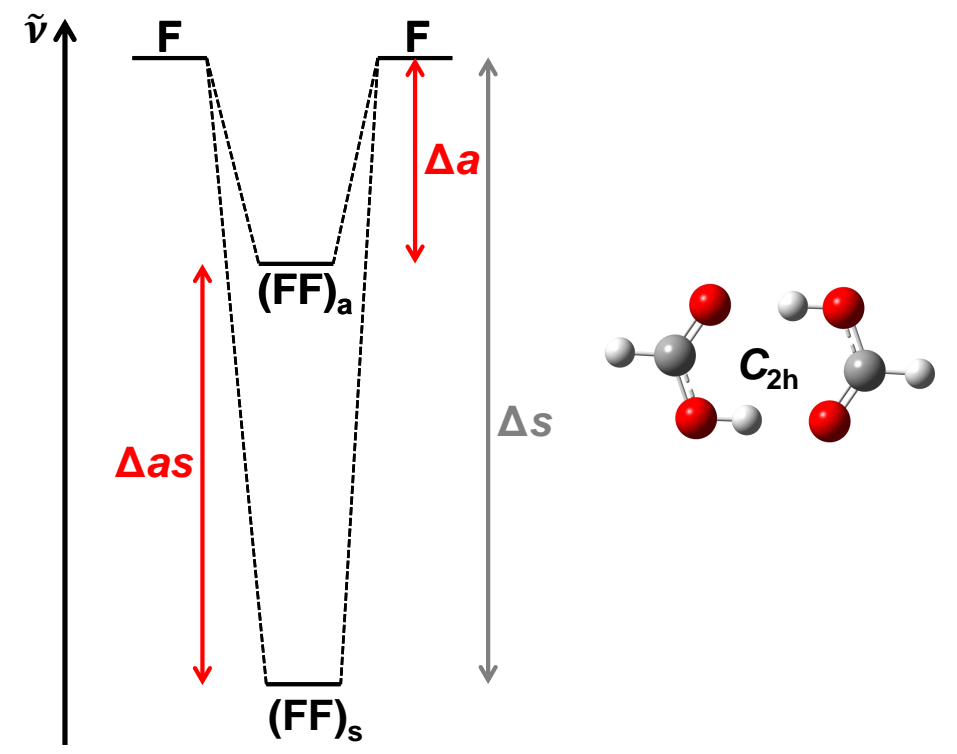

Figure 5.9: Coupling diagramm of the $C_{2 \mathrm{~h}}$ symmetric formic acid dimer. $\Delta a s$ represents the Davydov splitting. $\Delta a$ and $\Delta s$ illustrate the downshift of antisymmetric (a) and symmetric (s) $\mathrm{C}=\mathrm{O}$ stretching vibrations of the dimer with respect to the monomer.

overall aim of this section is therefore to examine these differences.

As mentioned previously, carboxylic acid dimers also serve as the simplest model system for double hydrogen bonding in biomolecules like the DNA. However, the hydrogen bonds present in these systems are predominantly asymmetric. One way of enhancing the comparability of such model systems is to study hetero dimers of two different acids. Since these exhibit a permanent electric dipole moment, they have been extensively studied with microwave spectroscopy. The plethora of studies include dimers of formic and acetic acid [419], formic and benzoic acid [106], formic and propiolic acid [420], formic and nitric acid [421], formic and cyclopropanecarboxylic acid [109], formic and

Table 5.3: Harmonically calculated and $(\mathrm{FF})_{\mathrm{a}}$-scaled band positions $\left(\mathrm{in}^{-1}\right.$ ) of the $\mathrm{C}=\mathrm{O}$ stretching vibrations of the homo and hetero dimers of formic, acetic, and pivalic acid as well as IR intensities $I$ (in $\mathrm{km} \mathrm{mol}^{-1}$ ) and Raman scattering cross-sections $\sigma$ (in $10^{-36} \mathrm{~m}^{2} \mathrm{sr}^{-1}$ ), calculated at the B3LYP-D3(BJ)/aVTZ level. The scaling factor amounts to 0.986 .

\begin{tabular}{cccccccc}
\hline \multirow{2}{*}{ Dimer } & \multicolumn{3}{c}{ symmetric } & & \multicolumn{3}{c}{ antisymmetric } \\
\cline { 2 - 3 } \cline { 7 - 9 } & $\nu_{\mathrm{s}}$ & $I$ & $\sigma$ & & $\nu_{\mathrm{a}}$ & $I$ & $\sigma$ \\
\hline$(\mathrm{FF})$ & 1664 & 0 & 91 & & 1741 & 822 & 0 \\
$(\mathrm{AA})$ & 1674 & 0 & 68 & & 1728 & 819 & 0 \\
$(\mathrm{PP})$ & 1661 & 0 & 78 & & 1712 & 774 & 0 \\
$(\mathrm{FA})$ & 1668 & 33 & 79 & & 1735 & 800 & 2 \\
$(\mathrm{AP})$ & 1666 & 12 & 70 & & 1721 & 781 & 2 \\
$(\mathrm{PF})$ & 1660 & 55 & 81 & & 1728 & 742 & 6 \\
\hline \hline
\end{tabular}


perfluorobutyric acid [110] formic and trifluoroacetic acid [101-105], acetic and trifluoroacetic acid [102, 104, 105], acrylic and trifluoroacetic acid [107], difluoroacetic and acrylic acid [108], formic and 3,3,3-trifluoro-2-(trifluoromethyl)propanoic acid [422], and very recently also the dimer of formic acid and C-deuterated formic acid (DCOOH) [111]. The advantage of microwave spectroscopy lies in its blindness towards the unpolar homo dimers. Hence, the hetero dimer can be examined without their interference.

In contrast, vibrational studies on hetero dimers have been surprisingly sparse. The first one was published in 1964 by Affsprung and co-workers, who measured near-infrared spectra of the acetic and trichloro acetic acid hetero dimer [112]. This was followed by a far-infrared study on the hetero dimers of formic and acetic acid with trifluoroacetic acid in 1970 by Clague and Novak [113]. Over 30 years later, the first mid-infrared spectrum was recorded by Keller in 2004, who studied the hetero dimer consisting of formic and trifluoro acetic acid [114]. The only vibrational study of a hetero dimer since then was published 14 years later by $\mathrm{Gu}$ and co-workers, who examined the hetero dimer of glycolic and 9-hydroxy-9-fluorene acid with IR-UV double resonance spectroscopy [115]. The aim of this work is not only to close this gap by studying the homo and hetero dimers of the three carboxylic acids introduced in the previous chapter, namely of formic, acetic, and pivalic acid, but to highlight their applicability for benchmarking quantum chemical methods. The quantities that will be exploited for this benchmark are the band position shifts $\Delta a$ and exciton splittings $\Delta a s$. Since anharmonic corrections are expected to largely cancel for $\Delta a$ and especially $\Delta a s$ [225], harmonic vibrational frequency calculations should already give a good estimate of these values, which will be tested within this section. A related aspect that will be addressed is whether the hetero dimer $\Delta a$ and $\Delta a s$ values can be predicted from those of the homo dimers, i.e., whether additivity applies for these quantities. The results of this study have been published in Ref. [329], which most of this section is loosely based on.

Firstly, the predicted dimer structures and their dissociation energies will be examined, followed by a discussion of their FTIR and Raman spectra. Due to the inversion centre in the homo dimers, the rule of mutual exclusion applies and both techniques are needed for a vibrational characterisation. By the introduction of asymmetry in the hetero dimers, the inversion symmetry is revoked, yet it still applies locally, as the Raman scattering cross-sections of the antisymmetric $\mathrm{C}=\mathrm{O}$ stretching vibrations are still fairly small and vice-versa the $\mathrm{IR}$ intensities of the symmetric $\mathrm{C}=\mathrm{O}$ stretching vibration, as can be seen in Table 5.3. For spectral assignments, scaled harmonic frequency calculations at the B3LYP-D3(BJ)/aVTZ level will be utilised, which were shown to yield good agreement for the rotamers of the formic acid monomer in Chapter 4. All band positions within this section are scaled to the antisymmetric $\mathrm{C}=\mathrm{O}$ stretching vibration of the formic acid dimer $(\mathrm{FF})_{\mathrm{a}}$ at $1741 \mathrm{~cm}^{-1}$. 

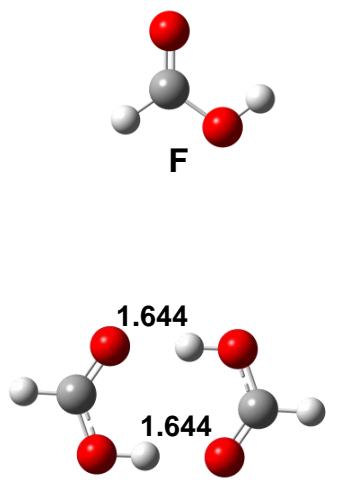

(FF)

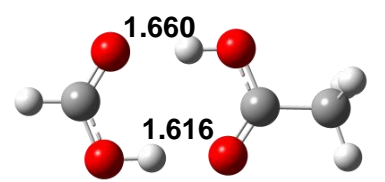

(FA)
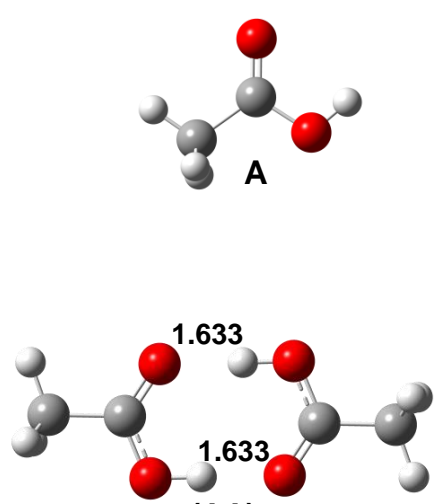

(AA)

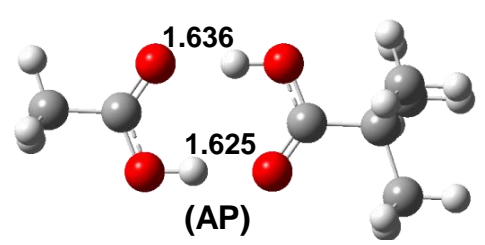

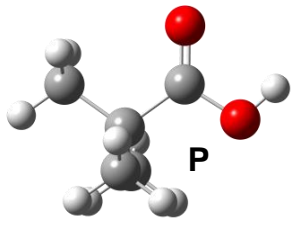
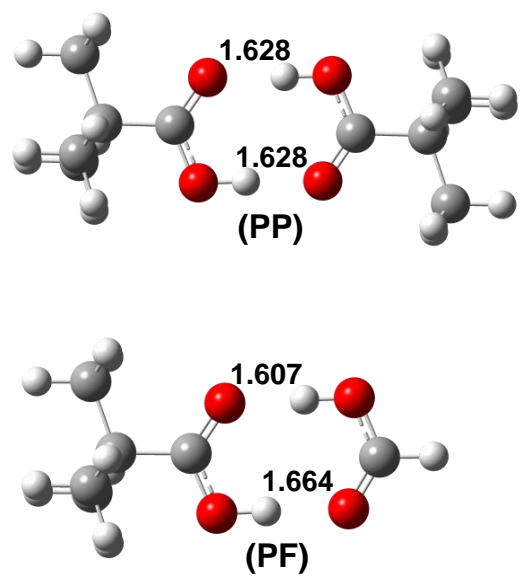

Figure 5.10: Global minimum structures of (homo and hetero) dimers composed of formic $(\mathrm{F})$, acetic $(\mathrm{A})$, and pivalic acid (P), calculated at the B3LYP-D3(BJ)/aVTZ level. Hydrogen bond lengths (in A) are displayed. Adapted from J. Chem. Phys. 149, 104307 (2018) (Ref. [329]), with the permission of AIP Publishing.

\subsubsection{Structures and Dissociation Energies of Homo and Hetero Dimers}

The structures of the formic $(\mathrm{F})$, acetic $(\mathrm{A})$, and pivalic acid $(\mathrm{P})$ monomers (all in transconfiguration) and all possible dimer combinations are depicted in Figure 5.6. The global minimum structure of the homo dimers is the doubly hydrogen bonded cyclic eightmembered ring [70, 117, 379, 400]. As expected, this binding motif remains the most favourable structure for the hetero dimers. The hydrogen bond lengths of the homo dimers decrease with size of the acid by about $1 \mathrm{pm}$, likely due to dispersion interactions of the residues that compress the dimer. Obviously, the two hydrogen bonds of the hetero dimers differ in length. In case of (AP), these differences are very small (about $1 \mathrm{pm}$ ), but can be as large as $6 \mathrm{pm}$ for (PF). (FA) lies in-between with $4 \mathrm{pm}$. Generally, the hydrogen bond where formic acid is the donor is shorter.

The least stable amongst the six homo and hetero dimers is the formic acid dimer with a predicted, zero-point corrected dissociation energy of $66.6 \mathrm{~kJ} \mathrm{~mol}^{-1}$ (Table 5.4). The pivalic acid dimer exhibits the largest dissociation energy of $73.3 \mathrm{~kJ} \mathrm{~mol}^{-1}$. The fact that $D_{0}^{\mathrm{h}}$ increases with the size of the residue implies that a large part of the stability increase is a result of dispersion interactions. And indeed, if the D3 dispersion energy gain [224] listed in Table 5.4 is subtracted from the electronic dissociation energy, an almost uniform binding energy of $65 \mathrm{~kJ} \mathrm{~mol}^{-1}$ is obtained, with formic acid as the only dimer that deviates by more than $1 \mathrm{~kJ} \mathrm{~mol}^{-1}$. 
Table 5.4: Harmonically calculated, zero-point corrected dissociation energies $D_{0}^{\mathrm{h}}\left(\mathrm{in} \mathrm{kJ} \mathrm{mol}{ }^{-1}\right)$ of homo and hetero dimers of formic, acetic, and pivalic acid, calculated at the B3LYPD3(BJ)/aVTZ level. In the second column, averaged homo dimer dissociation energies $\bar{D}_{0}^{\mathrm{h}}$ are shown, as an estimate for the hetero dimer dissociation energies. Further, the energy gain caused by dispersion interactions as well as the resulting binding energy are listed. The binding energy is determined via subtraction of the dispersion energy gain from the electronic dissociation energy $\left(D_{e}^{\mathrm{h}}\right)$. All values are given in $\mathrm{kJ} \mathrm{mol}^{-1}$. Adapted from J. Chem. Phys. 149, 104307 (2018) (Ref. [329]), with the permission of AIP Publishing.

\begin{tabular}{ccccc}
\hline \hline Dimer & $D_{0}^{\mathrm{h}}$ & $\bar{D}_{0}^{\mathrm{h}}$ & dispersion energy gain & binding energy \\
\hline$(\mathrm{FF})$ & 66.6 & & 10.4 & 63.9 \\
$(\mathrm{AA})$ & 71.3 & & 11.2 & 65.6 \\
$(\mathrm{PP})$ & 73.3 & & 13.0 & 65.3 \\
\hline$(\mathrm{FA})$ & 69.3 & 69.0 & 10.8 & 65.2 \\
$(\mathrm{AP})$ & 72.2 & 72.3 & 12.0 & 65.5 \\
$(\mathrm{PF})$ & 70.4 & 70.0 & 11.6 & 65.2 \\
\hline \hline
\end{tabular}

To check for computational additivity, the hetero dimer dissociation energies are predicted from the arithmetic averages of the respective homo dimer values. The agreement of $\bar{D}_{0}^{\mathrm{h}}$ (Table 5.4) with the computed hetero dimer dissociation energies is within $0.5 \mathrm{~kJ} \mathrm{~mol}^{-1}$.

Kollipost and co-workers [191] determined the dissociation energy of the formic acid dimer experimentally by measuring its low frequency modes as well as their combination bands, which significantly improved the accuracy of the rovibrational partition function. That, in combination with the room temperature equilibrium constant of the dissociation, yields a value of $59.5 \pm 0.5 \mathrm{~kJ} \mathrm{~mol}^{-1}$ for $D_{0}$ [191]. The harmonically calculated, zero-point corrected dissociation energy of the formic acid dimer (cf. Table 5.4) deviates by $12 \%$ from the (anharmonic) experimental value. Including anharmonic corrections increases this discrepancy just slightly to $13 \%\left(67.4 \mathrm{~kJ} \mathrm{~mol}^{-1}\right.$ at the B3LYP-D3(BJ)/aVTZ VPT2 level). For the acetic acid dimer, a semiexperimental value of the dissociation energy of $67-70 \mathrm{~kJ} \mathrm{~mol}^{-1}$ [423] also shows that the B3LYP-D3(BJ)/aVTZ estimate is likely too large. Altogether, these deviations clearly illustrate the necessity for experimental data to examine this mixing rule. In the following Section 5.3.2, the determination of experimental $\mathrm{C}=\mathrm{O}$ stretching vibrations of the hetero dimer is presented, followed by a vibrational benchmark in Section 5.3.4.

\subsubsection{FTIR and Raman Jet Spectra of Hetero Dimers}

The FTIR jet spectra of formic and acetic acid in the $\mathrm{C}=\mathrm{O}$ stretching region have been scrutinised in detail in Refs. [68, 71, 117, 118]. Their agreement with the band position determined within this work will be discussed below. The band positions of the $\mathrm{C}=\mathrm{O}$ stretching vibration of the pivalic acid monomer and the antisymmetric $\mathrm{C}=\mathrm{O}$ stretching vibration of the dimer were initially determined within the framework of the diploma 
thesis of C. Emmeluth [116]. FTIR jet spectra measured under similar conditions can be found in Figure 5.11. The monomer band $\mathrm{P}$ can be seen at $1774 \mathrm{~cm}^{-1}$ and the dimer band $(\mathrm{PP})_{\mathrm{a}}$ at $1718 \mathrm{~cm}^{-1}$. Both band positions agree reasonably well with the calculated, $(\mathrm{FF})_{\mathrm{a}}$-scaled band positions at the B3LYP-D3(BJ)/aVTZ level (deviations smaller than $\left.6 \mathrm{~cm}^{-1}\right)$. The band position of the symmetric $\mathrm{C}=\mathrm{O}$ stretching vibration of (PP) is known from a Raman jet study by Xue [68] and exhibits two maxima at $1661 \mathrm{~cm}^{-1}$ and $1658 \mathrm{~cm}^{-1}$.

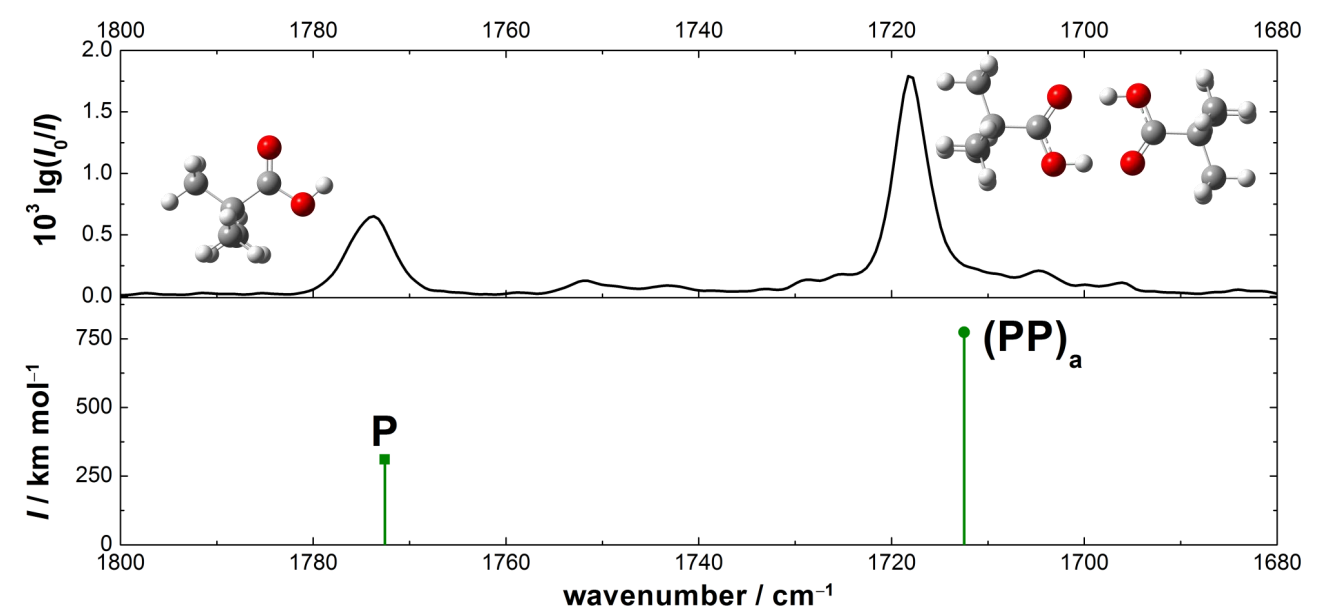

Figure 5.11: FTIR jet spectra of pivalic acid $(0.07 \%)$ in helium at a reservoir pressure of 300 mbar and 155 scans. Below the spectra, harmonically calculated, scaled band positions of the $\mathrm{C}=\mathrm{O}$ stretching vibration of the pivalic acid monomer and the antisymmetric $\mathrm{C}=\mathrm{O}$ stretching vibration of the dimer are shown. The calculations have been performed at the B3LYP-D3(BJ)/aVTZ level. The band positions are scaled to the antisymmetric $\mathrm{C}=\mathrm{O}$ stretching vibration of the formic acid dimer $(\mathrm{FF})_{\mathrm{a}}$. The scaling factor amounts to 0.986 .

FTIR jet spectra of all three possible mixtures of formic (F), acetic (A), and pivalic acid $(\mathrm{P})$ are shown in Figure 5.12 in panels A.I $(\mathrm{F}+\mathrm{A})$, B.I $(\mathrm{A}+\mathrm{P})$, and C.I $(\mathrm{P}+\mathrm{F})$ alongside scaled single substance (dashed lines) and monomer-corrected difference spectra (solid orange lines). In all three spectra of the mixtures, a new band emerges in between the homo dimer bands that persist if scaled single substance spectra are subtracted (cf. orange difference spectra). The band positions of $(\mathrm{FA})_{\mathrm{a}}\left(1736 \mathrm{~cm}^{-1}\right),(\mathrm{AP})_{\mathrm{a}}$ $\left(1726 \mathrm{~cm}^{-1}\right)$, and $(\mathrm{PF})_{\mathrm{a}}\left(1728 \mathrm{~cm}^{-1}\right)$ agree well with the B3LYP-D3(BJ)/aVTZ predictions plotted below the spectra. The deviations are below $6 \mathrm{~cm}^{-1}$.

The largest experimentally found spacing between homo and hetero dimer bands is observed for the (PF) hetero dimer, with differences of $13 \mathrm{~cm}^{-1}$ and $-10 \mathrm{~cm}^{-1}$ to the respective homo dimer bands. In both cases, the scaled B3LYP-D3(BJ)/aVTZ calculations match experiment. The overlap of the homo and hetero dimer bands in the mixed spectra increases for $(\mathrm{AP})_{\mathrm{a}}$, with distances of $-8 \mathrm{~cm}^{-1}$ to $(\mathrm{PP})_{\mathrm{a}}$ and $+6 \mathrm{~cm}^{-1}$ to $(\mathrm{AA})_{\mathrm{a}}$. For $(\mathrm{FA})_{\mathrm{a}}$, these spacings reduce even further down to $+5 \mathrm{~cm}^{-1}$ to $(\mathrm{FF})_{\mathrm{a}}$ and $-4 \mathrm{~cm}^{-1}$ to $(\mathrm{AA})_{\mathrm{a}}$. Nonetheless, the hetero dimer bands are all clearly seen and the separate analysis of difference spectra does not shift the band positions by more than $0.5 \mathrm{~cm}^{-1}$. The band 
positions of the $\mathrm{C}=\mathrm{O}$ stretching vibrations of the three monomers and of all homo and hetero dimers are summarised in Table 5.5 alongside literature values.

Table 5.5: Experimental band positions of the $\mathrm{C}=\mathrm{O}$ stretching vibrations of the formic, acetic, and pivalic acid monomers as well as the symmetric and antisymmetric $\mathrm{C}=\mathrm{O}$ stretching vibrations of their homo and hetero dimers in comparison to literature values (Refs. [68, 71, 116, 118]). The subscript $\mathrm{f}$ stands for free $\mathrm{C}=\mathrm{O}$ stretching vibration (monomer), whereas the subscripts of the dimer vibrations symbolise the symmetry of the vibration (antisymmetric and symmetric). Adapted from J. Chem. Phys. 149, 104307 (2018) (Ref. [329]), with the permission of AIP Publishing.

\begin{tabular}{cccccccc}
\hline \hline monomer & $\tilde{\nu_{\mathrm{f}}}$ & $\tilde{\nu}_{\mathrm{f}}$, literature & dimer & $\tilde{\nu}_{\mathrm{a}}$ & $\tilde{\nu}_{\mathrm{a}}$, literature & $\tilde{\nu}_{\mathrm{s}}$ & $\tilde{\nu}_{\mathrm{s}}$, literature \\
\hline $\mathrm{F}$ & 1777 & $1777^{[118]}$ & $(\mathrm{FF})$ & 1741 & $1741^{[118]}$ & 1666 & $1668^{[68]}$ \\
$\mathrm{A}$ & 1793 & $1793^{[71]}$ & $(\mathrm{AA})$ & 1732 & $1732^{[71]}$ & 1676 & $1680^{[68]}$ \\
$\mathrm{P}$ & 1774 & $1773^{[116]}$ & $(\mathrm{PP})$ & 1718 & $1718^{[116]}$ & 1660 & $1658 / 1661^{[68]}$ \\
\hline & & $(\mathrm{FA})$ & 1736 & & 1671 & \\
& & $(\mathrm{AP})$ & 1726 & & 1667 & \\
& & $(\mathrm{PF})$ & 1728 & & 1657 & \\
\hline \hline
\end{tabular}

Before discussing the corresponding Raman spectra of the three carboxylic acid mixtures, the homo dimer spectra will be briefly addressed. These are shown in Figure 5.13. The symmetric $\mathrm{C}=\mathrm{O}$ stretching bands of $(\mathrm{FF})$ and $(\mathrm{PP})$ exhibit two maxima and the band of the acetic acid dimer $(\mathrm{AA})_{\mathrm{S}}$ is broadened to the lower wavenumber side with several maxima at $1679 \mathrm{~cm}^{-1}, 1675 \mathrm{~cm}^{-1}, 1671 \mathrm{~cm}^{-1}$, and $1668 \mathrm{~cm}^{-1}$. All of this is indicative of Fermi resonances in the symmetric stretches. Such mode coupling has been predicted theoretically for the symmetric $\mathrm{C}=\mathrm{O}$ stretching vibration of the formic acid dimer by $\mathrm{Qu}$ and Bowman [78]. Under the assumption that the dark states have a negligible intrinsic intensity, i.e., all their intensity originates from the bright state, the experimental band positions of the $\mathrm{C}=\mathrm{O}$ stretching vibrations have been deconvoluted by taking the centre of mass of the integrated signal as band position, which yields $1666 \mathrm{~cm}^{-1}$ for $(\mathrm{FF})_{\mathrm{s}}$, $1676 \mathrm{~cm}^{-1}$ for $(\mathrm{AA})_{\mathrm{s}}$, and $1660 \mathrm{~cm}^{-1}$ for $(\mathrm{PP})_{\mathrm{s}}$. Compared to Raman jet spectra of Ref. [68] (cf. Table 5.5), the band position differ by $+2 \mathrm{~cm}^{-1}$ for $(\mathrm{FF})_{\mathrm{s}},+4 \mathrm{~cm}^{-1}$ for $(\mathrm{AA})_{\mathrm{s}}$, and $-2 /+1 \mathrm{~cm}^{-1}$ for $(\mathrm{PP})_{\mathrm{s}}$. Deviations on the order of $1-2 \mathrm{~cm}^{-1}$ are uncritical, as these coincide with the band position accuracy of the Raman jet set-up. The larger mismatch for the acetic acid dimer results from different band position criteria and concentrations chosen. Here, very low concentrations of $0.05 \%$ of acetic acid in helium were used to avoid contributions of larger clusters such as tetramers. These are composed of stacks of dimers, which is why their band positions overlap with those of the dimers [119]. More details on such dimer assemblies can be found in Chapter 7. In Ref. [68], distinctly higher concentrations of $0.7 \%$ were chosen. Additionally, the intensity maximum of the broad band of acetic acid extending from $1688-1662 \mathrm{~cm}^{-1}$ has been set as the band position. Hence, this band position difference is unsurprising.

The Raman jet spectra of the hetero dimers can be found in Figure 5.14. Compared to the antisymmetric $\mathrm{C}=\mathrm{O}$ stretching vibrations (Figure 5.12 ), the bands of the cor- 


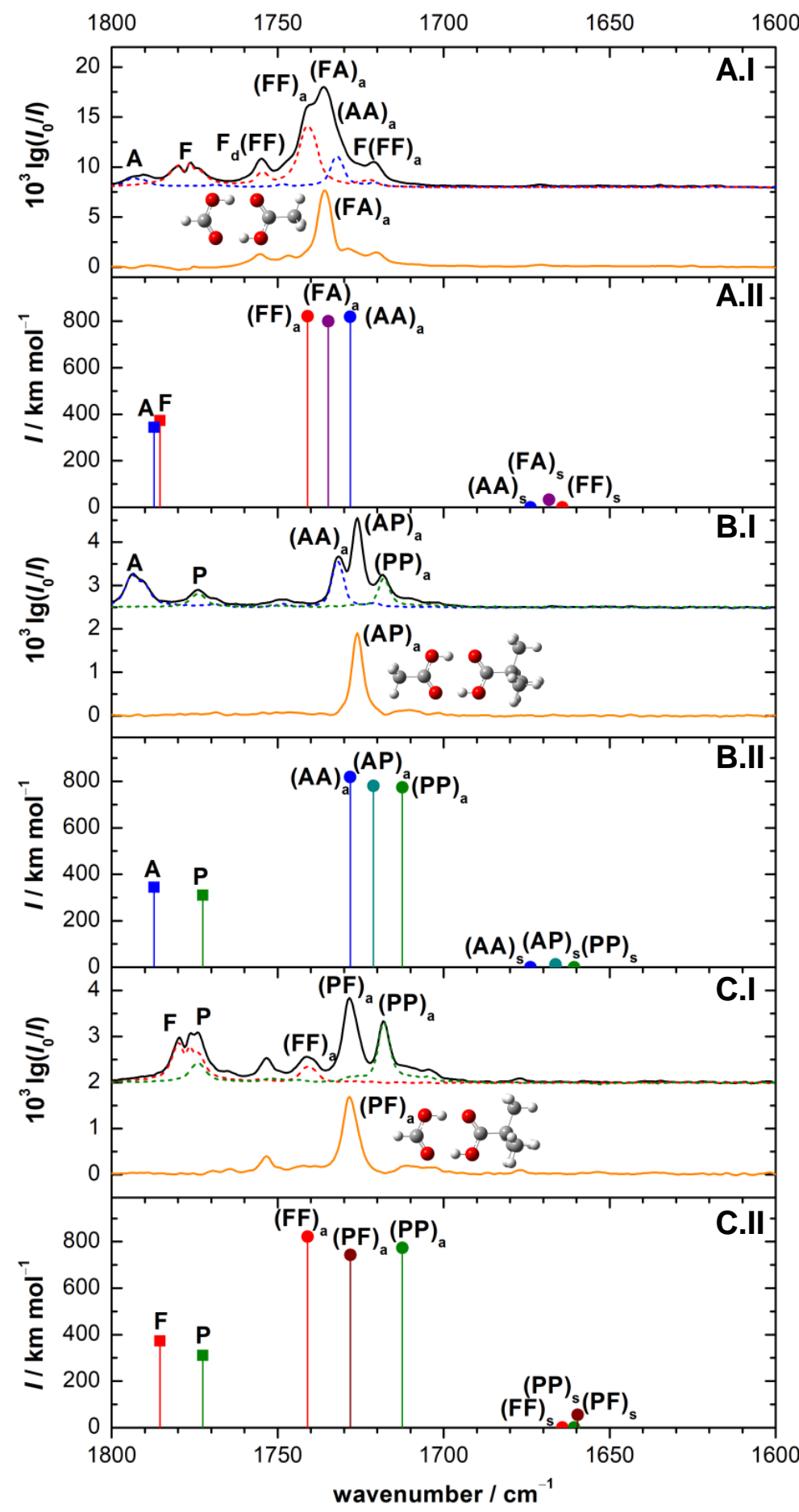

Figure 5.12: FTIR $\quad \mathrm{C}=\mathrm{O}$ stretching jet spectra of formic, $\mathrm{F}$, and acetic acid, $\mathrm{A}$ $\left(c_{\mathrm{F}}, c_{\mathrm{A}}<0.1 \%\right.$, 560 mbar reservoir pressure, A.I), acetic, A, and pivalic acid, $\mathrm{P}\left(c_{\mathrm{A}}, c_{\mathrm{P}}<0.05 \%\right.$, 750 mbar reservoir pressure, B.I), as well as pivalic, $\mathrm{P}$, and formic acid, $\mathrm{F}\left(c_{\mathrm{P}}, c_{\mathrm{F}} \leq 0.05 \%\right.$, 560 mbar reservoir pressure, C.I) in helium based on 50 pulses. Scaled single substance spectra are depicted with dashed lines (red: F, blue: A, green: P) and the corresponding monomercorrected difference spectra are shown in orange. The harmonic B3LYP-D3(BJ)/aVTZ band positions (scaled by 0.986 ) and IR intensities of the respective systems are shown below the spectra (A/B/C.II). F-containing expansions show additional trimer features such as $\mathrm{F}(\mathrm{FF})$, see Ref. [185]. Adapted from J. Chem. Phys. 149, 104307 (2018) (Ref. [329]), with the permission of AIP Publishing. 


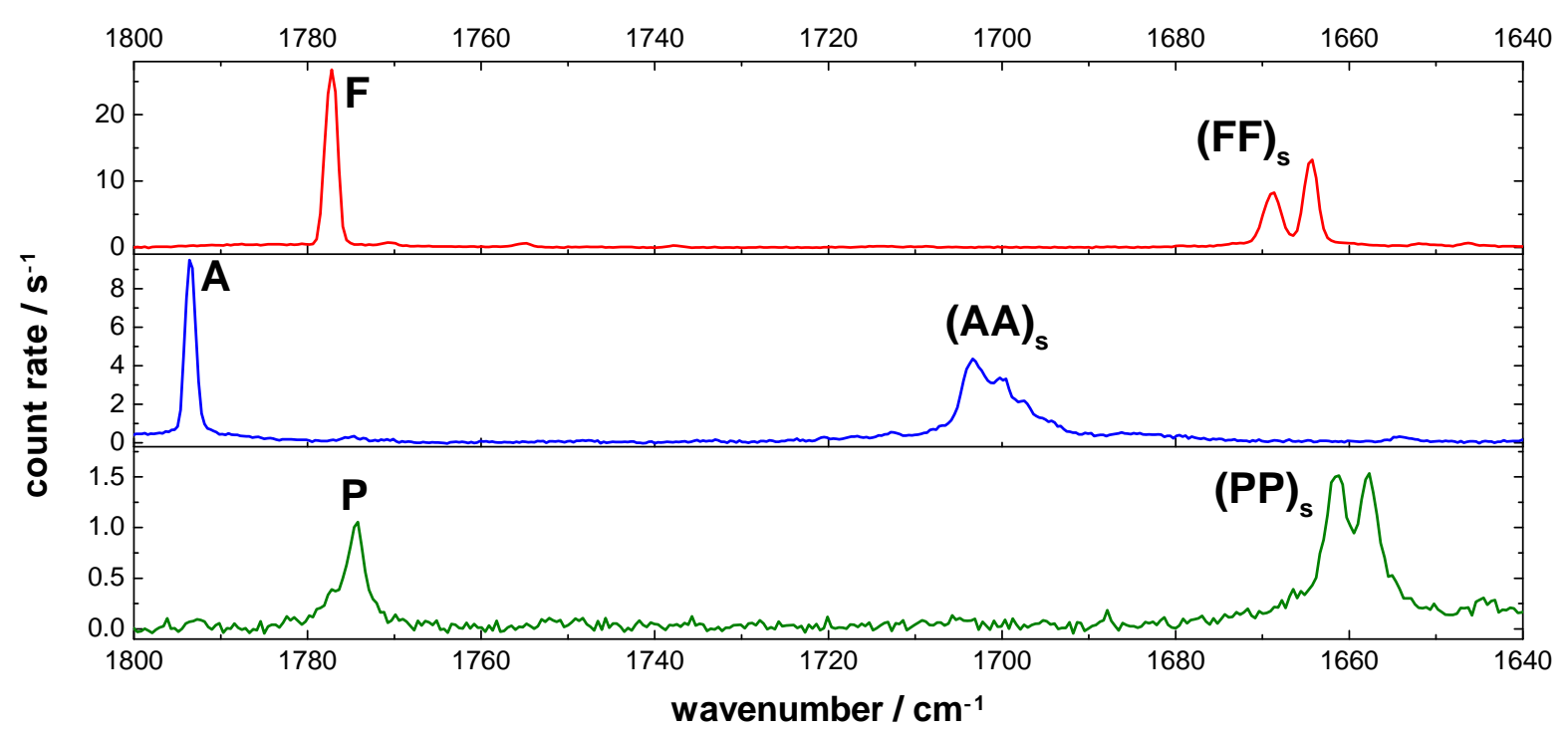

Figure 5.13: Raman jet spectra of formic (top), acetic (middle), and pivalic acid (bottom) recorded in the $\mathrm{C}=\mathrm{O}$ stretching region with concentrations of $0.08 \%$ of formic, $0.05 \%$ of acetic, and $0.04 \%$ of pivalic acid seeded in helium. Reservoir pressures of $700 \mathrm{mbar}$ (formic and acetic acid) and 1000 mbar (pivalic acid) were used. The data aquisition times amount to 30 (acetic acid) and 45 minutes (formic and pivalic acid).

responding symmetric modes are not as well separated. The predicted band position differences amount to $+6 \mathrm{~cm}^{-1}$ and $-4 \mathrm{~cm}^{-1}$ for $(\mathrm{FA})_{\mathrm{s}},+8 \mathrm{~cm}^{-1}$ and $-5 \mathrm{~cm}^{-1}$ for $(\mathrm{AP})_{\mathrm{s}}$, and $+4 \mathrm{~cm}^{-1}$ and $+1 \mathrm{~cm}^{-1}$ for $(\mathrm{PF})_{\mathrm{s}}$ (cf. Figure 5.14, panels A.II/B.II/C.II). Due to the mode coupling observed for the symmetric $\mathrm{C}=\mathrm{O}$ stretching vibrations of the homo dimers, the band position overlap is enhanced. Nonetheless, all three hetero dimer bands are clearly visible in the difference spectra. Their band positions are listed in Table 5.5. The overall agreement with the B3LYP-D3(BJ)/aVTZ calculations is even better. The largest mismatches are as low as $3 \mathrm{~cm}^{-1}$. This is surprising, as the calculated band positions have been scaled to the band of an antisymmetric $\mathrm{C}=\mathrm{O}$ stretching vibration. The Raman difference spectra exhibit a few irregularities such as residuals from homo dimer bands as seen for the formic acid dimer band $(\mathrm{FF})_{\mathrm{s}}$ in Figure 5.14 A.I or negative bands as seen in the same spectrum for the acetic acid dimer band $(\mathrm{AA})_{\mathrm{s}}$. Both are a result from imperfect subtraction of single substance spectra caused by varying monomer-todimer ratios. These, however, are not expected to perturb the band positions of the hetero dimer bands significantly. The band shape in the difference spectra, on the other hand, is affected, as seen for $(\mathrm{PF})_{\mathrm{s}}$, which overlaps with $(\mathrm{PP})_{\mathrm{s}}$ and is therefore broader. Moreover, similarly to the band of the acetic acid dimer, $(\mathrm{AP})_{\mathrm{s}}$ is broadened to one side, which might be a result of Fermi resonances. However, because of the spectral overlap, no exact conclusions are possible. 


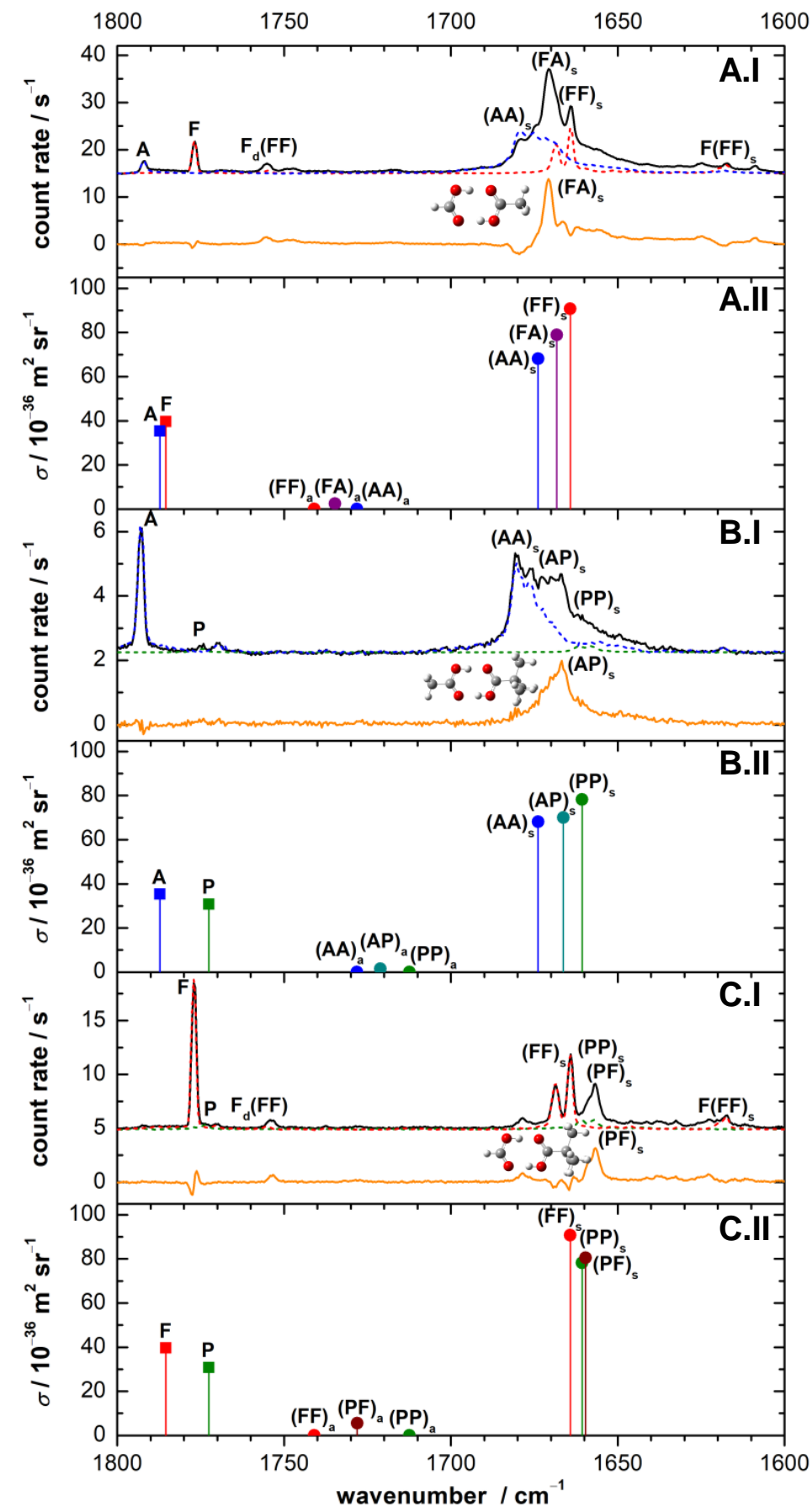

Figure 5.14: Raman $\mathrm{C}=\mathrm{O}$ stretching jet spectra of formic, $\mathrm{F}$, and acetic acid, $\mathrm{A} \quad\left(c_{\mathrm{F}}\right.$, $c_{\mathrm{A}}<0.4 \%, 700 \mathrm{mbar}$ reservoir pressure, A.I), acetic, A, and pivalic acid, $\mathrm{P}\left(c_{\mathrm{A}}, c_{\mathrm{P}}<\right.$ $0.05 \%, 1000$ mbar reservoir pressure, B.I), as well as pivalic, P, and formic acid, $\mathrm{F}\left(c_{\mathrm{P}}, c_{\mathrm{F}}<\right.$ $0.05 \%, 1500$ mbar reservoir pressure, C.I) in helium with recording times of $5 \times 180 \mathrm{~s}$. Scaled single substance spectra are depicted with dashed lines (red: $\mathrm{F}$, blue: A, green: P) and the corresponding monomer-corrected difference spectra are shown in orange. The harmonic B3LYP$\mathrm{D} 3(\mathrm{BJ}) / \mathrm{aVTZ}$ band positions (scaled by 0.986) and IR intensities of the respective systems are shown below the spec$\operatorname{tra}(\mathrm{A} / \mathrm{B} / \mathrm{C} . \mathrm{II})$. F-containing expansions show additional trimer features such as $\mathrm{F}(\mathrm{FF})$, see Ref. [185]. Adapted from J. Chem. Phys. 149, 104307 (2018) (Ref. [329]), with the permission of AIP Publishing. 


\subsubsection{Exciton Shifts and Couplings}

The experimental shifts $\Delta a$ and exciton splittings $\Delta a s$ are listed in Table 5.6 alongside the harmonic, $(\mathrm{FF})_{\mathrm{a}}$-scaled B3LYP-D3(BJ)/aVTZ values. The coupling diagrams are shown in Figure A.17 in the appendix. Compared to the calculated dissociation energies (Table 5.4), $\Delta a$ and $\Delta a s$ span a much wider range and are therefore more suitable for benchmarking purposes. $\Delta a$ varies from $36 \mathrm{~cm}^{-1}$ (formic acid dimer) to $61 \mathrm{~cm}^{-1}$ (acetic acid dimer). It is generally smaller for dimers containing formic acid, whereby (FA) and (PF) exhibit similar shifts of $49 \mathrm{~cm}^{-1}$ and $47 \mathrm{~cm}^{-1}$, respectively. Moreover, the $\Delta a$ values of (PP) and (AP) nearly coincide $\left(56 \mathrm{~cm}^{-1}\right.$ and $\left.57 \mathrm{~cm}^{-1}\right)$. For $\Delta a s$, the trend is reversed - the formic acid dimer has the largest Davydov splitting of $75 \mathrm{~cm}^{-1}$, followed by formic acid containing hetero dimers with $72 \mathrm{~cm}^{-1}(\mathrm{PF})$ and $65 \mathrm{~cm}^{-1}$ (FA). The acetic acid dimer exhibits the smallest $\Delta a s$ value of $56 \mathrm{~cm}^{-1}$. Besides, it is the only dimer where $\Delta a s$ is smaller than $\Delta a$.

If the hetero dimer shifts and Davydov splittings are estimated from the arithmetic average of the homo dimer values, a good agreement can be seen. The deviations are as low as $\pm 1 \mathrm{~cm}^{-1}$ for $\overline{\Delta a}$. For the Davydov splittings $\overline{\Delta a s}$, the largest mismatch amounts to $-5 \mathrm{~cm}^{-1}$ for $(\mathrm{PF})$. This could be due to a misassignment of the band, as the agreement for the other two hetero dimers is superior with $+1 \mathrm{~cm}^{-1}$ for (FA) and $-2 \mathrm{~cm}^{-1}$ for (AP). Nonetheless, the band of the antisymmetric $\mathrm{C}=\mathrm{O}$ stretching vibration of $(\mathrm{PF})$ is well separated from the homo dimer bands in the FTIR spectra (cf. Figure 5.12) and even though the band of the symmetric $\mathrm{C}=\mathrm{O}$ stretching vibration (cf. Raman spectra in Figure 5.14) overlaps with the corresponding band of the pivalic acid dimer, it is distinctly higher in intensity, due to the surplus of formic acid in the expansion. Therefore, the difference spectrum should not be highly affected by this overlap. One

Table 5.6: Experimental $(\Delta a)$, harmonically calculated $\left(\Delta a_{\mathrm{c}}\right)$, and homo dimer interpolated $\left(\overline{\Delta a}, \overline{\Delta a_{\mathrm{c}}}\right)$ downshift of the band of antisymmetric $\mathrm{C}=\mathrm{O}$ stretching vibration of the cyclic dimers compared to the monomer band position as well as experimental $(\Delta a s)$, harmonically calculated $\left(\Delta a s_{\mathrm{c}}\right)$, and homo dimer interpolated $\left(\overline{\Delta a s}, \overline{\Delta a s_{\mathrm{c}}}\right)$ excitonic splitting between the bands of the symmetric and antisymmetric $\mathrm{C}=\mathrm{O}$ stretching vibrations. The calculations have been performed at B3LYP-D3(BJ)/aVTZ level (scaling factor 0.986). Additionally, the downshift of the centre of the Davydov pair is listed in the last two columns. Adapted from J. Chem. Phys. 149, 104307 (2018) (Ref. [329]), with the permission of AIP Publishing.

\begin{tabular}{|c|c|c|c|c|c|c|c|c|c|c|}
\hline dimer & $\Delta a$ & $\overline{\Delta a}$ & $\Delta a_{\mathrm{c}}$ & $\overline{\Delta a_{\mathrm{c}}}$ & $\Delta a s$ & $\overline{\Delta a s}$ & $\Delta a s_{\mathrm{c}}$ & $\overline{\Delta a s_{\mathrm{c}}}$ & $\begin{array}{c}\Delta a+ \\
0.5 \Delta a s\end{array}$ & $\begin{array}{c}\Delta a_{\mathrm{c}}+ \\
0.5 \Delta a s_{\mathrm{c}}\end{array}$ \\
\hline$(\mathrm{FF})$ & 36 & & 44 & & 75 & & 77 & & 74 & 83 \\
\hline (AA) & 61 & & 59 & & 56 & & 54 & & 89 & 86 \\
\hline$(\mathrm{PP})$ & 56 & & 60 & & 58 & & 52 & & 85 & 86 \\
\hline$(\mathrm{FA})$ & 49 & 48 & 52 & 52 & 65 & 66 & 66 & 66 & 82 & 85 \\
\hline$(\mathrm{AP})$ & 57 & 58 & 59 & 60 & 59 & 57 & 55 & 53 & 87 & 87 \\
\hline$(\mathrm{PF})$ & 47 & 46 & 51 & 52 & 72 & 67 & 68 & 64 & 83 & 85 \\
\hline
\end{tabular}




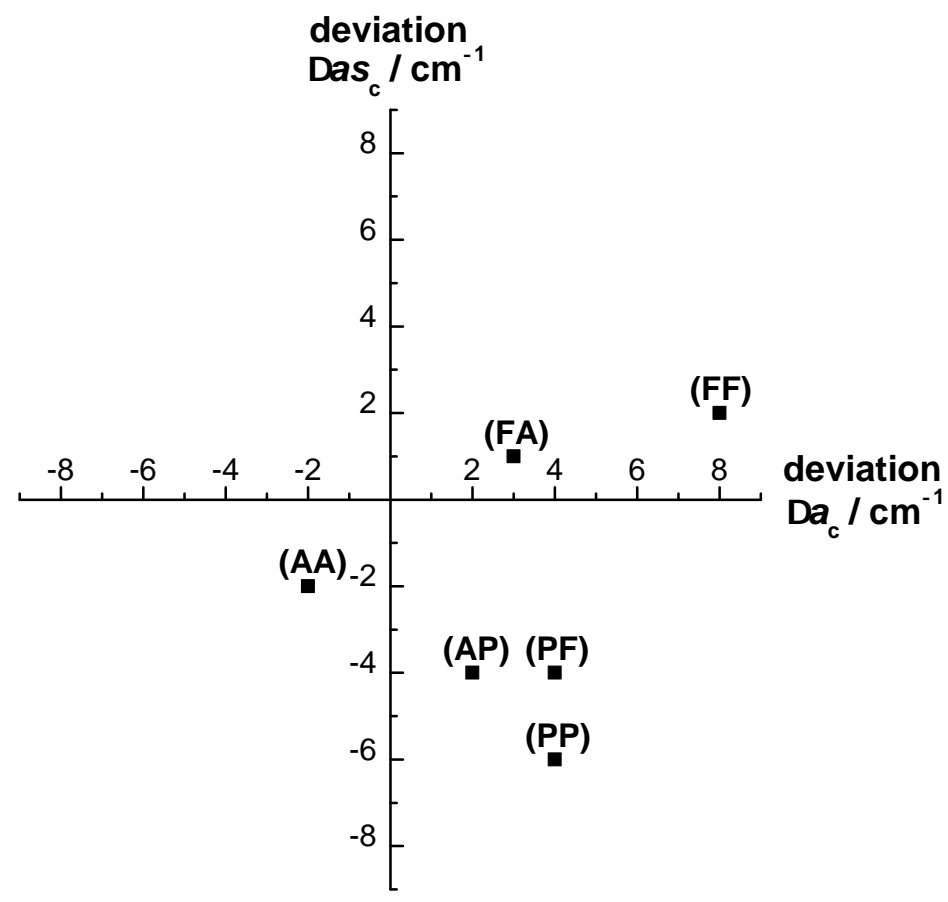

Figure 5.15: Deviations of harmonically calculated, $(\mathrm{FF})_{\mathrm{a}}$-scaled shifts $\Delta a_{\mathrm{c}}$ and Davydov splittings $\Delta a s_{\mathrm{c}}$ of the six homo and hetero dimers composed of formic, acetic, and pivalic acid from experiment. The calculations have been performed at the B3LYP-D3(BJ)/aVTZ level.

way to assess whether this mismatch is due to an experimental misassignment or simply the uncertainty of this mixing rule, is to apply it to the calculated shifts and exciton couplings. But initially, their general agreement with experiment will be compared.

In Figure 5.15, the deviations of the harmonically calculated shifts $\Delta a_{\mathrm{c}}$ and splittings $\Delta a s_{\mathrm{c}}$ are visualised with respect to (the anharmonic) experiment. Apart from $\Delta a_{\mathrm{c}}$ of acetic acid, the shifts $\Delta a_{\mathrm{c}}$ are overestimated. The largest discrepancy is observed for the formic acid dimer with $8 \mathrm{~cm}^{-1}$. All other differences between theory and experiment are below $5 \mathrm{~cm}^{-1}$. As seen in the previous section and chapter, the band position of the $\mathrm{C}=\mathrm{O}$ stretching vibration of the formic acid monomer is overestimated by B3LYP$\mathrm{D} 3(\mathrm{BJ})$, which is the main contributor to this mismatch. The exciton splittings $\Delta a s$ tend to be underestimated. The largest difference is seen for the pivalic acid dimer with $-6 \mathrm{~cm}^{-1}$. The other deviations are below $5 \mathrm{~cm}^{-1}$. If the calculated homo dimer shifts and exciton couplings are averaged, the corresponding $\overline{\Delta a_{\mathrm{c}}}$ and $\overline{\Delta a s_{\mathrm{c}}}$ values are as accurate as their $\overline{\Delta a}$ and $\overline{\Delta a s}$ counterparts. This also applies to the mismatch of $\Delta a s_{\mathrm{c}}$ and $\overline{\Delta a s_{\mathrm{c}}}$ of $(\mathrm{PF})$, which is nearly identical to the one observed for the experimental values (cf. Table 5.6). This suggests that this error is inherent in this additivity rule rather than an experimental assignment issue. In total, it amounts to solely $7 \%$. The overall good agreement of the harmonic, $(\mathrm{FF})_{\mathrm{a}}$-scaled B3LYP-D3(BJ)/aVTZ calculations with the anharmonic experiment as well as the reasonably good accuracy of the estimated hetero dimer shifts $\overline{\Delta a} / \overline{\Delta a_{\mathrm{c}}}$ and couplings $\overline{\Delta a s} / \overline{\Delta a s_{\mathrm{c}}}$ could indicate that the net anharmonic contributions are indeed small. Besides, the most evenly evolving quantity is the sum of 
the shift and half of the exciton splitting $\Delta a+0.5 \Delta a s$, i.e., the averaged dimer downshift with respect to the monomer. The experimental and calculated values are shown in Table 5.6. Experimentally, these range from $82-89 \mathrm{~cm}^{-1}$. The only exception is the formic acid dimer, whose $\Delta a+0.5 \Delta a s$ value is considerably lower $\left(74 \mathrm{~cm}^{-1}\right)$. This outlier cannot be reproduced by the calculations which might be attributed to the deficiency of B3LYP-D3(BJ)/aVTZ to predict the monomer band position correctly. This generally small range of the values again illustrates that the cohesion of the monomer units within the dimer is not largely affected by the substituents, as seen for the binding energies in Table 5.4 .

\subsubsection{Vibrational Benchmark}

Up until this point, the experimental shifts $\Delta a$ and exciton splittings $\Delta a s$ have been exclusively compared to harmonic, $(\mathrm{FF})_{\mathrm{a}}$-scaled B3LYP-D3(BJ)/aVTZ calculations, which seem to perform fairly well for these values. To test whether this also applies to other methods, additional harmonic frequency calculations have been carried out at the same levels of theory as for the formic acid monomer in Chapter 4, namely B2PLYP-D3(BJ), MP2, PBE0-D3, M06-2X, $\omega$ B97-XD, and PM3, all as implemented in Gaussian 09 [36]. Two further methods were tested with the ORCA program package (version 4.0.1) [37, 38]: Spin-component-scaled (SCS)-MP2 using the resolution of identity (RI) approximation and the PBEh-3c functional. Both have been used for the prediction of higherenergy formic acid dimers in the previous section. The basis set for all calculations is an augmented triple-zeta basis set (aVTZ). All harmonic frequency calculations have been scaled to the antisymmetric $\mathrm{C}=\mathrm{O}$ stretching vibration of the formic acid dimer $(\mathrm{FF})_{\mathrm{a}}$. Furthermore, exploratory anharmonic frequency calculations (VPT2) were performed for the formic acid dimer, to assess the overall impact of anharmonicity. For the larger acids such as $\mathrm{A}$ and $\mathrm{P}$, these are not worthwhile, as the nearly unhindered rotation of the methyl and tert-butyl groups is poorly described by VPT2 [79]. The results will be briefly discussed after the harmonic benchmark.

Rather than looking at the individual results for all dimers as done for the B3LYPD3(BJ)/aVTZ calculations in Figure 5.15, the individual deviations of each method are averaged, so that a mean deviation $\bar{D}$ for $\Delta a$ and $\Delta a s$ is obtained. For MP2, SCSMP2, and B2PLYP-D3(BJ), this has only been done for five of the six dimers, as (PP) is computationally too demanding. The results are plotted in Figure 5.16. The mean absolute deviation of $\bar{D}$ amounts to $(3 \pm 1) \mathrm{cm}^{-1}$ for all methods and is displayed as error bars. The experimental uncertainty of the shifts $\Delta a$ amounts to $\pm 4 \mathrm{~cm}^{-1}$ (twice the band position error of the Raman set-up discussed in the previous chapter). Since the band positions of symmetric $\mathrm{C}=\mathrm{O}$ stretching vibrations of the homo dimers were deconvoluted due to Fermi resonances, it is slightly larger for $\Delta a s$. As a conservative estimate, $\Delta(\Delta a s)$ is assumed to be $\pm 6 \mathrm{~cm}^{-1}$.

Apart from PM3, all methods predict the anharmonic experimental $\Delta a$ and $\Delta a s$ values on average with an accuracy of $\pm 20 \mathrm{~cm}^{-1}$, most even within $\pm 10 \mathrm{~cm}^{-1}$. Generally, underestimation dominates. While this particularly severe deviation of PM3 is expected, the large underestimation of SCS-MP2/aVTZ is somewhat more surprising, as 
this method has performed reasonably well in a study of formic and acetic acid clusters with nitrogen [79]. For the metastable formic acid dimer, the downshift of the bound $\mathrm{OH}$ stretching vibration with respect to the monomer was also underestimated within the double harmonic approximation with SCS-MP2 (cf. Figure 5.5). The MP2/aVTZ predictions are slightly better, yet not entirely satisfactory considering the computational effort. M06-2X/aVTZ and PBE0-D3(BJ)/aVTZ are the only methods that overestimate both the shift and the splitting. As seen for the VPT2 benchmark of the formic acid monomer (cf. Section 4.4), M06-2X as well as wB97-XD calculations are highly symmetry and integration grid dependent. Obviously, this will also affect the harmonic frequency calculations, yet the deviations are more than an order of magnitude smaller and for all vibrations below the experimental error intervals. B3LYP-D3(BJ)/aVTZ tends to predict $\Delta a$ on average too high and underestimates $\Delta a s$, yet the error is down to $\pm 5 \mathrm{~cm}^{-1}$. PBEh-3c performs similarly well. This fairly good agreement of scaled, harmonic frequency calculations at the B3LYP-D3(BJ)/aVTZ level with the (anharmonic) experimental band positions is seen in almost all chapters of this thesis, where these are used to support assignments. At the PBEh-3c level, it was also seen for the polar dimer

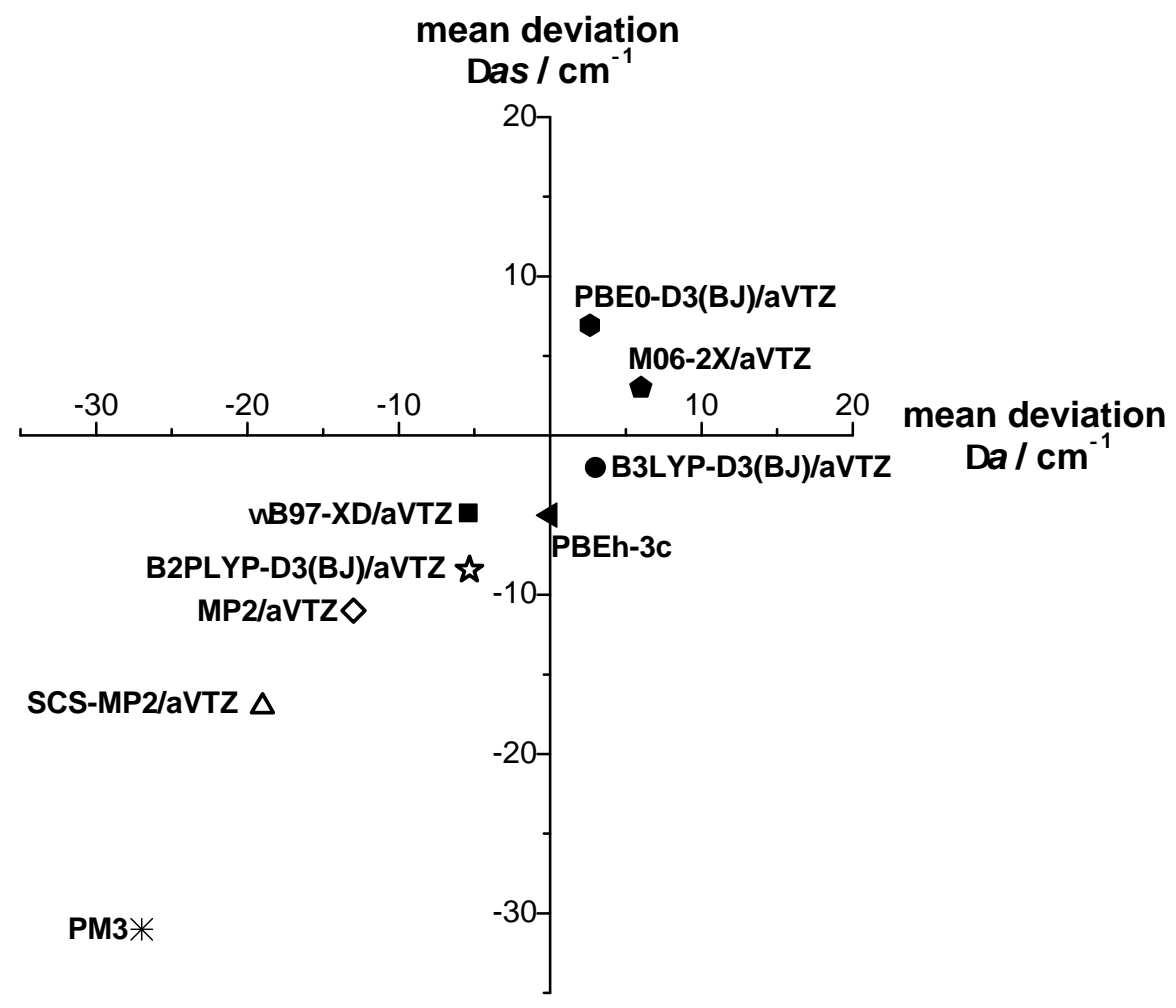

Figure 5.16: Mean deviation $(\bar{D})$ of calculated and $(\mathrm{FF})_{\mathrm{a}}$-scaled Davydov shifts $\Delta a$ and splittings $\Delta a s$, averaged over all six homo and hetero dimers of formic, acetic, and pivalic acid, with respect to experiment. The different levels of theory are displayed in the Figure. For SCS-MP2, MP2, and the B2PLYP-D3(BJ), only five of the six dimer values were averaged, as the pivalic acid dimer is computationally too costly. The experimental errors amount to $\pm 4 \mathrm{~cm}^{-1}$ for the shift $\Delta a$ and $\pm 6 \mathrm{~cm}^{-1}$ for the Davydov splitting $\Delta a s$. 


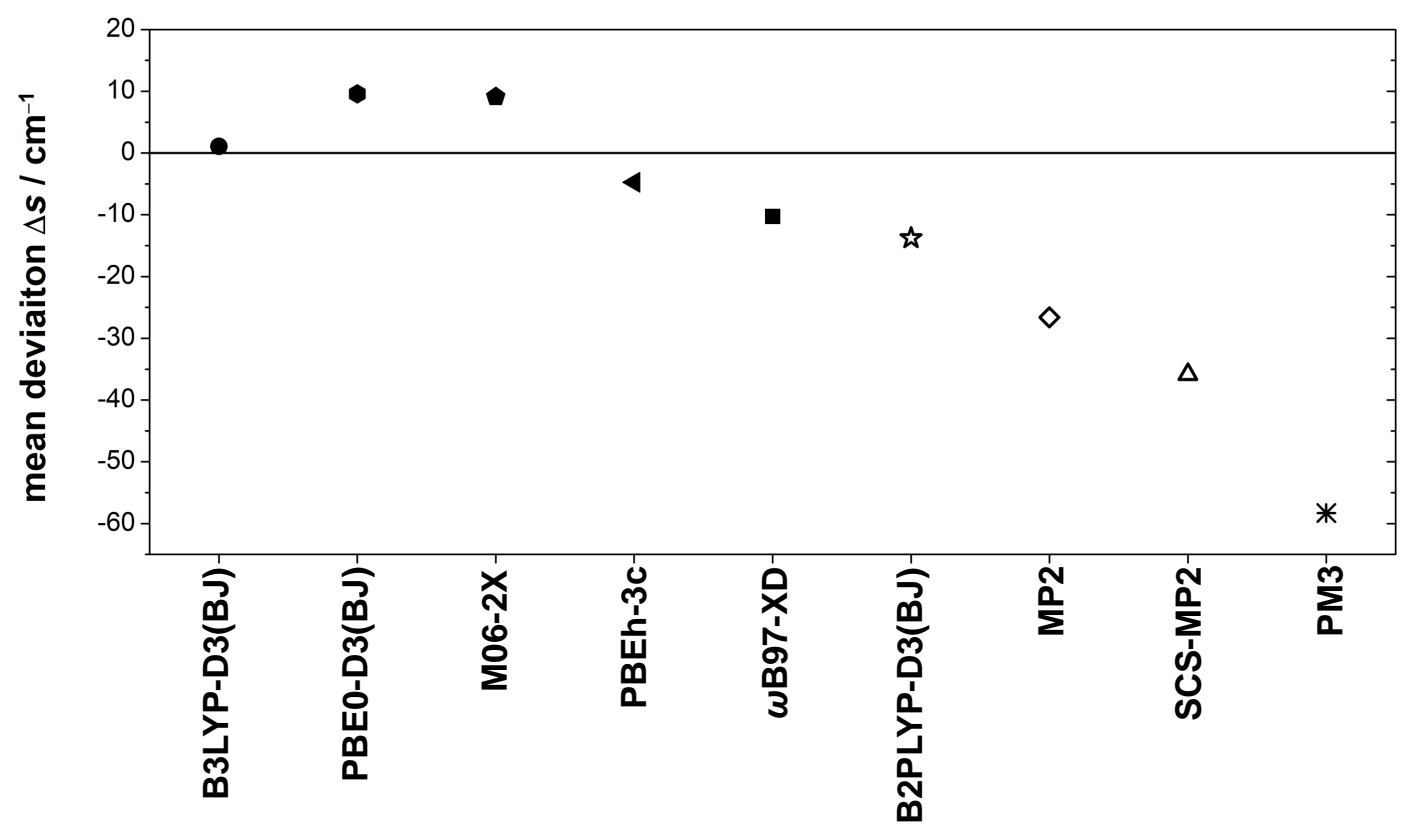

Figure 5.17: Mean deviation $(\bar{D})$ of calculated and $(\mathrm{FF})_{\mathrm{a}}$-scaled shifts of the symmetric $\mathrm{C}=\mathrm{O}$ stretching bands of carboxylic acid dimers with respect to the monomer $\Delta s$, averaged over all six homo and hetero dimers of formic, acetic, and pivalic acid, with respect to experiment. For SCS-MP2, MP2, and the B2PLYP-D3(BJ), only five of the six dimer values were averaged, as the pivalic acid dimer is computationally too costly. The experimental $\Delta s$ error amount to $\pm 6 \mathrm{~cm}^{-1}$.

$\mathrm{F}_{\mathrm{C}} \mathrm{F}$ in the previous section. Clearly, such good agreements are mostly based on error compensation. Altogether, this comparison of scaled harmonic frequency calculations with the (anharmonic) experimental $\Delta a$ and $\Delta a s$ values illustrates that these can be very valuable tools for relative band position assignments such as hydrogen bond induced downshifts or splittings between coupled modes such as the two $\mathrm{C}=\mathrm{O}$ oscillators, where anharmonic corrections tend to largely cancel [225].

As seen in Figure 5.9, the shift of the symmetric $\mathrm{C}=\mathrm{O}$ stretching vibration of the dimer with respect to the monomer $\Delta s$ corresponds to the sum of the exciton splitting $\Delta a s$ and the shift of the antisymmetric $\mathrm{C}=\mathrm{O}$ stretching band relative to the monomer $\Delta a$. As such, $\Delta s$ can serve as a valuable performance test, as the individual deviations of $\Delta a s$ and $\Delta a$ are either magnified or somewhat compensate each other. The averaged $\Delta s$ values of all methods are plotted in Figure 5.17. Expectedly, B3LYP-D3(BJ) is the best method for the determination of $\Delta s$, as a result of the error compensation of the slightly overestimated $\Delta a$ and underestimated $\Delta a s$ values. All other methods apart from PBEh-3c perform particularly poorly for $\Delta s$. Overall, it can therefore serve as a convenient performance assessment of the methods.

The large underestimation of $\Delta s$ by most methods is probably a result of the neglect of anharmonicity. It is likely that the dimer is more anharmonic than the monomer. Hence, the harmonic shifts $\Delta a$ will be somewhat too small. Additionally, the anharmonic cor- 
rection of the symmetric $\mathrm{C}=\mathrm{O}$ stretching vibration might be slightly larger, as indicated by the resonance pattern observed for the homo dimer bands in the Raman spectra (see Figure 5.13). Thus, the Davydov splittings $\Delta a s$ will also likely increase upon including anharmonicity. Overall, the impact of both effects is most pronounced for the $\Delta s$ values, which is why these are the most sensitive performance measure. To examine this in more detail, exploratory VPT2 calculations at the B3LYP-D3(BJ)/aVTZ, B2PLYP$\mathrm{D} 3(\mathrm{BJ}) / \mathrm{aVTZ}$, and at the MP2/aVTZ level have been performed for the formic acid dimer. As VPT2 calculations utilising DFT methods depend on the DFT integration grid size, symmetry, and optimisation criteria chosen (cf. Chapters 4.4.1 and 5.2.2), these were varied. This leads to eight anharmonic frequency calculations for B3LYP-D3(BJ) and B2PLYP-D3(BJ), and four for MP2 (no integration grid). The averaged fundamentals and their standard deviation are listed in Table A.9 in the appendix, alongside the respective harmonic band positions. Here, the focus is on the $\mathrm{C}=\mathrm{O}$ stretching vibrations $\nu_{\mathrm{C}=\mathrm{O}, \mathrm{s}}$ and $\nu_{\mathrm{C}=\mathrm{O}}$, a , which are summarised in Table 5.7 in addition to that of the formic acid monomer. All standard deviation below $0.1 \mathrm{~cm}^{-1}$ are omitted. This is seen for most harmonic frequency results.

At the B3LYP-D3(BJ)/aVTZ level, the standard deviations $\sigma$ of the band positions are largest, meaning that these results are most sensitive to the settings used. The largest $\sigma$ values are seen for the two $\mathrm{O}-\mathrm{H}$ stretching vibrations with $56 \mathrm{~cm}^{-1}$ and $17 \mathrm{~cm}^{-1}$ (cf. Table A.9). The B2PLYP-D3(BJ) standard deviations of the $\mathrm{C}=\mathrm{O}$ stretching vibrations are very similar to those at the B3LYP-D3(BJ) level, which holds true for most other modes. The MP2/aVTZ calculations, on the other hand, show barely any deviations with regard to the settings, as expected from Chapter 4.4. The most sensitive vibration is the lowest frequency mode, which is predicted to $(70 \pm 4) \mathrm{cm}^{-1}$. Generally, VPT2 is known to perform poorly for such intermolecular vibrations. However, this deviation is still moderate. In comparison, for B3LYP-D3(BJ)/aVTZ VPT2, the error is considerably larger $\left((71 \pm 14) \mathrm{cm}^{-1}\right)$.

As seen in Table 5.7, the predicted anharmonicity of the $\mathrm{C}=\mathrm{O}$ stretching vibration of the formic acid monomer is near-identical for all methods with $(-33 \pm 1) \mathrm{cm}^{-1}$ (B3LYPD3(BJ) and MP2) and $(-34 \pm 1) \mathrm{cm}^{-1}$ (B2PLYP-D3(BJ)). As expected, the anharmonic corrections of the dimer vibrations exceed those of the monomer and the anharmonicity

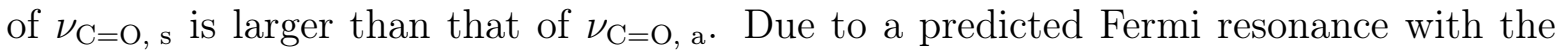
combination band of the $\mathrm{H}-\mathrm{O}-\mathrm{C}$ in plane bending vibration $\nu_{4}$ and the inter-monomer stretching vibration $\nu_{8}{ }^{1}$, the standard deviation of $\nu_{\mathrm{C}=\mathrm{O}, \mathrm{s}}$ is larger than that of $\nu_{\mathrm{C}=\mathrm{O}}$, a for B3LYP-D3(BJ) and B2PLYP-D3(BJ) (see Table 5.7). Altogether, both $\Delta a$ and $\Delta a s$ increase by including VPT2 corrections. To visualise the impact, the anharmonic $\Delta a$ and $\Delta a s$ values are plotted with their standard deviations as error bars in Figure 5.18. The respective harmonic values are shown as a reference with unfilled symbols. As before, the area of experimental agreement is illustrated as a green ellipsis. The best agreement of the harmonic $\Delta a$ and $\Delta a s$ results is exhibited by B2PLYP-D3(BJ), which agrees within the experimental uncertainty with both. Anharmonically, B2PLYP-D3(BJ)/aVTZ also

\footnotetext{
${ }^{1}$ The formic acid dimer vibrations are labelled according to the Herzberg nomenclature. Further details on the dimer nomenclature can be found in Appendix A.3.4.
} 
Table 5.7: Harmonically (harm) and anharmonically (anharm, VPT2) calculated band positions of the $\mathrm{C}=\mathrm{O}$ stretching vibrations of the formic acid monomer and dimer (symmetric $\nu_{\mathrm{C}=\mathrm{O}, \mathrm{s}}$ and antisymmetric $\nu_{\mathrm{C}=\mathrm{O} \text {, a }}$ ) at the B3LYP-D3(BJ)/aVTZ, B2PLYP-D3(BJ)/aVTZ, and MP2/aVTZ level. Additionally, $(\mathrm{FF})_{\mathrm{a}}$-scaled harmonic frequency calculations are shown (sc harm). The scaling factors amount to 0.986 (B3LYP-D3(BJ)), 0.990 (B2PLYP-D3(BJ)), and 0.983 (MP2). For all methods, the optimisation criteria (tight and very tight) and the symmetry $\left(C_{1}\right.$ and $\left.C_{2 \mathrm{~h}}\right)$ were varied and in case of B3LYP-D3(BJ) and B2PLYP-D3(BJ), additionally the DFT integration grid size (ultra fine and super fine). The averaged results are listed with their standard deviations $\sigma$, if these are $\geq 0.1$.

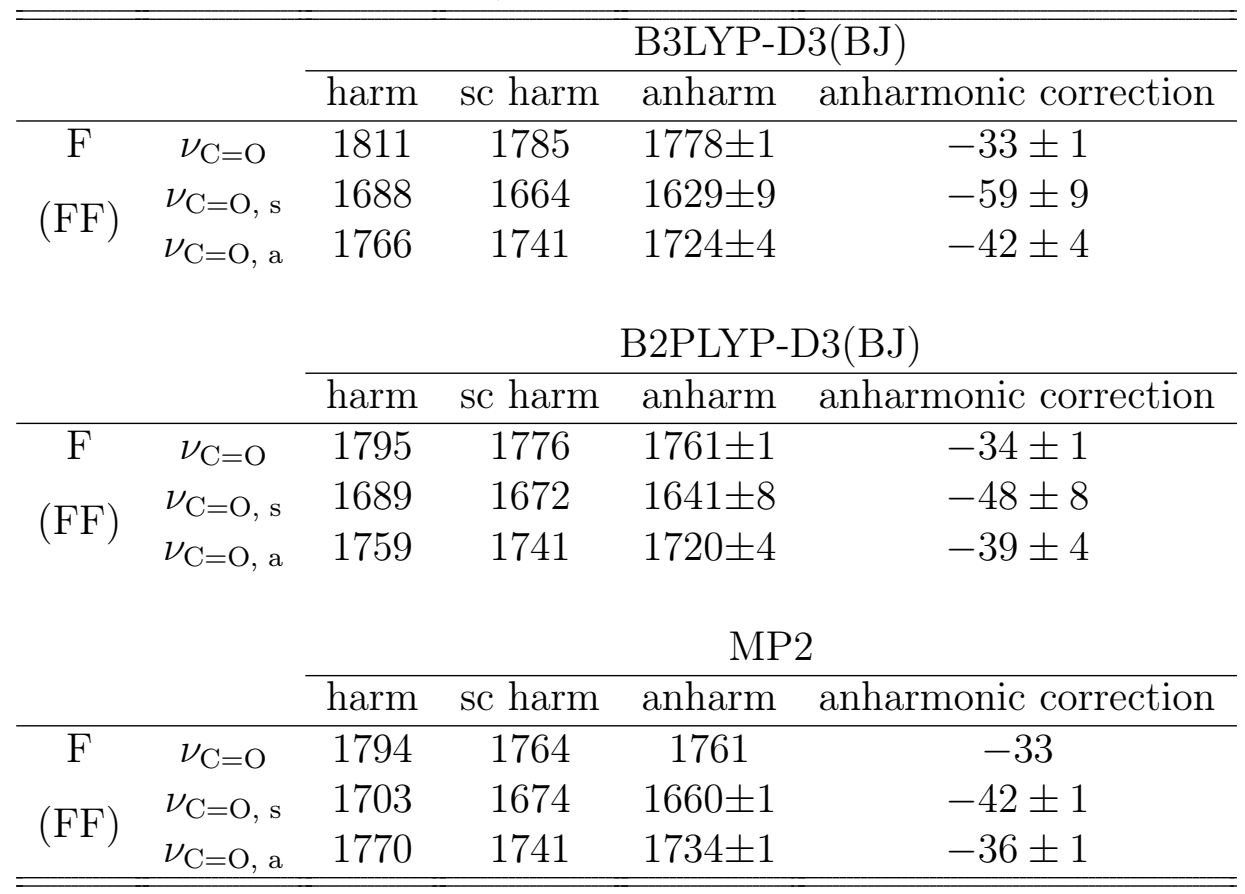

performs reasonably well, the MP2/aVTZ results move towards experiment, and B3LYPD3(BJ)/aVTZ overshoots massively. However, the uncertainty of the B3LYP-D3(BJ) and B2PLYP-D3(BJ) VPT2 calculations is also fairly high. Clearly, from these calculations hardly any predictions can be made about the extent of these effects - not only because these may vary for the different acids, but most importantly, because of the large DFT integration grid, symmetry, as well as optimisation sensitivity. Further details on the extent of these effects for the different vibrations of the formic acid monomer and dimer can be found in Appendix A.3.4. Altogether, high level ab initio anharmonic band positions of all three carboxylic acid dimers are crucially needed for a thorough characterisation.

Miliordos and Xantheas have calculated the fundamentals of $\mathrm{F}$ and (FF) harmonically with MP2/aVDZ and CCSD(T)/aVQZ and applied an MP2/aVDT VPT2 correction to both [396]. Their results are shown in red in Figure 5.18. With increasing basis set size, the agreement of the MP2 calculations improve, as expected. With the MP2 methodology, the extent of anharmonic correction is significantly smaller than that of 
B3LYP-D3(BJ). If applied to the harmonic CCSD(T)/aVQZ $\Delta a s$ and $\Delta a$ values, the agreement with experiment is slightly improved. The shift is met within the experimental uncertainty, with and without the anharmonic correction and $\Delta a s$ is slightly underestimated. The main reason for this mismatch is the band position of the symmetric $\mathrm{C}=\mathrm{O}$ stretching vibration, which is overestimated. $(\mathrm{FF})_{\mathrm{a}}$ is accurate to $1 \mathrm{~cm}^{-1}$. Kalescky, Kraka, and Cremer have calculated harmonic vibrational frequencies for $\mathrm{F}$ and $(\mathrm{FF})$ at the $\operatorname{CCSD}(\mathrm{T}) / \mathrm{aVDZ}$ and $\operatorname{CCSD}(\mathrm{T}) / \mathrm{aVTZ}$ level and performed a complete basis set (CBS) extrapolation [394]. The harmonic CCSD(T)/CBS values match experiment. Additionally, they have added a B3LYP/aVTZ VPT2 correction [394]. As the predicted anharmonicity of the dimer vibrations is identical, only $\Delta a$ is shifted by implementing it (see Figure 5.18). Similar to the B3LYP-D3(BJ)/aVTZ VPT2 results (cf. Table A.11), the anharmonic correction $\left(+9 \mathrm{~cm}^{-1}\right)$ is significantly larger than for MP2/aVDZ $\left(+4 \mathrm{~cm}^{-1}\right.$ for $\left.\Delta a\right)$ or MP2/aVTZ $\left(+4 \pm 1 \mathrm{~cm}^{-1}\right.$ for $\left.\Delta a\right)$, but overall still moderate.

As seen before, the downshift of the symmetric $\mathrm{C}=\mathrm{O}$ stretching band with respect to the monomer $\Delta s$ can be a helpful, overall performance measure, as it corresponds to the sum of $\Delta a$ and $\Delta a s$, which either results in magnification or, to some extent, cancellation of the deviations with respect to experiment. The $\Delta s$ predictions are plotted in Figure 5.19. Agreement with the experimental $\Delta s$ value of $(111 \pm 6) \mathrm{cm}^{-1}$ is achieved in two cases - for the B3LYP/aVTZ VPT2 corrected CCSD(T)/CBS value of Kalescky, Kraka, and Cremer [394], where also the harmonic value matches experiment, and with B2PLYP-D3(BJ)/aVTZ VPT2. In case of the former [394], $\Delta a s$ and $\Delta s$ seem to be particularly well described (cf. Figures 5.18 and 5.19). However, as the band positions of the monomer $\left(+25 \mathrm{~cm}^{-1}\right)$ and dimer $\mathrm{C}=\mathrm{O}$ stretches (antisymmetric: $+16 \mathrm{~cm}^{-1}$, symmetric: $+22 \mathrm{~cm}^{-1}$ ) are massively overestimated [394], this good agreement is likely somewhat fortuitous. As a result of the correlation of the $\Delta a$ and $\Delta a s$ deviations, the B3LYP-D3(BJ)/aVTZ VPT2 prediction overshoots the experimental value massively, whereas the MP2/aVDT VPT2 $\Delta s$ by Miliordos and Xantheas [396] exhibits the largest underestimation.

Two other groups have published anharmonic vibrational frequency calculations of the formic acid dimer, namely $\mathrm{Qu}$ and Bowman [78, 81] and Pitsevich and co-workers [395]. As the latter have performed these with B3LYP/aVTZ VPT2, these will not be discussed in detail. $\mathrm{Qu}$ and Bowman published a full-dimensional ab initio potential energy surface of the formic acid dimer in 2016 based on 13475 CCSD(T)-F12a/haTZ ${ }^{2}$ energies [78]. From their harmonic predictions of the $\mathrm{C}=\mathrm{O}$ stretching vibrations, a $\Delta$ as value of $65 \mathrm{~cm}^{-1}$ is obtained. In 2019, a full set of anharmonic VSCF and VCI band positions of the fundamentals of $(\mathrm{FF})$ was published [81]. The VCI $\Delta a s$ value overshoots the experimental value by $15 \mathrm{~cm}^{-1}\left(90 \mathrm{~cm}^{-1}\right)$, whereby the VSCF value agrees within the experimental uncertainty $\left(70 \mathrm{~cm}^{-1}\right)$, which is likely coincidental. All in all, these examples emphasise the shortcomings of current anharmonic vibrational frequency calculations of the formic acid dimer and highlight the need for further progress on this simple model system.

\footnotetext{
${ }^{2}$ haTZ refers to a mixed basis set where VTZ is used for all hydrogen atoms and aVTZ for carbon and oxygen atoms.
} 


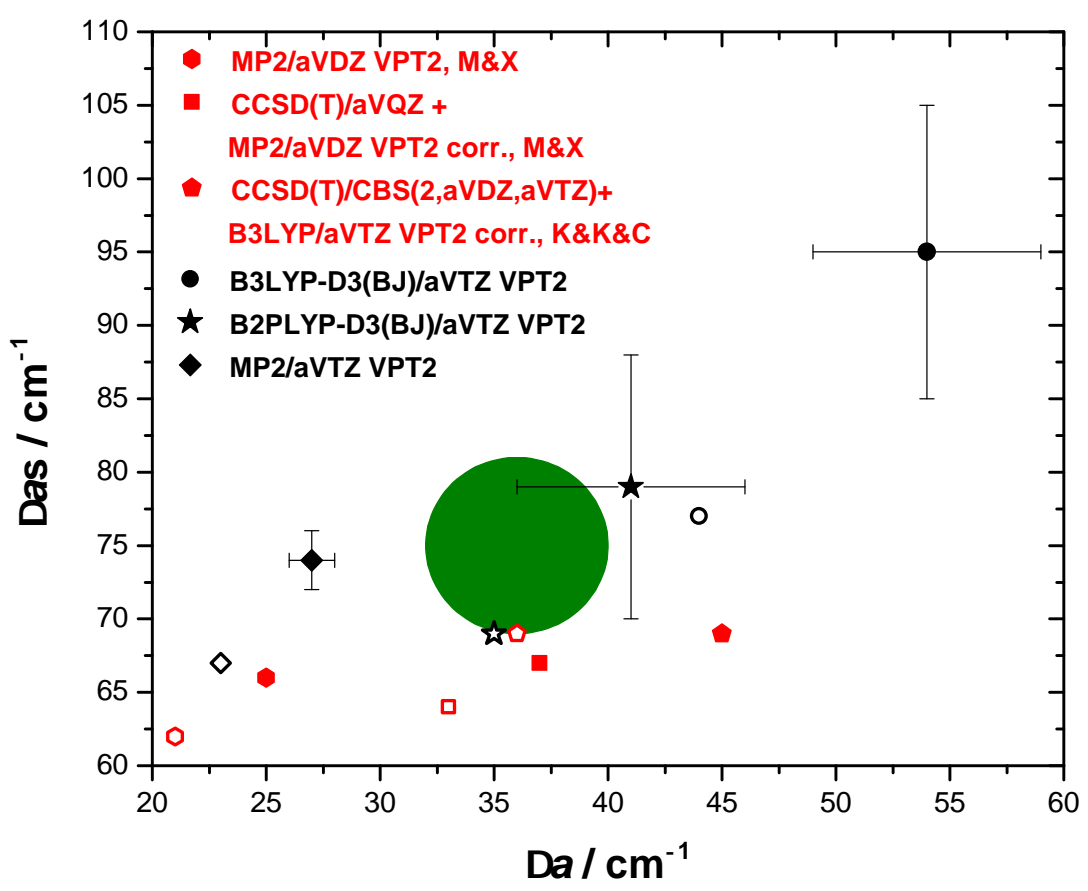

Figure 5.18: Anharmonically (VPT2) predicted shift $\Delta a$ and Davydov splitting $\Delta a s$ of the formic acid dimer. The methods tested are B3LYP-D3(BJ), B2PLYP-D3(BJ), and MP2, all with an aVTZ basis set. For all three, the symmetry $\left(C_{1}\right.$ and $\left.C_{2 \mathrm{~h}}\right)$, optimisation (tight and very tight), and for B3LYP-D3(BJ) and B2PLYP-D3(BJ) additionally the DFT integration grid (ultrafine and superfine) have been varied. The results were averaged and the standard deviation is displayed as an error bar. For reference, harmonic band positions are shown (open symbols). In addition, harmonically and anharmonically calculated $\Delta a$ and $\Delta a s$ values by Miliordos and Xantheas (M\&X) [396] as well as Kalescky, Kraka, and Cremer [394] (K\&K\&C) are shown in red. M\&X have calculated $\mathrm{F}$ and $(\mathrm{FF})$ band positions at the MP2/aVDZ VPT2 and CCSD(T)/aVQZ level and applied an anharmonic MP2/aVDZ VPT2 correction to both and K\&K\&C have corrected their harmonic CCSD(T)/CBS(2,aVDZ,aVTZ) frequencies with B3LYP/aVTZ VPT2. The area of experimental agreement is shown as a green elipsis. The error of the shift has been set to $\pm 4 \mathrm{~cm}^{-1}$ and that of the splitting to $\pm 6 \mathrm{~cm}^{-1}$. More details are found in the text.

Besides, Qu and Bowman [78] as well as Miliordos and Xantheas [396] compared their calculated band positions to experimental gas phase band positions by Bertie and Michaelian for the symmetric $\mathrm{C}=\mathrm{O}$ stretching vibration $\left(1670 \mathrm{~cm}^{-1}\right)$ [62] and for the antisymmetric vibration with IR data by Georges and co-workers $\left(1746 \mathrm{~cm}^{-1}\right)$ [385]. It is interesting to note that the predicted exciton coupling for these gas phase data is nearly the same $\left(76 \mathrm{~cm}^{-1}\right)$ as observed in this work $\left(75 \mathrm{~cm}^{-1}\right)$, even though the band positions of the two vibrations compared to the jet-cooled values vary by $4 \mathrm{~cm}^{-1}\left(1666 \mathrm{~cm}^{-1}\right)$ and $5 \mathrm{~cm}^{-1}\left(1741 \mathrm{~cm}^{-1}\right)$, meaning that thermal shifts effectively cancel for these two vibrations. 


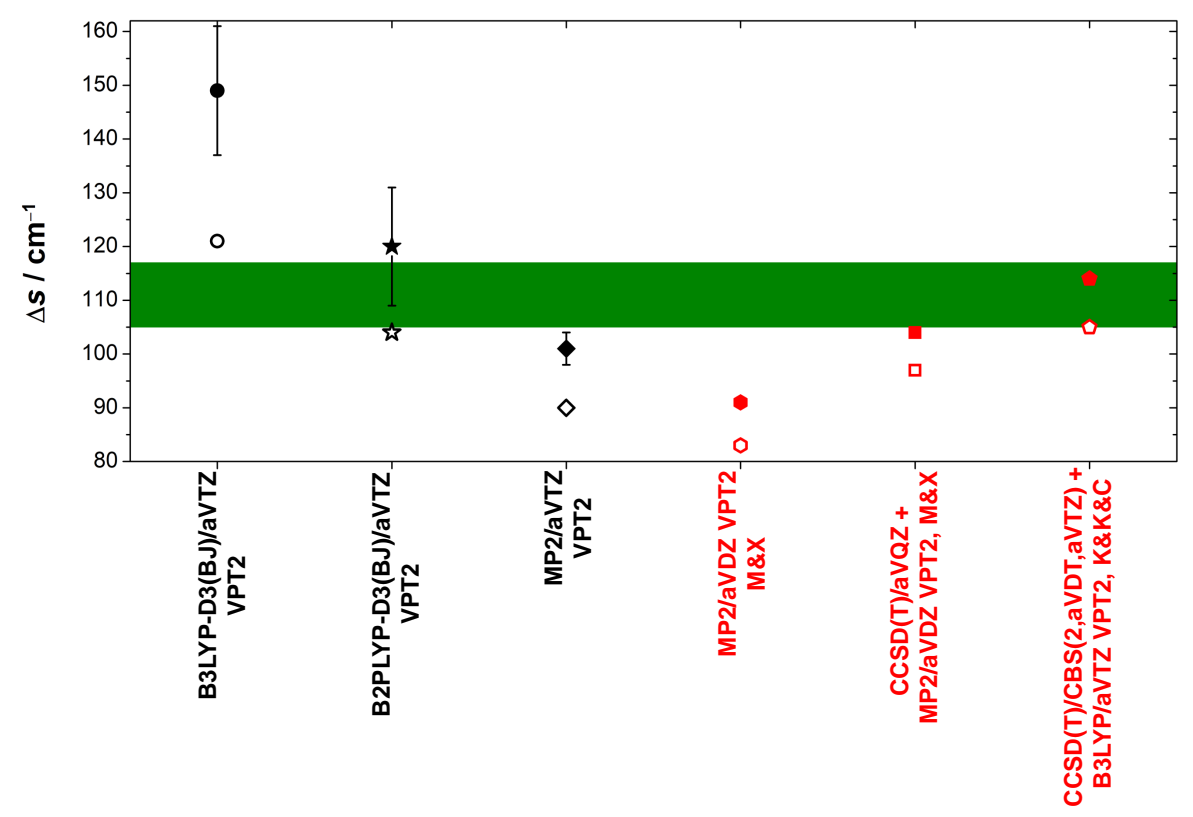

Figure 5.19: Anharmonically (VPT2) predicted shift of the symmetric $\mathrm{C}=\mathrm{O}$ stretching band of the formic acid dimer with respect to the monomer $\Delta s$. The methods tested are B3LYP-D3(BJ), B2PLYP-D3(BJ), and MP2, all with an aVTZ basis set. For all three, the symmetry $\left(C_{1}\right.$ and $C_{2 \mathrm{~h}}$ ), optimisation (tight and very tight), and for B3LYP-D3(BJ) and B2PLYP-D3(BJ) additionally the DFT integration grid (ultrafine and superfine) have been varied. The results were averaged and the standard deviation is displayed as an error bar. For reference, harmonic band positions are shown (open symbols). In addition, harmonically and anharmonically calculated $\Delta a$ and $\Delta a s$ values by Miliordos and Xantheas (M\&X) [396] as well as Kalescky, Kraka, and Cremer [394] (K\&K\&C) are shown in red. M\&X have calculated $\mathrm{F}$ and $(\mathrm{FF})$ band positions at the MP2/aVDZ VPT2 and CCSD(T)/aVQZ level and applied an anharmonic MP2/aVDZ VPT2 correction to both and K\&K\&C have corrected their harmonic CCSD(T)/CBS $(2, a V D Z, a V T Z)$ frequencies with B3LYP/aVTZ VPT2. The area of experimental agreement is shown in green. The error of the shift has been set to $\pm 6 \mathrm{~cm}^{-1}$. More details are found in the text.

\subsubsection{Outlook}

Compared to the formic acid dimer, both acetic and pivalic acid have electron donating groups. To scrutinise the effect of electron withdrawing groups on the exciton splitting $\Delta a s$ and shift $\Delta a$, fluorinated carboxylic acids are explored as another test system. Examples of those were given in the previous chapter. Out of these, 3,3,3-trifluoro-2(trifluoromethyl) propionic acid (TFTFPA) has been picked, as it is the least hazardous and corrosive amongst the acids. Harmonic frequency calculations of all homo and hetero dimers including TFTFPA can be found in Figure 5.20. As for the other three acids, the TFTFPA monomer is indicated by its first letter $(\mathrm{T})$. Correspondingly, the cyclic dimer is abbreviated to (TT).

In contrast to the previous carboxylic acid combinations, the monomer bands are well separated, as the $\mathrm{C}=\mathrm{O}$ stretching vibration of TFTFPA is predicted distinctly higher in wavenumber $\left(1813 \mathrm{~cm}^{-1}\right)$. The largest monomer separation is found for TFTFPA 

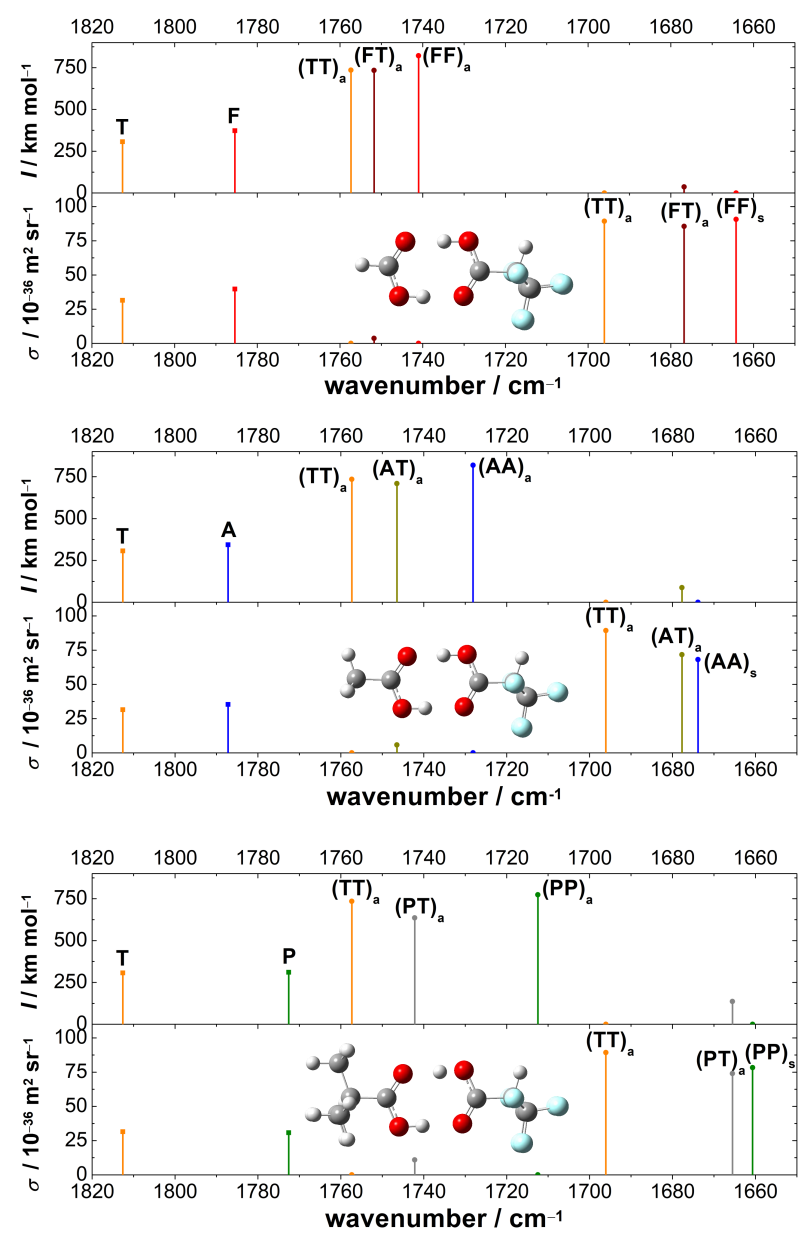

Figure 5.20: Predicted IR (top) and Raman spectra (bottom) of the three mixtures of formic $(\mathrm{F})$, acetic $(\mathrm{A})$, and pivalic acid $(\mathrm{P})$ with 3,3,3-trifluoro-2-(trifluoromethyl) propionic acid (T). The harmonically calculated band positions are scaled to the band position of the antisymmetric $\mathrm{C}=\mathrm{O}$ stretching vibration of the formic acid dimer (scaling factor 0.986). Top panel: Predicted IR and Raman spectra of the mixture of $\mathrm{T}$ and $\mathrm{F}$, middle: IR and Raman spectra of the mixture of $\mathrm{T}$ and $\mathrm{A}$, and bottom: IR and Raman spectra of the mixture of $\mathrm{T}$ and P. Additionally, the hetero dimer structures of the three mixtures are depicted.

and pivalic acid, whose band position difference amounts to $40 \mathrm{~cm}^{-1}$. Most importantly, the homo and hetero dimer band position differences are also satisfactory. The smallest spacing amounts to $3 \mathrm{~cm}^{-1}$ for $(\mathrm{AT})_{\mathrm{s}}$ and $(\mathrm{AA})_{\mathrm{s}}$. Consequently, these bands will likely overlap, as will $(\mathrm{PT})_{\mathrm{s}}$ and $(\mathrm{PP})_{\mathrm{s}}$, which differ by $5 \mathrm{~cm}^{-1}$. For the homo and hetero dimers of formic, acetic, and pivalic acid, an identical difference of $3 \mathrm{~cm}^{-1}$ was observed for $(\mathrm{PF})_{\mathrm{S}}$ and $(\mathrm{PP})_{\mathrm{s}}$, whose band position could be determined. Therefore, these differences should suffice.

The harmonically calculated and zero-point corrected dissociation energies of all homo and hetero dimers can be found in Table 5.8. The (TT) dimer is the second most stable homo dimer, only surpassed by the pivalic acid dimer, with an energy difference 
Table 5.8: Harmonically calculated, zero-point corrected dissociation energies $D_{0}^{\mathrm{h}}\left(\mathrm{in} \mathrm{kJ} \mathrm{mol}{ }^{-1}\right)$ of homo and hetero dimers of formic $(\mathrm{F})$, acetic $(\mathrm{A})$, pivalic $(\mathrm{P})$, and 3,3,3-trifluoro-2(trifluoromethyl) propionic acid (T), calculated at the B3LYP-D3(BJ)/aVTZ level. In the last column, averaged homo dimer dissociation energies $\bar{D}_{0}^{\mathrm{h}}$ are shown, as an estimate for the hetero dimer dissociation energies.

\begin{tabular}{ccc}
\hline \hline Dimer & $D_{0}^{\mathrm{h}}$ & $\bar{D}_{0}^{\mathrm{h}}$ \\
\hline$(\mathrm{FF})$ & 66.6 & \\
$(\mathrm{AA})$ & 71.3 & \\
$(\mathrm{PP})$ & 73.3 & \\
$(\mathrm{TT})$ & 72.2 & \\
\hline$(\mathrm{FA})$ & 69.3 & 69.0 \\
$(\mathrm{AP})$ & 72.2 & 72.3 \\
$(\mathrm{PF})$ & 70.4 & 70.0 \\
$(\mathrm{FT})$ & 70.1 & 69.4 \\
$(\mathrm{AT})$ & 74.6 & 71.7 \\
$(\mathrm{PT})$ & 76.3 & 72.8 \\
\hline \hline
\end{tabular}

of $1.1 \mathrm{~kJ} \mathrm{~mol}^{-1}$. Regarding the hetero dimers, the stability of the T-containing dimers increases with the size of the partner acid. (FT) is nearly as stable as (PF) with a predicted dissociation energy of $70.1 \mathrm{~kJ} \mathrm{~mol}^{-1}$. (PT) exhibits the by far largest calculated dissociation energy of all homo and hetero dimers with $76.3 \mathrm{~kJ} \mathrm{~mol}^{-1}$. Thus, the combination of an acid with electron donating and one with electron withdrawing groups seems to be best in terms of stability. Interestingly, the (AT) and (PT) hetero dimers are even more stable than the respective homo dimers, which was not the case for (FA), $(\mathrm{AP})$, and $(\mathrm{PF})$. This will likely favour their formation.

For the (FA), (AP), and (PF) hetero dimers, additivity of the predicted dissociation energies was found. The arithmetic averages of the homo dimer dissociation energies were accurate to $0.5 \mathrm{~kJ} \mathrm{~mol}^{-1}$. With the fluorinated TFTFPA, this is not the case. The (FT) dissociation energy predicted from those of (FF) and (TT) is $0.8^{\circ} \mathrm{kJ} \mathrm{mol}^{-1}$ smaller than the calculated value. This discrepancy increases up to $3.5 \mathrm{~kJ} \mathrm{~mol}^{-1}$ for $(\mathrm{PT})$. Whether this is due to a misprediction of the dissociation energy of (TT) or of the corresponding hetero dimers, or simply because this additivity rule does not apply for fluorinated species, remains to be explored.

The harmonically calculated shifts and Davydov splittings are listed in Table 5.9. The calculated hetero dimer shifts $\Delta a$ of (FT) and (AT) are smaller than their (PF) and (AP) counterparts, but overall similar to the other hetero dimers. Conversely, the predicted exciton splittings of the hetero dimers are larger with TFTFPA than with pivalic acid. In fact, $\Delta a s_{\mathrm{c}}$ of $(\mathrm{PT})$ is identical to the value of $(\mathrm{FF})$. The $\Delta a s_{\mathrm{c}}$ increase with TFTFPA is larger than the decrease of $\Delta a_{\mathrm{c}}$. As seen for the predicted dissociation energies, additivity of the shifts and Davydov splittings does not seem to hold true for the hetero dimers containing TFTFPA. For the shifts, the $\overline{\Delta a}_{\mathrm{c}}$ values differ by $+3 \mathrm{~cm}^{-1},+4 \mathrm{~cm}^{-1}$, and $+8 \mathrm{~cm}^{-1}$, the divergence increasing with the size of the carboxylic acid. The arithmetic 
averages of the exciton splittings differ by $-6 \mathrm{~cm}^{-1},-11 \mathrm{~cm}^{-1}$, and $-20 \mathrm{~cm}^{-1}$ from the calculated hetero dimer values. Whether this breakdown of the additivity rule is generally applicable or to be blamed on the calculations that perform particularly poorly for this acid, remains to be explored. The best way to test this is to measure the FTIR and Raman spectra of all three mixtures and determine the experimental values. Altogether, these discrepancies in addition to the good band separations make this acid an ideal test system for future studies.

Table 5.9: Calculated (B3LYP-D3(BJ)/aVTZ) shifts $\Delta a_{\mathrm{c}}$ and exciton splittings $\Delta a s_{\mathrm{c}}$ of all homo and hetero dimers of formic $(\mathrm{F})$, acetic $(\mathrm{A})$, pivalic $(\mathrm{P})$, and 3,3,3-trifluoro-2(trifluoromethyl) propionic acid $(\mathrm{T})$. The harmonic band positions used to calculate $\Delta a_{\mathrm{c}}$ and $\Delta a s_{\mathrm{c}}$ are scaled to the antisymmetric $\mathrm{C}=\mathrm{O}$ stretching vibration of the formic acid dimer. From the arithmetic average of the homo dimers approximated hetero dimer shifts $\overline{\Delta a_{\mathrm{c}}}$ and splittings $\overline{\Delta a s_{\mathrm{c}}}$ are also listed.

\begin{tabular}{ccccc}
\hline \hline dimer & $\boldsymbol{\Delta a}_{\mathbf{c}}$ & $\overline{\boldsymbol{\Delta \boldsymbol { a } _ { \mathbf { c } }}}$ & $\boldsymbol{\Delta a \boldsymbol { s } _ { \mathbf { c } }}$ & $\overline{\boldsymbol{\Delta a \boldsymbol { s } _ { \mathbf { c } }}}$ \\
\hline (FF) & 44 & & 77 & \\
$(\mathrm{AA})$ & 59 & & 54 & \\
$(\mathrm{PP})$ & 60 & & 52 & \\
$(\mathrm{TT})$ & 55 & & 61 & \\
$(\mathrm{FA})$ & 52 & 52 & 67 & 66 \\
$(\mathrm{AP})$ & 59 & 60 & 55 & 53 \\
$(\mathrm{PF})$ & 51 & 52 & 68 & 64 \\
$(\mathrm{FT})$ & 47 & 50 & 75 & 69 \\
$(\mathrm{AT})$ & 53 & 57 & 69 & 58 \\
$(\mathrm{PT})$ & 50 & 58 & 77 & 57 \\
\hline \hline
\end{tabular}

\subsubsection{Summary}

In this chapter, hydrogen bonded carboxylic acid dimers were studied in the $\mathrm{O}-\mathrm{H}$ and the $\mathrm{C}=\mathrm{O}$ stretching region. The $\mathrm{O}-\mathrm{H}$ stretching spectrum of the global minimum dimer extends over a few hundred wavenumbers and is very congested due to numerous resonances [70]. In order to improve the understanding of the onset of such resonances, a system was chosen where these are observed, yet on a distinctly smaller scale. Local minima of the acetic acid dimer studied in helium nanodroplets exhibit no resonances of the $\mathrm{O}-\mathrm{H}$ stretching vibrations [84], presumably, because the hydrogen bond induced downshifts of the $\mathrm{O}-\mathrm{H}$ stretching vibration are too small for sufficient wavefunction mixing with dark states (combination vibrations or overtones with near-zero IR intensities). Consequently, an ideal test system has to have a weaker hydrogen bond than the global minimum, but a stronger one than the metastable acetic acid dimers. Therefore, a polar formic acid dimer was studied in helium nanodroplets, as summarised in Figure 5.21. As the global minimum dimer does not form in helium nanodroplets [83], such local minima can be studied without its perturbation. In agreement with a previous study 


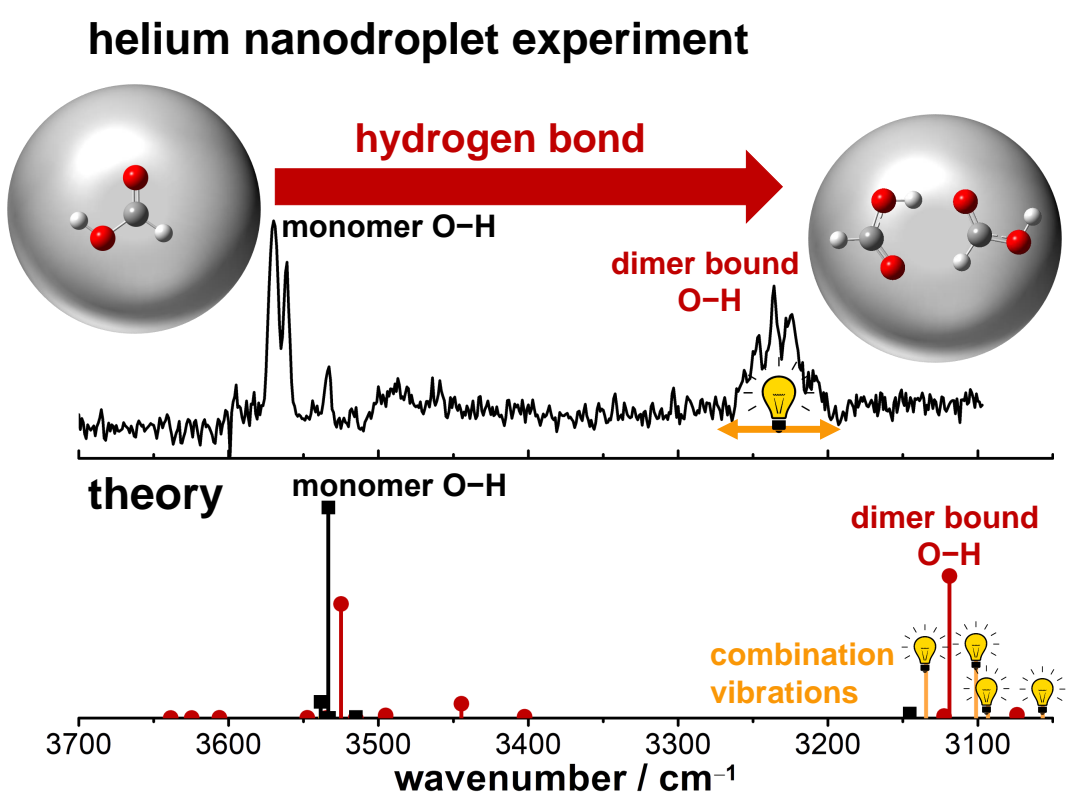

Figure 5.21: Helium nanodroplet spectra of a polar formic acid dimer show a broadened $\mathrm{OH}$ stretching band which is caused by wavefunction mixing of that fundamental with combination vibrations that light up. Adapted from Ref. [409] with permission from the PCCP Owner Societies.

of Madeja et al. [83], the spectral signatures were assigned to a polar dimer with one strong $\mathrm{O}-\mathrm{H} \cdots \mathrm{O}$ and one weak $\mathrm{C}-\mathrm{H} \cdots \mathrm{O}$ hydrogen bond (cf. Figure 5.21) on the basis of scaled harmonic vibrational frequency calculations at various levels of theory. The band of its bound $\mathrm{O}-\mathrm{H}$ stretching vibration is broadened. Additional anharmonic vibrational frequency calculations using vibrational perturbation theory showed that this broadening is likely due to lighting up of dark states, in this case, combination bands. This, however, is observed to a distinctly lower extent than for the global minimum dimer and provides a first onset of such resonances. A good starting point for a closer scrutiny of the resonance pattern are helium nanodroplet spectra of $\mathrm{DCOOH}$, as combination bands involving $\mathrm{C}-\mathrm{H}$ in-plane bending vibrations of $\mathrm{HCOOH}$ were predicted to be in Fermi resonance with the bound $\mathrm{O}-\mathrm{H}$ stretching fundamental, which are absent for both $\mathrm{DCOOH}$ and acetic acid and could therefore also be a reason why the observed acetic acid bands are sharper.

In the second part, the $\mathrm{C}=\mathrm{O}$ stretching vibrations of the global minimum dimers of formic, acetic, and pivalic acid were studied in a supersonic expansion with FTIR and Raman spectroscopy. In this spectral window, fewer Fermi resonances occur than for the $\mathrm{O}-\mathrm{H}$ stretching vibrations. Nonetheless, such resonances were observed for the bands of the symmetric $\mathrm{C}=\mathrm{O}$ stretching vibrations of the homo dimers. The main focus of this study was on the exciton coupling $\Delta a s$ and shifts $\Delta a$ of the $\mathrm{C}=\mathrm{O}$ oscillators and their change upon mixing different carboxylic acids, as summarised in Figure 5.22. Additivity of the hetero dimer $\Delta a s$ and $\Delta a$ values was identified, meaning that these can 


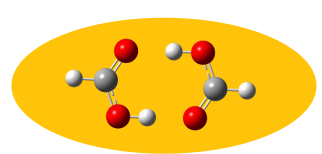

$(\mathrm{FF})$

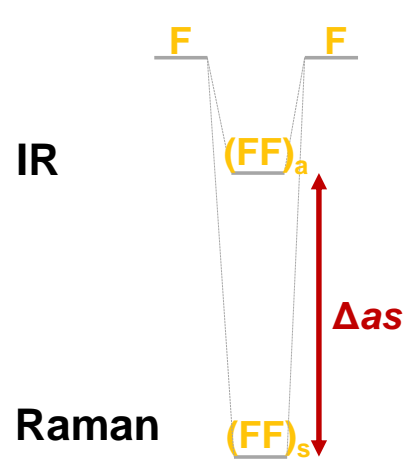

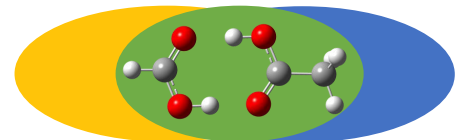

(FA)

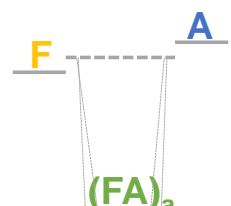

(FA)

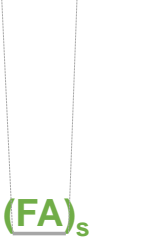

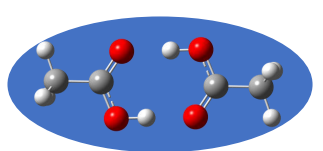

(AA)

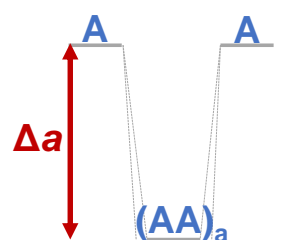

$(\mathrm{AA})_{\mathrm{a}}$

$(\underline{A A})_{s}$

Figure 5.22: The Davydov shifts $\Delta a$ and splittings $\Delta a s$ of weakly polar hetero dimers can be approximated from the arithmetic average of their respective homo dimer values.

be approximated from the respective homo dimer values with an accuracy of $\leq 5 \mathrm{~cm}^{-1}$, yet exploratory calculations using an acid with electron withdrawing groups indicate that this might be a special consequence of the similarly unpolar nature of the three residues. Nonetheless, these quantities are ideal for benchmarking quantum chemical methods, as net anharmonicity effects are likely small. Additionally, the values span over a relatively large range - larger than absolute quantities such as band positions, which is beneficial if comparing the results of different methods. Harmonic vibrational frequency calculations at the B3LYP-D3(BJ)/aVTZ and PBEh-3c level showed good agreement with experiment, although predominantly due to error compensation. Most other methods underestimated $\Delta a s$ and $\Delta a$. Exploratory anharmonic vibrational frequency calculations of the formic acid dimer indicate that the agreement of methods such as MP2/aVTZ and B2PLYP-D3(BJ)/aVTZ will be enhanced upon including anharmonicity. 



\section{Aggregation Dynamics: Trimers and Metastable Dimers}

\subsection{Introduction}

In addition to providing experimental reference data for benchmarking quantum chemical methods, another related motivation of this work is to examine the differences and similarities of the aggregation behaviour of carboxylic acids, in this case, formic, acetic, and pivalic acid. In the condensed phase, some acids were shown to exist as cyclic dimers, e.g., propionic [424], valeric [425], butyric [426] or benzoic acid [427], whereas others such as formic and acetic acid form long catemeric chains composed of strong $\mathrm{O}-\mathrm{H} \cdots \mathrm{O}=\mathrm{C}$ hydrogen bonds in addition to weaker $\mathrm{C}-\mathrm{H} \cdots \mathrm{O}=\mathrm{C}$ contacts $[428,429]$. The metastable $\mathrm{F}_{\mathrm{C}} \mathrm{F}$ dimer studied in helium nanodroplets in the previous chapter represents the building block of these chains (Figure 6.1). The same structure motif is also found in carboxylic acid trimers, which are composed of a cyclic dimer, to which another monomer is docking, or, changing the point of view, these consist of a monomer docking to an $\mathrm{F}_{\mathrm{C}} \mathrm{F}$ dimer, as shown in Figure 6.1. Studying these clusters for three carboxylic acids and scrutinising their differences and similarities might thus help to provide a better understanding of their condensed phase structures.

The vibrational characterisation of carboxylic acid clusters with an odd number of strong $\mathrm{O}-\mathrm{H} \cdots \mathrm{O}=\mathrm{C}$ hydrogen bonds in supersonic expansions is challenging, as their abundance compared to the doubly hydrogen bonded cyclic dimer (or assemblies of this dimer) is distinctly smaller, if present at all. Thus, the sheer necessity of multiexperimental approaches for these systems goes without saying. Here, FTIR and Raman jet spectroscopy are utilised in addition to a third technique called FTIR imaging, which will be first introduced in this chapter. It facilitates the space-resolved synchronous recording of 4096 FTIR spectra in a $(10 \times 10) \mathrm{mm}^{2}$ large area of the expansion and thus, enables a close examination of the cluster formation and decomposition. This kinetic perspective on the ongoing dynamics is particularly useful for the assignment of transient species, as will be shown in Section 6.2.3. Another important resource for spectral assignments throughout this work are scaled harmonic frequency calculations. These are carried out at the B3LYP-D3(BJ) level, as satisfactory performance was achieved for the carboxylic acid monomers and dimers studied in the previous chapters. However, in contrast to these studies, vibrational benchmarks of popular methods are not attempted here due to the limited applicability of vibrational perturbation theory (VPT2) calculations to these larger systems.

One of the first comparative FTIR jet studies of higher-energy dimers and trimers 


\section{Aggregation Dynamics: Trimers and Metastable Dimers}
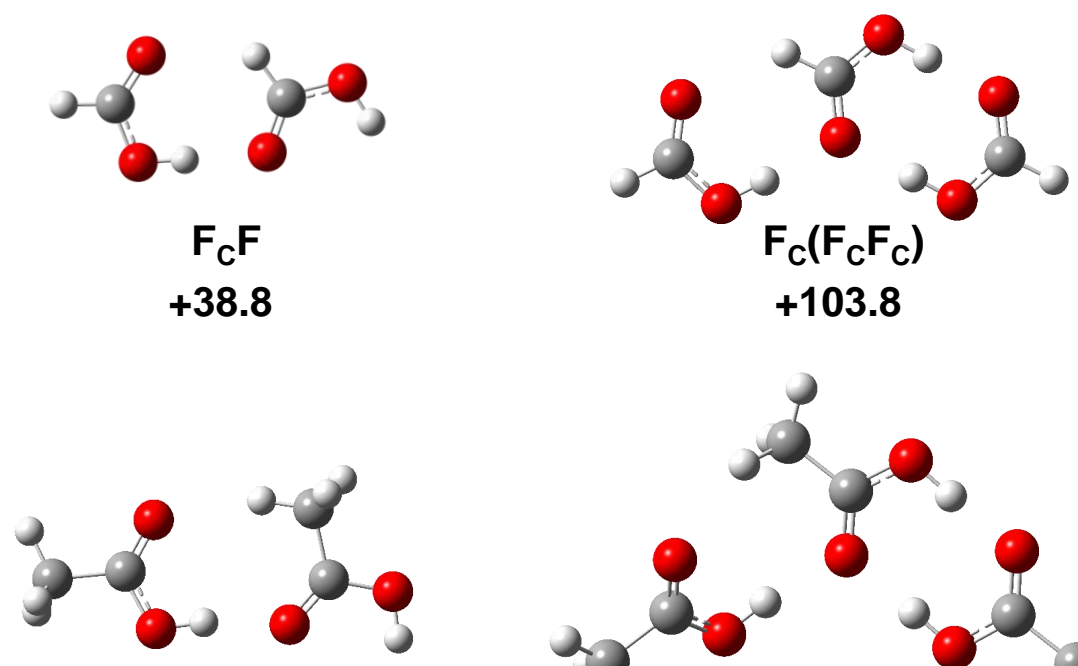

$\mathbf{A}_{\mathrm{C}} \mathrm{A}$

$+38.6$

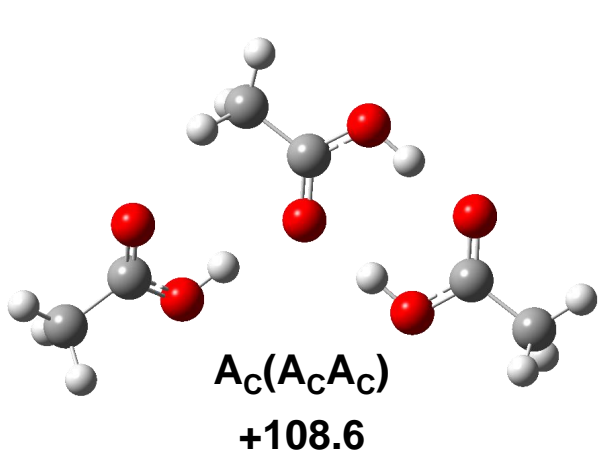

Figure 6.1: Calculated structures (B3LYP-D3(BJ)/def2-TZVP) of the metastable formic $\mathrm{F}_{\mathrm{C}} \mathrm{F}$ and acid dimers $A_{C} A$ as well as the global minimum trimers $F_{C}\left(F_{C} F_{C}\right)$ and $A_{C}\left(A_{C} A_{C}\right)$. The harmonically calculated, zero-point corrected dissociation energies into monomers are listed below.

of formic and acetic acid has been conducted by Oswald, Meyer, and Suhm in the $\mathrm{OH}$ stretching region in 2018 [79]. By adding small amounts of dinitrogen $(2.5-15 \%)$ to their formic/acetic acid in helium expansions, they could distinguish the two clusters, as the metastable dimer exhibits a free $\mathrm{OH}$ group that can be complexed by $\mathrm{N}_{2}$, whereas all $\mathrm{OH}$ groups of the trimer are involved in hydrogen bonding (see Figure 6.1). While an $\mathrm{OH}$ stretching band of both trimers was successfully assigned, a band of the higherenergy dimer was only found for acetic acid. These differences in aggregation behaviour of the two acids cannot be attributed to structural changes, as the trimer and higherenergy dimers of both are structurally (and energetically) similar [79] (cf. Figure 6.1). Therefore, these findings are unexpected. As seen in the previous chapter, the $\mathrm{OH}$ stretching region of carboxylic acids is very complex, so that weak bands are difficult to identify. Besides, by accounting for the hydrogen bond stiffening effect of helium nanodroplets, it was shown that the gas phase corrected band position of the bound $\mathrm{OH}$ stretching vibration of the $\mathrm{F}_{\mathrm{C}} \mathrm{F}$ dimer $\left(3252 \mathrm{~cm}^{-1}\right.$, see Section 3.1.6) is close to that of the formic acid trimer $\left(3258 \mathrm{~cm}^{-1}\right.$ [79]). Hence, both bands might overlap. Moreover, the bound $\mathrm{OH}$ stretching vibration of $\mathrm{F}_{\mathrm{C}} \mathrm{F}$ exhibits a $70 \mathrm{~cm}^{-1}$ wide broad resonance structure, whereas the corresponding acetic acid dimer signal is about five times narrower and much sharper and as such, easier to identify (cf. Figure 5.8). Hence, the acids might aggregate more similarly than the spectra might suggest. Another way of verifying this, is to study these in the spectrally less convoluted $\mathrm{C}=\mathrm{O}$ stretching region, which will be the focus of this chapter.

While the global (and local) energy mininum structures of carboxylic acids dimers have 


\section{Aggregation Dynamics: Trimers and Metastable Dimers}

been widely examined in the literature (for References, see Chapter 5), there are only a few studies on larger clusters such as trimers, most of which are theoretical $[219,220$, 430-433] or employed mass spectrometry [434-436]. The first spectroscopic evidence of higher aggregates was suggested by the Sander group in 1998, who attributed a $\mathrm{C}=\mathrm{O}$ stretching band $\left(1745 \mathrm{~cm}^{-1}\right)$ in-between the formic acid monomer $\left(1767 \mathrm{~cm}^{-1}\right)$ and dimer $\left(1728 \mathrm{~cm}^{-1}\right)$ in matrix isolation experiments to larger clusters [380]. A year later, Spinner [437] proposed that this band is more likely due to the polar dimer $\mathrm{F}_{\mathrm{C}} \mathrm{F}$, as the overall cluster abundance in the experiments was too low. In a follow-up work, the Sander group studied the growth kinetics in a solid argon matrix by increasing the temperature of the host from 7 to $40 \mathrm{~K}$ [82]. At low temperatures, the diffusion of the formic acid monomer is prevented and therefore, no clusters are observed. Upon increasing the temperature, the band in-between monomer and the dimer increases while the dimer band remains constant. At higher temperatures, the band of this unknown species decreases while the dimer band increases. From these growth dynamics, it was deduced that the unknown species is indeed the polar $\mathrm{F}_{\mathrm{C}} \mathrm{F}$ dimer that is an intermediate species in the cyclic dimer formation [82]. In a more recent matrix isolation IR study by Ito from 2015 [119], this band was re-assigned to a trimer band, though without commenting on the previous studies of the Sander group. Thus, an additional motivation of this chapter is to shed more light on this assignment controversy in the $\mathrm{C}=\mathrm{O}$ stretching region by providing additional experimental reference data.

The structure of this chapter is as follows: Firstly, FTIR and Raman spectra of the formic acid trimer and potential contributions of the higher-energy dimer will be discussed in the $\mathrm{C}=\mathrm{O}$ stretching region, followed by an analysis of the aggregation dynamics in supersonic expansions studied with FTIR imaging. These results have been published in Reference [185], which parts of this chapter are based on. Afterwards, the trimers and higher-energy dimers of the larger carboxylic acids are examined and their differences and similarities to formic acid are addressed.

\subsection{Formic Acid Trimers}

\subsubsection{Trimer structures}

Roy and Thakkar explored the conformational hypersurface of the formic acid trimer and were able to identify 33 minima [432]. The three most stable structures and their relative energies with respect to the global minimum are shown in Figure 6.2. The calculations have been performed at the B3LYP-D3(BJ)/def2-TZVP level. This smaller basis set is chosen so that all trimer calculations within this chapter are computationally feasible with same method and basis set. In a study of carboxylic acid dimers, B3LYPD3(BJ)/def2-TZVP was shown to exhibit a similar performance compared to B3LYPD3(BJ)/aVTZ [329], which was used previously. As opposed to Chapters 4 and 5, these calculations have been performed with the ORCA program package (version 4.0.1) [37, 38]. Differences to corresponding calculations with Gaussian 09 amount to $1-2 \mathrm{~cm}^{-1}$ for the $\mathrm{C}=\mathrm{O}$ stretching vibrations, but can be larger for other fundamentals, which can 


\section{Aggregation Dynamics: Trimers and Metastable Dimers}

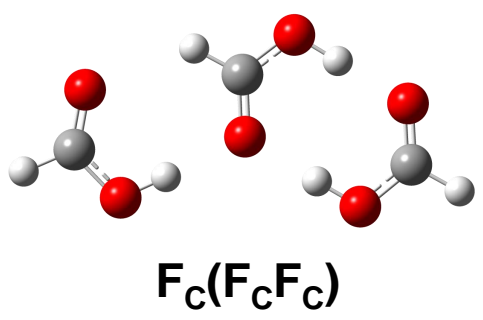

0

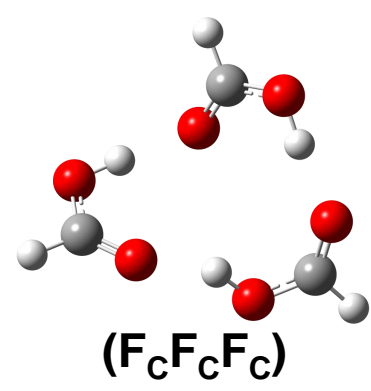

$+5.7$

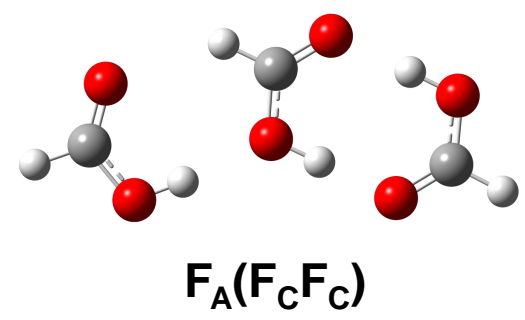

$+7.7$

Figure 6.2: Calculated global and local minimum structures (B3LYP-D3(BJ)/def2-TZVP) of the formic acid trimer. The zero-point corrected, harmonic energy differences (in $\mathrm{kJ} \mathrm{mol}^{-1}$ ) with respect to the global minimum are listed below the structures.

mainly be attributed to the different implementation of B3LYP in both programs. As before, the experimental (anharmonic) band positions are compared to scaled, harmonic frequency calculations. For consistency, all bands are scaled to the antisymmetric $\mathrm{C}=\mathrm{O}$ stretching vibration of the formic acid dimer at $1741 \mathrm{~cm}^{-1}$.

As seen in Figure 6.2, the trimer nomenclature has been adapted from the polar dimer in the previous chapter, namely an $\mathrm{O}-\mathrm{H} \cdots \mathrm{O}=\mathrm{C}$ hydrogen bond is indicated by the index $\mathrm{C}$ (carbonyl) and an $\mathrm{O}-\mathrm{H} \cdots \mathrm{O}-\mathrm{H}$ interaction has the index $\mathrm{A}$ (alcohol). Cyclic subunits are enclosed by parentheses. The three most favourable trimers from the study of Roy and Thakkar [432] are called $\mathrm{F}_{\mathrm{C}}\left(\mathrm{F}_{\mathrm{C}} \mathrm{F}_{\mathrm{C}}\right),\left(\mathrm{F}_{\mathrm{C}} \mathrm{F}_{\mathrm{C}} \mathrm{F}_{\mathrm{C}}\right)$, and $\mathrm{F}_{\mathrm{A}}\left(\mathrm{F}_{\mathrm{C}} \mathrm{F}_{\mathrm{C}}\right)$. The $\left(\mathrm{F}_{\mathrm{C}} \mathrm{F}_{\mathrm{C}} \mathrm{F}_{\mathrm{C}}\right)$ trimer forms a non-planar cycle with all $\mathrm{OH}$ groups involved in strong hydrogen bonds (Figure 6.2). The other two are composed of a cyclic dimer with a monomer docking to it, either to the carbonyl $\left(\mathrm{F}_{\mathrm{C}}\left(\mathrm{F}_{\mathrm{C}} \mathrm{F}_{\mathrm{C}}\right)\right)$ or the alcohol group $\left(\mathrm{F}_{\mathrm{A}}\left(\mathrm{F}_{\mathrm{C}} \mathrm{F}_{\mathrm{C}}\right)\right)$. In line with the findings of Roy and Thakkar [432], the most stable trimer at the B3LYP$\mathrm{D} 3(\mathrm{BJ}) /$ def2-TZVP level is predicted to be $\mathrm{F}_{\mathrm{C}}\left(\mathrm{F}_{\mathrm{C}} \mathrm{F}_{\mathrm{C}}\right)$, which matches the structure observed in microwave spectroscopy measurements by Neill in 2011 [218]. The agreement between the calculated equilibrium and observed vibrationally averaged rotational constants is satisfactory with overestimations on the order of $1-2 \%$ (see Table A.12). The cyclic trimer is $5.7 \mathrm{~kJ} \mathrm{~mol}^{-1}$ higher in energy and $\mathrm{F}_{\mathrm{A}}\left(\mathrm{F}_{\mathrm{C}} \mathrm{F}_{\mathrm{C}}\right)$ is energetically the most unfavourable structure of the three $\left(+7.7 \mathrm{~kJ} \mathrm{~mol}^{-1}\right)$. Due to these fairly large energy differences compared to the global minimum, it is unlikely that the local minima are formed to a significant extent in supersonic expansions. Perhaps more importantly, kinetic trapping is unlikely for $\mathrm{F}_{\mathrm{A}}\left(\mathrm{F}_{\mathrm{C}} \mathrm{F}_{\mathrm{C}}\right)$ due to an easy proton tunneling pathway to the global minimum and also for $\left(\mathrm{F}_{\mathrm{C}} \mathrm{F}_{\mathrm{C}} \mathrm{F}_{\mathrm{C}}\right)$ due to the absence of a stable dimer subunit precursor. Therefore, these are discarded from the following analysis. The higher-energy dimer $\mathrm{F}_{\mathrm{C}} \mathrm{F}$ was examined in the previous chapter and is displayed in Figure 6.1.

As for the dimers, the indices $\mathrm{C}$ are omitted for the rest of this section, so that $\mathrm{F}_{\mathrm{C}}\left(\mathrm{F}_{\mathrm{C}} \mathrm{F}_{\mathrm{C}}\right)$ shortens to $\mathrm{F}(\mathrm{FF})$ and correspondingly, $\mathrm{F}_{\mathrm{C}} \mathrm{F}$ to $\mathrm{FF}$. Instead, indices are used to indicate the type of vibration. The trimer has three $\mathrm{C}=\mathrm{O}$ stretching vibrations, the symmetric (index s) and antisymmetric (a) $\mathrm{C}=\mathrm{O}$ stretching vibrations of the dimer subunit and the $\mathrm{C}=\mathrm{O}$ stretching vibration of the docking monomer, which is marked by 


\section{Aggregation Dynamics: Trimers and Metastable Dimers}

the index d. The two $\mathrm{C}=\mathrm{O}$ stretching vibrations of $\mathrm{FF}$ are labelled accordingly. Note that in contrast to the type of hydrogen bond, the indices indicating the symmetry are written in lowercase letters.

\subsubsection{FTIR and Raman Spectra}

FTIR and Raman spectra of formic acid recorded under trimer-optimised conditions are shown in Figure 6.3. Initially, the focus is on the FTIR spectra. These feature three distinct bands in addition to the formic acid monomer $\mathrm{F}\left(1777 \mathrm{~cm}^{-1}\right)$ and the band of the antisymmetric $\mathrm{C}=\mathrm{O}$ stretching vibration of the cyclic dimer $(\mathrm{FF})_{\mathrm{a}}\left(1741 \mathrm{~cm}^{-1}\right)$. Two of these are located adjacent to $(\mathrm{FF})_{\mathrm{a}}$ at $1755 \mathrm{~cm}^{-1}$ and $1725 / 1722 \mathrm{~cm}^{-1}$. The third band at $1619 \mathrm{~cm}^{-1}$ is distinctly weaker.

Below the experimental spectra, $(\mathrm{FF})_{\mathrm{a}}$-scaled harmonic band positions at the B3LYPD3(BJ)/def2-TZVP level are plotted alongside the corresponding IR intensities (and Raman scattering cross-sections). As expected from the structural similarities of the higher-energy dimer and the trimer, the predicted band positions of both are very similar. The band of the antisymmetric $\mathrm{C}=\mathrm{O}$ stretching vibration of $\mathrm{FF}$ nearly coincides with that of the docking vibration of the formic acid trimer $\mathrm{F}_{\mathrm{d}}(\mathrm{FF})\left(2 \mathrm{~cm}^{-1}\right.$ difference $)$ and the symmetric $\mathrm{C}=\mathrm{O}$ stretching band $\mathrm{FF}_{\mathrm{s}}$ differs by just $5 \mathrm{~cm}^{-1}$ from $\mathrm{F}(\mathrm{FF})_{\mathrm{a}}$. As such, both bands adjacent to $(\mathrm{FF})_{\mathrm{a}}$ could be due to the trimer $\mathrm{F}(\mathrm{FF})$, the higher energy dimer $\mathrm{FF}$, or both. The band of the third $\mathrm{C}=\mathrm{O}$ stretching vibration of the trimer $\mathrm{F}(\mathrm{FF})_{\mathrm{s}}$ is predicted at $1630 \mathrm{~cm}^{-1}$. The third, weaker experimental band $\left(1619 \mathrm{~cm}^{-1}\right)$ is located $11 \mathrm{~cm}^{-1}$ downshifted from it. A similar mismatch is observed for the formic acid monomer band, which is predicted to $1787 \mathrm{~cm}^{-1}$ and observed at $1777 \mathrm{~cm}^{-1}$.

As expected for the symmetric $\mathrm{C}=\mathrm{O}$ stretching vibration of the trimer, the potential $\mathrm{F}(\mathrm{FF})_{\mathrm{s}}$ band is more intense in the corresponding Raman spectra shown in Figure 6.3. The Raman band exhibits two maxima at $1617 / 1619 \mathrm{~cm}^{-1}$ and is broadened to the higher wavenumber side. The antisymmetric $\mathrm{C}=\mathrm{O}$ stretching vibrations of $\mathrm{FF}$ and $\mathrm{F}(\mathrm{FF})$ have a negligible Raman scattering cross-section, so that only the bands of symmetric stretching vibrations of all clusters are featured in addition to the docking vibration of the trimer. Thus, the band at $1755 \mathrm{~cm}^{-1}$ in the Raman spectra can be solely attributed to $\mathrm{F}_{\mathrm{d}}(\mathrm{FF})$. The predicted scattering cross-section ratio of $\mathrm{F}_{\mathrm{d}}(\mathrm{FF})\left(1755 \mathrm{~cm}^{-1}\right)$ with respect to $\mathrm{F}(\mathrm{FF})_{\mathrm{s}}\left(1617 / 1619 \mathrm{~cm}^{-1}\right)$ confirms the trimer assignment, as it roughly matches the experimental band integral ratio. Near the predicted band position of the symmetric $\mathrm{C}=\mathrm{O}$ stretching vibration of the polar dimer $\mathrm{FF}_{\mathrm{s}}\left(1717 \mathrm{~cm}^{-1}\right)$, a weak, broad signal is observed at around $1715 \mathrm{~cm}^{-1}$. The deviation to $\mathrm{FF}_{\mathrm{s}}$ amounts to $2 \mathrm{~cm}^{-1}$. Thus, there might be a small amount of the polar dimer present in the Raman jet spectra, though its abundance is much lower than that of the trimer and global minimum dimer (cf. Figure A.20).

For comparison of the FTIR and Raman spectra, one needs to keep in mind that the part of the expansion that is probed in both experiments differs (see Section 3.1.4). The Raman spectra are measured close to the nozzle (1 mm distance), whereas a large area of the expansion is captured with the FTIR jet set-up (10 mm nozzle distance, 


\section{Aggregation Dynamics: Trimers and Metastable Dimers}

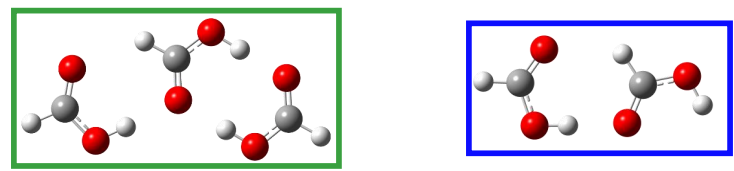

FTIR

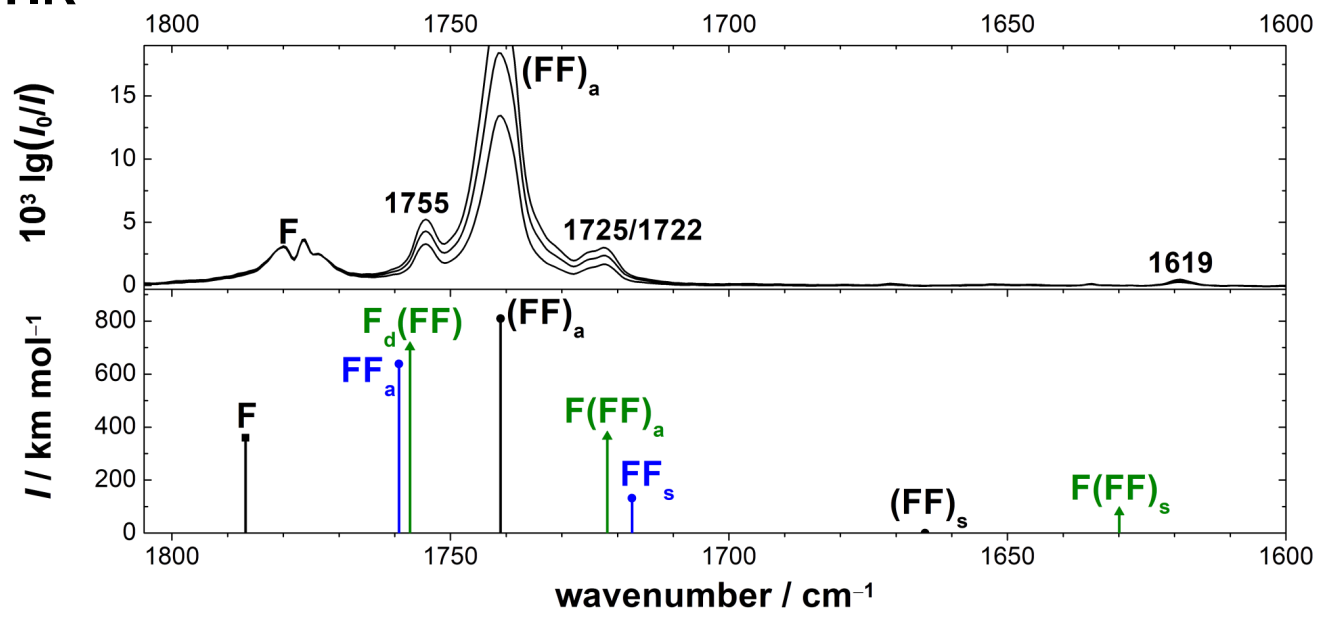

Raman

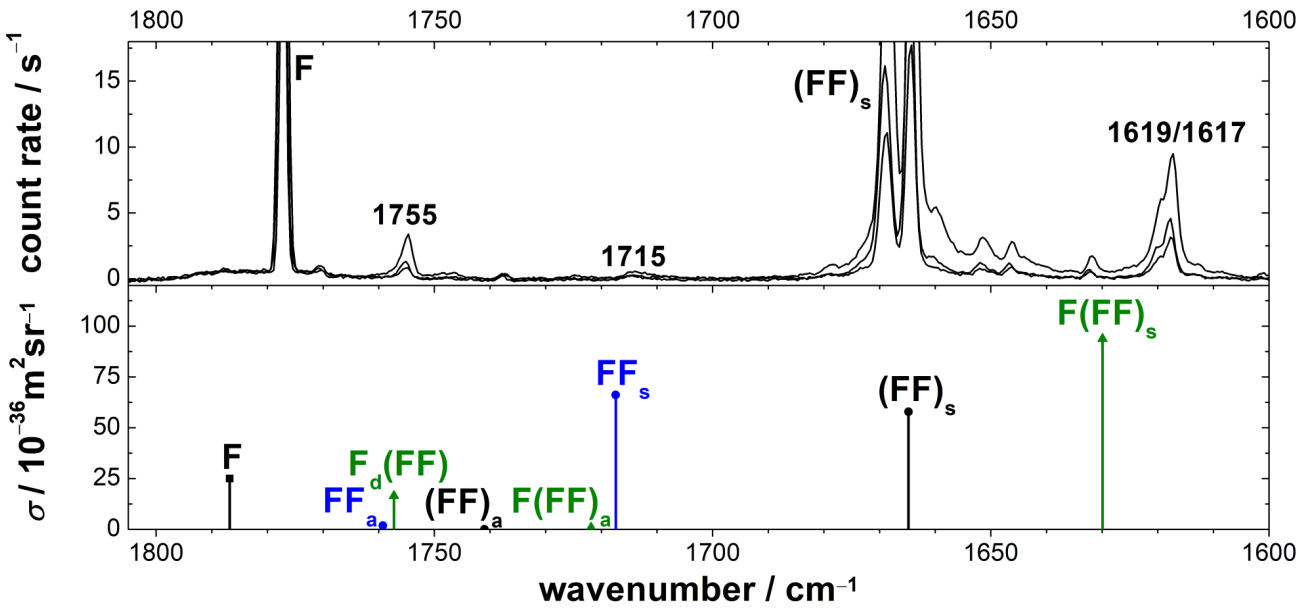

Figure 6.3: Top: FTIR jet spectra of formic acid in helium in the $\mathrm{C}=\mathrm{O}$ stretching region at a reservoir pressure of 560 mbar with increasing concentrations of $<0.1-0.4 \%$. Each spectrum has been averaged over 50 scans. Bottom: Raman jet spectra of formic acid in helium in the $\mathrm{C}=\mathrm{O}$ stretching region at a reservoir pressure of $700 \mathrm{mbar}$. The concentrations increase from bottom to top from $<0.1-0.3 \%$. The acquisition times amount to $30-45$ minutes. The spectra have been intensity-scaled to the monomer band of lowest intensity (spectrum of highest cluster concentration). Below the FTIR and Raman spectra, harmonically calculated and (FF) ${ }_{\mathrm{a}}$-scaled band position (scaling factor 0.983 ) of the formic acid monomer F, cyclic (FF) and polar dimer $\mathrm{FF}$, and the formic acid trimer $\mathrm{F}(\mathrm{FF})$ are shown. The calculations have been performed at the B3LYP-D3(BJ)/def2-TZVP level. In two insets, the global minimum structures of the trimer and the polar dimer are shown. 


\section{Aggregation Dynamics: Trimers and Metastable Dimers}

$10 \mathrm{~mm}$ beam diameter ${ }^{1}$; both values refer to the focus point). Hence, the FTIR spectra give an overview over the cluster formation, whereas the Raman spectra correspond to a centre line snapshot close to the nozzle. The potential signal of the polar dimer in the Raman spectra might diminish at larger distances as it is converted to the global minimum dimer, as shown in the study of the growth dynamics by the Sander group [82]. However, the signal decrease with distance in the Raman experiment is too steep for a closer analysis, as the band is overall fairly weak (cf. Figure A.20). In the FTIR spectra, both clusters could be featured. Whether the deduced ratio in the Raman spectra is the upper limit due to the interconversion to $(\mathrm{FF})$ cannot be quantified so far, as the areas of highest stability of FF in the expansions are not known. These could be determined by recording space-resolved spectra, as will be detailed in the next section.

Due to the overlap of the bands at $1755 \mathrm{~cm}^{-1}$ and $1725 / 1722 \mathrm{~cm}^{-1}$ with the antisymmetric $\mathrm{C}=\mathrm{O}$ stretching band of the cyclic dimer $(\mathrm{FF})_{\mathrm{a}}$ in the FTIR spectra, the experimental intensity ratio with respect to the third $\mathrm{C}=\mathrm{O}$ stretching band is not as exact, yet it matches the predicted ratio for the trimer rather well, which confirms that its fraction is larger, if the polar dimer is present at all. Altogether, this lack of spectral separation in the FTIR spectra is similar to the aforementioned possible overlap of the $\mathrm{O}-\mathrm{H}$ docking vibration of the trimer and the bound $\mathrm{O}-\mathrm{H}$ stretching vibration of the polar dimer.

The band positions of the three $\mathrm{C}=\mathrm{O}$ stretching vibrations of $\mathrm{F}(\mathrm{FF})$ are summarised alongside the $\mathrm{F}$ and $(\mathrm{FF})$ bands in Table 6.1 and agree well with the harmonic, $(\mathrm{FF})_{\mathrm{a}^{-}}$ scaled B3LYP-D3(BJ)/def2-TZVP prediction (deviations below $5 \mathrm{~cm}^{-1}$ ) except for $\mathrm{F}(\mathrm{FF})_{\mathrm{s}}$, where a mismatch of $+11 / 13 \mathrm{~cm}^{-1}$ is observed. As mentioned above, a similar discrepancy $\left(+10 \mathrm{~cm}^{-1}\right)$ is seen for the formic acid monomer. As the assignment of the polar dimer to the band at $1715 \mathrm{~cm}^{-1}$ needs further confirmation, it is not included.

Table 6.1: Experimental $\tilde{\nu}_{\exp }$ as well as harmonic, $(\mathrm{FF})_{\mathrm{a}}$-scaled band position (in $\left.\mathrm{cm}^{-1}\right)$ of the $\mathrm{C}=\mathrm{O}$ stretching vibrations of the formic acid monomer $\mathrm{F}$, dimer $(\mathrm{FF})$, and trimer $\mathrm{F}(\mathrm{FF})$, calculated at the B3LYP-D3(BJ)/def2-TZVP level. The scaling factor amounts to 0.983. The three vibrations are the docking vibration (index $\mathrm{d}$ ) of the monomer unit, as well as the symmetric (s) and antisymmetric (a) $\mathrm{C}=\mathrm{O}$ stretching vibration of the dimer unit within the trimer. In the fourth column, the deviations of the predicted band positions are listed with respect to experiment.

\begin{tabular}{cccc}
\hline \hline & $\tilde{\nu}_{\text {exp }}$ & B3LYP-D3(BJ) & \\
\hline $\mathrm{F}$ & 1777 & 1787 & +10 \\
$(\mathrm{FF})_{\mathrm{a}}$ & 1741 & 1741 & \\
$(\mathrm{FF})_{\mathrm{s}}$ & 1666 & 1665 & -1 \\
$\mathrm{~F}_{\mathrm{d}}(\mathrm{FF})$ & 1755 & 1757 & +2 \\
$\mathrm{~F}(\mathrm{FF})_{\mathrm{a}}$ & $1722 / 1725$ & 1722 & $0 /-3$ \\
$\mathrm{~F}(\mathrm{FF})_{\mathrm{s}}$ & $1617 / 1619$ & 1630 & $+13 /+11$ \\
\hline \hline
\end{tabular}

\footnotetext{
${ }^{1}$ The beam diameter of the Raman laser, is three orders of magnitude smaller.
} 


\section{Aggregation Dynamics: Trimers and Metastable Dimers}

\subsubsection{Aggregation Dynamics in Supersonic Expansions}

One way of studying the aggregation dynamics of carboxylic acids in supersonic expansions in more detail is to map the expansion area, i.e., to record several such snap shots similar to the Raman spectra, as opposed to having an averaged FTIR view. Raman mapping is one way of realising this, which is favourable due to the high spatial resolution achievable $(\approx 2 \mu \mathrm{m})[438,439]$. On the downside, substance consumption and recording times are high. An alternative is FTIR imaging, where mapping is parallelised by using an area detector. Here, a $64 \times 64$ pixel mid-infrared focal plane array detector (FPA) is employed that enables the synchronous recording of 4096 FTIR spectra per scan. Typically for the mid-infrared, the detector elements are composed of mercury cadmium telluride (MCT). Each detector element (pixel) has a size of $(40 \times 40) \mu \mathrm{m}^{2}$ resulting in a total detector size of $(2.56 \times 2.56) \mathrm{mm}^{2}$. Together with the fourfold magnification of the IR lens used (cf. Section 3.1.1), a $(10 \times 10) \mathrm{mm}^{2}$ large area of the expansion is mapped. The spatial resolution depends on the data preprocessing described in Section 3.1.1 and is set to $<0.4 \mathrm{~mm}$ throughout this work. In addition to the lower substance and time consumption, the reproducibility of the measurements is enhanced, as the relative position of the detector elements with respect to each other is fixed.

To examine cluster formation in supersonic expansions, the experimental conditions need to be changed, so that their effect on the cluster distributions and areas of formation can be probed. Here, the reservoir pressure is enhanced from 300 mbar to 700 mbar. A higher reservoir pressure results in more collisions when the particles are accelerated towards the vacuum chamber during the expansion. To visualise the impact of these, the bands of interest are integrated, namely monomer, dimer, and trimer. For the latter, the band at $1755 \mathrm{~cm}^{-1}$ has been chosen, as it exhibits the highest intensity (see Figure 6.3). Besides, one should note that the formic acid dimer signal (FF) at $1741 \mathrm{~cm}^{-1}$ is not necessarily exclusively due to dimers, but can also contain contributions of larger dimer assemblies, such as tetramers. These are composed of stacks of dimers [433], which is why their $\mathrm{C}=\mathrm{O}$ stretching bands are barely separated from those of the dimer [119]. A closer examination of such dimer assemblies can be found in the next chapter. The integrated intensity of the monomer and cluster bands is then plotted for each pixel. The results are depicted in Figure 6.4.

As expected, the highest monomer signal can be seen at the nozzle exit. It diminishes with increasing nozzle distance, due to the density decrease in the supersonic expansion. With increasing reservoir pressure, the monomer signal depletes along the top-down propagation axis of flow. At the highest reservoir pressure, it is only present left and right from the nozzle centre line. The reason for this depletion becomes apparent when looking at the dimer signal in the middle row of Figure 6.4. Expectedly, the number of dimers (and dimer assemblies $(\mathrm{FF})_{n}$ ) rises with the number of collisions. Since the $(\mathrm{FF})_{n}$ formation consumes monomers, these are only present where fewer collisions occur, i.e., at the lower density sides of the flow.

The most interesting carboxylic acid species are those with an odd number of hydrogen bonds, as these are more reactive towards aggregation. An example is the higher-energy 


\section{Aggregation Dynamics: Trimers and Metastable Dimers}

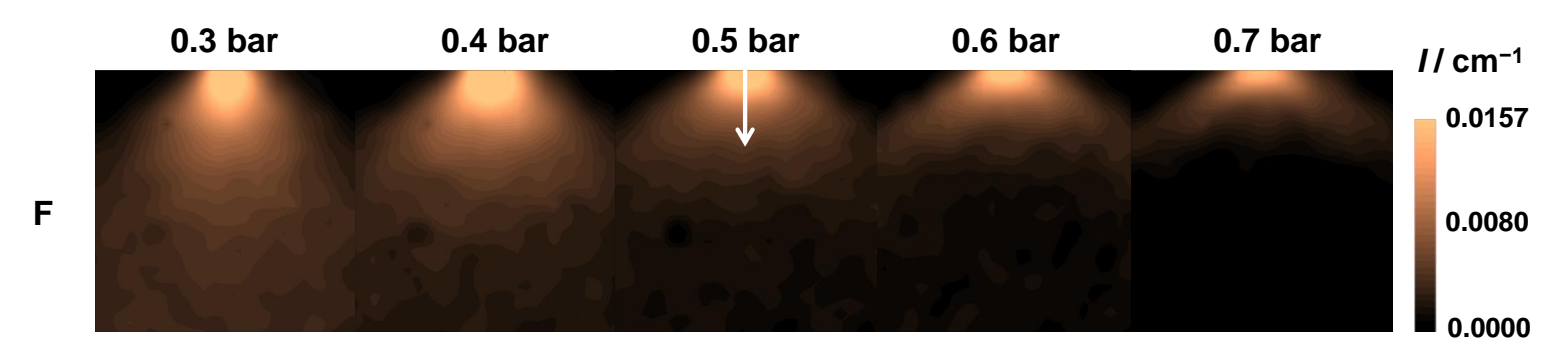

$(\mathrm{FF})_{n}$
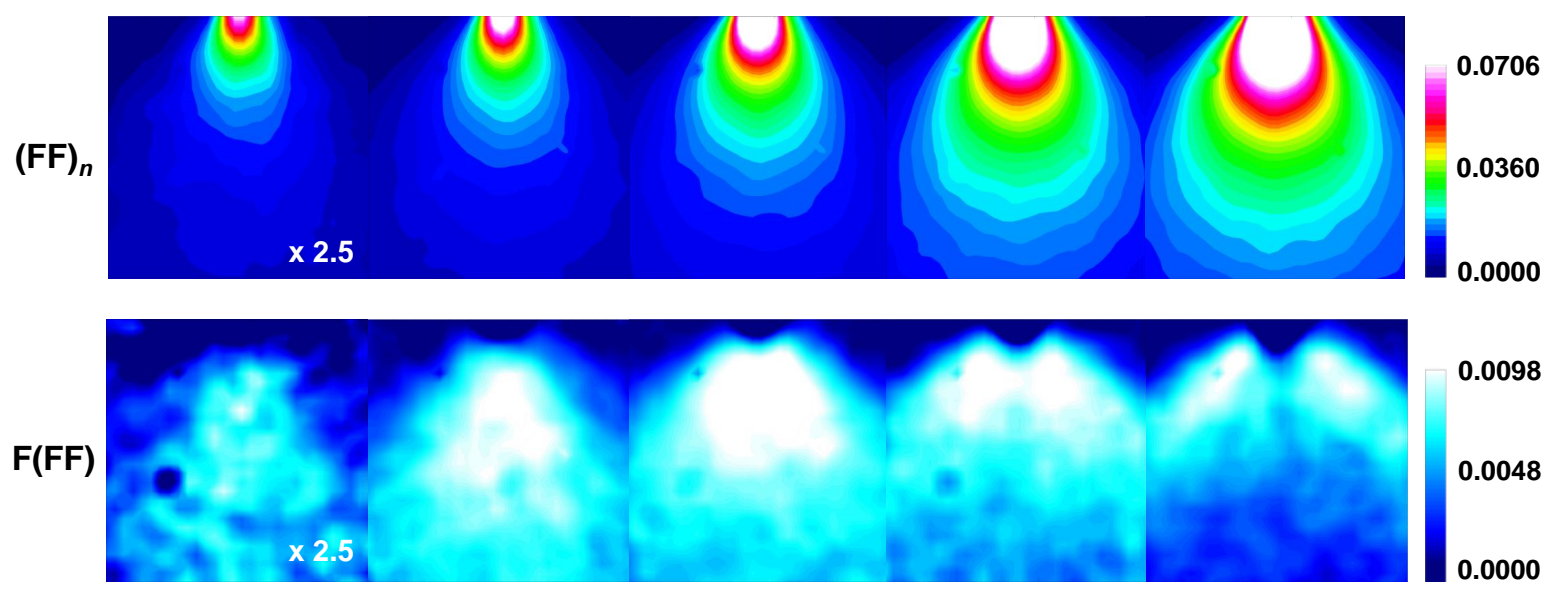

Figure 6.4: Two dimensional (2D) space-resolved integrated band intensity of bands of formic acid monomers $\mathrm{F}$, dimers and dimer assemblies $(\mathrm{FF})_{n}$, and trimers $\mathrm{F}(\mathrm{FF})$, measured in FTIR jet spectra of formic acid $(c<0.2 \%)$ seeded in helium in the $\mathrm{C}=\mathrm{O}$ stretching region. The reservoir pressure increases from left to right from $0.3-0.7$ bar. The propagation of the supersonic flow is indicated by an arrow. The image size is $(10 \times 10) \mathrm{mm}^{2}$. Adapted from J. Chem. Phys. 147, 144305 (2017) (Ref. [185]), with the permission of AIP Publishing.

dimer FF, which is an intermediate in the cyclic dimer formation [82]. Another example of such a "reactive" species is the trimer $\mathrm{F}(\mathrm{FF})$. The spatially resolved integrated intensity of the band attributed to its docking vibration is plotted in the last row of Figure 6.4. Contrary to the monomer and dimer, the potential trimer signal is not seen directly behind the nozzle. This species forms with some distance to it, that varies only slightly with reservoir pressure. Its signal maximum is reached at a reservoir pressure of 500 mbar. If the pressure is increased further, a depletion along the expansion centre is observed. Similar to the monomer, it is only present at the lower density sides of the flow at 700 mbar. This area of stability is expected for trimers, as the number of collisions is lower and predominantly monomers and dimers are present, out of which the trimers are formed. If the collision frequency is too high, the trimers likely aggregate further to form more stable, even-numbered (dimer containing) clusters, as observed along the propagation axis of the flow. As the polar dimer is an intermediate in the cyclic dimer formation [82], its spatial distribution in the expansion should resemble that of (FF) more closely. Thus, the aggregation behaviour fully supports a dominant trimer assignment.

As detailed in Section 2.2, supersonic expansions exhibit several areas that vary distinctly in Mach number (ratio of flow velocity with respect to sonic velocity). So far, only 


\section{Aggregation Dynamics: Trimers and Metastable Dimers}

the high collision frequency region behind the nozzle has been probed. It is followed by the zone of silence where the number of collisions approaches zero and the flow reaches its terminal velocity (largest Mach number). That part of the expansion is usually chosen for spectroscopic interrogation. As a result of its supersonic speed, the supersonic flow is blind towards the boundaries imposed by the background it is expanded into (cf. Section 2) [56]. Once its density falls below $p_{\mathrm{BG}}$ (overexpansion), it is rapidly confined to these boundaries via collisions with the background gas molecules [56, 123]. As a consequence, the supersonic flow is re-compressed and a shock wave is formed. Shock waves enclose the flow sideways and in propagation direction (see Figure 2.2). The former are called lateral and the latter normal (or frontal) shock waves. These areas are characterised by a rapid change in various quantities such as the entropy, particle density, number of collisions, and temperature, all of which increase. The simultaneous increase of particle density and temperature makes shock waves particularly interesting for studying aggregation dynamics, because the former is promoting cluster formation, whereas the latter is antagonising cluster stability.

In order to examine shock waves with FTIR imaging, these need to be inside the imaged $(10 \times 10) \mathrm{mm}^{2}$ area. As detailed in Section 2.2, at small pressure ratios ${ }^{2}$, the distance depends approximately linearly on the ratio of reservoir and background pressure $p_{0} / p_{\mathrm{BG}}[120,125]$ (Equation 2.10). Hence, increasing the background pressure while lowering the reservoir pressure is one way of moving the shock waves into the image. Here, $p_{0}$ is lowered to $300 \mathrm{mbar}$ and $p_{\mathrm{BG}}$ is increased to $10-20 \mathrm{mbar}$ by manually restricting the pumping speed with a butterfly valve. According to Equation 2.10, this yields shock wave distances of $y_{\mathrm{M}}=5.6 \mathrm{~mm}(10 \mathrm{mbar})$ and $2.8 \mathrm{~mm}(20 \mathrm{mbar})$, which are inside the probed area of $(10 \times 10) \mathrm{mm}^{2}$.

In Figure 6.5, the two-dimensional (2D) spatial evolution of the monomer and dimer densities are shown for the two background pressures. The total integrated intensity over the whole $\mathrm{C}=\mathrm{O}$ stretching region is additionally displayed as a measure of the overall substance density (third column of Figure 6.5). The nozzle is located at $x=5 \mathrm{~mm}$ and $y=0 \mathrm{~mm}$. As already seen in Figure 6.4, the particle density is highest at the nozzle and reaches a minimum in the zone of silence. Due to the collisions with background molecules, the density reaches a maximum at the frontal shock wave. With respect to the zone of silence, this increase is approximately three-fold (cf. Figure 6.5). Behind it, the density declines as the particles re-expand. The estimated shock wave distance $\left(y_{\mathrm{M}}=5.6 \mathrm{~mm}\right)$ roughly matches the onset of the density increase. At the higher background pressure, the zone of silence has shrunken considerably. The expansion is compressed to a distinctly smaller area and several density maxima can be seen, i.e., several re-expansions and re-compressions. Further details on such shock wave oscillations can be found in Refs. [124, 137-141]. The distance of the normal shock wave again roughly matches its prediction $(2.8 \mathrm{~mm})$. The density maximum at the nozzle is smaller than at the first shock wave in case of the 20 mbar expansion. This is a result of the data preprocessing rather than a physical effect. For smoothing of the data,

\footnotetext{
${ }^{2} \mathrm{~A}$ small pressure ratio is defined via the shock wave distance $y_{\mathrm{M}}$ with $y_{\mathrm{M}}<(L / D)^{0.94} \cdot D$ in Ref.
} [125]. With the nozzle used here $(L=20 \mathrm{~mm}$ and $D=0.5 \mathrm{~mm})$, one obtains $y_{\mathrm{M}}<16 \mathrm{~mm}$. 


\section{Aggregation Dynamics: Trimers and Metastable Dimers}
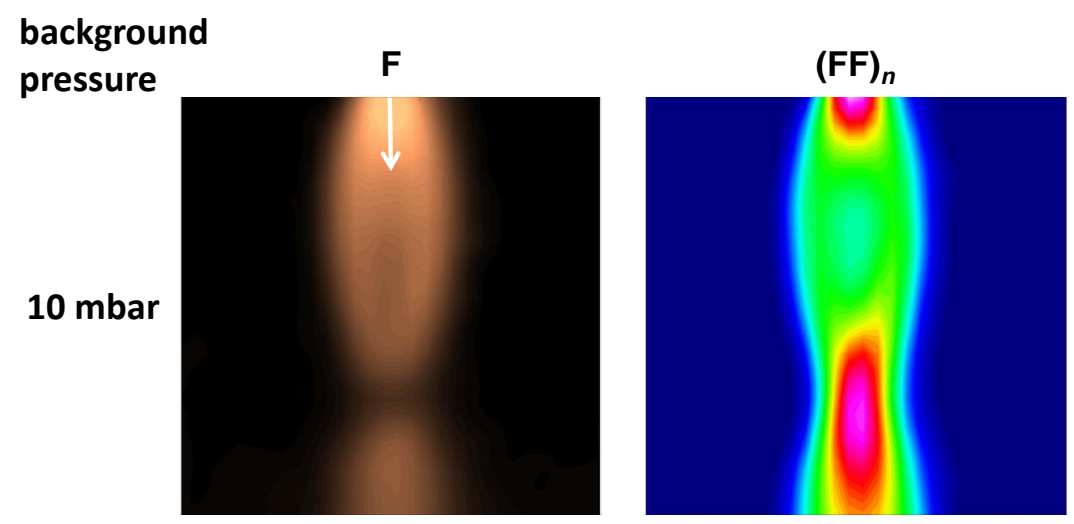

\section{total integrated intensity}
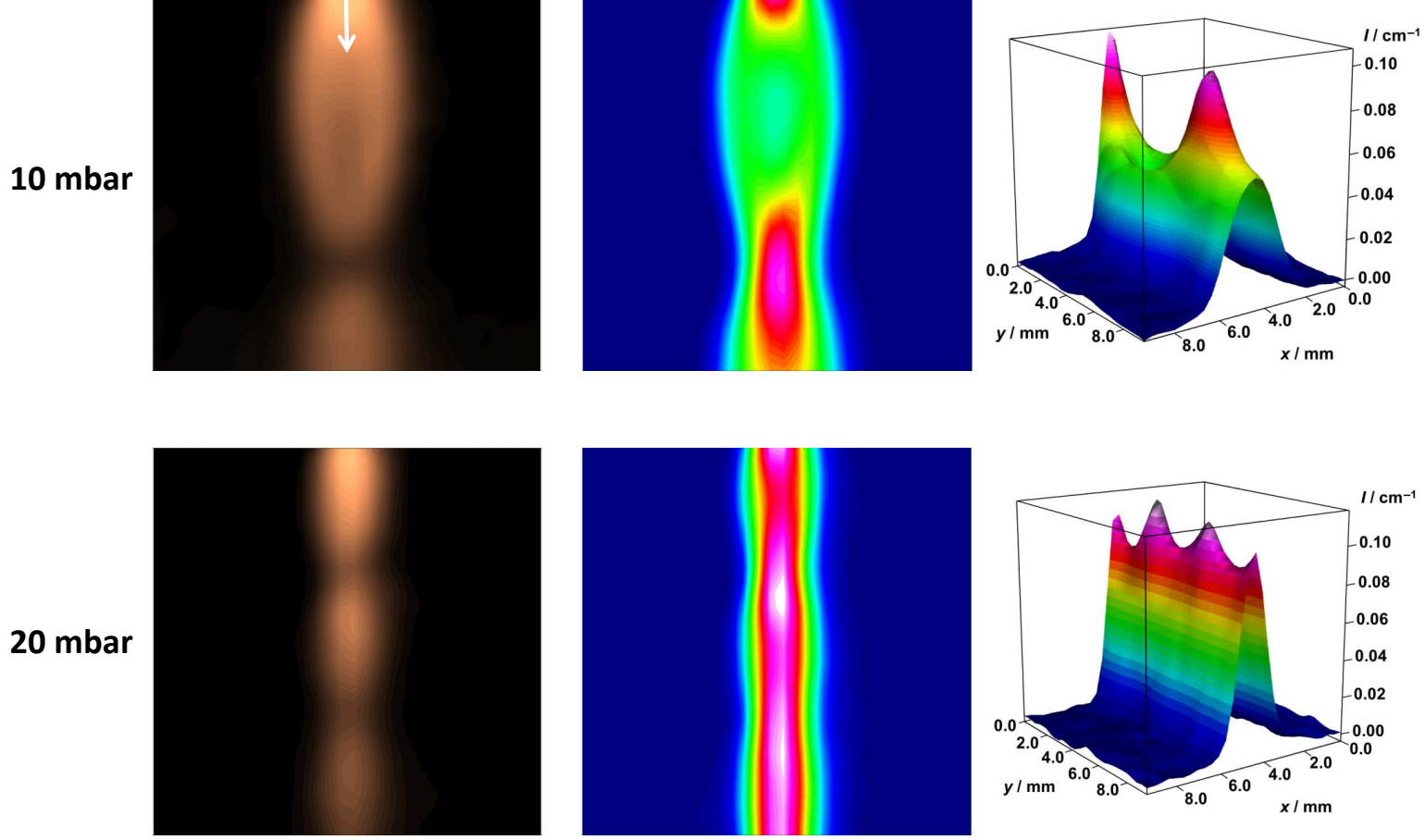

Figure 6.5: $2 \mathrm{D}$ evolution of the integrated band intensity of a formic acid monomer band $\mathrm{F}$ and a band of dimers and larger dimer assemblies $(\mathrm{FF})_{n}$ in the $\mathrm{C}=\mathrm{O}$ stretching region. The third column shows the spatial evolution of the band integral over the whole $\mathrm{C}=\mathrm{O}$ stretching region in a three dimensional representation. The space-resolved FTIR jet spectra have been recorded at a reservoir pressure of 300 mbar, a formic acid concentration of $c<0.2 \%$, and background pressures of $10 \mathrm{mbar}$ (top row) and $20 \mathrm{mbar}$ (bottom row). The image size amounts to $(10 \times 10) \mathrm{mm}^{2}$. The arrow illustrates the propagation direction of the supersonic expansion. Adapted from J. Chem. Phys. 147, 144305 (2017) (Ref. [185]), with the permission of AIP Publishing.

a Gauss binning is performed (Figure 3.6, in Section 3.1.1), where the spectra of the pixel are weighted with their neighbours with Gauss-type scaling factors. The spectra of the four nearest neighbours are weighed by 0.7 and those of the four diagonal pixels by 0.5 . As the outmost pixel ring has no adjacent pixels, these are neglected, which reduces the number of pixels/spectra. This applies to the spectra at the nozzle. Thus, the seemingly lower density at the nozzle is a direct result of discarding these spectra.

The spatially resolved integrated intensity of the formic acid monomer is highest at the nozzle and reaches a minimum at the frontal shock wave, whereas the dimer band integral reaches a maximum. Dimer formation is favoured at the shock wave, as it depends quadratically on pressure. This re-formation of dimers consumes monomers, which causes the phase shift of their densities. The associated temperature increase at the shock wave has been studied in more detail by Zischang and Suhm in expansions 


\section{Aggregation Dynamics: Trimers and Metastable Dimers}

of nitrous oxide in helium [120]. The estimated rotational temperature observed at the normal shock wave under similar conditions is about $230 \mathrm{~K}$, which corresponds to a $150 \mathrm{~K}$ temperature increase with respect to the zone of silence [120]. At 20 mbar, it seems that the density minimum of the monomer at the shock wave is slightly shifted from the density maximum of the dimer, likely because the re-cooling after the compression also favours cluster formation. Altogether, vibrational spectroscopy at shock waves is beneficial for scrutinising cluster stabilities. Unfortunately, the formic acid trimer does not form significantly under these adjusted conditions (smaller reservoir pressure and higher background pressure). Studying this species would be particularly interesting, as it will likely show a very different aggregation behaviour than the dimer.

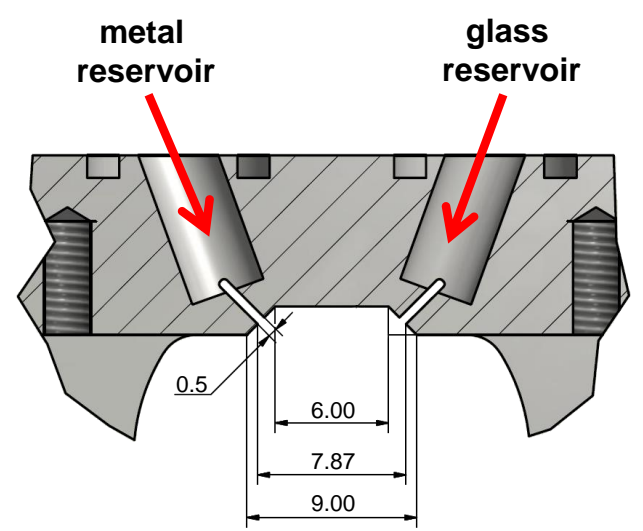

Figure 6.6: Technical drawing (by R. Hildebrandt) of the double slit nozzle, displayed previously in Section 3.1.1 as part of Figure 3.2. All dimensions are given in mm. Adapted from J. Chem. Phys. 147, 144305 (2017) (Ref. [185]), with the permission of AIP Publishing.

For this purpose, a double slit nozzle was developed, which is shown in Figure 6.6. The two slits are converging in a $90^{\circ}$ angle and each of them can be connected to a different reservoir, so that two substance-in-helium mixtures can be expanded at the same time. The distance of the two slits $(7.5 \mathrm{~mm})$ has been chosen such that both expansions collide within the $(10 \times 10) \mathrm{mm}^{2}$ large imaged area. When the two expansions interfere, the density and consequently also the number of collisions and the temperature increase, as their two lateral shock waves interact, as detailed in Section 3.1.1. In this collision zone, cluster stability can be probed without adjusting the conditions such as reservoir or background pressure. In fact, the optimised conditions for trimer formation from Figure 6.4 (reservoir pressure of $500 \mathrm{mbar}$ ) have been chosen. The results are depicted in Figure 6.7. In the left column of that Figure, an unimpaired expansion of formic acid in helium is shown. As expected from the chosen conditions, the $2 \mathrm{D}$ evolutions of the monomer, dimer, and trimer densities match those in Figure 6.4. For the simultaneous expansion of two supersonic jets, a pure helium jet is chosen as the counterpart, so that the flow rates are comparable and the expansion of interest can be studied without spectral interference.

The formic acid dimer band integral is by far largest and does not diminish in the shock wave, so that it is the best measure of overall density. In Figure 6.7, it can 


\section{Aggregation Dynamics: Trimers and Metastable Dimers}

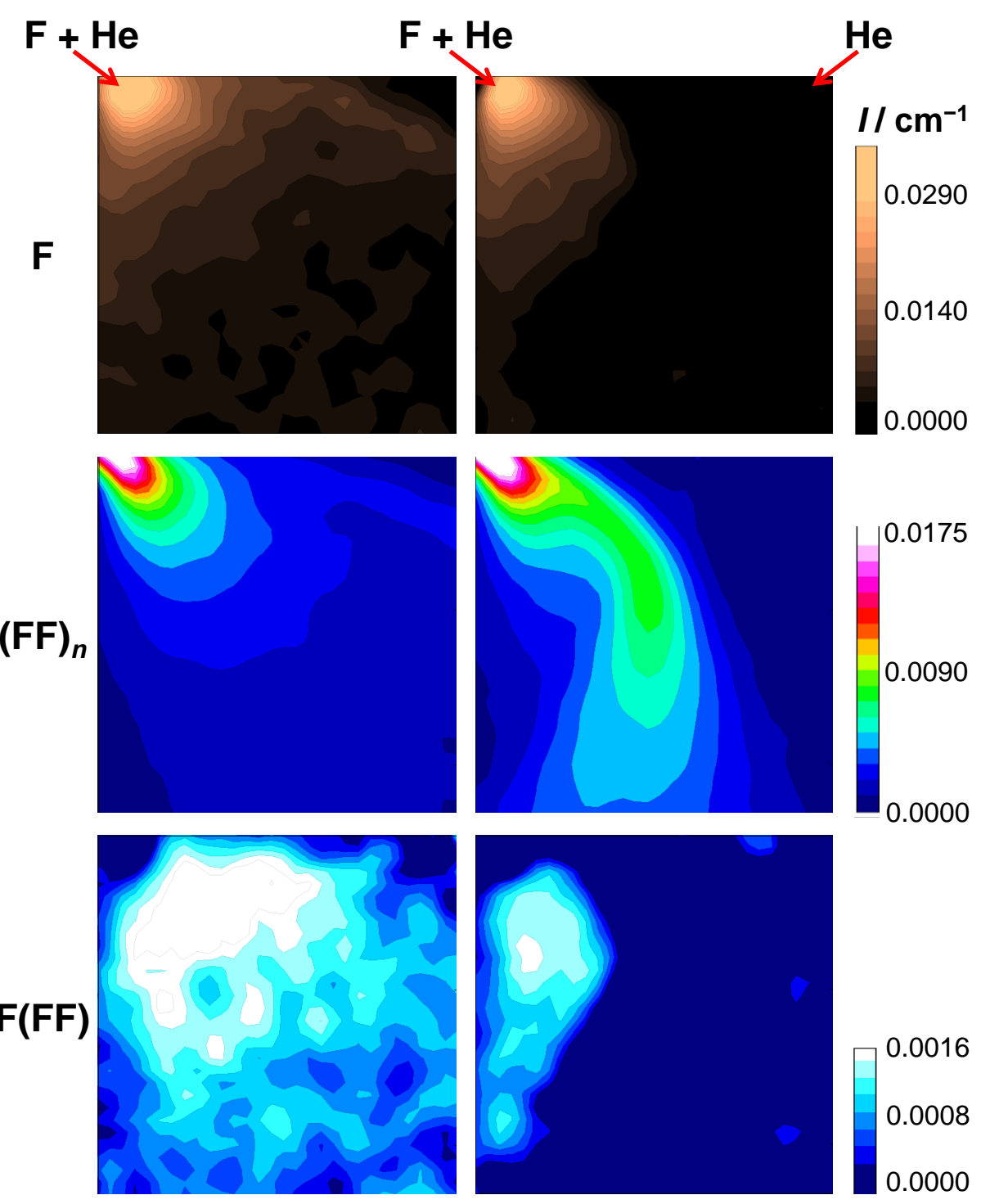

Figure 6.7: Space-resolved two dimensional evolution of the integrated band intensity of formic acid monomers $\mathrm{F}$, dimers and dimer assemblies $(\mathrm{FF})_{n}$, and trimers $\mathrm{F}(\mathrm{FF})$ measured in FTIR jet spectra of formic acid $(c<0.2 \%)$ in helium at a reservoir pressure of 500 mbar averaged over 260 (left columm) and 150 scans (right column). The first column shows a single slit expansion and the second column the interference of that with a second, pure helium expansion. The size of each image amounts to $(10 \times 10) \mathrm{mm}^{2}$. Adapted from J. Chem. Phys. 147, $144305(2017)$ (Ref. [185]), with the permission of AIP Publishing.

be seen that the substance-in-helium expansion is deflected by the second expansion. Thus, mixing of both is unlikely due to viscous flow, as mentioned in Section 3.1.1. At the point where the deflection starts, i.e., the collision zone, the dimer density remains approximately constant. In the single slit experiment, it has dropped considerably due to the overall density decrease. The compression by the second expansion results in a 


\section{Aggregation Dynamics: Trimers and Metastable Dimers}

higher number of collisions so that the formation dimers and larger dimer assemblies is favoured. The monomer density drops to zero, as it is consumed in the formation of these larger clusters. Monomer density can only be found directly behind the nozzle and in the unimpaired left side of the expansion, where both temperature and density are lower. In fact, this unperturbed left part of the substance expansion is analogous to the single slit expansion, also in terms of density decrease.

The increase of the rotational temperature in the collision zone of the double expansion can be estimated from the distance of the maxima of the rotational branches $\Delta \tilde{\nu}_{\mathrm{PR}}^{\max }$ of the formic acid monomer band, which is proportional to the square root of the temperature [127], as detailed in Section 2. At room temperature, it amounts to $29 \mathrm{~cm}^{-1}$ (Figure A.19 in Appendix A.4). In Figure 6.8, two spectra of formic acid are shown, which have been averaged over the expansion areas shown as black and red boxes in the insets. $\Delta \tilde{\nu}_{\mathrm{PR}}^{\max }$ amounts to $8 \mathrm{~cm}^{-1}$ in the colder spectrum of the single slit expansion and increases to $20 \mathrm{~cm}^{-1}$ just before the interaction zone in the double slit expansion. Similar changes are seen for the formic acid dimer $\mathrm{P}$ and $\mathrm{R}$ branches. Assuming a room temperature of $300 \mathrm{~K}$, the $\Delta \tilde{\nu}_{\mathrm{PR}}^{\max }$ values of the monomer translate into rotational temperatures of $23 \mathrm{~K}$ and $143 \mathrm{~K}$, respectively. As the formic acid monomer band diminishes when both jets interfere, the temperature in that zone has to be determined with another reference system. Here, nitrous oxide $\mathrm{N}_{2} \mathrm{O}$ is used, as outlined in Section 3.1.1. The temperature was found to rise to about $(230 \pm 30) \mathrm{K}$ upon collision of the two jets (cf. Figure 3.3), which is similar to the temperature estimated in normal shock waves [120].

The formic acid trimer density approaches zero when the two expansions collide -

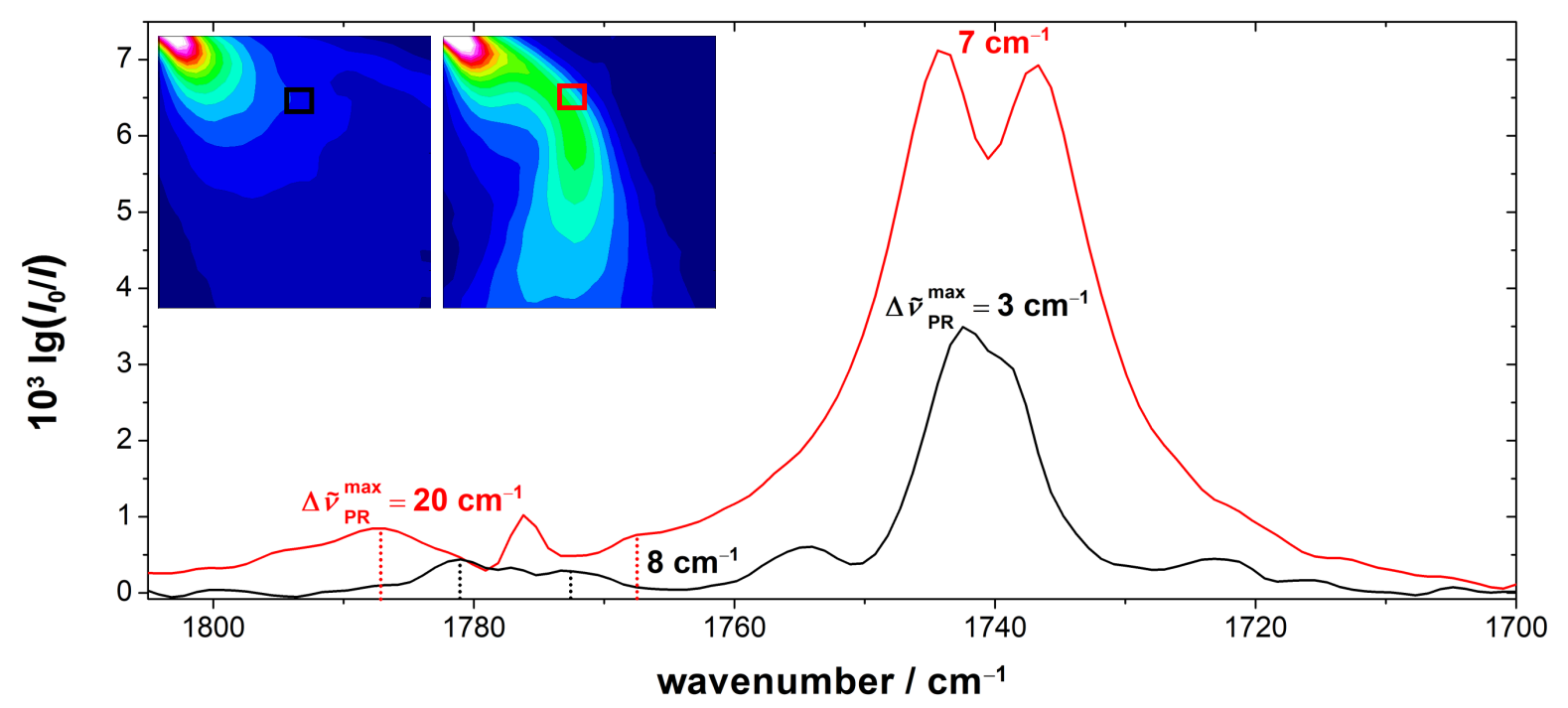

Figure 6.8: FTIR jet spectra of formic acid $(c<0.2 \%)$ in helium at a reservoir pressure of 500 mbar spatially averaged over $3 \times 3$ binned pixel blocks shown as black and red boxes in the insets. The number of scans amounts to 260 (black spectrum) and 150 (red spectrum). Additionally, the distance of the maxima of the $\mathrm{P}$ and $\mathrm{R}$ branches of the formic acid monomer and dimer are listed. 


\section{Aggregation Dynamics: Trimers and Metastable Dimers}

presumably as a result of the high number of collisions that favour the formation of dimer assemblies $(\mathrm{FF})_{n}$ and/or the temperature increase that causes its dissociation. Therefore, these collision experiments can be used to distinguish metastable from stable clusters. In line with the single slit expansions at high reservoir pressures (Figure 6.4), the trimer is stable in the less dense and colder parts of the expansion, in this case, the unperturbed, left side of the flow.

Altogether, the space-resolved visualisation of the monomer, dimer, and trimer densities in supersonic expansions allows for a more in-depth understanding of aggregation processes that helps to validate assignments, like in this case assignment of the formic acid trimer $\mathrm{F}(\mathrm{FF})$. Several more examples are given in the next chapter. With conventional FTIR jet spectroscopy only the average over a larger expansion area is obtained. If spectra under the conditions shown in Figure 6.4 were recorded at a reservoir pressures of 700 mbar, trimer bands might not have been observed or just very weakly, even though the trimer is present in the expansions, yet not necessarily in the field of view. Thus, FTIR imaging can also serve as a guide as to where to record spectra in supersonic expansions and can help to optimise conditions, which is particularly useful for experiments that do not cover a large expansion area like the Raman jet spectra.

\subsection{Acetic and Pivalic Acid Trimers}

\subsubsection{Comparison to the Formic Acid Spectra}

After the thorough discussion of the FTIR and Raman spectra of the formic acid, the rest of this chapter is focussed on understanding the differences and similarities to acetic and pivalic acid. The FTIR and Raman jet spectra of the three acids are shown in Figure 6.9.

In an analysis of the FTIR jet spectra of acetic acid by Häber and co-workers [117, 440] as well as Emmeluth and co-workers [71, 116], three types of unknown bands were identified in several spectral regions, labelled $\mathrm{X}, \mathrm{Y}$, and $\mathrm{Z}$. In the $\mathrm{C}=\mathrm{O}$ stretching region, these are located at $1834 \mathrm{~cm}^{-1}(\mathrm{Z}), 1769 \mathrm{~cm}^{-1}(\mathrm{X}), 1749 \mathrm{~cm}^{-1}(\mathrm{Y})$, and $1721 \mathrm{~cm}^{-1}(\mathrm{X})$, i.e., one band $(\mathrm{Z})$ is upshifted with respect to the monomer band $\mathrm{A}\left(1793 \mathrm{~cm}^{-1}\right)$, two are located in-between $\mathrm{A}$ and $(\mathrm{AA})_{\mathrm{a}}(\mathrm{X}$ and $\mathrm{Y})$, and one is found on the lower-wavenumber side of $(\mathrm{AA})_{\mathrm{a}}(\mathrm{X})$. As seen in Figure 6.9, the formic acid trimer bands can be found at similar positions, with the difference that the acetic acid spectrum exhibits an additional peak between monomer and dimer. The same applies to pivalic acid, but its spectrum exhibits two bands downshifted from $(\mathrm{PP})_{\mathrm{a}}$. In conclusion, the most common features of the FTIR spectra of the three acids are the bands upshifted from the monomer, hereinafter called $\mathrm{Z}$ bands, a band about $20 \mathrm{~cm}^{-1}$ downshifted from it (X band in case of acetic acid, docking vibration of $\mathrm{F}(\mathrm{FF})$ ), and at least one band on the lower-wavenumber side of the antisymmetric $\mathrm{C}=\mathrm{O}$ stretching vibration of the cyclic dimer (second $\mathrm{X}$ band of acetic acid, $\left.\mathrm{F}(\mathrm{FF})_{\mathrm{a}}\right)$.

In all Raman spectra (Figure 6.9), the band shifted by about $-20 \mathrm{~cm}^{-1}$ from the monomer is also present. Moreover, all spectra feature a weak broad signal between 


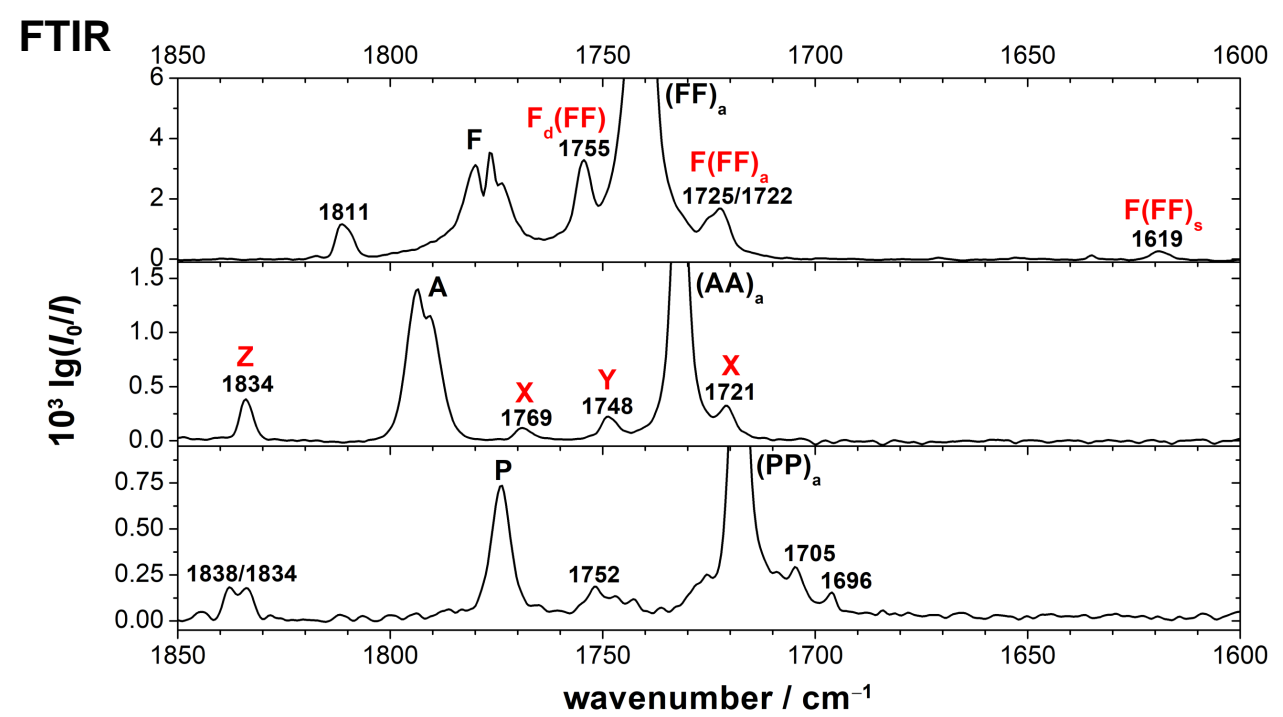

\section{Raman}

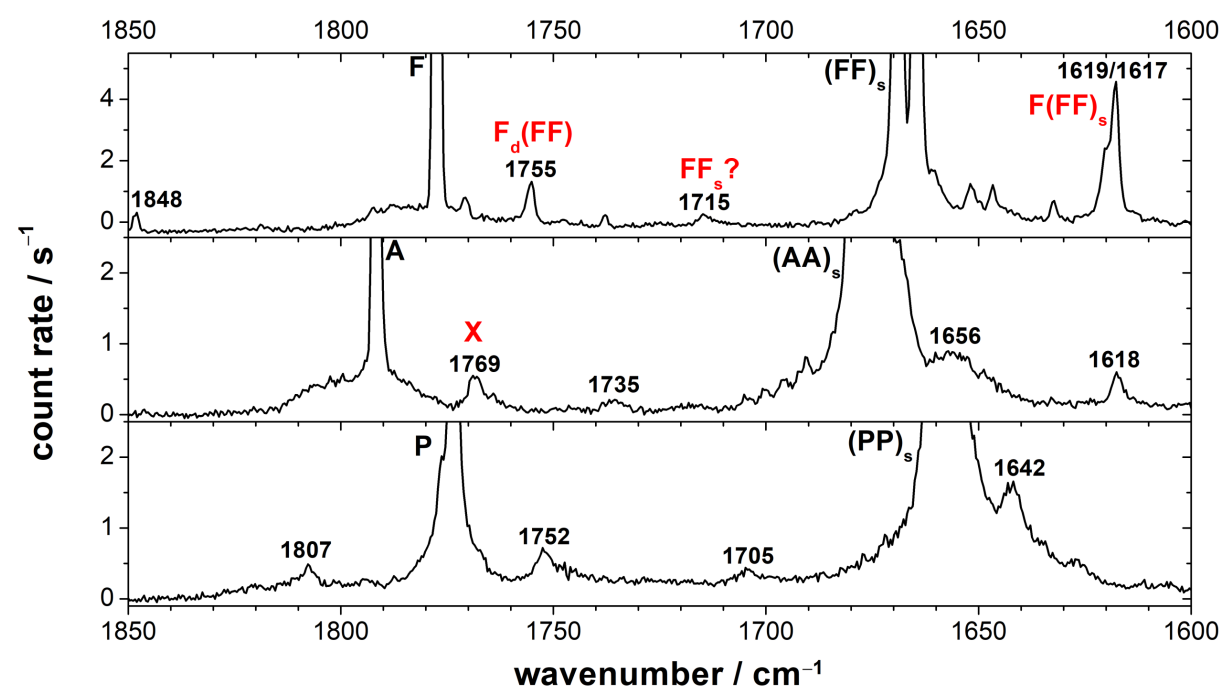

Figure 6.9: Top: FTIR jet spectra of formic $(c<0.1 \%, 560$ mbar, 50 scans $)$, acetic $(c<0.1 \%$, 750 mbar, 90 scans), and pivalic acid ( $c<0.1 \%, 500$ mbar, 100 scans $)$ in the $\mathrm{C}=\mathrm{O}$ stretching region. Bottom: Raman jet spectra of formic $(c<0.1 \%, 700 \mathrm{mbar}$, data acquisition time: $10 \times 180 \mathrm{~s})$, acetic $(c<0.1 \%, 700 \mathrm{mbar}$, data acquisition time: $6 \times 300 \mathrm{~s})$, and pivalic acid $\left(c<0.2 \%, 500\right.$ mbar, data acquisition time: $\left.14 \times 120 \mathrm{~s}, \theta_{\mathrm{D}}=60{ }^{\circ} \mathrm{C}\right)$ in the $\mathrm{C}=\mathrm{O}$ stretching region. The X, Y, Z labels are taken from Refs. [71, 116]. Previously assigned formic acid bands are shown in red. ? marks tentative assignments.

$1740-1700 \mathrm{~cm}^{-1}$. In case of acetic and pivalic acid, a lower-wavenumber shoulder of the symmetric $\mathrm{C}=\mathrm{O}$ stretching vibration of the cyclic dimer is seen. Overall, this means that the formed clusters of the acids must bear at least some resemblance. The $\mathrm{Z}$ bands are not due to the higher-energy dimer or trimer, as the bands of these clusters are predicted downshifted from the monomer band, rather than upshifted. As such, the Z bands will be discussed separately at the end of this chapter. 


\section{Aggregation Dynamics: Trimers and Metastable Dimers}

\subsubsection{Acetic Acid Trimers}

As mentioned in the previous chapter and section, the higher-energy acetic acid dimer $\mathrm{A}_{\mathrm{C}} \mathrm{A}$ exhibits the same structure motif as the formic acid dimer $\mathrm{F}_{\mathrm{C}} \mathrm{F}$ (Figure 6.1). The same applies to the global minimum of the acetic acid trimer (Figure 6.1) [79]. Contrary to the formic acid trimer, however, the $\mathrm{A}_{\mathrm{C}}\left(\mathrm{A}_{\mathrm{C}} \mathrm{A}_{\mathrm{C}}\right)$ trimer is not planar, as the docking monomer binds to an out-of-plane hydrogen atom of the methyl group (see inlet of Figure 6.10). Local minimum structures of the acetic acid trimer were calculated, but are about $6 \mathrm{~kJ} \mathrm{~mol}^{-1}$ higher in energy and are kinetically not likely to be trapped and therefore not considered further. The structures and their relative energies can be found in Appendix A.4.2 alongside those of the dimer. Again, $\mathrm{A}_{C}\left(\mathrm{~A}_{\mathrm{C}} \mathrm{A}_{\mathrm{C}}\right)$ is shortened to $\mathrm{A}(\mathrm{AA})$ for the rest of this section. The same applies for $\mathrm{A}_{\mathrm{C}} \mathrm{A}$, which reduces to $\mathrm{AA}$.

The FTIR and Raman jet spectra of acetic acid are shown in Figure 6.10. To distinguish cluster sizes, the intensity of all spectra has been scaled to the respective acetic acid dimer band $(\mathrm{AA})_{\mathrm{a}} /(\mathrm{AA})_{\mathrm{s}}$ in the spectrum of lowest concentration. All bands that increase faster in intensity than the dimer can be attributed to larger clusters, whereas the monomer intensity shows the reverse order - it exhibits the highest intensity in the spectrum of lowest concentration. From the scaling behaviour, it seems that the $\mathrm{X}$ bands in the FTIR spectra at $1769 \mathrm{~cm}^{-1}$ and $1721 \mathrm{~cm}^{-1}$ can be attributed to clusters larger than the dimer. Considering the relatively low concentrations, presumably trimers. The $\mathrm{Y}$ band at $1749 \mathrm{~cm}^{-1}$ shows the scaling behaviour of a dimer.

For further validation, harmonically calculated, $(\mathrm{FF})_{\mathrm{a}}$-scaled band positions of the acetic acid monomer A, local AA and global minimum dimer (AA), as well as trimer $\mathrm{A}(\mathrm{AA})$ are plotted below the spectra. The band positions of the monomer $\mathrm{A}$ as well as the global minimum dimer $(\mathrm{AA})_{\mathrm{a}}$ agree reasonably well with experiment, though both are slightly underestimated by the $(\mathrm{FF})_{\mathrm{a}}$-scaling (cf. Table 6.2). For some bands like (AA) the $(\mathrm{FF})_{\mathrm{a}}$-scaling shows a superior agreement. Thus, it is kept for consistency throughout this chapter. The two dominantly IR active trimer vibrations $\mathrm{A}_{\mathrm{d}}(\mathrm{AA})$ and $\mathrm{A}(\mathrm{AA})_{\mathrm{a}}$ are predicted at $1754 \mathrm{~cm}^{-1}$ and $1714 \mathrm{~cm}^{-1}$, respectively. The former deviates by $-15 \mathrm{~cm}^{-1}$ from the band at $1769 \mathrm{~cm}^{-1}$ and the latter by $-7 \mathrm{~cm}^{-1}$ from the band downshifted from $(\mathrm{AA})_{\mathrm{a}}$ at $1721 \mathrm{~cm}^{-1}$. As mentioned above, the agreement could be slightly improved by $(\mathrm{AA})_{\mathrm{a}}$-scaling. These discrepancies are large, however, the prediction of the distance between both bands exhibits a moderate deviation of $-8 \mathrm{~cm}^{-1}$ from the experimental value $\left(48 \mathrm{~cm}^{-1}\right)$. The band at $1769 \mathrm{~cm}^{-1}$ is also featured in the Raman spectra (bottom panel of Figure 6.10), which endorses the $\mathrm{A}_{\mathrm{d}}(\mathrm{AA})$ assignment, as $\mathrm{AA}_{\mathrm{a}}$ exhibits a nearzero Raman scattering cross-section. The symmetric $\mathrm{C}=\mathrm{O}$ stretching vibration of the trimer is predominantly Raman active. Near its predicted band position $\left(1643 \mathrm{~cm}^{-1}\right)$, two broad shoulders of the dimer band $(\mathrm{AA})_{\mathrm{s}}$ can be seen that increase faster in intensity than the dimer. These are located between $1673-1664 \mathrm{~cm}^{-1}$ and $1660-1637 \mathrm{~cm}^{-1}$. The latter is more pronounced at lower concentrations and the experimental band integral ratio with respect to $A_{d}(A A)$ at $1769 \mathrm{~cm}^{-1}$ roughly matches the expected ratio of about three, so that it is tentatively assigned to $\mathrm{A}(\mathrm{AA})_{\mathrm{s}}$. The band between $1673-1664 \mathrm{~cm}^{-1}$ could be due to a larger cluster such as a tetramer, which are composed of stacked dimers, so that their $\mathrm{C}=\mathrm{O}$ stretching vibrations are expected to be similar to those of 


\section{Aggregation Dynamics: Trimers and Metastable Dimers}

the dimers [119]. The band at $1618 \mathrm{~cm}^{-1}$ is close to the experimental band position of $\mathrm{F}(\mathrm{FF})_{\mathrm{s}}\left(1619 / 1617 \mathrm{~cm}^{-1}\right)$, yet its intensity with respect to the band at $1769 \mathrm{~cm}^{-1}$ is much smaller than expected. It could be a combination band or overtone of the acetic acid dimer. VPT2 calculations of the acetic acid dimer are not very reliable due to the large amplitude methyl torsions [79] and predict several combination bands in that spectral region. Thus, the origin of this band remains unknown. The tentatively assigned acetic acid trimer A(AA) band positions are summarised in Table 6.2.

The calculated band position of the antisymmetric $\mathrm{C}=\mathrm{O}$ stretching vibration of the metastable dimer AA $\left(1762 \mathrm{~cm}^{-1}\right)$ deviates by $+13 \mathrm{~cm}^{-1}$ from the $\mathrm{Y}$ band at $1749 \mathrm{~cm}^{-1}$. The symmetric $\mathrm{C}=\mathrm{O}$ stretching vibration of that cluster $\mathrm{AA}_{\mathrm{s}}$ is predominantly Raman active. Around its predicted band position $\left(1735 \mathrm{~cm}^{-1}\right)$, a very broad, weak band can be seen with maximum at $1736 \mathrm{~cm}^{-1}$, which would partially overlap with the $(\mathrm{AA})_{\mathrm{a}}$ band in the FTIR spectra. A similarly small band position deviation was seen for the corresponding formic acid cluster. Hence, the band position difference of the two AA bands $\left(13 \mathrm{~cm}^{-1}\right)$ might be severely overestimated $\left(+14 \mathrm{~cm}^{-1}\right.$, a factor of 2$)$ by the B3LYP-D3(BJ)/def2-TZVP calculations. Another possible explanation of the Y band $\left(1749 \mathrm{~cm}^{-1}\right)$ in the FTIR spectra could be a Fermi resonance with $(\mathrm{AA})_{\mathrm{a}}$. Exploratory VPT2 calculations of the dimer (AA) did not predict such a resonance, yet their validity is somewhat limited, as aforementioned. Perhaps the more convincing argument is that Oswald and co-workers have identified a significant amount of the metastable dimer AA in FTIR jet expansions via the shift of the $\mathrm{OH}$ band upon nitrogen complexation. These spectra have been recorded at the same set-up (filet jet, Section 3.1.2) and similar concentrations, so that its spectral features must also be present in the $\mathrm{C}=\mathrm{O}$ stretching region, if the assignment was correct. Hence, for further verification, the abundance of that cluster in the $\mathrm{OH}$ stretching region can be compared to that in the $\mathrm{C}=\mathrm{O}$ stretching region.

As discussed before, the $\mathrm{OH}$ stretching region of carboxylic acid dimers exhibits a very complex, broad resonance structure, which overlaps with the $\mathrm{CH}$ stretching vibrations (cf. Figure 5.3). To get an estimate of the abundance of AA with respect to (AA) from their $\mathrm{OH}$ stretching bands, the contribution of the $\mathrm{CH}$ bands must be subtracted. An upper bound of their contribution to the total band integral between $3350-2500 \mathrm{~cm}^{-1}$ (cf. Figure A.23) is estimated to about $22 \%$ via comparison with the OD stretching spectrum of $\mathrm{CH}_{3} \mathrm{COOD}$, which has been measured by Zielke in 2007 [194]. The band of the bound $\mathrm{OH}$ stretching vibration of AA at $3304 \mathrm{~cm}^{-1}$ [79] is overlapping with the onset of the broad $\mathrm{OH}$ stretching region of (AA) (cf. Figure 5.8). To approximate its share, the band at $3304 \mathrm{~cm}^{-1}$ is integrated based on the band width $\left(13 \mathrm{~cm}^{-1}\right)$ of AA observed in the corresponding helium nanodroplet spectrum (Figure 5.8). Overall, this yields roughly $3 \%$ of AA with respect to $(\mathrm{AA})$, if the obtained band integral ratio $(\approx 1 \%)$ is corrected for the predicted IR intensity differences $\left(I_{(\mathrm{AA})} / I_{\mathrm{AA}}=3.00\right)$. In the $\mathrm{C}=\mathrm{O}$ stretching region, the amount of $\mathrm{AA}$ is estimated to roughly $6 \%$ of (AA) based on the tentative assignment. Considering the crudity of the approximation, the agreement is satisfactory, which reinforces the higher-energy dimer assignment of the $\mathrm{Y}$ band. However, in view of the grossly overestimated theoretical harmonic band splitting and the uncertainties of anharmonic calculations for $\mathrm{A}$ and its complexes, alternative 
Table 6.2: Experimental $\tilde{\nu}_{\exp }$ as well as harmonic, $(\mathrm{FF})_{\mathrm{a}}$-scaled band position $\left(\mathrm{in}_{\mathrm{cm}}{ }^{-1}\right)$ of the $\mathrm{C}=\mathrm{O}$ stretching vibrations of acetic acid monomer, the metastable AA and the cyclic dimer (AA) as well as the trimer A(AA), calculated at the B3LYP-D3(BJ)/def2-TZVP level. The scaling factor amounts to 0.983 . The three vibrations are the docking vibration (index d) of the monomer unit of the trimer, as well as the symmetric (s) and antisymmetric (a) $\mathrm{C}=\mathrm{O}$ stretching vibration of the dimer (units). Tentative assignments are printed in italics. In the fourth column, the band position deviations with respect to experiment are listed.

\begin{tabular}{cccc}
\hline \hline & $\tilde{\nu}_{\text {exp }}$ & B3LYP-D3(BJ) & \\
\hline $\mathrm{A}$ & 1793 & 1788 & -5 \\
$(\mathrm{AA})_{\mathrm{a}}$ & 1732 & 1728 & -4 \\
$(\mathrm{AA})_{\mathrm{s}}$ & 1676 & 1673 & -3 \\
$\mathrm{AA}_{\mathrm{a}}$ & 1749 & 1762 & -13 \\
$\mathrm{AA}_{\mathrm{s}}$ & 1736 & 1735 & -1 \\
$\mathrm{~A}_{\mathrm{d}}(\mathrm{AA})$ & 1769 & 1754 & -15 \\
$\mathrm{~A}(\mathrm{AA})_{\mathrm{a}}$ & 1721 & 1714 & -7 \\
$\mathrm{~A}(\mathrm{AA})_{\mathrm{s}}$ & $1660-1637$ & 1641 & \\
\hline \hline
\end{tabular}

and in particular more complex explanations should not be ruled out. The tentatively assigned band positions of AA are summarised in Table 6.2.

The main difference between the FTIR and Raman jet spectra of acetic acid is the differing amount of polar dimer present in the expansions. While similar concentrations of AA and $\mathrm{A}(\mathrm{AA})$ are observed in the FTIR spectra, an order of magnitude less polar dimer can be seen in the corresponding Raman spectra (cf. Figures 6.10 and A.24). This is at first counterintuitive, as this dimer has shown to be an intermediate in the cyclic dimer formation in the case of formic acid [82]. As such, one would expect its highest abundance at low nozzle distances, i.e., in the expansion area probed by the Raman experiment, rather than in the FTIR spectra, which have been averaged over a large expansion area. However, these might capture areas of higher stability of the AA dimers, as mentioned above, which would explain these differences. For further conclusions, an FTIR imaging study of the X and Y bands of acetic acid would be particularly insightful. Unfortunately, the signals are too weak for an analysis under trimer optimised conditions. If the concentrations are significantly enhanced, dimer assemblies start to dominate, which will be discussed in the next chapter. Altogether, this highlights the distinct advantages of formic acid for such aggregation dynamic studies.

The corresponding Raman jet spectra of formic acid (Figure 6.3) look fairly similar to those of acetic acid - the main difference is the well-separated band of the symmetric $\mathrm{C}=\mathrm{O}$ stretching vibration of the formic acid trimer $\mathrm{F}(\mathrm{FF})_{\mathrm{s}}$. The FTIR spectra of both acids, on the other hand, differ more pronouncedly due to the strong additional Y band. However, since $\mathrm{FF}$ and $\mathrm{F}(\mathrm{FF})$ are predicted to overlap, these spectral differences might not necessarily reflect changes in aggregation behaviour. 


\section{Aggregation Dynamics: Trimers and Metastable Dimers}
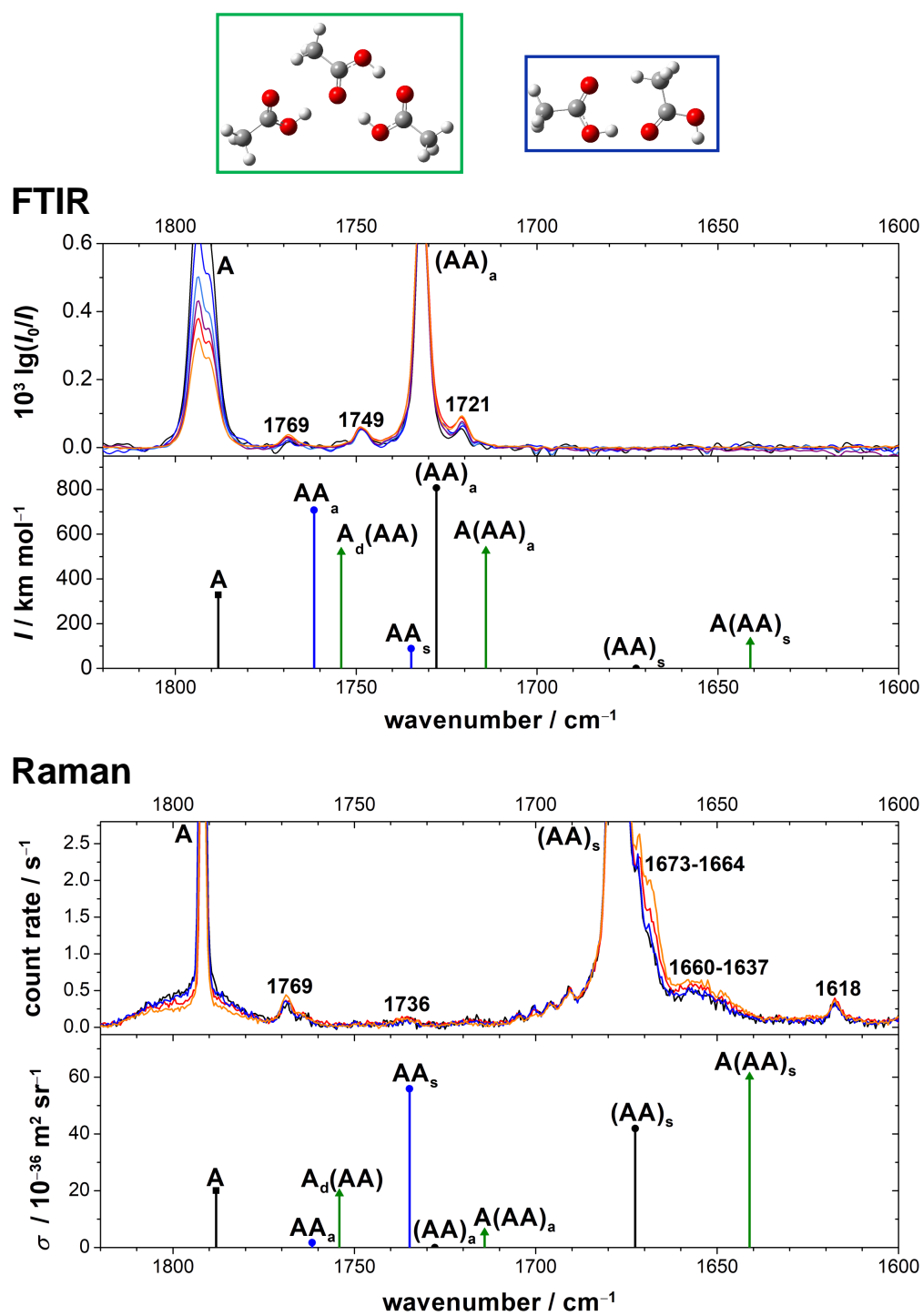

Figure 6.10: Top: FTIR jet spectra of acetic acid in helium in the $\mathrm{C}=\mathrm{O}$ stretching region at a reservoir pressure of $560-750$ mbar with concentrations of $<0.02-0.06 \%$. Each spectrum has been averaged over $40-50$ scans. The intensity of all spectra has been scaled to (AA)a. The scaling factors amount to 1.0 (black spectrum), 0.65 (blue), 0.45 (light blue), 0.35 (purple), 0.27 (red), and 0.22 (orange), whereby the smallest scaling factor corresponds to the spectrum of highest concentration. Bottom: Raman jet spectra of acetic acid in helium in the $\mathrm{C}=\mathrm{O}$ stretching region at reservoir pressures of 500-1000 mbar. The concentrations increase from $<0.05-0.12 \%$. The acquisition times amount to 30-35 minutes. The spectra have been intensity-scaled to the dimer band in the spectrum with the lowest concentration. The scaling factors amount to 1.0 (black spectrum), 0.87 (blue), 0.65 (red), and 0.55 (orange). Below the FTIR and Raman spectra, harmonically calculated and $(\mathrm{FF})_{\mathrm{a}}$-scaled band positions (scaling factor 0.983 ) of the acetic acid monomer A, cyclic (AA) and polar dimer AA, and the acetic acid trimer A(AA) are shown. The calculations have been performed at the B3LYP-D3(BJ)/def2TZVP level. In two insets, the global minimum structures of the trimer and the polar dimer are shown. 


\subsubsection{Pivalic Acid Trimers}

The three most stable pivalic acid trimer structures and their harmonically calculated, zero-point corrected relative energy differences are depicted in Figure 6.11. The global minimum trimer is $\mathrm{P}_{\mathrm{C}}\left(\mathrm{P}_{\mathrm{C}} \mathrm{P}_{\mathrm{C}}\right)$, which exhibits the same structure motif as that of the other two acids. Due to the bulky tert-butyl groups, the monomer docks out-of-plane to the dimer. Most interesting is the cyclic trimer $\left(\mathrm{P}_{\mathrm{C}} \mathrm{P}_{\mathrm{C}} \mathrm{P}_{\mathrm{C}}\right)$. Its energy penalty relative to the global minimum amounts to only a third of that for the other carboxylic acids $\left(1.8 \mathrm{~kJ} \mathrm{~mol}^{-1}\right)$. Thus, it might be possible to observe both pivalic acid trimers in the supersonic expansions. The third trimer $\mathrm{P}_{\mathrm{A}}\left(\mathrm{P}_{\mathrm{C}} \mathrm{P}_{\mathrm{C}}\right)$ is $9.3 \mathrm{~kJ} \mathrm{~mol}^{-1}$ less stable and expected to interconvert easily into the global minimum structure via concerted proton transfer. It is therefore discarded from further discussions. The structure of the higher-energy pivalic acid dimer $\mathrm{P}_{\mathrm{C}} \mathrm{P}$ is depicted in Figure 6.12. Its energy difference to the second local minimum is similar to that of the other acids. Further details on these dimers can be found in Appendix A.4.3. As before, the indices $\mathrm{C}$ are omitted for the rest of this section.

Compared to formic and acetic acid, the vapour pressure of pivalic acid is considerably lower (cf. Section 3.1.5). As a consequence, signal intensities in the spectra are generally smaller, despite similar data acquisition times (see Figure 6.9). A further complication is the broadness of some bands, e.g., that at $1752 \mathrm{~cm}^{-1}$. Therefore, the spectral analysis of the aggregation behaviour of pivalic acid is particularly challenging.

The FTIR spectra of pivalic acid are depicted in Figure 6.12. Aside from the monomer $\mathrm{P}\left(1774 \mathrm{~cm}^{-1}\right)$ and dimer band $(\mathrm{PP})_{\mathrm{a}}\left(1718 \mathrm{~cm}^{-1}\right)$, three distinct bands at $1752 \mathrm{~cm}^{-1}$, $1705 \mathrm{~cm}^{-1}$, and $1696 \mathrm{~cm}^{-1}$ can be seen in addition to a smaller peak at $1743 \mathrm{~cm}^{-1}$. The first two are also present in the Raman spectra alongside two bands downshifted from $(\mathrm{PP})_{\mathrm{s}}$ at $1656 \mathrm{~cm}^{-1}$ and at $1627 \mathrm{~cm}^{-1}$. In Figure 6.9, one can also see that there is a very weak broad feature at $1705 \mathrm{~cm}^{-1}$, which is more pronounced at low acid concentrations (black and blue spectra in Figure 6.12). Intensity scaling to the respective dimer bands
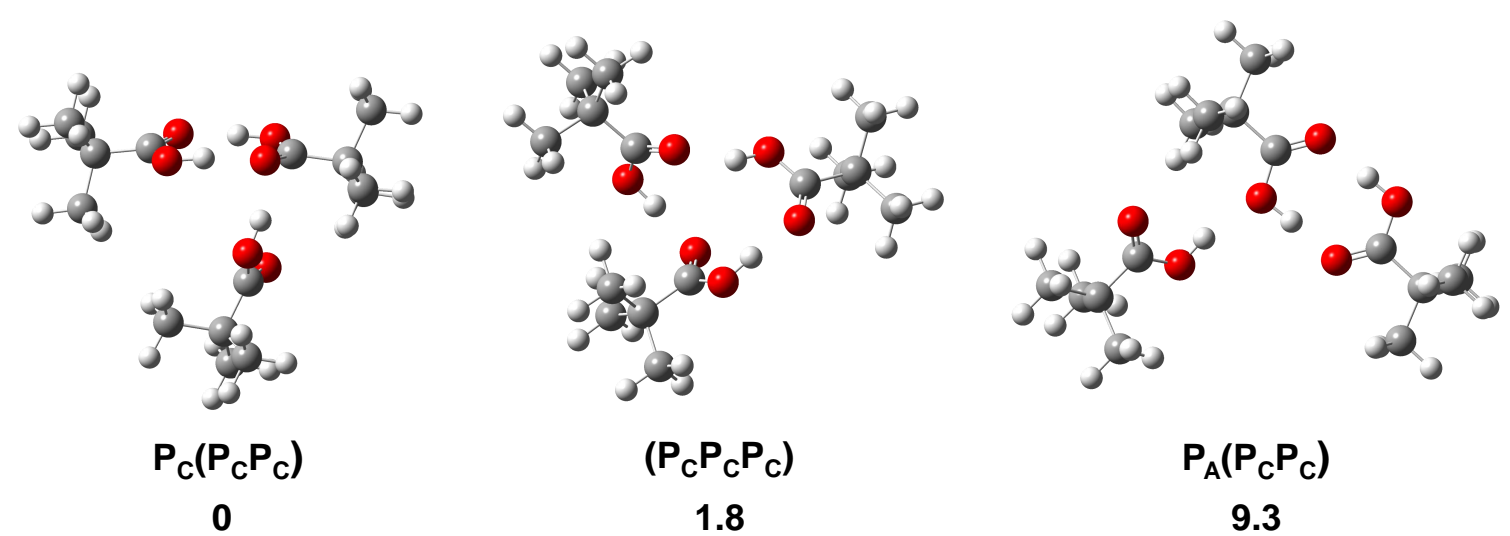

Figure 6.11: Calculated global and local minimum structures of pivalic acid trimers alongside their harmonic, zero-point corrected energy differences relative to the global minimum (in $\mathrm{kJ} \mathrm{mol}^{-1}$ ). The harmonic calculations have been performed at the B3LYP-D3(BJ)/def2-TZVP level. 


\section{FTIR}
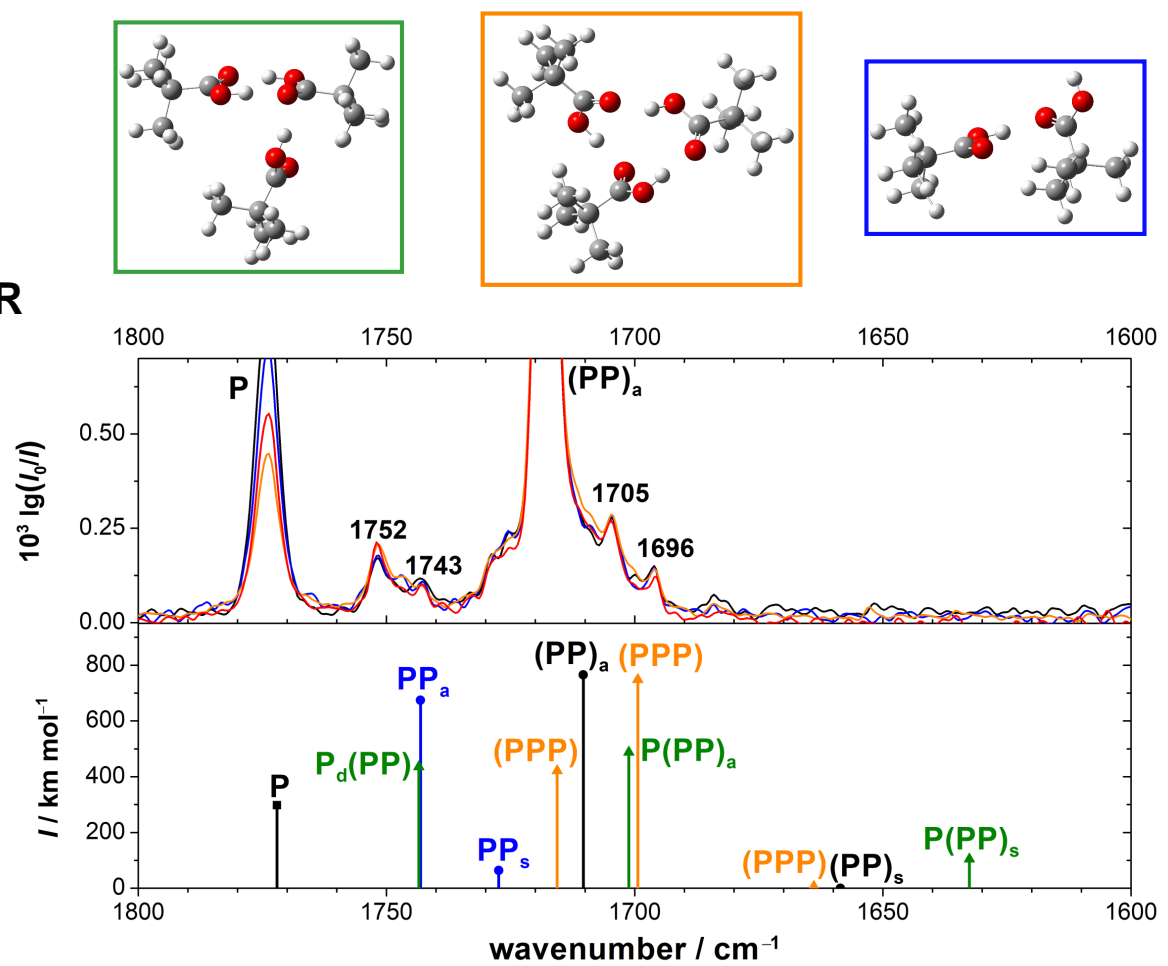

Raman

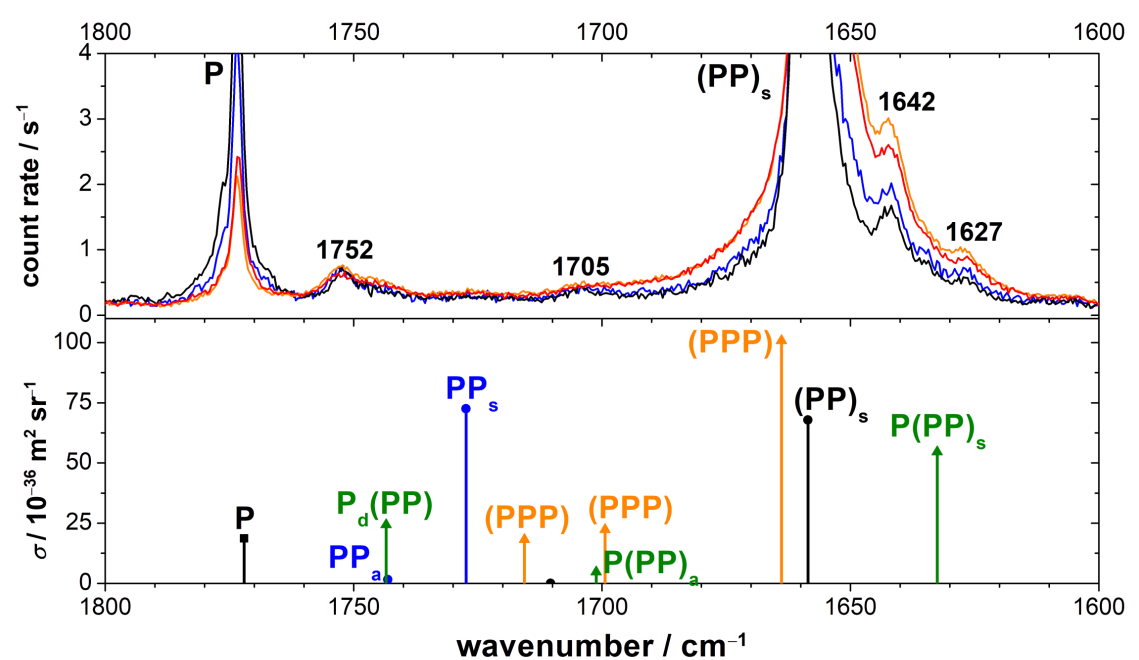

Figure 6.12: Top: FTIR jet spectra of pivalic acid in the $\mathrm{C}=\mathrm{O}$ stretching region at a concentration of $c<0.07 \%$ in helium and increasing reservoir pressures of $300-900$ mbar. The spectra were averaged over 50-155 scans. Bottom: Raman jet spectra of pivalic acid in the $\mathrm{C}=\mathrm{O}$ stretching region. The two highest concentrated spectra (red and orange) were recorded at an acid concentration of $c<0.22 \%$ in helium, nozzle temperatures of $60-130{ }^{\circ} \mathrm{C}$, reservoir pressures of $0.5-1.0 \mathrm{bar}$, and data aquisition times of 14-46 minutes. The spectra displayed in black and blue were measured at a concentration of $c<0.04 \%$, without heating the nozzle, reservoir pressures of 1.0-1.5 bar, and data acquisition times of 30 minutes. Below the FTIR and Raman spectra, harmonically calculated and $(\mathrm{FF})_{\mathrm{a}}$-scaled band position (scaling factor 0.983 ) of the pivalic acid monomer $\mathrm{P}$, cyclic (PP) and polar dimer PP, and the pivalic acid trimers $\mathrm{P}(\mathrm{PP})$ and $(\mathrm{PPP})$ are shown. The calculations have been performed at the B3LYPD3(BJ)/def2-TZVP level. The predicted structures of $\mathrm{P}(\mathrm{PP}),(\mathrm{PPP})$, and $\mathrm{PP}$ are shown in the insets above the spectra. 
$(\mathrm{PP})_{\mathrm{a}} /(\mathrm{PP})_{\mathrm{s}}$ is not conclusive, as the concentration differences are small, the bands of interest broad, and the signal-to-noise ratio poor. Moreover, the dimer band $(\mathrm{PP})_{\mathrm{s}}$ in the Raman spectra significantly broadens at higher concentrations (red and orange spectra in Figure 6.12), which can most likely be attributed to the formation of larger, dimer containing clusters (dimer assemblies) such as tetramers, which will be addressed in the next chapter. Overall, the analysis of the FTIR and Raman spectra of pivalic acid can therefore only aim to provide a likely explanation for the bands, but no definite assignments.

The predicted band position of the docking vibration of the trimer $\mathrm{P}_{\mathrm{d}}(\mathrm{PP})$ coincides with the band of the antisymmetric $\mathrm{C}=\mathrm{O}$ stretching of the polar dimer $\mathrm{PP}_{\mathrm{a}}$. In the FTIR and Raman spectra, a band at $1752 \mathrm{~cm}^{-1}$ is present, which seems to overlap with a weaker feature at $1743 \mathrm{~cm}^{-1}$ in the FTIR spectra, but as it is near noise level, no conclusion can be drawn. The aforementioned similar downshift with respect to the monomer band of $22 \mathrm{~cm}^{-1}$ compared to the other acids $\left(22 \mathrm{~cm}^{-1}\right.$ for formic and $24 \mathrm{~cm}^{-1}$ for acetic acid), suggests the assumption that the band at $1752 \mathrm{~cm}^{-1}$ is, at least partially, due to $\mathrm{P}_{\mathrm{d}}(\mathrm{PP})$. As for the other acids, the Raman band cannot feature contributions of the higher-energy dimer PP, whereas the FTIR band could contain $\mathrm{PP}_{\mathrm{a}}$ and $\mathrm{P}_{\mathrm{d}}(\mathrm{PP})$. The deviation of the predicted band positions of $\mathrm{P}_{\mathrm{d}}(\mathrm{PP})$ amounts to $-9 \mathrm{~cm}^{-1}$, yet the agreement could be improved if the band were scaled to $(\mathrm{PP})_{\mathrm{a}}$ instead of $(\mathrm{FF})_{\mathrm{a}}$. The second very weak feature at $1743 \mathrm{~cm}^{-1}$ could be due to the higher-energy dimer PP, which was observed at a similar band position in case of acetic acid $\left(1749 \mathrm{~cm}^{-1}\right)$, yet this is highly speculative. The corresponding symmetric $\mathrm{C}=\mathrm{O}$ stretching vibration of $\mathrm{PP}$ is not seen in the Raman spectra, though this might be a result of its broadness.

Other than for formic and acetic acid, there are two bands downshifted from $(\mathrm{PP})_{\mathrm{a}}$ at $1705 \mathrm{~cm}^{-1}$ and $1696 \mathrm{~cm}^{-1}$ in the FTIR spectra. The intensity of the former matches the predicted intensity ratio of $\mathrm{P}_{\mathrm{d}}(\mathrm{PP})$ and $\mathrm{P}(\mathrm{PP})_{\text {a }}$ of nearly 1:1 better, if the band at $1752 \mathrm{~cm}^{-1}$ was predominantly due to $\mathrm{P}_{\mathrm{d}}(\mathrm{PP})$. The weak band at $1705 \mathrm{~cm}^{-1}$ in the Raman spectra supports this conjecture, as its intensity matches the expected intensity of $\mathrm{P}(\mathrm{PP})_{\mathrm{a}}$ with respect to $\mathrm{P}_{\mathrm{d}}(\mathrm{PP})$ and $\mathrm{P}(\mathrm{PP})_{\mathrm{s}}$. Another explanation of one of these two bands could be a $\mathrm{C}=\mathrm{O}$ stretching band of a cyclic trimer (PPP). However, as the dominantly Raman active (PPP) band at $1663 \mathrm{~cm}^{-1}$ would overlap with the symmetric $\mathrm{C}=\mathrm{O}$ stretching vibration of the cyclic dimer at $1660 \mathrm{~cm}^{-1}$, if featured, the Raman spectra do not allow for any conclusions about this cluster. As for the other two acids, the symmetric $\mathrm{C}=\mathrm{O}$ stretching vibration of the global minimum trimer is predicted downshifted from the dimer band in the Raman spectra. At $1642 \mathrm{~cm}^{-1}$, there is a broad band, which is upshifted by $9 \mathrm{~cm}^{-1}$ from the predicted band position $\mathrm{P}(\mathrm{PP})_{\mathrm{s}}\left(1633 \mathrm{~cm}^{-1}\right)$ in addition to a smaller shoulder at $1627 \mathrm{~cm}^{-1}$. The relative band position of the former with respect to $(\mathrm{PP})_{\mathrm{S}}$ is similar to the tentatively assigned band of the acetic acid trimer and its intensity with respect to the band at $1752 \mathrm{~cm}^{-1}$ roughly matches the expected scattering cross-section ratio, so that an assignment to $\mathrm{P}(\mathrm{PP})_{\mathrm{s}}$ seems conceivable. The second band at $1627 \mathrm{~cm}^{-1}$ is overall too weak. The most plausible band positions of the $\mathrm{C}=\mathrm{O}$ stretching vibrations of the pivalic acid trimer are listed in Table 6.3.

For definite assignments, additional experimental reference data, ideally with a better signal-to-noise ratio, as well as more reliable quantum chemical band position predictions 


\section{Aggregation Dynamics: Trimers and Metastable Dimers}

Table 6.3: Experimental $\tilde{\nu}_{\exp }$ as well as harmonic, $(\mathrm{FF})_{\mathrm{a}}$-scaled band position $\left(\mathrm{in}_{\mathrm{cm}}{ }^{-1}\right)$ of the $\mathrm{C}=\mathrm{O}$ stretching vibrations of pivalic acid monomer, the cyclic dimer $(\mathrm{PP})$ as well as the trimer $\mathrm{P}(\mathrm{PP})$, calculated at the B3LYP-D3(BJ)/def2-TZVP level. The scaling factor amounts to 0.983 . The three vibrations are the docking vibration (index $\mathrm{d}$ ) of the monomer unit of the trimer, as well as the symmetric (s) and antisymmetric (a) $\mathrm{C}=\mathrm{O}$ stretching vibration of the dimer (units). Tentative assignments are printed in italics. In the fourth column, the band position deviations with respect to experiment are listed.

\begin{tabular}{cccc}
\hline \hline & $\tilde{\nu}_{\exp }$ & B3LYP-D3(BJ) & \\
\hline $\mathrm{P}$ & 1774 & 1772 & -2 \\
$(\mathrm{PP})_{\mathrm{a}}$ & 1718 & 1710 & -8 \\
$(\mathrm{PP})_{\mathrm{s}}$ & 1660 & 1659 & -1 \\
$\mathrm{P}_{\mathrm{d}}(\mathrm{PP})$ & 1752 & 1743 & -9 \\
$\mathrm{P}(\mathrm{PP})_{\mathrm{a}}$ & 1705 & 1701 & -4 \\
$\mathrm{P}(\mathrm{PP})_{\mathrm{s}}$ & 1642 & 1633 & -9 \\
\hline \hline
\end{tabular}

are needed. Higher concentrated FTIR spectra are not feasible with the current set-up due to the limited vapour pressure at room temperature. For that, another set-up has to be used, called the popcorn jet $[8,441]$ in laboratory jargon. It allows for substances to be heated up to $200{ }^{\circ} \mathrm{C}$ [441], yet the absorption path $(6 \mathrm{~cm})$ [8] is 10 times smaller than that of the FTIR set-up used (cf. Section 3.1.2), so that long measurement times are necessary to achieve an adequate signal-to-noise ratio. The higher concentrated Raman spectra were measured with a heatable saturator (Figure 3.10), yet the balance between high signal intensities and low concentrations of clusters larger than the dimer is slim, which limits spectral interpretation. One way to exclude or confirm certain structures in the supersonic expansion is to record microwave spectra, as both trimers as well as the higher-energy dimer exhibit a permanent electric dipole moment. Another option would be to investigate the hetero clusters of carboxylic acids, i.e., the band position shifts if one pivalic acid molecule in the trimer is replaced with formic or acetic acid in analogy to the deuteration experiments discussed in Chapter 4.

\section{$6.4 \mathrm{Z}$ bands}

As previously mentioned, the FTIR spectra of formic, acetic, and pivalic acid each feature a band upshifted with respect to the monomer at $1811 \mathrm{~cm}^{-1}, 1834 \mathrm{~cm}^{-1}$, and $1838 / 1834 \mathrm{~cm}^{-1}$, respectively. To distinguish monomer from cluster bands and determine cluster sizes, the FTIR spectra of each of the acids have been (intensity-) scaled to the antisymmetric $\mathrm{C}=\mathrm{O}$ stretching vibration of the cyclic dimer. The results are shown in Figure 6.13. In all three cases, the bands scale like the dimer band. This leaves two possible explanations - these bands could be either due to combination or overtone vibrations of the cyclic dimer, potentially in Fermi resonance with the fundamental, or due to a metastable dimer. 
Table 6.4: Harmonically calculated and $\left(\mathrm{F}_{\mathrm{C}} \mathrm{F}_{\mathrm{C}}\right)_{\mathrm{a}}$-scaled band positions $\tilde{\nu}$ (in $\mathrm{cm}^{-1}$ ) as well as IR intensities $I$ (in $\mathrm{km} \mathrm{mol}^{-1}$ ) and Raman scattering cross-sections $\sigma$ (in $10^{-36} \mathrm{~m}^{2} \mathrm{sr}^{-1}$ ) of the formic acid monomer, the cyclic dimer and three higher-energy dimers. The zero-point corrected dissociation energies $D_{0}^{h}$ into monomers (in $\mathrm{kJ} \mathrm{mol}^{-1}$ ) are also listed. The calculations have been performed at the B3LYP-D3(BJ)/def2-TZVP level.

\begin{tabular}{c|c|ccc|ccc}
\hline \hline & $D_{0}^{h}$ & $\tilde{\nu}$ & $I$ & $\sigma$ & $\tilde{\nu}$ & $I$ & $\sigma$ \\
\hline $\mathrm{F}$ & & 1787 & 360 & 25 & & & \\
$\left(\mathrm{~F}_{\mathrm{C}} \mathrm{F}_{\mathrm{C}}\right)$ & 68.4 & 1741 & 814 & 0 & 1664 & 0 & 58 \\
$\mathrm{~F}_{\mathrm{C}} \mathrm{F}$ & 39.0 & 1760 & 643 & 2 & 1718 & 131 & 66 \\
$\mathrm{~F}_{\mathrm{A}} \mathrm{F}_{\mathrm{C}}$ & 30.2 & 1792 & 304 & 23 & 1746 & 498 & 42 \\
$\mathrm{~F}_{\mathrm{A}} \mathrm{F}$ & 24.1 & 1797 & 400 & 34 & 1763 & 330 & 22 \\
\hline \hline
\end{tabular}

The structures of metastable formic acid dimers were explored in the previous chapter. The local minimum closest in energy to the global minimum dimer $\left(\mathrm{F}_{\mathrm{C}} \mathrm{F}_{\mathrm{C}}\right)$ is $\mathrm{F}_{\mathrm{C}} \mathrm{F}$. Energetically next-higher are $\mathrm{F}_{\mathrm{A}} \mathrm{F}_{\mathrm{C}}$ and $\mathrm{F}_{\mathrm{A}} \mathrm{F}$ (cf. Figure 5.6). The energy differences with respect to the global minimum dimer are fairly large $-\mathrm{F}_{\mathrm{A}} \mathrm{F}_{\mathrm{C}}$ and $\mathrm{F}_{\mathrm{A}} \mathrm{F}$ are $38.2 \mathrm{~kJ} \mathrm{~mol}^{-1}$ and $44.3 \mathrm{~kJ} \mathrm{~mol}^{-1}$ higher in energy (see Table 6.4), respectively. It is therefore improbable to see these two structures in the supersonic expansion unless they are kinetically trapped. The bands of the $\mathrm{C}=\mathrm{O}$ stretching vibrations of these metastable dimers are both IR and Raman active (cf. Table 6.4). Due to the structural similarities of the acids seen before, this likely also applies to the corresponding acetic and pivalic acid dimers. The Raman spectra shown in Figure 6.9 feature no peaks at the band position of the FTIR bands. Moreover, both $\mathrm{F}_{\mathrm{A}} \mathrm{F}_{\mathrm{C}}$ and $\mathrm{F}_{\mathrm{A}} \mathrm{F}$ exhibit a non-hydrogen bonded $\mathrm{C}=\mathrm{O}$ group, whose $\mathrm{C}=\mathrm{O}$ stretching band is predicted upshifted with respect to the monomer (Table 6.4), yet these upshifts $\left(5 \mathrm{~cm}^{-1}\right.$ and $\left.10 \mathrm{~cm}^{-1}\right)$ are distinctly smaller than the experimentally observed difference of $34 \mathrm{~cm}^{-1}$. All of this contradicts a higher-energy dimer band assignment.

As shown for formic acid trimers, studying the aggregation dynamics in supersonic expansions with FTIR imaging can help to distinguish clusters based on their area of highest stability in the expansion. In case of acetic acid expansions, the band at $1836 \mathrm{~cm}^{-1}$ is very broad and weak in the FTIR imaging spectra and the band of pivalic acid not seen due to the low signal-to-noise ratio. These two acids are therefore not suitable for such analysis. In the formic acid expansions, the $\mathrm{Z}$ band is clearly visible and most importantly, its intensity is high enough for integration, at least at higher reservoir pressures. From the single slit expansions of formic acid shown in Figure 6.4, reservoir pressures of 500-700 mbar have been chosen. The resulting integrated intensities of the monomer, dimer, trimer, as well as the $\mathrm{Z}$ band at $1811 \mathrm{~cm}^{-1}$ are visualised in Figure 6.14 .

The $\mathrm{Z}$ band shows the same aggregation behaviour as the formic acid dimer - it is present directly behind the nozzle and is seen in areas of highest number of collisions. The density of a metastable cluster is not expected to increase with increasing number of collisions as seen for the $\mathrm{Z}$ band here, but rather show the opposite behaviour. Consequently, these can be ruled out and the band is attributed to a combination band or 

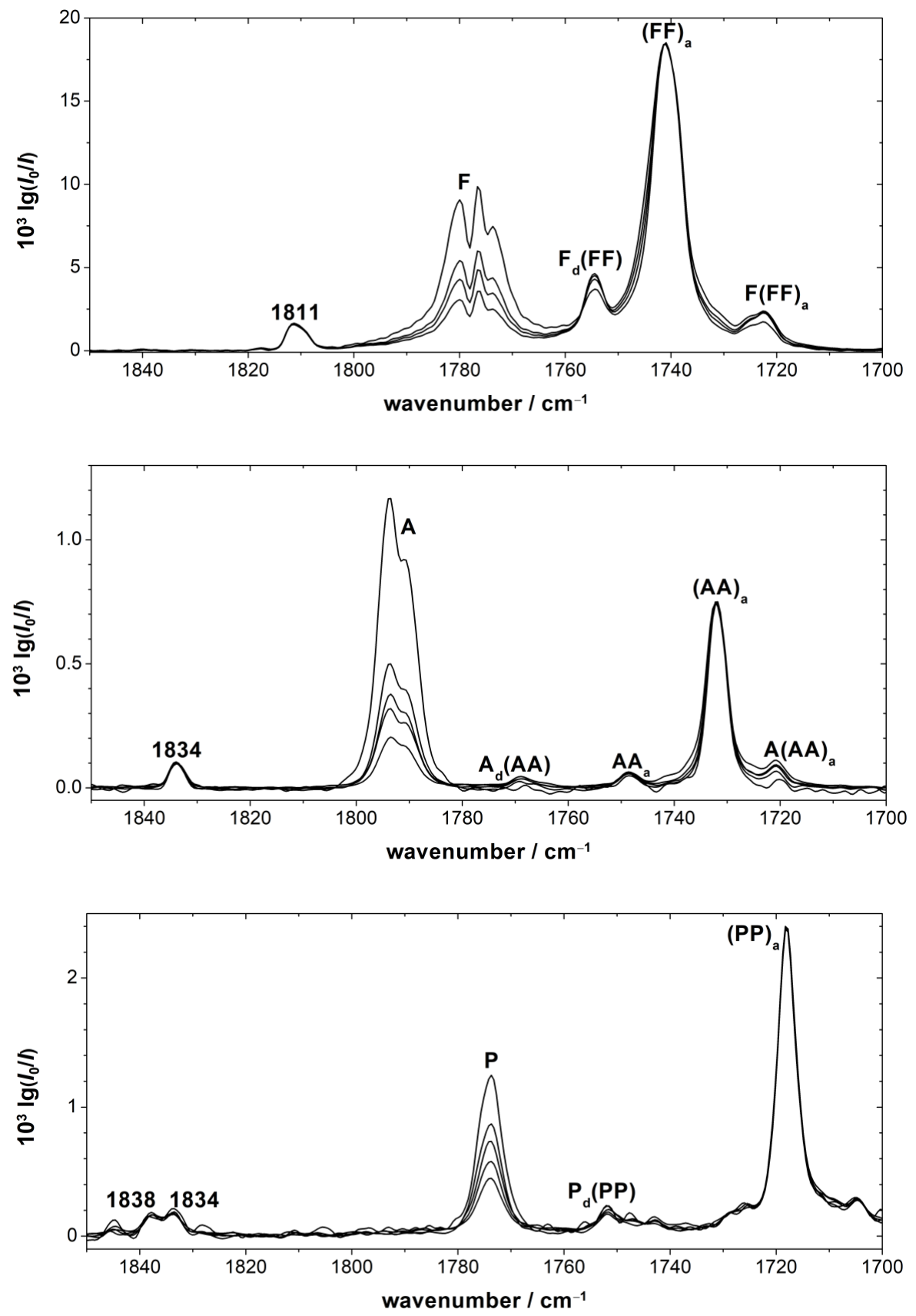

Figure 6.13: Dimer-scaled FTIR spectra of formic, acetic, and pivalic acid in the $\mathrm{C}=\mathrm{O}$ stretching region. The experimental conditions are listed in Figures 6.3, 6.10, and 6.12. 
6 Aggregation Dynamics: Trimers and Metastable Dimers

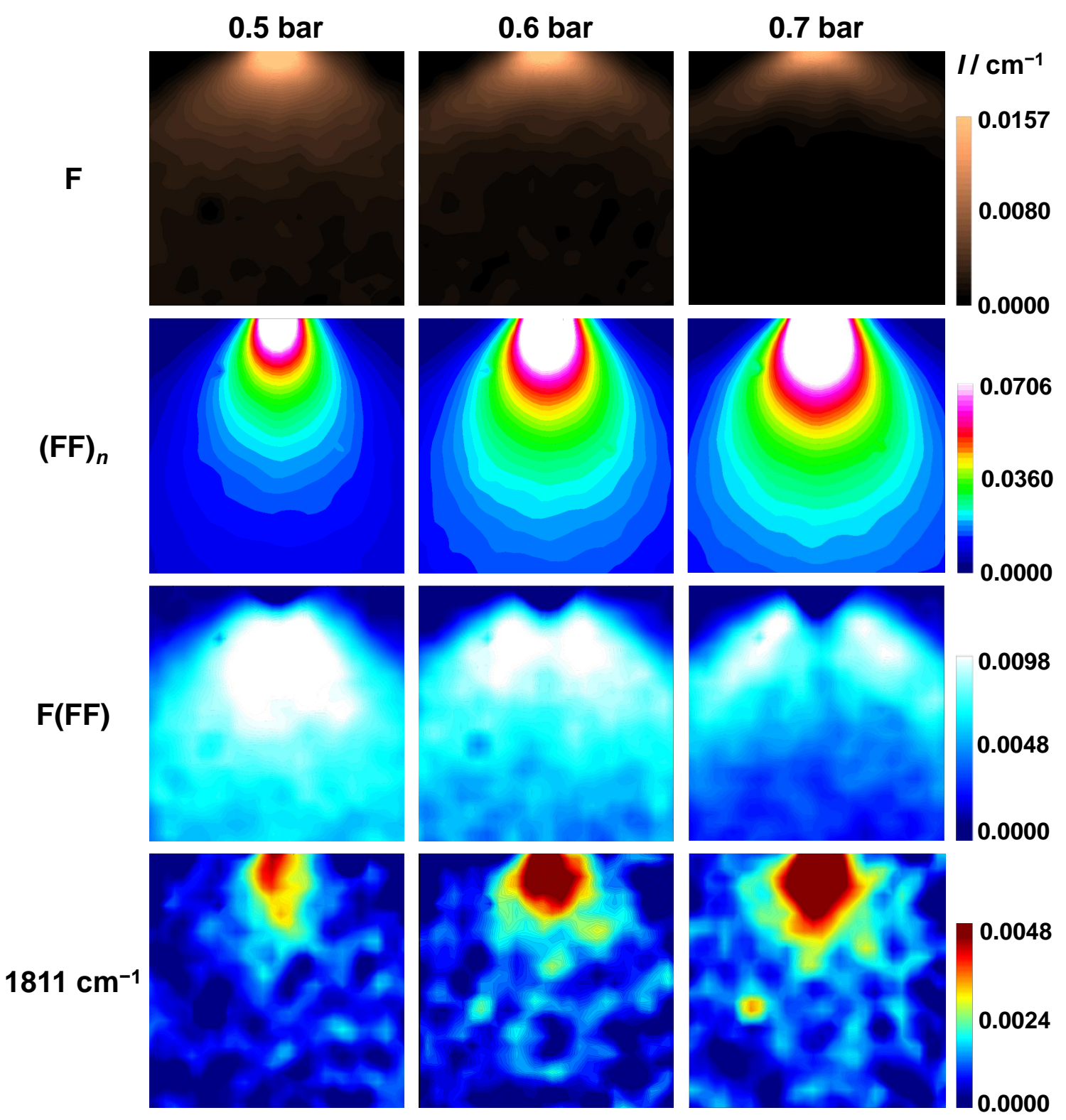

Figure 6.14: $2 \mathrm{D}$ space-resolved integrated intensity of an unknown band at $1811 \mathrm{~cm}^{-1}$ in FTIR spectra of formic acid in helium $(c<0.2 \%)$ at reservoir pressures of $0.5-0.7$ bar in comparison to the integrated intensity of monomer, dimer, and trimer bands, previously shown in Figure 6.4 . 


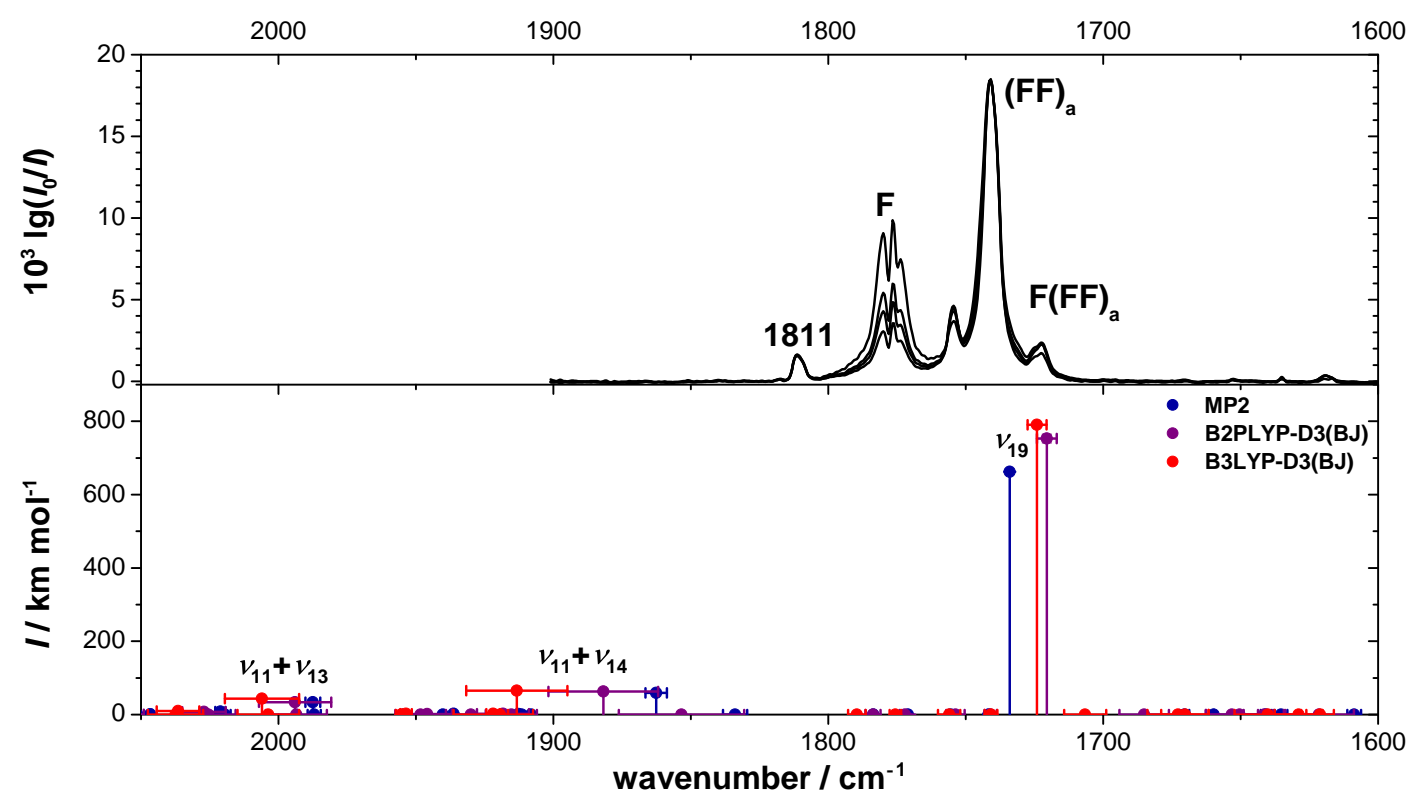

Figure 6.15: Top: Dimer-scaled FTIR spectra of formic acid in helium in the $\mathrm{C}=\mathrm{O}$ stretching region. The experimental conditions are identical to those of Figure 6.3. Bottom: Anharmonically (VPT2) calculated band positions of the antisymmetric $\mathrm{C}=\mathrm{O}$ stretching vibration $\nu_{19}$ of the formic acid dimer and dimer combination bands between $2015-1600 \mathrm{~cm}^{-1}$. The calculations have been performed at the MP2/aVTZ (blue), B2PLYP-D3(BJ)/aVTZ (purple), and B3LYP-D3(BJ)/aVTZ level (red). Eight settings have been tested - two DFT integration grid sizes (ultrafine and superfine), $C_{1}$ and $C_{2 h}$ symmetry, as well as tight and very tight optimisation. The eight (four for MP2) anharmonic frequency calculations have been averaged and are displayed with their standard deviation as error bars. The error bars of the intensities are barely visible due to their small size. Apart from $I\left(\nu_{19}\right)$ of B2PLYP-D3(BJ) $\left( \pm 12 \mathrm{~km} \mathrm{~mol}^{-1}\right)$, these are below $\pm 5 \mathrm{~km} \mathrm{~mol}^{-1}$ within this spectral region.

overtone of the global minimum dimer with particularly high intensity.

VPT2 calculations of the global as well as local minimum dimer of formic acid were shown in the previous chapter. For $(\mathrm{FF})$, the anharmonic frequency calculations were performed at the MP2, B2PLYP-D3(BJ), and B3LYP-D3(BJ) level. For all methods, an aVTZ basis set was employed. The DFT integration grid (ultrafine and superfine), the optimisation criteria (tight and very tight), and symmetry $\left(C_{1}\right.$ or $\left.C_{2 \mathrm{~h}}\right)$ were varied. The resulting eight frequency calculations (four for MP2) were averaged. The averaged values and their standard deviation for all 24 fundamentals are listed in Table A.9 in the appendix. The antisymmetric $\mathrm{C}=\mathrm{O}$ stretching vibration of the formic acid dimer $(\mathrm{FF})_{\mathrm{a}}$ corresponds to $\nu_{19}$. To examine whether there are potential combination or overtone vibrations with sufficient intensity near the band at $1811 \mathrm{~cm}^{-1}$, these and the fundamental are displayed in Figure 6.15 alongside the dimer scaled FTIR jet spectra of formic acid. As for the $\mathrm{F}_{\mathrm{C}} \mathrm{F}$ dimer in Figure 5.7, the six intermolecular vibrations are omitted for this analysis, as these are not well described by VPT2.

Two combination bands with remarkably high intensities can be seen upshifted from 

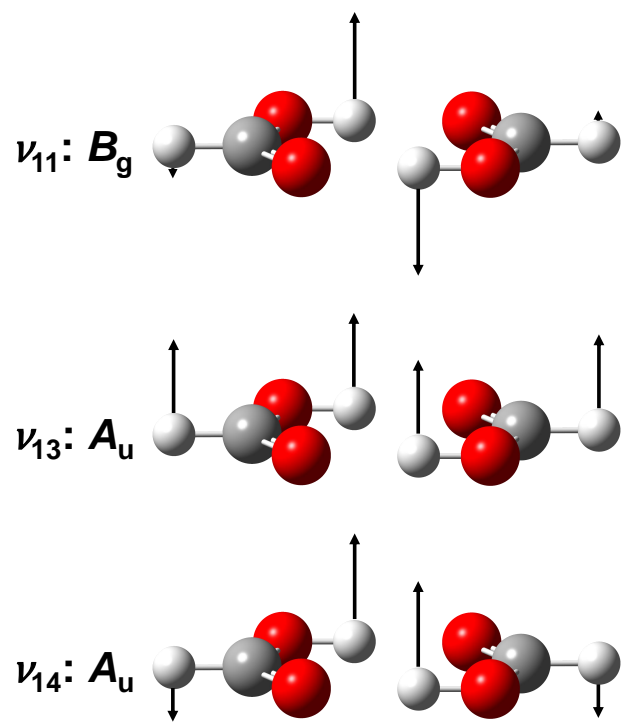

Figure 6.16: $\mathrm{O}-\mathrm{H}$ and $\mathrm{C}-\mathrm{H}$ out-of-plane bending vibrations $\nu_{11}, \nu_{13}$, and $\nu_{14}$ of the formic acid dimer, calculated at the B3LYP-D3(BJ)/aVTZ VPT2 level. Additionally, the symmetry of the modes are listed.

the fundamental. $\left(\nu_{11}+\nu_{13}\right)$ and $\left(\nu_{11}+\nu_{14}\right)$ are combinations of $\mathrm{O}-\mathrm{H}$ out-of-plane bending vibrations, which are depicted in Figure 6.16. The relative intensity of $\left(\nu_{11}+\nu_{14}\right)$ with respect to the fundamental $\nu_{19}$ agrees well with experiment for all methods, yet the band position difference varies distinctly. Experimentally, it amounts to $70 \mathrm{~cm}^{-1}$. The predicted differences are distinctly larger. These amount to $129 \pm 4 \mathrm{~cm}^{-1}$ (MP2), $161 \pm 21 \mathrm{~cm}^{-1}$ (B2PLYP-D3(BJ)), $189 \pm 19 \mathrm{~cm}^{-1}$ (B3LYP-D3(BJ)). A Fermi resonance between $\left(\nu_{11}+\nu_{14}\right)\left(B_{\mathrm{u}}\right.$ symmetry) and $\nu_{19}\left(B_{\mathrm{u}}\right.$ symmetry) (cf. Figure 6.16 and Table A.9) is not predicted by the methods and seems improbable considering the larger wavenumber difference of the bands. Overall, a severe overestimation of the band position of $\left(\nu_{11}+\nu_{14}\right)$ by the VPT2 calculations seems most likely. This overestimation is also seen for the band positions of the respective fundamentals $\nu_{11}$ and $\nu_{14}$, as shown in Table 6.5.

The reason why a similar band is seen for dimers can be easily rationalised by looking

Table 6.5: Experimental $[68,118]$ and anharmonically calculated (VPT2) band positions (in $\mathrm{cm}^{-1}$ ) of the two $\mathrm{O}-\mathrm{H}$ out-of-plane bending vibrations $\nu_{11}$ and $\nu_{14}$ of the formic acid dimer at the B3LYP-D3(BJ)/aVTZ, B2PLYP-D3(BJ)/aVTZ, and MP2/aVTZ level. The standard deviations resulting from the variation of the symmetry, optimisation settings, and DFT integration grid are listed alongside the predicted band positions.

\begin{tabular}{cccc}
\hline \hline experiment & B3LYP-D3(BJ) & B2PLYP-D3(BJ) & MP2 \\
\hline $911^{[68]}$ & $957 \pm 11$ & $942 \pm 12$ & $932 \pm 3$ \\
$939^{[118]}$ & $986 \pm 7$ & $973 \pm 9$ & $964 \pm 2$ \\
\hline \hline
\end{tabular}




\section{Aggregation Dynamics: Trimers and Metastable Dimers}

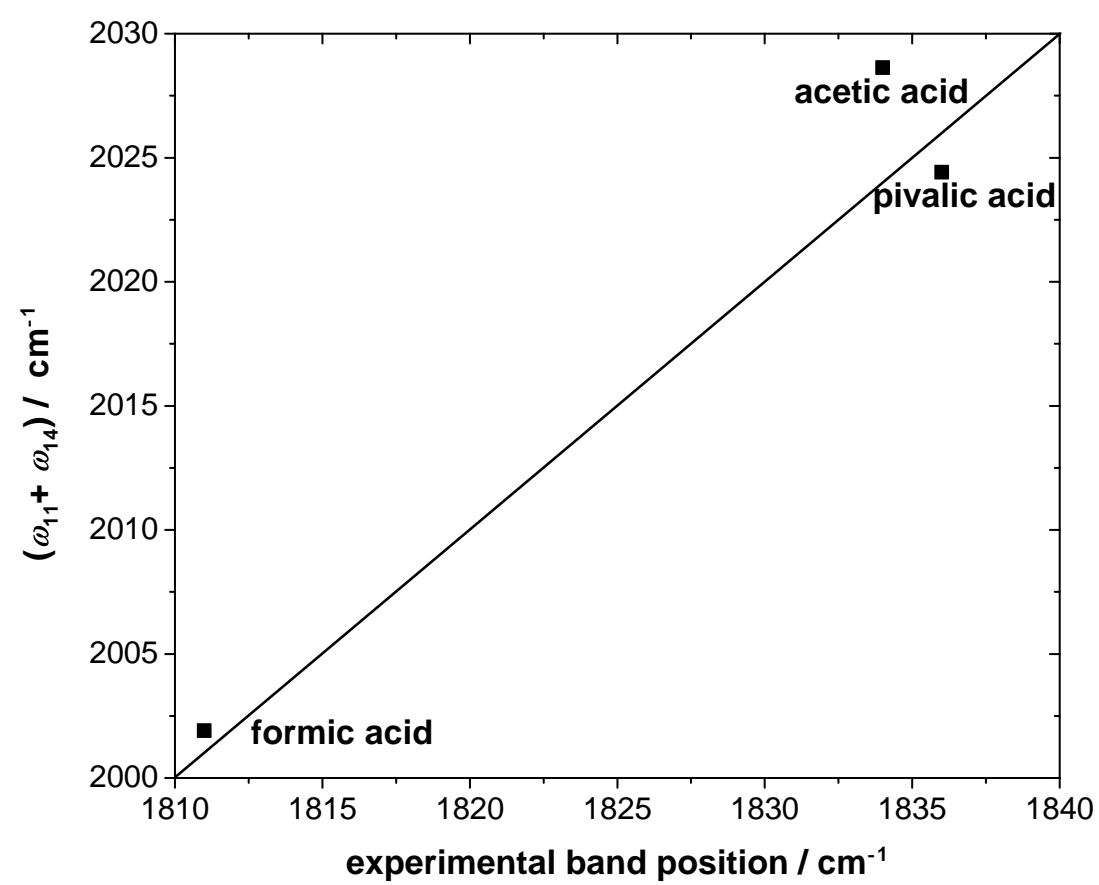

Figure 6.17: Sum of the harmonically predicted band positions of the two O-H out-of-plane bending vibrations $\omega_{11}$ and $\omega_{14}$ of the formic, acetic, and pivalic acid dimers plotted against the (anharmonic) experimental band position of the $\mathrm{Z}$ band observed in the spectra of each acid. The bands $\left(\omega_{11}\right.$ and $\left.\omega_{14}\right)$ are labelled according to those of the formic acid dimer.

at the vibrations displayed in Figure 6.16. The $\mathrm{CH}$ moiety moves only slightly for $\nu_{11}$ and $\nu_{14}$ and as such, its change to methyl or tert-butyl groups will not largely affect the band position of the corresponding vibrations. A plot of the sum of the predicted harmonic band positions of the corresponding modes of all three acids at the B3LYPD3(BJ)/aVTZ level against the experimental band position under the assumption of similar anharmonicities endorses this suspicion (see Figure 6.17). Thus, it seems likely that the combination band is featured at a similar band position for all acids.

Unfortunately, there are no high level anharmonic vibrational frequencies of combination bands reported for any of the carboxylic acid dimers. As briefly mentioned in the previous chapter, anharmonic band positions of the fundamentals of the formic acid dimer were calculated by Miliordos and Xantheas by applying a MP2/aVDZ VPT2 correction to their harmonic $\operatorname{CCSD}(\mathrm{T}) / \mathrm{aVQZ}$ values [396] and by $\mathrm{Qu}$ and Bowman [81], who constructed a potential hypersurface out of 13475 CCSD(T)-F12a/haVTZ energies ${ }^{3}$ and calculated anharmonic frequencies with the VSCF/VCI methodology. Two other groups performed DFT VPT2 calculations, which are similar to the ones shown here [394, 395]. So overall, this combination band assignment is just one of many examples that can profit from further progress of anharmonic vibrational frequency calculations of the formic acid dimer.

\footnotetext{
${ }^{3}$ haVTZ is an abbreviation which stands for an aVTZ basis used for C and O atoms and a VTZ basis employed for hydrogen atoms.
} 


\subsection{Summary and Outlook}
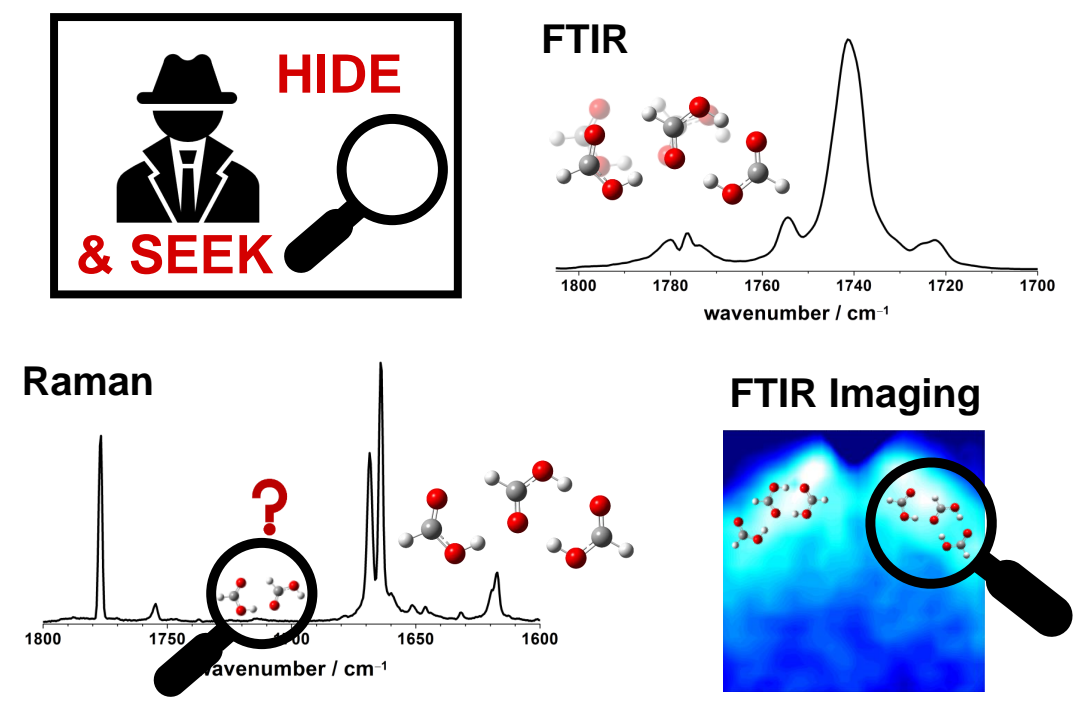

Figure 6.18: Schematic visualisation of trimer and higher-energy dimer assignments of formic acid.

Within this chapter, the trimer formation of three carboxylic acids (formic, acetic and pivalic acid) was studied in the $\mathrm{C}=\mathrm{O}$ stretching region and, following up on the previous chapter, compared with the formation of higher-energy dimers by the combination of FTIR and Raman jet spectroscopy as well as FTIR imaging, as schematically visualised for formic acid in Figure 6.18. Overall, the aggregation behaviour of the three acids is fairly similar. Bands of the $\mathrm{C}=\mathrm{O}$ stretching vibrations of the formic and acetic acid trimers were identified and most likely pivalic acid trimer assignments discussed. The most pronounced spectral difference is seen for the FTIR spectra of acetic acid, where the band attributed to the predominantly IR active vibration of higher-energy dimer is wellseparated from the docking vibration of trimer. In case of formic acid, FTIR and Raman spectra as well as FTIR imaging experiments confirm that the trimer is featured in the expansions, yet a metastable dimer contribution cannot be quantified due to a potential overlap of their bands (see Figure 6.18). To validate that the metastable formic acid dimer is indeed also present in the supersonic expansions, other spectral regions need to be explored. The harmonically calculated band positions as well as IR intensities and Raman scattering cross-sections of both structures are displayed in Figure 6.19.

Most $\mathrm{O}-\mathrm{H}$ and $\mathrm{C}-\mathrm{H}$ stretching vibrations of $\mathrm{FF}$ and $\mathrm{F}(\mathrm{FF})$ are predicted to be well-separated, although as mentioned before, that does not necessarily hold true for the docking $\mathrm{O}-\mathrm{H}$ vibration of the trimer and the bound $\mathrm{OH}$ stretching vibration of the higher-energy dimer. In addition, the $\mathrm{OH}$ stretching region is congested by the broad resonance structure of the formic acid dimer and therefore, not convenient for cluster band assignments. The most suitable vibration for verification of the presence of a metastable dimer of formic acid is the $\mathrm{C}-\mathrm{O}$ stretching vibration of $\mathrm{FF}$ predicted at $1154 \mathrm{~cm}^{-1}$, as it is the most intense, well separated IR band. Other options are 


\section{Aggregation Dynamics: Trimers and Metastable Dimers}

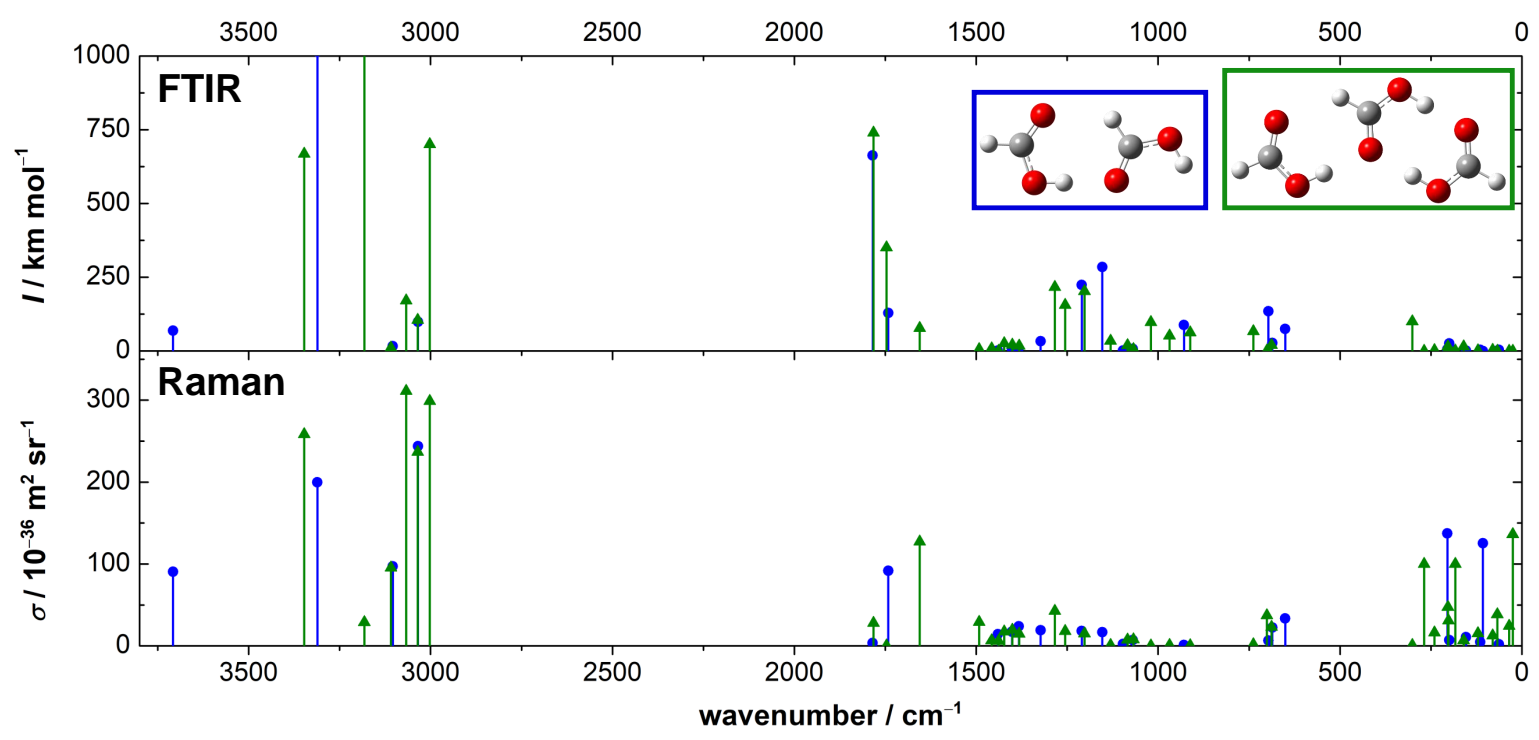

Figure 6.19: Harmonically calculated band positions, IR intensities, and Raman scattering cross-sections of all fundamentals of the formic acid trimer $\mathrm{F}(\mathrm{FF})$ (green) and higher-energy dimer FF (blue). The calculations have been performed at the B3LYP-D3(BJ)/def2-TZVP level.

the $\mathrm{O}-\mathrm{C}=\mathrm{O}$ in-plane-bending vibrations predicted around $700 \mathrm{~cm}^{-1}$ by the B3LYPD3(BJ)/def2-TZVP calculations. For Raman spectroscopic scrutiny, the $\mathrm{C}=\mathrm{O}$ stretching vibrations or the inter-monomer modes are ideal, yet the amount of higher-energy dimer seen in the Raman spectra was surprisingly small, if present at all. These differences in FTIR and Raman spectra are attributed to the different parts of the expansions probed - Raman spectra are recorded close to the nozzle (1 mm difference), whereas the FTIR spectra are averaged over a large expansion area due to the large beam diameter and nozzle distance $(10 \mathrm{~mm}$ in the focus point). Therefore, Raman jet spectroscopy cannot necessarily serve to explain the differences in the FTIR jet spectra.

Another limiting factor for the study of the aggregation behaviour of the three carboxylic acids is the performance of the scaled harmonic frequency calculations at the B3LYP-D3(BJ) level, which varies vastly for the different acids and vibrations. Part of this problem was already seen for the exciton shifts $\Delta a$ in the previous chapter where the large overestimation of the formic acid monomer $\mathrm{C}=\mathrm{O}$ stretching band position caused particularly large errors for the shifts of the antisymmetric $\mathrm{C}=\mathrm{O}$ stretching vibration of the dimer with respect to the monomer $\Delta a$ in case of $(\mathrm{FF})$ and $(\mathrm{FA})$. As both the exciton splittings $\Delta a s$ and shifts $\Delta a$ are relative quantities, the performance difference is somewhat compensated and not as clearly visible as for absolute values. To visualise this effect, the band position differences of the $(\mathrm{FF})_{\mathrm{a}}$-scaled harmonic frequency calculations with respect to experiment are plotted for the $\mathrm{C}=\mathrm{O}$ stretching vibrations of the monomers, dimers, and trimers of all three acids. The results are shown in Figure 6.20. Additionally, that of the hetero dimers (FA), (AP), and (PF) is included. Large performance differences are seen for the monomer bands with an underestimation of 


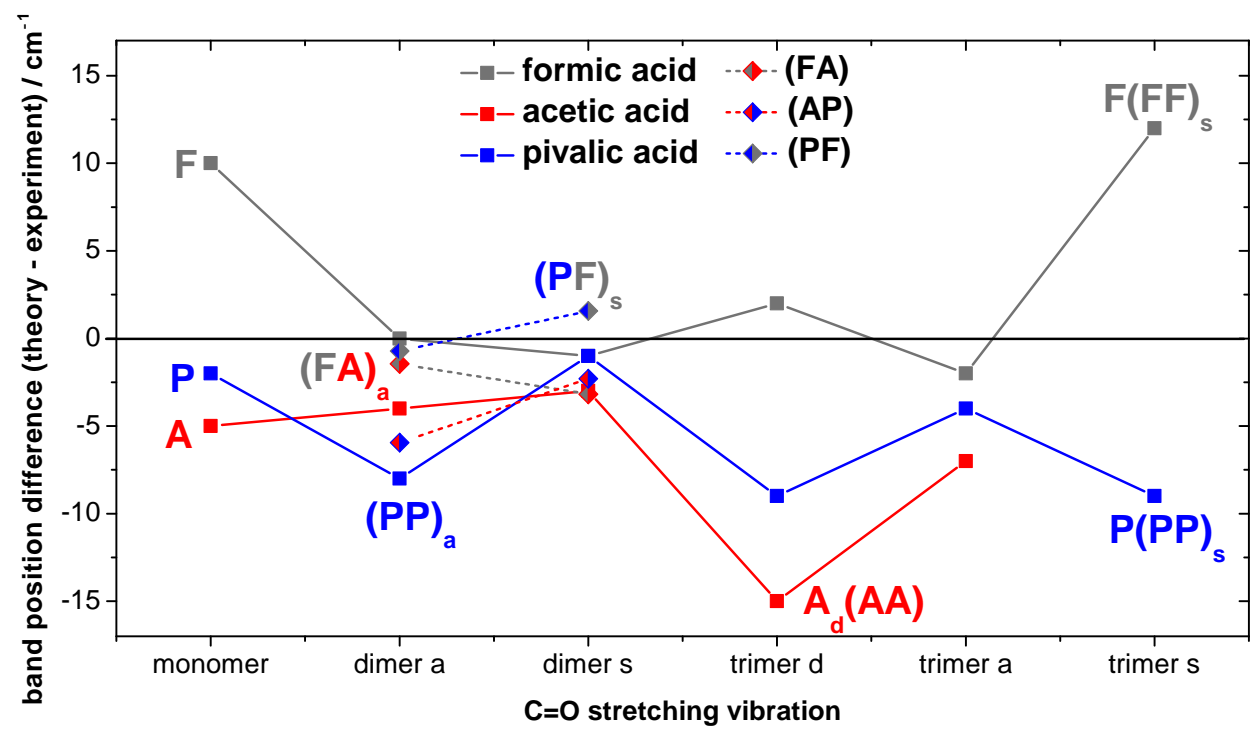

Figure 6.20: Band position differences of $(\mathrm{FF})_{\mathrm{a}}$-scaled harmonic frequency calculations at the B3LYP-D3(BJ)/def2-TZVP level with respect to experiment for the $\mathrm{C}=\mathrm{O}$ stretching vibrations of carboxylic acid monomers, the antisymmetric (a) and symmetric ( $\mathrm{s}$ ) $\mathrm{C}=\mathrm{O}$ stretching vibrations of the dimers and trimers and for the latter, additionally that of the docking vibration (d). The three carboxylic acids are formic F (grey), acetic A (red), and pivalic acid P (blue). The differences obtained for the six hetero dimer bands are also listed. For bands with two maxima, the averaged band position mismatch is plotted.

$-5 \mathrm{~cm}^{-1}$ for acetic and an overestimation of $+10 \mathrm{~cm}^{-1}$ for formic acid with respect to experiment. A similar divergence is seen for the docking vibrations of the trimers, which endorses their assignment, as these corresponds to the $\mathrm{C}=\mathrm{O}$ stretching vibration of the monomer unit of the trimer. Especially peculiar is the large discrepancy between the B3LYP-D3(BJ) prediction of the symmetric $\mathrm{C}=\mathrm{O}$ stretching vibration of the trimers. The corresponding acetic acid value is not listed here, as the exact band position could not be determined due the broadness of the band and its overlap with (AA). However, it is in any case underestimated, if the assignment was correct (cf. Figure 6.10). While those of $\mathrm{P}(\mathrm{PP})_{\mathrm{s}}$ and $\mathrm{A}(\mathrm{AA})_{\mathrm{s}}$ are tentative, though plausible, the formic acid band position assignment of $\mathrm{F}(\mathrm{FF})_{\mathrm{s}}$ seems to be the most certain of the three acids, as the band is well separated from the symmetric $\mathrm{C}=\mathrm{O}$ stretching vibration of the formic acid dimer in the Raman spectra and its intensity matches that of the other trimer bands in both the FTIR and the Raman spectra. A good validation, whether this discrepancy is inherent in harmonic B3LYP-D3(BJ) or caused by a misassignment, is the measurement of the corresponding mixed trimer bands, as the B3LYP-D3(BJ) performance for the hetero dimers lies mostly in-between that of the homo dimers, as shown in Figure 6.20. The only exception is $(\mathrm{PF})$, particularly its symmetric $\mathrm{C}=\mathrm{O}$ stretching vibration. This outlier was already seen in the previous chapter, where the prediction of the hetero dimer exciton splittings $\Delta$ as from the homo dimer values exhibited a larger deviation for that cluster, both for the experimental as well as the B3LYP-D3(BJ) predictions.

Assignments of hetero clusters beyond the cyclic dimer, i.e., higher-energy dimers or 


\section{Aggregation Dynamics: Trimers and Metastable Dimers}

trimers, is particularly difficult due to the richness of possible conformers. In the simplest case of mixing two acids, one is more abundant than the other in the trimer. Within the global minimum structure motif of the homo dimers, six hetero trimer conformations are feasible, three for each 1:2 and 2:1 type trimer, depending on which molecule is substituted by the other acid. Additionally, there are two conformations of the higherenergy dimers, which were predicted to be overlapping with trimer bands in the spectra of formic, acetic, and pivalic acid, if present. For spectroscopic scrutiny, it is therefore crucial to reduce the number of conformers, e.g., by having a surplus of one of the acids in the expansion.

The best test system out of the three carboxylic acids studied in this work is the combination of formic and pivalic acid, as the vapour pressure of pivalic acid is by far the smallest so that large concentration differences are feasible without interference of clusters larger than the trimer (cf. Section 3.1.5). Equally important, the predicted dissociation energy differences of $(\mathrm{FF}),(\mathrm{PP})$, and $(\mathrm{PF})$ are the largest (see Table 5.4 in Chapter 5.3). As such, the number of possible trimer conformers that need to be considered reduces significantly, as described in Appendix A.4.4. The spectra of all three mixtures optimised for trimer formation were measured throughout the course of this work, yet for spectral assignments, a more reliable quantum chemical method than B3LYP-D3(BJ) is needed and the single substance spectra need to be better understood. The reliability of scaled harmonic frequency calculations of the other methods tested for the homo and hetero trimers in Chapter 5, does not seem to be significantly better, as shown in Figure A.27. For the $\mathrm{F}, \mathrm{A}$, and $\mathrm{P} \mathrm{C}=\mathrm{O}$ stretching vibrations, most methods show a similar behaviour to B3LYP. The only two methods that exhibit a similar performance for all three monomers are MP2 and SCS-MP2, which are computationally too expensive for the trimer calculations, as even the pivalic acid dimer (PP) was out of reach with a moderately sized basis set, in this case, aVTZ. Additionally, the band position deviations of scaled harmonic frequency calculations at the MP2 and SCS-MP2 level are particularly severe, as already discussed in Chapter 5. Overall, this example clearly highlights that for benchmarking quantum chemical methods, experiment and theory heavily rely on each other - without further progress on the theoretical side, the experimental data presented here cannot be fully interpreted, but more experimental reference data sets are necessary for further progress of quantum chemical method development. Thus, only via close collaboration further growth can be achieved.

In addition to the bands of metastable dimers and the trimers, another band was identified in the FTIR spectra upshifted with respect to the monomer band for all carboxylic acids tested. Through the synergy of FTIR and Raman jet spectroscopy, FTIR imaging, and anharmonic vibrational frequency calculations, it was shown that this band can most likely be attributed to a combination band of the cyclic dimer rather than another cluster. For further validation, high level anharmonic vibrational frequency calculations are needed, which are strikingly sparse, even for the formic acid dimer (for further details, see Section 5.3.4). A fundamental of another cluster was ruled out based on FTIR imaging experiments that show identical aggregation behaviour for that band and the cyclic dimer. The high applicability of this technique for distinguishing different clusters will be highlighted for dimer assemblies in the following chapter. 


\section{Dimer Assemblies}

\subsection{Introduction}
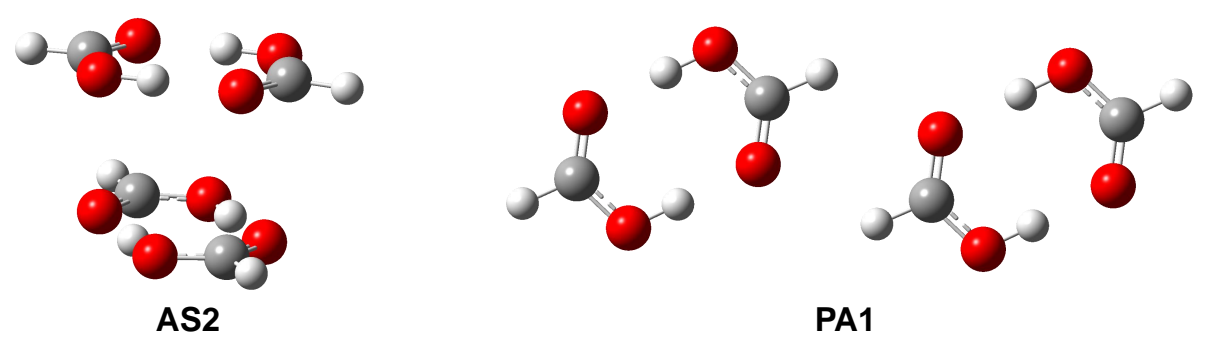

Figure 7.1: Calculated global minimum structures of the formic acid tetramer with (AS2) and without dispersion correction (PA1) [220, 433]. AS refers to aligned stack and PA to planar association. The nomenclature is adapted from Ref. [220].

Similar to carboxylic acid trimers, larger, even numbered clusters such as tetramers were predominantly studied theoretically [119, 219, 220, 430, 431, 433, 442-448]. For the quantum chemical description of such systems, typically DFT methods are used. The predicted structure of the global minimum depends decisively on whether a dispersion correction is applied. An example is the formic acid tetramer: Without it, a planar structure composed of two (FF) units is predicted to be most stable with B3LYP [119, 219, 220], as shown in Figure 7.1. It is called PA1 in Ref. [220], in which PA refers to planar association. In 2006, Karpfen and Thakkar calculated binding energies of formic acid tetramers at the MP2 level with increasing basis set sizes (VDZ, VTZ, aVTZ, and VQZ) and compared the performance to the B3LYP results. With MP2, the global minimum is a $\pi-\pi$ stacked dimer (Figure 7.1), which is consistent with works of Wang [443] and Zhao and Truhlar [444]. This structure is abbreviated to AS2 (aligned stack). In more recent studies of Thakkar [433] and Ito [119], the dispersion-correcting potentials (DCP) by Torres and DiLabo [449] were employed to improve the performance of B3LYP. With this correction, the global minimum prediction is analogous to MP2 (AS2 in Figure 7.1). Other examples of hydrogen bonded clusters where the implementation of dispersion corrections has a profound effect on the obtained global minimum structure are trans- $N$-methylacetamide trimers, which are predicted as chains rather than cyclic as found experimentally, if calculated without it [450]. The same is observed for imidazole [2] and 1,1,1,3,3,3-hexafluoro-propan-2-ol trimers [10]. Altogether, these examples highlight the importance of including such long range corrections for hydrogen bonded systems of a certain size. 


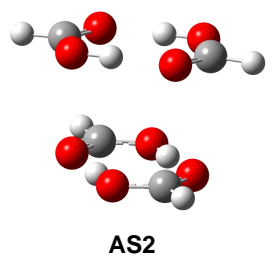

0

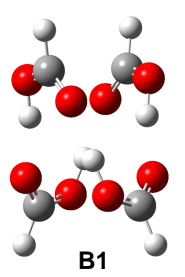

3.4

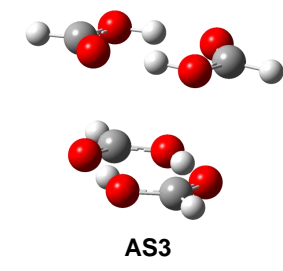

1.2

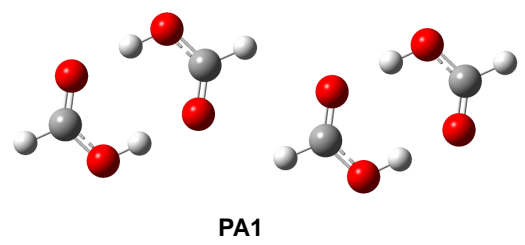

4.1

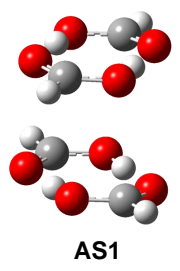

2.9
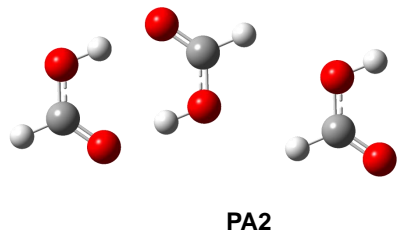

7.7

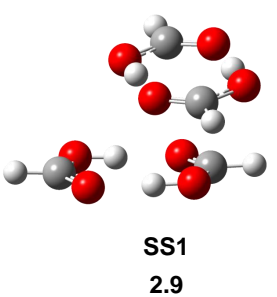

2.9

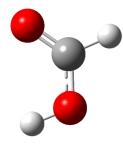

Figure 7.2: Calculated global and local minimum structures (B3LYP-D3(BJ)/aVTZ) of the formic acid tetramer. Their relative energies (in $\mathrm{kJ} \mathrm{mol}^{-1}$ ) are listed with respect to the global minimum (AS2). The structures and nomenclature were taken from Ref. [220]. AS refers to aligned stacks, SS to shifted stacks, PA to planar associations, and B to bowls.

Here, the global minimum and the six next-higher local minima of the formic acid tetramer from Refs. [220, 433] were calculated with B3LYP using Grimme's D3 dispersion correction [451]. The structures and their relative energy difference with respect to the global minimum are depicted in Figure 7.2. The predicted energetic order is in line with the B3LYP-DCP approach [433] as well as with the MP2 calculations [220], apart from B1 and PA1, which are energetically switched, and AS1 and SS1, where the latter is predicted to be isoenergetic with B3LYP-D3 (Figure 7.2) and lower in energy relative to AS1 with the other two methodologies. The four lowest-energy tetramers are all $\pi-\pi$ stacked structures. Their relative energy differences are small (within $3 \mathrm{~kJ} \mathrm{~mol}^{-1}$ ), meaning that the potential energy surface for rotating one dimer on top of the other is presumably relatively flat $[220]$.

Table 7.1: Harmonically calculated, $(\mathrm{FF})_{\mathrm{a}}$-scaled band position $\left(\right.$ in $\left.\mathrm{cm}^{-1}\right)$ of the $\mathrm{C}=\mathrm{O}$ stretching vibrations of the formic acid dimer and the four most stable tetramers (Figure 7.2) at the B3LYP-D3(BJ)/aVTZ level. The scaling factor amounts to 0.986. In addition, the predicted Raman scattering-cross sections $\sigma$ (in $10^{-36} \mathrm{~m}^{2} \mathrm{sr}^{-1}$ ) and IR intensities $I$ (in $\mathrm{km} \mathrm{mol}^{-1}$ ) are listed.

\begin{tabular}{ccccccc|cccccc}
\hline \hline & $\tilde{\nu}$ & $\sigma$ & $I$ & $\tilde{\nu}$ & $\sigma$ & $I$ & $\tilde{\nu}$ & $\sigma$ & $I$ & $\tilde{\nu}$ & $\sigma$ & $I$ \\
\hline$(\mathrm{FF})$ & 1664 & 91 & 0 & & & & 1741 & 0 & 822 & & & \\
$\mathrm{AS} 2$ & 1654 & 0 & 2 & 1659 & 144 & 0 & 1720 & 0 & 10 & 1747 & 0 & 1352 \\
$\mathrm{AS} 3$ & 1657 & 75 & 3 & 1668 & 79 & 0 & 1727 & 0 & 291 & 1747 & 1 & 1071 \\
$\mathrm{AS} 1$ & 1667 & 0 & 4 & 1670 & 163 & 0 & 1736 & 2 & 668 & 1746 & 0 & 675 \\
$\mathrm{SS} 1$ & 1661 & 160 & 5 & 1663 & 0 & 4 & 1739 & 1 & 1080 & 1739 & 3 & 325 \\
\hline \hline
\end{tabular}


The harmonically calculated and $(\mathrm{FF})_{\mathrm{a}}$-scaled band positions of the $\mathrm{C}=\mathrm{O}$ stretching vibrations of the four stacked tetramers are listed in Table 7.1 in addition to the corresponding dimer vibrations. As expected, the predicted tetramer band positions are close to those of the dimer - in most cases one $\mathrm{C}=\mathrm{O}$ stretching vibration of the tetramer is up- and one downshifted with respect to each of the two dimer vibrations. Their predicted Raman scattering cross-sections and IR intensities vary - either there is one dominantly IR and Raman active vibration as for AS2, or the intensities are each split over two modes as seen for AS3. This close proximity of dimer and tetramer $\mathrm{C}=\mathrm{O}$ stretching vibrations will also hold true for larger stacks of dimers and is the reason for the lack of experimental data on the formic acid tetramer and larger dimer assemblies [119]. Evidence that bands of larger clusters overlap with dimer bands was already seen in the Raman jet spectra in Chapter 6 . The symmetric $\mathrm{C}=\mathrm{O}$ stretching vibrations of acetic and pivalic acid broaden at the lower-wavenumber side at higher concentrations (Figures 6.10 and 6.12).

Within this chapter, the vibrational characterisation of these dimer assemblies shall be explored experimentally. Since the vapour pressure of pivalic acid is too small for such measurements, this study is focussed on formic and acetic acid. The relative energy differences of acetic acid tetramers calculated in the structure motifs predicted for the four lowest-energy formic acid tetramers are shown in Figure A.28 in the appendix. The lowest-energy structure out of the four is additionally displayed in Figure 7.3 and exhibits an analogous structure motif (AS2) than the formic acid global minimum tetramer. Rather than assigning vibrational bands to specific structures, which is not feasible due to their overlap, the overall aim of this chapter is to examine the formation of dimer assemblies from a dynamical point of view by studying their aggregation behaviour spatially resolved with FTIR imaging. A two-fold approach is employed for this study - first of all, their formation is enhanced by utilising high substance concentrations and monitored space-resolved. As before, the region behind the nozzle as well as shock waves are examined. Secondly, the carrier gas is varied. Heavier rare gases than helium are more efficient cooling agents and accelerate cluster formation (cf. Section 2.2). Obviously, the polarisability of rare gas atoms also rises with their size. As a consequence, these tend to form van-der-Waals complexes with the seeded substances [55]. This is typically observed for argon and the heavier homologues [146-148]. Excessive condensation of

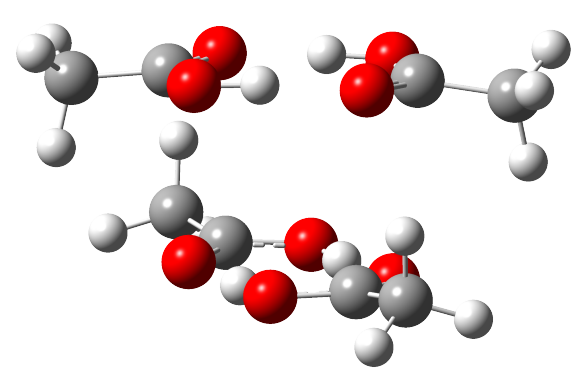

Figure 7.3: Predicted acetic acid tetramer structure in the AS2 structure motif of formic acid, calculated at the B3LYP-D3(BJ)/def2-TZVP level. 
carrier gas on monomers and clusters causes nanocoating of these. In the vibrational spectra, this manifests in band position shifts relative to isolated molecules or clusters in the gas phase. A recent study by Oswald and co-workers illustrates that this shift approaches the one found in cryomatrices, if the molecules are fully coated [149]. In their study, the incremental shift of the $\mathrm{N}-\mathrm{H}$ stretching frequency of the pyrrole monomer was examined by adding increasing amounts of dinitrogen to helium expansions [149]. Other examples of such nanocoating studies include small alcohol dimers such as the methanol [452], ethanol [146, 452, 453], and tert-butyl alcohol dimers [452], as well as the $n$-alkanol dimers of 1-propanol, 1-butanol, and 1-hexanol [148].

Here, a different strategy will be explored: Instead of measuring several acid-in-helium expansions with increasing amounts of nitrogen or rare gas, the sample substance is purely expanded in the respective additive and the complexation is observed spaceresolved with FTIR imaging. Close to the nozzle, the rotational temperature is still high and decreases with distance via collisions. The weakly bound van-der-Waals complexes with the carrier gas will only form if the temperature is sufficiently low, i.e., with some distance to the nozzle. From there on, the progressing nanosolvation can then be observed in detail. For these experiments, argon will be utilised as carrier gas. Two aspects will be examined - firstly nanosolvation with argon and secondly, enhanced cluster formation. The purpose of the latter is to increase the amount of clusters without having to use extremely high acid concentrations. To ensure that the band positions of these are not perturbed by argon, the interference of two supersonic expansions will be exploited. The accompanying temperature increase will likely be high enough to break the weak van-der-Waals interactions to argon.

The nomenclature of the clusters is adapted from the previous chapters. Since the size and exact structure of the dimer stacks is unknown, these are abbreviated to $(\mathrm{FF})_{n}$ and $(\mathrm{AA})_{n}$, whereby $n$ is larger than one $(n>1)$. To avoid ambiguity, the symmetries of the vibrations are not displayed as an index.

The results presented in this chapter were obtained as parts of the bachelor theses of Sophie Schweer (initial performance test of the applicability of formic and acetic acid for the characterisation of tetramers shown in Figure A.29) [179] and Lea Leibold (acetic acid tetramer measurements) [454], and during a research project of Michael Schwarzer (argon and helium carrier gas experiments with formic acid).

\subsection{High Concentration Measurements}

Both formic and acetic acid expansions were carried out at high concentrations of $1-2 \%$ in helium. The FTIR jet spectra are shown in Figure A.29 in the Appendix. For reference, all spectra shown in the previous chapters were measured at concentrations below $0.2 \%$. Since the band of dimer assemblies is more pronounced for acetic acid, despite the lower concentrations used, these will be scrutinised in more detail.

In Figure 7.4, FTIR jet spectra of a single slit expansion of acetic acid in helium are displayed with increasing nozzle distance. The spectra have been spatially averaged over $3 \times 3$ binned pixels. The acetic acid monomer band position increases with distance from 


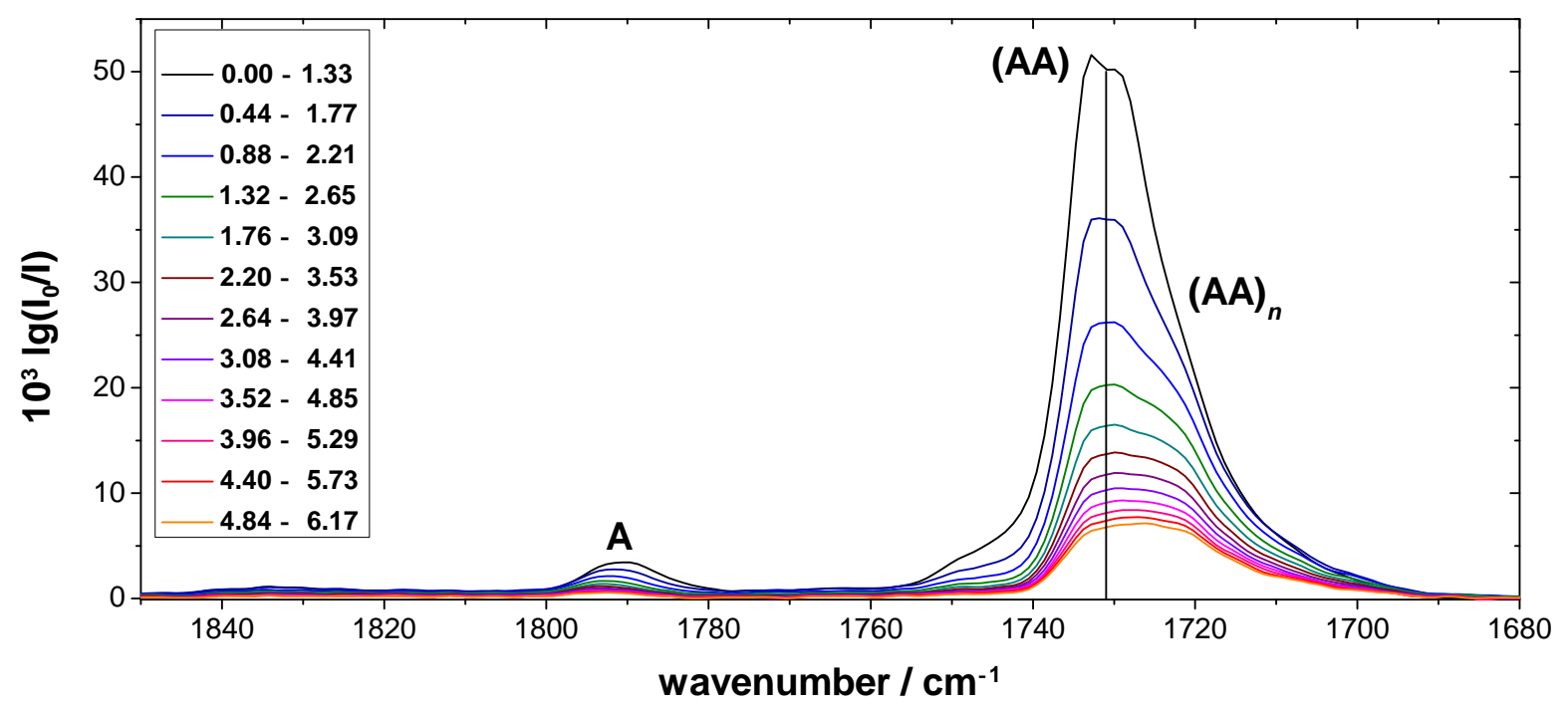

Figure 7.4: FTIR spectra of acetic acid $(c<1.0 \%$ in helium, 500 mbar reservoir pressure, 160 scans) in the $\mathrm{C}=\mathrm{O}$ stretching region averaged over $3 \times 3$ binned pixels with increasing nozzle distance. The nozzle distance intervals (in $\mathrm{mm}$ ) are show in the inlet.

$1790 \mathrm{~cm}^{-1}$ (spectrum directly at the nozzle) to $1793 \mathrm{~cm}^{-1}$ (from $2 \mathrm{~mm}$ nozzle distance onwards), which is attributed to rotational cooling. At a nozzle distance of about $1 \mathrm{~mm}$, a small shoulder forms in the (AA)-attributed band profile, that is approximately equal in intensity at a nozzle distance of about $3 \mathrm{~mm}$. Considering the band positions in Table 7.1 , this band is likely due to dimer assemblies (AA) ${ }_{n}$.

To probe their stability in shock waves, converging expansions of two supersonic jets have been realised similar to those of formic acid (Figure 6.7). The results are displayed in Figure 7.5. The monomer and dimer densities in the single slit expansion of acetic acid resemble those of the formic acid monomer and dimer in Figure 6.7. Additionally, the shoulder of the dimer band has been integrated (bottom row of Figure 7.5). As seen in the corresponding FTIR spectra (Figure 7.4), these larger clusters form at some distances to the nozzle. The density maximum is located along the propagation axis where the number of collisions is highest. In the double expansion, the two flows are pushed inwards due to the Bernoulli effect (see Section 3.1.1). Expectedly, the acetic acid dimer signal remains high when the two expansions interfere. Dimer band intensity is also seen at low nozzle distances in-between the two slits, which was not the case for formic acid. This area is filled with background gas, which typically consists of carrier gas (helium) and a small fraction of warm monomer. In case of carboxylic acids, it is also composed of warm dimers. Their high abundance at the concentrations used here (five-fold increase compared to the formic acid expansions in Chapter 6) is the reason for the higher intensity in this area. There also seems to be density of larger dimer assemblies in the warm region between the slits, yet this is likely due to the broadness of the gas phase dimer band.

The signal of dimer assemblies $(\mathrm{AA})_{n}$ is most interesting. In contrast to the formic acid trimer, the density of these dimer assemblies reaches a maximum when both expansions 


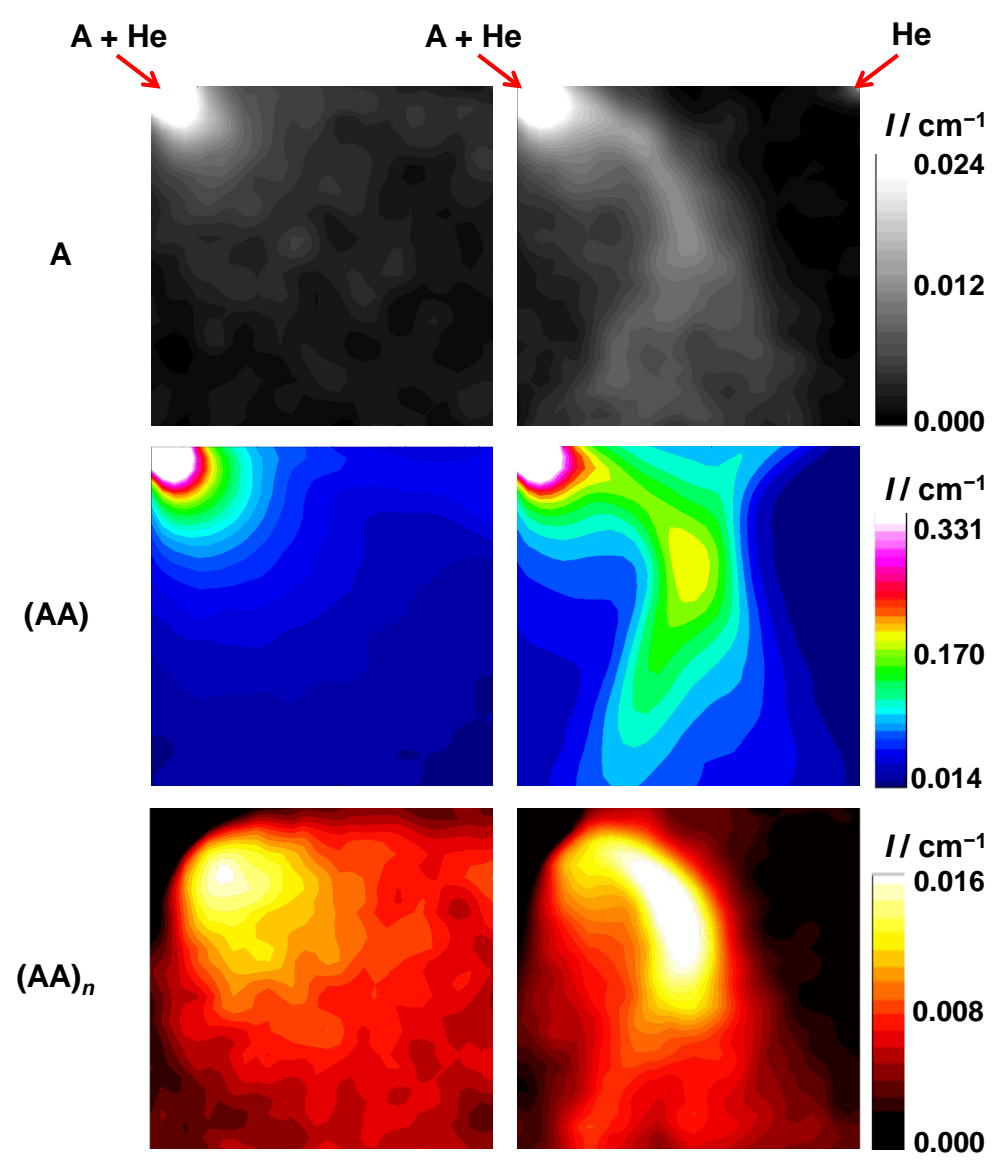

Figure 7.5: 2D spatial evolution of the integrated intensity $I$ of the acetic acid monomer A, dimer (AA), and dimer assembly bands $(\mathrm{AA})_{n}$ with $n>1$ in a supersonic expansion of acetic acid in helium in the $\mathrm{C}=\mathrm{O}$ stretching region $(\mathrm{c}<1 \%$, left column). The interference of that expansion with a second, pure helium expansion is visualised in the right column. The reservoir pressures used amount to 500 mbar. The spectra have been averaged over 160 and 154 scans, respectively. The positions of the nozzle slits are marked with arrows.

interfere (Figure 7.5). An FTIR spectrum in the collision zone is depicted in Figure 7.6 and shows that the $(\mathrm{AA})_{n}$ band is clearly enhanced. The band maximum is centred at $1727 \mathrm{~cm}^{-1}$. The temperature increase is evident by the smaller wavenumber of the acetic acid monomer band $\left(1790 \mathrm{~cm}^{-1}\right)$ as well as the overall broadness of all bands. The enhanced (AA $)_{n}$ formation is likely a result of the compression by the second expansion that results in a high number of collisions and therefore, favours the formation of these larger dimer assemblies. As the spectral features of different dimer assemblies cannot be separated, their size in shock waves remains unknown. The bands of trimers, on the other hand, are well separated from those of dimers and dimer assemblies so that their decline in shock waves in favour of dimer assembly formation can be observed, which explains their different areas of stability in the expansions. 


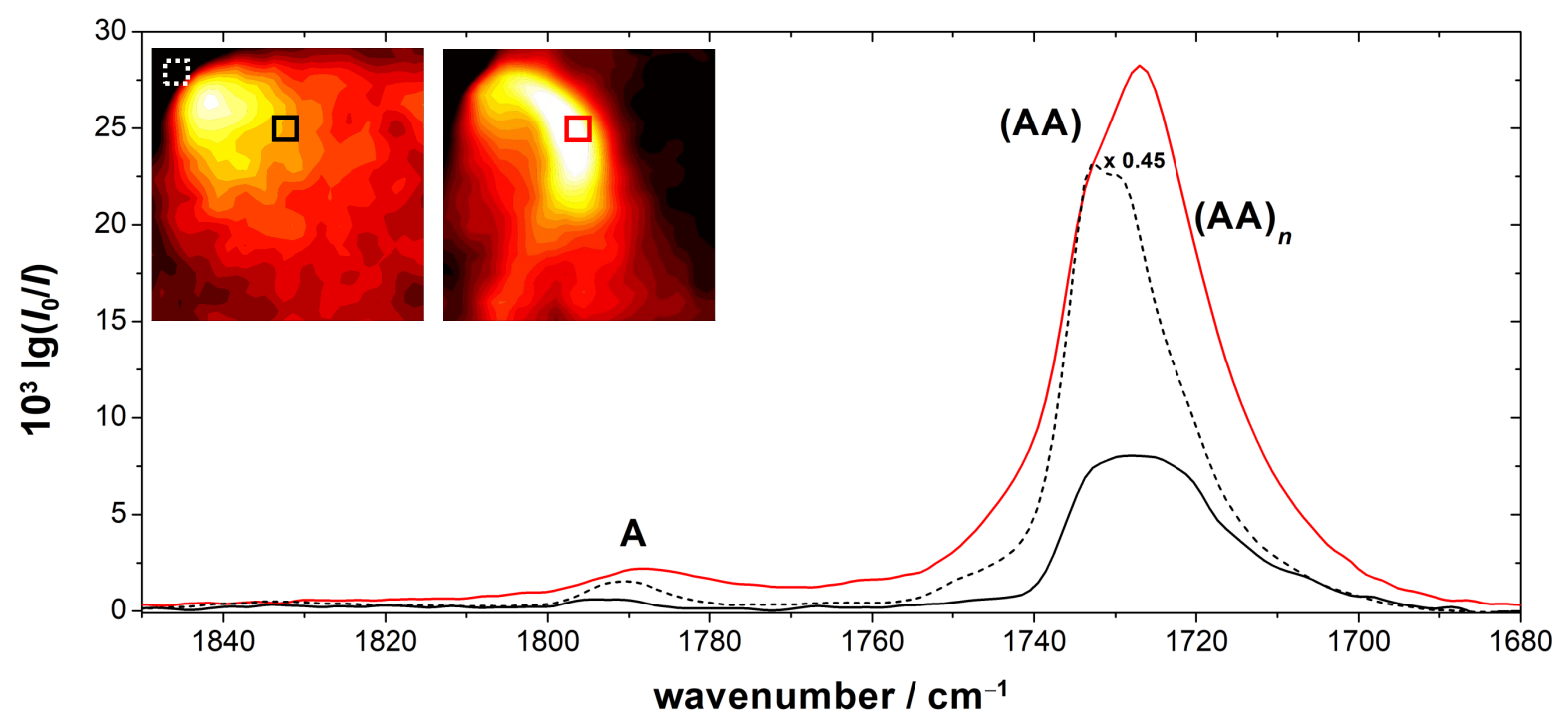

Figure 7.6: FTIR jet spectra of acetic acid $(c<1 \%$ in helium) averaged over $3 \times 3$ binned pixels. The spectra have been recorded at a reservoir pressure of $500 \mathrm{mbar}$ in a single slit expansion (black) and in interfering expansions (red). The number of scans amounts to 160 (black) and 154 (red). The spatial location of the $3 \times 3$ binned pixels in the expansion is marked by black and red boxes in the inlets. The dashed spectrum has been averaged over the area marked with the white dashed square and has been scaled by 0.45 . The $2 \mathrm{D}$ spatially resolved evolutions of the integrated intensity of the monomer A, dimer (AA), and dimer assembly bands $(\mathrm{AA})_{n}$ in the $(10 \times 10) \mathrm{mm}^{2}$ wide expansion area can be found in Figure 7.5, the latter one being repeated as an inset in this figure.

\subsection{Influence of the Carrier Gas}

As a next step, the carrier gas is varied. The study of its impact is focussed on two aspects - firstly, argon coating of formic acid clusters will be scrutinised to simulate the transition from gas phase spectra to those recorded in cryomatrices and secondly, the enhanced cluster formation with the heavier carrier gas. To ensure that the band positions of these are not perturbed by argon, these will be studied in shock waves between converging jets, as the accompanying temperature increase will likely be high enough to break the weak interactions to argon. Since the dimer assemblies were shown to be stabilised in shock waves, these are expected to remain.

To quantify the impact of argon on the formic acid spectra, these were measured under identical conditions in helium and argon. The results are summarised in Figure 7.7. As before, spectra with increasing nozzle distance are shown. The distance intervals are visualised as squares in the plots of the $2 \mathrm{D}$ spatial evolution of the overall density (band integral over the whole $\mathrm{C}=\mathrm{O}$ stretching region). The spectra of formic acid in helium feature the monomer $\left(1777 \mathrm{~cm}^{-1}\right)$ and dimer band $\left(1741 \mathrm{~cm}^{-1}\right)$. Due to the low signal intensities, the pixel-spanning noise mentioned in the Section 3.1.1 can be clearly seen. By performing a single expansion with the double slit nozzle, barely any substance-free areas exist that can be utilised for its reduction. With argon as a carrier gas, the dimer 

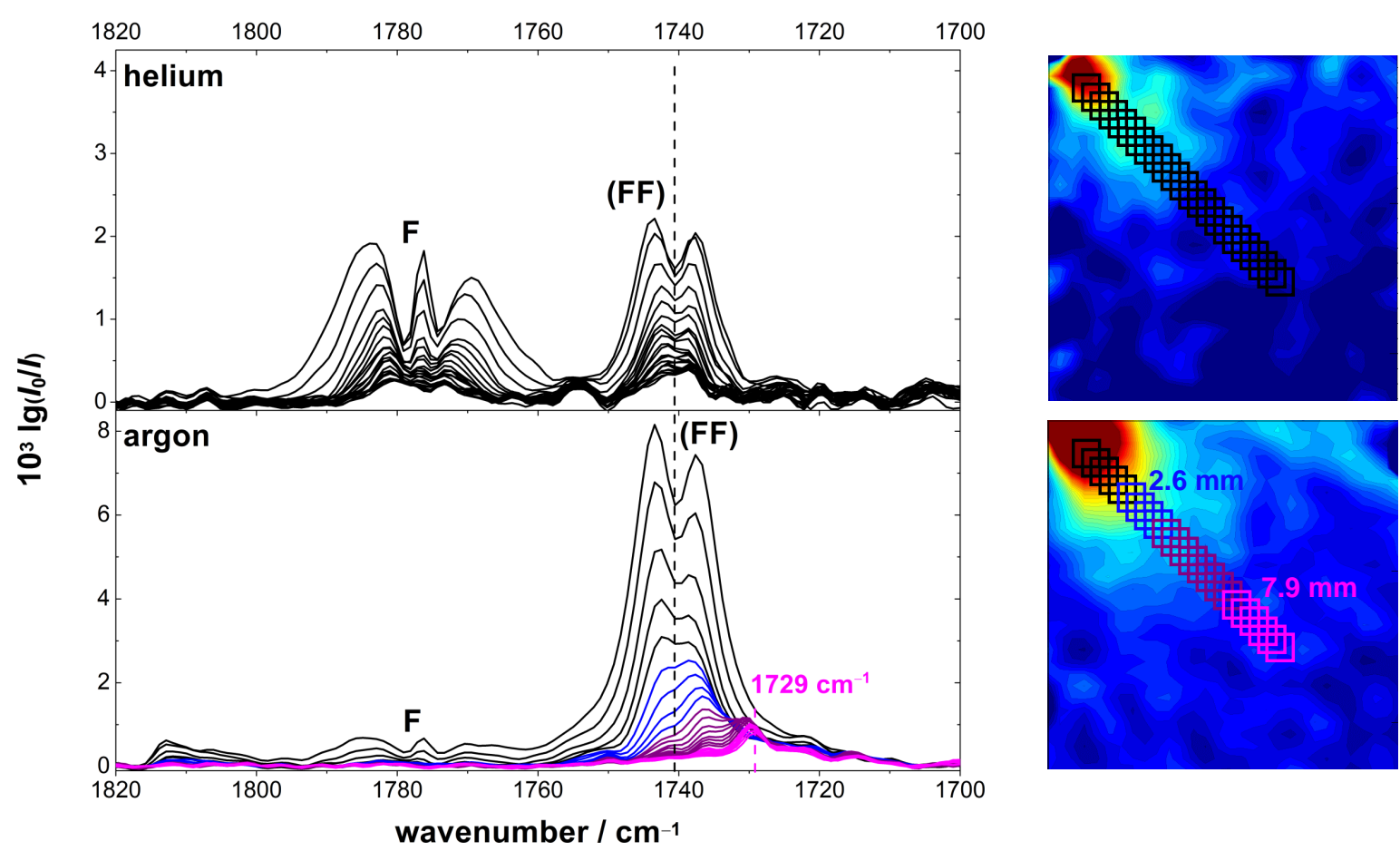

Figure 7.7: FTIR jet spectra of formic acid $(c<0.1 \%)$ seeded in helium (top) and argon (bottom) recorded at a reservoir pressure of 500 mbar averaged over 100 and 205 scans, respectively. The spectra have been averaged over $3 \times 3$ binned pixels illustrated as black, blue, and pink boxes in the spatially resolved images of the overall density in the expansions (integrated intensities over the whole $\mathrm{C}=\mathrm{O}$ stretching region). The integrated intensity is increasing from blue, over green and yellow up to red. Randomisation of the pixel-spanning noise is not carried out, as the signal loss is too severe.

signal increases significantly compared to the monomer. In addition, the combination band $\left(\nu_{11}+\nu_{14}\right)$ of the dimer (Section 6.4) is seen at $1811 \mathrm{~cm}^{-1}$. In the helium spectra, its intensity is too low for detection. Starting from a nozzle distance of about $3 \mathrm{~mm}$ (blue spectra in Figure 7.7), the band of the formic acid dimer shifts towards lower wavenumbers. At that point, the distance of the maxima of the $\mathrm{P}$ and $\mathrm{R}$ branches of the formic acid monomer band amounts to approximately $13 \mathrm{~cm}^{-1}$, which corresponds to a rotational temperature of about $60 \mathrm{~K} .{ }^{1}$ At the nozzle, it is roughly $20-40 \mathrm{~K}$ higher. The comparatively low rotational temperature at the nozzle exit is a result of collisions already taking place inside it (cf. Figure 3.1). Overall, this temperature analysis is error prone due to the low monomer signal in the argon expansions and should only be taken as a rough estimate. An increase in cooling efficiency by using argon instead of helium is not seen for the small nozzle distances analysed here and it is likely counteracted by the stronger aggregation due to the associated heat of condensation. From a nozzle

\footnotetext{
${ }^{1}$ The rotational temperature can be estimated according to Equation 2.8 (see Section 2.2). The distance of the maxima of the $\mathrm{P}$ and $\mathrm{R}$ branches $\Delta \tilde{\nu}_{\mathrm{PR}, \mathrm{RT}}^{\max }$ of the $\mathrm{C}=\mathrm{O}$ stretching band of the formic acid monomer amounts to $29 \mathrm{~cm}^{-1}$ in the gas phase at room temperature (see Figure A.19).
} 
distance of $8 \mathrm{~mm}$ onwards, the band position of the shifted dimer $\left(1729 \mathrm{~cm}^{-1}\right)$ remains constant. It nearly coincides with the argon matrix values measured by Gantenberg and co-workers $\left(1728.3 \mathrm{~cm}^{-1}\right)$ [82] and Marushkevich et al. $\left(1728 \mathrm{~cm}^{-1}\right)$ [399] and matches one of the two bands $\left(1729 \mathrm{~cm}^{-1}\right.$ and $\left.1730 \mathrm{~cm}^{-1}\right)$ observed by Ito [455]. Hence, (FF) is fully surrounded (nanocoated) by argon. The shifted band exhibits several side maxima on the higher-wavenumber side. Whether these are due to clusters or the pixel-spanning noise is hard to specify due to the low signal caused by the density decrease.

Overall, this experiment nicely illustrates that the shift of vibrational bands in cryomatrices can be simulated by recording space-resolved FTIR spectra of the sample substance seeded in the respective carrier gas. The advantage compared to conventional FTIR spectroscopy as used in Refs. [79, 149] is that the incremental shift of the formic acid dimer band can be observed space-resolved rather than in several snap shots, which requires fewer measurements. Since the formic acid dimer has recently also been studied in a nitrogen matrix where an identical shift $\left(-13 \mathrm{~cm}^{-1}\right)$ was found for the antisymmetric $\mathrm{C}=\mathrm{O}$ stretching vibration [389], similar experiments with $\mathrm{N}_{2}$ might be very interesting.

\subsubsection{Comparison to Quantum Chemical Calculations}

The comparison of quantum chemical calculations with matrix isolation data in form of a benchmark is very demanding, as the matrix environment has to be accurately captured on the theory side. This includes host-guest and host-host interactions, several solvation shells, and potentially even different trapping sides of the guest. An attempt to simulate the matrix shifts of the fundamentals of the formic acid dimer (FF) relative to the gas phase has been presented by Ito in 2019 [397] for an argon matrix. It is based on a previous study of the most likely structure of the first solvation shell [456].

(FF) was determined to be located on an Ar (111) plane of the cubic lattice with four substitutional sides [456]. The first solvation shell around (FF) was modelled with 26 argon atoms and the second with 68 [397]. While the structural relaxation caused by the host-host interactions between the first two shells was found to have a critical influence on the results, additional solvation shells were shown to have barely any impact on the predicted matrix shifts [397]. The calculations were carried out in a two layer ONIOM scheme, which separates the atoms into two layers - a high layer, where calculations are performed at a higher level of theory, in this case B971/6-31++G(3df,3pd), and a low layer, where the atoms are treated with universal force fields [397]. The overall best agreement with experiment was achieved, when (FF) was regarded as the high and the argon shells as the low layer [397].

The thus predicted matrix shifts by Ito are listed in Table 7.2 alongside the experimental values obtained from jet [66, 68, 118, 191, 387] and matrix data [65, 455]. Gas phase band positions are not used here to exclude effects from thermal shifts. As aforementioned, the fundamentals of (FF) are labelled according to the Herzberg nomenclature throughout this work, but with the order of $A_{\mathrm{u}}$ and $B_{\mathrm{g}}$ switched, so that the Raman active (symmetric) vibrations correspond to $\nu_{1}-\nu_{12}$ and the IR active (antisymmetric) vibrations to $\nu_{13}-\nu_{24}$. Further details can be found in Ref. [62] (assignment of the modes) and in Appendix A.3.4. The $\mathrm{O}-\mathrm{H}\left(\nu_{1}\right.$ and $\left.\nu_{17}\right)$ and $\mathrm{C}-\mathrm{H}$ stretching vibrations 
$\left(\nu_{2}\right.$ and $\left.\nu_{18}\right)$ are omitted for this analysis due to the aforementioned complex resonance pattern of $(\mathrm{FF})$. The antisymmetric $\mathrm{H}-\mathrm{O}-\mathrm{C}$ in plane bending vibration $\nu_{20}$ is also excluded, as a re-assignment of the gas phase band position has been suggested by a recent FTIR jet study of Kollipost [118] that might also affect the argon matrix assignment. FTIR imaging experiments scrutinising the shift of this band in an argon expansion could help to examine this further.

The comparison of the experimental and predicted matrix shifts clearly underlines the difficulty of accurately simulating vibrational spectra of molecules or molecular clusters stored in cryogenic matrices. Most of the experimentally determined matrix shifts listed in Table 7.2 are below $\pm 10 \mathrm{~cm}^{-1}$, thus, the impact of the environment on the spectra can be very subtle for some modes. Furthermore, two different trapping sites of the formic acid dimer were identified in the IR matrix experiments by Ito [455], which were not captured with the model described in Ref. [397]. The agreement of the predicted matrix shifts is within $\pm 5 \mathrm{~cm}^{-1}$ for most vibrations, yet the relative errors are fairly large. For some fundamentals, particularly pronounced deviations are seen (see $\nu_{4}, \nu_{5}, \nu_{19}$, and $\nu_{22}$ in Table 7.2). These amount to $-10 \mathrm{~cm}^{-1}$ and $-20 \mathrm{~cm}^{-1}$ for symmetric $\mathrm{H}-\mathrm{O}-\mathrm{C}$ and $\mathrm{H}-\mathrm{C}-\mathrm{O}$ in plane bending vibrations $\nu_{4}$ and $\nu_{5}$ and to $+12 /+13 \mathrm{~cm}^{-1}$ and $+5 /+9 \mathrm{~cm}^{-1}$ for the antisymmetric $\mathrm{C}=\mathrm{O}$ and $\mathrm{C}-\mathrm{O}$ stretching vibrations $\nu_{19}$ and $\nu_{22}$, respectively. The matrix shifts of the out-of-plane bending vibrations $\nu_{10}, \nu_{12}$, and $\nu_{13}$, on the other hand, are particularly well captured by Ito's model. Whether this is coincidence or based on a good description of their host-guest interaction, can only be decided with additional experimental reference data on the three missing modes $\nu_{11}, \nu_{15}$, and $\nu_{16}$. For $\nu_{14}$ (antisymmetric $\mathrm{O}-\mathrm{H}$ out of plane bending vibration), the deviations are already larger.

In conclusion, in addition to the complexity of the simulation of matrix isolation spectra, this example clearly highlights the need for further progress on the matter, which can only be achieved by vigorous efforts on both the theoretical as well as the experimental side [457]. Another good reference system for such a systematic study would be the formic acid monomer, where matrix shifts are on average larger and a full set of matrix and jet data is available (cf. Table 4.2). Besides, F was examined in various matrix hosts such as neon [298, 335], nitrogen [345], or molecular hydrogen [309, 336], which enables a comparison of the different host-host and host-guest interactions. Due to the new jet-cooled reference data on the higher-energy cis-formic acid obtained in the course of this thesis, one could also contrast differences between the host-guest interactions of both rotamers (to some extent). 
7 Dimer Assemblies

Table 7.2: Comparison of experimental and predicted argon matrix shifts (in $\mathrm{cm}^{-1}$ ) of the fundamentals of the formic acid dimer. The bands are labelled according to the Herzberg nomenclature, yet the order of the $A_{\mathrm{u}}$ and $B_{\mathrm{g}}$ modes is switched. Jet-cooled values serve as the experimental argon-free reference. Details on the calculated values (prediction) can be found in Ref. [397] and in the text.

\begin{tabular}{|c|c|c|c|c|c|c|c|}
\hline \multirow{2}{*}{ symmetry } & \multirow{2}{*}{$\nu_{i}$} & \multicolumn{3}{|c|}{ experiment } & \multicolumn{3}{|c|}{ prediction ${ }^{[397]}$} \\
\hline & & jet & $\operatorname{argon}^{b, c}$ & matrix shift & free & argon & matrix shift \\
\hline \multirow{7}{*}{$A_{\mathrm{g}}$} & $\nu_{3}$ & $1666^{\mathrm{a}}$ & $1671 / 1660$ & $+5 /-6$ & 1703 & 1703 & 0 \\
\hline & $\nu_{4}$ & $1431^{[68]}$ & 1439 & +8 & 1486 & 1484 & -2 \\
\hline & $\nu_{5}$ & $1376^{[68]}$ & 1397 & +21 & 1399 & 1400 & +1 \\
\hline & $\nu_{6}$ & $1224^{[68]}$ & 1229 & +5 & 1267 & 1268 & +1 \\
\hline & $\nu_{7}$ & $682^{[68]}$ & 679 & -3 & 688 & 689 & +1 \\
\hline & $\nu_{8}$ & $194^{[66]}$ & 196 & +2 & 206 & 212 & +6 \\
\hline & $\nu_{9}$ & $161^{[66]}$ & 165 & +4 & 170 & 177 & +7 \\
\hline \multirow{3}{*}{$B_{\mathrm{g}}$} & $\nu_{10}$ & $1060^{[68]}$ & 1062 & +2 & 1081 & 1083 & +2 \\
\hline & $\nu_{11}$ & $911^{[68]}$ & - & & 998 & 1001 & +3 \\
\hline & $\nu_{12}$ & $242^{[66]}$ & $251 / 243$ & $+9 /+1$ & 264 & 273 & +9 \\
\hline \multirow{4}{*}{$A_{\mathrm{u}}$} & $\nu_{13}$ & $1069^{[118]}$ & $1072 / 1068$ & $+3 /-1$ & 1109 & 1111 & +2 \\
\hline & $\nu_{14}$ & $939^{[118]}$ & $947 / 940$ & $+8 /+1$ & 1013 & 1016 & +3 \\
\hline & $\nu_{15}$ & - & - & & 187 & 202 & +15 \\
\hline & $\nu_{16}$ & - & - & & 77 & 96 & +19 \\
\hline \multirow{5}{*}{$B_{\mathrm{u}}$} & $\nu_{19}$ & $1741^{\mathrm{a}}$ & $1730 / 1729$ & $-11 /-12$ & 1779 & 1780 & +1 \\
\hline & $\nu_{21}$ & $1372^{[118]}$ & $1373 / 1372$ & $+1 / 0$ & 1398 & 1399 & +1 \\
\hline & $\nu_{22}$ & $1230^{[387]}$ & $1227 / 1223$ & $-3 /-7$ & 1268 & 1270 & +2 \\
\hline & $\nu_{23}$ & $708^{[118]}$ & - & & 722 & 724 & +2 \\
\hline & $\nu_{24}$ & $264^{[191]}$ & - & & 277 & 284 & +7 \\
\hline $\begin{array}{l}{ }^{\mathrm{a}} \text { this work } \\
\mathrm{b} \text { Raman: [65] } \\
{ }^{\mathrm{c}} \text { IR: }[455]\end{array}$ & & & & & & & \\
\hline
\end{tabular}




\subsubsection{Formation of Dimer Assemblies}

In order to detect dimer assemblies in argon jet expansions without any band position perturbation, argon has to be evaporated from the clusters. One area of a supersonic expansion where this can be achieved are shock waves. The enhanced number of collisions additionally benefits the formation of $(\mathrm{FF})_{n}$. Here, the interfering lateral shock waves in a double slit expansion are chosen. To maximise the cluster formation, a reservoir pressure of 700 mbar has been employed for the substance in argon expansion, whereas for the second pure helium expansion a lower reservoir pressure of $100 \mathrm{mbar}$ is used. This asymmetry shifts the collision zone to the helium-side. As such, the pathway of cluster formation is prolonged before both jets interfere.

The FTIR imaging results of the double expansion are displayed in Figure 7.8 alongside those of an unperturbed flow. Three images are shown - the two-dimensional spatial evolution of the integrated intensity over the whole $\mathrm{C}=\mathrm{O}$ stretching region as a measure of overall density (top row), the integral over the argon complexed formic acid dimer band at $1729 \mathrm{~cm}^{-1}$ (middle row), and a third band integral of argon-free formic acid dimer assemblies (bottom row). Initially, the focus is on the first two rows.

Due to the compression by the second expansion, the overall density is remaining high when both collide. This area of high density is narrower compared to Figure 7.5, as the reservoir pressure of the second expansion is lower. The argon induced downshift of the dimer band occurs at some distance to the nozzle, which is somewhat smaller than for the 500 mbar expansion (Figure 7.7). Expectedly, the signal at $1729 \mathrm{~cm}^{-1}$ diminishes when the lateral shock waves of both expansions interfere. This is attributed to the evaporation of argon from the formic acid clusters. Intensity-scaled FTIR spectra cutting through the shock wave are shown in Figure 7.9. In the unperturbed part of the expansion (black spectrum), the band of the argon complexed formic acid dimer is clearly seen. It declines with decreasing distance to the density maximum, where it is completely gone. Simultaneously, a shoulder grows in on the higher-wavenumber side of the band. It can be partially attributed to warm, argon-free formic acid dimer ( $\mathrm{R}$ and $\mathrm{P}$ branches at $1744 / 1737 \mathrm{~cm}^{-1}$, dashed, grey line in the lower trace of Figure 7.9 ). However, the asymmetry and broadness of the band indicate that there are additional contributions, likely bands of larger dimer assemblies, as seen in the high concentration spectra in Figure A.29 in the appendix. The spatially resolved 2D evolution of the integrated intensity of this higher wavenumber shoulder is shown in the bottom row of Figure 7.8. As expected, it is highest in the collision zone. The high signal directly behind the nozzle is solely due to warm dimer. In the single slit expansion, such signal can be also seen at the second, non-used slit, as the flow approaches the walls of the apparatus so that likely a shock wave with the background gas is formed that similarly causes argon evaporation. This is illustrated by the corresponding spectra in Figure 7.10. The scattered intensity maxima at higher distances are due to noise integration rather than actual signal of warm clusters.

All in all, these experiments nicely illustrate that the advantages of heavier carrier gases such as argon can be effectively exploited in shock waves without the downsides of band position shifts due to nanocoating. 


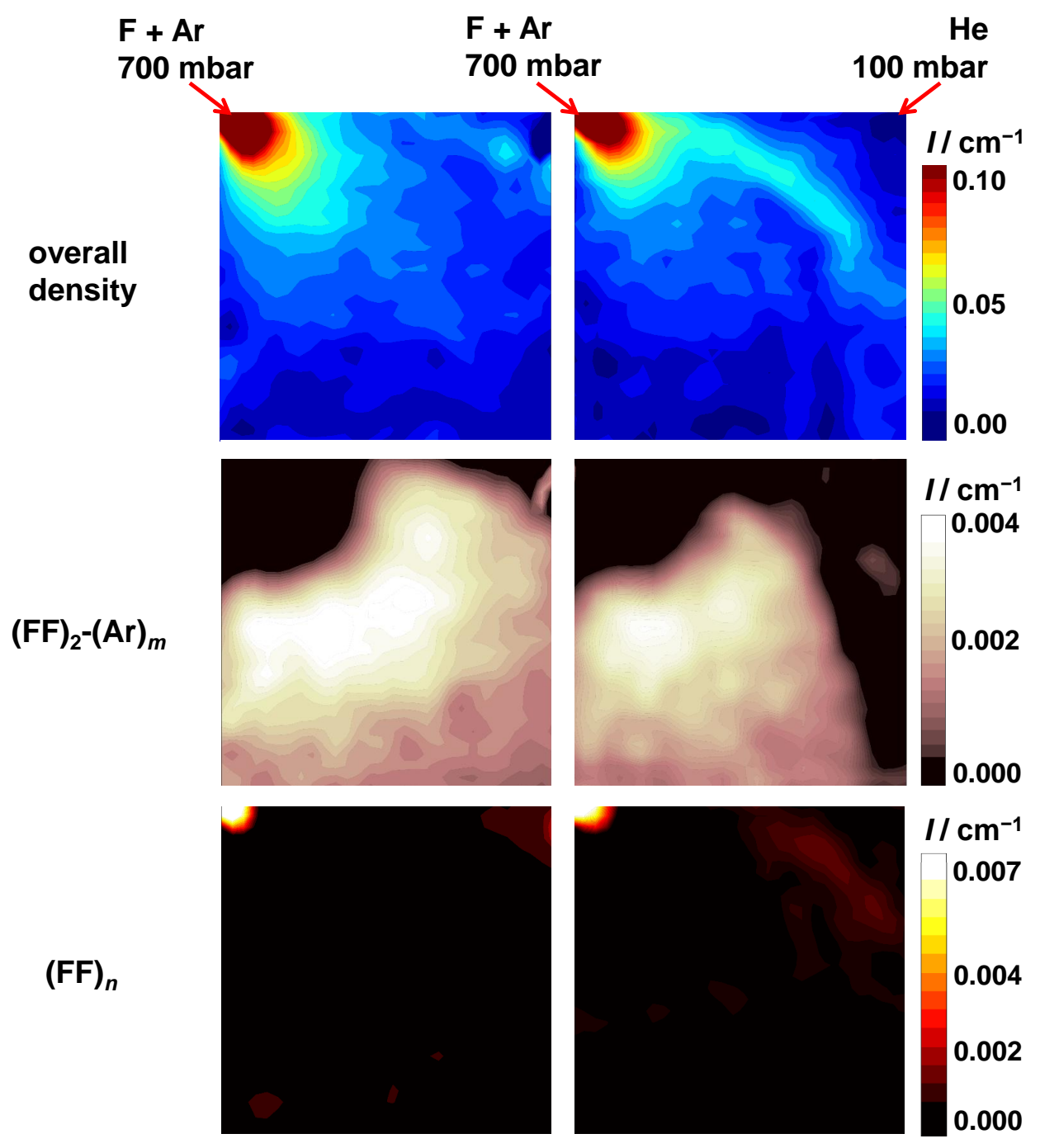

Figure 7.8: FTIR imaging experiments of formic acid $(c<0.1 \%)$ in argon expansions at a reservoir pressure of 700 mbar. In the left column, the unperturbed expansion is shown and in the right column, its interference with a pure helium jet expanded at a lower reservoir pressure of 100 mbar. The FTIR spectra have been averaged over 150 and 170 scans, respectively. The $2 \mathrm{D}$ spatially resolved images have been obtained by integrating over the whole $\mathrm{C}=\mathrm{O}$ stretching region $\left(1800-1650 \mathrm{~cm}^{-1}\right.$, top), a band of argon coated formic acid dimers (FF)$(\mathrm{Ar})_{m}\left(1733-1726 \mathrm{~cm}^{-1}\right.$, middle $)$, and a band of dimer assemblies $\left(1752-1742 \mathrm{~cm}^{-1}\right.$, bottom $)$ $(\mathrm{FF})_{n}$, where $m \geq 1$ and $n>1$. The band integrals chosen for the latter two are visualised in Figure A.30 in the appendix. 

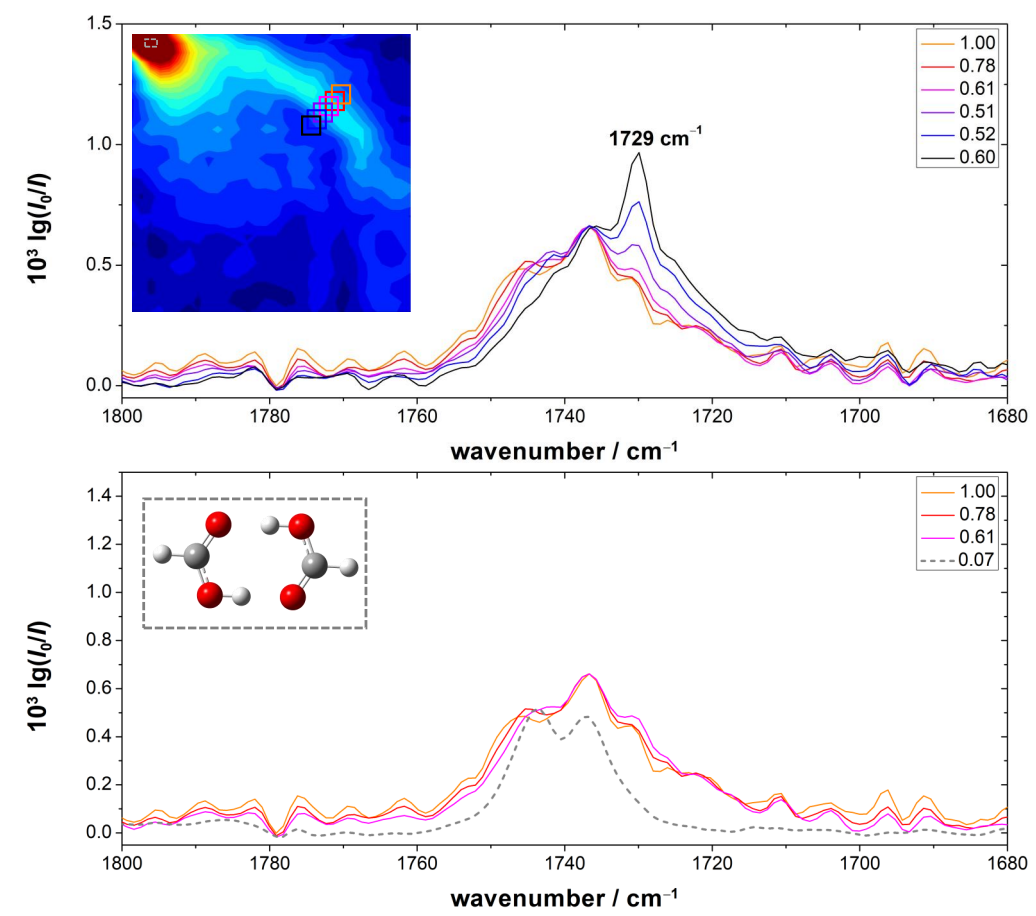

Figure 7.9: FTIR spectra of a supersonic expansions of formic acid $(c<0.1 \%)$ in argon (700 mbar reservoir pressure) interfering with a pure helium expansion (100 mbar reservoir pressure) averaged over 170 scans. The FTIR spectra are spatially averaged over $3 \times 3$ binned pixels shown as coloured boxes in the inset. The colours correspond to those of the spectra. All spectra have been intensity-scaled to the band at $1736 \mathrm{~cm}^{-1}$. The scaling factors are listed in the legend. Below, the FTIR spectra of the shock wave are displayed alongside a scaled FTIR spectrum averaged over $1 \times 2$ binned pixel directly at the nozzle (grey box in the inset).

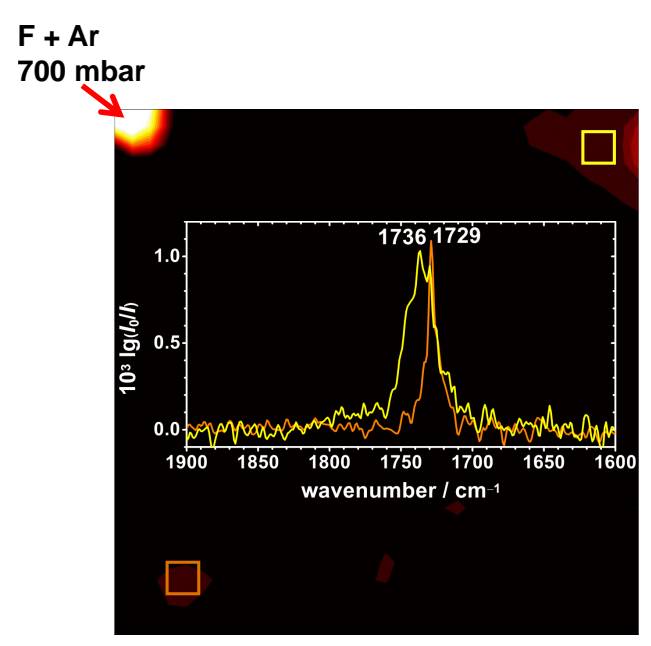

Figure 7.10: FTIR spectra of single slit expansion of formic acid $(c<0.1 \%)$ in argon at a reservoir pressure of 700 mbar spatially averaged over $3 \times 3$ binned pixels. The number of scans amounts to 150 . The area over which the spectra have been averaged are illustrated as orange and yellow boxes. The spatial evolution of $(\mathrm{FF})_{n}$ is shown in the background, which was previously shown in Figure 7.8. 


\subsection{Outlook}

Examples of promising systems for future studies are 1,1,1,3,3,3-hexafluoro-propan-2ol (abbreviated to HFIP) or pyrrole. The $\mathrm{O}-\mathrm{H} / \mathrm{N}-\mathrm{H}$ stretching vibrations of the monomers and their clusters were shown to incrementally shift upon nitrogen and argon complexation [149, 458, 459]. With such systematic studies, one can significantly improve the interpretation of gas phase and matrix isolation spectra. Besides, the dominant monomer and dimer conformations of HFIP exhibit particularly strong IR bands in the OH stretching region [193, 458], which is beneficial for FTIR imaging experiments, as these suffer from a low signal-to-noise ratio (cf. Section 3). Furthermore, due to its clearly identified and sufficiently separated trimer bands [10] and the less populated monomer and dimer conformations [193, 458], HFIP is also an exciting system for aggregation behaviour studies with helium or heavier carrier gases using FTIR imaging.

So far, FTIR imaging experiments have only been conducted in the $\mathrm{C}=\mathrm{O}$ stretching region, as the transmittance of the employed IR lens $(3-5 \mu \mathrm{m}$, ASIO Series, Janos Technology) reduces significantly above $3000 \mathrm{~cm}^{-1}$ (cf. Figure 7.11 ), which combines transmittance and focussing effects and should therefore only be viewed on a relative scale. The band of the $\mathrm{N}-\mathrm{H}$ stretching vibration of the pyrrole monomer is located at $3531 \mathrm{~cm}^{-1}$ and is shifted to $3514 \mathrm{~cm}^{-1}$ upon nitrogen complexation [149]. The $\mathrm{O}-\mathrm{H}$ stretching region of HFIP monomers, clusters, and their nitrogen aggregates extends from $3650-3450 \mathrm{~cm}^{-1}$ [459]. Unfortunately, both coincide with a transmittance minimum of the IR lens, shown in Figure 7.11. To quantify the severity of this dip, FTIR spectra of the $\mathrm{OH}$ stretching region of acetic acid have been recorded. The results are shown in Figure 7.11 alongside an FTIR jet spectrum recorded with the conventional FTIR jet-up (filet jet) for reference. Even though the substance concentration has been significantly enhanced for the FTIR imaging (chilli jet) measurements, neither the monomer A $\left(3585 \mathrm{~cm}^{-1}\right)$ nor the onset of the broad resonance structure overlapping with signals of the polar dimer AA $\left(3304 \mathrm{~cm}^{-1}\right)$ and the trimer A(AA) $\left(3257 \mathrm{~cm}^{-1}\right)^{2}$ are observable due to the high noise between $3300-3600 \mathrm{~cm}^{-1}$. The increase in broadness of the resonance structure of (AA) compared to the filet spectrum can be attributed to higher rotational temperatures at low nozzle distances.

An effective way to enhance the sensitivity of the chilli jet is to utilise narrow band pass filters. The measurements shown in Figure 7.11 were recorded with a $1600 \mathrm{~cm}^{-1}$ wide IR filter $\left(\mathrm{F} 13,4100-2500 \mathrm{~cm}^{-1}\right)$. To avoid overexposure of the imaging detector (MCT FPA), two light attenuators had to be placed inside the beam path, a perforated metal piece and five layers of fly screen. Their transmittance curves can be found in Figure A.4 in the appendix. By reducing the spectral window to approximately $440 \mathrm{~cm}^{-1}$ via the combination of two IR filters (F1: $4000-2860 \mathrm{~cm}^{-1}$ and F2: $3300-2000 \mathrm{~cm}^{-1}$ ), one attenuator (perforated metal piece) could be omitted. The FTIR spectrum recorded under the same experimental conditions is shown in red in Figure 7.11. The spectral noise is significantly reduced, clearly highlighting the potential for improvement. The lower substance signal can be attributed to the concentration uncertainty imposed by

\footnotetext{
${ }^{2}$ The $\mathrm{A}, \mathrm{AA}$, and $\mathrm{A}(\mathrm{AA})$ assignments were taken from Ref. [79].
} 

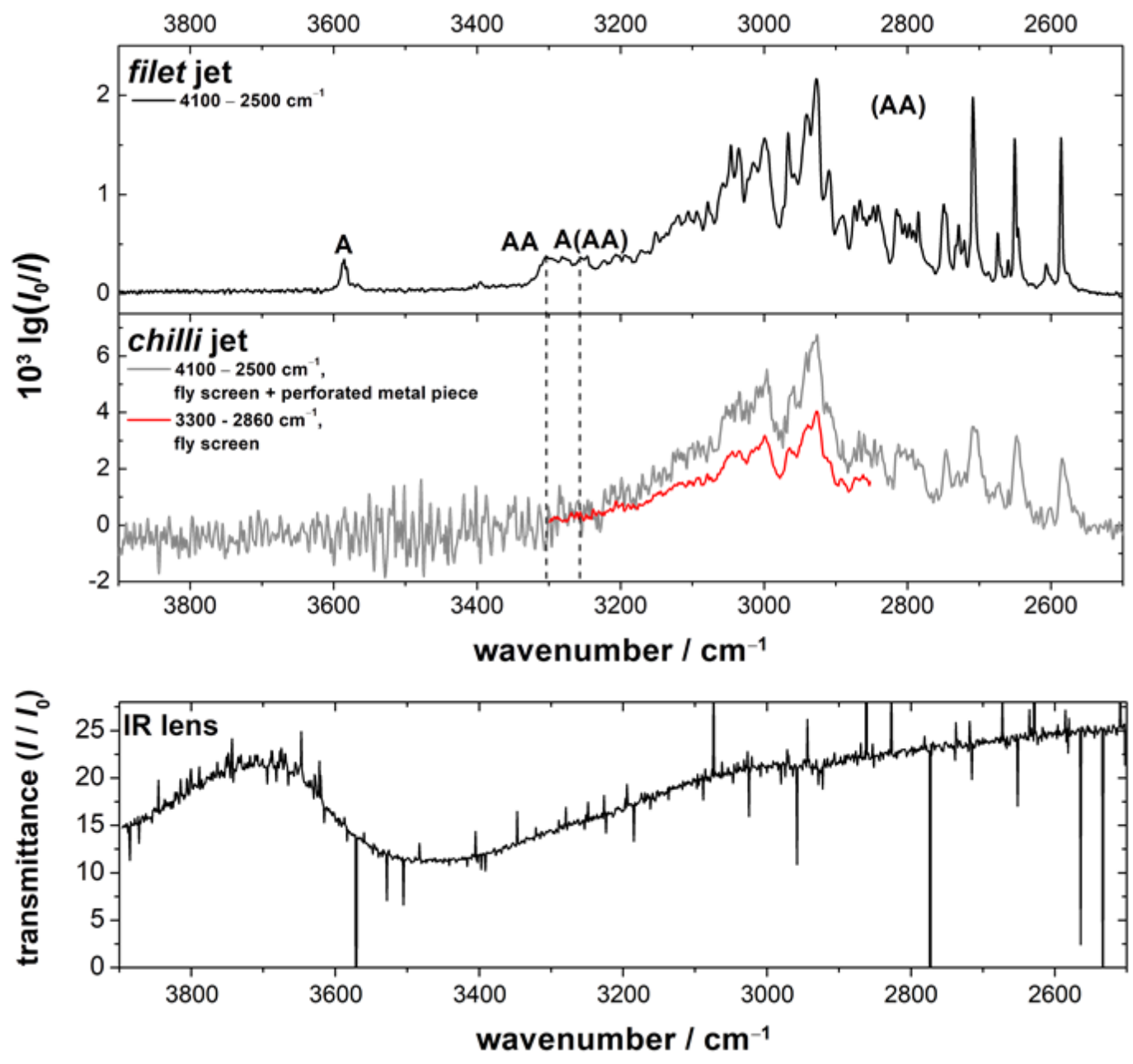

Figure 7.11: FTIR jet spectra of acetic acid in helium recorded with the filet (top) and the chilli jet (middle). The filet jet spectrum has been recorded at an acid concentration of $c<0.1 \%$ in helium, a reservoir pressure of $550 \mathrm{mbar}$, with the band pass filter F13 $\left(4100-2500 \mathrm{~cm}^{-1}\right)$ and has been averaged over 50 scans. The chilli jet spectra have been measured at a substance concentration of $c<0.4 \%$ in helium and a reservoir of 500 mbar. The number of scans amounts to 135 for both spectra shown. For the grey spectrum, the F13 band pass filter has been used in combination with both light attenuators (fly screen and perforated metal piece) and the red spectrum has been recorded with the combination of two band pass filters F1 $\left(4000-2860 \mathrm{~cm}^{-1}\right)$ and F2 $\left(3300-2000 \mathrm{~cm}^{-1}\right)$, leading to an effective transparent range of $3300-2860 \mathrm{~cm}^{-1}$ and only the fly screen attenuator. Both chilli jet spectra have been spatially averaged over $3 \times 3$ binned pixels at the nozzle exit. The transmittance spectrum of the utilised IR lens is shown below. The $I_{0}$ measurement was carried out without the IR lens and correspondingly, the $I$ measurement with it. 


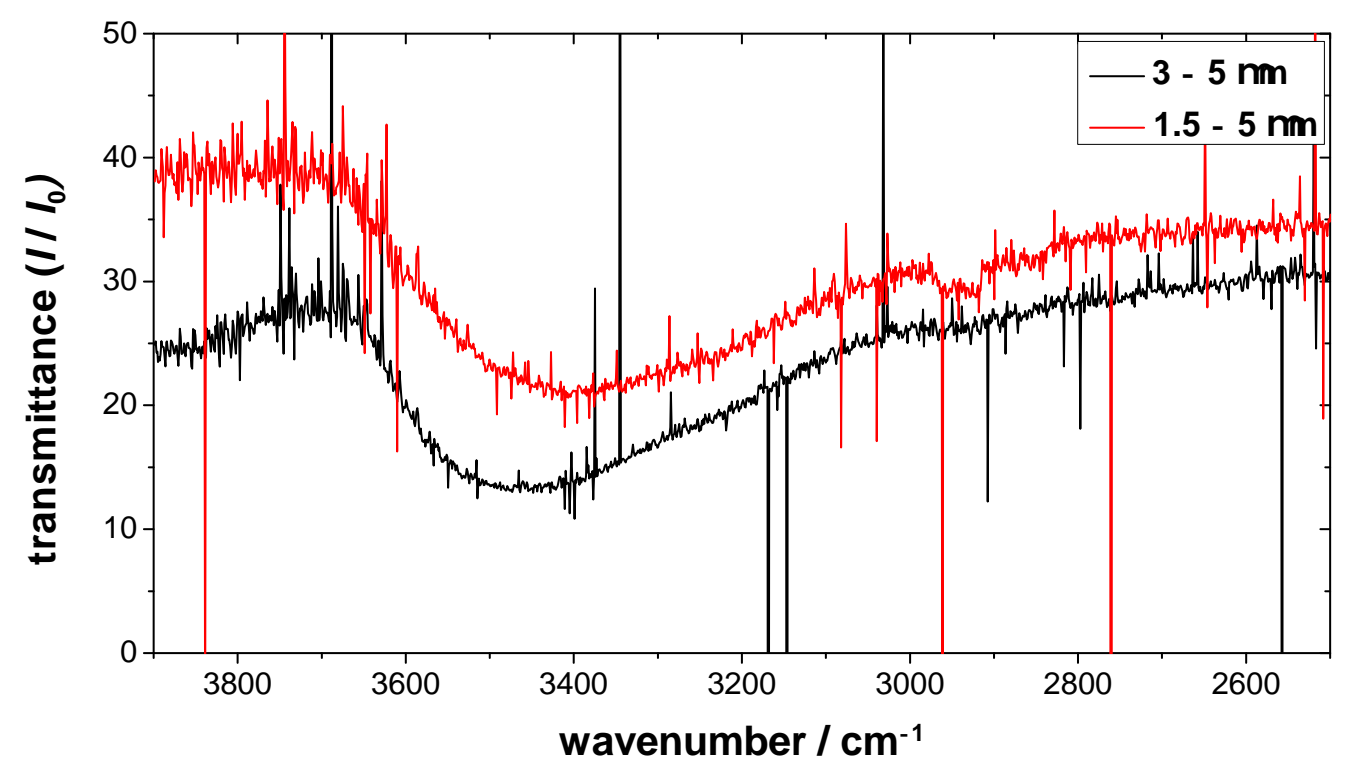

Figure 7.12: Transmittance of the IR lens used throughout this work $(3-5 \mu \mathrm{m})$ in comparison to a newly purchased lens with an extended spectral coverage $(1.5-5 \mu \mathrm{m})$ measured with filter F13 $\left(4100-2500 \mathrm{~cm}^{-1}\right) . I_{0}$ has been recorded without the IR lens and correspondingly, $I$ with it.

the measurement of solids. It limits the reproducibility of the planned measurements, as the $\mathrm{OH}$ stretching region would have to be sampled with several narrow-band pass filters such as F19 $\left(3400-3200 \mathrm{~cm}^{-1}\right)$ for a full spectrum in the relevant region. Therefore, the substances have to be measured above their melting point by increasing the helium dilution.

Another resource for optimisation is the IR lens itself. A new IR lens $(1.5-5 \mu \mathrm{m}$, NYCTEA Series, Janos Technology) with a higher transmittance $T$ in the OH stretching region has been purchased (see Figure 7.12). Even though it also exhibits a minimum between $3650-3000 \mathrm{~cm}^{-1}$, its transmittance is consistently higher than that of the $3-5 \mu \mathrm{m}$ IR lens. The $T$ difference of both is approximately constant between $2500-$ $3000 \mathrm{~cm}^{-1}$. Above $3000 \mathrm{~cm}^{-1}$, it is increasing in favour of the $1.5-5 \mu \mathrm{m}$ IR lens.

In conclusion, the spectral extension of the FTIR imaging apparatus to the $\mathrm{O}-\mathrm{H} / \mathrm{N}-\mathrm{H}$ stretching region via the combination of a new IR lens and spectral mapping with several narrow band pass filters is promising. Further adaptations that are currently being implemented include a change of the magnification ratio $A$ of the IR lens (currently $0.25)$. Increasing it to unity reduces the imaged area to the dimensions of the detector $\left((2.56 \times 2.56) \mathrm{mm}^{2}\right)$ and therefore, improves the spatial resolution attainable. This enables a closer scrutiny of the high collision region behind the nozzle and the area of interfering shock waves, where spectral changes occur more rapidly due to the steep density and temperature gradient (cf. Figures 2.1 and 2.2). The magnification ratio of the IR lens can be modified by changing the relative distances between the detector, IR lens, and nozzle. Therefore, the MCT FPA detector and the IR lens will be placed on rails (Figure 7.13). Previously, they were mounted at a fixed position (see Figure 

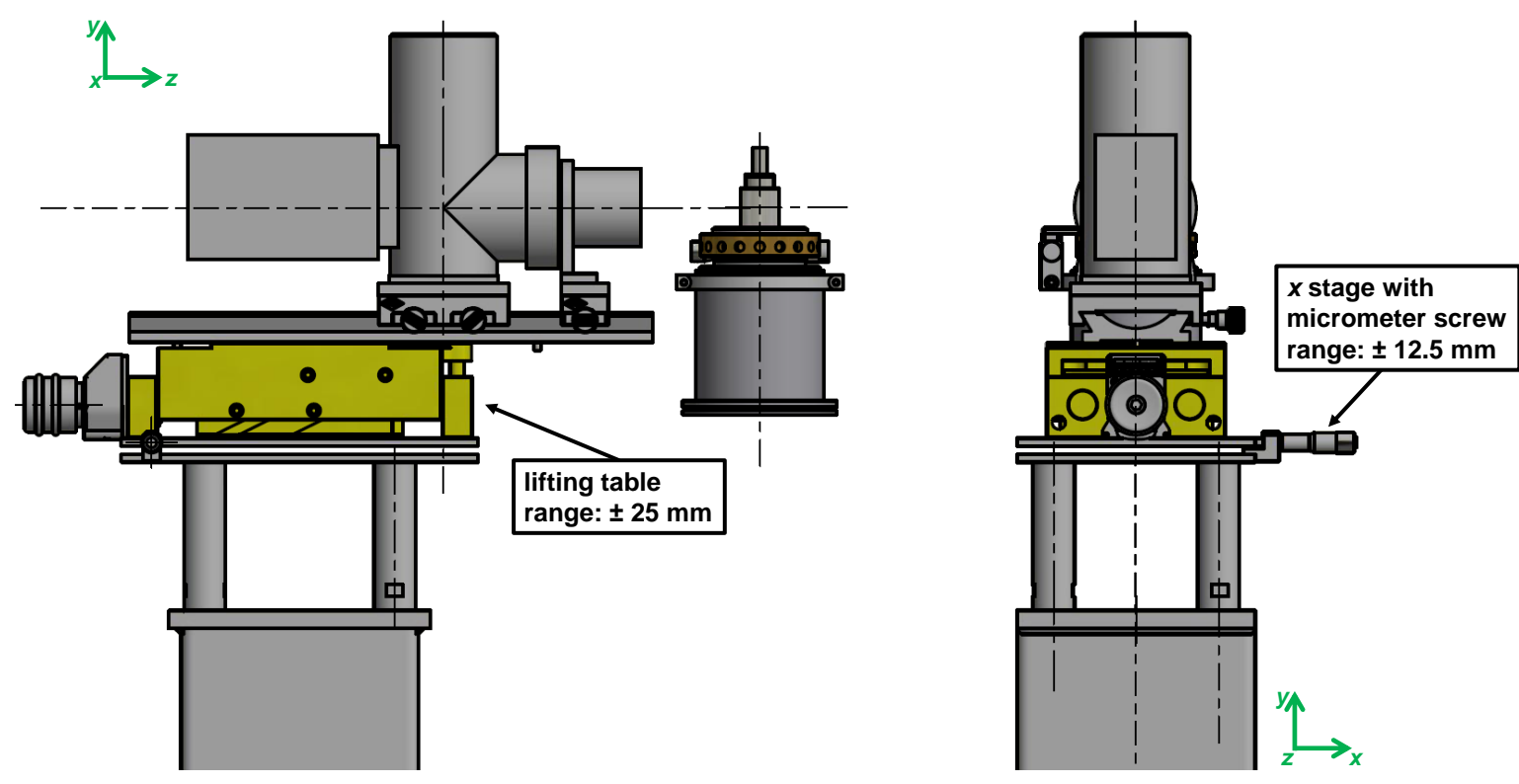

Figure 7.13: Technical drawing of the modifications to the detector table of the FTIR imaging set-up, provided by R. Hildebrandt from the mechanical workshop.

3.4). In order to move the imaged area to the location of interest in the expansion, the $x$ and $y$ positions need to be mappable (cf. Figure 3.4). This is achieved by placing the detector and IR lens on a lifting table ( $y$ axis) with a flexible range of $\pm 25 \mathrm{~mm}$, which is mounted to a movable $x$ stage $( \pm 12.5 \mathrm{~mm})$ that replace the current detector table (Figure 7.13). Finally, to enhance the comparability between the three jet set-ups used throughout this work, the nozzle width of the chilli jet is reduced to match that of the other two $(0.2 \mathrm{~mm})$. All of these changes are currently being implemented and will hopefully increase the flexibility and versatility of the technique for such dynamic gas phase studies to somewhat close the large gap to the condensed phase, where FTIR imaging is already a well-established technique [150-163, 166, 168, 169, 175, 176].

\subsection{Summary}

Within this chapter, dimer assemblies of formic and acetic acid were studied with FTIR imaging. By using relatively high acid concentrations ( $1 \%$ in helium), the space-resolved formation of acetic acid dimer assemblies was observed. Directly behind the nozzle of the expansion, warm dimer (and monomer) can be seen. The band attributed to dimer assemblies forms with some distance to the nozzle, which depends on the concentrations and reservoir pressures chosen. The overlap of both bands is a result of their structural similarities - the dimer assemblies are predicted to be composed of stacked dimers [119, 220, 433].

Additionally, the stability of dimer assemblies of acetic acid $(\mathrm{AA})_{n}$ was probed in shock waves. Similar to the global minimum dimer, these were shown to be stable in this high 


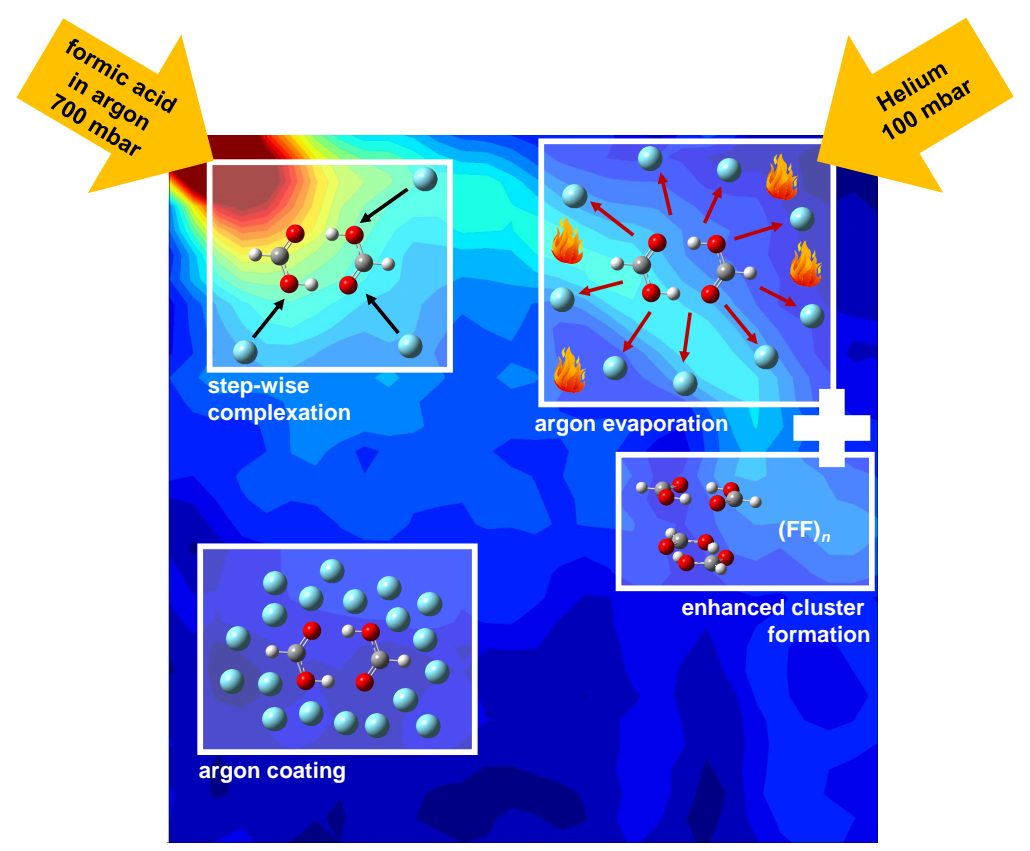

Figure 7.14: Schematic representation of the aggregation processes in supersonic expansions of formic acid seeded in argon in interference with a pure helium expansion.

temperature and density region, as the compression by the second expansion promotes their formation. Unfortunately, no information about the size $n$ of these dimer assemblies in the shock wave is obtainable, as their spectral features are not well separated.

Another approach to enhance cluster formation is the variation of the carrier gas. The spatially resolved IR spectra show that argon coated carboxylic acid clusters form at some distance to the nozzle, as schematically illustrated in Figure 7.14. The associated shift of carboxylic acid band, in this case, the formic acid dimer, reaches the value in an argon cryomatrix relative to the gas phase, when the (FF) is fully surrounded by argon (Figure 7.14). Thus, these experiments can be exploited to simulate the shift of vibrational bands in solid rare gas matrices with respect to the gas phase. Argon can easily be evaporated from the clusters by increasing the temperature in a shock wave (Figure 7.14), while the dimer assemblies remain stable. To promote their formation, the formic acid concentration can be increased. Here, just $0.07 \%$ of formic acid in argon was used compared to the $1 \%$ of acetic acid in helium. Altogether, these carrier gas experiments are particularly versatile and can be easily extended to other molecular systems, for which the set-up applicability of the chilli jet is currently being extended. 



\section{Summary and Outlook}

Due to the richness of experimental studies and the easily extendable system size, carboxylic acid monomers and their hydrogen bonded clusters are an established reference system for the performance test of quantum chemical methods. However, not all experimental data is suitable for such a benchmark, as the theoretical models typically assume the molecules to be isolated in the gas phase at $0 \mathrm{~K}$, whereas many pioneering studies of carboxylic acids were performed for molecules embedded in solid rare gas matrices [59, $82,302,338]$. Thus, the focus of this work was not only on the extension of the vibrational data base, but also on the development of strategies to characterise particularly relevant structures such as local minima in an environment that can be easily exploited for benchmarking, i.e., without the implementation of a challenging description of the surroundings on the theory side.

The smallest example of such a higher-energy structure studied in this work is the cisrotamer of formic acid. Via the combination of thermal excitation, rapid conformational freezing in a supersonic expansion, and Raman spectroscopic detection, the band positions of its four stretching vibrations $(\mathrm{O}-\mathrm{H}, \mathrm{C}-\mathrm{H}, \mathrm{C}=\mathrm{O}$, and $\mathrm{C}-\mathrm{O})$ were determined. Prior to this work, only its $\mathrm{OH}$ torsion had been assigned in the gas phase [57]. This lack of data restricted a thorough evaluation of the globality of potential energy surface (PES) predictions of the monomer, especially as the divergence between different models tends to increase in higher-energy regimes of the PES [327, 328]. With the new reference data, the first sensible vibrational benchmark of high-level ab initio predictions [327, 328] as well as vibrational perturbation theory (VPT2) calculations was feasible. For the latter, specifically in combination with DFT methods, several insufficiencies were revealed, such as a distinct integration grid size and symmetry dependency of the anharmonic band positions, which was found to be particularly pronounced for $\omega$ B97-XD and M06-2X and is well known for the latter [230, 231]. In an exploratory study, deuteration was shown to be an effective starting point for future studies, as a new vibration becomes accessible with the approach used here, namely the $\mathrm{O}-\mathrm{D}$ in-plane-bending vibration. Hence, the vibrational data base of this simple model system can be significantly extended and therefore also its applicability as a benchmark reference system.

Another higher-energy structure examined in the course of this work is a metastable formic acid dimer. In a collaboration with the group of Prof. Andrew Ellis at the University of Leicester, its helium nanodroplet spectrum was revisited, as the band of its bound $\mathrm{OH}$ stretching vibration reported previously exhibited a poor signal-to-noise ratio [83]. The newly measured spectra enabled a thorough examination of the resonance broadened band $\left(72 \mathrm{~cm}^{-1}\right.$ band width). To utilise the helium nanodroplet spectra for benchmarking, the stiffening of hydrogen bonds by the surrounding helium needs to be accounted for, which was done by compressing the spectra accordingly, thus obtaining 
a simulation of a gas phase spectrum. With anharmonic vibrational frequency calculations using VPT2, it was shown that the spectral broadening can be attributed to wavefunction mixing of the bright $\mathrm{OH}$ stretching fundamental with nearby dark states, presumably combination vibrations. While for a qualitative analysis of this coupling, VPT2 calculations might suffice, a high-level ab initio treatment is vital for a quantitative understanding. Unsurprisingly, the theoretical interest in carboxylic acid dimers has so far mainly focussed on the global minimum dimer of formic acid [32, 78, 80, 81, 394-396], as it is the smallest and the experimentally best characterised one (see Chapter 5 for references), which leaves higher-energy structures highly under-represented. With the new data presented here, this can hopefully be changed.

However, even the quantum chemical description of the global minimum dimers remains challenging, an example of which was seen in an examination of their $\mathrm{C}=\mathrm{O}$ stretching vibrations. The purpose of this study was to determine the exciton coupling $\Delta a s$ of these dimers, i.e., the splitting of vibrational modes as a result of the interaction of the $\mathrm{C}=\mathrm{O}$ oscillators in the $C_{2 \mathrm{~h}}$ symmetric dimers. In addition, the shift of the dimer bands with respect to the monomer $\Delta a / \Delta s$ was examined. By extending this study to the binary mixtures of the three acids, namely formic, acetic, and pivalic acid, a benchmarking reference data set of six exciton splittings $\Delta a s$ and shifts $\Delta a / \Delta s$ was obtained. Scaled harmonic frequency calculations at various levels of theory were found to suffice for the prediction of these quantities with an accuracy of $\pm 10 \mathrm{~cm}^{-1}$. For a more accurate description, anharmonicity needs to be accounted for. An anharmonic vibrational benchmark is only feasible for the formic acid dimer, as high-level literature data exists $[78,81$, 394-396] and VPT2 calculations are worthwhile due to the absence of large amplitude methyl or tert-butyl torsions. Nonetheless, even the anharmonic frequencies from vibrational configuration interaction (VCI) calculations based on a CCSD(T)-F12a/haTZ PES fitted to 13475 energies [78, 81] or an anharmonic MP2/aVDZ VPT2 correction applied to the currently best harmonic prediction at the CCSD $(\mathrm{T}) /$ aVQZ level [396] exhibit several shortcomings in describing this system. Simpler anharmonic approaches such as VPT2 might be of qualitative help for spectral analysis, as seen in several examples in this work, but ultimately cannot provide a quantitative understanding in most cases, which heavily relies on more elaborate methods. Hence, further progress of these is vitally needed.

Vibrational characterisation of carboxylic acids beyond the dimer is particularly sparse. Trimers as the simplest, odd-numbered clusters are spectrally similar to the aforementioned higher-energy dimers, as the monomer unit of the trimer is bound via the same hydrogen bonds, which has resulted in assignment controversies in the literature [82, $119,380,437]$. To tackle these more challenging assignments, the interplay of three experimental techniques was used in this work, namely conventional FTIR and Raman jet spectroscopy as well as FTIR imaging. In the $\mathrm{C}=\mathrm{O}$ stretching spectra of all three acids, spectral signatures of trimers were identified. The formation of the higher-energy dimers in the expansions remains somewhat elusive, yet indications for their presence were found, which were most evident for acetic acid. To advance the interpretation of the vibrational spectra, the quantum chemical description needs to be significantly improved, as all DFT methods tested were shown to perform highly non-uniformly for 
formic, acetic, and pivalic acid as well as for the different vibrations characterised, which ultimately limits further experimental progress.

In addition to the vibrational characterisation of small carboxylic acids and their clusters in environments that can be easily used for benchmarking, the transition from perturbation-free gas phase spectra to those recorded in cryogenic matrices was modelled by expanding formic acid in argon as a carrier gas instead of helium. These argon jet expansions allow for an observation of the progressing nanosolvation of molecules or molecular clusters, in this case, the formic acid dimer, as a result of van-der-Waals interactions between seed and carrier. The resulting band position shift of the antisymmetric $\mathrm{C}=\mathrm{O}$ stretching vibration of the formic acid dimer was found to resemble the shift observed in cryogenic matrices with respect to the gas phase. By utilising collision experiments in a double expansion of two converging jets, it was shown that the argon nano-shell formed around the cluster can be easily evaporated as a result of the temperature increase in the interfering expansions. Particularly when gas phase or matrix assignments are in doubt, this approach can bridge the gap between the two techniques, which is challenging to model theoretically [397].

In conclusion, the newly determined experimental reference data on carboxylic acid monomers and their hydrogen bonded clusters (see Appendix A.6 for a summary of key experimental observables) cannot only be utilised for a performance test of high-level $a b$ initio quantum chemical calculations, where rigorous benchmarking is particularly crucial to point out obvious flaws and trigger constant improvements of the methods, but the data also illustrates that further experimental progress heavily relies on a more consistent vibrational description by cost-efficient methodologies that can be employed for systems larger than dimers. 



\section{Bibliography}

[1] J. Altnöder, A. Bouchet, J. J. Lee, K. E. Otto, M. A. Suhm, A. ZehnackerRentien, "Chirality-dependent balance between hydrogen bonding and London dispersion in isolated ( \pm )-1-indanol clusters", Phys. Chem. Chem. Phys. 2013, 15, 10167-10180.

[2] T. Forsting, J. Zischang, M. A. Suhm, M. Eckhoff, B. Schröder, R. A. Mata, "Strained hydrogen bonding in imidazole trimer: A combined infrared, Raman, and theory study", Phys. Chem. Chem. Phys. 2019, 21, 5989-5998.

[3] A. Zehnacker, M. A. Suhm, "Chirality recognition between neutral molecules in the gas phase", Angew. Chem. Int. Ed. 2008, 47, 6970-6992.

[4] M. Albrecht, A. Borba, K. Le Barbu-Debus, B. Dittrich, R. Fausto, S. Grimme, A. Mahjoub, M. Nedić, U. Schmitt, L. Schrader, M. A. Suhm, A. Zehnacker-Rentien, J. Zischang, "Chirality influence on the aggregation of methyl mandelate", New J. Chem. 2010, 34, 1266.

[5] C. Medcraft, S. Zinn, M. Schnell, A. Poblotzki, J. Altnöder, M. Heger, M. A. Suhm, D. Bernhard, A. Stamm, F. Dietrich, M. Gerhards, "Aromatic embedding wins over classical hydrogen bonding - a multi-spectroscopic approach for the diphenyl ether-methanol complex", Phys. Chem. Chem. Phys. 2016, 18, 2597525983.

[6] D. M. Hewett, S. Bocklitz, D. P. Tabor, E. L. Sibert III, M. A. Suhm, T. S. Zwier, "Identifying the first folded alkylbenzene via ultraviolet, infrared, and Raman spectroscopy of pentylbenzene through decylbenzene", Chem. Sci. 2017, 8, 5305-5318.

[7] D. Bernhard, F. Dietrich, M. Fatima, C. Perez, A. Poblotzki, G. Jansen, M. A. Suhm, M. Schnell, M. Gerhards, "Multi-spectroscopic and theoretical analyses on the diphenyl ether-tert-butyl alcohol complex in the electronic ground and electronically excited state", Phys. Chem. Chem. Phys. 2017, 19, 18076-18088.

[8] D. Bernhard, M. Fatima, A. Poblotzki, A. L. Steber, C. Pérez, M. A. Suhm, M. Schnell, M. Gerhards, "Dispersion-controlled docking preference: multi-spectroscopic study on complexes of dibenzofuran with alcohols and water", Phys. Chem. Chem. Phys. 2019, 21, 16032-16046.

[9] F. Dietrich, D. Bernhard, M. Fatima, C. Pérez, M. Schnell, M. Gerhards, "The Effect of Dispersion on the Structure of Diphenyl Ether Aggregates", Angew. Chem. Int. Ed. 2018, 57, 9534-9537. 
[10] S. Oswald, N. A. Seifert, F. Bohle, M. Gawrilow, S. Grimme, W. Jäger, Y. Xu, M. A. Suhm, "The Chiral Trimer and a Metastable Chiral Dimer of Achiral Hexafluoroisopropanol: A Multi-Messenger Study", Angew. Chem. Int. Ed. 2019, $58,5080-5084$.

[11] K. K. Irikura, R. D. Johnson, R. N. Kacker, "Uncertainties in scaling factors for ab initio vibrational frequencies", J. Phys. Chem. A 2005, 109, 8430-8437.

[12] M. D. Halls, J. Velkovski, H. B. Schlegel, "Harmonic frequency scaling factors for Hartree-Fock, S-VWN, B-LYP, B3-LYP, B3-PW91 and MP2 with the Sadlej pVTZ electric property basis set", Theor. Chem. Acc. 2001, 105, 413-421.

[13] R. D. Johnson, K. K. Irikura, R. N. Kacker, R. Kessel, "Scaling Factors and Uncertainties for ab Initio Anharmonic Vibrational Frequencies", J. Chem. Theory Comput. 2010, 6, 2822-2828.

[14] J. A. Pople, H. B. Schlegel, R. Krishnan, D. J. Defrees, J. S. Binkley, M. J. Frisch, R. A. Whiteside, R. F. Hout, W. J. Hehre, "Molecular orbital studies of vibrational frequencies", Int. J. Quantum Chem. 1981, 20, 269-278.

[15] M. W. Wong, "Vibrational frequency prediction using density functional theory", Chem. Phys. Lett. 1996, 256, 391-399.

[16] P. Sinha, S. E. Boesch, C. Gu, R. A. Wheeler, A. K. Wilson, "Harmonic Vibrational Frequencies: Scaling Factors for HF, B3LYP, and MP2 Methods in Combination with Correlation Consistent Basis Sets", J. Phys. Chem. A 2004, 108, 9213-9217.

[17] J. P. Merrick, D. Moran, L. Radom, "An evaluation of harmonic vibrational frequency scale factors", J. Phys. Chem. A 2007, 111, 11683-11700.

[18] I. M. Alecu, J. Zheng, Y. Zhao, D. G. Truhlar, "Computational Thermochemistry: Scale Factor Databases and Scale Factors for Vibrational Frequencies Obtained from Electronic Model Chemistries", J. Chem. Theory Comput. 2010, 6, 28722887.

[19] M. K. Kesharwani, B. Brauer, J. M. L. Martin, "Frequency and zero-point vibrational energy scale factors for double-hybrid density functionals (and other selected methods): can anharmonic force fields be avoided?", J. Phys. Chem. A 2015, 119, 1701-1714.

[20] P. Pulay, W. Meyer, "Comparison of the ab initio force constants of ethane, ethylene and acetylene", Mol. Phys. 1974, 27, 473-490.

[21] P. Pulay, F. Török, "Force constants, vibrational assignment and geometry of methyl amine from hartree -fock calculations", J. Mol. Struct. 1975, 29, 239246.

[22] P. Botschwina, "An ab initio calculation of the force field and vibrational frequencies of $\mathrm{H}_{2} \mathrm{CNH}$ ", Chem. Phys. Lett. 1974, 29, 580-584. 
[23] K. Kozmutza, P. Pulay, "Semiempirical calculation of harmonic force constants: CNDO $/ 2$ and MINDO/2 study of $\mathrm{C}_{2} \mathrm{H}_{6}, \mathrm{C}_{2} \mathrm{H}_{4}$ and $\mathrm{C}_{2} \mathrm{H}_{2}$ ", Theoret. Chim. Acta 1975, 37, 67-75.

[24] C. E. Blom, C. Altona, "Application of self-consistent-field ab initio calculations to organic molecules", Mol. Phys. 1976, 31, 1377-1391.

[25] F. Török, Á. Hrgedüs, K. Kósa, P. Pulay, "Prediction of vtbrational spectra by the CNDO/2 force method", J. Mol. Struct. 1976, 32, 93-99.

[26] G. Fogarasi, P. Pulay, K. Molt, W. Sawodny, "Ab initio Hartree-Fock calculation of the force constants and geometry of $\mathrm{HNF}_{2}$ and $\mathrm{H}_{2} \mathrm{NF}^{\prime \prime}$, Mol. Phys. 1977, 33, 1565-1570.

[27] G. Rauhut, P. Pulay, "Transferable Scaling Factors for Density Functional Derived Vibrational Force Fields", J. Phys. Chem. 1995, 99, 3093-3100.

[28] J. Baker, A. A. Jarzecki, P. Pulay, "Direct Scaling of Primitive Valence Force Constants: An Alternative Approach to Scaled Quantum Mechanical Force Fields", J. Phys. Chem. A 1998, 102, 1412-1424.

[29] C. Fábri, T. Szidarovszky, G. Magyarfalvi, G. Tarczay, "Gas-phase and Ar-matrix SQM scaling factors for various DFT functionals with basis sets including polarization and diffuse functions", J. Phys. Chem. A 2011, 115, 4640-4649.

[30] M. Sibaev, D. L. Crittenden, "PyVCI: A flexible open-source code for calculating accurate molecular infrared spectra", Comput. Phys. Commun. 2016, 203, 290297.

[31] T. Carrington, "Perspective: Computing (ro-)vibrational spectra of molecules with more than four atoms", J. Chem. Phys. 2017, 146, 120902.

[32] V. Barone, M. Biczysko, J. Bloino, "Fully anharmonic IR and Raman spectra of medium-size molecular systems: Accuracy and interpretation", Phys. Chem. Chem. Phys. 2014, 16, 1759-1787.

[33] H. H. Nielsen, "The Vibration-Rotation Energies of Molecules", Rev. Mod. Phys. 1951, 23, 90-136.

[34] V. Barone, "Anharmonic vibrational properties by a fully automated second-order perturbative approach", J. Chem. Phys. 2005, 122, 14108.

[35] V. Barone, M. Biczysko, J. Bloino, M. Borkowska-Panek, I. Carnimeo, P. Panek, "Toward anharmonic computations of vibrational spectra for large molecular systems", Int. J. Quantum Chem. 2012, 112, 2185-2200. 
[36] M. J. Frisch, G. W. Trucks, H. B. Schlegel, G. E. Scuseria, M. A. Robb, J. R. Cheeseman, G. Scalmani, V. Barone, B. Mennucci, G. A. Petersson, H. Nakatsuji, M. Caricato, X. Li, H. P. Hratchian, A. F. Izmaylov, J. Bloino, G. Zheng, J. L. Sonnenberg, M. Hada, M. Ehara, K. Toyota, R. Fukuda, J. Hasegawa, M. Ishida, T. Nakajima, Y. Honda, O. Kitao, H. Nakai, T. Vreven, Montgomery, J. A., Jr., J. E. Peralta, F. Ogliaro, M. Bearpark, J. J. Heyd, E. Brothers, K. N. Kudin, V. N. Staroverov, R. Kobayashi, J. Normand, K. Raghavachari, A. Rendell, J. C. Burant, S. S. Iyengar, J. Tomasi, M. Cossi, N. Rega, J. M. Millam, M. Klene, J. E. Knox, J. B. Cross, V. Bakken, C. Adamo, J. Jaramillo, R. Gomperts, R. E. Stratmann, O. Yazyev, A. J. Austin, R. Cammi, C. Pomelli, J. W. Ochterski, R. L. Martin, K. Morokuma, V. G. Zakrzewski, G. A. Voth, P. Salvador, J. J. Dannenberg, S. Dapprich, A. D. Daniels, Ö. Farkas, J. B. Foresman, J. V. Ortiz, J. Cioslowski, D. J. Fox, Gaussian 09 Revision E.01 2009.

[37] F. Neese, "The ORCA program system", WIREs Comput. Mol. Sci. 2012, 2, 7378.

[38] F. Neese, "Software update: the ORCA program system, version 4.0", WIREs Comput. Mol. Sci. 2018, 8, e1327.

[39] H.-J. Werner, P. J. Knowles, G. Knizia, F. R. Manby, M. Schütz, P. Celani, W. Györffy, D. Kats, T. Korona, R. Lindh, A. Mitrushenkov, G. Rauhut, K. R. Shamasundar, T. B. Adler, R. D. Amos, S. J. Bennie, A. Bernhardsson, A. Berning, D. L. Cooper, M. J. O. Deegan, A. J. Dobbyn, F. Eckert, E. Goll, C. Hampel, A. Hesselmann, G. Hetzer, T. Hrenar, G. Jansen, C. Köppl, S. J. R. Lee, Y. Liu, A. W. Lloyd, Q. Ma, R. A. Mata, A. J. May, S. J. McNicholas, W. Meyer, T. F. Miller III, M. E. Mura, A. Nicklass, D. P. O’Neill, P. Palmieri, D. Peng, K. Pflüger, R. Pitzer, M. Reiher, T. Shiozaki, H. Stoll, A. J. Stone, R. Tarroni, T. Thorsteinsson, M. Wang, M. Welborn, MOLPRO, version 2019.2, a package of ab initio programs, 2019.

[40] J. F. Stanton, J. Gauss, L. Cheng, M. E. Harding, D. A. Matthews, P. G. Szalay, CFOUR, Coupled-Cluster techniques for Computational Chemistry, a quantumchemical program package, With contributions from A.A. Auer, R.J. Bartlett, U. Benedikt, C. Berger, D.E. Bernholdt, Y.J. Bomble, O. Christiansen, F. Engel, R. Faber, M. Heckert, O. Heun, M. Hilgenberg, C. Huber, T.-C. Jagau, D. Jonsson, J. Jusélius, T. Kirsch, K. Klein, W.J. Lauderdale, F. Lipparini, T. Metzroth, L.A. Mück, D.P. O’Neill, D.R. Price, E. Prochnow, C. Puzzarini, K. Ruud, F. Schiffmann, W. Schwalbach, C. Simmons, S. Stopkowicz, A. Tajti, J. Vázquez, F. Wang, J.D. Watts and the integral packages MOLECULE (J. Almlöf and P.R. Taylor), PROPS (P.R. Taylor), ABACUS (T. Helgaker, H.J. Aa. Jensen, P. Jørgensen, and J. Olsen), and ECP routines by A. V. Mitin and C. van Wüllen. For the current version, see http://www.cfour.de.

[41] G. D. Carney, L. L. Sprandel, C. W. Kern in Advances in chemical physics, (Eds.: I. Prigogine, S. A. Rice), Advances in Chemical Physics, John Wiley, New York and Chichester, 1978, pp. 305-379. 
[42] J. M. Bowman, "Self-consistent field energies and wavefunctions for coupled oscillators", J. Chem. Phys. 1978, 68, 608-610.

[43] J. M. Bowman, "The self-consistent-field approach to polyatomic vibrations", Acc. Chem. Res. 1986, 19, 202-208.

[44] D. M. Benoit, "Fast vibrational self-consistent field calculations through a reduced mode-mode coupling scheme", J. Chem. Phys. 2004, 120, 562-573.

[45] M. B. Hansen, M. Sparta, P. Seidler, D. Toffoli, O. Christiansen, "New Formulation and Implementation of Vibrational Self-Consistent Field Theory", J. Chem. Theory Comput. 2010, 6, 235-248.

[46] Y. Scribano, D. M. Lauvergnat, D. M. Benoit, "Fast vibrational configuration interaction using generalized curvilinear coordinates and self-consistent basis", $J$. Chem. Phys. 2010, 133, 094103.

[47] L. S. Norris, M. A. Ratner, A. E. Roitberg, R. B. Gerber, "Møller-Plesset perturbation theory applied to vibrational problems", J. Chem. Phys. 1996, 105, 11261-11267.

[48] J. O. Jung, R. B. Gerber, "Vibrational wave functions and spectroscopy of $\left(\mathrm{H}_{2} \mathrm{O}\right)_{n}$, $n=2,3,4,5$ : Vibrational self-consistent field with correlation corrections", J. Chem. Phys. 1996, 105, 10332-10348.

[49] O. Christiansen, "Møller-Plesset perturbation theory for vibrational wave functions", J. Chem. Phys. 2003, 119, 5773-5781.

[50] O. Christiansen, "Vibrational coupled cluster theory", J. Chem. Phys. 2004, 120, 2149-2159.

[51] P. Seidler, O. Christiansen, "Automatic derivation and evaluation of vibrational coupled cluster theory equations", J. Chem. Phys. 2009, 131, 234109.

[52] O. Christiansen, "Selected new developments in vibrational structure theory: potential construction and vibrational wave function calculations", Phys. Chem. Chem. Phys. 2012, 14, 6672-6687.

[53] J. M. Bowman, T. Carrington, H.-D. Meyer, "Variational quantum approaches for computing vibrational energies of polyatomic molecules", Mol. Phys. 2008, 106, 2145-2182.

[54] R. E. Smalley, L. Wharton, D. H. Levy, "Molecular optical spectroscopy with supersonic beams and jets", Acc. Chem. Res. 1977, 10, 139-145.

[55] J. M. Hayes, "Analytical spectroscopy in supersonic expansions", Chem. Rev. 1987, 87, 745-760.

[56] M. Herman, R. Georges, M. Hepp, D. Hurtmans, "High resolution Fourier transform spectroscopy of jet-cooled molecules", Int. Rev. Phys. Chem. 2000, 19, 277325 . 
[57] O. I. Baskakov, V.-M. Horneman, J. Lohilahti, S. Alanko, "High resolution FTIR spectra of the $\nu_{9}$ vibrational band of cis-rotamers $\mathrm{HCOOH}$ and $\mathrm{H}^{13} \mathrm{COOH}$ ", $J$. Mol. Struct. 2006, 795, 49-53.

[58] W. H. Hocking, "The Other Rotamer of Formic Acid, cis-HCOOH", Z. Naturforsch. A 1976, 31, 1113-1121.

[59] M. Pettersson, J. Lundell, L. Khriachtchev, M. Räsänen, "IR Spectrum of the Other Rotamer of Formic Acid, cis-HCOOH", J. Am. Chem. Soc. 1997, 119, 11715-11716.

[60] E. M. Maçôas, J. Lundell, M. Pettersson, L. Khriachtchev, R. Fausto, M. Räsänen, "Vibrational spectroscopy of cis- and trans-formic acid in solid argon", J. Mol. Spectrosc. 2003, 219, 70-80.

[61] L. G. Bonner, J. S. Kirby-Smith, "The Raman spectrum of formic acid vapor", Phys. Rev. 1940, 57, 1078.

[62] J. E. Bertie, K. H. Michaelian, "The Raman spectra of gaseous formic acid $-h_{2}$ and $-d_{2} "$, J. Chem. Phys. 1982, 76, 886-894.

[63] J. E. Bertie, K. H. Michaelian, H. H. Eysel, D. Hager, "The Raman-active O-H and $\mathrm{O}-\mathrm{D}$ stretching vibrations and Raman spectra of gaseous formic acid- $d_{1}$ and -OD", J. Chem. Phys. 1986, 85, 4779-4789.

[64] P. Zielke, M. A. Suhm, "Raman jet spectroscopy of formic acid dimers: low frequency vibrational dynamics and beyond", Phys. Chem. Chem. Phys. 2007, 9, 4528-4534.

[65] A. Olbert-Majkut, J. Ahokas, J. Lundell, M. Pettersson, "Raman spectroscopy of formic acid and its dimers isolated in low temperature argon matrices", Chem. Phys. Lett. 2009, 468, 176-183.

[66] Z. Xue, M. A. Suhm, "Probing the stiffness of the simplest double hydrogen bond: the symmetric hydrogen bond modes of jet-cooled formic acid dimer", J. Chem. Phys. 2009, 131, 54301.

[67] Z. Xue, M. A. Suhm, "Adding more weight to a molecular recognition unit: The low-frequency modes of carboxylic acid dimers", Mol. Phys. 2010, 108, 2279 2288.

[68] Z. Xue, Raman spectroscopy of carboxylic acid and water aggregates, Logos Berlin, Berlin, 2011.

[69] G. M. Florio, T. S. Zwier, E. M. Myshakin, K. D. Jordan, E. L. Sibert III, "Theoretical modeling of the $\mathrm{OH}$ stretch infrared spectrum of carboxylic acid dimers based on first-principles anharmonic couplings", J. Chem. Phys. 2003, 118,1735 .

[70] C. Emmeluth, M. A. Suhm, D. Luckhaus, "A monomers-in-dimers model for carboxylic acid dimers", J. Chem. Phys. 2003, 118, 2242. 
[71] C. Emmeluth, M. A. Suhm, "A chemical approach towards the spectroscopy of carboxylic acid dimer isomerism", Phys. Chem. Chem. Phys. 2003, 5, 3094.

[72] K. Heyne, N. Huse, J. Dreyer, E. T. J. Nibbering, T. Elsaesser, S. Mukamel, "Coherent low-frequency motions of hydrogen bonded acetic acid dimers in the liquid phase", J. Chem. Phys. 2004, 121, 902-913.

[73] J. Dreyer, "Hydrogen-bonded acetic acid dimers: Anharmonic coupling and linear infrared spectra studied with density-functional theory", J. Chem. Phys. 2005, 122, 184306.

[74] P. Blaise, M. J. Wojcik, O. Henri-Rousseau, "Theoretical interpretation of the line shape of the gaseous acetic acid cyclic dimer", J. Chem. Phys. 2005, 122, 064306 .

[75] D. Luckhaus, "Concerted Hydrogen Exchange Tunneling in Formic Acid Dimer", J. Phys. Chem. A 2006, 110, 3151-3158.

[76] M. Boczar, Ł. Boda, M. J. Wójcik, "Theoretical modeling of the O-H stretching IR bands of hydrogen-bonded dimers of benzoic acid in $S_{0}$ and $S_{1}$ electronic states", J. Chem. Phys. 2007, 127, 084307.

[77] G. L. Barnes, E. L. Sibert III, "Elucidating energy disposal pathways following excitation of the symmetric $\mathrm{OH}$ stretching band in formic acid dimer", Chem. Phys. Lett. 2008, 460, 42-45.

[78] C. Qu, J. M. Bowman, "An ab initio potential energy surface for the formic acid dimer: Zero-point energy, selected anharmonic fundamental energies, and groundstate tunneling splitting calculated in relaxed 1-4-mode subspaces", Phys. Chem. Chem. Phys. 2016, 18, 24835-24840.

[79] S. Oswald, E. Meyer, M. A. Suhm, "Dinitrogen as a Sensor for Metastable Carboxylic Acid Dimers and a Weak Hydrogen Bond Benchmarking Tool", J. Phys. Chem. A 2018, 122, 2933-2946.

[80] C. Qu, J. M. Bowman, "High-dimensional fitting of sparse datasets of CCSD(T) electronic energies and MP2 dipole moments, illustrated for the formic acid dimer and its complex IR spectrum", J. Chem. Phys. 2018, 148, 241713.

[81] C. Qu, J. M. Bowman, "Quantum approaches to vibrational dynamics and spectroscopy: Is ease of interpretation sacrificed as rigor increases?", Phys. Chem. Chem. Phys. 2019, 21, 3397-3413.

[82] M. Gantenberg, M. Halupka, W. Sander, "Dimerization of Formic Acid-An Example of a "Noncovalent" Reaction Mechanism", Chem. Eur. J. 2000, 6, 18651869.

[83] F. Madeja, M. Havenith, K. Nauta, R. E. Miller, J. Chocholousova, P. Hobza, "Polar isomer of formic acid dimers formed in helium nanodroplets", J. Chem. Phys. 2004, 120, 10554-10560. 
[84] J. A. Davies, M. W. D. Hanson-Heine, N. A. Besley, A. Shirley, J. Trowers, S. Yang, A. M. Ellis, "Dimers of acetic acid in helium nanodroplets", Phys. Chem. Chem. Phys. 2019, 21, 13950-13958.

[85] J. P. Toennies, A. F. Vilesov, "Superfluid Helium Droplets: A Uniquely Cold Nanomatrix for Molecules and Molecular Complexes", Angew. Chem. Int. Ed. 2004, 43, 2622-2648.

[86] S. Yang, A. M. Ellis, "Helium droplets: A chemistry perspective", Chem. Soc. Rev. 2013, 42, 472-484.

[87] M. Y. Choi, G. E. Douberly, T. M. Falconer, W. K. Lewis, C. M. Lindsay, J. M. Merritt, P. L. Stiles, R. E. Miller, "Infrared spectroscopy of helium nanodroplets: Novel methods for physics and chemistry", Int. Rev. Phys. Chem. 2006, 25, 1575 .

[88] G. E. Douberly, R. E. Miller, "The isomers of HF-HCN formed in helium nanodroplets: Infrared spectroscopy and ab initio calculations", J. Chem. Phys. 2005, 122, 024306.

[89] C. R. Keedy, "The rotational-vibrational spectra of HCN and DCN: A physical chemistry experiment", J. Chem. Educ. 1992, 69, A296.

[90] K. W. Jucks, R. E. Miller, "Infrared spectroscopy of the hydrogen cyanide dimer", J. Chem. Phys. 1988, 88, 6059-6067.

[91] K. Nauta, R. E. Miller, "Solvent mediated vibrational relaxation: Superfluid helium droplet spectroscopy of HCN dimer", J. Chem. Phys. 1999, 111, 3426-3433.

[92] T. Häber, U. Schmitt, M. A. Suhm, "FTIR-spectroscopy of molecular clusters in pulsed supersonic slit-jet expansions", Phys. Chem. Chem. Phys. 1999, 1, 55735582 .

[93] M. I. Sulaiman, S. Yang, A. M. Ellis, "Infrared Spectroscopy of Methanol and Methanol/Water Clusters in Helium Nanodroplets: The OH Stretching Region", J. Phys. Chem. A 2017, 121, 771-776.

[94] K. E. Otto, Z. Xue, P. Zielke, M. A. Suhm, "The Raman spectrum of isolated water clusters", Phys. Chem. Chem. Phys. 2014, 16, 9849-9858.

[95] P. E. Fraley, K. Narahari Rao, "High resolution infrared spectra of water vapor: $\nu_{1}$ and $\nu_{3}$ bands of $\mathrm{H}_{2}^{16} \mathrm{O}$ ", J. Mol. Spectrosc. 1969, 29, 348-364.

[96] C. J. Burnham, S. S. Xantheas, M. A. Miller, B. E. Applegate, R. E. Miller, "The formation of cyclic water complexes by sequential ring insertion: Experiment and theory", J. Chem. Phys. 2002, 117, 1109-1122.

[97] G. A. Kuipers, D. F. Smith, A. H. Nielsen, "Infrared Spectrum of Hydrogen Fluoride", J. Chem. Phys. 1956, 25, 275-279.

[98] A. S. Pine, W. J. Lafferty, B. J. Howard, "Vibrational predissociation, tunneling, and rotational saturation in the HF and DF dimers", J. Chem. Phys. 1984, 81, 2939-2950. 
[99] K. Nauta, R. E. Miller, "The hydrogen fluoride dimer in liquid helium: A prototype system for studying solvent effects on hydrogen bonding", J. Chem. Phys. 2000, 113, 10158-10168.

[100] M. Y. Choi, R. E. Miller, "Infrared laser spectroscopy of imidazole complexes in helium nanodroplets: Monomer, dimer, and binary water complexes", J. Phys. Chem. A 2006, 110, 9344-9351.

[101] C. C. Costain, G. P. Srivastava, "Study of Hydrogen Bonding. The Microwave Rotation Spectrum of $\mathrm{CF}_{3} \mathrm{COOH}-\mathrm{HCOOH}$ ", J. Chem. Phys. 1961, 35, 1903.

[102] C. C. Costain, G. P. Srivastava, "Microwave Rotation Spectra of HydrogenBonded Molecules", J. Chem. Phys. 1964, 41, 1620.

[103] G. P. Srivastava, M. L. Golay, "Microwave absorption in the hydrogen-bonded molecule $\mathrm{CF}_{3} \mathrm{COOH}-\mathrm{HCOOH}$ ", J. Phys. B 1971, 4, 886.

[104] E. M. Bellott, E. Wilson, "Hydrogen bonded bimolecular complexes of carboxylic acids in the vapor phase", Tetrahedron 1975, 31, 2896-2898.

[105] L. Martinache, W. Kresa, M. Wegener, U. Vonmont, A. Bauder, "Microwave spectra and partial substitution structure of carboxylic acid bimolecules", Chem. Phys. 1990, 148, 129-140.

[106] L. Evangelisti, P. Ecija, E. J. Cocinero, F. Castano, A. Lesarri, W. Caminati, R. Meyer, "Proton Tunneling in Heterodimers of Carboxylic Acids: A Rotational Study of the Benzoic Acid-Formic Acid Bimolecule", J. Phys. Chem. Lett. 2012, 3, 3770-3775.

[107] Q. Gou, G. Feng, L. Evangelisti, W. Caminati, "Rotational Study of cis- and trans-Acrylic Acid-Trifluoroacetic Acid", J. Phys. Chem. A 2013, 117, 1350013503.

[108] G. Feng, Q. Gou, L. Evangelisti, W. Caminati, "Frontiers in rotational spectroscopy: shapes and tunneling dynamics of the four conformers of the acrylic acid-difluoroacetic acid adduct", Angew. Chem. Int. Ed. 2014, 53, 530-534.

[109] A. M. Pejlovas, W. Lin, S. G. Kukolich, "Microwave spectra and structure of the cyclopropanecarboxylic acid-formic acid dimer", J. Chem. Phys. 2015, 143, 124311 .

[110] J. Thomas, M. J. Carrillo, A. Serrato, W. Lin, W. Jäger, Y. Xu, "Rotational spectroscopic and theoretical study of the perfluorobutyric acid $\cdots$ formic acid complex", J. Mol. Spectrosc. 2017, 335, 88-92.

[111] W. Li, L. Evangelisti, Q. Gou, W. Caminati, R. Meyer, "Barrier to Proton Transfer in the Dimer of Formic Acid: A Pure Rotational Study", Angew. Chem. 2018, 52, 803.

[112] H. E. Affsprung, S. D. Christian, A. M. Melnick, "Hetero-dimerization of acetic and trichloroacetic acids in carbon tetrachloride solution", Spectrochim. Acta 1964, 20, 285-290. 
[113] D. Clague, A. Novak, "Far infrared spectra of homogeneous and heterogeneous dimers of some carboxylic acids", J. Mol. Struct. 1970, 5, 149-152.

[114] J. W. Keller, "The Formic Acid-Trifluoroacetic Acid Bimolecule. Gas-Phase Infrared Spectrum and Computational Studies", J. Phys. Chem. A 2004, 108, 46104618.

[115] Q. Gu, Y. Xia, S. Chen, P. Su, Z. Yang, C. O. Trindle, J. L. Knee, "Infrared spectroscopy of gas phase alpha hydroxy carboxylic acid homo and hetero dimers", Phys. Chem. Chem. Phys. 2018, 20, 29601-29609.

[116] C. Emmeluth, Schwingungsdynamik von Carbonsäureaggregaten, diploma thesis, Georg-August-Universität Göttingen, Göttingen, 2001.

[117] T. Häber, U. Schmitt, C. Emmeluth, M. A. Suhm, "Ragout-jet FTIR spectroscopy of cluster isomerism and cluster dynamics: From carboxylic acid dimers to $\mathrm{N}_{2} \mathrm{O}$ nanoparticles", Faraday Discuss. 2001, 118, 331-359.

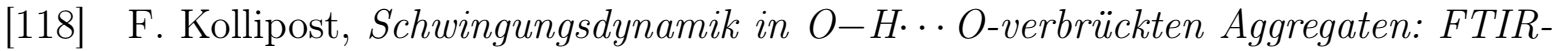
Spektroskopie vom Nah- bis zum Ferninfraroten, Ph.D. thesis, Georg-AugustUniversität Göttingen, Göttingen, 2015.

[119] F. Ito, "Infrared spectra of formic acid clusters in noble gas matrices", J. Mol. Struct. 2015, 1091, 203-209.

[120] J. Zischang, M. A. Suhm, "Infrared absorption imaging of 2D supersonic jet expansions: Free expansion, cluster formation, and shock wave patterns", J. Chem. Phys. 2013, 139, 24201.

[121] J. M. Hollas, Modern spectroscopy, 4. ed., reprinted, Wiley, Chichester, 2010.

[122] L. G. Bonner, "The Vibrational Spectrum of Water Vapor", Phys. Rev. 1934, 46, 458-464.

[123] D. R. Miller, Atomic and molecular beam methods, (Ed.: G. Scoles), Oxford University Press, New York, 1988.

[124] J. Zischang, Strukturdynamik wasserhaltiger Aggregate in Überschallexpansionen, Ph.D. thesis, Georg-August-Universität Göttingen, Göttingen, 2014.

[125] A. E. Beylich, "Struktur von Überschall-Freistrahlen aus Schlitzblenden", Z. Flugwiss. Weltraumforsch. 1979, 3, 48-58.

[126] G. Tejeda, B. Maté, J. M. Fernández-Sánchez, S. Montero, "Temperature and density mapping of supersonic jet expansions using linear Raman spectroscopy", Phys. Rev. Lett. 1996, 76, 34-37.

[127] G. Herzberg, Molecular Spectra and Molecular Structure. I. Spectra of Diatomic Molecules, 2. ed., van Nostrand, New York, 1950.

[128] J. Zischang, J. J. Lee, M. A. Suhm, "Communication: Where does the first water molecule go in imidazole?", J. Chem. Phys. 2011, 135, 061102.

[129] S. Tanimura, Y. Okada, K. Takeuchi, "FTIR Spectroscopy of UF $_{6}$ Clustering in a Supersonic Laval Nozzle", J. Phys. Chem. 1996, 100, 2842-2848. 
[130] A. Benidar, R. Georges, R. Le Doucen, J. Boissoles, S. Hamon, A. Canosa, B. R. Rowe, "Uniform Supersonic Expansion for FTIR Absorption Spectroscopy: The $\nu_{5}$ Band of $\mathrm{NO}_{2}$ at $26 \mathrm{~K}$ ", J. Mol. Spectrosc. 2000, 199, 92-99.

[131] R. Georges, A. Bonnamy, A. Benidar, M. Decroi, J. Boissoles, "FTIR free-jet set-up for the high resolution spectroscopic investigation of condensable species", Mol. Phys. 2002, 100, 1551-1558.

[132] J. Arnó, J. W. Bevan in Jet Spectroscopy and Molecular Dynamics, (Eds.: J. M. Hollas, D. Phillips), Springer Netherlands, Dordrecht, s.l., 1995, pp. 29-73.

[133] D. J. Nesbitt, "High-Resolution, Direct Infrared Laser Absorption Spectroscopy in Slit Supersonic Jets: Intermolecular Forces and Unimolecular Vibrational Dynamics in Clusters", Annu. Rev. Phys. Chem. 1994, 45, 367-399.

[134] M. Sulkes, C. Jouvet, S. A. Rice, "Theoretical and experimental characterization of supersonic expansions from slit sources", Chem. Phys. Lett. 1982, 87, 515-519.

[135] K. Veeken, J. Reuss, "Infrared line narrowing and cluster absorption in a planar jet", Appl. Phys. B 1985, 38, 117-124.

[136] M. D. Morse in Atomic, Molecular, and Optical Physics: Atoms and Molecules, Experimental Methods in the Physical Sciences, Elsevier, 1996, pp. 21-47.

[137] M. Nishida, T. Nakagawa, H. Kobayashi, "Density contours of a supersonic freejet", Exp. Fluids 1985, 3, 181-183.

[138] B. E. Djakov, R. Enikov, D. Oliver, "Supersonic Jets with Periodic Structure due to Arc plasma Expansion into a Low pressure ambient", Contrib. Plasma Phys. 1997, 37, 57-76.

[139] J. Panda, "Shock oscillation in underexpanded screeching jets", J. Fluid Mech. 1998, 363, 173-198.

[140] J. Panda, R. G. Seasholtz, "Measurement of shock structure and shock-vortex interaction in underexpanded jets using Rayleigh scattering", Phys. Fluids 1999, 11, 3761-3777.

[141] A. Broc, S. Benedictis, G. Dilecce, M. Vigliotti, R. G. Sharafutdinov, P. A. Skovorodko, "Experimental and numerical investigation of an $\mathrm{O}_{2} / \mathrm{NO}$ supersonic free jet expansion", J. Fluid Mech. 2004, 500, 211-237.

[142] R. A. Larsen, S. K. Neoh, D. R. Herschbach, "Seeded supersonic alkali atom beams", Rev. Sci. Instrum. 1974, 45, 1511-1516.

[143] A. Amirav, U. Even, J. Jortner, "Cooling of large and heavy molecules in seeded supersonic beams", Chem. Phys. 1980, 51, 31-42.

[144] G. M. McClelland, K. L. Saenger, J. J. Valentini, D. R. Herschbach, "Vibrational and rotational relaxation of iodine in seeded supersonic beams", J. Phys. Chem. 1979, 83, 947-959.

[145] M. V. Johnston, "Supersonic jet expansions in analytical spectroscopy", Trends Anal. Chem. 1984, 3, 58-61. 
[146] C. Emmeluth, V. Dyczmons, T. Kinzel, P. Botschwina, M. A. Suhm, M. Yáñez, "Combined jet relaxation and quantum chemical study of the pairing preferences of ethanol", Phys. Chem. Chem. Phys. 2005, 7, 991-997.

[147] T. N. Wassermann, P. Zielke, J. J. Lee, C. Cézard, M. A. Suhm, "Structural preferences, argon nanocoating, and dimerization of n-alkanols as revealed by OH stretching spectroscopy in supersonic jets", J. Phys. Chem. A 2007, 111, $7437-7448$.

[148] J. J. Lee, S. Höfener, W. Klopper, T. N. Wassermann, M. A. Suhm, "Origin of the Argon Nanocoating Shift in the OH Stretching Fundamental of $n$-Propanol: A Combined Experimental and Quantum Chemical Study", J. Phys. Chem. C 2009, 113, 10929-10938.

[149] S. Oswald, M. A. Suhm, S. Coussan, "Incremental NH stretching downshift through stepwise nitrogen complexation of pyrrole: a combined jet expansion and matrix isolation study", Phys. Chem. Chem. Phys. 2019, 21, 1277-1284.

[150] H.-Y. N. Holman, M. C. Martin, W. R. McKinney, "Tracking Chemical Changes in a Live Cell: Biomedical Applications of SR-FTIR Spectromicroscopy", J. Spectrosc. 2003, 17, 139-159.

[151] S. G. Kazarian, K. L. A. Chan, "Applications of ATR-FTIR spectroscopic imaging to biomedical samples", Biochim. Biophys. Acta 2006, 1758, 858-867.

[152] C. Krafft, D. Codrich, G. Pelizzo, V. Sergo, "Raman mapping and FTIR imaging of lung tissue: congenital cystic adenomatoid malformation", Analyst 2008, 133, $361-371$.

[153] E. Ly, O. Piot, R. Wolthuis, A. Durlach, P. Bernard, M. Manfait, "Combination of FTIR spectral imaging and chemometrics for tumour detection from paraffinembedded biopsies", Analyst 2008, 133, 197-205.

[154] G. Steiner, E. Koch, "Trends in Fourier transform infrared spectroscopic imaging", Anal. Bioanal. Chem. 2009, 394, 671-678.

[155] M. K. Kuimova, K. L. A. Chan, S. G. Kazarian, "Chemical imaging of live cancer cells in the natural aqueous environment", Appl. Spectrosc. 2009, 63, 164-171.

[156] M. J. Nasse, M. J. Walsh, E. C. Mattson, R. Reininger, A. Kajdacsy-Balla, V. Macias, R. Bhargava, C. J. Hirschmugl, "High-resolution Fourier-transform infrared chemical imaging with multiple synchrotron beams", Nat. Methods 2011, 8, 413-416.

[157] B. R. Wood, K. R. Bambery, M. W. A. Dixon, L. Tilley, M. J. Nasse, E. Mattson, C. J. Hirschmugl, "Diagnosing malaria infected cells at the single cell level using focal plane array Fourier transform infrared imaging spectroscopy", Analyst 2014, 139, 4769-4774.

[158] K. L. Andrew Chan, S. G. Kazarian, "Attenuated total reflection Fourier-transform infrared (ATR-FTIR) imaging of tissues and live cells", Chem. Soc. Rev. 2016, $45,1850-1864$. 
[159] H. Shinzawa, B. Turner, J. Mizukado, S. G. Kazarian, "Protein hydration in living cells probed by Fourier transform infrared (FT-IR) spectroscopic imaging", Analyst 2017, 142, 2475-2483.

[160] C. L. Song, M. Ryu, J. Morikawa, A. Kothari, S. G. Kazarian, "Thermal effect on dispersive infrared spectroscopic imaging of prostate cancer tissue", J. Biophotonics 2018, 11, e201800187.

[161] D. Lizoňová, J. Mužík, M. Šoltys, J. Beránek, S. G. Kazarian, F. Štěpánek, "Molecular-level insight into hot-melt loading and drug release from mesoporous silica carriers", Eur. J. Pharm. Biopharm. 2018, 130, 327-335.

[162] C. L. Song, S. G. Kazarian, "Three-dimensional depth profiling of prostate tissue by micro ATR-FTIR spectroscopic imaging with variable angles of incidence", Analyst 2019, 144, 2954-2964.

[163] C. L. Song, M. Z. Vardaki, R. D. Goldin, S. G. Kazarian, "Fourier transform infrared spectroscopic imaging of colon tissues: evaluating the significance of amide I and $\mathrm{C}-\mathrm{H}$ stretching bands in diagnostic applications with machine learning", Anal. Bioanal. Chem. 2019, DOI 10.1007/s00216-019-02069-6.

[164] R. Bhargava, S.-Q. Wang, J. L. Koenig in Liquid Chromatography / FTIR Microspectroscopy / Microwave Assisted Synthesis, (Eds.: A. Abe, A.-C. Albertsson, H.-J. Cantow, K. Dusek, S. Edwards, H. Höcker, J. F. Joanny, H.-H. Kausch, S. Kobayashi, K.-S. Lee, I. Manners, O. Nuyken, S. I. Stupp, U. W. Suter, G. Wegner), Advances in Polymer Science, Springer, Berlin, Heidelberg, 2003, pp. 137191.

[165] A. Gupper, S. G. Kazarian, "Study of Solvent Diffusion and Solvent-Induced Crystallization in Syndiotactic Polystyrene Using FT-IR Spectroscopy and Imaging", Macromolecules 2005, 38, 2327-2332.

[166] X. Zhou, P. Zhang, Z. LI, G. Rao, "Miscibility Behavior of Ethylene/Vinyl Acetate and C5 Petroleum Resin by FTIR Imaging", Anal. Sci. 2007, 23, 877-880.

[167] C. Vogel, E. Wessel, H. W. Siesler, "FT-IR Spectroscopic Imaging of Anisotropic Poly(3-hydroxybutyrate)/Poly(lactic acid) Blends with Polarized Radiation", Macromolecules 2008, 41, 2975-2977.

[168] M. Ryu, J. A. Kimber, T. Sato, R. Nakatani, T. Hayakawa, M. Romano, C. Pradere, A. A. Hovhannisyan, S. G. Kazarian, J. Morikawa, "Infrared thermospectroscopic imaging of styrene radical polymerization in microfluidics", Chem. Eng. J. 2017, 324, 259-265.

[169] Y. Hikima, J. Morikawa, S. G. Kazarian, "Analysis of molecular orientation in polymeric spherulite using polarized micro attenuated total reflection Fourier transform infrared (ATR-FTIR) spectroscopic imaging", Anal. Chim. Acta 2019, 1065, 79-89. 
[170] C. Ricci, K. L. Andrew Chan, S. G. Kazarian, "Combining the Tape-Lift Method and Fourier Transform Infrared Spectroscopic Imaging for Forensic Applications", Appl. Spectrosc. 2006, 60, 1013-1021.

[171] N. J. Crane, E. G. Bartick, R. S. Perlman, S. Huffman, "Infrared spectroscopic imaging for noninvasive detection of latent fingerprints", J. Forensic Sci. 2007, 52, 48-53.

[172] A. V. Ewing, S. G. Kazarian, "Infrared spectroscopy and spectroscopic imaging in forensic science", Analyst 2017, 142, 257-272.

[173] M. Spring, C. Ricci, D. A. Peggie, S. G. Kazarian, "ATR-FTIR imaging for the analysis of organic materials in paint cross sections: case studies on paint samples from the National Gallery, London", Anal. Bioanal. Chem. 2008, 392, 37-45.

[174] E. Joseph, C. Ricci, S. G. Kazarian, R. Mazzeo, S. Prati, M. Ioele, "MacroATR-FT-IR spectroscopic imaging analysis of paint cross-sections", Vib. Spectrosc. 2010, 53, 274-278.

[175] C. Schmidt Patterson, D. Carson, A. Phenix, H. Khanjian, K. Trentelman, J. Mass, C. J. Hirschmugl, "Synchrotron-based Imaging FTIR Spectroscopy in the Evaluation of Painting Cross-sections", e-PS 2013, 1-9.

[176] F. Gabrieli, F. Rosi, A. Vichi, L. Cartechini, L. Pensabene Buemi, S. G. Kazarian, C. Miliani, "Revealing the Nature and Distribution of Metal Carboxylates in Jackson Pollock's Alchemy (1947) by Micro-Attenuated Total Reflection FT-IR Spectroscopic Imaging", Anal. Chem. 2017, 89, 1283-1289.

[177] A. Vichi, G. Eliazyan, S. G. Kazarian, "Study of the Degradation and Conservation of Historical Leather Book Covers with Macro Attenuated Total ReflectionFourier Transform Infrared Spectroscopic Imaging", ACS Omega 2018, 3, 71507157.

[178] M. Heger, Diagonal and Off-Diagonal Anharmonicity in Hydrogen-Bonded Systems, Ph.D. thesis, Georg-August-Universität Göttingen, Göttingen, 2015.

[179] S. M. Schweer, Chemische Bildgebung von Überschallexpansionen, bachelor thesis, Georg-August-Universität Göttingen, Göttingen, 2017.

[180] D. Bernoulli, Hydrodynamica, sive de viribus et motibus fluidorum commentarii : opus academicum ab auctore, dum Petropoli ageret, congestum, sumptibus Johannis Reinholdi Dulseckeri and Typis Joh. Deckeri, typographi Basiliensis, 1738.

[181] W. Demtröder, Experimentalphysik 1, Vierte Auflage, Springer-Verlag Berlin Heidelberg, Berlin, Heidelberg, 2006.

[182] G. Herzberg, Molecular Spectra and Molecular Structure. II. Infrared and Raman Spectra of Polyatomic Molecules, van Nostrand, New York, 1945.

[183] A. Simon, J. Gast, A. Keens, Fourier Spectrometer, United States Patent 5,309,217, 1994. 
[184] Bruker Optics, The Bruker ROCKSOLID ${ }^{\mathrm{TM}}$ Interferometer: Technical Note, www. orgchm . bas . bg / irlab / Site _ BG / Laboratory / rocksolid . pdf, last accessed 04.11.2019.

[185] K. A. E. Meyer, M. A. Suhm, "Formic acid aggregation in 2D supersonic expansions probed by FTIR imaging", J. Chem. Phys. 2017, 147, 144305.

[186] R. Bhargava, I. W. Levin, "Effective Time Averaging of Multiplexed Measurements: A Critical Analysis", Anal. Chem. 2002, 74, 1429-1435.

[187] D. A. Scribner, M. R. Kruer, J. M. Killiany, "Infrared focal plane array technology", Proc. IEEE 1991, 79, 66-85.

[188] N. Borho, Chirale Erkennung in Molekülclustern: Maßgeschneiderte Aggregation von alpha-Hydroxyestern, 1st ed., Cuvillier Verlag, Göttingen, 2005.

[189] T. Scharge, D. Luckhaus, M. A. Suhm, "Observation and quantification of the hydrogen bond effect on $\mathrm{O}-\mathrm{H}$ overtone intensities in an alcohol dimer", Chem. Phys. 2008, 346, 167-175.

[190] R. Wugt Larsen, M. A. Suhm, "The benefits of alternation and alkylation: large amplitude hydrogen bond librational modes of alcohol trimers and tetramers", Phys. Chem. Chem. Phys. 2010, 12, 8152-8157.

[191] F. Kollipost, R. Wugt Larsen, A. V. Domanskaya, M. Nörenberg, M. A. Suhm, "Communication: The highest frequency hydrogen bond vibration and an experimental value for the dissociation energy of formic acid dimer", J. Chem. Phys. 2012, 136, 151101.

[192] F. Kollipost, K. Papendorf, Y.-F. Lee, Y.-P. Lee, M. A. Suhm, "Alcohol dimershow much diagonal OH anharmonicity?", Phys. Chem. Chem. Phys. 2014, 16, 15948-15956.

[193] M. A. Suhm, F. Kollipost, "Femtisecond single-mole infrared spectroscopy of molecular clusters", Phys. Chem. Chem. Phys. 2013, 15, 10702-10721.

[194] P. Zielke, Ramanstreuung am Überschallstrahl: Wasserstoffbrückendynamik aus neuer Perspektive, Cuvillier Verlag, 2007.

[195] T. Wassermann, Umgebungseinflüsse auf die CC-und CO-Torsionsdynamik in Molekülen und Molekülaggregaten: Schwingungsspektroskopie bei tiefen Temperaturen, Logos Verlag Berlin GmbH, 2010.

[196] N. O. B. Lüttschwager, Raman Spectroscopy of Conformational Rearrangements at Low Temperatures: Folding and stretching of alkanes in supersonic jets, Springer, 2014.

[197] K. Otto, Raman-Spektroskopie kleiner Moleküle und Molekülaggregate im Überschallstrahl nach thermischer Anregung, Ph.D. thesis, Georg-August-Universität Göttingen, Göttingen, 2015.

[198] S. Bocklitz, Conformational spectroscopy of flexible chain molecules near the folding limit, Ph.D. thesis, Georg-August-Universität Göttingen, Göttingen, 2017. 
[199] T. Forsting, PhD thesis, Georg-August-Universität Göttingen, Göttingen, expected 2019.

[200] National Institute of Standards and Technology, NIST Atomic Spectra Database, https:// physics.nist.gov / PhysRefData/ASD/lines_form.html, last accessed 04.11.2019.

[201] T. Forsting, M. A. Suhm, Curry-Jet setup, https://figshare.com/articles/CurryJet_SETUP/6395840/1, 2019.

[202] D. A. Long, The Raman effect: A unified treatment of the theory of Raman scattering by molecules, Wiley, Chichester and New York, 2002.

[203] G. W. A. Kahlbaum, "Studies of vapor pressure measurements", Z. Phys. Chem. 1894, 13, 14-55.

[204] D. Ambrose, N. Ghiassee, "Vapour pressures and critical temperatures and critical pressures of some alkanoic acids: $\mathrm{C}_{1}$ to $\mathrm{C}_{10}$ ", J. Chem. Thermodyn. 1987, 19, 505519.

[205] S. P. Verevkin, "Measurement and Prediction of the Monocarboxylic Acids Thermochemical Properties", J. Chem. Eng. Data 2000, 45, 953-960.

[206] J. W. Stout, L. H. Fisher, "The Entropy of Formic Acid. The Heat Capacity from 15 to $300^{\circ} \mathrm{K}$. Heats of Fusion and Vaporization", J. Chem. Phys. 1941, 9, $163-168$.

[207] M. D. Taylor, J. Bruton, "The Vapor Phase Dissociation of Some Carboxylic Acids. II. Formic and Propionic Acids", J. Am. Chem. Soc. 1952, 74, 4151-4152.

[208] National Institute of Standards and Technology, Formic Acid: Phase Change Data, https://webbook.nist.gov/cgi/cbook.cgi?ID=C64186\&Mask=4ref-20, last accessed 04.11.2019.

[209] D. R. Stull, "Vapor Pressure of Pure Substances. Organic and Inorganic Compounds", Ind. Eng. Chem. 1947, 39, 517-540.

[210] R. C. Wilhoit, J. Chao, K. R. Hall, "Thermodynamic Properties of Key Organic Oxygen Compounds in the Carbon Range $\mathrm{C}_{1}$ to $\mathrm{C}_{4}$. Part 1 . Properties of Condensed Phases", J. Phys. Chem. Ref. Data 1985, 14, 1-175.

[211] D. Ambrose, J. H. Ellender, C. Sprake, R. Townsend, "Thermodynamic properties of organic oxygen compounds XLV. The vapour pressure of acetic acid", J. Chem. Thermodyn. 1977, 9, 735-741.

[212] R. A. McDonald, S. A. Shrader, D. R. Stull, "Vapor Pressures and Freezing Points of Thirty Pure Organic Compounds", J. Chem. Eng. Data 1959, 4, 311-313.

[213] ILO International Chemical Safety Cards, Pivalic Acid, http://www.ilo.org/dyn/ icsc/showcard.display?p_version $=2 \& p \_c a r d \_i d=0486$, last accessed 04.11.2019.

[214] J. Tandy, C. Feng, A. Boatwright, G. Sarma, A. M. Sadoon, A. Shirley, N. D. N. Rodrigues, E. M. Cunningham, S. Yang, A. M. Ellis, "Communication: Infrared spectroscopy of salt-water complexes", J. Chem. Phys. 2016, 144, 121103. 
[215] A. M. Sadoon, G. Sarma, E. M. Cunningham, J. Tandy, M. W. D. Hanson-Heine, N. A. Besley, S. Yang, A. M. Ellis, "Infrared Spectroscopy of $\mathrm{NaCl}\left(\mathrm{CH}_{3} \mathrm{OH}\right)_{n}$ Complexes in Helium Nanodroplets", J. Phys. Chem. A 2016, 120, 8085-8092.

[216] Z. Yang, Q. Gu, C. O. Trindle, J. L. Knee, "Communication: The ionization spectroscopy of mixed carboxylic acid dimers", J. Chem. Phys. 2013, 139, 151101.

[217] M. Winnewisser, B. P. Winnewisser, M. Stein, M. Birk, G. Wagner, G. Winnewisser, K. M. Yamada, S. P. Belov, O. I. Baskakov, "Rotational Spectra of cis$\mathrm{HCOOH}$, trans-HCOOH, and trans- ${ }^{13} \mathrm{COOH}$ ", J. Mol. Spectrosc. 2002, 216, 259-265.

[218] Justin Lindsay Neill, A New Generation of Chirped Pulse Rotational Spectroscopy with Applications to Structure Determination and Astrochemistry, Ph.D. thesis, University of Virginia, Charlottesville, Virginia, 2011.

[219] A. K. Roy, A. J. Thakkar, "Formic acid tetramers: A structural study", Chem. Phys. Lett. 2004, 393, 347-354.

[220] A. Karpfen, A. J. Thakkar, "Does the most stable formic acid tetramer have pi stacking or C-H...O interactions?", J. Chem. Phys. 2006, 124, 224313.

[221] A. D. Becke, "Density-functional thermochemistry. III. The role of exact exchange", J. Chem. Phys. 1993, 98, 5648.

[222] C. Lee, W. Yang, R. G. Parr, "Development of the Colle-Salvetti correlationenergy formula into a functional of the electron density", Phys. Rev. B 1988, 3\%, $785-789$.

[223] S. Grimme, J. Antony, S. Ehrlich, H. Krieg, "A consistent and accurate ab initio parametrization of density functional dispersion correction (DFT-D) for the 94 elements H-Pu", J. Chem. Phys. 2010, 132, 154104.

[224] S. Grimme, S. Ehrlich, L. Goerigk, "Effect of the damping function in dispersion corrected density functional theory", J. Comput. Chem. 2011, 32, 1456-1465.

[225] S. Oswald, M. A. Suhm, "Soft experimental constraints for soft interactions: A spectroscopic benchmark data set for weak and strong hydrogen bonds", Phys. Chem. Chem. Phys. 2019, 21, 18799.

[226] T. H. Dunning, "Gaussian basis sets for use in correlated molecular calculations. I. The atoms boron through neon and hydrogen", J. Chem. Phys. 1989, 90, 10071023.

[227] R. A. Kendall, T. H. Dunning, R. J. Harrison, "Electron affinities of the first-row atoms revisited. Systematic basis sets and wave functions", J. Chem. Phys. 1992, 96, 6796-6806.

[228] D. E. Woon, T. H. Dunning, "Gaussian basis sets for use in correlated molecular calculations. III. The atoms aluminum through argon", J. Chem. Phys. 1993, 98, $1358-1371$. 
[229] F. Weigend, R. Ahlrichs, "Balanced basis sets of split valence, triple zeta valence and quadruple zeta valence quality for $\mathrm{H}$ to $\mathrm{Rn}$ : Design and assessment of accuracy", Phys. Chem. Chem. Phys. 2005, 7, 3297-3305.

[230] S. E. Wheeler, K. N. Houk, "Integration Grid Errors for Meta-GGA-Predicted Reaction Energies: Origin of Grid Errors for the M06 Suite of Functionals", J. Chem. Theory Comput. 2010, 6, 395-404.

[231] N. Mardirossian, M. Head-Gordon, "How Accurate Are the Minnesota Density Functionals for Noncovalent Interactions, Isomerization Energies, Thermochemistry, and Barrier Heights Involving Molecules Composed of Main-Group Elements?", J. Chem. Theory Comput. 2016, 12, 4303-4325.

[232] S. Grimme, "Semiempirical GGA-type density functional constructed with a longrange dispersion correction", J. Comput. Chem. 2006, 27, 1787-1799.

[233] J.-D. Chai, M. Head-Gordon, "Long-range corrected hybrid density functionals with damped atom-atom dispersion corrections", Phys. Chem. Chem. Phys. 2008, 10, 6615-6620.

[234] C. Møller, M. S. Plesset, "Note on an Approximation Treatment for ManyElectron Systems", Phys. Rev. 1934, 46, 618-622.

[235] M. Head-Gordon, J. A. Pople, M. J. Frisch, "MP2 energy evaluation by direct methods", Chem. Phys. Lett. 1988, 153, 503-506.

[236] S. Grimme, "Improved second-order Møller-Plesset perturbation theory by separate scaling of parallel- and antiparallel-spin pair correlation energies", Phys. Chem. Chem. Phys. 2003, 118, 9095-9102.

[237] C. Adamo, V. Barone, "Toward reliable density functional methods without adjustable parameters: The PBE0 model", J. Chem. Phys. 1999, 110, 6158-6170.

[238] Y. Zhao, D. G. Truhlar, "The M06 suite of density functionals for main group thermochemistry, thermochemical kinetics, noncovalent interactions, excited states, and transition elements: Two new functionals and systematic testing of four M06class functionals and 12 other functionals", Theor. Chem. Acc. 2008, 120, 215241.

[239] S. Grimme, "Semiempirical hybrid density functional with perturbative secondorder correlation", J. Chem. Phys. 2006, 124, 034108.

[240] S. Grimme, J. G. Brandenburg, C. Bannwarth, A. Hansen, "Consistent structures and interactions by density functional theory with small atomic orbital basis sets", J. Chem. Phys. 2015, 143, 054107.

[241] J. J. P. Stewart, "Optimization of parameters for semiempirical methods I. Method", J. Comput. Chem. 1989, 10, 209-220.

[242] J. J. P. Stewart, "Optimization of parameters for semiempirical methods II. Applications", J. Comput. Chem. 1989, 10, 221-264. 
[243] M. Born, R. Oppenheimer, "Zur Quantentheorie der Molekeln", Ann. Phys. 1927, 389, 457-484.

[244] H. Eyring, M. Polanyi, "Über einfache Gasreaktionen", Z. Phys. Chem. B 1931, 12, 279.

[245] J. C. Polanyi, "Concepts in reaction dynamics", Acc. Chem. Res. 1972, 5, 161168.

[246] H. Pelzer, E. Wigner, "The speed constansts of the exchange reactions", Z. Phys. Chem. B 1932, 15, 445.

[247] X. Huang, D. W. Schwenke, S. A. Tashkun, T. J. Lee, "An isotopic-independent highly accurate potential energy surface for $\mathrm{CO}_{2}$ isotopologues and an initial ${ }^{12} \mathrm{C}^{16} \mathrm{O}_{2}$ infrared line list", J. Chem. Phys. 2012, 136, 124311.

[248] X. Huang, R. S. Freedman, S. A. Tashkun, D. W. Schwenke, T. J. Lee, "Semiempirical ${ }^{12} \mathrm{C}^{16} \mathrm{O}_{2}$ IR line lists for simulations up to $1500 \mathrm{~K}$ and $20,000 \mathrm{~cm}^{-1 ",} J$. Quant. Spectrosc. Radiat. Transfer 2013, 130, 134-146.

[249] H. Partridge, D. W. Schwenke, "The determination of an accurate isotope dependent potential energy surface for water from extensive $a b$ initio calculations and experimental data", J. Chem. Phys. 1997, 106, 4618-4639.

[250] S. V. Shirin, O. L. Polyansky, N. F. Zobov, P. Barletta, J. Tennyson, "Spectroscopically determined potential energy surface of $\mathrm{H}_{2}{ }^{16} \mathrm{O}$ up to $25000 \mathrm{~cm}^{-1}$ ", Chem. Phys. Lett. 2003, 118, 2124-2129.

[251] S. V. Shirin, O. L. Polyansky, N. F. Zobov, R. I. Ovsyannikov, A. G. Császár, J. Tennyson, "Spectroscopically determined potential energy surfaces of the $\mathrm{H}_{2}{ }^{16} \mathrm{O}$, $\mathrm{H}_{2}{ }^{17} \mathrm{O}$, and $\mathrm{H}_{2}{ }^{18} \mathrm{O}$ isotopologues of water", J. Mol. Spectrosc. 2006, 236, 216-223.

[252] I. I. Bubukina, N. F. Zobov, O. L. Polyansky, S. V. Shirin, S. N. Yurchenko, "Optimized semiempirical potential energy surface for $\mathrm{H}_{2}{ }^{16} \mathrm{O}$ up to $26000 \mathrm{~cm}^{-1}$ ", Opt. Spectrosc. 2011, 110, 160-166.

[253] O. L. Polyansky, R. I. Ovsyannikov, A. A. Kyuberis, L. Lodi, J. Tennyson, N. F. Zobov, "Calculation of rotation-vibration energy levels of the water molecule with near-experimental accuracy based on an ab initio potential energy surface", $J$. Phys. Chem. A 2013, 117, 9633-9643.

[254] I. I. Mizus, A. A. Kyuberis, N. F. Zobov, V. Y. Makhnev, O. L. Polyansky, J. Tennyson, "High-accuracy water potential energy surface for the calculation of infrared spectra", Philos. Trans. A Math. Phys. Eng. Sci. 2018, 376, 20170149.

[255] X. Huang, B. J. Braams, J. M. Bowman, "Ab initio potential energy and dipole moment surfaces of $\left(\mathrm{H}_{2} \mathrm{O}\right)_{2}$ ", J. Phys. Chem. A 2006, 110, 445-451.

[256] A. Shank, Y. Wang, A. Kaledin, B. J. Braams, J. M. Bowman, "Accurate ab initio and "hybrid" potential energy surfaces, intramolecular vibrational energies, and classical ir spectrum of the water dimer", J. Chem. Phys. 2009, 130, 144314. 
[257] Y. Wang, S. Carter, B. J. Braams, J. M. Bowman, "MULTIMODE quantum calculations of intramolecular vibrational energies of the water dimer and trimer using ab initio-based potential energy surfaces", J. Chem. Phys. 2008, 128, 071101.

[258] T. Rajamäki, A. Miani, L. Halonen, "Vibrational energy levels for symmetric and asymmetric isotopomers of ammonia with an exact kinetic energy operator and new potential energy surfaces", J. Chem. Phys. 2003, 118, 6358-6369.

[259] S. N. Yurchenko, J. Zheng, H. Lin, P. Jensen, W. Thiel, "Potential-energy surface for the electronic ground state of $\mathrm{NH}_{3}$ up to $20,000 \mathrm{~cm}^{-1}$ above equilibrium", $J$. Chem. Phys. 2005, 123, 134308.

[260] S. N. Yurchenko, M. Carvajal, H. Lin, J. Zheng, W. Thiel, P. Jensen, "Dipole moment and rovibrational intensities in the electronic ground state of $\mathrm{NH}_{3}$ : bridging the gap between $a b$ initio theory and spectroscopic experiment", J. Chem. Phys. 2005, 122, 104317.

[261] R. Marquardt, K. Sagui, W. Klopper, M. Quack, "Global analytical potential energy surface for large amplitude nuclear motions in ammonia", J. Phys. Chem. $B$ 2005, 109, 8439-8451.

[262] X. Huang, D. W. Schwenke, T. J. Lee, "An accurate global potential energy surface, dipole moment surface, and rovibrational frequencies for $\mathrm{NH}_{3}$ ", J. Chem. Phys. 2008, 129, 214304.

[263] B. Kuhn, T. R. Rizzo, D. Luckhaus, M. Quack, M. A. Suhm, "A new sixdimensional analytical potential up to chemically significant energies for the electronic ground state of hydrogen peroxide", J. Chem. Phys. 1999, 111, 2565-2587.

[264] B. Fehrensen, D. Luckhaus, M. Quack, "Stereomutation dynamics in hydrogen peroxide", Chem. Phys. 2007, 338, 90-105.

[265] P. Małyszek, J. Koput, “Accurate ab initio potential energy surface and vibrationrotation energy levels of hydrogen peroxide", J. Comput. Chem. 2013, 34, 337345.

[266] X. Zhang, S. Zou, L. B. Harding, J. M. Bowman, "A Global ab Initio Potential Energy Surface for Formaldehyde", J. Phys. Chem. A 2004, 108, 8980-8986.

[267] Q. K. Wang, J. M. Bowman, "Two-component, ab initio potential energy surface for $\mathrm{CO}_{2}-\mathrm{H}_{2} \mathrm{O}$, extension to the hydrate clathrate, $\mathrm{CO}_{2} @\left(\mathrm{H}_{2} \mathrm{O}\right)_{20}$, and VSCF/VCI vibrational analyses of both", J. Chem. Phys. 2017, 147, 161714.

[268] M. Kofranek, H. Lischka, A. Karpfen, "Coupled pair functional study on the hydrogen fluoride dimer. I. Energy surface and characterization of stationary points", Chem. Phys. 1988, 121, 137-153.

[269] M. Quack, M. A. Suhm, "Potential energy surfaces, quasiadiabatic channels, rovibrational spectra, and intramolecular dynamics of $(\mathrm{HF})_{2}$ and its isotopomers from quantum Monte Carlo calculations", J. Chem. Phys. 1991, 95, 28-59. 
[270] W. Klopper, M. Quack, M. A. Suhm, "A new ab initio based six-dimensional semi-empirical pair interaction potential for HF", Chem. Phys. Lett. 1996, 261, $35-44$.

[271] W. Klopper, M. Quack, M. A. Suhm, "HF dimer: Empirically refined analytical potential energy and dipole hypersurfaces from ab initio calculations", J. Chem. Phys. 1998, 108, 10096-10115.

[272] A. V. Nikitin, M. Rey, V. G. Tyuterev, "Rotational and vibrational energy levels of methane calculated from a new potential energy surface", Chem. Phys. Lett. 2011, 501, 179-186.

[273] S. N. Yurchenko, J. Tennyson, "ExoMol line lists - IV. The rotation-vibration spectrum of methane up to 1500 K", Mon. Notices Royal Astron. Soc. 2014, 440, 1649-1661.

[274] M. Majumder, S. E. Hegger, R. Dawes, S. Manzhos, X.-G. Wang, C. Tucker, J. Li, H. Guo, "Explicitly correlated MRCI-F12 potential energy surfaces for methane fit with several permutation invariant schemes and full-dimensional vibrational calculations", Mol. Phys. 2015, 113, 1823-1833.

[275] A. Owens, S. N. Yurchenko, A. Yachmenev, J. Tennyson, W. Thiel, "A highly accurate $a b$ initio potential energy surface for methane", J. Chem. Phys. 2016, $145,104305$.

[276] V. Hänninen, L. Halonen, "Calculation of spectroscopic parameters and vibrational overtones of methanol", Mol. Phys. 2003, 101, 2907-2916.

[277] E. L. Sibert III, J. Castillo-Chará, "Theoretical studies of the potential surface and vibrational spectroscopy of $\mathrm{CH}_{3} \mathrm{OH}$ and its deuterated analogs", J. Chem. Phys. 2005, 122, 194306.

[278] J. M. Bowman, X. Huang, N. C. Handy, S. Carter, "Vibrational levels of methanol calculated by the reaction path version of MULTIMODE, using an ab initio, fulldimensional potential", J. Phys. Chem. A 2007, 111, 7317-7321.

[279] Z. Bačić, J. C. Light, "Accurate localized and delocalized vibrational states of HCN/HNC", J. Chem. Phys. 1987, 86, 3065-3077.

[280] G. E. Scuseria, H. F. Schaefer, "The photodissociation of formaldehyde: A coupled cluster study including connected triple excitations of the transition state barrier height for $\mathrm{H}_{2} \mathrm{CO} \rightarrow \mathrm{H}_{2}+\mathrm{CO}$ ", J. Chem. Phys. 1989, 90, 3629-3636.

[281] J. Altnöder, K. Krüger, D. Borodin, L. Reuter, D. Rohleder, F. Hecker, R. A. Schulz, X. T. Nguyen, H. Preiß, M. Eckhoff, M. Levien, M. A. Suhm, "The Guinness molecules for the carbohydrate formula", Chem. Rec. 2014, 14, 1116-1133.

[282] W. J. Pietenpol, J. D. Rogers, D. Williams, "Microwave Spectra of Asymmetric Top Molecules", Phys. Rev. 1950, 78, 480-481.

[283] R. G. Lerner, B. P. Dailey, J. P. Friend, "Microwave Spectrum and Structure of Formic Acid", J. Chem. Phys. 1957, 26, 680-683. 
[284] G. Erlandsson, "Millimeter-Wave Spectrum of Formic Acid", J. Chem. Phys. 1958, 28, 71-75.

[285] R. Trambarulo, A. Clark, C. Hearns, "Planarity of the Formic Acid Monomer", J. Chem. Phys. 1958, 28, 736-737.

[286] H. Kim, R. Keller, W. D. Gwinn, "Dipole Moment of Formic Acid, HCOOH and HCOOD", Phys. Rev. 1962, 37, 2748-2750.

[287] E. Willemot, D. Dangoisse, J. Bellet, "Microwave spectrum of formic acid and its isotopic species in $\mathrm{D},{ }^{13} \mathrm{C}$ and ${ }^{18} \mathrm{O}$. Study of Coriolis resonances between $\nu_{7}$ and $\nu_{9}$ vibrational excited states", J. Mol. Spectrosc. 1978, 73, 96-119.

[288] E. Willemot, D. Dangoisse, N. Monnanteuil, J. Bellet, "Microwave spectra of molecules of astrophysical interest. XVIII. Formic acid", J. Phys. Chem. Ref. Data 1980, 9, 59-160.

[289] R. Davis, A. G. Robiette, M. Gerry, E. Bjarnov, G. Winnewisser, "Microwave spectra and centrifugal distortion constants of formic acid containing ${ }^{13} \mathrm{C}$ and ${ }^{18} \mathrm{O}$ : Refinement of the harmonic force field and the molecular structure", J. Mol. Spectrosc. 1980, 81, 93-109.

[290] J. Vander Auwera, "High-resolution investigation of the far-infrared spectrum of formic acid", J. Mol. Spectrosc. 1992, 155, 136-142.

[291] O. I. Baskakov, "Rotational Spectrum of the Excited Vibrational States of DCOOH and Assignment of Optically Pumped Laser Transitions", J. Mol. Spectrosc. 1996, 180, 266-276.

[292] G. Cazzoli, C. Puzzarini, S. Stopkowicz, J. Gauss, "Hyperfine structure in the rotational spectra of trans-formic acid: Lamb-dip measurements and quantumchemical calculations", Astron. Astrophys. 2010, 520, A64.

[293] Z. van Williams, "Infra-Red Spectra of Monomeric Formic Acid and Its Deuterated Forms. II. Low Frequency Region $\left(2200-800 \mathrm{~cm}^{-1}\right)$ ", J. Chem. Phys. 1947, $15,243-251$.

[294] Z. van Williams, "Infra-Red Spectra of Monomeric Formic Acid and Its Deuterated Forms. I. High Frequency Region", J. Chem. Phys. 1947, 15, 232-242.

[295] R. C. Millikan, K. S. Pitzer, "Infrared Spectra and Vibrational Assignment of Monomeric Formic Acid", J. Chem. Phys. 1957, 27, 1305-1308.

[296] T. Miyazawa, K. S. Pitzer, "Internal Rotation and Infrared Spectra of Formic Acid Monomer and Normal Coordinate Treatment of Out-of-Plane Vibrations of Monomer, Dimer, and Polymer", J. Chem. Phys. 1959, 30, 1076-1086.

[297] I. C. Hisatsune, J. Heicklen, "Are There 2 Structural Isomers of Formic-Acid", Can. J. Spectrosc. 1973, 18, 135-142.

[298] R. L. Redington, "Vibrational spectra and normal coordinate analysis of isotopically labeled formic acid monomers", J. Mol. Spectrosc. 1977, 65, 171-189. 
[299] J.-C. Deroche, J. Kauppinen, E. Kyrö, " $\nu_{7}$ and $\nu_{9}$ bands of formic acid near 16 um", J. Mol. Spectrosc. 1979, 78, 379-394.

[300] R. E. Bumgarner, J.-I. Choe, S. G. Kukolich, R. J. Butcher, "High-resolution spectroscopy of the $\nu_{6}$ and $\nu_{8}$ bands of formic acid", J. Mol. Spectrosc. 1988, 132, 261-276.

[301] J. Notholt, F. Cappellani, H. Roesdahl, G. Restelli, "Absolute infrared band intensities and air broadening coefficient for spectroscopic measurements of formic acid in air", Spectrochim. Acta A 1991, 47, 477-483.

[302] L. Khriachtchev, M. Pettersson, M. Räsanen, "Conformational Memory in Photodissociation of Formic Acid", J. Am. Chem. Soc. 2002, 124, 10994-10995.

[303] O. I. Baskakov, J. Demaison, "Spectroscopic Study of the $v_{6}=1$ and $v_{8}=1$ Vibrational States of Formic Acid, HCOOH: New Assignments of Laser Transitions", J. Mol. Spectrosc. 2002, 211, 262-272.

[304] A. Perrin, J.-M. Flaud, B. Bakri, J. Demaison, O. Baskakov, S. Sirota, M. Herman, J. Auwera, "New High-Resolution Analysis of the $\nu_{7}$ and $\nu_{9}$ Fundamental Bands of trans-Formic Acid by Fourier Transform Infrared and Millimeter-Wave Spectroscopy", J. Mol. Spectrosc. 2002, 216, 203-213.

[305] M. Freytes, D. Hurtmans, S. Kassi, J. Liévin, J. Vander Auwera, A. Campargue, M. Herman, "Overtone spectroscopy of formic acid", Chem. Phys. 2002, 283, 4761.

[306] O. I. Baskakov, E. A. Alekseev, R. A. Motiyenko, J. Lohilahti, V.-M. Horneman, S. Alanko, B. P. Winnewisser, I. R. Medvedev, F. C. de Lucia, "FTIR and millimeter wave investigation of the $7^{1}$ and $9^{1}$ states of formic acid $\mathrm{HCOOH}$ and $\mathrm{H}^{13} \mathrm{COOH} "$, J. Mol. Spectrosc. 2006, 240, 188-201.

[307] O. I. Baskakov, I. A. Markov, E. A. Alekseev, R. A. Motiyenko, J. Lohilahti, V.-M. Horneman, B. P. Winnewisser, I. R. Medvedev, F. C. de Lucia, "Simultaneous analysis of rovibrational and rotational data for the $4^{1}, 5^{1}, 6^{1}, 7^{2}, 8^{1}, 7^{1} 9^{1}$ and $9^{2}$ states of HCOOH", J. Mol. Struct. 2006, 795, 54-77.

[308] J. Vander Auwera, K. Didriche, A. Perrin, F. Keller, "Absolute line intensities for formic acid and dissociation constant of the dimer", J. Chem. Phys. 2007, 126, 124311 .

[309] L. O. Paulson, D. T. Anderson, "High-resolution vibrational spectroscopy of trans-formic acid in solid parahydrogen", J. Phys. Chem. A 2009, 113, 17701778 .

[310] A. Perrin, J. Vander Auwera, Z. Zelinger, "High-resolution Fourier transform study of the $\nu_{3}$ fundamental band of trans-formic acid", J. Quant. Spectrosc. Radiat. Transfer 2009, 110, 743-755. 
[311] L. O. Paulson, D. T. Anderson, J. Lundell, K. Marushkevich, M. Melavuori, L. Khriachtchev, "Conformation resolved induced infrared activity: Trans- and cisformic acid isolated in solid molecular hydrogen", J. Phys. Chem. A 2011, 115, $13346-13355$.

[312] D. Golebiowski, T. Földes, T. Vanfleteren, M. Herman, A. Perrin, "Complementary cavity-enhanced spectrometers to investigate the $\mathrm{OH}+\mathrm{CH}$ combination band in trans-formic acid", J. Chem. Phys. 2015, 143, 014201.

[313] W. Luo, Y. Zhang, W. Li, C. Duan, "Jet-cooled infrared absorption spectrum of the $\nu_{4}$ fundamental band of HCOOH and HCOOD", J. Mol. Spectrosc. 2017, $334,22-25$.

[314] S. Lopes, R. Fausto, L. Khriachtchev, "Formic acid in deuterium and hydrogen matrices", Mol. Phys. 2019, 117, 1708-1718.

[315] K. Hull, T. Wells, B. E. Billinghurst, H. Bunn, P. L. Raston, "Synchrotron-based infrared spectroscopy of formic acid: Confirmation of the reassignment of Fermicoupled $8 \mu \mathrm{m}$ states", AIP Adv. 2019, 9, 015021.

[316] L. G. Bonner, R. Hofstadter, "Vibration Spectra and Molecular Structure IV. The Infra-Red Absorption Spectra of the Double and Single Molecules of Formic Acid", J. Chem. Phys. 1938, 6, 531-534.

[317] R. Hofstadter, "Vibration Spectra and Molecular Structure VI. Infra-Red Absorption Spectrum of Heavy Formic Acid", Phys. Rev. 1938, 6, 540-543.

[318] K. Nakamoto, S. Kishida, "Normal Coordinate Analyses of Hydrogen-Bonded Compounds. I. Monomeric Formic Acid and Acetic Acid", J. Chem. Phys. 1964, 41, 1554-1558.

[319] H. Susi, J. R. Scheker, "The normal vibrations of formic acid and methyl formate", Spectrochim. Acta A 1969, 25, 1243-1263.

[320] K. Fukushima, J. Chao, B. J. Zwolinski, "Normal coordinate treatment and thermodynamic properties of the cis-trans isomers of formic acid and its deuteroanalog", J. Chem. Thermodyn. 1971, 3, 553-562.

[321] C. W. Bock, M. Trachtman, P. George, "An ab initio study of the geometries, anharmonic force fields and fundamental vibration frequencies of cis- and transformic acid", J. Mol. Spectrosc. 1980, 80, 131-144.

[322] J. H. van Lenthe, F. B. van Duijneveldt, M. M. van Schaik, "Hartree-Fock and MCSCF calculations on the cis-trans isomerism of formic acid", J. Mol. Struct. 1982, 88, 333-341.

[323] A. G. Császár, W. D. Allen, H. F. Schaefer, "In pursuit of the ab initio limit for conformational energy prototypes", J. Chem. Phys. 1998, 108, 9751-9764.

[324] J. Demaison, M. Herman, J. Liévin, "Anharmonic force field of cis- and transformic acid from high-level ab initio calculations, and analysis of resonance polyads", J. Chem. Phys. 2007, 126, 164305. 
[325] H. Hirao, "Theoretical study of formic acid: A new look at the origin of the planar Z conformation and C-O rotational barrier", Chem. Phys. 2008, 344, 213-220.

[326] L. O. Paulson, J. Kaminský, D. T. Anderson, P. Bouř, J. Kubelka, "Theoretical Study of Vibrationally Averaged Dipole Moments for the Ground and Excited $\mathrm{C}=\mathrm{O}$ Stretching States of trans-Formic Acid", J. Chem. Theory Comput. 2010, $6,817-827$.

[327] D. P. Tew, W. Mizukami, "Ab Initio Vibrational Spectroscopy of cis- and transFormic Acid from a Global Potential Energy Surface", J. Phys. Chem. A 2016, 120, 9815-9828.

[328] F. Richter, P. Carbonnière, "Vibrational treatment of the formic acid double minimum case in valence coordinates", J. Chem. Phys. 2018, 148, 064303.

[329] K. A. E. Meyer, M. A. Suhm, "Vibrational exciton coupling in homo and hetero dimers of carboxylic acids studied by linear infrared and Raman jet spectroscopy", J. Chem. Phys. 2018, 149, 104307.

[330] K. A. E. Meyer, M. A. Suhm, "Stretching of cis-formic acid: warm-up and cooldown as molecular work-out", Chem. Sci. 2019, 10, 6285-6294.

[331] E. Bjarnov, W. H. Hocking, "The Structure of the Other Rotamer of Formic Acid, cis-HCOOH", Z. Naturforsch. A 1978, 33, 610-618.

[332] M. Pettersson, E. M. S. Maçôas, L. Khriachtchev, J. Lundell, R. Fausto, M. Räsänen, "Cis $\rightarrow$ trans conversion of formic acid by dissipative tunneling in solid rare gases: Influence of environment on the tunneling rate", J. Chem. Phys. 2002, 117, 9095-9098.

[333] M. Pettersson, E. M. S. Maçôas, L. Khriachtchev, R. Fausto, M. Räsänen, "Conformational isomerization of formic acid by vibrational excitation at energies below the torsional barrier", J. Am. Chem. Soc. 2003, 125, 4058-4059.

[334] E. M. S. Maçôas, L. Khriachtchev, M. Pettersson, R. Fausto, M. Räsänen, "Rotational isomerization of small carboxylic acids isolated in argon matrices: Tunnelling and quantum yields for the photoinduced processes", Phys. Chem. Chem. Phys. 2005, 7, 743-749.

[335] K. Marushkevich, L. Khriachtchev, M. Räsänen, "High-energy conformer of formic acid in solid neon: giant difference between the proton tunneling rates of cis monomer and trans-cis dimer", J. Chem. Phys. 2007, 126, 241102.

[336] K. Marushkevich, L. Khriachtchev, M. Räsänen, "High-energy conformer of formic acid in solid hydrogen: conformational change promoted by host excitation", Phys. Chem. Chem. Phys. 2007, 9, 5748.

[337] L. Khriachtchev, "Rotational isomers of small molecules in noble-gas solids: From monomers to hydrogen-bonded complexes", J. Mol. Struct. 2008, 880, 14-22.

[338] E. M. S. Maçôas, L. Khriachtchev, M. Pettersson, R. Fausto, M. Räsänen, "Rotational isomerism in acetic acid: The first experimental observation of the highenergy conformer", J. Am. Chem. Soc. 2003, 125, 16188-16189. 
[339] E. M. S. Maçôas, L. Khriachtchev, M. Pettersson, R. Fausto, M. Räsänen, "Rotational isomerism of acetic acid isolated in rare-gas matrices: Effect of medium and isotopic substitution on IR-induced isomerization quantum yield and cis $\rightarrow$ trans tunneling rate", J. Chem. Phys. 2004, 121, 1331-1338.

[340] E. Isoniemi, L. Khriachtchev, M. Makkonen, M. Räsänen, "UV photolysis products of propiolic acid in noble-gas solids", J. Phys. Chem. A 2006, 110, 11479 11487 .

[341] S. Lopes, A. V. Domanskaya, R. Fausto, M. Räsänen, L. Khriachtchev, "Formic and acetic acids in a nitrogen matrix: Enhanced stability of the higher-energy conformer", J. Chem. Phys. 2010, 133, 144507.

[342] S. Lopes, T. Nikitin, R. Fausto, "Propiolic Acid in Solid Nitrogen: NIR- and UV-Induced cis $\rightarrow$ trans Isomerization and Matrix-Site-Dependent trans $\rightarrow$ cis Tunneling", J. Phys. Chem. A 2019, 123, 1581-1593.

[343] G. Giubertoni, O. O. Sofronov, H. J. Bakker, "Observation of Distinct Carboxylic Acid Conformers in Aqueous Solution", J. Phys. Chem. Lett. 2019, 10, 3217-3222.

[344] J. Bloino, V. Barone, "A second-order perturbation theory route to vibrational averages and transition properties of molecules: General formulation and application to infrared and vibrational circular dichroism spectroscopies", J. Chem. Phys. 2012, 136, 124108.

[345] J. Lundell, M. Räsänen, Z. Latajka, "Matrix isolation FTIR and ab initio study of complexes between formic acid and nitrogen", Chem. Phys. 1994, 189, 245-260.

[346] K. G. Goroya, Y. Zhu, P. Sun, C. Duan, "High resolution jet-cooled infrared absorption spectra of the formic acid dimer: a reinvestigation of the $\mathrm{C}-\mathrm{O}$ stretch region", J. Chem. Phys. 2014, 140, 164311.

[347] C. Domingo, R. Escribano, W. F. Murphy, S. Montero, "Raman intensities of overtones and combination bands of $\mathrm{C}_{2} \mathrm{H}_{2}, \mathrm{C}_{2} \mathrm{HD}$, and $\mathrm{C}_{2} \mathrm{D}_{2}$ ", J. Chem. Phys. 1982, 77, 4353-4359.

[348] L. N. Vidal, P. A. M. Vazquez, "CCSD study of anharmonic Raman cross sections of fundamental, overtone, and combination transitions", Int. J. Quantum Chem. 2012, 112, 3205-3215.

[349] F. Madeja, P. Markwick, M. Havenith, K. Nauta, R. E. Miller, "Rotationally resolved infrared spectroscopy of $h_{2^{-}}$and $d_{1^{-}}$formic acid monomer in liquid He droplets", J. Chem. Phys. 2002, 116, 2870-2878.

[350] K. Goh, P. Ong, T. Tan, W. Wang, H. Teo, "The High-Resolution Infrared Spectrum of the $\nu_{5}$ Band of Deuterated Formic Acid (DCOOH)", J. Mol. Spectrosc. 1998, 190, 125-129.

[351] K. L. Goh, P. P. Ong, T. L. Tan, H. H. Teo, W. F. Wang, "High-Resolution FTIR Spectrum of the $v_{5}$ Band of HCOOD", J. Mol. Spectrosc. 1998, 191, 343-347.

[352] T. L. Tan, K. L. Goh, P. P. Ong, H. H. Teo, "FTIR Spectrum of the $\nu_{4}$ Band of DCOOD", J. Mol. Spectrosc. 1999, 195, 324-327. 
[353] Goh, Ong, Teo, Tan, "Improved Rovibrational Constants for the $\nu_{3}$ Infrared Band of HCOOD", J. Mol. Spectrosc. 1999, 197, 322-323.

[354] T. L. Tan, K. L. Goh, P. P. Ong, H. H. Teo, "Rovibrational Constants for the $v_{6}$ and $2 \nu_{9}$ Bands of HCOOD by Fourier Transform Infrared Spectroscopy", J. Mol. Spectrosc. 1999, 198, 110-114.

[355] T. L. Tan, K. L. Goh, P. P. Ong, H. H. Teo, "Rovibrational Analysis of $\nu_{2}$ and $2 \nu_{5}$ Bands of DCOOH by High Resolution FTIR Spectroscopy", J. Mol. Spectrosc. 1999, 198, 387-392.

[356] K. Goh, P. Ong, T. Tan, H. Teo, "High resolution FTIR spectrum of the $\nu_{6}$ band of deuterated formic acid (DCOOH)", Spectrochim. Acta A 1999, 55, 1309-1318.

[357] K. L. Goh, P. P. Ong, T. L. Tan, "The $\nu_{3}$ band of DCOOH", Spectrochim. Acta A 1999, 55, 2601-2614.

[358] O. I. Baskakov, H. Bürger, W. Jerzembeck, "The Coriolis-Coupled States $v_{7}=1$ and $v_{9}=1$ of trans-HCOOD and trans-DCOOD", J. Mol. Spectrosc. 1999, 193, $33-45$.

[359] O. I. Baskakov, S. Alanko, M. Koivusaari, "The Coriolis-Coupled States $v_{6}=1$ and $v_{8}=1$ of DCOOH", J. Mol. Spectrosc. 1999, 198, 40-42.

[360] K. Goh, P. Ong, H. Teo, T. Tan, "High resolution FTIR spectrum of the $\nu_{1}$ band of DCOOD", Spectrochim. Acta A 2000, 56, 991-1001.

[361] O. I. Baskakov, "The Vibrational State $v_{5}=1$ of HCOOD", J. Mol. Spectrosc. 2001, 208, 194-196.

[362] O. I. Baskakov, "FTIR Spectrum of the $\nu_{3}$ Band of HCOOD", J. Mol. Spectrosc. 2002, 213, 1-7.

[363] O. I. Baskakov, J. Lohilahti, V.-M. Horneman, "High resolution analysis of the $\nu_{7}$ and $\nu_{9}$ bands of DCOOH", J. Mol. Spectrosc. 2003, 219, 191-199.

[364] A. Domanskaya, K. Marushkevich, L. Khriachtchev, M. Räsänen, "Spectroscopic study of cis-to-trans tunneling reaction of HCOOD in rare gas matrices", J. Chem. Phys. 2009, 130, 154509.

[365] I. Bhattacharya, P. Banerjee, J. Sadhukhan, T. Chakraborty, "Modulations of $\nu_{\mathrm{O}-\mathrm{H}}$ and $\nu_{\mathrm{C}=\mathrm{O}}$ Stretching Frequencies of Difluoroacetic Acid with Internal Rotation of $\mathrm{CHF}_{2}$ Rotor: A Combined Vapor Phase and Matrix Isolation Infrared Spectroscopy Study", J. Phys. Chem. A 2019, 123, 2771-2779.

[366] Chemical Book, 3,3,3-trifluoro-2-(trifluoromethyl) propionic acid, https://www. chemicalbook.com/CASEN_564-10-3.htm, last accessed 04.11.2019.

[367] B. A. Shiekh, D. Kaur, "The role of torsional motion on the properties of propiolic acid and its H/D isotopic analogs: A density functional study using SCTST and a full anharmonic VPT2 model", Chem. Phys. Lett. 2016, 646, 168-173.

[368] H. J. Bakker, J. L. Skinner, "Vibrational spectroscopy as a probe of structure and dynamics in liquid water", Chem. Rev. 2010, 110, 1498-1517. 
[369] W. W. Cleland, M. M. Kreevoy, "Low-barrier hydrogen bonds and enzymic catalysis", Science 1994, 264, 1887-1890.

[370] C. L. Perrin, J. B. Nielson, " "Strong" hydrogen bonds in chemistry and biology", Annu. Rev. Phys. Chem. 1997, 48, 511-544.

[371] M. S. Taylor, E. N. Jacobsen, "Asymmetric catalysis by chiral hydrogen-bond donors", Angew. Chem. Int. Ed. 2006, 45, 1520-1543.

[372] S. J. Benkovic, S. Hammes-Schiffer, "A perspective on enzyme catalysis", Science 2003, 301, 1196-1202.

[373] L. Pauling, R. B. Corey, H. R. Branson, "The structure of proteins: Two hydrogenbonded helical configurations of the polypeptide chain", Proc. Nat. Acad. Sci. 1951, 37, 205-211.

[374] G. N. Ramachandran, G. Kartha, "Structure of collagen", Nature 1954, 174, 269270 .

[375] J. D. Watson, F. H. C. Crick, "A structure for deoxyribose nucleic acid", Nature 1953, 171, 737-738.

[376] C. Herrmann, M. Reiher in Atomistic Approaches in Modern Biology: From Quantum Chemistry to Molecular Simulations, (Ed.: M. Reiher), Springer Berlin Heidelberg, Berlin, Heidelberg, 2007, pp. 85-132.

[377] T. R. Rizzo, J. A. Stearns, O. v. Boyarkin, "Spectroscopic studies of cold, gasphase biomolecular ions", Int. Rev. Phys. Chem. 2009, 28, 481-515.

[378] R. C. Millikan, K. S. Pitzer, "The Infrared Spectra of Dimeric and Crystalline Formic Acid", J. Am. Chem. Soc. 1958, 80, 3515-3521.

[379] Y. Maréchal, "IR spectra of carboxylic acids in the gas phase: A quantitative reinvestigation", J. Chem. Phys. 1987, 87, 6344.

[380] M. Halupka, W. Sander, "A simple method for the matrix isolation of monomeric and dimeric carboxylic acids", Spectrochim. Acta A 1998, 54, 495-500.

[381] A. Winkler, P. Hess, "Study of the Energetics and Dynamics of Hydrogen Bond Formation in Aliphatic Carboxylic Acid Vapors by Resonant Photoacoustic Spectroscopy", J. Am. Chem. Soc. 1994, 116, 9233-9240.

[382] F. Ito, T. Nakanaga, "A jet-cooled infrared spectrum of the formic acid dimer by cavity ring-down spectroscopy", Chem. Phys. Lett. 2000, 318, 571-577.

[383] F. Ito, T. Nakanaga, "Jet-cooled infrared spectra of the formic acid dimer by cavity ring-down spectroscopy: Observation of the $\mathrm{O}-\mathrm{H}$ stretching region", Chem. Phys. 2002, 277, 163-169.

[384] M. Hippler, "Proton relaxation and intermolecular structure of liquid formic acid: A nuclear magnetic resonance study Dedicated to the memory of the late Hermann Gerhard Hertz", Phys. Chem. Chem. Phys. 2002, 4, 1457-1463. 
[385] R. Georges, M. Freytes, D. Hurtmans, I. Kleiner, J. Vander Auwera, M. Herman, "Jet-cooled and room temperature FTIR spectra of the dimer of formic acid in the gas phase", Chem. Phys. 2004, 305, 187-196.

[386] K. Marushkevich, L. Khriachtchev, J. Lundell, M. Räsänen, "cis-trans formic acid dimer: experimental observation and improved stability against proton tunneling", J. Am. Chem. Soc. 2006, 128, 12060-12061.

[387] F. Ito, "Jet-cooled infrared spectra of the formic acid dimer by cavity ring-down spectroscopy: Observation of the $\mathrm{C}-\mathrm{O}$ stretching region and vibrational analysis of the Fermi-triad system", Chem. Phys. Lett. 2007, 447, 202-207.

[388] O. Birer, M. Havenith, "High-resolution infrared spectroscopy of the formic acid dimer", Annu. Rev. Phys. Chem. 2009, 60, 263-275.

[389] S. Lopes, R. Fausto, L. Khriachtchev, "Formic acid dimers in a nitrogen matrix", J. Chem. Phys. 2018, 148, 034301.

[390] N. Shida, P. F. Barbara, J. Almlöf, "A reaction surface Hamiltonian treatment of the double proton transfer of formic acid dimer", J. Chem. Phys. 1991, 94, 3633.

[391] S. Miura, M. E. Tuckerman, M. L. Klein, "An ab initio path integral molecular dynamics study of double proton transfer in the formic acid dimer", J. Chem. Phys. 1998, 109, 5290-5299.

[392] H. Ushiyama, K. Takatsuka, "Successive mechanism of double-proton transfer in formic acid dimer: A classical study", J. Chem. Phys. 2001, 115, 5903.

[393] J. Chocholoušová, J. Vacek, P. Hobza, "Potential energy and free energy surfaces of the formic acid dimer: Correlated ab initio calculations and molecular dynamics", Phys. Chem. Chem. Phys. 2002, 4, 2119-2122.

[394] R. Kalescky, E. Kraka, D. Cremer, "Accurate determination of the binding energy of the formic acid dimer: the importance of geometry relaxation", J. Chem. Phys. 2014, 140, 084315.

[395] G. A. Pitsevich, A. E. Malevich, E. N. Kozlovskaya, I. Y. Doroshenko, V. Sablinskas, V. E. Pogorelov, D. Dovgal, V. Balevicius, "Anharmonic analysis of C-H and O-H stretching vibrations of the formic acid dimer", Vib. Spectrosc. 2015, $79,67-75$.

[396] E. Miliordos, S. S. Xantheas, "On the validity of the basis set superposition error and complete basis set limit extrapolations for the binding energy of the formic acid dimer", J. Chem. Phys. 2015, 142, 094311.

[397] F. Ito, "Modeling and spectral simulation of formic acid dimer in Ar matrix using ONIOM calculations", Comput. Theor. Chem. 2019, 1161, 18-25.

[398] D. Luckhaus, "Hydrogen exchange in formic acid dimer: tunnelling above the barrier", Phys. Chem. Chem. Phys. 2010, 12, 8357-8361.

[399] K. Marushkevich, L. Khriachtchev, J. Lundell, A. Domanskaya, M. Rasanen, "Matrix isolation and ab initio study of trans-trans and trans-cis dimers of formic acid", J. Phys. Chem. A 2010, 114, 3495-3502. 
[400] A. Olbert-Majkut, J. Ahokas, J. Lundell, M. Pettersson, "Raman spectroscopy of acetic acid monomer and dimers isolated in solid argon", J. Raman Spectrosc. 2011, 42, 1670-1681.

[401] K. Marushkevich, M. Siltanen, M. Räsänen, L. Halonen, L. Khriachtchev, "Identification of New Dimers of Formic Acid: The Use of a Continuous-Wave Optical Parametric Oscillator in Matrix Isolation Experiments", J. Phys. Chem. Lett. 2011, 2, 695-699.

[402] K. Marushkevich, L. Khriachtchev, M. Rasanen, M. Melavuori, J. Lundell, "Dimers of the higher-energy conformer of formic acid: experimental observation", J. Phys. Chem. A 2012, 116, 2101-2108.

[403] S. Lopes, A. V. Domanskaya, M. Rasanen, L. Khriachtchev, R. Fausto, "Acetic acid dimers in a nitrogen matrix: Observation of structures containing the higherenergy conformer", J. Chem. Phys. 2015, 143, 104307.

[404] J. Tang, Y. Xu, A. R. W. McKellar, W. Jäger, "Quantum solvation of carbonyl sulfide with helium atoms", Science 2002, 297, 2030-2033.

[405] A. R. W. McKellar, Y. Xu, W. Jäger, "Spectroscopic exploration of atomic scale superfluidity in doped helium nanoclusters", Phys. Rev. Lett. 2006, 97, 183401.

[406] A. R. W. McKellar, "Infrared spectra of $\mathrm{CO}_{2}$-doped ${ }^{4}$ He clusters, ${ }^{4} \mathrm{He}_{N^{-}} \mathrm{CO}_{2}$, with $N=1-60 "$, J. Chem. Phys. 2008, 128, 044308.

[407] G. Guelachvili, "Absolute wavenumber measurements of 1-0, 2-0, HF and 2-0, $\mathrm{H}^{35} \mathrm{Cl}, \mathrm{H}^{37} \mathrm{Cl}$ absorption bands", Opt. Commun. 1976, 19, 150-154.

[408] P. Das, C. J. Knapp, W. Jäger, "Ro-vibrational spectroscopy of the formic acid$d_{1}$ monomer embedded in helium nanodroplets", J. Mol. Spectrosc. 2017, 341, $17-22$.

[409] K. A. E. Meyer, J. A. Davies, A. M. Ellis, "Shifting formic acid dimers into perspective: vibrational scrutiny in helium nanodroplets", Phys. Chem. Chem. Phys. 2020, 22, 9637-9646.

[410] A. Poblotzki, J. Altnöder, M. A. Suhm, "Subtle solvation behaviour of a biofuel additive: the methanol complex with 2,5-dimethylfuran", Phys. Chem. Chem. Phys. 2016, 18, 27265-27271.

[411] M. Heger, M. A. Suhm, R. A. Mata, "Communication: Towards the binding energy and vibrational red shift of the simplest organic hydrogen bond: harmonic constraints for methanol dimer", J. Chem. Phys. 2014, 141, 101105.

[412] R. M. Balabin, "Polar (acyclic) isomer of formic acid dimer: gas-phase Raman spectroscopy study and thermodynamic parameters", J. Phys. Chem. A 2009, 113, 4910-4918.

[413] A. S. Davydov, "The Theory of Molecular Excitons", Sov. Phys. Usp. 1964, 7, $145-178$. 
[414] V. M. Agranovich, Excitations in organic solids, Oxford University Press, Oxford and New York, 2009.

[415] Y. Marechal, "Infrared Spectra of H-Bonded Systems", J. Chem. Phys. 1968, 48, 3697.

[416] D. Chamma, O. Henri-Rousseau, "IR theory of weak H-bonds: Davydov coupling, Fermi resonances and direct relaxations. I. Basis equations within the linear response theory", Chem. Phys. 1999, 248, 53-70.

[417] K. Heyne, N. Huse, E. T. Nibbering, T. Elsaesser, "Ultrafast coherent nuclear motions of hydrogen bonded carboxylic acid dimers", Chem. Phys. Lett. 2003, $369,591-596$.

[418] P. Ottiger, H. Köppel, S. Leutwyler, "Excitonic splittings in molecular dimers: Why static ab initio calculations cannot match them", Chem. Sci. 2015, 6, 60596068 .

[419] M. C. D. Tayler, B. Ouyang, B. J. Howard, "Unraveling the spectroscopy of coupled intramolecular tunneling modes: a study of double proton transfer in the formic-acetic acid complex", J. Chem. Phys. 2011, 134, 54316.

[420] A. M. Daly, K. O. Douglass, L. C. Sarkozy, J. L. Neill, M. T. Muckle, D. P. Zaleski, B. H. Pate, S. G. Kukolich, "Microwave measurements of proton tunneling and structural parameters for the propiolic acid-formic acid dimer", J. Chem. Phys. 2011, 135, 154304.

[421] R. B. Mackenzie, C. T. Dewberry, K. R. Leopold, "The formic acid-nitric acid complex: microwave spectrum, structure, and proton transfer", J. Phys. Chem. A 2014, 118, 7975-7985.

[422] J. Thomas, M. J. Carrillo, A. Serrato, F. Xie, W. Jäger, Y. Xu, W. Lin, "Microwave spectrum of the complex of 3,3,3-trifluoro-2-(trifluoromethyl)propanoic acid and formic acid", Mol. Phys. 2019, 117, 1193-1199.

[423] M. Goubet, P. Soulard, O. Pirali, P. Asselin, F. Réal, S. Gruet, T. R. Huet, P. Roy, R. Georges, "Standard free energy of the equilibrium between the trans-monomer and the cyclic-dimer of acetic acid in the gas phase from infrared spectroscopy", Phys. Chem. Chem. Phys. 2015, 17, 7477-7488.

[424] F. J. Strieter, D. H. Templeton, R. F. Scheuerman, R. L. Sass, "The crystal structure of propionic acid", Acta Crystallogr. 1962, 15, 1233-1239.

[425] R. F. Scheuerman, R. L. Sass, "The crystal structure of valeric acid", Acta Crystallogr. 1962, 15, 1244-1247.

[426] F. J. Strieter, D. H. Templeton, "Crystal structure of butyric acid", Acta Crystallogr. 1962, 15, 1240-1244.

[427] G. Bruno, L. Randaccio, "A refinement of the benzoic acid structure at room temperature", Acta Crystallogr. B 1980, 36, 1711-1712. 
[428] F. Holtzberg, B. Post, I. Fankuchen, "The crystal structure of formic acid", Acta Crystallogr. 1953, 6, 127-130.

[429] P. G. Jönsson, "Hydrogen bond studies. XLIV. Neutron diffraction study of acetic acid", Acta Crystallogr. B 1971, 27, 893-898.

[430] M. Stein, J. Sauer, "Formic acid tetramers: Structure isomers in the gas phase", Chem. Phys. Lett. 1997, 267, 111-115.

[431] J. M. Hermida Ramón, M. A. Ríos, "A new intermolecular polarizable potential for cis-formic acid. Introduction of many-body interactions in condensed phases", Chem. Phys. 1999, 250, 155-169.

[432] A. K. Roy, A. J. Thakkar, "Structures of the formic acid trimer", Chem. Phys. Lett. 2004, 386, 162-168.

[433] A. J. Thakkar, "Small clusters of formic acid: Tests and applications of density functional theory with dispersion-correcting potentials", Chem. Phys. Lett. 2013, $560,71-74$.

[434] N. I. Butkovskaya, I. I. Morozov, "Electric-field focusing of small formic acid, methanol and ethanol clusters in supersonic modulated beam", Chem. Phys. Lett. 1990, 168, 345-348.

[435] W. Y. Feng, C. Lifshitz, "Evaporation of Dimers from Proton-Bound Formic Acid Clusters", J. Phys. Chem. 1994, 98, 6075-6081.

[436] S. Heinbuch, F. Dong, J. J. Rocca, E. R. Bernstein, "Single photon ionization of hydrogen bonded clusters with a soft x-ray laser: $(\mathrm{HCOOH})_{\mathrm{x}}$ and $(\mathrm{HCOOH})_{\mathrm{y}}\left(\mathrm{H}_{2} \mathrm{O}\right)_{\mathrm{z}}$ ", J. Chem. Phys. 2007, 126, 244301.

[437] E. Spinner, "Comment on 'A simple method for the matrix isolation of monomeric and dimeric carboxylic acids'. Possible implications of findings, in regard to liquid formic acid", Spectrochim. Acta A 1999, 55, 1819-1825.

[438] G. Tejeda, B. Maté, J. M. Fernández-Sánchez, S. Montero, "Temperature and density mapping of supersonic jet expansions using linear Raman spectroscopy", Phys. Rev. Lett. 1996, 76, 34-37.

[439] B. Maté, I. A. Graur, T. Elizarova, I. Chirokov, G. Tejeda, J. M. Fernández, S. Montero, "Experimental and numerical investigation of an axisymmetric supersonic jet", J. Fluid Mech. 2001, 426, 177-197.

[440] T. Häber, Ragout-Jet-FTIR-Spektroskopie: Eine neuartige Methode zur Untersuchung der Dynamik von Oligomeren und nanometer-großen Molekülclustern, 1. Auflage, Cuvillier, Göttingen, 2001.

[441] J. Altnöder, Untersuchungen zum Einfluss von London-Dispersionswechselwirkungen auf die Molekülaggregation, Ph.D. thesis, Georg-August-Universität Göttingen, Göttingen, 2015.

[442] L. Turi, "Ab Initio Molecular Orbital Analysis of Dimers of cis-Formic Acid. Implications for Condensed Phases", J. Phys. Chem. 1996, 100, 11285-11291. 
[443] W. Wang, "Method-dependent relative stability of hydrogen bonded and $\pi-\pi$ stacked structures of the formic acid tetramer", Chem. Phys. Lett. 2005, 402, $54-56$.

[444] Y. Zhao, D. G. Truhlar, "Infinite-basis calculations of binding energies for the hydrogen bonded and stacked tetramers of formic acid and formamide and their use for validation of hybrid DFT and ab initio methods", J. Phys. Chem. A 2005, 109, 6624-6627.

[445] A. K. Roy, A. J. Thakkar, "Pentamers of formic acid", Chem. Phys. 2005, 312, 119-126.

[446] S. A. Blair, A. J. Thakkar, "Structural characteristics of formic acid dodecamers, $(\mathrm{HCOOH})_{12}$ ", Chem. Phys. Lett. 2008, 450, 258-262.

[447] S. P. McCarthy, A. K. Roy, S. Kazachenko, A. J. Thakkar, "A dispersion-corrected density functional theory study of hexamers of formic acid", Can. J. Chem. 2013, 91, 527-528.

[448] D. Di Tommaso, K. L. Watson, "Density functional theory study of the oligomerization of carboxylic acids", J. Phys. Chem. A 2014, 118, 11098-11113.

[449] E. Torres, G. A. DiLabio, "A (Nearly) Universally Applicable Method for Modeling Noncovalent Interactions Using B3LYP", J. Phys. Chem. Lett. 2012, 3, 17381744 .

[450] T. Forsting, H. C. Gottschalk, B. Hartwig, M. Mons, M. A. Suhm, "Correcting the record: The dimers and trimers of trans-N-methylacetamide", Phys. Chem. Chem. Phys. 2017, 19, 10727-10737.

[451] S. Grimme, "Density functional theory with London dispersion corrections", WIREs Comput. Mol. Sci. 2011, 1, 211-228.

[452] S. Oswald, M. Wallrabe, M. A. Suhm, "Cooperativity in Alcohol-Nitrogen Complexes: Understanding Cryomatrices through Slit Jet Expansions", J. Phys. Chem. A 2017, 121, 3411-3422.

[453] T. N. Wassermann, M. A. Suhm, "Ethanol monomers and dimers revisited: a Raman study of conformational preferences and argon nanocoating effects", $J$. Phys. Chem. A 2010, 114, 8223-8233.

[454] L. C. Leibold, Chemische Bildgebung von Clusterstabilität in Stoßfronten, bachelor thesis, Georg-August-Universität Göttingen, Göttingen, 2018.

[455] F. Ito, "Infrared spectra of $(\mathrm{HCOOH})_{2}$ and $(\mathrm{DCOOH})_{2}$ in rare gas matrices: a comparative study with gas phase spectra", J. Chem. Phys. 2008, 128, 114310.

[456] F. Ito, "Modeling and spectral simulation of matrix-isolated molecules by density functional calculations: a case study on formic acid dimer", J. Chem. Phys. 2010, 133, 214502.

[457] R. A. Mata, M. A. Suhm, "Benchmarking Quantum Chemical Methods: Are We Heading in the Right Direction?", Angew. Chem. Int. Ed. 2017, 56, 11011-11018. 


\section{Bibliography}

[458] S. Oswald, M. A. Suhm, "Experimental Reference Data for Hexafluorinated Propanol by Exploring an Unusual Intermolecular Torsional Balance", Angew. Chem. Int. Ed. 2017, 56, 12672-12676.

[459] S. Oswald, Weak Hydrogen Bonds to Molecular Nitrogen and Oxygen as Experimental Benchmarks for Quantum Chemistry, Ph.D. thesis, Georg-AugustUniversität Göttingen, Göttingen, 2019. 
A Appendix

\section{A Appendix}

\section{A.1 Experimental and Theoretical Methods}

\section{A.1.1 Chilli Jet}
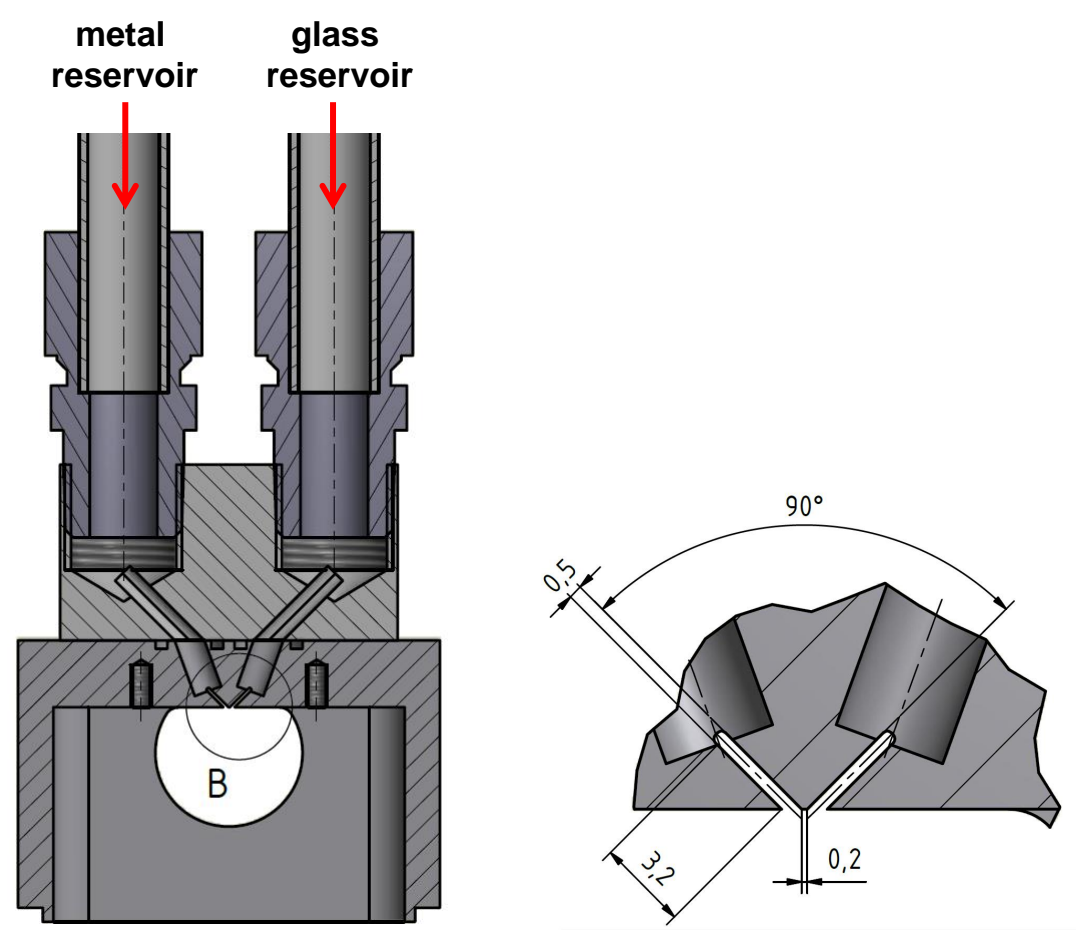

Figure A.1: Technical drawing of the prototype of the double slit nozzle, provided by R. Hildebrandt from the mechanical workshop. The slit length and width amount to $20 \mathrm{~mm}$ and $0.5 \mathrm{~mm}$. Its design is based on the $2 \times 10$ slit nozzle of Ref. [124]. All dimensions are given in $\mathrm{mm}$. 

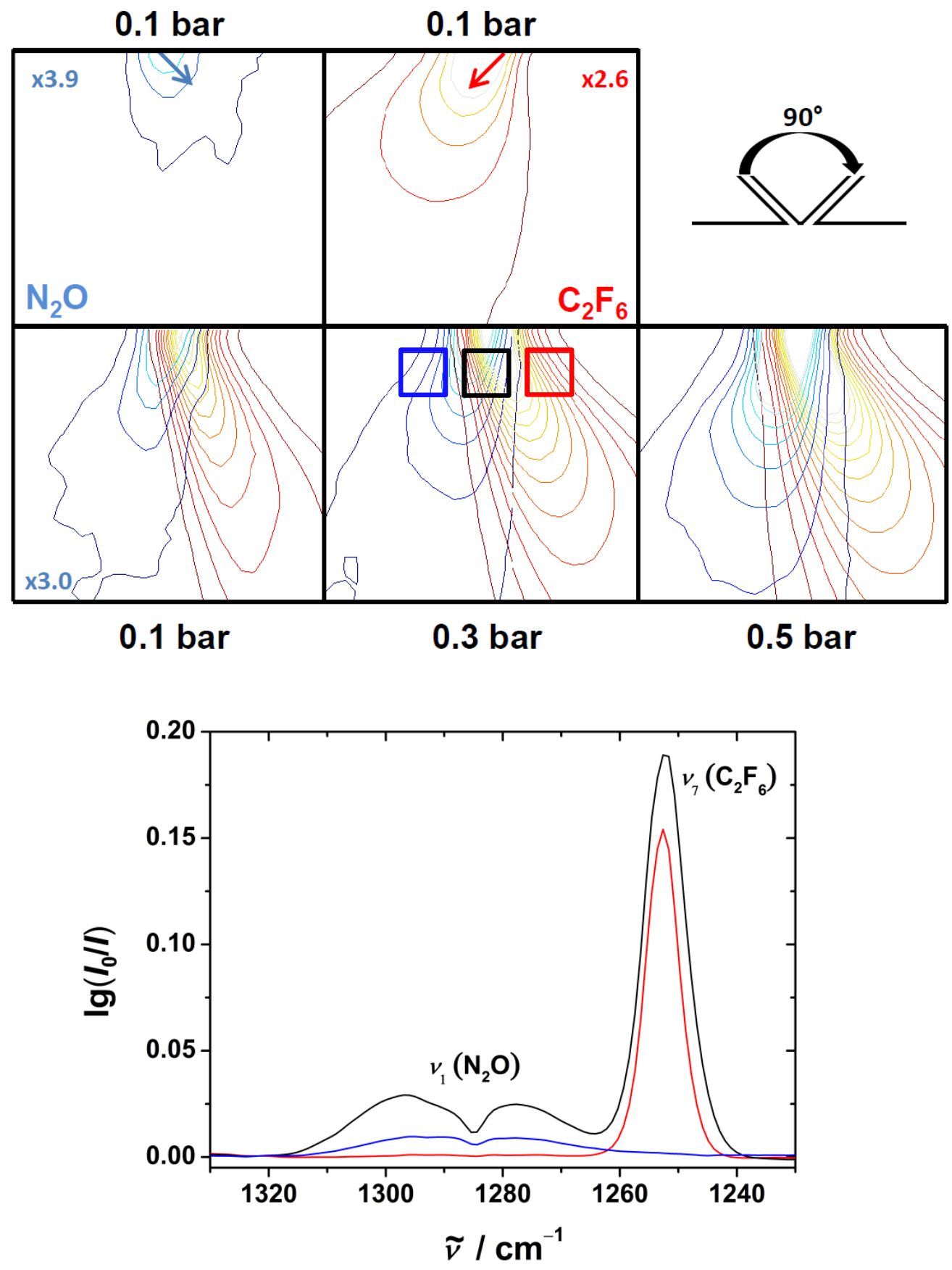

Figure A.2: Space-resolved two dimensional evolution of the integrated intensity of the $\nu_{1}$ band of nitrous oxide (blue) and the $\nu_{7}$ band of hexafluoroethane (red) in single substance (upper part) and mixed supersonic expansions (lower part). The single substance expansions have been recorded at a reservoir pressure of 0.1 bar, the mixed expansions between $0.1-0.5$ bar. $\mathrm{C}_{2} \mathrm{~F}_{6}$ has been further diluted with helium (ratio 1:12). The arrows indicate the propagation direction of the supersonic flows. A schematic drawing of the double slit nozzle $(20 \mathrm{~mm}$ length and $0.5 \mathrm{~mm}$ width) can be found on the upper right-hand side and a technical drawing in Figure A.1. FTIR jet spectra averaged over $16 \times 16$ pixel blocks depicted in black, red, and blue are shown at the bottom. The colours of the spectra correspond to those of the blocks. 


\section{A.1.2 FTIR Components and Settings}

Table A.1: Parameters of FTIR jet measurements with the chilli jet.

\begin{tabular}{|c|c|c|}
\hline setting & value & comment \\
\hline light source & globar & \\
\hline aperture & $8 \mathrm{~mm}$ & $\begin{array}{l}\text { necessary for a full } \\
\text { illumination of the detector }\end{array}$ \\
\hline beam splitter & $\mathrm{KBr}$ & \\
\hline mirror speed & $5 \mathrm{kHz}$ & $\begin{array}{l}\text { limited by slow } \\
\text { read-out of the detector }\end{array}$ \\
\hline resolution & $2 \mathrm{~cm}^{-1}$ & continuous expansions: $4 \mathrm{~cm}^{-1}$ \\
\hline acquisition & single sided & \\
\hline detector & $64 \times 64 \mathrm{MCT}$ FPA & \\
\hline exposure time & between $0.1891-0.2395 \mathrm{~ms}$ & \\
\hline IR lens & $\begin{array}{l}50 \mathrm{~mm}, 3-5 \mu \mathrm{m}, \\
\text { circular field of view (FOV) } 23^{\circ}\end{array}$ & Nyctea (ASIO series) \\
\hline apodisation & Norton-Beer, medium & \\
\hline zero filling factor & 4 & \\
\hline
\end{tabular}

Table A.2: Parameters of FTIR jet measurements with the filet jet.

\begin{tabular}{ccc}
\hline \hline setting & value & comment \\
\hline light source & globar & \\
aperture & $3.5-4.0 \mathrm{~mm}$ & \\
beam splitter & $\mathrm{KBr}$ & \\
mirror speed & $80 \mathrm{kHz}$ & \\
resolution & $2 \mathrm{~cm}^{-1}$ & \\
acquisition & single sided fast return & pre-amplifier: $R=1.0 \mathrm{k} \Omega, V_{\mathrm{c}}=2.0 \mathrm{~V}$ \\
detector & $\mathrm{MCT}$ & \\
apodisation & Norton-Beer, medium & \\
zero filling factor & 4 & \\
\hline \hline
\end{tabular}


Table A.3: List of filters used throughout this work. Their transparent spectral window is given in $\mathrm{cm}^{-1}$. The transmittance curves of two newly purchased filters (F18 and F19) can be found in Figure A.3. The filter numbering (F1-F19) represents the order of purchase.

\begin{tabular}{ccc}
\hline \hline filter & transparent spectral window & comment \\
\hline F13 & $4100-2500$ & \\
F1 & $4000-2860$ & \\
F19 & $3400-3200$ & \\
F2 & $3300-2000$ & \\
F4 & $2260-1100$ & used for Figures 3.3 and A.2 \\
F5 & $1800-1700$ & now replaced by filter F18 \\
F18 & $1900-1600$ & used for most FTIR spectra \\
& & in the C=O stretching region \\
\hline \hline
\end{tabular}

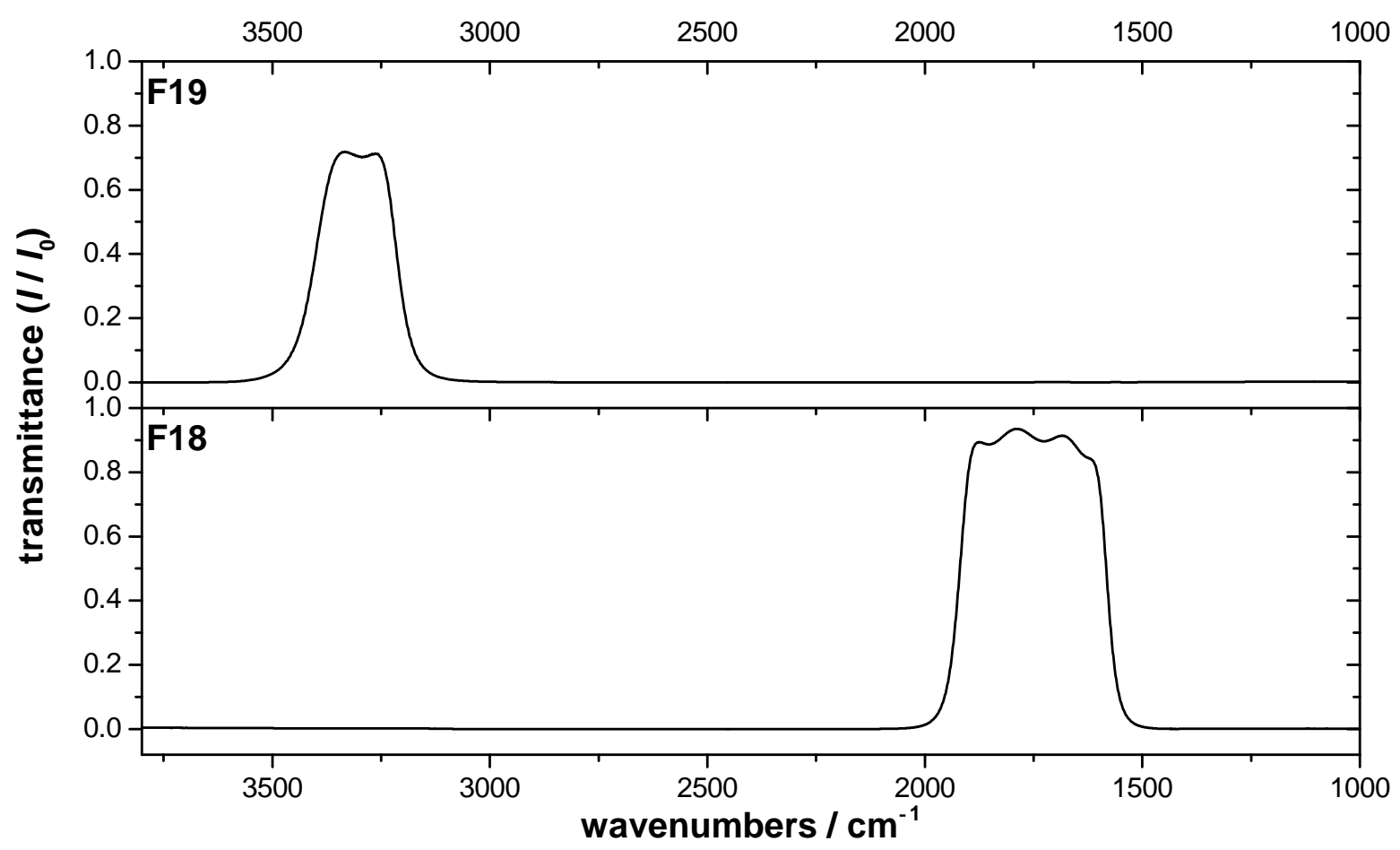

Figure A.3: Measured transmittance of two narrow IR band pass filters (F18, F19) between $3800-1000 \mathrm{~cm}^{-1}$, recorded with a DTGS detector. More information on the filters can be found in Table A.3. 
A Appendix

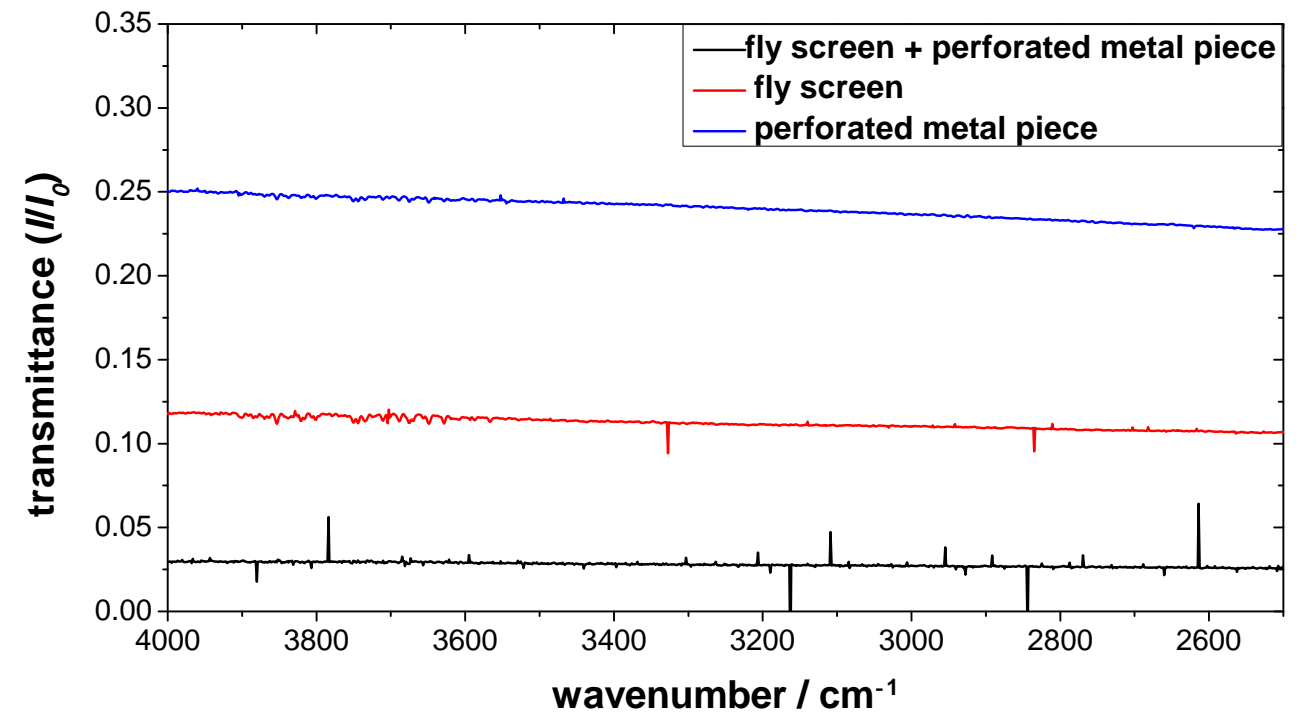

Figure A.4: Transmittance $\left(I / I_{0}\right)$ of the light attenuators (perforated metal piece, five layers of fly screen, and the combination of both) measured with band pass filter F13 $\left(4100-2500 \mathrm{~cm}^{-1}\right)$. $I$ has been recorded with the light attenuator(s) and correspondingly, $I_{0}$ without it.

Table A.4: List of faulty pixels of the $64 \times 64$ focal plane array detector. The pixel are numbered from 0-4095 in the spectrometer software (OPUS), whereby the first row contains pixels $0-63$, the second row $64-127$, and so on. Three additional pixels are faulty compared to those reported in Ref. [124]. These are marked new.

\begin{tabular}{cc}
\hline \hline pixel number & comment \\
\hline 201 & \\
207 & \\
270 & new \\
457 & \\
713 & \\
969 & \\
1225 & \\
1481 & \\
1737 & \\
1993 & \\
2249 & \\
2505 & \\
2543 & new \\
2761 & \\
3017 & \\
3273 & \\
3529 & \\
3785 & \\
4041 & new \\
\hline \hline
\end{tabular}




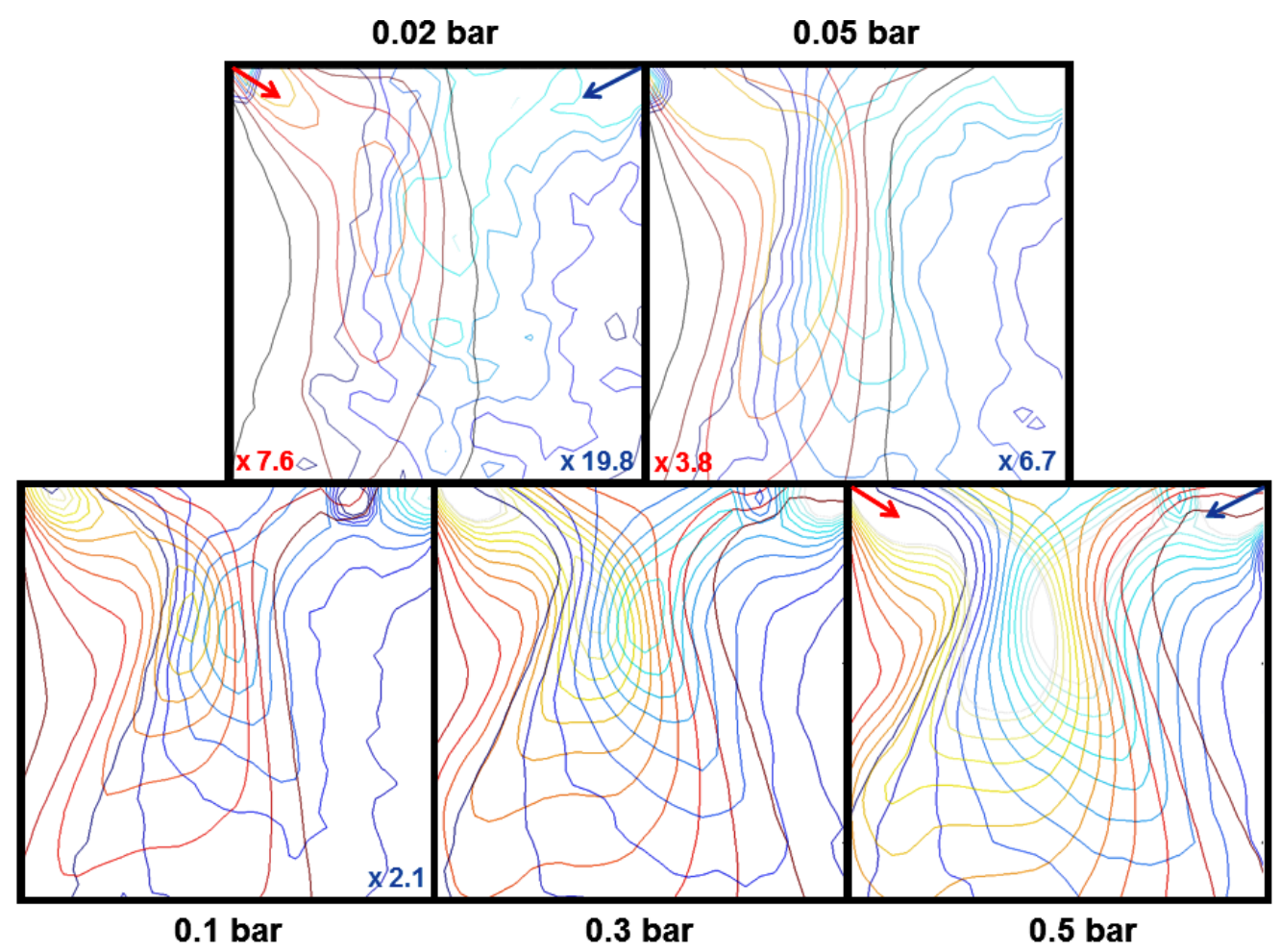

Figure A.5: Space-resolved two dimensional evolution of the integrated band intensity of the $\nu_{1}$ band of nitrous oxide (blue) and the $\nu_{7}$ band of hexafuoroethane (red) in mixed supersonic expansions of varying reservoir pressures $(0.02-0.5 \mathrm{bar}) . \mathrm{C}_{2} \mathrm{~F}_{6}$ has been further diluted with helium (ratio 1:12). The arrows indicate the propagation direction of the supersonic flows. A technical drawing of the double slit nozzle $(20 \mathrm{~mm}$ length and $0.5 \mathrm{~mm}$ width, average nozzle distance $7.5 \mathrm{~mm}$ ) used for the measurements can be found in Figure 3.2. 


\section{A.2 Carboxylic Acid Monomers}

\section{A.2.1 Scaled Harmonic Frequency Calculations}

Table A.5: Scaled harmonic band positions of the four stretching vibrations of cis- (cF) and trans-formic acid (F). The calculations have been performed at the B3LYP-D3(BJ)/aVTZ level. All band positions are given in $\mathrm{cm}^{-1}$. Additionally, harmonically calculated Raman scattering cross-sections (in $10^{-36} \mathrm{~m}^{2} \mathrm{sr}^{-1}$ ) are listen in parentheses behind the band positions.

\begin{tabular}{ccccc}
\hline \hline$\nu_{i}$ & assignment & scaling factor & $\mathrm{F}$ & $\mathrm{cF}$ \\
\hline$\nu_{1}$ & $\mathrm{O}-\mathrm{H}$ & 0.961 & $3570(76)$ & $3632(104)$ \\
$\nu_{2}$ & $\mathrm{C}-\mathrm{H}$ & 0.965 & $2942(172)$ & $2859(172)$ \\
$\nu_{3}$ & $\mathrm{C}=\mathrm{O}$ & 0.981 & $1777(40)$ & $1821(47)$ \\
$\nu_{6}$ & $\mathrm{C}-\mathrm{O}$ & 0.985 & $1105(12)$ & $1088(51)$ \\
\hline \hline
\end{tabular}

\section{A.2.2 Vibrational Benchmark: $\mathrm{C}-\mathrm{H}$ Stretching Vibration}

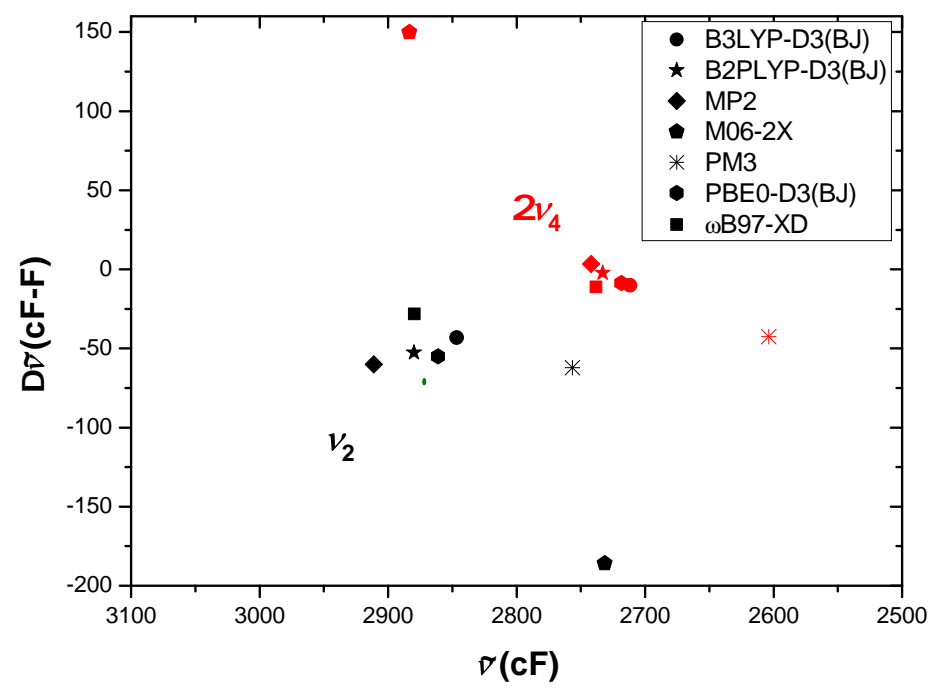

Figure A.6: Anharmonic (VPT2) prediction of the band position shift between the cis- and trans-rotamer of formic acid against the band position of the cis-rotamer for the $\mathrm{C}-\mathrm{H}$ stretching vibration $\nu_{2}$ (black symbols) as well as the overtone of the $\mathrm{C}-\mathrm{H}$ bending vibration $\nu_{4}$ (red symbols). The employed methods are listed in the inset. For all, an aVQZ basis set has been used. The experimental band position of $\nu_{2}$ of cis-HCOOD as well as the band position shift $\Delta \nu_{2}$ between the cis- and trans-rotamers is marked by a green ellipsis - the dimensions representing the experimental uncertainties of the band position $\left( \pm 2 \mathrm{~cm}^{-1}\right)$ and the shift $\left( \pm 4 \mathrm{~cm}^{-1}\right)$.

\section{A.2.3 Instabilities of DFT Functionals}


A Appendix
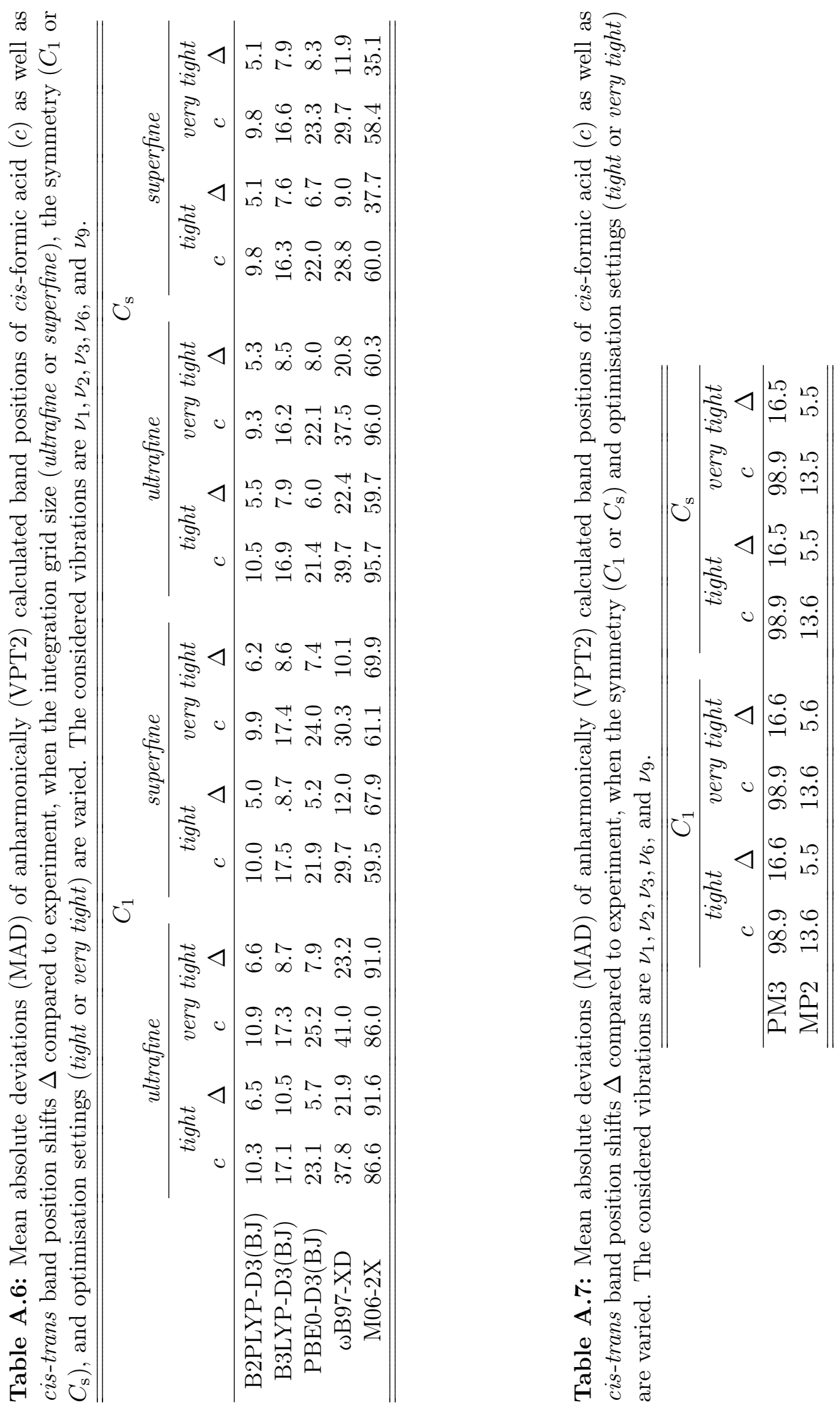


\section{A.2.4 $\mathrm{C}-\mathrm{H}$ stretching vibration of $\mathrm{HCOOD}$}

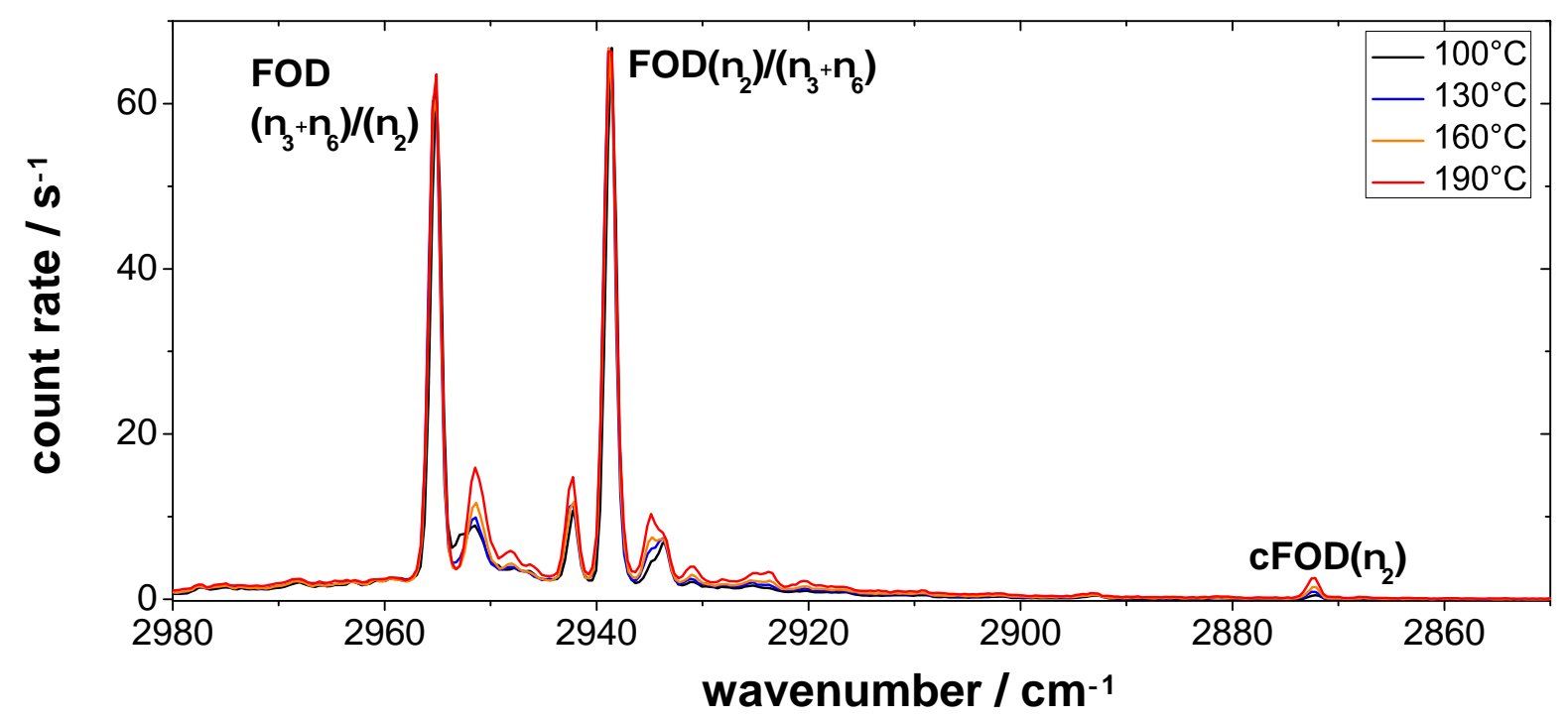

Figure A.7: Raman jet spectra of HCOOD $(<0.2 \%)$ in helium, recorded at a reservoir pressure of 0.3 bar, increasing nozzle temperature of $100-190{ }^{\circ} \mathrm{C}$ and acquisition times of $27-33$ minutes. The spectra have been intensity scaled to the band at $2939 \mathrm{~cm}^{-1}$. The band at $2942 \mathrm{~cm}^{-1}$ corresponds to $\nu_{2}$ of $\mathrm{HCOOH}$ (impurity of about $5 \%$ ). 


\section{A.2.5 Fully Deuterated Formic Acid}

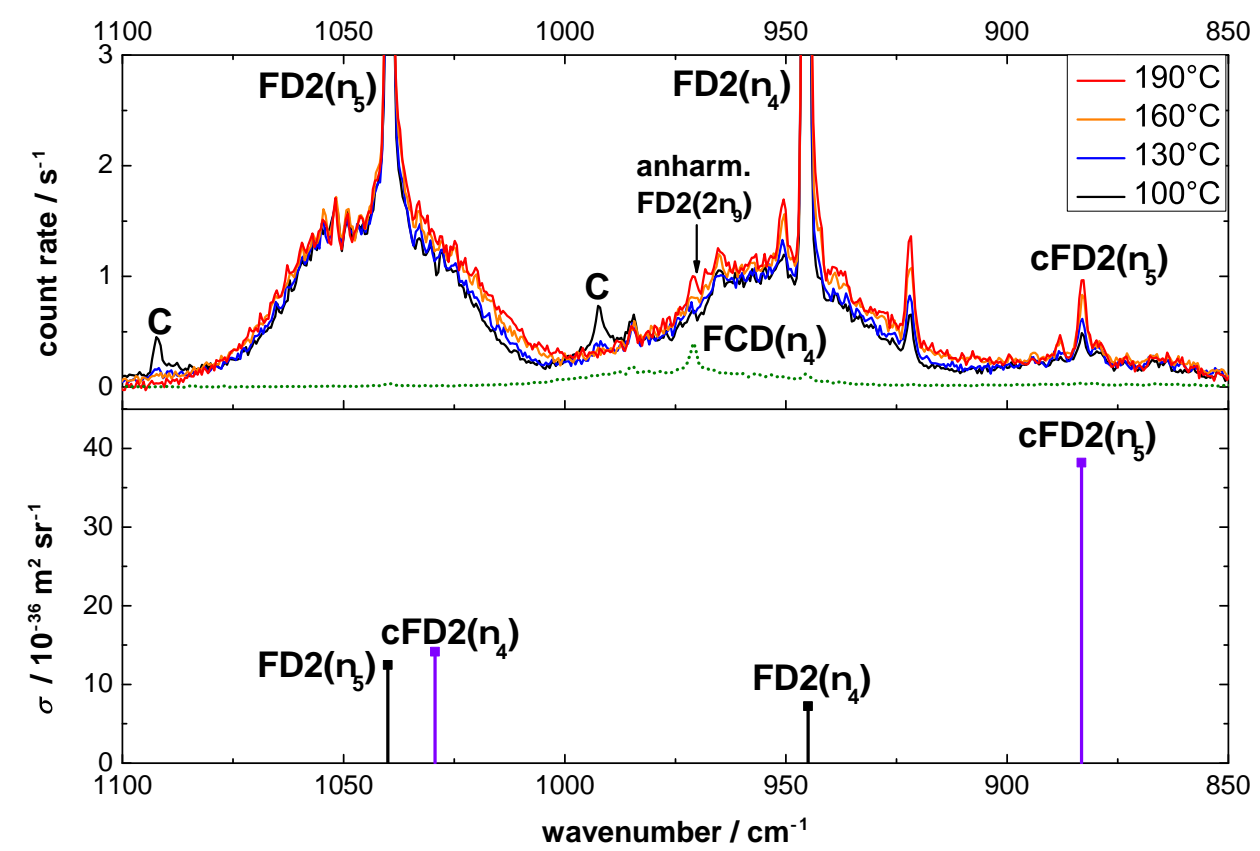

Figure A.8: Raman jet spectra of DCOOD $(c<0.2 \%$ in helium) at a reservoir pressure of $0.3 \mathrm{bar}$, nozzle temperatures $\theta_{\mathrm{n}}$ of $100-190{ }^{\circ} \mathrm{C}$, and data acquisition times of $40-50$ minutes. The spectra have been intensity-scaled to the spectrum at the highest nozzle temperature. Additionally, a scaled DCOOH spectrum has been plotted (green, dotted line). DCOOD is abbreviated to FD2 and DCOOH to FCD. Cluster bands are marked by a C. Below the spectra, harmonically calculated and FD2 $\left(\nu_{4}\right)$-scaled band positions as well as Raman scattering cross-sections of the fundamentals of cis- (cFD2) and trans-DCOOD (FD2) are shown. The calculations have been performed at the B3LYP-D3(BJ)/aVTZ level. The anharmonically (VPT2) calculated band position (B3LYP-D3(BJ)/aVTZ) of the overtone of the O-D out-ofplane bending vibration $2 \nu_{9}$ of DCOOD is shown by the tip of an arrow.

\section{A.2.6 $\mathrm{C}-\mathrm{O}$ stretching vibration of HCOOD}

To validate that $\sigma\left(\nu_{6}\right)$ of cis-HCOOD is in fact too low to be detectable with the combination of thermal excitation, rapid jet-cooling, and Raman spectroscopic detection as used throughout this work, the corresponding spectra have been recorded and are displayed in Figure A.9. Expectedly, the $\mathrm{C}-\mathrm{O}$ stretching region of HCOOD is very similar to $\mathrm{HCOOH}$ with several hot bands downshifted with respect to the fundamental at $1177 \mathrm{~cm}^{-1}$, yet the intensity pattern is different - the strongest hot band is located directly next to the fundamental at $1173 \mathrm{~cm}^{-1}$ and has an intensity of $17 \%$ of $\nu_{6}$ of transHCOOD. The second hot band at $1168 \mathrm{~cm}^{-1}$ has an intensity of $4 \%$ of $\nu_{6}$. Hence, both are too intense to be cis-HCOOD bands, even if the (though very similar) scattering cross-sections of both rotamers are accounted for. The remaining hot bands are too small for an intensity analysis. Therefore, $\nu_{6}$ of cis-HCOOD cannot be assigned. One 


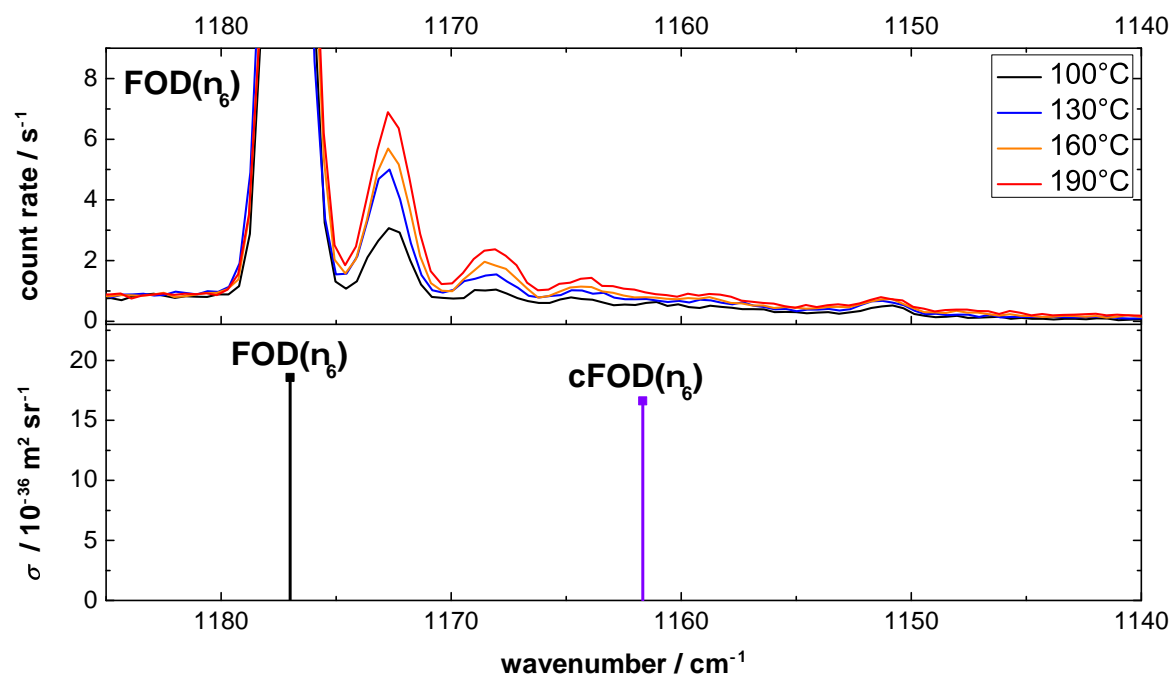

Figure A.9: Raman jet spectra of HCOOD $(c<0.2 \%$ in helium) at a reservoir pressure of 0.3 bar, increasing nozzle temperatures $\left(\theta_{\mathrm{n}}=100-190{ }^{\circ} \mathrm{C}\right)$, and data acquisition times of 25-35 minutes in the $\mathrm{C}-\mathrm{O}$ stretching region. The spectra have been intensity-scaled to the spectrum at the highest nozzle temperature. Below the spectra, harmonically calculated and $\operatorname{FOD}\left(\nu_{6}\right)$-scaled band positions as well as Raman scattering cross-sections of the fundamentals of cis- (cFOD) and trans-HCOOD (FOD) are shown. The calculations have been performed at the B3LYP-D3(BJ)/aVTZ level.

way of determining $\nu_{6}$ would be to enhance the thermal population further by employing higher nozzle temperatures, which is currently not possible with the set-up. 


\section{A.2.7 Cis-Acetic and -Pivalic Acid Measurements}

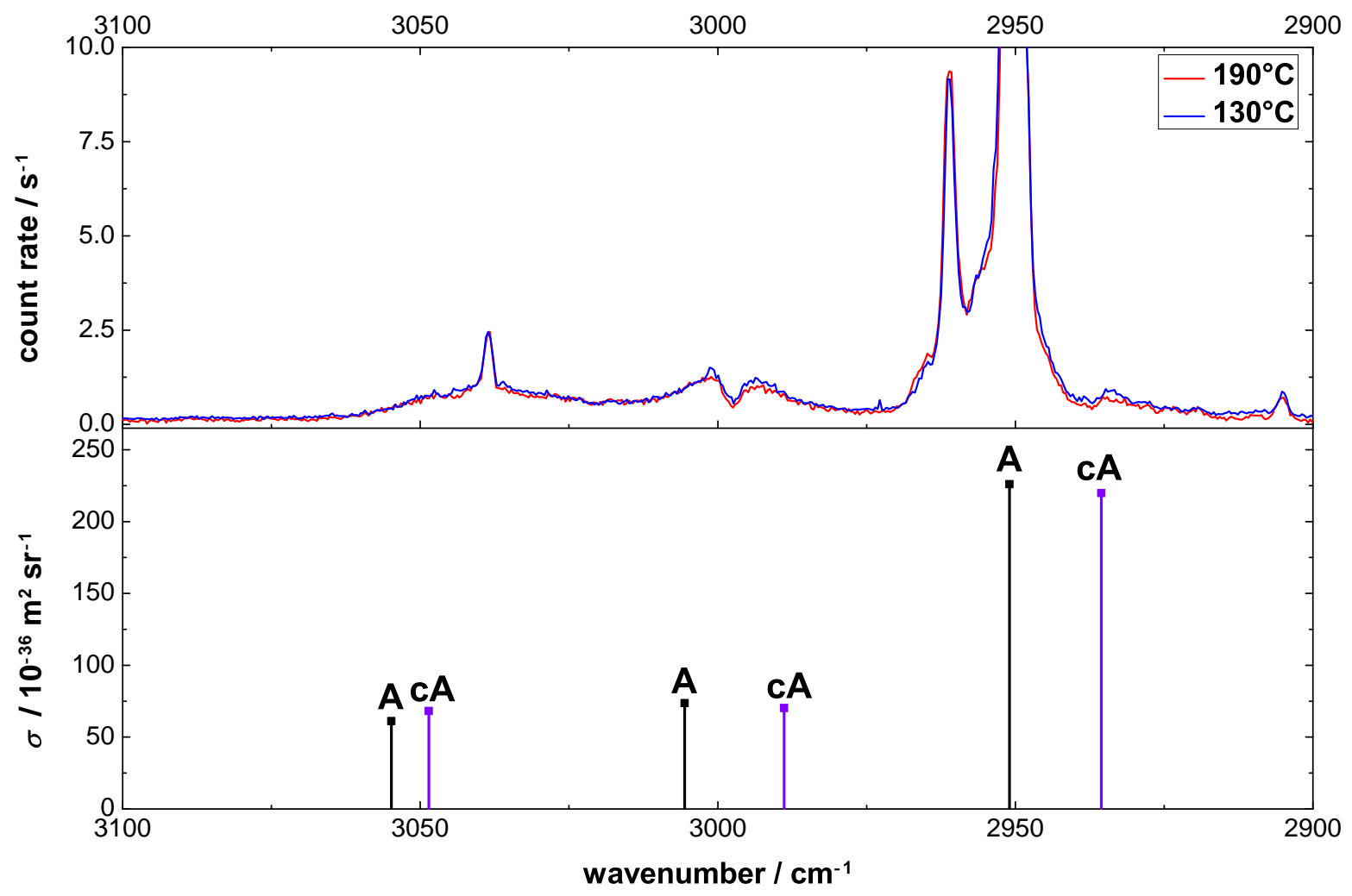

Figure A.10: Raman jet spectra of acetic acid $(c<0.3 \%$ in helium) in the $\mathrm{C}-\mathrm{H}$ stretching region recorded at a reservoir pressure of $1.0 \mathrm{bar}$, nozzle temperatures of $130{ }^{\circ} \mathrm{C}$ and $190{ }^{\circ} \mathrm{C}$, and data acquisition times of $25\left(130{ }^{\circ} \mathrm{C}\right)$ and 30 minutes $\left(190{ }^{\circ} \mathrm{C}\right)$. The band intensity has been scaled to the spectrum measured at $190{ }^{\circ} \mathrm{C}$. Below the spectra, harmonically calculated and scaled band positions as well as Raman scattering cross-sections of the fundamentals of cis- (cA) and trans-acetic acid (A) are shown. The calculations have been performed at the B3LYP-D3(BJ)/aVTZ level. 
A Appendix
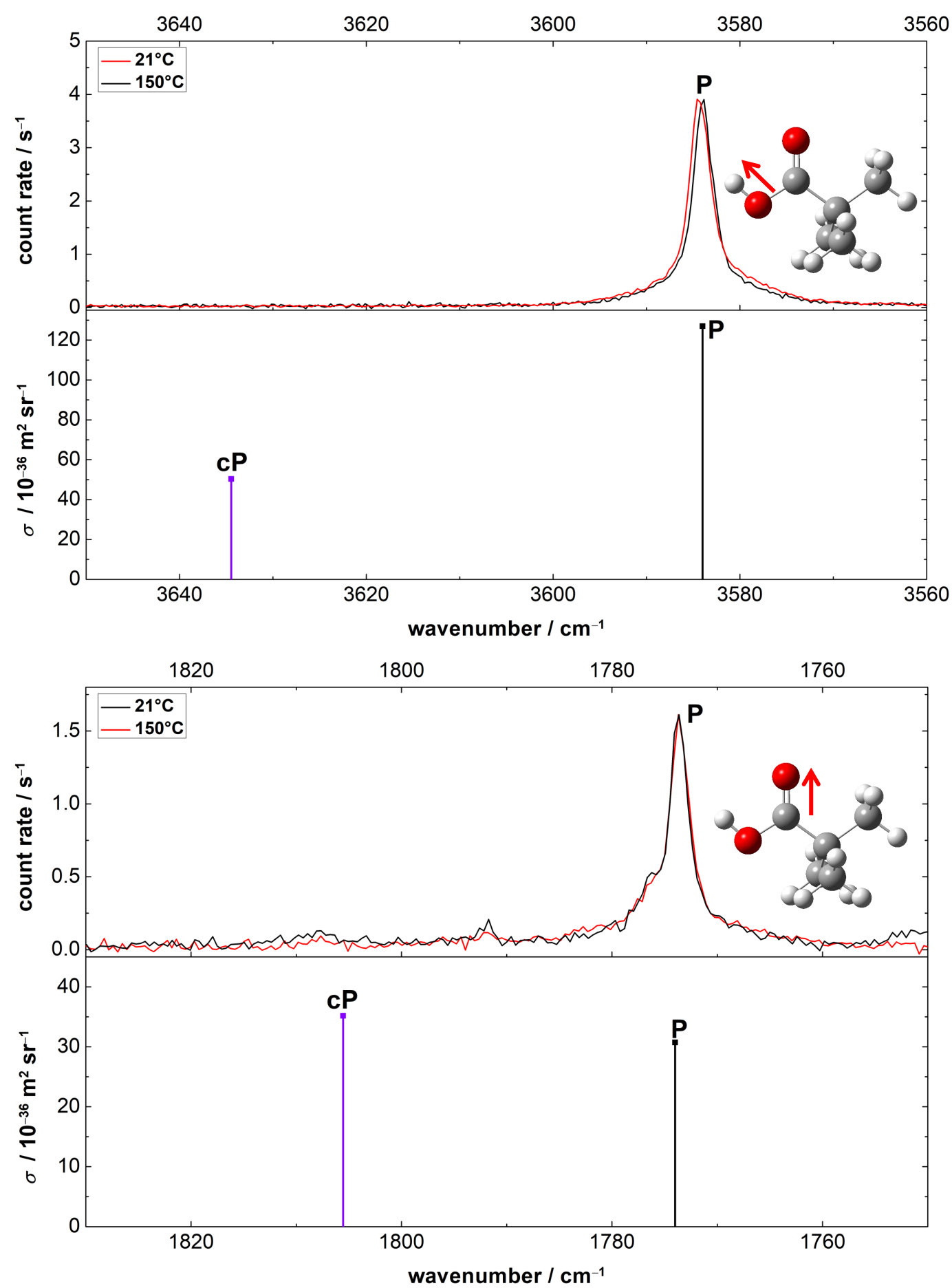

Figure A.11: Raman jet spectra of pivalic acid $(c<0.3 \%$ in helium) at a reservoir pressure of $1.0 \mathrm{bar}$, nozzle temperatures of room temperature $\left(21^{\circ} \mathrm{C}\right)$ and $150{ }^{\circ} \mathrm{C}$, and data acquisition times of 30-40 minutes in the $\mathrm{O}-\mathrm{H}$ (top) and $\mathrm{C}=\mathrm{O}$ stretching regions (bottom). The band intensity has been scaled to the spectrum measured at $190{ }^{\circ} \mathrm{C}$. Below the spectra, harmonically calculated and trans-pivalic acid scaled band positions as well as (unscaled) Raman scattering cross-sections of the fundamentals of cis- $(\mathrm{cP})$ and trans-pivalic acid $(\mathrm{P})$ are shown. The calculations have been performed at the B3LYP-D3(BJ)/aVTZ level. 


\section{A.3 Carboxylic Acid Dimers}

\section{A.3.1 Setting Sensitivity of VPT2 Calculations}

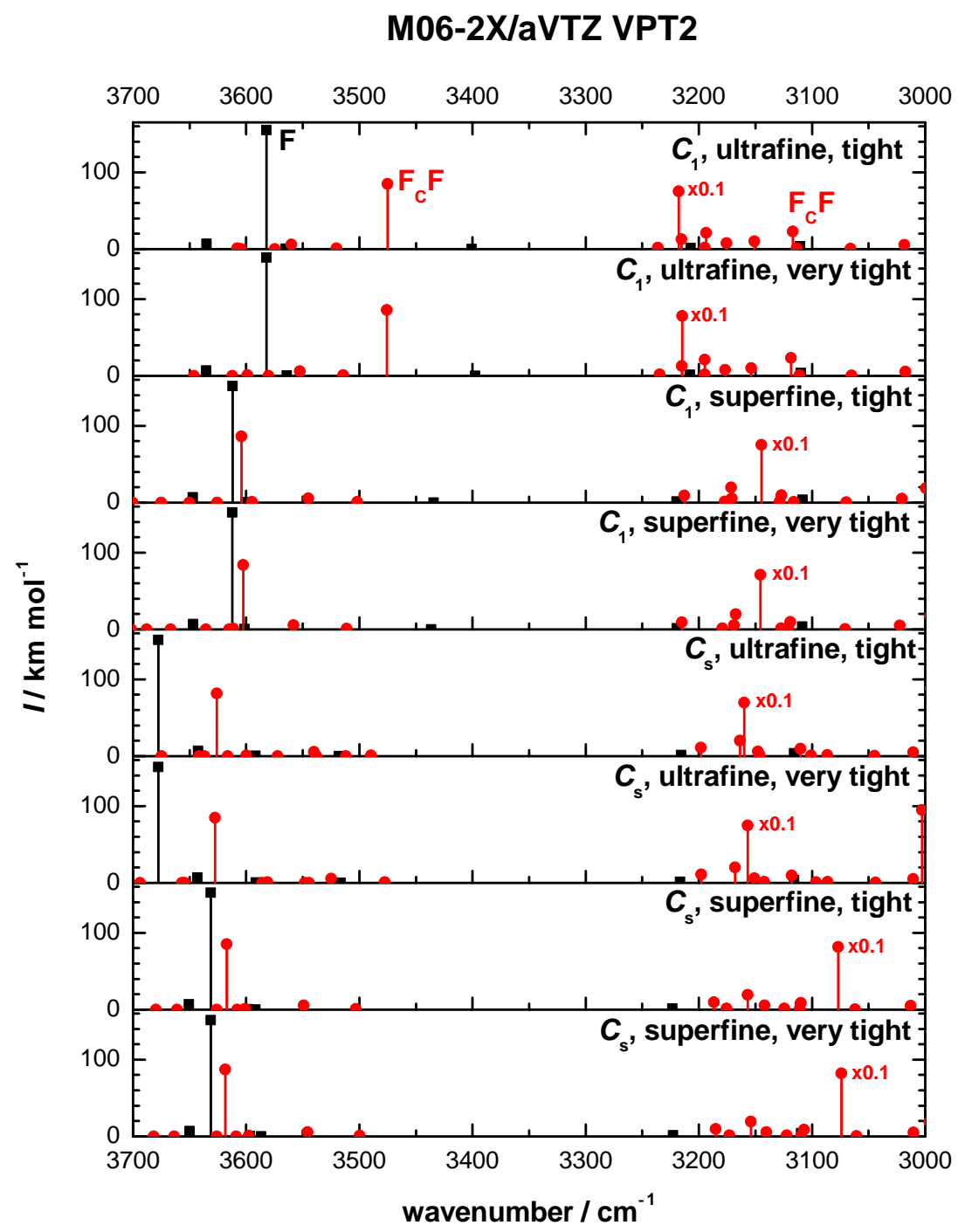

Figure A.12: Anharmonic (VPT2) band positions of the formic acid monomer (black squares) and metastable dimer $\mathrm{F}_{\mathrm{C}} \mathrm{F}$ calculated at the M06-2X/aVTZ level. The optimisation criteria (tight and very tight), the DFT integration grid (ultrafine and superfine), as well as symmetry $\left(C_{1}\right.$ and $\left.C_{\mathrm{s}}\right)$ have been varied. Adapted from Ref. [409] with permission from the PCCP Owner Societies. 
A Appendix

\section{B3LYP-D3(BJ)/aVTZ VPT2}

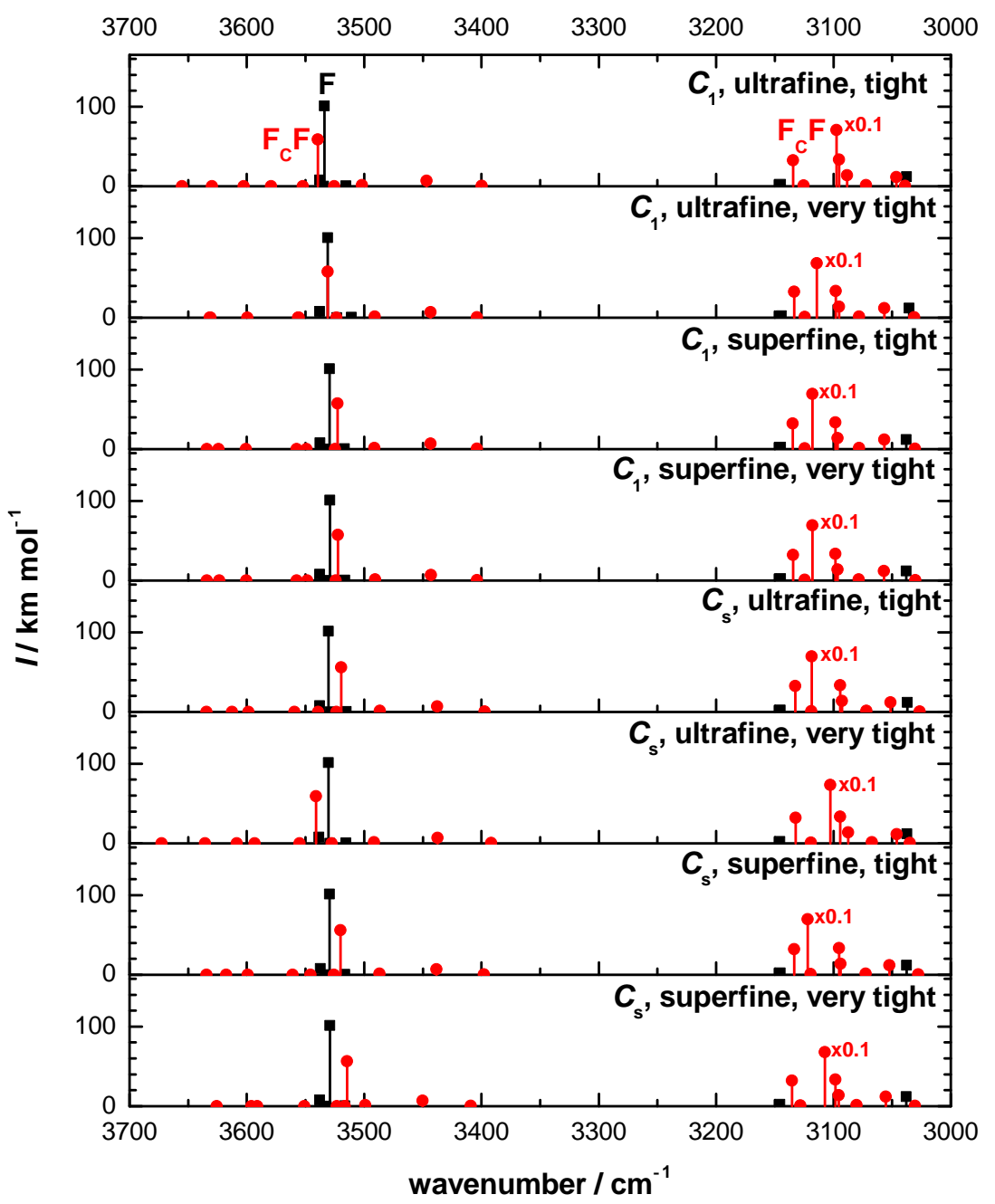

Figure A.13: Anharmonic (VPT2) band positions of the formic acid monomer (black squares) and metastable dimer $\mathrm{F}_{\mathrm{C}} \mathrm{F}$ calculated at the B3LYP-D3(BJ)/aVTZ level. The optimisation criteria (tight and very tight), the DFT integration grid (ultrafine and superfine), as well as symmetry $\left(C_{1}\right.$ and $\left.C_{\mathrm{s}}\right)$ have been varied. Adapted from Ref. [409] with permission from the PCCP Owner Societies. 


\section{B2PLYP-D3(BJ)/aVTZ VPT2}

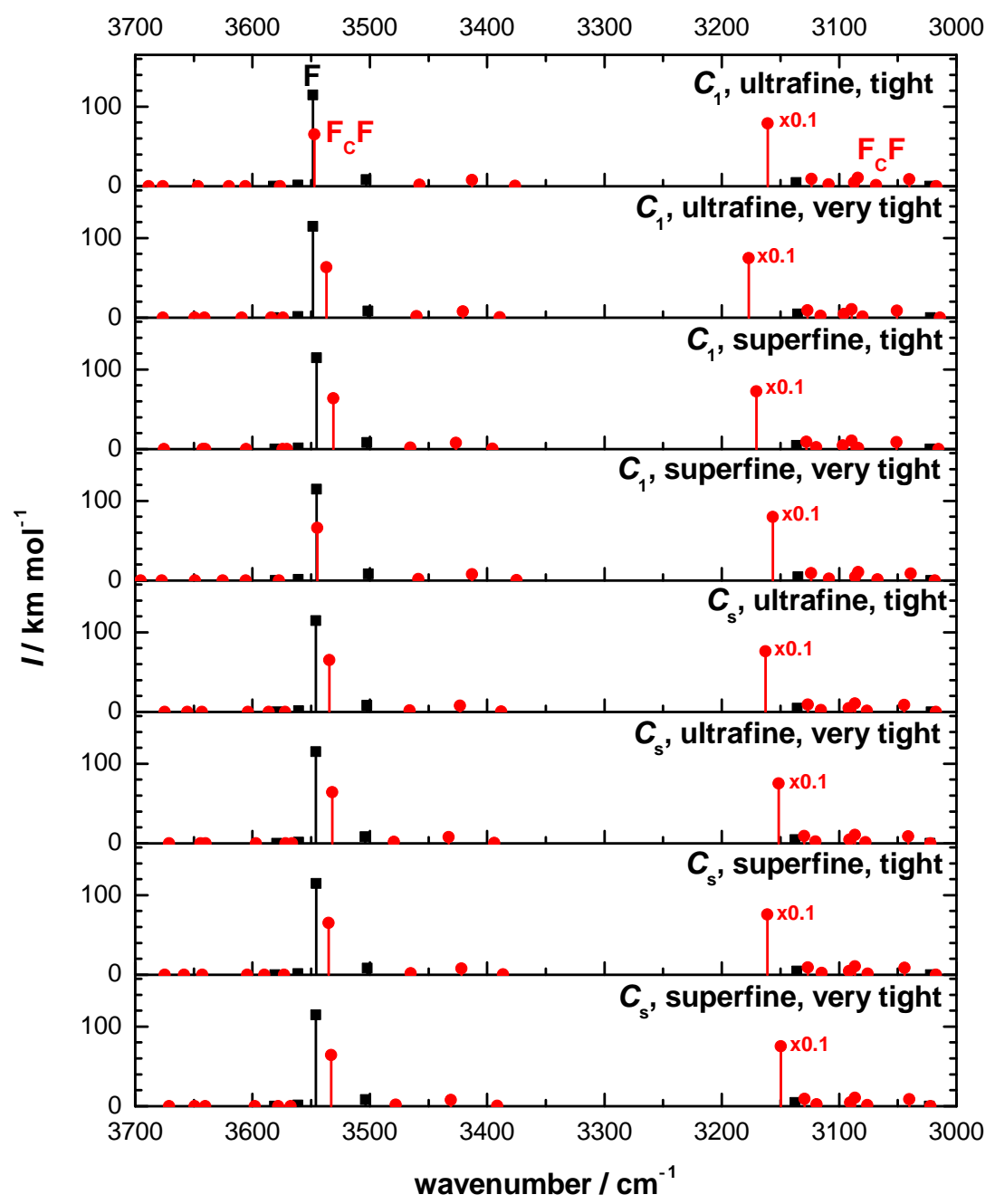

Figure A.14: Anharmonic (VPT2) band positions of the formic acid monomer (black squares) and metastable dimer $\mathrm{F}_{\mathrm{C}} \mathrm{F}$ calculated at the B2PLYP-D3(BJ)/aVTZ level. The optimisation criteria (tight and very tight), the DFT integration grid (ultrafine and superfine), as well as symmetry $\left(C_{1}\right.$ and $\left.C_{\mathrm{S}}\right)$ have been varied. Adapted from Ref. [409] with permission from the PCCP Owner Societies. 
A Appendix

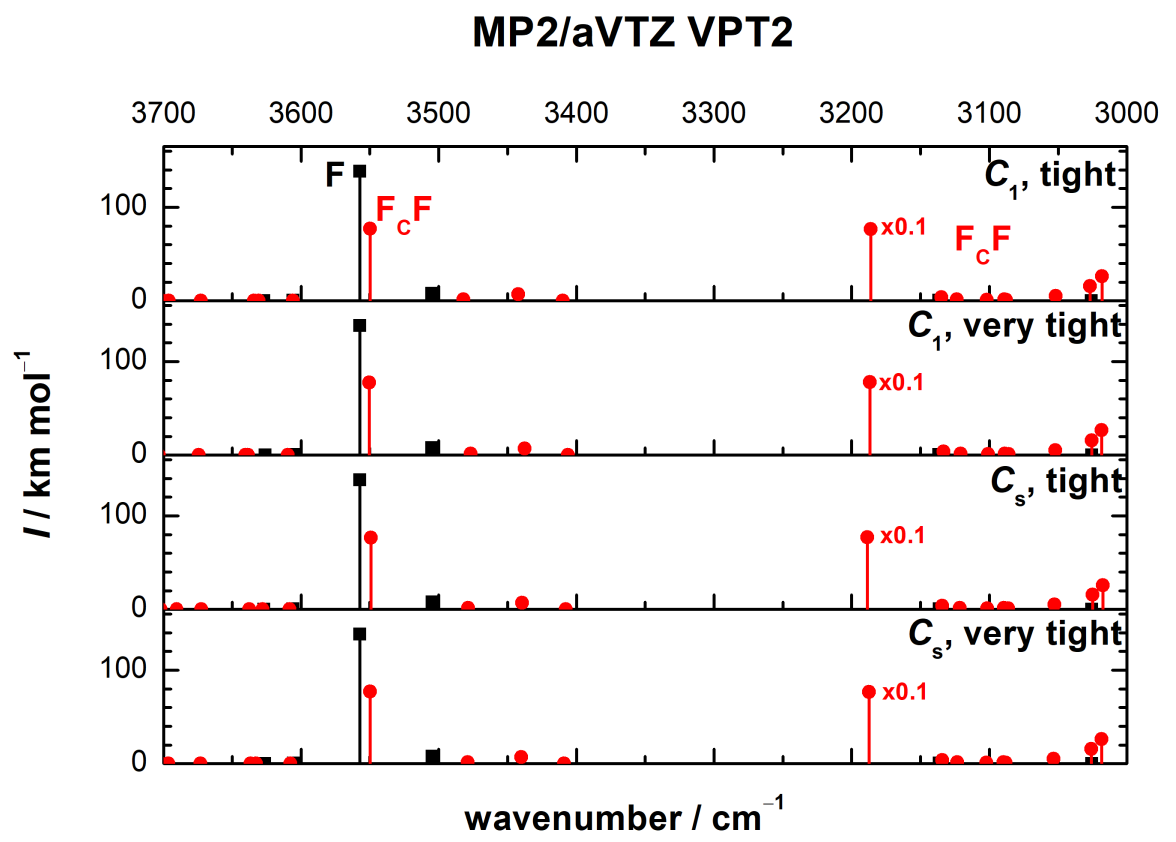

Figure A.15: Anharmonic (VPT2) band positions of the formic acid monomer (black squares) and metastable dimer $\mathrm{F}_{\mathrm{C}} \mathrm{F}$ calculated at the MP2/aVTZ level. The optimisation criteria (tight and very tight) and symmetry $\left(C_{1}\right.$ and $\left.C_{\mathrm{s}}\right)$ have been varied. Adapted from Ref. [409] with permission from the PCCP Owner Societies. 


\section{A.3.2 Combination Vibrations of the $F_{C} F$ dimer}

Table A.8: Anharmonically (VPT2) calculated and F( $\left.\nu_{1}\right)$-scaled band positions (in $\mathrm{cm}^{-1}$ ) of combination vibrations and the corresponding fundamentals of the $\mathrm{F}_{\mathrm{C}} \mathrm{F}$ dimer. The fundamentals are labelled in Herzberg nomenclature [182]. The calculations have been performed at the B3LYP-D3(BJ)/aVTZ level. Adapted from Ref. [409] with permission from the PCCP Owner Societies.

\begin{tabular}{cc|cc}
\hline \hline$\nu_{i}+\nu_{j}$ & & $\nu_{i}$ & \\
\hline$\nu_{5}+\nu_{7}$ & $3169 \pm 1$ & $\nu_{5}$ & $1771 \pm 3$ \\
$\nu_{5}+\nu_{9}$ & $3131 \pm 2$ & $\nu_{6}$ & $1726 \pm 3$ \\
$\nu_{6}+\nu_{7}$ & $3128 \pm 4$ & $\nu_{7}$ & $1404 \pm 3$ \\
$\nu_{6}+\nu_{9}$ & $3087 \pm 5$ & $\nu_{9}$ & $1357 \pm 5$ \\
\hline \hline
\end{tabular}
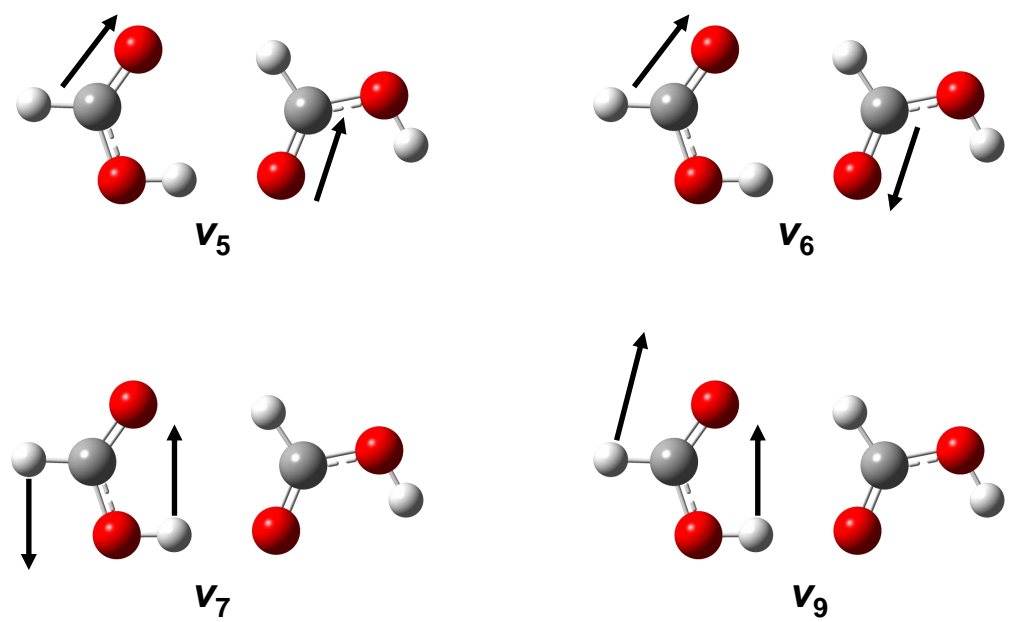

Figure A.16: $\mathrm{C}=\mathrm{O}$ stretching $\left(\nu_{5}\right.$ and $\left.\nu_{6}\right)$ and $\mathrm{C}-\mathrm{H}$ in-plane bending vibrations $\left(\nu_{7}\right.$ and $\left.\nu_{9}\right)$ of the higher-energy $\mathrm{F}_{\mathrm{C}} \mathrm{F}$ dimer of formic acid. Reproduced from Ref. [409] with permission from the PCCP Owner Societies. 


\section{A.3.3 Coupling Diagrams of the Homo and Hetero Dimers}
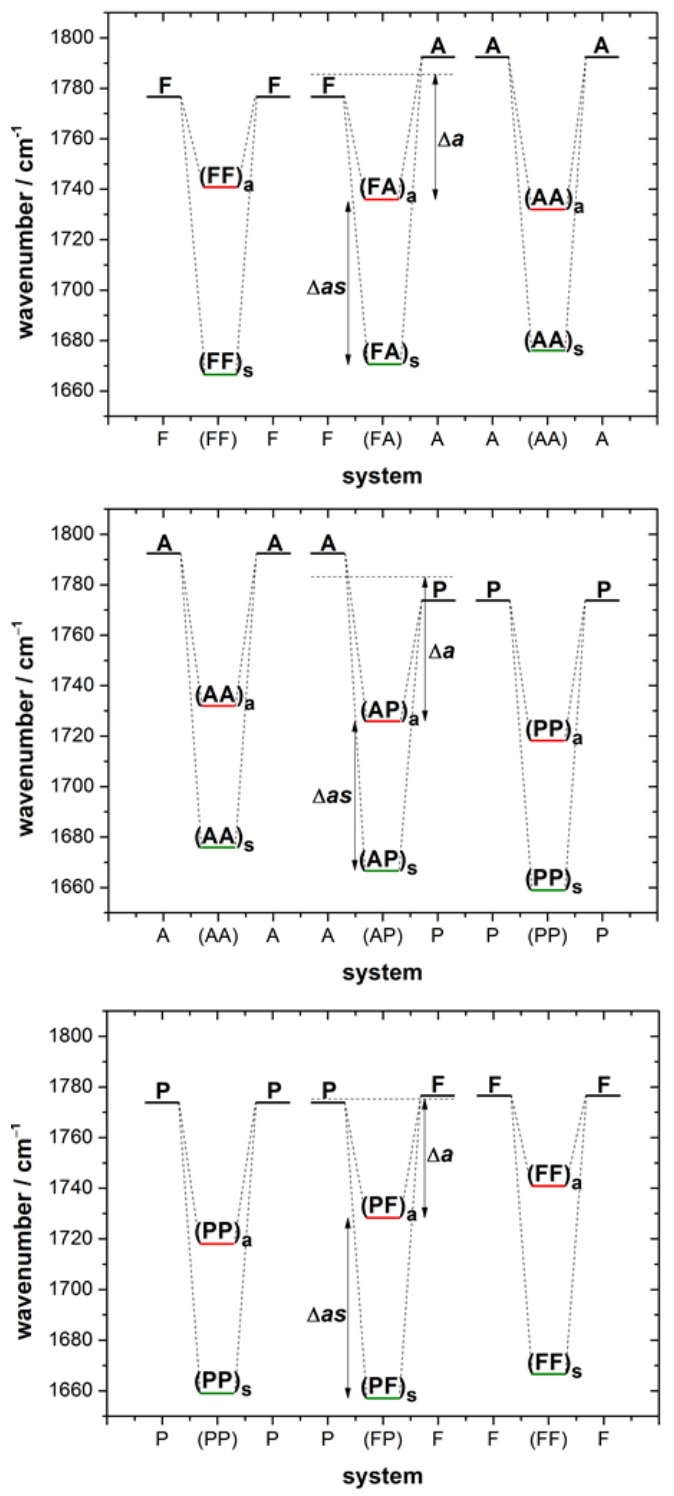

Figure A.17: Exciton coupling diagrams of the symmetric and antisymmetric $\mathrm{C}=\mathrm{O}$ stretching vibration of all six homo and hetero dimers of formic, acetic, and pivalic acid. Adapted from J. Chem. Phys. 149, 104307 (2018) (Ref. [329]), with the permission of AIP Publishing.

\section{A.3.4 Anharmonic Frequency Calculations of the Formic Acid Dimer}

The formic acid dimer forms a planar eight-membered ring of $C_{2 \mathrm{~h}}$ point group symmetry. The vibrations of the formic acid dimer can be divided into four symmetry groups $A_{\mathrm{g}}, B_{\mathrm{g}}, A_{\mathrm{u}}$, and $B_{\mathrm{u}}$. The symmetric (gerade) vibrations are Raman active, and the 
antisymmetric (ungerade) IR active. The labelling of the vibrations is done according to the Herzberg nomenclature with one difference - the order of the $A_{\mathrm{u}}$ and $B_{\mathrm{g}}$ vibrations is switched, so that the Raman active vibrations are $\nu_{1}-\nu_{12}$ and accordingly, the IR active vibrations $\nu_{13}-\nu_{24}$.

Harmonically as well as anharmonically (VPT2) calculated band positions at the B3LYP-D3(BJ), B2PLYP-D3(BJ), and the MP2 level of all fundamentals are listed in Table A.9. As in Chapter 4.4, the DFT integration grid size (ultrafine or superfine) and the symmetry $\left(C_{1}\right.$ or $\left.C_{2 \mathrm{~h}}\right)$ have been varied. In addition, the optimisation criteria have been enhanced from tight to very tight. In Table A.9, the averages over the resulting eight calculations (four in case of MP2) are summarised. The harmonic frequency calculations show nearly no symmetry, DFT integration grid size, or optimisation dependencies with three exceptions for B3LYP-D3(BJ), where the averaged band position of $\nu_{4}, \nu_{11}$, and $\nu_{17}$ has a standard deviation $\sigma$ of $\pm 1 \mathrm{~cm}^{-1}$. For the anharmonic B3LYP-D3(BJ) calculations, the deviations are obviously larger. Seven of the twenty-four vibrations have standard deviations above $5 \mathrm{~cm}^{-1}$. Expectedly, the largest $\sigma$ values can be seen for the two $\mathrm{O}-\mathrm{H}$ stretching vibrations $\nu_{1}\left( \pm 17 \mathrm{~cm}^{-1}\right)$ and $\nu_{17}\left( \pm 59 \mathrm{~cm}^{-1}\right)$, which show strong Fermi resonances. Other modes include the two $\mathrm{O}-\mathrm{H}$ out-of-plane bending vibrations $\nu_{11}$ and $\nu_{14}$, the symmetric $\mathrm{C}=\mathrm{O}$ stretching vibration $\nu_{3}$, as well as two inter-monomer modes, namely the stretching vibration $\nu_{8}$ and the lowest frequency vibration (twisting mode) $\nu_{16}$. The latter has the largest relative error of $20 \%$ and is the only vibration where the sign of the total anharmonicity changes. However, it is not surprising, as the lowest frequency inter-monomer modes are known to be poorly described by VPT2, whereas for the other modes, the high sensitivity towards the settings is mainly caused by Fermi resonances. In case of MP2, this poor description of the lowest frequency vibration $\nu_{17}$ is also seen. On average, the sign of the anharmonicity is positive and the standard deviation is largest with $\pm 4 \mathrm{~cm}^{-1}$. As expected, setting sensitivity is far less pronounced for MP2. Only five vibrations have a $\sigma$ larger than one, three of which are the intermonomer modes $\left(\nu_{8}, \nu_{15}\right.$, and $\left.\nu_{16}\right)$. The remaining two are the two $\mathrm{O}-\mathrm{H}$ out-of-plane bending vibrations $\nu_{11}$ and $\nu_{14}$.

According calculations for the formic acid monomer can be found in Table A.10. As detailed in Chapter 4.4, B3LYP-D3(BJ) is most sensitive towards the settings, however, the resulting deviations are still moderate and smaller than for the dimer. The only standard deviation above $5 \mathrm{~cm}^{-1}$ is seen for $\nu_{5}$ with $35 \mathrm{~cm}^{-1}$. This is due to the strong Fermi resonance of that vibration with the overtone of the $\mathrm{O}-\mathrm{H}$ out-of-plane bending vibration $2 \nu_{9}$ (cf. also Chapter 4.2). The predicted energetic order varies for the settings, which causes these differences. If both band positions are plotted for the different settings (see Figure A.18), the agreement is on the same order as for the other vibrations. Again, the overall smallest deviations with regard to the settings is seen for the MP2 calculations, but the overall setting sensitivity is reasonable compared to the formic acid dimer. 
Table A.9: Harmonically and anharmonically (VPT2) calculated fundamentals (in $\mathrm{cm}^{-1}$ ) of the formic acid dimer at the B3LYP-D3(BJ)/aVTZ, B2PLYP-D3(BJ)/aVTZ, and at the MP2/aVTZ level. For all methods, the optimisation criteria (tight and very tight) and the symmetry $\left(C_{1}\right.$ and $\left.C_{2 \mathrm{~h}}\right)$ were varied. In case of B3LYP-D3(BJ) and B2PLYP-D3(BJ), the integration grid size (ultrafine and superfine) was also altered. The averaged results for all fundamentals are listed with their standard deviations $\sigma$, if $\sigma \geq 0.1 \mathrm{~cm}^{-1}$. All band positions with standard deviations above $5 \mathrm{~cm}^{-1}$ are printed in bold font.

\begin{tabular}{|c|c|c|c|c|c|c|c|}
\hline \multirow{2}{*}{ symmetry } & \multirow{2}{*}{$\nu_{i}$} & \multicolumn{2}{|c|}{ B3LYP-D3(BJ) } & \multicolumn{2}{|c|}{ B2PLYP-D3(BJ) } & \multicolumn{2}{|c|}{ MP2 } \\
\hline & & harm & anharm & harm & anharm & harm & anharm \\
\hline \multirow{9}{*}{$A_{\mathrm{g}}$} & 1 & $3001 \pm 1$ & $2609 \pm 17$ & 3107 & $2933 \pm 1$ & 3109 & $2763 \pm 1$ \\
\hline & 2 & 3065 & $2903 \pm 1$ & 3077 & $2685 \pm 13$ & 3139 & $2963 \pm 1$ \\
\hline & 3 & 1688 & $1629 \pm 9$ & 1689 & $1641 \pm 8$ & 1703 & $1660 \pm 1$ \\
\hline & 4 & $1485 \pm 1$ & $1433 \pm 3$ & 1483 & $1433 \pm 4$ & 1483 & $1430 \pm 1$ \\
\hline & 5 & 1405 & $1372 \pm 2$ & 1409 & $1375 \pm 2$ & 1408 & $1373 \pm 1$ \\
\hline & 6 & 1262 & $1237 \pm 2$ & 1256 & $1234 \pm 6$ & 1262 & $1232 \pm 1$ \\
\hline & 7 & 691 & $685 \pm 2$ & 687 & $682 \pm 2$ & 685 & $679 \pm 1$ \\
\hline & 8 & 221 & $211 \pm 7$ & 216 & $203 \pm 2$ & 213 & $198 \pm 2$ \\
\hline & 9 & 178 & $162 \pm 5$ & 173 & $162 \pm 3$ & 169 & $161 \pm 1$ \\
\hline \multirow{3}{*}{$B_{\mathrm{g}}$} & 10 & 1080 & $1056 \pm 2$ & 1084 & $1059 \pm 2$ & 1089 & $1063 \pm 1$ \\
\hline & 11 & $992 \pm 1$ & $957 \pm 11$ & 982 & $941 \pm 12$ & 980 & $932 \pm 3$ \\
\hline & 12 & 261 & $251 \pm 3$ & 258 & $248 \pm 3$ & 258 & $246 \pm 1$ \\
\hline \multirow{4}{*}{$A_{\mathrm{u}}$} & 13 & 1106 & $1072 \pm 3$ & 1108 & $1073 \pm 2$ & 1117 & $1078 \pm 1$ \\
\hline & 14 & 1009 & $986 \pm 7$ & 1001 & $973 \pm 9$ & 998 & $964 \pm 2$ \\
\hline & 15 & 185 & $183 \pm 5$ & 182 & $178 \pm 4$ & 179 & $173 \pm 2$ \\
\hline & 16 & 75 & $71 \pm 14$ & 73 & $76 \pm 5$ & 69 & $70 \pm 4$ \\
\hline \multirow{8}{*}{$B_{\mathrm{u}}$} & 17 & $3132 \pm 1$ & $2787 \pm 59$ & 3198 & $2892 \pm 13$ & 3230 & 2962 \\
\hline & 18 & 3051 & $2874 \pm 2$ & 3096 & $2925 \pm 2$ & 3129 & $2963 \pm 1$ \\
\hline & 19 & 1766 & $1724 \pm 4$ & 1759 & $1720 \pm 4$ & 1770 & $1734 \pm 1$ \\
\hline & 20 & 1454 & $1408 \pm 3$ & 1456 & $1410 \pm 4$ & 1456 & $1407 \pm 1$ \\
\hline & 21 & 1404 & $1372 \pm 2$ & 1405 & $1372 \pm 3$ & 1404 & $1369 \pm 1$ \\
\hline & 22 & 1265 & $1239 \pm 4$ & 1260 & $1238 \pm 12$ & 1266 & $1242 \pm 1$ \\
\hline & 23 & 726 & $721 \pm 2$ & 719 & $713 \pm 2$ & 715 & 708 \\
\hline & 24 & 294 & $288 \pm 4$ & 286 & $279 \pm 4$ & 283 & $274 \pm 1$ \\
\hline
\end{tabular}


Table A.10: Harmonically and anharmonically (VPT2) calculated fundamentals (in $\mathrm{cm}^{-1}$ ) of the formic acid monomer at the B3LYP-D3(BJ)/aVTZ, B2PLYP-D3(BJ)/aVTZ, and the MP2/aVTZ level. For all methods, the optimisation criteria (tight and very tight) and the symmetry $\left(C_{1}\right.$ and $\left.C_{2 \mathrm{~h}}\right)$ were varied. In case of B3LYP-D3(BJ) and B2PLYP-D3(BJ), the integration grid size (ultrafine and superfine) was also altered. The averaged results for all fundamentals are listed with their standard deviations $\sigma$. Standard deviations below $0.1 \mathrm{~cm}^{-1}$ are not listed.

\begin{tabular}{|c|c|c|c|c|c|c|c|}
\hline \multirow{2}{*}{ symmetry } & \multirow{2}{*}{$\nu_{i}$} & \multicolumn{2}{|c|}{$\overline{\text { B3LYP-D3(BJ) }}$} & \multicolumn{2}{|c|}{ "B2PLYP-D3(BJ) } & \multicolumn{2}{|c|}{ MP2 } \\
\hline & & harm & anharm & harm & anharm & harm & anharm \\
\hline \multirow{7}{*}{$A^{\prime}$} & 1 & $3716 \pm 1$ & $3530 \pm 2$ & $3733 \pm 1$ & $3546 \pm 2$ & 3741 & 3557 \\
\hline & 2 & 3049 & $2893 \pm 1$ & 3124 & $2934 \pm 1$ & 3124 & 2969 \\
\hline & 3 & 1811 & $1778 \pm 1$ & 1795 & $1761 \pm 1$ & 1794 & 1761 \\
\hline & 4 & 1402 & $1374 \pm 1$ & 1408 & $1379 \pm 1$ & 1409 & $1381 \pm 1$ \\
\hline & 5 & 1299 & $1245 \pm 35$ & 1303 & $1221 \pm 2$ & 1302 & $1222 \pm 1$ \\
\hline & 6 & 1122 & $1089 \pm 1$ & 1125 & $1091 \pm 1$ & 1131 & $1097 \pm 1$ \\
\hline & 7 & 629 & $622 \pm 2$ & 628 & $621 \pm 1$ & 626 & $619 \pm 1$ \\
\hline \multirow{2}{*}{$A^{\prime \prime}$} & 8 & 1052 & $1031 \pm 1$ & 1056 & $1035 \pm 1$ & 1059 & $1037 \pm 1$ \\
\hline & 9 & 675 & $641 \pm 3$ & 676 & $644 \pm 1$ & 675 & $643 \pm 1$ \\
\hline
\end{tabular}




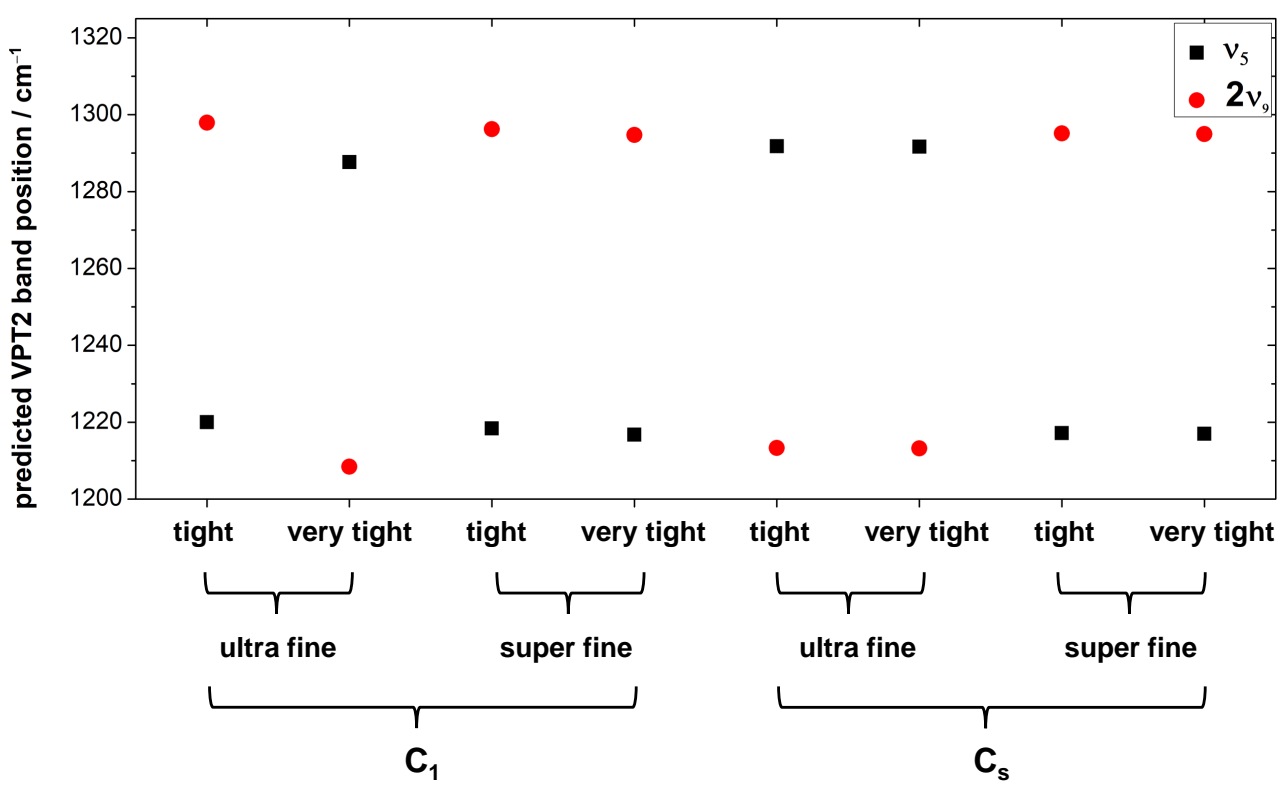

Figure A.18: Anharmonically (VPT2) calculated band positions (in $\mathrm{cm}^{-1}$ ) of the $\mathrm{O}-\mathrm{H}$ inplane bending vibration $\nu_{5}$ and the overtone of the $\mathrm{O}-\mathrm{H}$ out-of-plane bending vibration $2 \nu_{9}$ of the formic acid monomer at the B3LYP-D3(BJ)/aVTZ level. The symmetry $\left(C_{s}\right.$ or $\left.C_{1}\right)$, the DFT integration grid (ultrafine or superfine), and the optimisation criteria (tight or very tight) were varied.

Table A.11: Harmonically (harm), harmonically and ( $\mathrm{FF})_{\mathrm{a}}$-scaled (sc harm), and anharmonically (anharm, VPT2) calculated shifts $(\Delta a)$ and exciton splittings $(\Delta a s)$ at the B3LYPD3(BJ), B2PLYP-D3(BJ), and MP2 level. For all methods, an augmented triple-zeta basis set (aVTZ) has been used.

\begin{tabular}{ccccccccc}
\hline \hline & \multicolumn{2}{c}{ B3LYP-D3(BJ) } & & \multicolumn{2}{c}{ B2PLYP-D3(BJ) } & & \multicolumn{2}{c}{ MP2 } \\
\cline { 2 - 3 } \cline { 8 - 9 } \cline { 8 - 9 } & $\Delta a$ & $\Delta a s$ & & $\Delta a$ & $\Delta a s$ & & $\Delta a$ & $\Delta a s$ \\
\hline harm & 45 & 78 & & 36 & 70 & & 24 & 67 \\
sc harm & 44 & 77 & & 35 & 69 & & 23 & 67 \\
anharm & $54 \pm 5$ & $95 \pm 10$ & & $41 \pm 5$ & $79 \pm 9$ & & $27 \pm 1$ & $74 \pm 2$ \\
\hline \hline
\end{tabular}




\section{A.4 Carboxylic Acid Trimers}

\section{A.4.1 Formic Acid Trimers}

Table A.12: Experimental (Ref. [218]) and harmonically calculated (B3LYP-D3(BJ)/def2TZVP) rotational constants (in $\mathrm{MHz}$ ) of the formic acid trimer. The relative deviations (in \%) are given in the third column.

\begin{tabular}{cccc}
\hline \hline rotational constant & experiment $^{[218]}$ & B3LYP-D3(BJ) & relative deviation \\
\hline$A$ & $2936.5116(4)$ & 2994.1892 & +2.0 \\
$B$ & $595.07077(7)$ & 598.4994 & +0.6 \\
$C$ & $495.25989(6)$ & 498.7967 & +0.7 \\
\hline \hline
\end{tabular}

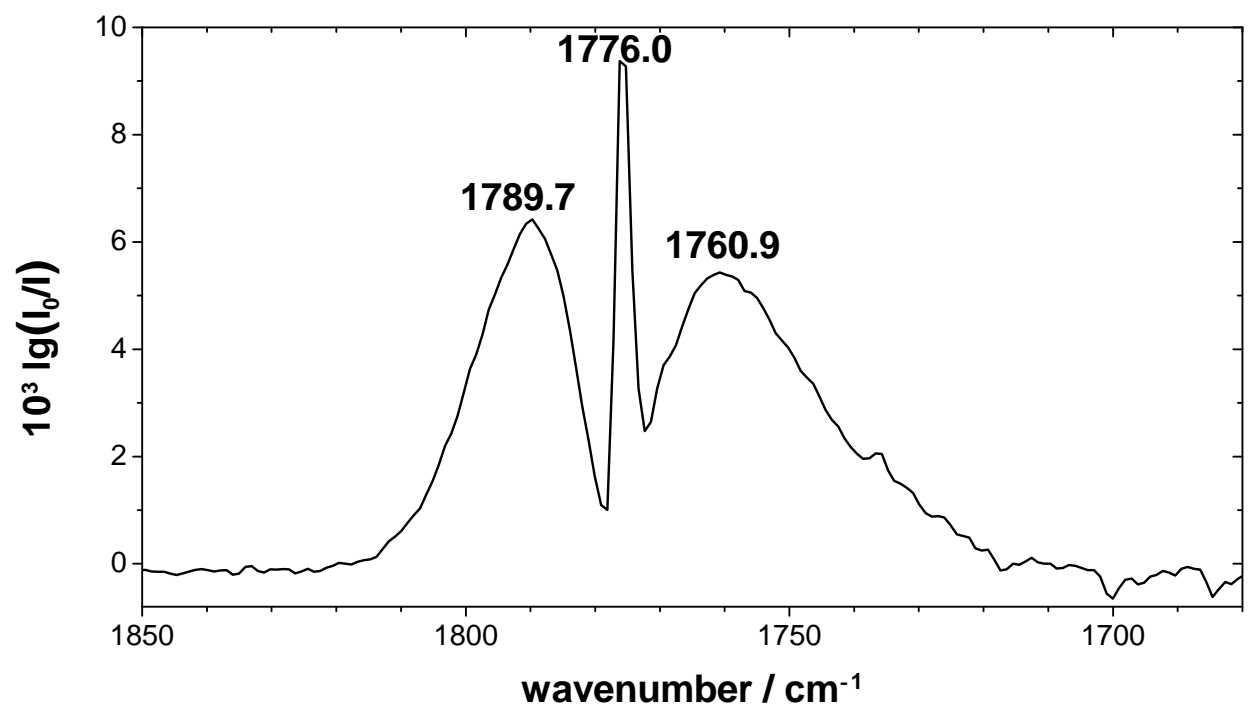

Figure A.19: Gas phase spectrum of the $\mathrm{C}=\mathrm{O}$ stretching vibration of the formic acid monomer. The spectrum has been recorded with the chilli jet set-up. The number of scans amounts to 100. The band positions of the maxima of the P, Q, and R branches are listed. 


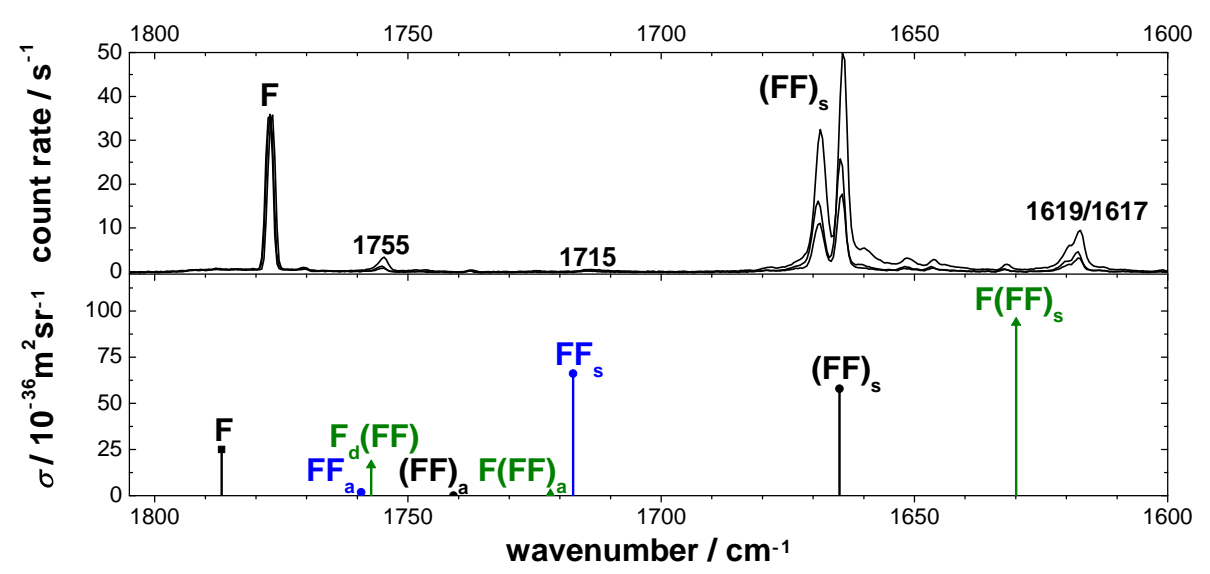

Figure A.20: Raman jet spectra of formic acid in helium in the $\mathrm{C}=\mathrm{O}$ stretching region at a reservoir pressure of 700 mbar. The concentrations increase from bottom to top from $<0.1-$ $0.3 \%$. The acquisition times amount to $30-45$ minutes. The spectra have been intensity-scaled to the monomer band of lowest intensity (spectrum of highest cluster concentration). Below the Raman spectra, harmonically calculated and $(\mathrm{FF})_{\mathrm{a}}$-scaled band position (scaling factor 0.983 ) of the formic acid monomer F, cyclic (FF) and polar dimer FF, and the formic acid trimer $\mathrm{F}(\mathrm{FF})$ are shown. The calculations have been performed at the B3LYP-D3(BJ)/def2-TZVP level.

\section{A.4.2 Acetic Acid Higher-Energy Dimers and Trimers}
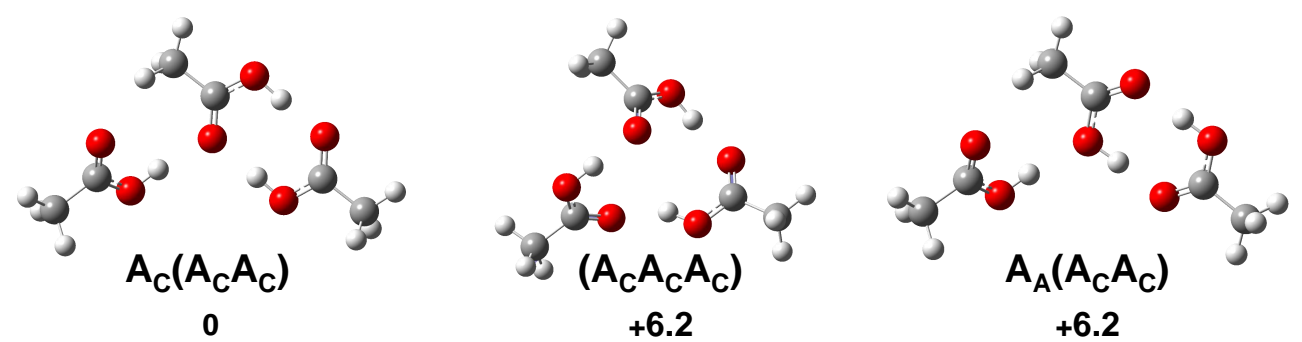

Figure A.21: Global and local minimum structures of the acetic acid trimer, calculated at the B3LYP-D3(BJ)/def2-TZVP level. The harmonically calculated, zero-point corrected relative energy differences with respect to the global minimum are listed in $\mathrm{kJ} \mathrm{mol}^{-1}$.

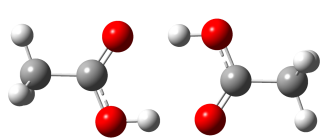

$\left(A_{c} A_{c}\right)$

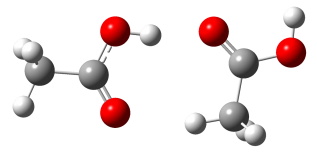

$A_{C} A$

34.2

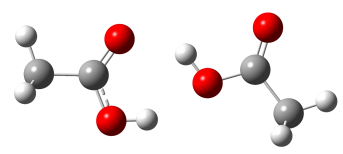

$\left(A_{A} A_{C}\right)$

40.0

Figure A.22: Global and local minimum structures of the acetic acid dimer, calculated at the B3LYP-D3(BJ)/def2-TZVP level. The harmonically calculated, zero-point corrected relative energy differences with respect to the global minimum are listed in $\mathrm{kJ} \mathrm{mol}^{-1}$. 


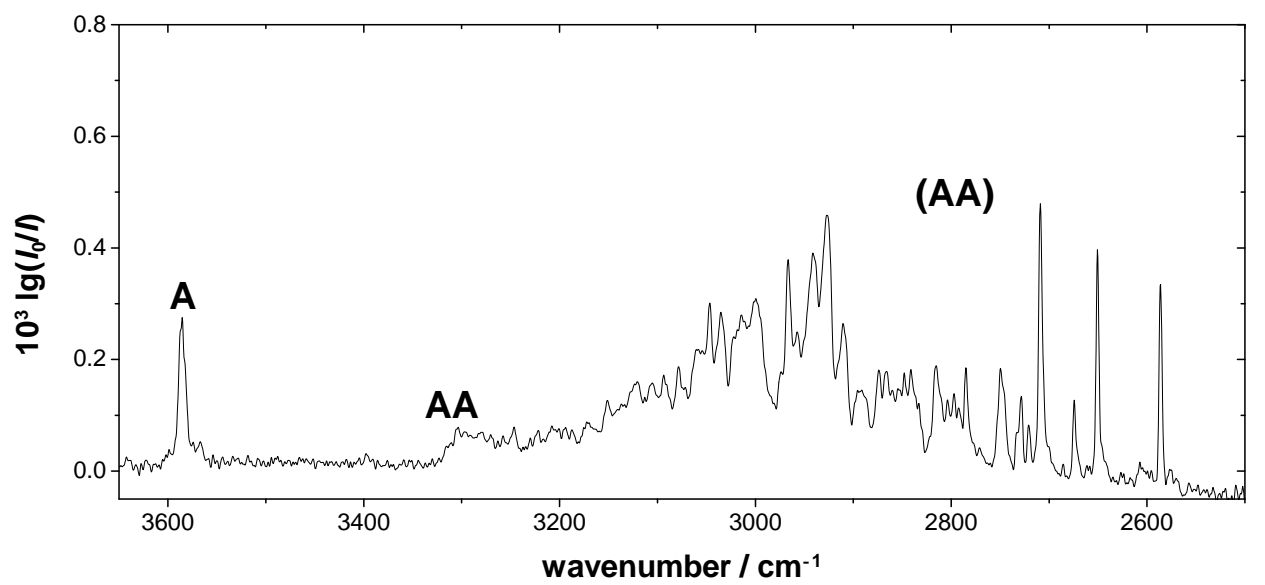

Figure A.23: FTIR spectrum of acetic acid in helium $(c<0.06 \%)$ recorded at a reservoir pressure of 560 mbar and 50 scans in the $\mathrm{OH}$ stretching region.

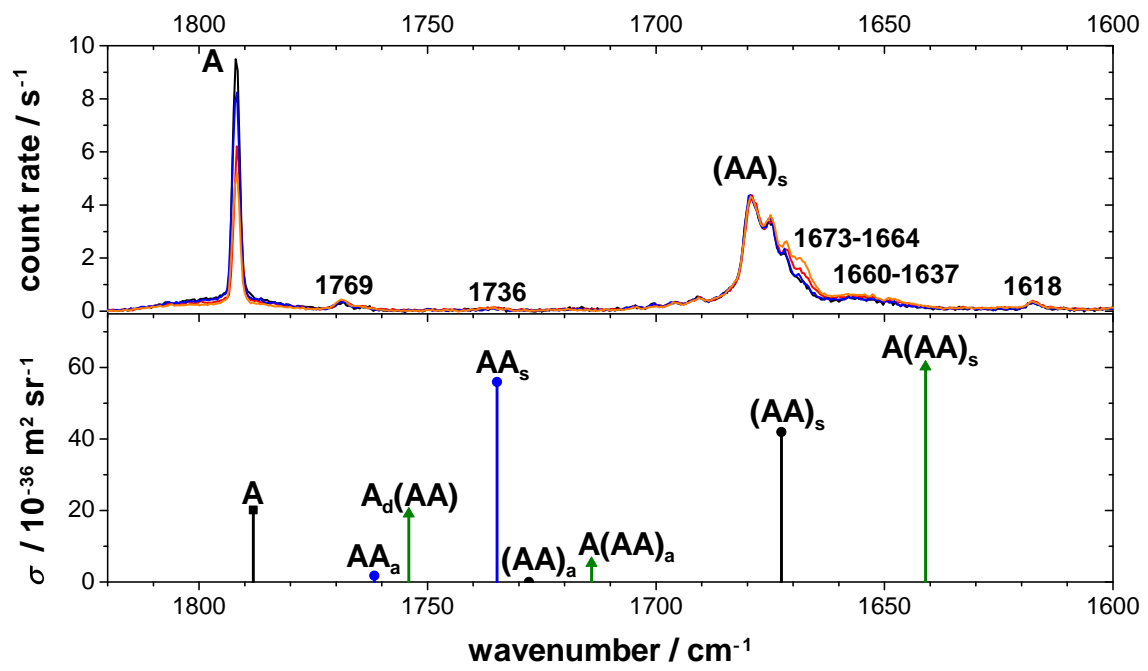

Figure A.24: Raman jet spectra of acetic acid in helium in the $\mathrm{C}=\mathrm{O}$ stretching region at reservoir pressures of 500-1000 mbar, which were previously shown as part of Figure 6.10. The concentrations increase from $<0.05-0.12 \%$. The acquisition times amount to 30-35 minutes. The spectra have been intensity-scaled to the dimer band in the spectrum with the lowest concentration. The scaling factors amount to 1.0 (black spectrum), 0.87 (blue), 0.65 (red), and 0.55 (orange). Below Raman spectra, harmonically calculated and $(\mathrm{FF})_{\mathrm{a}}$-scaled band positions (scaling factor 0.983 ) of the acetic acid monomer A, cyclic (AA) and polar dimer AA, and the acetic acid trimer $\mathrm{A}(\mathrm{AA})$ are shown. The calculations have been performed at the B3LYPD3(BJ)/def2-TZVP level. 


\section{A.4.3 Pivalic Acid Higher-Energy Dimers}

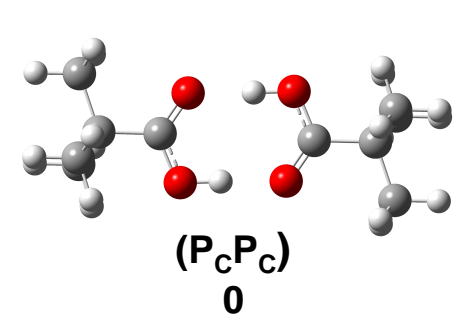

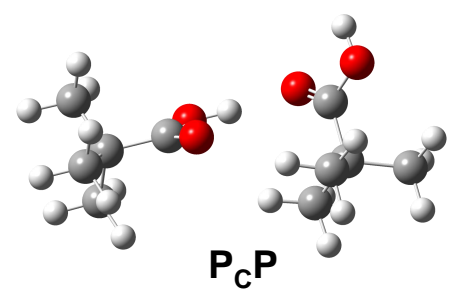

36.3

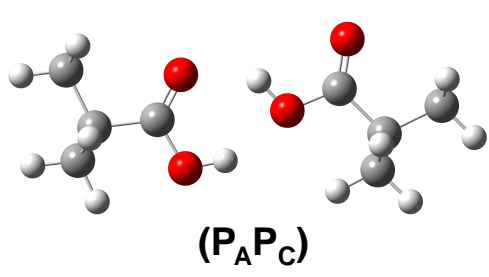

40.1

Figure A.25: Global and local minimum structures of the pivalic acid dimer calculated at the B3LYP-D3(BJ)/def2-TZVP level. The harmonic, zero-point corrected relative energy difference with respect to the global minimum $\left(\mathrm{P}_{\mathrm{C}} \mathrm{P}_{\mathrm{C}}\right)$ are listed below the structures.

Table A.13: (FF) $\mathrm{a}^{\text {-scaled }}$ (0.983) harmonic band positions (in $\mathrm{cm}^{-1}$ ) of global and local minimum structures of the pivalic acid dimer as well as their predicted IR intensities $I$ (in $\mathrm{km} \mathrm{mol}^{-1}$ ) and Raman scattering cross-sections (in $10^{-36} \mathrm{~m}^{2} \mathrm{sr}^{-1}$ ), calculated at the B3LYPD3(BJ)/def2-TZVP level.

\begin{tabular}{ccccccc}
\hline \hline dimer & $\tilde{\nu}$ & $I$ & $\sigma$ & $\tilde{\nu}$ & $I$ & $\sigma$ \\
\hline$\left(\mathrm{P}_{\mathrm{C}} \mathrm{P}_{\mathrm{C}}\right)$ & 1659 & 0 & 68 & 1710 & 765 & 0 \\
$\mathrm{P}_{\mathrm{C}} \mathrm{P}$ & 1727 & 64 & 73 & 1743 & 674 & 2 \\
$\left(\mathrm{P}_{\mathrm{A}} \mathrm{P}_{\mathrm{C}}\right)$ & 1776 & 205 & 15 & 1730 & 427 & 38 \\
\hline \hline
\end{tabular}

\section{A.4.4 Mixed Trimers}

To distinguish the six conformers depicted in Figure A.26, the two docking sides of a hetero dimer are implemented in the nomenclature by the sequence the hetero dimer is displayed. For example, the monomer of the $\mathrm{F}(\mathrm{PF})$ hetero trimer docks to the pivalic acid side and correspondingly, to the formic acid side in case of $\mathrm{F}(\mathrm{FP})$.

The harmonically predicted zero-point corrected dissociation energies (B3LYP-D3(BJ)/def2TZVP) of the hetero trimers are listed below the respective structures in A.26. The two most stable structures $\mathrm{F}(\mathrm{PP})$ and $\mathrm{F}(\mathrm{FP})$ are near-isoenergetic, followed by $\mathrm{P}(\mathrm{FP})$, which is $1 \mathrm{~kJ} \mathrm{~mol}^{-1}$ less stable. The dissociation energies of all other trimers are distinctly (at least $4 \mathrm{~kJ} \mathrm{~mol}^{-1}$ ) lower. A large part of the stability of $\mathrm{F}(\mathrm{PP})$ is a result of the large dissociation energy of the pivalic acid dimer with respect to (FF) and (FP) (cf. Table 5.4). The dissociation energy of the (FP) hetero dimer is smaller compared to (PP), yet the docking to the formic acid side of a dimer is preferred over the docking to pivalic acid. The energy difference amounts to $4-5 \mathrm{~kJ} \mathrm{~mol}^{-1}$ for the hetero trimers containing (FP). As the two effects are largely cancelling out, $\mathrm{F}(\mathrm{PP})$ and $\mathrm{F}(\mathrm{FP})$ are energetically similar. In summary, the three likeliest structures to be featured in a supersonic expansion are $\mathrm{F}(\mathrm{PP}), \mathrm{P}(\mathrm{FP})$, and $\mathrm{F}(\mathrm{FP})$. 

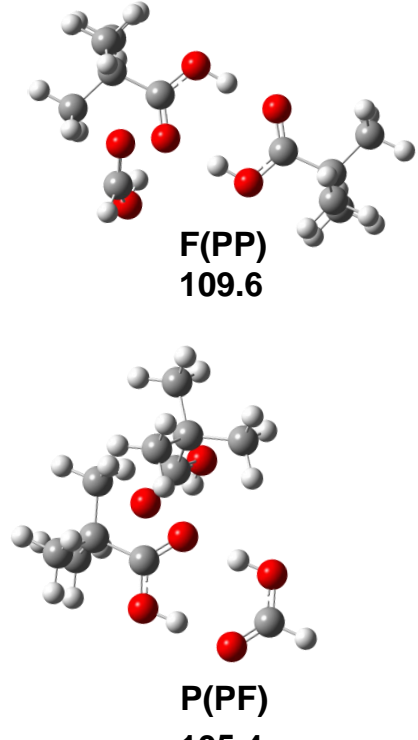

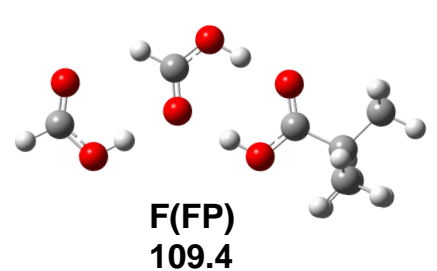

109.4

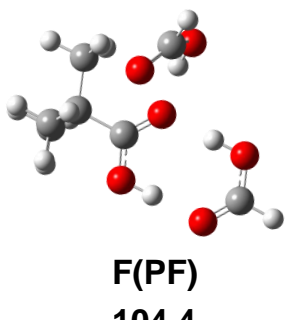

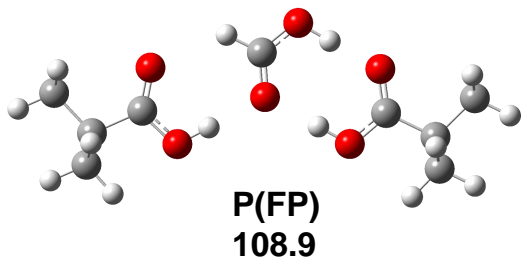

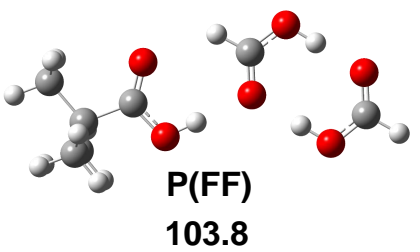

Figure A.26: Conformers of mixed formic and pivalic acid trimers, harmonically calculated at the B3LYP-D3(BJ)/def2-TZVP level. Below each structure, the zero-point corrected dissociation energy (in $\mathrm{kJ} \mathrm{mol}^{-1}$ ) into monomers is listed. 


\section{A.4.5 Performance of Quantum Chemical Methods}
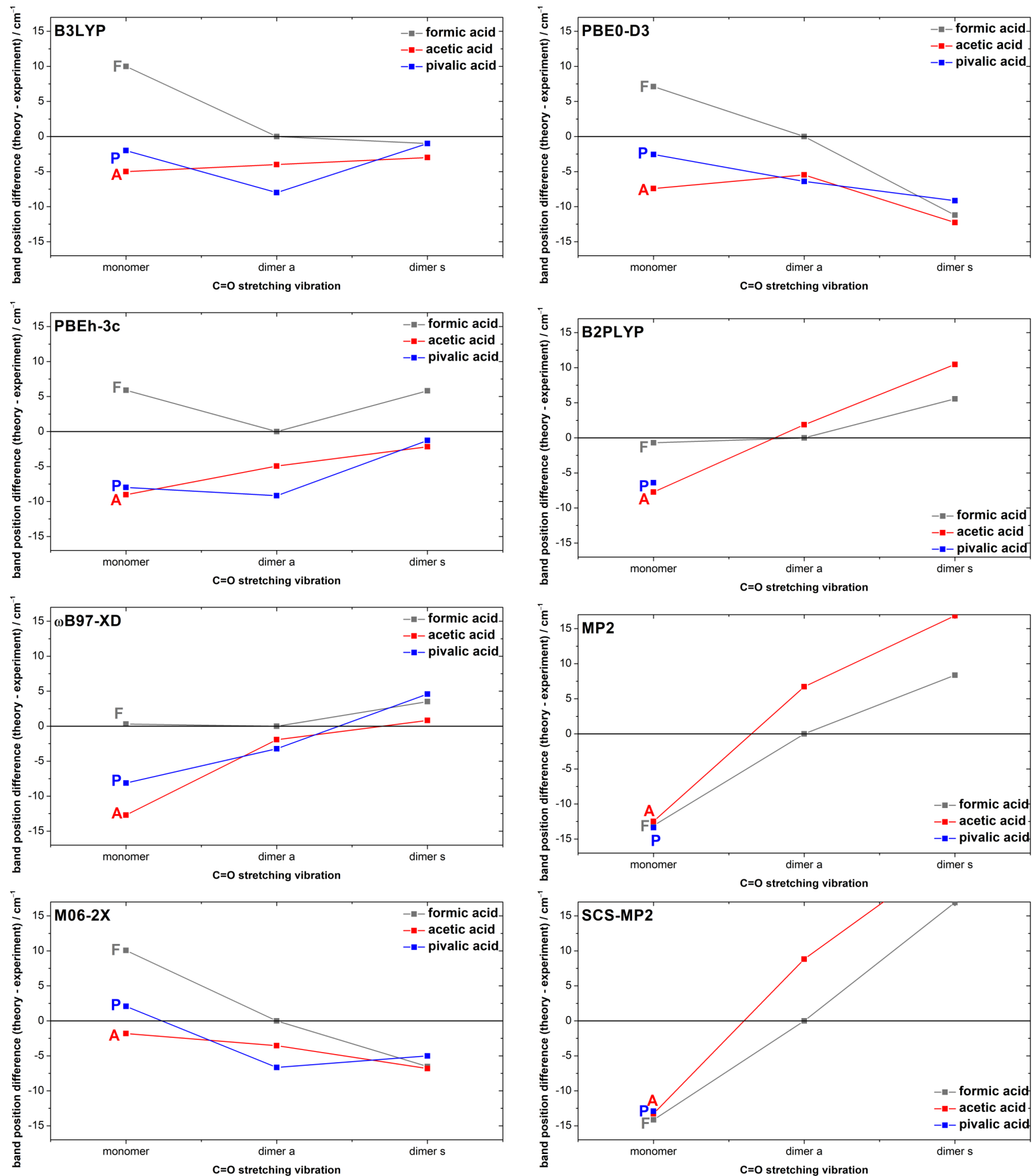

Figure A.27: Band position differences of $(\mathrm{FF})_{\mathrm{a}}$-scaled harmonic frequency calculations at the various levels of theory with respect to experiment for the $\mathrm{C}=\mathrm{O}$ stretching vibrations of carboxylic acid monomers and the antisymmetric (a) and symmetric ( $\mathrm{s}$ ) $\mathrm{C}=\mathrm{O}$ stretching vibrations of the dimers. The three carboxylic acids are formic F (grey), acetic A (red), and pivalic acid P (blue). All calculations have been performed with an aVTZ basis set. 


\section{A.5 Dimer Assemblies}

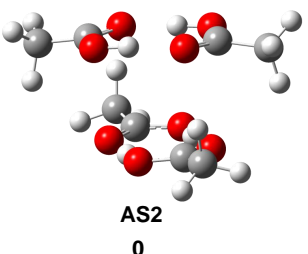

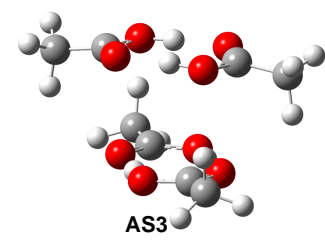

2.3
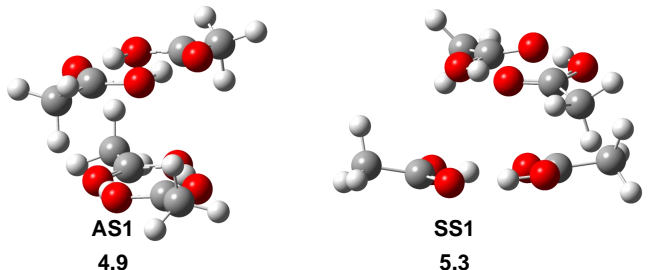

5.3

Figure A.28: Relative energy differences (in $\mathrm{kJ} \mathrm{mol}^{-1}$ ) of acetic acid tetramers calculated at the B3LYP-D3(BJ)/def2-TZVP level, predicted within the structure motifs found for formic acid by Roy and Thakkar [219].

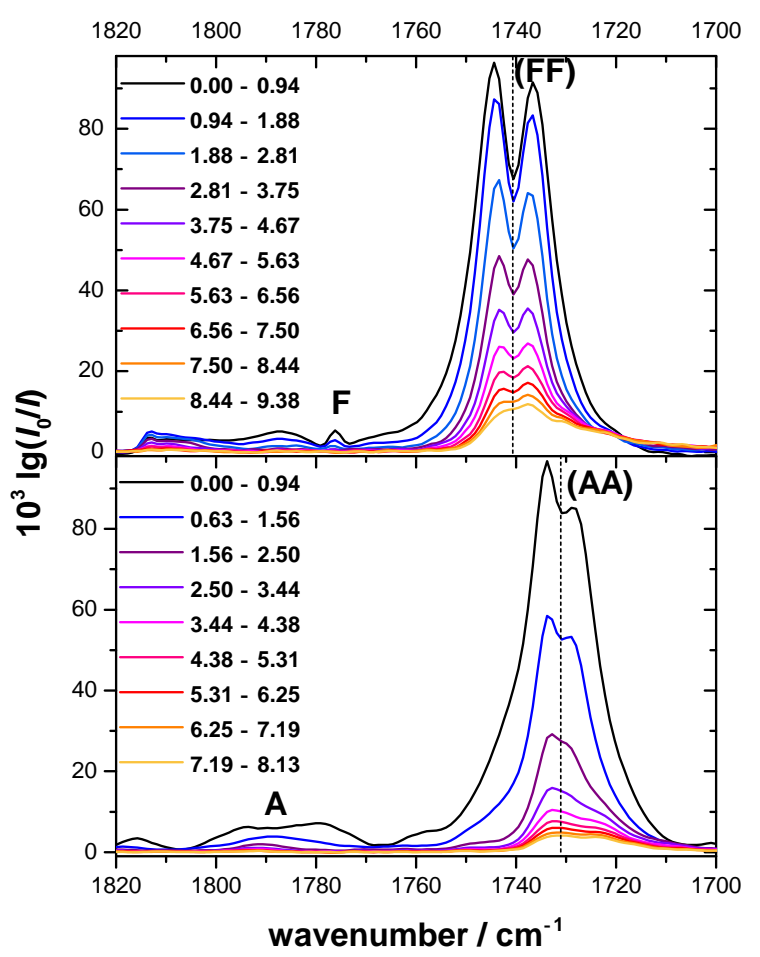

Figure A.29: FTIR jet spectra of formic $(c<2.3 \%$ in helium, 20 scans, top panel) and acetic acid ( $c<1.0 \%$ in helium, 152 scans, bottom) in the $\mathrm{C}=\mathrm{O}$ stretching region at a reservoir pressure of 300 mbar. The spectra shown have been spatially averaged over $3 \times 3$ binned pixel with increasing nozzle distance. The distance intervals (in $\mathrm{mm}$ ) are listed in the Figure. 


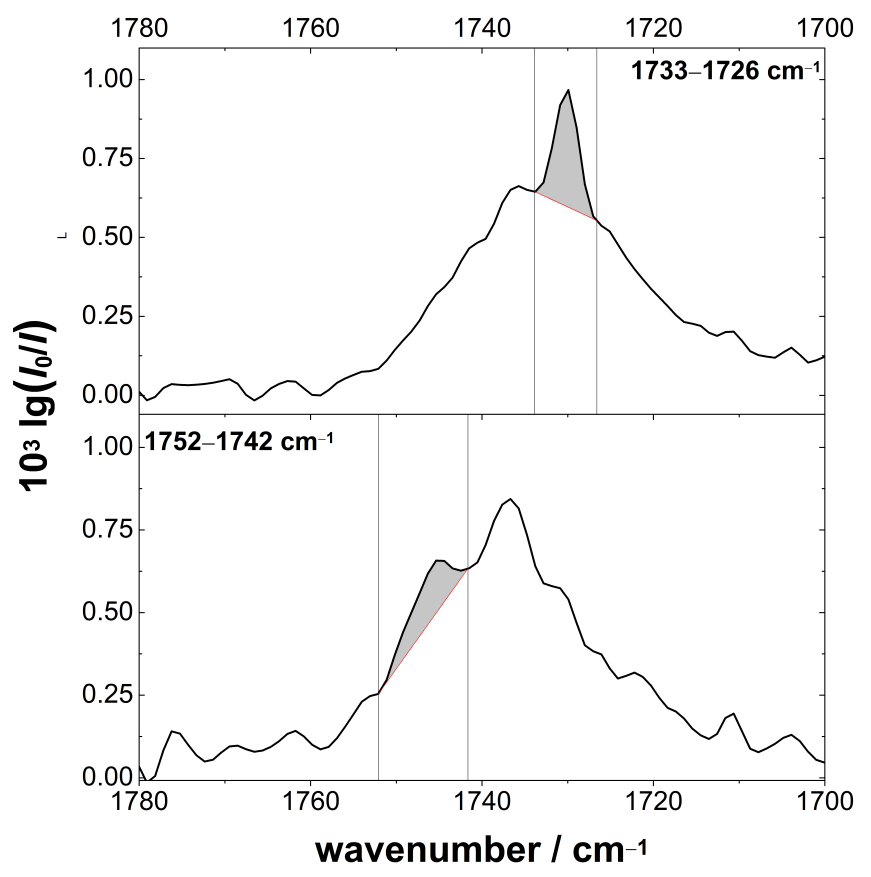

Figure A.30: Visualisation of the band integral used to obtain the 2D evolution of the integrated intensity of the argon nanocoated formic acid dimer band $(\mathrm{FF})_{2}-(\mathrm{Ar})_{m}$ (top) and the band of dimer assemblies (FF) $)_{2}$ (bottom) in Figure 7.8.

\section{A.6 Summary of Key Experimental Observables for Benchmarking Quantum Chemical Methods}

\section{A.6.1 The Formic Acid Monomer}

\section{The cis-Rotamer of the Formic Acid Monomer}

In this work, perturbation-free band positions of eight fundamentals of cis-HCOOH and cis-HCOOD were determined with Raman jet spectroscopy, which are listed alongside their experimental uncertainties in Table A.14. Additionally, the spectral shift of these bands with respect to the corresponding band of the global minumum trans-structure is given (cis-trans-shift). For $\nu_{2}$ of trans-HCOOD, a Fermi resonance doublet with an intensity ratio close to $1: 1$ is observed at $2939 / 2955 \mathrm{~cm}^{-1}$. Bertie and Michaelian attributed the resonance partner to the $\left(\nu_{3}+\nu_{6}\right)$ combination band, but incorrectly assigned it to an $\mathrm{HCOOH}$ impurity (at $2941.8 \mathrm{~cm}^{-1}$ ) in their spectra [63]. The second band of the resonance doublet $\left(2955 \mathrm{~cm}^{-1}\right)$ observed in this work is likely overshadowed by a dimer band $\left(2951.4 \mathrm{~cm}^{-1}\right)$ in their spectra. In Table A.14, the cis-trans-shifts to the bands of both resonance partners of trans-HCOOD are listed. The cis-formic acid bands observed in this work did not appear to be resonance split or broadened, though this might be attributed to the relatively low intensity of these in the spectra. Therefore, no conclusions about weaker Fermi resonances of the cis-rotamers can be drawn from 
Table A.14: Raman jet band positions of vibrational fundamentals in cis-rotamers of $\mathrm{HCOOH}$ and HCOOD as well as the shift with respect to the trans-rotamer $\left(\mathrm{in}^{-1} \mathrm{~cm}^{-1}\right.$ ). The vibrational modes are labelled according to the Herzberg nomenclature of $\mathrm{HCOOH}$, which is adapted for HCOOD. For $\nu_{2}$ of HCOOD, a Fermi resonance doublet with an intensity ratio of nearly 1:1 is observed for the trans-isomer and the cis-trans-shift to both bands is listed. Experimental error bars are given in parentheses. See text for further details.

\begin{tabular}{cccccc}
\hline \multirow{2}{*}{$\nu_{i}$} & \multicolumn{2}{c}{ cis-rotamer } & & \multicolumn{2}{c}{ cis-trans-shift } \\
\cline { 2 - 3 } \cline { 5 - 6 } & HCOOH & HCOOD & & HCOOH & HCOOD \\
\hline 1 & $3637(2)$ & $2686(2)$ & & $+67(4)$ & $+55(4)$ \\
2 & $2873(2)$ & $2872(2)$ & & $-69(4)$ & $-67(4) /-83(4)$ \\
3 & $1818(2)$ & $1820(2)$ & & $+41(4)$ & $+46(4)$ \\
5 & & $905(2)$ & & $-68(4)$ \\
6 & $1093(2)$ & & & $-11(4)$ & \\
\hline
\end{tabular}

the data presented in this work. The most uncertain of all eight cis-assignments is $\nu_{6}$ of $\mathrm{HCOOH}$ due to an overlap with non-isomeric (trans-)hot band structure (cf. Figure 4.7).

All band positions were determined from the maximum of the Q branch in the Raman spectra. As the spectral uncertainty of the Raman jet set-up amounts to $1-2 \mathrm{~cm}^{-1}$ (cf. Section 3.1.3), band position errors of $\pm 2 \mathrm{~cm}^{-1}$ are employed. The experimental error bar of the shift has been generously assigned to twice the band position uncertainty $\left( \pm 4 \mathrm{~cm}^{-1}\right)$.

\section{Non-Isomeric Hot Bands}

In addition to cis-formic acid bands, non-isomeric hot bands, i.e., those originating from thermally populated low-lying energy levels localised on trans-formic acid were observed in the Raman jet spectra. Table A.15 summarises the band positions for the most pronounced ones of trans-HCOOH alongside the trans-fundamentals and possible assignments based on anharmonicity matrix elements $x_{i j}$ from B3LYP-D3(BJ)/aVTZ VPT2 calculations and comparison to VCI [327] and MCTDH calculations [328]. The assignments are tentative and therefore have to be treated very carefully. As for cisformic acid, the band positions listed in Table A.15 correspond to the band maxima and the experimental uncertainty of these amounts to $\pm 2 \mathrm{~cm}^{-1}$. 
Table A.15: Raman jet band positions of hot bands of trans-HCOOH alongside the respective trans-fundamentals (in $\mathrm{cm}^{-1}$ ). Experimental error bars are given in parentheses. Tentative assignments of main and possible lower-intensity contributions are listed.

\begin{tabular}{cccc}
\hline$\nu_{i}$ & trans-fundamental & hot band & tentative assignment \\
\hline 2 & $2942(2)$ & $2925(2)$ & $\left(\nu_{2}+\nu_{8}-\nu_{8}\right)$ \\
& & $2938(2)$ & $\left(\nu_{2}+\nu_{9}-\nu_{9}\right),\left(\nu_{2}+\nu_{6}-\nu_{6}\right)$ \\
3 & $1777(2)$ & $1770(2)$ & $\left(\nu_{3}+\nu_{7}-\nu_{7}\right),\left(\nu_{3}+\nu_{8}-\nu_{8}\right)$ \\
& & $1101(2)$ & $\left(\nu_{6}+\nu_{7}-\nu_{7}\right),\left(\nu_{6}+\nu_{8}-\nu_{8}\right)$ \\
6 & $1105(2)$ & $1097(2)$ & $\left(\nu_{6}+\nu_{9}-\nu_{9}\right)$ \\
\hline
\end{tabular}

\section{A.6.2 Dimers}

\section{The Higher-Energy Dimer of Formic Acid in Helium Nanodroplets}

The $\mathrm{OH}$ stretching vibrations of a higher-energy formic acid dimer were characterised in helium nanodroplets (see Figure 5.21 for structure). Band position deviations with respect to the gas phase are generally known to be on the order of just a few $\mathrm{cm}^{-1}$ in these droplets [85]. As the largest deviation reported for a variety of different substances, amongst others $\mathrm{HCOOH}, \mathrm{HF}$, and $\mathrm{HCN}$, in Ref. [85] amounts to $-2.36 \mathrm{~cm}^{-1}(\mathrm{C}-\mathrm{H}$ stretching vibration of the formic acid monomer), a conservative solvent shift error bar of $\pm 3 \mathrm{~cm}^{-1}$ is assumed for free (non-hydrogen bonded) stretching vibrations in this work. For hydrogen bonded clusters, the shift with respect to the gas phase, also known as helium solvent shift, was found to correlate linearly with the downshift of the respective bound stretching vibration of that cluster [87]. An upper bound of the gas phase band position has been estimated to about $5 \%$ of the hydrogen bond induced downshift from the shifts reported in Ref. [87] under the assumption of an intercept at zero (see Section 3.1.6 for further details). For the bound $\mathrm{OH}$ stretching vibration of the higher-energy dimer of formic acid, this yields an error bar of up to $+16 \mathrm{~cm}^{-1}$ for the estimated gas phase band position (cf. Table A.16).

Table A.16: Band maxima (with error bars in parentheses) and full widths down to the baseline $\left(\right.$ in $\mathrm{cm}^{-1}$ ) of the $\mathrm{OH}$ stretching vibrations ( $\mathrm{f}=$ free, $\mathrm{b}=$ bound) of the higher-energy formic acid dimer measured in helium nanodroplets.

\begin{tabular}{ccc}
\hline assignment & maximum & width \\
\hline$\nu(\mathrm{OH})^{\mathrm{f}}$ & $3561(3)$ & $3574-3555$ \\
$\nu(\mathrm{OH})^{\mathrm{b}}$ & $3236(+16)$ & $3194-3266$ \\
\hline
\end{tabular}




\section{Exciton Coupling in Homo and Hetero Dimers of Carboxylic Acids}

The $\mathrm{C}=\mathrm{O}$ stretching vibrations of the six homo and hetero dimers of formic, acetic, and pivalic acid were characterised with FTIR and Raman jet spectroscopy. From the band positions, the exciton splitting $\Delta a s$ (band position difference between the antisymmetric and symmetric $\mathrm{C}=\mathrm{O}$ stretching vibration) was determined as well as the shift of the antisymmetric (a)/symmetric ( $\mathrm{s}$ ) $\mathrm{C}=\mathrm{O}$ stretching vibration of the dimer with respect to the monomer $\Delta a / \Delta s$ (cf. Table A.17). The experimental uncertainty of the band position shift $\Delta a$ is assumed to be $\pm 4 \mathrm{~cm}^{-1}$. The bands of the symmetric $\mathrm{C}=\mathrm{O}$ stretching vibrations of all three homo dimers are resonance broadened (acetic acid) or split (formic and pivalic acid), as shown in Figure 5.13. Under the assumption that the dark state has a negligible intensity, the centre of mass of the integrated band is taken as the band position. Due to the higher uncertainty that comes with this deconvolution, larger experimental error bars of $\pm 6 \mathrm{~cm}^{-1}$ are employed for the exciton splitting $\Delta a s$ and the shift $\Delta s . \Delta a, \Delta s$, and in particular $\Delta a s$ profit from partial compensation of anharmonic effects, also due to the empirical deperturbation of Fermi resonances.

Table A.17: Experimental downshift of the band of antisymmetric (a)/symmetric (s) $\mathrm{C}=\mathrm{O}$ stretching vibration of the cyclic dimers compared to the monomer band position $\Delta a / \Delta s$ as well as the experimental $(\Delta a s)$ exciton splitting between the bands of the symmetric and antisymmetric $\mathrm{C}=\mathrm{O}$ stretching vibrations. Experimental uncertainties are given in parentheses. See text for further details.

\begin{tabular}{cccc}
\hline \hline dimer & $\boldsymbol{\Delta} \boldsymbol{a}$ & $\boldsymbol{\Delta} \boldsymbol{a s}$ & $\boldsymbol{\Delta} \boldsymbol{s}$ \\
\hline$(\mathrm{FF})$ & $36(4)$ & $75(6)$ & $110(6)$ \\
$(\mathrm{AA})$ & $61(4)$ & $56(6)$ & $117(6)$ \\
$(\mathrm{PP})$ & $56(4)$ & $58(6)$ & $115(6)$ \\
\hline$(\mathrm{FA})$ & $49(4)$ & $65(6)$ & $114(6)$ \\
$(\mathrm{AP})$ & $57(4)$ & $59(6)$ & $116(6)$ \\
$(\mathrm{PF})$ & $47(4)$ & $72(6)$ & $118(6)$ \\
\hline \hline
\end{tabular}




\title{
Curriculum Vitae
}

\author{
Katharina Meyer Stadthagen, Germany \\ Nationality German
}

\section{Education}

2016-2019

Ph.D. candidate, Georg-August-Universität Göttingen, Germany

2013-2016

Master of Science in Chemistry, Georg-AugustUniversität Göttingen, Germany

master thesis - "Spectroscopy of Carboxylic Acid Dimers"

2010-2013 Bachelor of Science in Chemistry, Georg-AugustUniversität Göttingen, Germany

bachelor thesis - "Infrared Spectroscopic Characterisation of Multi-Slit Nozzle Expansions"

2004-2010

June 2010

Advanced Secondary Education, Ratsgymnasium Stadthagen, Germany

Allgemeine Hochschulreife (Abitur)

\section{Research Stays}

Nov $2018 \quad$ University of Leicester, United Kingdom

helium nanodroplet measurements of formic acid in collaboration with Dr. Julia A. Davies and Prof. Dr. Andrew M. Ellis

Oct 2014 - Mar 2015 University of Oxford, United Kingdom project - "The Development of a MS Technique for the Analysis of Cold Ion-Molecule Reactions in Coulomb Crystals" supervisors: Dr. Brianna R. Heazlewood, Prof. Dr. Timothy P. Softley 


\section{Publications}

I. Ejection of Coulomb Crystals from a Linear Paul Ion Trap for Ion-Molecule Reaction Studies

K. A. E. Meyer, L. L. Pollum, L. S. Petralia, A. Tauschinsky, C. J. Rennick, T. P. Softley, B. R. Heazlewood, J. Phys. Chem. A 2015, 119, 12449-12456.

II. Formic acid aggregation in 2D supersonic expansions probed by FTIR imaging

K. A. E. Meyer, M. A. Suhm, J. Chem. Phys. 2017, 147, 144305, Ref. [185].

III. Vibrational exciton coupling in homo and hetero dimers of carboxylic acids studied by linear infrared and Raman jet spectroscopy

K. A. E. Meyer, M. A. Suhm, J. Chem. Phys. 2018, 149, 104307, Ref. [329].

IV. Stretching of cis-formic acid: warm-up and cool-down as molecular work-out

K. A. E. Meyer, M. A. Suhm, Chem. Sci. 2019, 10, 6285-6294, Ref. [330].

V. Shifting formic acid dimers into perspective: vibrational scrutiny in helium nanodroplets

K. A. E. Meyer, J. A. Davies, A. M. Ellis, Phys. Chem. Chem. Phys. 2020, 22, 9637-9646, Ref. [409]. 